Supporting Information

\title{
Iridium-catalyzed highly efficient and site-selective deoxygenation of alcohols
}

Shiyi Yang, † Weiping Tang, $\ddagger$,§ Zhanhui Yang*,† and Jiaxi Xu*,†

\author{
† State Key Laboratory of Chemical Resource Engineering, College of Science, Beijing University \\ of Chemical Technology, Beijing 100029, P. R. China \\ ‡ School of Pharmacy, University of Wisconsin-Madison, 777 Highland Avenue, Madison, WI \\ 53705, USA \\ $\S$ Department of Chemistry, University of Wisconsin-Madison, Madison, WI, 53706, USA
}

Corresponding authors:

zhyang@mail.buct.edu.cn (to Z. Yang), ixxu@mail.buct.edu.cn (to J. Xu) 


\section{Contents}

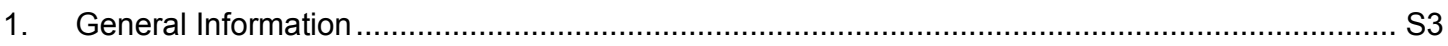

2. General procedure for directing-group screening ................................................................. 3

2.1. Initial screening of directing group with primary alcohols............................................... S3

2.2. Initial screening of directing group with secondary alcohols. ................................................ 3

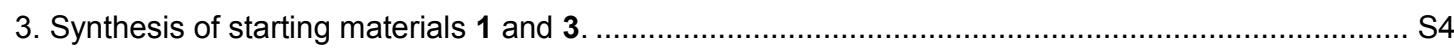

3.1 Preparation of commercially unavailable ( $\mathrm{N}$-substituted amino) aryl bromides...................... S4

3.2 Preparation of the ( $\mathrm{N}$-substituted amino) aryl magnesium bromide solution (1.0 M in THF).... S5

3.3 General procedure for synthesis of starting materials 1 and $3 \ldots \ldots \ldots \ldots \ldots \ldots \ldots \ldots \ldots \ldots \ldots \ldots \ldots \ldots . . .55$

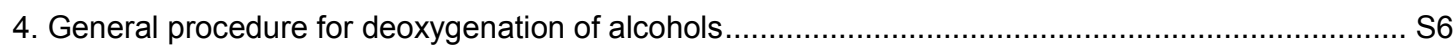

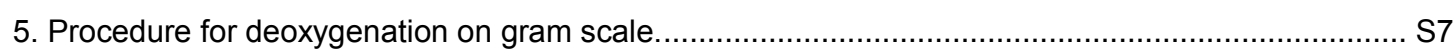

6. General procedure for studying the efficiency of transfer deoxygenation against $\mathrm{pH}$ values ............. S7

7. General procedure for structure modification of steroids .......................................................... 8

7.1 Structural modifications of pregnenolone (7a), estrone (7b), and epiandrosterone (7c)......... S8

7.2 Structural modifications of cholesterol 9 ........................................................................... S8

8. Procedures for further transformation of the directing group..................................................... S9

8.1 Procedure for Preparation of Aryltrimethylammonium Triflates............................................. S9

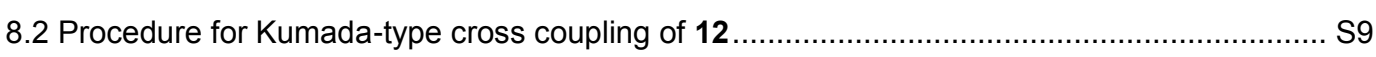

8.3 Procedure for Suzuki-type cross coupling of 12 ……....................................................... 9

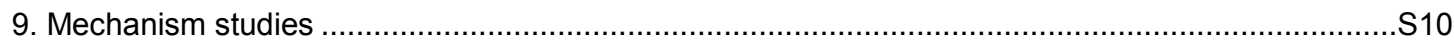

9.1 Carbocation probing experiments ...............................................................................

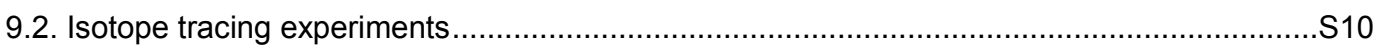

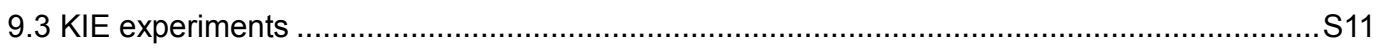

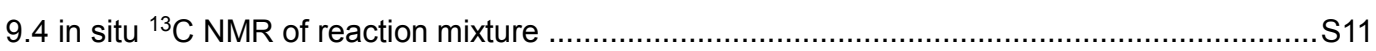

9.5 Selected ESI-HRMS spectra of alcohols ....................................................................... 13

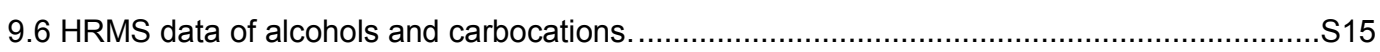

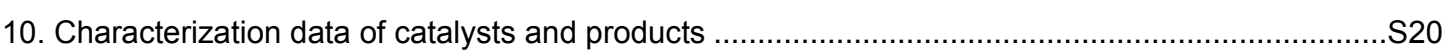

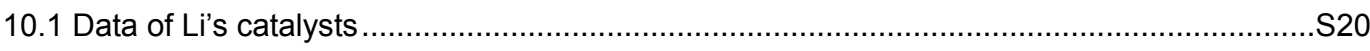

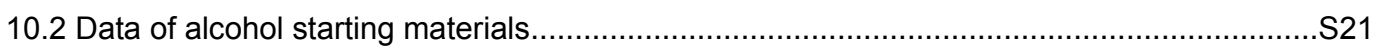

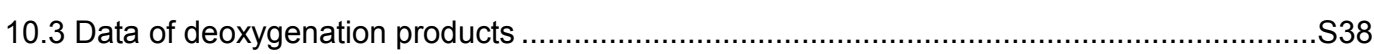

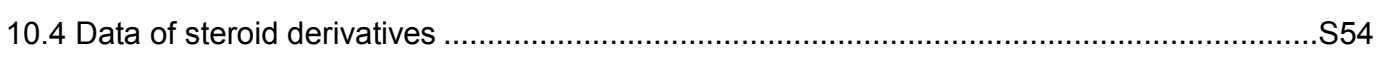

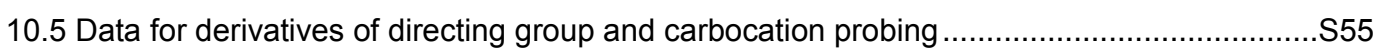

11. Copies of ${ }^{1} \mathrm{H}$ NMR and ${ }^{13} \mathrm{C}$ NMR spectra of catalysts and products.......................................55

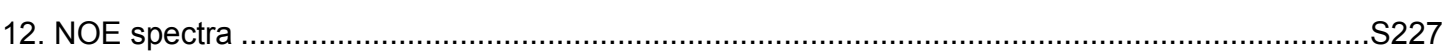

13. ${ }^{1} \mathrm{H}$ NMR of the crude reaction mixtures in optimization of the reaction conditions, $\mathrm{pH}$-yield correlation,

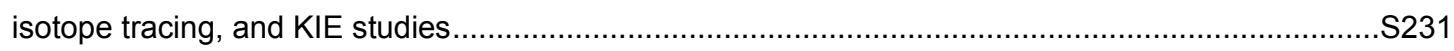

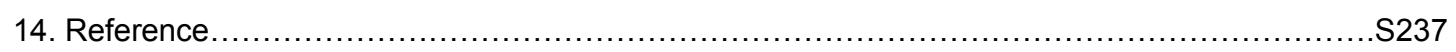




\section{General Information}

Except otherwise stated, all the chemicals were used directly as commercially received. ${ }^{1} \mathrm{H}$ and ${ }^{13} \mathrm{C}$ NMR spectra and the NOE spectra were recorded on a Bruker $(400 \mathrm{MHz})$ NMR spectrometer, with acetone- $d_{6}$, dimethyl sulfoxide- $d_{6}$, or $\mathrm{CDCl}_{3}$ as the solvent and tetramethylsilane as internal standard. TLC analyses were performed on silica gel plates, and the plates were visualized with UV light. PE and EA are the abbreviations for petroleum ether $\left(60-90^{\circ} \mathrm{C}\right)$ and ethyl acetate, respectively.

Tang's catalysts (TCs) were prepared according to our previous publications. ${ }^{1}$ Li's catalysts (LCs) were prepared according to Li's and co-workers' publications. ${ }^{2}$

\section{General procedure for directing-group screening}

\subsection{Initial screening of directing group with primary alcohols.}

To a mixture of formic acid $(75 \mu \mathrm{L}, 2 \mathrm{mmol})$ and primary alcohols $(0.5 \mathrm{mmol})$ in deionized water $(1 \mathrm{~mL})$ at $80{ }^{\circ} \mathrm{C}$ was added the aqueous solution of catalyst TC-3 $(20 \mu \mathrm{L}, 0.005$ $\left.\mathrm{mol}^{-1} \mathrm{~L}\right)$. The resulting suspension was stirred for $2 \mathrm{~h}$. Then, water $(5 \mathrm{~mL})$ was added, followed by addition of ethyl acetate $(5 \mathrm{~mL})$ to extract organic substances. The organic phase was dried over $\mathrm{Na}_{2} \mathrm{SO}_{4}$, and analysed with TLC and GC-MS to screen the optimal directing group. The results are summarized as below in Table S1.

\subsection{Initial screening of directing group with secondary alcohols.}

To a $5-\mathrm{mL}$ tube was sequentially added alcohol $(0.25 \mathrm{mmol}), 0.5 \mathrm{~mL}$ of the $T C-3$ catalyst solution in deionized water $(0.000005 \mathrm{~mol} / \mathrm{L}$ for $S / C=100,000)$. The tube was immersed in a preheated $80^{\circ} \mathrm{C}$ oil-bath for $5 \mathrm{~min}$, followed by slow addition of formic acid (38 $\mu \mathrm{L}, 1$ $\mathrm{mmol}$ ) in $1 \mathrm{~min}$. The resultant reaction mixture was stirred for indicated time (see the tables in main text). After cooling to room temperature, diluting with saturated sodium bicarbonate solution $(5 \mathrm{~mL})$, extracting with ethyl acetate $(5 \mathrm{~mL} \times 3)$, and removal of solvent under vacuum, the obtained residue was submitted to ${ }^{1} \mathrm{H}$ NMR to detect the desired dehydrogenation product.

Table S1. Initial screening of directing group with primary alcohols. 


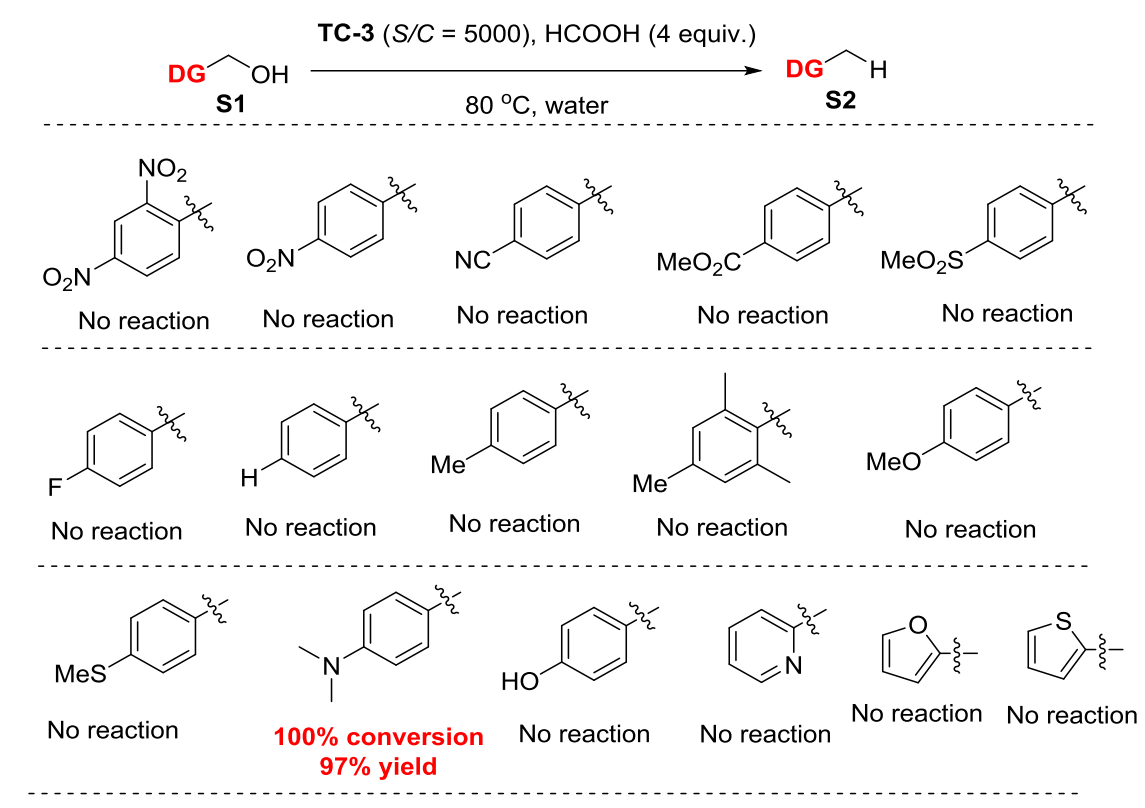

Table S2. Initial screening of directing group with secondary alcohols.

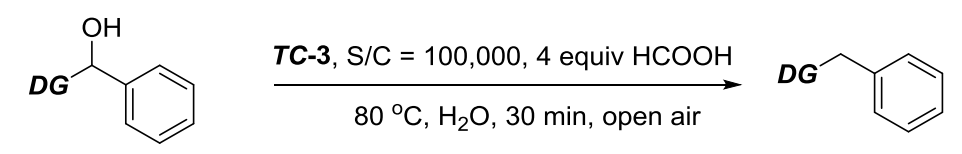

not observed

\section{Synthesis of starting materials 1 and 3 .}

\subsection{Preparation of commercially unavailable ( $N$-substituted amino) aryl bromides}

As listed below in Figure S1, 1-(4-bromophenyl)pyrrolidine (S3) and 1-(4-bromophenyl)piperidine (S4) were prepared from of 4-bromoaniline and 1,4-dibromobutane and 1,5-dibromopentane, respectively, following Patel's procedure. ${ }^{3}$ 1-(4-Bromophenyl)-1,2,3,4-tetrahydroquinoline (S5) and 2-(4-bromophenyl)-1,2,3,4tetrahydroisoquinoline (S6) were prepared from (4-bromophenyl)boronic acid and tetrahydroquinoline and tetrahydroisoquinoline, respectively, following Buchwald's procedure. $^{4}$ 
<smiles>Brc1ccc(N2CCCC2)cc1</smiles>

S3<smiles>Brc1ccc(N2CCCCC2)cc1</smiles>

S4<smiles>Sc1ccc(N2CCCc3ccccc32)cc1</smiles><smiles>O=S(=O)(O)c1ccc(Br)cc1</smiles>

Figure S1. Four commercially unavailable N-substituted 4-aminoaryl bromides

\subsection{Preparation of the ( $\mathrm{N}$-substituted amino) aryl magnesium bromide solution (1.0 M in THF).}

To an oven dried $25 \mathrm{~mL}$ two-necked round bottom flask equipped with a magnetic stir bar and a constant-pressure dropping funnel, activated magnesium turnings $(12.5 \mathrm{mmol}, 0.30$ g) was added. The equipment was sealed with rubber septum, evacuated, and back filled with nitrogen. To the funnel was added $1 \mathrm{M}$ solution of ( $\mathrm{N}$-substituted amino) aryl bromide (see Table S3) $(12 \mathrm{mmol})$ in THF (12 mL) by syringe. The above solution was added dropwise at r.t. within $30 \mathrm{~min}$. When $1 \mathrm{~mL}$ of the solution was added, 1 drop of 1,2-dibromoethane was added via syringe to initiate the reaction. Upon addition, the flask was immersed in a preheated $50{ }^{\circ} \mathrm{C}$ oil-bath for $2 \mathrm{~h}$. The prepared ( $\mathrm{N}$-substituted amino) aryl magnesium bromide solution (1.0 M in THF) was stored in nitrogen atmosphere and used in the next step.

Table S3. Prepared Grignard reagents<smiles>CN(C)c1ccc(NBr)cc1</smiles>

SG1<smiles>CN(C)c1ccccc1N(Br)Br</smiles>

SG2<smiles>CN(C)c1cccc(N(Br)Br)c1</smiles>

SG3<smiles></smiles>

SG4<smiles>Br[Mg]c1ccc(N2CCCC2)cc1</smiles>

SG5<smiles>CC(C)(O)[Sn](Br)(Br)c1ccc(N2CCCCC2)cc1</smiles>

SG6<smiles>Br[Mg]c1ccc(N2CCc3ccccc3C2)cc1</smiles>

SG7<smiles>Br[Mg]c1ccc(N2CCCc3ccccc32)cc1</smiles>

\subsection{General procedure for synthesis of starting materials 1 and 3.}

\section{Procedure A:}

To a $25 \mathrm{~mL}$ dry flask equipped with a magnetic stir bar was added aldehydes or ketones (2.5 mmol) under nitrogen atmosphere and $5 \mathrm{~mL}$ of dry THF was added via syringe. The resultant mixture was stirred for 5 minutes at $0{ }^{\circ} \mathrm{C}$. Then the corresponding Grignard reagent $(1.0 \mathrm{M}$ solution in THF, $3.0 \mathrm{~mL}$ ) was added dropwise. The solution was stirred at $0{ }^{\circ} \mathrm{C}$ for another $30 \mathrm{~min}$ and warmed to r.t. overnight. The reaction mixture was quenched by adding saturated $\mathrm{NH}_{4} \mathrm{Cl}$ solution and extracted with ethyl acetate. The organic extract was dried $\left(\mathrm{Na}_{2} \mathrm{SO}_{4}\right)$, filtered, and concentrated. The crude product was purified by flash chromatography on silica gel (for tertiary alcohols containing $\alpha-\mathrm{H}, \mathrm{Al}_{2} \mathrm{O}_{3}$ was used to avoid 
elimination) to give products 1 or 3 .

\section{Procedure $B$ :}

To a $25 \mathrm{~mL}$ dry flask equipped with a magnetic stir bar was added the corresponding ( $N$-substituted amino) aryl aldehydes $(2.5 \mathrm{mmol})$ under nitrogen atmosphere and $5 \mathrm{~mL}$ of dry THF was added via syringe. The resultant mixture was stirred for 5 minutes at $0{ }^{\circ} \mathrm{C}$. Then phenyl magnesium bromide $(1.0 \mathrm{M}$ solution in THF, $6.0 \mathrm{~mL})$ was added dropwise. The solution was stirred at $0{ }^{\circ} \mathrm{C}$ for another $30 \mathrm{~min}$ and warmed to r.t. overnight. The reaction mixture was quenched by adding saturated $\mathrm{NH}_{4} \mathrm{Cl}$ solution and extracted with ethyl acetate. The organic extract was dried $\left(\mathrm{Na}_{2} \mathrm{SO}_{4}\right)$, filtered, and concentrated. The crude product was purified by flash chromatography on silica gel to give products 1 .

\section{Procedure $\mathrm{C}$ (for aldehydes or ketones containing active $\mathrm{OH}$ group):}

To a solution of 4-bromo- $\mathbf{N}, \mathbf{N}$-dimethylaniline $(1.2 \mathrm{~g}, 6 \mathrm{mmol})$ in THF $(80 \mathrm{~mL})$ at $-78^{\circ} \mathrm{C}$ was added dropwise a solution of $n$-butyllithium $(2.5 \mathrm{~mL}, 2.4 \mathrm{M}$ in hexanes). The mixture was stirred at $-78^{\circ} \mathrm{C}$ for 30 minutes, and then, $2.5 \mathrm{mmol}$ of aldehydes or ketones in THF ( 5 $\mathrm{mL}$ ) was added dropwise. The resulting mixture was stirred for a further 30 minutes at $-78^{\circ} \mathrm{C}$, followed by removal of the cooling bath and warming to room temperature overnight. Then water $(5 \mathrm{~mL})$ was added to quench the reaction. Saturated aqueous $\mathrm{NH}_{4} \mathrm{Cl}$ solution $(100 \mathrm{~mL}$ ) was added and the mixture was extracted with ethyl acetate $(3 \mathrm{x}$ $30 \mathrm{~mL}$ ). The combined organic extracts were dried over $\mathrm{Na}_{2} \mathrm{SO}_{4}$ and concentrated under vacuum. The residue was purified by flash chromatography on neutral $\mathrm{Al}_{2} \mathrm{O}_{3}$ to give products 1 or 3 .

\section{Procedure $D$ (for bulky ketones):}

To a solution of 4-bromo- $\mathbf{N}, \mathbf{N}$-dimethylaniline $(1.2 \mathrm{~g}, 6 \mathrm{mmol})$ in THF $(80 \mathrm{~mL})$ at $-78^{\circ} \mathrm{C}$ was added dropwise a solution of $\mathrm{n}$-butyllithium $(2.5 \mathrm{~mL}, 2.4 \mathrm{M}$ in hexanes). The mixture was stirred at $-78^{\circ} \mathrm{C}$ for 30 minutes, and then, $5.0 \mathrm{mmol}$ of ketones in THF $(10 \mathrm{~mL})$ was added dropwise. Similar workup as in Procedure $C$ afforded desired products 3 .

\section{General procedure for deoxygenation of alcohols}

To a $5-\mathrm{mL}$ tube was sequentially added alcohol $(0.25 \mathrm{mmol}), 0.5 \mathrm{~mL}$ of the $\mathbf{T C}-3$ catalyst solution in deionized water $(0.002 \mathrm{~mol} / \mathrm{L}$ for $S / C=1000 ; 0.0001 \mathrm{~mol} / \mathrm{L}$ for $S / C=20,000$; $0.000005 \mathrm{~mol} / \mathrm{L}$ for $S / C=100,000$ ) and, if needed, $0.5 \mathrm{~mL}$ of TFE or HFIP was used as the co-solvent (see the tables in main text, or analytical data section in $\mathrm{SI}$ ). The tube was immersed in a preheated $80^{\circ} \mathrm{C}$ oil-bath for $5 \mathrm{~min}$, followed by slow addition of formic acid $(38 \mu \mathrm{L}, 1 \mathrm{mmol}$ ) in $1 \mathrm{~min}$. The resultant reaction mixture was stirred for indicated time (see the tables in main text). After cooling to room temperature, diluting with saturated sodium bicarbonate solution $(5 \mathrm{~mL})$, extracting with ethyl acetate $(5 \mathrm{~mL} \times 3)$, and drying over $\mathrm{Na}_{2} \mathrm{SO}_{4}$, concentration of the organic phase under reduced pressure followed by purification by column chromatography on silica gel with afforded desired products. 


\section{Procedure for deoxygenation on gram scale.}

To a $25-\mathrm{mL}$ flask was sequentially added $1 \mathrm{~b}(1.0 \mathrm{~g}, 4.4 \mathrm{mmol})$ and $9.0 \mathrm{~mL}$ of the $T C-3$ catalyst solution in deionized water $(0.000005 \mathrm{~mol} / \mathrm{L}$ for $S / C=100,000)$. The flask was immersed in a preheated $80^{\circ} \mathrm{C}$ oil-bath for $5 \mathrm{~min}$, followed by slow addition of formic acid $(0.6 \mathrm{~mL}, 15.8 \mathrm{mmol})$ within $5 \mathrm{~min}$. The resultant reaction mixture was stirred for $1 \mathrm{~h}$. After cooling to room temperature, diluting with saturated sodium bicarbonate solution (10 $\mathrm{mL})$, and extracting with ethyl acetate $(10 \mathrm{~mL} \times 3)$, concentration of the organic phase under reduced pressure, purification by column chromatography on silica gel afforded desired product $\mathbf{2} \mathbf{b}$ as a colorless liquid. Yield: $0.75 \mathrm{~g}, 80 \%$.

\section{General procedure for studying the efficiency of transfer deoxygenation against $\mathrm{pH}$ values}

To a 5-mL tube was sequentially added (4-(dimethylamino)phenyl)(phenyl)methanol $\mathbf{1 b}$ $(0.25 \mathrm{mmol}), 0.5 \mathrm{~mL}$ of the $T C-3$ catalyst solution in deionized water $(0.000005 \mathrm{~mol} / \mathrm{L}$ for $S / C=100,000$ ) The tube was immersed in a preheated $80^{\circ} \mathrm{C}$ oil-bath for $5 \mathrm{~min}$, followed by slow addition of formic acid and $\mathrm{HCOONa} \cdot 2 \mathrm{H}_{2} \mathrm{O}$. The resultant reaction mixture was stirred for $30 \mathrm{~min}$. Then $1 \mathrm{~mL}$ of EA was added. After cooling to room temperature, diluting with saturated sodium bicarbonate solution $(2 \mathrm{~mL})$, extracting with ethyl acetate $(2 \mathrm{~mL} \times 3)$, and concentration under reduced pressure, the crude residue was submitted to ${ }^{1} \mathrm{H}$ NMR to determine the Conversions (calculated from the ratio of deoxygenation product and starting material). The results were summarized in Table S4.

Table S4. The efficiency of transfer deoxygenation against $\mathrm{pH}$ values

\begin{tabular}{|c|l|l|l|l|l|l|l|}
\hline \multicolumn{2}{|c|}{$p H$ of the reaction media } & 1.56 & 3.03 & 3.52 & 3.95 & 4.44 & 7.15 \\
\hline \multirow{2}{*}{$\mathrm{HCOOH}$} & $\mu \mathrm{L}$ & 38 & 32 & 24 & 16 & 8 & 0 \\
\cline { 2 - 8 } & $\mathrm{mmol}$ & 1.0 & 0.8 & 0.6 & 0.4 & 0.2 & 0 \\
\hline \multirow{2}{*}{$\mathrm{HCOONa} \cdot 2 \mathrm{H}_{2} \mathrm{O}$} & $\mathrm{mg}$ & 0 & 21 & 42 & 63 & 84 & 104 \\
\cline { 2 - 8 } & $\mathrm{mmol}$ & 0 & 0.2 & 0.4 & 0.6 & 0.8 & 1.0 \\
\hline \multicolumn{2}{|c|}{ Conversion (\%) } & 92 & 70 & 68 & 50 & 32 & 1 \\
\hline
\end{tabular}




\section{General procedure for structure modification of steroids}

\subsection{Structural modifications of pregnenolone (7a), estrone (7b), and epiandrosterone (7c).}

To a solution of 1-(4-bromophenyl)piperidine $(130 \mathrm{mg}, 0.54 \mathrm{mmol})$ in THF $(10 \mathrm{~mL})$ at $-78^{\circ} \mathrm{C}$ was added dropwise a solution of $n$-butyllithium $(0.25 \mathrm{~mL}, 2.4 \mathrm{M}$ in hexanes, 2.4 equiv.). The mixture was stirred at $-78^{\circ} \mathrm{C}$ for 30 minutes, and then, $0.25 \mathrm{mmol}$ of steroid $(7 \mathrm{a}, 7 \mathrm{~b}$, or $\mathbf{7 c})$ in THF $(2 \mathrm{~mL})$ was added dropwise. The resulting mixture was stirred for a further 30 minutes at $-78^{\circ} \mathrm{C}$, followed by removal of the cooling bath and warming to room temperature overnight. Then water $(2 \mathrm{~mL})$ was added to quench the reaction. Saturated aqueous $\mathrm{NH}_{4} \mathrm{Cl}$ solution $(5 \mathrm{~mL})$ was added and the mixture was extracted with ethyl acetate $(3 \times 10 \mathrm{~mL})$. The combined organic extracts were dried over $\mathrm{Na}_{2} \mathrm{SO}_{4}$ and concentrated under reduced pressure. The residue was purified by flash chromatography on neutral $\mathrm{Al}_{2} \mathrm{O}_{3}$ to give the crude tertiary alcohols, which were directly submitted to the above deoxygenation procedure described in Section 4 with water-HFIP $(1: 1, v / v)$ as solvent. $^{5}$

\subsection{Structural modifications of cholesterol 9}

According to a procedure published by Reeves and coworkers. ${ }^{6}$

To a $25-\mathrm{mL}$ round-bottom flask charged with cholesterol $(387 \mathrm{mg}, 1 \mathrm{mmol}$ ) and DCM (10 $\mathrm{mL}$ ), was added one drop of saturated sodium bicarbonate solution. Dess-Martin periodinane $\left(940 \mathrm{mg}, 2.2 \mathrm{mmol}\right.$ ) was added in 4 portions within $0.5 \mathrm{~h}$ at $0{ }^{\circ} \mathrm{C}$. The mixture was stirred for another 3 hours, and was warmed to room temperature. The reaction was quenched with saturated sodium bicarbonate solution. After extracting with $\mathrm{DCM}$, washing by brine, drying over $\mathrm{Na}_{2} \mathrm{SO}_{4}$, and concentrating under reduced pressure, the crude mixture was purified by column chromatography (PE/EA $=25: 1$ ) to afford the ketone product, which was directly used in the next step.

To a solution of 4-bromo- $N, N$-dimethylaniline $(200 \mathrm{mg}, 1.0 \mathrm{mmol})$ in THF $(20 \mathrm{~mL})$ at $-78^{\circ} \mathrm{C}$ was added dropwise $n$-butyllithium $(0.42 \mathrm{~mL}, 1.0 \mathrm{mmol}, 2.4 \mathrm{M}$ in hexane). The mixture was stirred at $-78^{\circ} \mathrm{C}$ for 30 minutes, followed by dropwise addition of the above ketone product in $5 \mathrm{~mL}$ of THF. The resulting mixture was stirred for a further 30 minutes at $-78^{\circ} \mathrm{C}$, followed by removal of the cooling bath and warming to room temperature overnight. Then water $(5 \mathrm{~mL})$ was added to quench the reaction. Saturated aqueous $\mathrm{NH}_{4} \mathrm{Cl}$ solution $(10 \mathrm{~mL})$ was added and the mixture was extracted with ethyl acetate $(3 \mathrm{x}$ $15 \mathrm{~mL}$ ). The combined organic extracts were dried over $\mathrm{Na}_{2} \mathrm{SO}_{4}$ and concentrated under reduced pressure. The residue was purified by chromatography on neutral $\mathrm{Al}_{2} \mathrm{O}_{3}(\mathrm{PE}: \mathrm{EA}=$ $20: 1$ to $10: 1$ ) to give the crude tertiary alcohol 10 as a pale yellow solid (348 mg, two-step yield $69 \%)$. 
Deoxygenation of $\mathbf{1 1}$ was performed according to the procedure described in Section 4 with water-HFIP $(1: 1, v / v)$ as solvent.

\section{Procedures for further transformation of the directing} group.

\subsection{Procedure for Preparation of Aryltrimethylammonium}

\section{Triflates.}

According to a procedure published by Reeves and coworkers. ${ }^{7}$

To a $25 \mathrm{~mL}$ dry round bottom flask equipped with a magnetic stir bar was charged $\mathrm{N}, \mathrm{N}$-dimethyl-4-(9H-xanthen-9-yl)aniline (4I) (301 mg, $1 \mathrm{mmol})$ and $\mathrm{CH}_{2} \mathrm{Cl}_{2}(5 \mathrm{~mL})$. To the resultant stirring solution was added dropwise at r.t. methyl trifluoromethanesulfonate (150 $\mu \mathrm{L}, 1.3 \mathrm{mmol}$ ). The solution was stirred at r.t. for $2 \mathrm{~h}$, and TLC analysis indicated complete consumption of $4 \mathrm{I}$. The reaction mixture was concentrated to remove $\mathrm{CH}_{2} \mathrm{Cl}_{2}$ and the residue was treated with diethyl ether $(3 \mathrm{~mL})$. The resultant solid was filtered, washed with hexanes, and dried under vacuum to give 12 (442 $\mathrm{mg}, 95 \%$ yield) as a pale yellow solid.

\subsection{Procedure for Kumada-type cross coupling of $12^{8}$}

According to a procedure published by MacMillan and coworkers. ${ }^{8}$

To a dry Schlenk tube equipped with a magnetic stir bar was added $\mathrm{N}, \mathrm{N}, \mathrm{N}$-trimethyl-4-(9H-xanthen-9-yl)benzenaminiumtrifluoromethanesulfonate (12) (140 $\mathrm{mg}, 0.3 \mathrm{mmol})$ and $\mathrm{PdCl}_{2}\left(\mathrm{PPh}_{3}\right)_{2}(2.1 \mathrm{mg}, 0.003 \mathrm{mmol}, 1 \mathrm{~mol} \%)$. The tube was sealed with a rubber septum, evacuated, and back filled with nitrogen. THF $(2.0 \mathrm{~mL})$ was added via syringe, and the resultant mixture was stirred for 5 minutes. Then phenylmagnesium bromide ( $0.32 \mathrm{~mL}, 0.32 \mathrm{mmol}, 1.05$ equiv, $1.0 \mathrm{M}$ solution in THF) was added dropwise at r.t. The mixture was stirred at r.t. for $2 \mathrm{~h}$. The reaction mixture was quenched by addition of water $(1 \mathrm{~mL})$ and $6 \mathrm{M} \mathrm{HCl}(1 \mathrm{~mL})$, and extracted with methyl tert-butyl ether. The organic extract was dried $\left(\mathrm{Na}_{2} \mathrm{SO}_{4}\right)$, filtered, and concentrated, and the crude product was purified by column chromatography on silica gel (PE : EA = 100:1 to 50:1) to give 14 (94 mg, 94\% yield) as a white solid.

\subsection{Procedure for Suzuki-type cross coupling of 12}

According to a procedure published by MacMillan and coworkers. ${ }^{8}$

To a 10-mL dry Schlenk tube equipped with a magnetic stir bar was charged $\mathrm{N}, \mathrm{N}, \mathrm{N}$-trimethyl-4-(9H-xanthen-9-yl)benzenaminiumtrifluoromethanesulfonate (10) (140 $\mathrm{mg}, 0.3 \mathrm{mmol}, 1.0$ equiv), $\mathrm{Ni}(\mathrm{COD})_{2}(8.2 \mathrm{mg}, 0.03 \mathrm{mmol}, 10 \mathrm{~mol} \%)$, IMes $\cdot \mathrm{HCl}(10.3 \mathrm{mg}$, 
$0.03 \mathrm{mmol}, 10 \mathrm{~mol} \%)$, dibenzo[b, d]furan-4-ylboronic acid (13) (92 mg, $0.36 \mathrm{mmol}, 1.2$ equiv), and CsF (140 mg, $0.9 \mathrm{mmol}, 3$ equiv). The tube was sealed with a rubber septum, evacuated, and back filled with nitrogen quickly. Then $2.0 \mathrm{~mL}$ of dry dioxane was added via syringe. The heterogeneous mixture was heated to $80^{\circ} \mathrm{C}$ and stirred for $12 \mathrm{~h}$. Upon cooling to r.t., the mixture was filtered through a pad of silica, and washed with ethyl acetate. The solvent was removed under reduced pressure and the residue was purified by column chromatography on silica gel (PE:EA $=100: 1$ to $60: 1)$ to afford the product 16 (106 $\mathrm{mg}, 83 \%$ yield) as a white solid.

\section{Mechanism studies}

\subsection{Carbocation probing experiments}

To a $5-\mathrm{mL}$ tube was sequentially added $0.25 \mathrm{mmol}$ of $\mathbf{3 i}$ and $0.5 \mathrm{~mL}$ of deionized water. The tube was immersed in a preheated $80{ }^{\circ} \mathrm{C}$ oil-bath for $5 \mathrm{~min}$, followed by slow addition of formic acid ( $38 \mu \mathrm{L}, 1.0 \mathrm{mmol}$ ) dropwise. The resultant reaction mixture was stirred for $30 \mathrm{~min}$. After cooling to room temperature, diluting with saturated sodium bicarbonate solution $(5 \mathrm{~mL})$, and extracting with ethyl acetate $(5 \mathrm{~mL} \times 3)$, drying over $\mathrm{Na}_{2} \mathrm{SO}_{4}$, concentration of the organic phase under reduced pressure, the residue was obtained as a pale yellow liquid without further purification to give the quantitative yield of 5 .

To a $5-\mathrm{mL}$ tube was sequentially added $0.25 \mathrm{mmol}$ of $1 \mathbf{b}, 0.3 \mathrm{mmol}$ of 4-bromothiophenol and $0.5 \mathrm{~mL}$ of deionized water. The tube was immersed in a preheated $80^{\circ} \mathrm{C}$ oil-bath for $5 \mathrm{~min}$, followed by slow addition of formic acid (38 $\mu \mathrm{L}, 1.0$ $\mathrm{mmol}$ ) dropwise. The resultant reaction mixture was stirred for $30 \mathrm{~min}$. After cooling to room temperature, diluting with saturated sodium bicarbonate solution $(5 \mathrm{~mL})$, and extracting with ethyl acetate $(5 \mathrm{~mL} \times 3)$, drying over $\mathrm{Na}_{2} \mathrm{SO}_{4}$, concentration of the organic phase under reduced pressure, the residue was purified by column chromatography on silica gel (PE:DCM $=20: 1$ to $\mathrm{PE}: \mathrm{EA}=30: 1)$ to afford the product 6 (95 mg, 96\% yield) as a white solid.

\subsection{Isotope tracing experiments}

To a $5-\mathrm{mL}$ tube was sequentially added $0.25 \mathrm{mmol}$ of 1 aa and $0.5 \mathrm{~mL}$ of the TC-3 catalyst solution in deuterium water $(0.0002 \mathrm{~mol} / \mathrm{L}$ for $S / C=10,000)$. The tube was immersed in a preheated $80{ }^{\circ} \mathrm{C}$ oil-bath for $5 \mathrm{~min}$, followed by slow addition of formic acid (38 $\mu \mathrm{L}, 1.0$ $\mathrm{mmol}$ ) in $1 \mathrm{~min}$. The resultant reaction mixture was stirred for $30 \mathrm{~min}$. After cooling to room temperature, diluting with saturated sodium bicarbonate solution $(5 \mathrm{~mL})$, and extracting with ethyl acetate $(5 \mathrm{~mL} \times 3)$, concentration of the organic phase under reduced pressure followed by purification by column chromatography on silica gel with afforded desired products 2 aa ( $83 \mathrm{mg}$, yield $98 \%$ ) as a white solid. The H/D ratio was obtained from its ${ }^{1} \mathrm{H}$ NMR spectrum. 
To a $5-\mathrm{mL}$ tube was sequentially added $0.25 \mathrm{mmol}$ of 1 aa and $0.5 \mathrm{~mL}$ of the TC-3 catalyst solution in deionized water $(0.0002 \mathrm{~mol} / \mathrm{L}$ for $S / C=10,000)$. The tube was immersed in a preheated $80^{\circ} \mathrm{C}$ oil-bath for $5 \mathrm{~min}$, followed by slow addition of DCOOD ( $\left.38 \mu \mathrm{L}, 1.0 \mathrm{mmol}\right)$ in $1 \mathrm{~min}$. The resultant reaction mixture was stirred for $30 \mathrm{~min}$. After cooling to room temperature, diluting with saturated sodium bicarbonate solution $(5 \mathrm{~mL})$, and extracting with ethyl acetate $(5 \mathrm{~mL} \times 3)$, concentration of the organic phase under reduced pressure followed by purification by column chromatography on silica gel with afforded desired products 2 aa ( $81 \mathrm{mg}$, yield $95 \%$ ) as a white solid. The $\mathrm{H} / \mathrm{D}$ ratio was obtained from its ${ }^{1} \mathrm{H}$ NMR spectrum.

\subsection{KIE experiments}

To a $5-\mathrm{mL}$ tube was sequentially added $0.25 \mathrm{mmol}$ of $1 \mathrm{~b}$ and $0.5 \mathrm{~mL}$ of the TC-3 catalyst solution in deionized water $(0.00002 \mathrm{~mol} / \mathrm{L}$ for $S / C=100,000)$. The tube was immersed in a preheated $80{ }^{\circ} \mathrm{C}$ oil-bath for $5 \mathrm{~min}$, followed by addition of DCOOD $(19 \mu \mathrm{L}, 0.5 \mathrm{mmol})$ and $\mathrm{HCOOH}(19 \mu \mathrm{L}, 0.5 \mathrm{mmol})$ dropwise simultaneously. The resultant reaction mixture was stirred for $10 \mathrm{~min}$, diluted immediately with $5 \mathrm{~mL}$ of water and extracted with ethyl acetate $(2 \mathrm{~mL} \times 3)$. Then 4-nitroacetophenone $(13.8 \mathrm{mg}, 0.083 \mathrm{mmol}$ ) was added as the internal standard. After concentration of the organic phase under reduced pressure, the residue was submitted to ${ }^{1} \mathrm{H}$ NMR to determine yield and the ratio of $\mathbf{2} \mathbf{b}$ to $\mathbf{d}-\mathbf{2} \mathbf{b}$.

To a 5 - $\mathrm{mL}$ tube was sequentially added $0.125 \mathrm{mmol}$ of $1 \mathbf{b}, 0.125 \mathrm{mmol}$ of $\boldsymbol{d}-1 \mathbf{b}$, and 0.5 $\mathrm{mL}$ of the TC-3 catalyst solution in deionized water $(0.00002 \mathrm{~mol} / \mathrm{L}$ for $S / C=100,000)$. The tube was immersed in a preheated $80{ }^{\circ} \mathrm{C}$ oil-bath for $5 \mathrm{~min}$, followed by addition of $\mathrm{HCOOH}(38 \mu \mathrm{L}, 1.0 \mathrm{mmol})$ dropwise. The resultant reaction mixture was stirred for $10 \mathrm{~min}$. Similar workup procedure as described above giave the ratio of $\mathbf{2} \mathbf{b}$ to $\mathbf{d} \mathbf{d - 2} \mathbf{b}$.

\section{4 in situ ${ }^{13} \mathrm{C}$ NMR of reaction mixture}

To an NMR tube was sequentially added $0.25 \mathrm{mmol}$ of $1 \mathrm{~b}, 0.1 \mathrm{~mL}$ of HFIP, and $0.4 \mathrm{~mL}$ of deuterium water, followed by addition of DCOOD (38 $\mu \mathrm{L}, 0.5 \mathrm{mmol})$ dropwise into the mixture. Since the deoxygenation occurred so fast (within $30 \mathrm{~min}$ ), in our NMR test, catalyst was not added. The tube was submitted to ${ }^{13} \mathrm{C}$ NMR immediately after shaken vigorously for several times. Data were collected for every 10 minutes per cycle (128 scans, relaxation time of 2 seconds for one cycle of ${ }^{13} \mathrm{C}$ NMR test). 


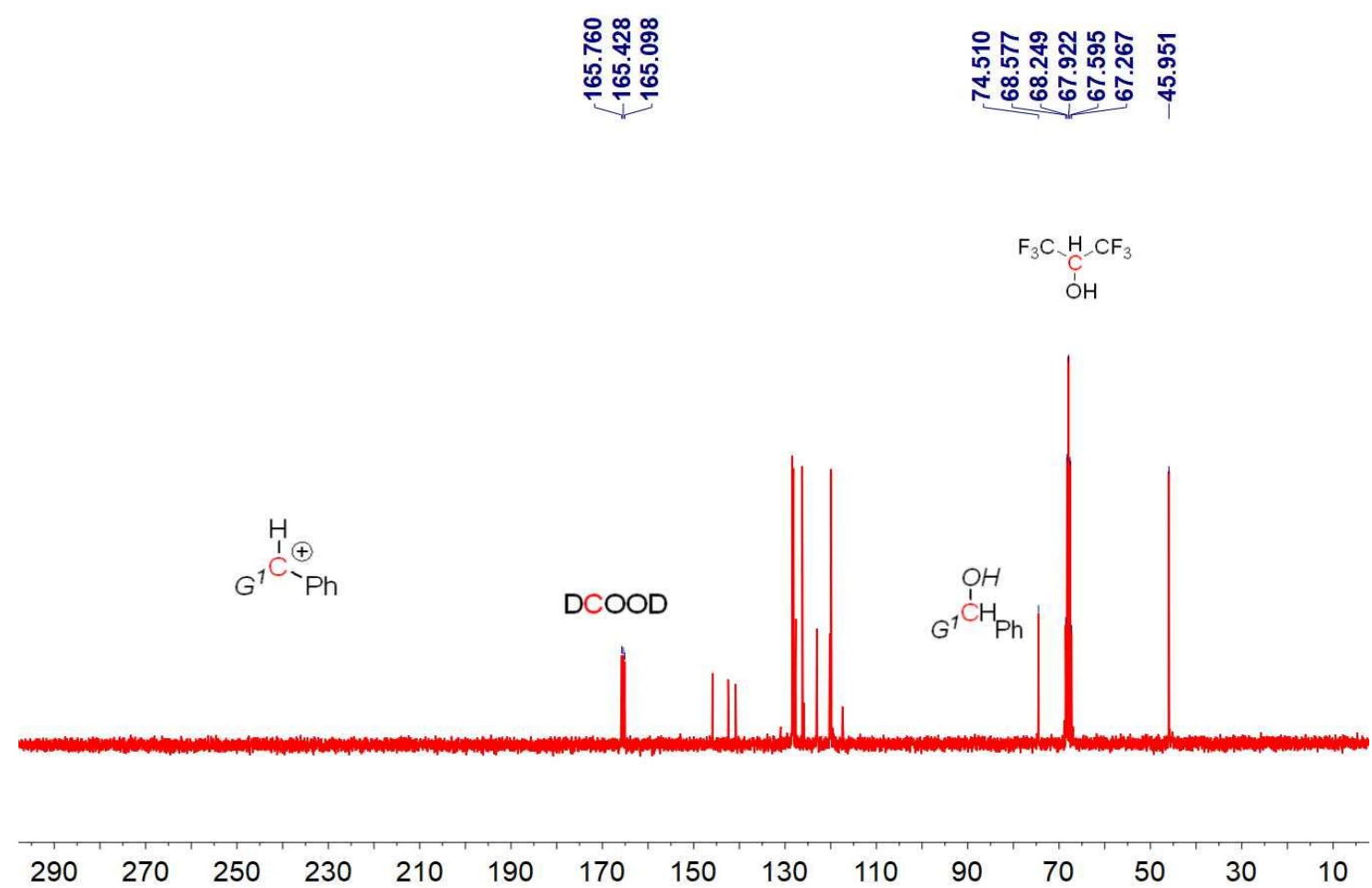

Figure S2. in situ ${ }^{13} \mathrm{C}$ NMR spectrum (0-30 $\mathrm{min}$, the $3^{\text {rd }}$ cycle, almost no change was observed; the carbocation cannot be detected).
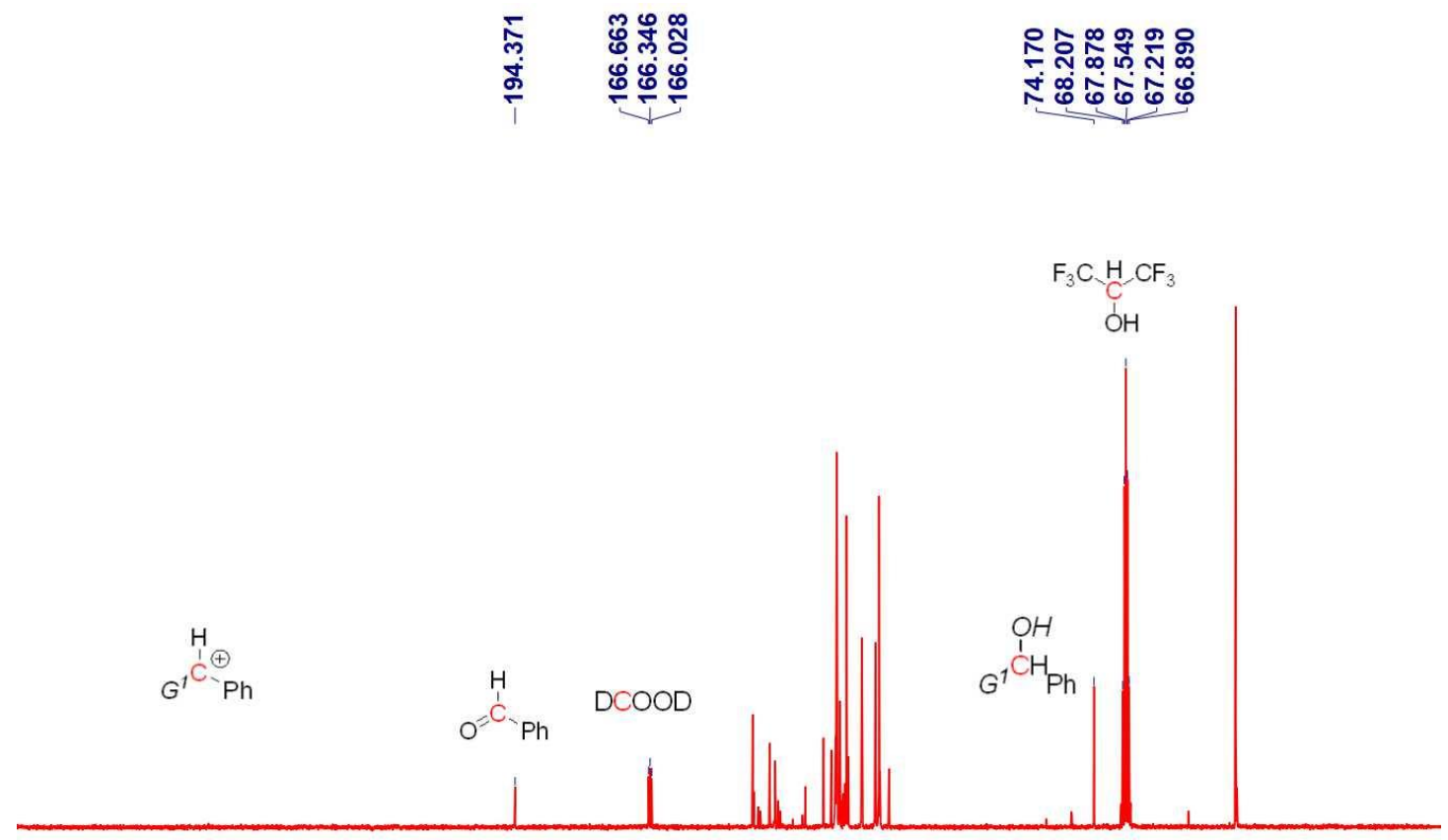

$\begin{array}{lllllllllllllll}290 & 270 & 250 & 230 & 210 & 190 & 170 & 150 & 130 & 110 & 90 & 70 & 50 & 30 & 10\end{array}$

Figure S3. in situ ${ }^{13} \mathrm{C}$ NMR (30-60 min, by product benzaldehyde appeared at the $4^{\text {th }}$ cycle; still, the carbocation cannot be detected). 


\subsection{Selected ESI-HRMS spectra of alcohols}

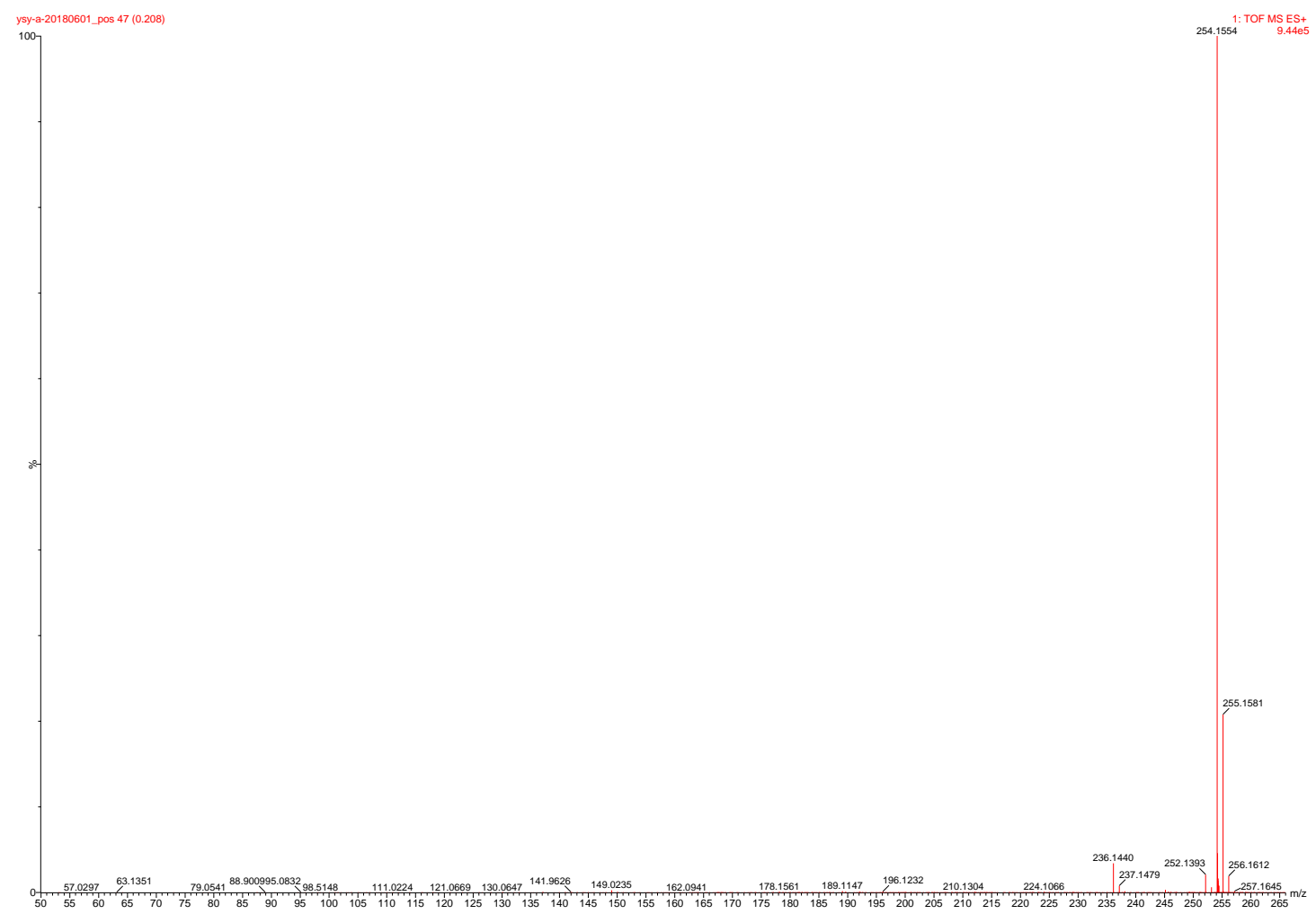

Figure S4. HRMS spectra of 1b-4.

HRMS (positive ESI): $\mathrm{m} / \mathrm{z}$ calculated for $\mathrm{C}_{17} \mathrm{H}_{20} \mathrm{NO}[\mathrm{M}+\mathrm{H}]^{+}$: calcd: 254.1539 ; found: 254.1554;

$\mathrm{m} / \mathrm{z}$ calculated for carbocation, $\mathrm{C}_{17} \mathrm{H}_{19} \mathrm{~N}^{+}[\mathrm{M}-17.0027]^{+}$: calcd: 236.1434 ; found: 236.1440.<smiles>OC(c1ccccc1)c1ccc(N2CCCC2)cc1</smiles><smiles>O=C(c1ccccc1)c1ccc(N2CCCC2)cc1</smiles> 


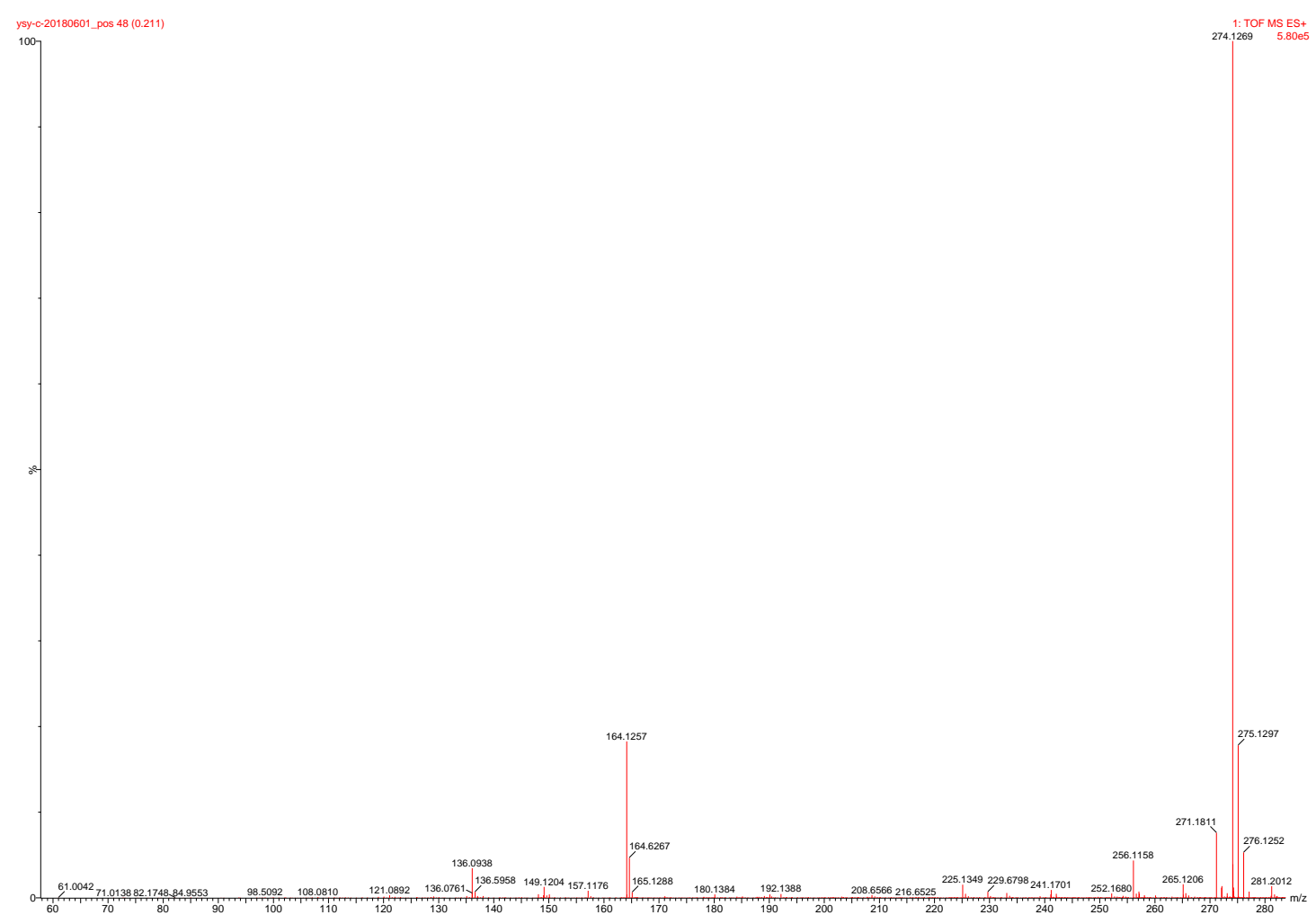

Figure S5. HRMS spectra of 10.

HRMS (positive ESI): $\mathrm{m} / \mathrm{z}$ calculated for $\mathrm{C}_{16} \mathrm{H}_{20} \mathrm{NOS}[\mathrm{M}+\mathrm{H}]^{+}$: calcd: 274.1260 ; found: 274.1269 .

$\mathrm{m} / \mathrm{z}$ calculated for carbocation, $\mathrm{C}_{16} \mathrm{H}_{19} \mathrm{NS}^{+}[\mathrm{M}-17.0027]^{+}$: calcd: 256.1154 ; found: 256.1158 .<smiles>CSc1ccc(C(O)c2ccc(N(C)C)cc2)cc1</smiles><smiles>CSc1ccc(C(=O)c2ccc(N(C)C)cc2)cc1</smiles> 


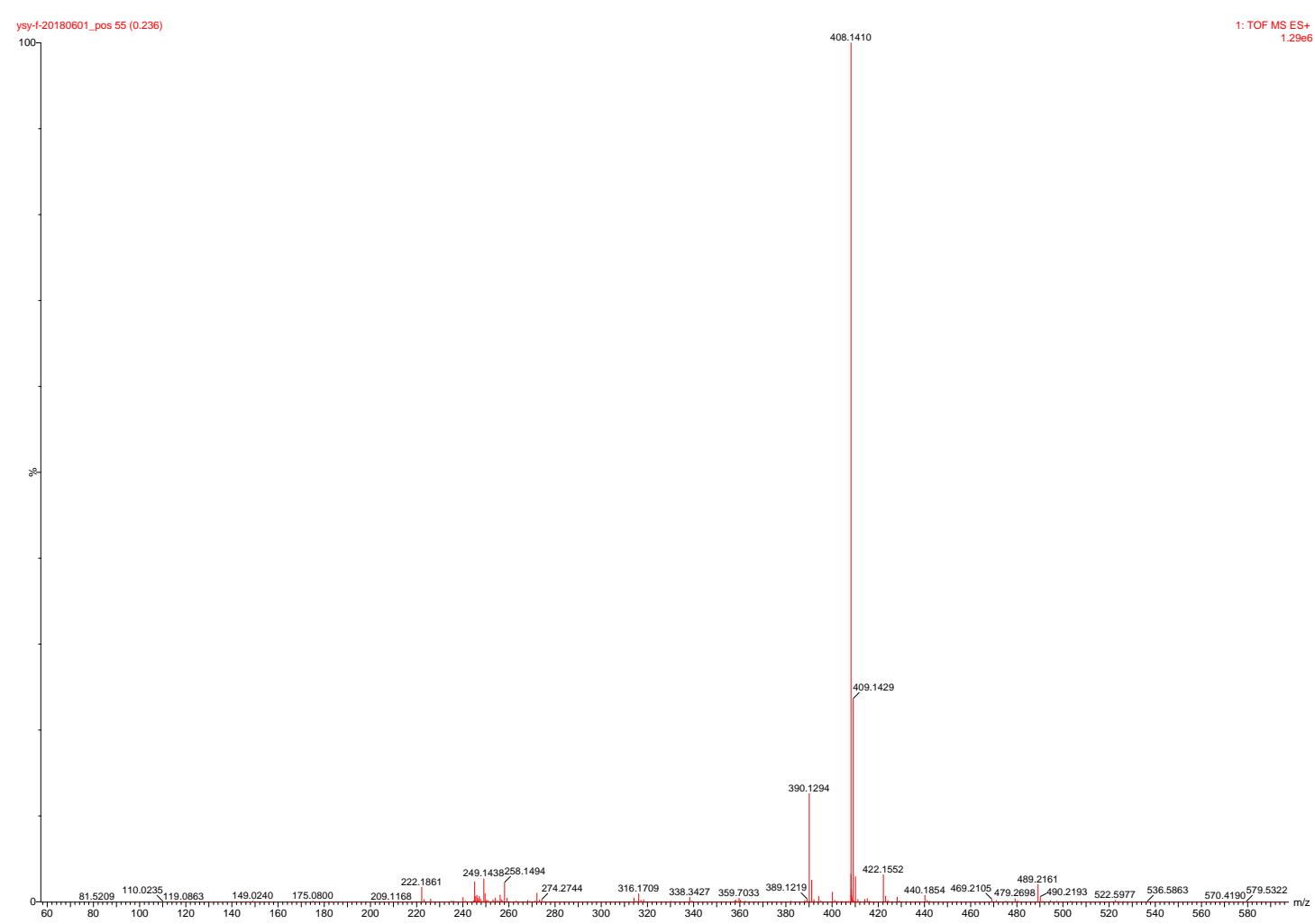

Figure S6. HRMS spectra of $\mathbf{3 e .}$

HRMS (positive ESI): $\mathrm{m} / \mathrm{z}$ calculated for $\mathrm{C}_{19} \mathrm{H}_{20} \mathrm{NO}_{2} \mathrm{~F}_{6}[\mathrm{M}+\mathrm{H}]^{+}$: calcd: 408.1393 ; found: 408.1410 .

$\mathrm{m} / \mathrm{z}$ calculated for carbocation, $\mathrm{C}_{19} \mathrm{H}_{19} \mathrm{NOF}_{6}{ }^{+}$[M-17.0027] $]^{+}$calcd: 390.1287 ; found: 390.1294 .

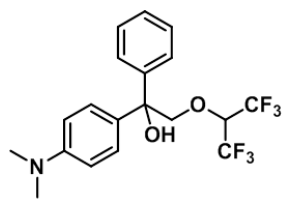<smiles></smiles>

\subsection{HRMS data of alcohols and carbocations.}

HRMS data of other alcohols and resultant carbocations generated in ESI-HRMS spectrometer.

(1b-6): HRMS (positive ESI): $\mathrm{m} / \mathrm{z}$ for $\mathrm{C}_{22} \mathrm{H}_{22} \mathrm{NO}[\mathrm{M}+\mathrm{H}]^{+}$: calcd: 316.1696 ; found: 316.1708. $\mathrm{m} / \mathrm{z}$ for carbocation, $\mathrm{C}_{22} \mathrm{H}_{21} \mathrm{~N}^{+}[\mathrm{M}-17.0027]^{+}$: calcd: 298.1591; found: 298.1602.
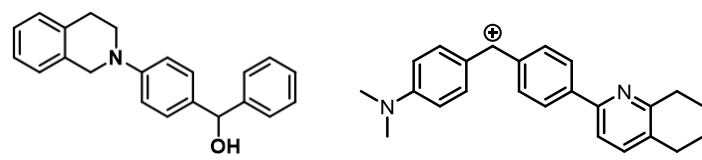

(1b-7): HRMS (positive ESI): $\mathrm{m} / \mathrm{z}$ for $\mathrm{C}_{22} \mathrm{H}_{22} \mathrm{NO}[\mathrm{M}+\mathrm{H}]^{+}$: calcd: 316.1696 ; found: 316.1703 . $\mathrm{m} / \mathrm{z}$ for carbocation, $\mathrm{C}_{22} \mathrm{H}_{21} \mathrm{~N}^{+}$[M-17.0027] $]^{+}$: calcd: 298.1591; found: 298.1599. 

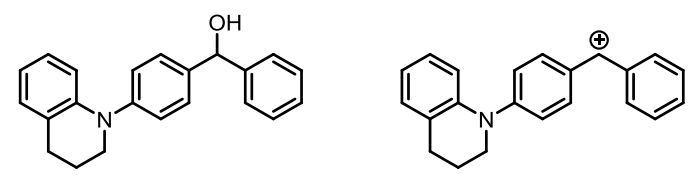

(1h): HRMS (positive ESI): $\mathrm{m} / \mathrm{z}$ for $\mathrm{C}_{16} \mathrm{H}_{28} \mathrm{NO}[\mathrm{M}+\mathrm{H}]^{+}$: calcd: 250.2165; found: 250.2168. $\mathrm{m} / \mathrm{z}$ for carbocation, $\mathrm{C}_{16} \mathrm{H}_{27} \mathrm{~N}^{+}$[M-17.0027] $]^{+}$calcd: 232.2060; found: 232.2060.<smiles>CCCCCC(O)c1ccc(N(CC)CC)cc1</smiles><smiles>CCCCCOc1ccc(N(CC)CC)cc1</smiles>

(1k): HRMS (positive ESI): $\mathrm{m} / \mathrm{z}$ for $\mathrm{C}_{18} \mathrm{H}_{30} \mathrm{NO}[\mathrm{M}+\mathrm{H}]^{+}$: calcd: 276.2322 ; found: 276.2322 . $\mathrm{m} / \mathrm{z}$ for carbocation, $\mathrm{C}_{18} \mathrm{H}_{29} \mathrm{~N}^{+}$[M-17.0027] $]^{+}$calcd: 258.2217; found: 258.2221.<smiles>CC(C)=CCCC(C)CC(O)c1ccc(N(C)C)cc1</smiles><smiles>CC(C)=CCCC(C)COc1ccc(N(C)C)cc1</smiles>

(11): $\mathrm{HRMS}$ (positive $\mathrm{ESI}$ ): $\mathrm{m} / \mathrm{z}$ for $\mathrm{C}_{16} \mathrm{H}_{18} \mathrm{NO}_{3}[\mathrm{M}+\mathrm{H}]^{+}$: calcd: 272.1281 ; found: 272.1284 . $\mathrm{m} / \mathrm{z}$ for carbocation, $\mathrm{C}_{16} \mathrm{H}_{17} \mathrm{NO}_{2}{ }^{+}[\mathrm{M}-17.0027]^{+}$: calcd: 254.1176; found: 254.1173.<smiles>CN(C)c1ccc(C(O)c2ccc3c(c2)OCO3)cc1</smiles><smiles>CN(C)c1ccc([C+]c2ccc3c(c2)OCO3)cc1</smiles>

(1m): HRMS (positive ESI): $\mathrm{m} / \mathrm{z}$ for $\mathrm{C}_{20} \mathrm{H}_{29} \mathrm{~N}_{2} \mathrm{O}$ [M+H] $]^{+}$calcd: 313.2274 ; found: 313.2274 . $\mathrm{m} / \mathrm{z}$ for carbocation, $\mathrm{C}_{20} \mathrm{H}_{28} \mathrm{~N}_{2}{ }^{+}$[M-17.0027] ${ }^{+}$: calcd: 295.2169; found: 295.2178.<smiles>CCCCN(C)c1ccc(C(O)c2ccc(N(C)C)cc2)cc1</smiles><smiles>CCCCN(C)c1ccc([C+]c2ccc(N(C)C)cc2)cc1</smiles>

(1y): HRMS (positive ESI): $\mathrm{m} / \mathrm{z}$ for $\mathrm{C}_{15} \mathrm{H}_{17} \mathrm{NOBr}[\mathrm{M}+\mathrm{H}]^{+}$: calcd: 306.0488 ; found: 306.0493. $\mathrm{m} / \mathrm{z}$ for carbocation, $\mathrm{C}_{15} \mathrm{H}_{16} \mathrm{NBr}^{+}[\mathrm{M}-17.0027]^{+}$: calcd: 288.0383; found: 288.1424.<smiles>CN(C)c1ccc(C(O)c2cccc(Br)c2)cc1</smiles><smiles>CN(C)c1ccc([C+](O)c2cccc(Br)c2)cc1</smiles>

(1aa): HRMS (positive $\mathrm{ESI}$ ): $\mathrm{m} / \mathrm{z}$ for $\mathrm{C}_{15} \mathrm{H}_{17} \mathrm{NOI}[\mathrm{M}+\mathrm{H}]^{+}$: calcd: 354.0349 ; found: 354.0355 . $\mathrm{m} / \mathrm{z}$ for carbocation, $\mathrm{C}_{15} \mathrm{H}_{16} \mathrm{NI}^{+}$[M-17.0027] $]^{+}$: calcd: 336.0244 ; found: 336.0253 . 
<smiles>CN(C)c1ccc(C(O)c2ccc(I)cc2)cc1</smiles><smiles>CN(C)c1ccc([C+]c2ccc(I)cc2)cc1</smiles>

(1ak): HRMS (positive ESI): $\mathrm{m} / \mathrm{z}$ for $\mathrm{C}_{24} \mathrm{H}_{27} \mathrm{~N}_{2} \mathrm{O}$ [M+H]+: calcd: 359.2118; found: 359.2123. $\mathrm{m} / \mathrm{z}$ for carbocation, $\mathrm{C}_{24} \mathrm{H}_{26} \mathrm{~N}_{2}{ }^{+}$[M-17.0027] $]^{+}$calcd: 341.2083; found: 341.2080 .<smiles>CN(C)c1ccc(C(O)c2ccc(-c3ccc4c(n3)CCCC4)cc2)cc1</smiles><smiles>CN(C)c1ccc([C+]c2ccc(-c3ccc4c(n3)CCCC4)cc2)cc1</smiles>

(1al): HRMS (positive ESI): $\mathrm{m} / \mathrm{z}$ for $\mathrm{C}_{18} \mathrm{H}_{21} \mathrm{~N}_{2} \mathrm{O}$ [M+H] $]^{+}$: calcd: 281.1648; found: 281.1653 $\mathrm{m} / \mathrm{z}$ for carbocation, $\mathrm{C}_{18} \mathrm{H}_{20} \mathrm{~N}_{2}{ }^{+}$[M-17.0027] $]^{+}$: calcd: 263.1543; found:263.1550.<smiles>CN(C)c1ccc(C(O)c2c[nH]c3ccccc23)cc1</smiles><smiles>CN(C)c1ccc([C+]c2c[nH]c3ccccc23)cc1</smiles>

(1ao): HRMS (positive ESI): $\mathrm{m} / \mathrm{z}$ for $\mathrm{C}_{17} \mathrm{H}_{22} \mathrm{NOS}[\mathrm{M}+\mathrm{H}]^{+}$: calcd: 288.1417 ; found: 288.1421.

$\mathrm{m} / \mathrm{z}$ for carbocation, $\mathrm{C}_{17} \mathrm{H}_{21} \mathrm{NS}^{+}$[M-17.0027] $]^{+}$calcd: 270.1312 ; found: 270.1464 .<smiles>CSCc1ccc(C(O)c2ccc(N(C)C)cc2)cc1</smiles><smiles>CSCc1ccc([C+]c2ccc(N(C)C)cc2)cc1</smiles>

(1ap): HRMS (positive ESI): $\mathrm{m} / \mathrm{z}$ for $\mathrm{C}_{17} \mathrm{H}_{22} \mathrm{NO}_{2}[\mathrm{M}+\mathrm{H}]^{+}$: calcd: 272.1645; found: 272.1646 .

$\mathrm{m} / \mathrm{z}$ for carbocation, $\mathrm{C}_{17} \mathrm{H}_{21} \mathrm{NO}^{+}[\mathrm{M}-17.0027]^{+}$: calcd: 254.1540 ; found: 254.1545.<smiles>COCc1ccc(C(O)c2ccc(N(C)C)cc2)cc1</smiles><smiles>COCc1ccc([C+]([O-])c2ccc(N(C)C)cc2)cc1</smiles>

(1aq): HRMS (positive ESI): $\mathrm{m} / \mathrm{z}$ for $\mathrm{C}_{18} \mathrm{H}_{25} \mathrm{~N}_{2} \mathrm{O}[\mathrm{M}+\mathrm{H}]^{+}$: calcd: 285.1961; found: 285.1965.

m/z for carbocation, $\mathrm{C}_{18} \mathrm{H}_{24} \mathrm{~N}_{2}{ }^{+}$[M-17.0027] $]^{+}$calcd: 267.1856; found: 267.1851.<smiles>CN(C)Cc1ccc(C(O)c2ccc(N(C)C)cc2)cc1</smiles><smiles>CN(C)Cc1ccc([C+]c2ccc(N(C)C)cc2)cc1</smiles>

(1au): HRMS (positive ESI): $\mathrm{m} / \mathrm{z}$ for $\mathrm{C}_{16} \mathrm{H}_{20} \mathrm{NO}_{2}[\mathrm{M}+\mathrm{H}]^{+}$: calcd: 258.1489; found: 
258.1494.

$\mathrm{m} / \mathrm{z}$ for carbocation, $\mathrm{C}_{16} \mathrm{H}_{19} \mathrm{NO}^{+}[\mathrm{M}-17.0027]^{+}$: calcd: 240.1384 ; found: 240.1380 .<smiles>CN(C)c1ccc(C(O)c2ccc(CO)cc2)cc1</smiles><smiles>CN(C)c1ccc([C+](O)c2ccc(CO)cc2)cc1</smiles>

(3d): HRMS (positive ESI): $\mathrm{m} / \mathrm{z}$ for $\mathrm{C}_{14} \mathrm{H}_{24} \mathrm{NO}[\mathrm{M}+\mathrm{H}]^{+}$: calcd: 222.1852; found: 222.1858. $\mathrm{m} / \mathrm{z}$ for carbocation, $\mathrm{C}_{14} \mathrm{H}_{23} \mathrm{~N}^{+}$[M-17.0027] $]^{+}$: calcd: 204.1747; found: 204.1698.<smiles>CN(C)c1ccc(C(C)(O)C(C)(C)C)cc1</smiles><smiles>CN(C)c1ccc(C(C)(C)C)cc1</smiles>

(3f): HRMS (positive ESI): $\mathrm{m} / \mathrm{z}$ for $\mathrm{C}_{22} \mathrm{H}_{24} \mathrm{NO}_{2}[\mathrm{M}+\mathrm{H}]^{+}$: calcd: 334.1802 ; found: 334.1812 . $\mathrm{m} / \mathrm{z}$ for carbocation, $\mathrm{C}_{22} \mathrm{H}_{23} \mathrm{NO}^{+}$[M-17.0027] $]^{+}$: calcd: 316.1697; found: 316.1706.<smiles>CN(C)c1ccc(C(=O)Oc2ccccc2)cc1</smiles><smiles>CN(C)c1ccc(C(OCc2ccccc2)c2ccccc2)cc1</smiles>

(3g): HRMS (positive ESI): $\mathrm{m} / \mathrm{z}$ for $\mathrm{C}_{12} \mathrm{H}_{18} \mathrm{NO}[\mathrm{M}+\mathrm{H}]^{+}$: calcd: 192.1383; found: 192.1385 . $\mathrm{m} / \mathrm{z}$ for carbocation, $\mathrm{C}_{12} \mathrm{H}_{17} \mathrm{~N}^{+}$[M-17.0027] $]^{+}$calcd: 174.1278; found: 174.1281.<smiles>CN(C)c1ccc(C2(O)CCC2)cc1</smiles><smiles>CN(C)c1ccc([C+]2CCC2)cc1</smiles>

(3j): HRMS (positive $\mathrm{ESI}$ ): $\mathrm{m} / \mathrm{z}$ for $\mathrm{C}_{15} \mathrm{H}_{24} \mathrm{NO}[\mathrm{M}+\mathrm{H}]^{+}$: calcd: 234.1852; found: 234.1848. $\mathrm{m} / \mathrm{z}$ for carbocation, $\mathrm{C}_{15} \mathrm{H}_{23} \mathrm{~N}^{+}$[M-17.0027] $]^{+}$calcd: 216.1747 ; found: 216.1753 .<smiles>CN(C)c1ccc(C2(O)CCCCCC2)cc1</smiles><smiles>CN(C)c1ccc(C2CCCCCC2)cc1</smiles>

(3k): HRMS (positive ESI): $\mathrm{m} / \mathrm{z}$ for $\mathrm{C}_{20} \mathrm{H}_{34} \mathrm{NO}$ [M+H] $]^{+}$calcd: 304.2635 ; found: 304.2651 . $\mathrm{m} / \mathrm{z}$ for carbocation, $\mathrm{C}_{20} \mathrm{H}_{33} \mathrm{~N}^{+}$[M-17.0027] : calcd: 286.2530; found: 286.2538<smiles>CN(C)c1ccc(C2(O)CCCCCCCCCC2)cc1</smiles><smiles>CCCCCCCCCCCCCCc1ccc(N(C)C)cc1</smiles>

(3m): HRMS (positive ESI): m/z for $\mathrm{C}_{18} \mathrm{H}_{22} \mathrm{NO}[\mathrm{M}+\mathrm{H}]^{+}$: calcd: 268.1696; found: 268.1634 . 
$\mathrm{m} / \mathrm{z}$ for carbocation, $\mathrm{C}_{18} \mathrm{H}_{23} \mathrm{~N}^{+}[\mathrm{M}-17.0027]^{+}$: calcd: 250.1591 ; found: 250.1607 .<smiles>CN(C)c1ccc(C2(O)CCCc3ccccc32)cc1</smiles><smiles>CN(C)c1ccc(C2CCCc3ccccc32)cc1</smiles>

(3o): HRMS (positive ESI): $\mathrm{m} / \mathrm{z}$ for $\mathrm{C}_{18} \mathrm{H}_{28} \mathrm{NO}[\mathrm{M}+\mathrm{H}]^{+}$: calcd: 274.2165 ; found: 274.2170 . $\mathrm{m} / \mathrm{z}$ for carbocation, $\mathrm{C}_{18} \mathrm{H}_{27} \mathrm{~N}^{+}$[M-17.0027] $]^{+}$: calcd: 256.2060; found: 256.2067
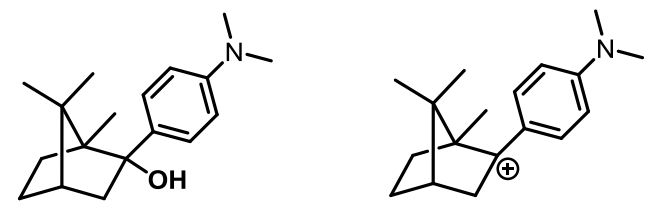

(3p): HRMS (positive ESI): $\mathrm{m} / z$ for $\mathrm{C}_{21} \mathrm{H}_{30} \mathrm{NO}[\mathrm{M}+\mathrm{H}]^{+}$: calcd: 312.2322 ; found: 312.2325 . $\mathrm{m} / \mathrm{z}$ for carbocation, $\mathrm{C}_{21} \mathrm{H}_{29} \mathrm{~N}^{+}[\mathrm{M}-17.0027]^{+}$: calcd: 294.2217 ; found: 294.2222.<smiles>C=C(C)[C@@H]1CC=C(C)C(O)(c2ccc(N3CCCCC3)cc2)C1</smiles><smiles>C=C(C)[C@H]1CC=C(C)[C@H](c2ccc(N3CCCCC3)cc2)C1</smiles>

(3q): HRMS (positive ESI): $\mathrm{m} / \mathrm{z}$ for $\mathrm{C}_{24} \mathrm{H}_{42} \mathrm{NO}[\mathrm{M}+\mathrm{H}]^{+}$: calcd: 360.3261 ; found: 360.3272 . $\mathrm{m} / \mathrm{z}$ for carbocation, $\mathrm{C}_{24} \mathrm{H}_{41} \mathrm{~N}^{+}$[M-17.0027] $]^{+}$: calcd: 342.3156 ; found: 342.3168 .
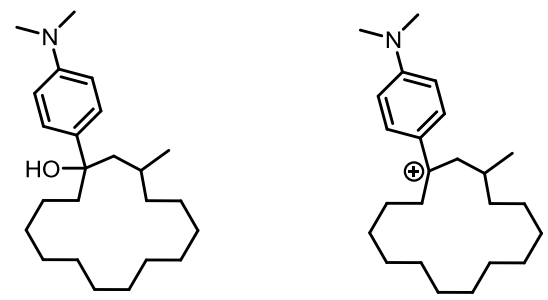

(3r): HRMS (positive ESI): $\mathrm{m} / \mathrm{z}$ for $\mathrm{C}_{35} \mathrm{H}_{41} \mathrm{~N}_{3} \mathrm{OF}$ [M+H] $]^{+}$calcd: 538.3228 ; found: 538.3240 . $\mathrm{m} / \mathrm{z}$ for carbocation, $\mathrm{C}_{35} \mathrm{H}_{40} \mathrm{~N}_{3} \mathrm{~F}^{+}$[M-17.0027] $]^{+}$calcd: 520.3123 ; found: 520.3130 .
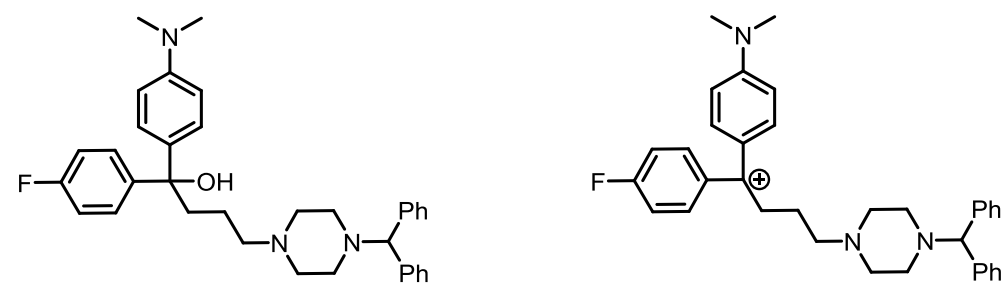

(3t): HRMS (positive ESI): $\mathrm{m} / \mathrm{z}$ for $\mathrm{C}_{14} \mathrm{H}_{22} \mathrm{NO}_{2}[\mathrm{M}+\mathrm{H}]^{+}$: calcd: 236.1645 ; found: 236.1654 . $\mathrm{m} / \mathrm{z}$ for carbocation, $\mathrm{C}_{14} \mathrm{H}_{21} \mathrm{NO}^{+}[\mathrm{M}-17.0027]^{+}$: calcd: 218.1540; found: 218.1546.<smiles>CN(C)c1ccc(C2(O)CCC(O)CC2)cc1</smiles><smiles>CN(C)c1ccc(C2CCC(O)CC2)cc1</smiles> 
(3u): HRMS (positive ESI): $\mathrm{m} / \mathrm{z}$ for $\mathrm{C}_{14} \mathrm{H}_{24} \mathrm{NO}_{2}[\mathrm{M}+\mathrm{H}]^{+}$: calcd: 238.1802; found: 238.1810. $\mathrm{m} / \mathrm{z}$ for carbocation, $\mathrm{C}_{14} \mathrm{H}_{23} \mathrm{NO}^{+}[\mathrm{M}-17.0027]^{+}$: calcd: 220.1697 ; found: 220.1699 .<smiles>CN(C)c1ccc(C(C)(O)CC(C)(C)O)cc1</smiles><smiles>CC(CC(C)(C)O)c1ccc(N(C)C)cc1</smiles>

(3v): HRMS (positive ESI): $\mathrm{m} / \mathrm{z}$ for $\mathrm{C}_{12} \mathrm{H}_{18} \mathrm{NO}_{2}[\mathrm{M}+\mathrm{H}]^{+}$: calcd: 208.1332; found: 208.1334. $\mathrm{m} / \mathrm{z}$ for carbocation, $\mathrm{C}_{12} \mathrm{H}_{17} \mathrm{NO}^{+}[\mathrm{M}-17.0027]^{+}$: calcd: 190.1227; found: 190.1223.<smiles>CC(=O)C(C)(O)c1ccc(N(C)C)cc1</smiles><smiles>CC(=O)c1ccc(N(C)C)cc1</smiles>

\section{Characterization data of catalysts and products}

\subsection{Data of Li's catalysts}

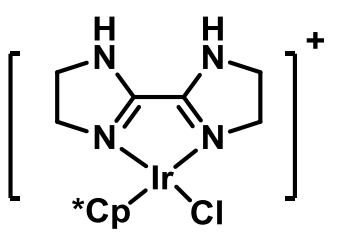

Complex LC-1: yellow powder; yield: $98 \mathrm{mg}, 82 \%$; ${ }^{1} \mathrm{H}$ NMR (400 MHz, $\left.\mathrm{D}_{2} \mathrm{O}\right) \delta(\mathrm{ppm}): 4.13-4.07(\mathrm{~m}, 4 \mathrm{H}), 4.02-3.96(\mathrm{~m}, 4 \mathrm{H})$, cl $1.78(\mathrm{~s}, 15 \mathrm{H}) ;{ }^{13} \mathrm{C}$ NMR $\left(101 \mathrm{MHz}, \mathrm{D}_{2} \mathrm{O}\right) \delta(\mathrm{ppm}):$ 161.3, 87.7, $51.7,46.6,8.5$.

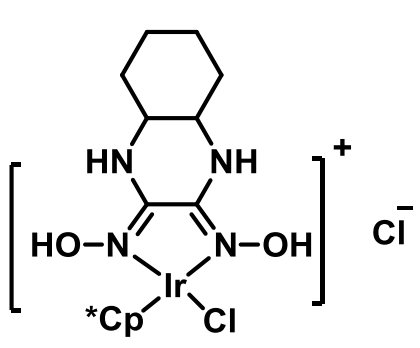

Complex LC-2: yellow powder; yield: $89 \mathrm{mg}, 74 \%$; ${ }^{1} \mathrm{H}$ NMR (400 MHz, $\left.\mathrm{D}_{2} \mathrm{O}\right) \delta$ (ppm): 3.29 (s, 2H), 2.05-2.01 (m, 2H), 1.78-1.74 (s, 2H), $1.74(\mathrm{~s}, 15 \mathrm{H}), 1.37-1.35(\mathrm{~m}, 4 \mathrm{H}) ;{ }^{13} \mathrm{C} \mathrm{NMR}$ (101 MHz, $\left.\mathrm{D}_{2} \mathrm{O}\right) \delta(\mathrm{ppm}):$ 152.0, 89.1, 55.1, 28.8, 23.2, 8.3.<smiles>CC1CNC2=C(O)N(O)I(Cl)(Cl)(C[C@H]2Cl)N1O</smiles>

Complex LC-3: yellow powder; yield: $85 \mathrm{mg}, 76 \% ;{ }^{1} \mathrm{H}$ NMR (400 MHz, $\mathrm{D}_{2} \mathrm{O}$ ) $\delta(\mathrm{ppm})$ : 3.90-3.78 (s, 2H), 3.52 (dd, $J=12.4$ $\mathrm{Hz}, 4.0 \mathrm{~Hz}, 1 \mathrm{H}$ ), 3.23 (dd, $J=12.0 \mathrm{~Hz}, 8.8 \mathrm{~Hz}, 1 \mathrm{H}), 1.74$ (s, $15 \mathrm{H}), 1.27(\mathrm{~d}, J=6.4 \mathrm{~Hz}, 3 \mathrm{H}) ;{ }^{13} \mathrm{C}$ NMR $\left(101 \mathrm{MHz}, \mathrm{D}_{2} \mathrm{O}\right) \delta$ (ppm): 151.6, 89.1, 45.9, 44.7, 17.3, 8.3. 


\subsection{Data of alcohol starting materials}<smiles>COc1ccc(C(O)c2ccccc2)cc1</smiles>

(4-Methoxyphenyl)(phenyl)methanol (S1a): CAS No. 720-44-5.

Prepared according to procedure $B$ from the aldehyde

4-methoxybenzaldehyde. White solid; m.p.: $68^{\circ} \mathrm{C}$ (reported 68 $\left.{ }^{\circ} \mathrm{C}\right) ;{ }^{9}$ yield: $974 \mathrm{mg}, 91 \% ;{ }^{1} \mathrm{H}$ NMR $\left(400 \mathrm{MHz}, \mathrm{CDCl}_{3}\right) \delta(\mathrm{ppm}): 7.41-7.27(\mathrm{~m}, 7 \mathrm{H}), 6.90(\mathrm{~d}$, $J=8.8 \mathrm{~Hz}, 2 \mathrm{H}), 5.81(\mathrm{~s}, 1 \mathrm{H}), 3.81(\mathrm{~s}, 3 \mathrm{H}), 2.44$ (brs, $1 \mathrm{H}) ;{ }^{13} \mathrm{C} \mathrm{NMR}\left(101 \mathrm{MHz}, \mathrm{CDCl}_{3}\right) \delta$ (ppm): 158.9, 144.0, 136.1, 128.4, 127.9, 127.3, 126.3, 113.8, 75.7, 55.2.<smiles>OC(c1ccccc1)c1ccc2c(c1)OCO2</smiles>

Benzo[d][1,3]dioxol-5-yl(phenyl)methanol (S1b): CAS No. 4382-91-6

Prepared according to procedure $B$ from the aldehyde benzo[d][1,3]dioxole-5-carbaldehyde. Pale yellow oil; yield: $1.01 \mathrm{~g}, 89 \%$; ${ }^{1} \mathrm{H}$ NMR $\left(400 \mathrm{MHz}, \mathrm{CDCl}_{3}\right) \delta(\mathrm{ppm}):$ 7.39-7.28 (m, 5H), 6.88-6.77 (m, 4H), 5.93-5.90 (m, 2H), $5.70(\mathrm{~d}, J=2.8 \mathrm{~Hz}, 1 \mathrm{H}), 3.07(\mathrm{~d}, J=3.2 \mathrm{~Hz}, 1 \mathrm{H}) ;{ }^{13} \mathrm{C}$ NMR $(101 \mathrm{MHz}$, $\left.\mathrm{CDCl}_{3}\right) \delta(\mathrm{ppm}): 147.5,146.7,143.7,137.9,128.3,127.3,126.2,119.9,107.9,107.1$, 100.8, 75.6.<smiles>Oc1ccc(C(O)c2ccccc2)cc1</smiles>
4-(Hydroxy(phenyl)methyl)phenol (S1c): CAS No. 833-39-6 Prepared according to procedure $C$ from 4-bromoaniline and the aldehyde 4-hydroxybenzaldehyde. White solid; m.p.: 158-159 ${ }^{\circ} \mathrm{C}$ (reported $161{ }^{\circ} \mathrm{C}$ ) 10 ; yield: $933 \mathrm{mg}, 93 \% ;{ }^{1} \mathrm{H}$ NMR (400 MHz, DMSO- $\left.d_{6}\right) \delta(\mathrm{ppm}): 9.23(\mathrm{~s}, 1 \mathrm{H}), 7.34-6.69(\mathrm{~m}, 9 \mathrm{H}), 5.68(\mathrm{~s}, 1 \mathrm{H}), 5.59(\mathrm{~s}, 1 \mathrm{H})$; ${ }^{13} \mathrm{C}$ NMR $\left(101 \mathrm{MHz}\right.$, DMSO-d $\left.d_{6}\right) \delta$ (ppm): 156.2, 146.2, 136.2, 128.0, 127.5, 126.5, 126.1 , 114.8, 74.0, 39.5.<smiles>OC(c1ccccc1)c1ccco1</smiles>

Furan-2-yl(phenyl)methanol (S1d): CAS No. 4484-57-5

Prepared according to procedure $B$ from the aldehyde furan-2-carbaldehyde. Pale yellow oil; yield: $716 \mathrm{mg}, 82 \%$; ${ }^{1} \mathrm{H}$ NMR $\left(400 \mathrm{MHz}, \mathrm{CDCl}_{3}\right) \delta(\mathrm{ppm}): 7.44-7.33(\mathrm{~m}, 6 \mathrm{H}), 6.32(\mathrm{dd}, J=3.2 \mathrm{~Hz}, 2.0$ $\mathrm{Hz}, 1 \mathrm{H}), 6.12(\mathrm{~d}, J=3.2 \mathrm{~Hz}, 1 \mathrm{H}), 5.80(\mathrm{~s}, 1 \mathrm{H}) ;{ }^{13} \mathrm{C} \mathrm{NMR}\left(101 \mathrm{MHz}, \mathrm{CDCl}_{3}\right) \delta(\mathrm{ppm}): 155.9$, $142.4,140.8,128.3,127.9,126.5,110.1,107.3,70.0$.<smiles>OC(c1ccccc1)c1cccs1</smiles>

Phenyl(thiophen-2-yl)methanol (S1e): CAS No. 26059-21-2

Prepared according to procedure $B$ from the aldehyde thiophene-2-carbaldehyde. Pale yellow oil; yield: $828 \mathrm{mg}, 87 \% ;{ }^{1} \mathrm{H}$ NMR (400 MHz, $\left.\mathrm{CDCl}_{3}\right) \delta(\mathrm{ppm}): 7.50-7.30(\mathrm{~m}, 6 \mathrm{H}), 6.97(\mathrm{dd}, J=4.8$ $\mathrm{Hz}, 3.6 \mathrm{~Hz}, 1 \mathrm{H}), 6.94-6.89(\mathrm{~m}, 1 \mathrm{H}), 6.07(\mathrm{~d}, J=3.6 \mathrm{~Hz}, 1 \mathrm{H}) ; 2.61(\mathrm{~d}, J=4.0 \mathrm{~Hz}, 1 \mathrm{H}) ;{ }^{13} \mathrm{C}$ NMR (101 MHz, $\left.\mathrm{CDCl}_{3}\right) \delta(\mathrm{ppm}): 148.1,143.1,128.5,127.9,126.6,126.3,125.4,124.8$, 72.4 .

1-(4-(N,N-Dimethylamino)phenyl)-2-methylpropan-1-ol (1a): CAS No. 4278-81-3. 
Prepared according to procedure A from Grignard reagent SG1.<smiles>CC(C)C(O)c1ccc(N(C)C)cc1</smiles>

White solid; m.p.: $37-38^{\circ} \mathrm{C}$ (reported $39{ }^{\circ} \mathrm{C}$ ); ${ }^{11} 10 \mathrm{mmol}$ scale, yield: $1.72 \mathrm{~g}, 89 \%$; ${ }^{1} \mathrm{H}$ NMR $\left(400 \mathrm{MHz}, \mathrm{CDCl}_{3}\right) \delta(\mathrm{ppm}): 7.18$ (d, $J=7.6 \mathrm{~Hz}$ 2H), $6.73(\mathrm{~d}, J=7.6 \mathrm{~Hz}, 2 \mathrm{H}), 4.21(\mathrm{~d}, J=7.6 \mathrm{~Hz}, 1 \mathrm{H}), 2.95(\mathrm{~s}, 6 \mathrm{H})$, 2.16 (brs, 1H), 2.01-1.88 (m, 1H), $1.04(\mathrm{~d}, J=6.8 \mathrm{~Hz}, 3 \mathrm{H}), 0.78(\mathrm{~d}, J$ $=6.8 \mathrm{~Hz}, 3 \mathrm{H}) ;{ }^{13} \mathrm{C} \mathrm{NMR}\left(101 \mathrm{MHz}, \mathrm{CDCl}_{3}\right) \delta$ (ppm): 149.9, 131.8,

$127.3,112.3,79.8,40.6,35.0,18.9,18.7$.<smiles>CN(C)c1ccc(C(O)c2ccccc2)cc1</smiles>

4-Benzyl-N,N-dimethylaniline (1b): CAS No. 7494-77-1.

Prepared according to procedure $A$ from Grignard reagent SG1. White solid; m.p.: $68-70{ }^{\circ} \mathrm{C}$ (reported $69-70{ }^{\circ} \mathrm{C}$ ) 12 ; $10 \mathrm{mmol}$ scale, yield: $2.00 \mathrm{~g}, 88 \%$; ${ }^{1} \mathrm{H}$ NMR (400 MHz, $\mathrm{CDCl}_{3}$ ) $\delta$ (ppm): 7.43-7.28 $(\mathrm{m}, 5 \mathrm{H}), 7.24(\mathrm{~d}, J=8.4 \mathrm{~Hz}, 2 \mathrm{H}), 6.73(\mathrm{~d}, J=8.4 \mathrm{~Hz}, 2 \mathrm{H}), 5.76(\mathrm{~s}$, 1H), 2.95 (s, 6H), 2.59 (brs, 1H); ${ }^{13} \mathrm{C}$ NMR (101 MHz, $\left.\mathrm{CDCl}_{3}\right) \delta$ (ppm): 150.0, 142.3, 132.1, $128.1,127.6,126.9,126.2,112.4,75.7,40.5$.<smiles>CN(C)c1ccccc1C(O)c1ccccc1</smiles>

(4-(Dimethylamino)phenyl)(phenyl)methanol (1b-1): CAS No. 34599-89-8.

Prepared according to procedure $A$ from Grignard reagent SG2.

White solid; m.p.: $70-71{ }^{\circ} \mathrm{C}$; yield: $471 \mathrm{mg}, 83 \%$; ${ }^{1} \mathrm{H}$ NMR $(400 \mathrm{MHz}$, $\left.\mathrm{CDCl}_{3}\right) \delta(\mathrm{ppm}): 7.32(\mathrm{~d}, J=7.6 \mathrm{~Hz}, 2 \mathrm{H}), 7.26-7.14(\mathrm{~m}, 5 \mathrm{H}), 7.04(\mathrm{t}, J=7.2 \mathrm{~Hz}, 1 \mathrm{H}), 6.96$ (d, $J=7.6 \mathrm{~Hz}, 1 \mathrm{H}), 5.92(\mathrm{~s}, 1 \mathrm{H}), 2.52(\mathrm{~s}, 6 \mathrm{H}) ;{ }^{13} \mathrm{C} \mathrm{NMR}\left(101 \mathrm{MHz}, \mathrm{CDCl}_{3}\right) \delta$ (ppm): 151.6, $144.3,138.5,129.1,128.2,128.1,126.9,126.5,125.3,122.0,75.6,45.7$.<smiles>CN(C)c1cccc(C(O)c2ccccc2)c1</smiles>

(3-(Dimethylamino)phenyl)(phenyl)methanol (1b-2): CAS No. 86997-96-8.

Prepared according to procedure $A$ from Grignard reagent SG3.

White solid; m.p.: $97-98{ }^{\circ} \mathrm{C}$; yield: $516 \mathrm{mg}, 91 \%$; ${ }^{1} \mathrm{H}$ NMR $(400 \mathrm{MHz}$, $\left.\mathrm{CDCl}_{3}\right) \delta(\mathrm{ppm}): 7.30(\mathrm{~d}, J=7.6 \mathrm{~Hz}, 2 \mathrm{H}), 7.25(\mathrm{t}, J=7.4 \mathrm{~Hz}, 2 \mathrm{H})$, 7.17-7.08 (m, 2H), $6.71(\mathrm{~s}, 1 \mathrm{H}), 6.61(\mathrm{~d}, J=7.6 \mathrm{~Hz}, 1 \mathrm{H}), 6.57(\mathrm{~d}, J=8.0 \mathrm{~Hz}, 1 \mathrm{H}), 5.66(\mathrm{~s}$, $1 \mathrm{H}), 2.83$ (s, 6H), 2.33 (brs, $1 \mathrm{H}) ;{ }^{13} \mathrm{C} \mathrm{NMR}\left(101 \mathrm{MHz}, \mathrm{CDCl}_{3}\right) \delta$ (ppm): 150.6, 144.8, 143.9, $129.2,128.3,127.3,126.4,115.1,111.9,110.8,76.5,40.7$.<smiles>CCN(CC)c1ccc(C(O)c2ccccc2)cc1</smiles>

(4-(Diethylamino)phenyl)(phenyl)methanol (1b-3): CAS No. 279675-41-1.

Prepared according to procedure $A$ from Grignard reagent SG4.

Pale yellow oil; yield: $567 \mathrm{mg}, 89 \% ;{ }^{1} \mathrm{H}$ NMR $\left(400 \mathrm{MHz}, \mathrm{CDCl}_{3}\right)$ $\delta$ (ppm): 7.31 (d, $J=7.2 \mathrm{~Hz}, 2 \mathrm{H}), 7.23(\mathrm{t}, J=7.2 \mathrm{~Hz}, 2 \mathrm{H}), 7.16-7.13(\mathrm{~m}, 1 \mathrm{H}), 7.08(\mathrm{~d}, J=$ $8.4 \mathrm{~Hz}, 2 \mathrm{H}), 6.54(\mathrm{~d}, J=8.0 \mathrm{~Hz}, 2 \mathrm{H}), 5.65(\mathrm{~d}, J=3.2 \mathrm{~Hz}, 1 \mathrm{H}), 3.24(\mathrm{q}, J=7.2 \mathrm{~Hz}, 4 \mathrm{H})$, 2.08 (brd, $J=2.4 \mathrm{~Hz}, 1 \mathrm{H}), 1.05(\mathrm{t}, J=7.2 \mathrm{~Hz}, 6 \mathrm{H}) ;{ }^{13} \mathrm{C} \mathrm{NMR}\left(101 \mathrm{MHz}, \mathrm{CDCl}_{3}\right) \delta(\mathrm{ppm})$ : $147.3,144.3,130.7,128.2,128.0,127.0,126.3,111.5,75.9,44.3,12.5$.

\section{Phenyl(4-(pyrrolidin-1-yl)phenyl)methanol (1b-4):}


Prepared according to procedure A from Grignard reagent SG5.

White solid; m.p.: $86-88{ }^{\circ} \mathrm{C}$; yield: $519 \mathrm{mg}, 82 \%$; ${ }^{1} \mathrm{H}$ NMR $(400$<smiles>OC(c1ccccc1)c1ccc(N2CCCC2)cc1</smiles>
$\left.\mathrm{MHz}, \mathrm{CDCl}_{3}\right) \delta(\mathrm{ppm}): 7.40-7.19(\mathrm{~m}, 7 \mathrm{H}), 6.52(\mathrm{~d}, J=8.4 \mathrm{~Hz}$, $2 \mathrm{H}), 5.78(\mathrm{~s}, 1 \mathrm{H}), 3.27(\mathrm{t}, J=6.0 \mathrm{~Hz}, 4 \mathrm{H}), 2.07(\mathrm{brs}, 1 \mathrm{H}), 1.99(\mathrm{t}$, $J=6.0 \mathrm{~Hz}, 4 \mathrm{H}) ;{ }^{13} \mathrm{C}$ NMR $\left(101 \mathrm{MHz}, \mathrm{CDCl}_{3}\right) \delta(\mathrm{ppm}): 147.5$, $144.4,130.8,128.2,127.9,127.0,126.3,111.5,76.1,47.6$, 25.4 .

HRMS (positive ESI): $\mathrm{m} / \mathrm{z}$ calculated for $\mathrm{C}_{17} \mathrm{H}_{20} \mathrm{NO}[\mathrm{M}+\mathrm{H}]^{+}: 254.1539$; found: 254.1555 .<smiles>OC(c1ccccc1)c1ccc(N2CCCCC2)cc1</smiles>

Phenyl(4-(piperidin-1-yl)phenyl)methanol (1b-5): CAS No. 1184918-36-2.

Prepared according to procedure $A$ from Grignard reagent SG6.

White solid; m.p.: $90-91{ }^{\circ} \mathrm{C}$; yield: $587 \mathrm{mg}, 88 \%$; ${ }^{1} \mathrm{H}$ NMR $(400$ $\left.\mathrm{MHz}, \mathrm{CDCl}_{3}\right) \delta(\mathrm{ppm}): 7.34-7.20(\mathrm{~m}, 5 \mathrm{H}), 7.17(\mathrm{~d}, J=8.4 \mathrm{~Hz}, 2 \mathrm{H}), 6.84(\mathrm{~d}, J=8.8 \mathrm{~Hz}, 2 \mathrm{H})$, $5.71(\mathrm{~d}, J=3.6 \mathrm{~Hz}, 1 \mathrm{H}$ ), 3.09 (t, $J=5.4 \mathrm{~Hz}, 4 \mathrm{H}$ ), 2.28-2.25 (brm, 1H), 1.65 (qui, $J=5.6 \mathrm{~Hz}$, $4 \mathrm{H}$ ), 1.52 (qui, $J=5.6 \mathrm{~Hz}, 2 \mathrm{H}$ ); ${ }^{13} \mathrm{C}$ NMR $\left(101 \mathrm{MHz}, \mathrm{CDCl}_{3}\right) \delta$ (ppm): 151.6, 144.1, 134.4, $128.3,127.5,127.2,126.4,116.2,75.8,50.5,25.7,24.2$.<smiles>OC(c1ccccc1)c1ccc(N2CCc3ccccc3C2)cc1</smiles>

(4-(3,4-Dihydroisoquinolin-2(1H)-yl)phenyl)(phenyl)m ethanol (1b-6):

Prepared according to procedure $A$ from Grignard reagent SG7.

Brown oil; yield: $622 \mathrm{mg}, 79 \%$; ${ }^{1} \mathrm{H}$ NMR $\left(400 \mathrm{MHz}, \mathrm{CDCl}_{3}\right) \delta(\mathrm{ppm}): 7.40$ (d, J = $7.2 \mathrm{~Hz}$, $2 \mathrm{H}), 7.34(\mathrm{~d}, J=7.6 \mathrm{~Hz}, 2 \mathrm{H}), 7.28-7.14(\mathrm{~m}, 7 \mathrm{H}), 6.94(\mathrm{~d}, J=8.8 \mathrm{~Hz}, 2 \mathrm{H}), 5.80(\mathrm{~d}, J=3.6$ $\mathrm{Hz}, 1 \mathrm{H}), 4.41(\mathrm{~s}, 2 \mathrm{H}), 3.56(\mathrm{t}, J=6.0 \mathrm{~Hz}, 2 \mathrm{H}), 2.98(\mathrm{t}, J=6.0 \mathrm{~Hz}, 2 \mathrm{H}), 2.16(\mathrm{~d}, J=3.6 \mathrm{~Hz}$, $1 \mathrm{H}) ;{ }^{13} \mathrm{C}$ NMR $\left(101 \mathrm{MHz}, \mathrm{CDCl}_{3}\right) \delta$ (ppm): 149.9, 144.4, 134.8, 134.3, 133.9, 128.5, 128.3, $127.7,127.2,126.5,126.4,126.3,126.0,114.8,75.9,50.6,46.3,29.0$.

HRMS (positive ESI): $\mathrm{m} / \mathrm{z}$ calculated for $\mathrm{C}_{22} \mathrm{H}_{22} \mathrm{NO}[\mathrm{M}+\mathrm{H}]^{+}: 316.1696$; found: 316.1708 .

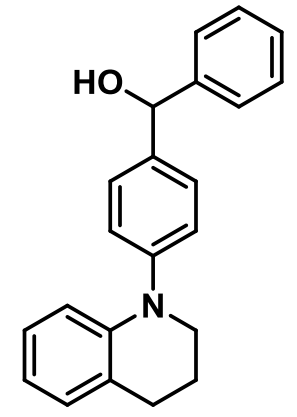

(4-(3,4-Dihydroquinolin-1(2H)-yl)phenyl)(phenyl)methanol (1b-7): Prepared according to procedure $A$ from Grignard reagent SG8.

Yellow oil; yield: $610 \mathrm{mg}, 77 \%$; ${ }^{1} \mathrm{H}$ NMR $\left(400 \mathrm{MHz}, \mathrm{CDCl}_{3}\right) \delta(\mathrm{ppm})$ : 7.45-7.28 (m, 7H), $7.21(\mathrm{~d}, J=8.8 \mathrm{~Hz}, 2 \mathrm{H}), 7.06(\mathrm{~d}, J=7.2 \mathrm{~Hz}, 1 \mathrm{H})$, $6.94(\mathrm{td}, J=7.8 \mathrm{~Hz}, 1.6 \mathrm{~Hz}, 1 \mathrm{H}), 6.80(\mathrm{~d}, J=8.0 \mathrm{~Hz}, 1 \mathrm{H}), 6.72(\mathrm{td}, J=$ $7.2 \mathrm{~Hz}, 0.8 \mathrm{~Hz}, 1 \mathrm{H}), 5.84(\mathrm{~d}, J=2.8 \mathrm{~Hz}, 1 \mathrm{H}), 3.62(\mathrm{t}, J=6.0 \mathrm{~Hz}, 2 \mathrm{H})$, 2.85 (t, $J=6.0 \mathrm{~Hz}, 2 \mathrm{H}$ ), 2.30 (d, $J=3.6 \mathrm{~Hz}, 1 \mathrm{H}$ ), 2.04 (qui, $J=6.2 \mathrm{~Hz}$, $2 \mathrm{H}) ;{ }^{13} \mathrm{C}$ NMR $\left(101 \mathrm{MHz}, \mathrm{CDCl}_{3}\right) \delta$ (ppm): 147.7, 144.0, 143.8, 138.6, $129.3,128.5,127.6,127.5,126.4,126.3,124.9,124.1,118.5,116.0,75.9,50.6,27.7$, 22.7.

HRMS (positive ESI): m/z calculated for $\mathrm{C}_{22} \mathrm{H}_{22} \mathrm{NO}[\mathrm{M}+\mathrm{H}]^{+}:$316.1696; found: 316.1703 .

(4-(Methylamino)phenyl)(phenyl)methanol (1b-8): CAS No. 1824251-28-6 
Prepared according to procedure $B$ from the aldehyde 4-(Methylamino)benzaldehyde.<smiles>CNc1ccc(C(O)c2ccccc2)cc1</smiles>

Pale yellow liquid; yield: $394 \mathrm{mg}, 74 \%$; ${ }^{1} \mathrm{H}$ NMR $(400 \mathrm{MHz}$, $\left.\mathrm{CD}_{3} \mathrm{COCD}_{3}\right) \delta(\mathrm{ppm}): 7.40(\mathrm{~d}, J=7.2 \mathrm{~Hz}, 2 \mathrm{H}), 7.28(\mathrm{t}, J=7.6$ $\mathrm{Hz}, 2 \mathrm{H}), 7.20-7.17(\mathrm{~m}, 1 \mathrm{H}), 7.12(\mathrm{~d}, J=8.4 \mathrm{~Hz}, 2 \mathrm{H}), 5.69(\mathrm{~d}, J=$ $2.8 \mathrm{~Hz}, 1 \mathrm{H}), 4.83(\mathrm{brs}, 1 \mathrm{H}), 4.48(\mathrm{~d}, J=3.6 \mathrm{~Hz}, 0 \mathrm{H}), 2.75(\mathrm{~s}, 3 \mathrm{H})$ ${ }^{13} \mathrm{C}$ NMR $\left(101 \mathrm{MHz}, \mathrm{CD}_{3} \mathrm{COCD}_{3}\right) \delta(\mathrm{ppm}): 150.1,147.1,134.1$, $128.7,128.4,127.2,127.1,112.4,76.1,30.6$.<smiles>CC(=O)Nc1ccc(C(O)c2ccccc2)cc1</smiles>

\section{N-(4-(hydroxy(phenyl)methyl)phenyl)acetamide}

(1b-9): CAS No. 108714-83-6.

Prepared according to procedure $B$ from the aldehyde $\mathrm{N}$-(4-formylphenyl)acetamide.

White solid; m.p.: $142-143{ }^{\circ} \mathrm{C}$ (reported $\left.147{ }^{\circ} \mathrm{C}\right) ;{ }^{13}$ yield: 470 mg, 78\%; ${ }^{1} \mathrm{H}$ NMR (400 MHz, DMSO- $\left.d_{6}\right) \delta(\mathrm{ppm}): 9.88(\mathrm{~s}, 1 \mathrm{H}), 7.50(\mathrm{~d}, J=8.4 \mathrm{~Hz}, 2 \mathrm{H}$ ), 7.36-7.17 (m, 7H), $5.81(\mathrm{~d}, J=4.0 \mathrm{~Hz}, 1 \mathrm{H}), 5.64(\mathrm{~d}, J=2.8 \mathrm{~Hz}, 1 \mathrm{H}), 2.02(\mathrm{~s}, 3 \mathrm{H}) ;{ }^{13} \mathrm{C}$ NMR $\left(101 \mathrm{MHz}, \mathrm{DMSO}-d_{6}\right) \delta(\mathrm{ppm}): 168.1,145.8,140.3,137.9,128.0,126.6,126.5,126.2$, $118.8,73.9,23.9$.<smiles>CN(C)c1ccc(C(O)C2CCCCC2)cc1</smiles>

Cyclohexyl(4-(dimethylamino)phenyl)methanol (1g): CAS No. 101355-74-2.

Prepared according to procedure $A$ from Grignard reagent SG1. White solid; m.p.: $86-87^{\circ} \mathrm{C}$ (reported $86-87^{\circ} \mathrm{C}$ ) ${ }^{14}$; yield: $513 \mathrm{mg}$, $88 \% ;{ }^{1} \mathrm{H}$ NMR $\left(400 \mathrm{MHz}, \mathrm{CDCl}_{3}\right) \delta(\mathrm{ppm}): 7.17(\mathrm{~d}, J=8.8 \mathrm{~Hz}$,

2H), $6.72(\mathrm{~d}, J=8.8 \mathrm{~Hz}, 2 \mathrm{H}), 4.24(\mathrm{~d}, J=7.6 \mathrm{~Hz}, 1 \mathrm{H}), 2.95$ (s, 6H), 1.85 (brs, 1H), 1.68-0.85 (m, 11H); $\left.{ }^{13} \mathrm{C} \mathrm{NMR} \mathrm{(101} \mathrm{MHz,} \mathrm{CDCl}_{3}\right) \delta$ (ppm): 150.0, 131.7, 127.5, 112.3, 79.2, $44.8,40.6,29.3,26.4,26.1,26.0$.

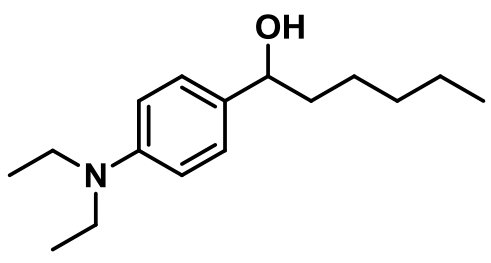

1-(4-(Diethylamino)phenyl)hexan-1-ol (1h):

Prepared according to procedure $A$ from Grignard reagent SG4.

Yellow oil; yield: $517 \mathrm{mg}, 83 \%$; ${ }^{1} \mathrm{H}$ NMR $\left(400 \mathrm{MHz}, \mathrm{CDCl}_{3}\right)$ $\delta(\mathrm{ppm}): 7.20(\mathrm{~d}, J=8.8 \mathrm{~Hz}, 2 \mathrm{H}), 6.70(\mathrm{~d}, J=8.4 \mathrm{~Hz}, 2 \mathrm{H})$, $4.54(\mathrm{t}, J=6.4 \mathrm{~Hz}, 1 \mathrm{H}), 3.37(\mathrm{t}, J=7.2 \mathrm{~Hz}, 4 \mathrm{H}), 1.90-1.67(\mathrm{~m}, 3 \mathrm{H}), 1.48-1.31(\mathrm{~m}, 6 \mathrm{H}), 1.18$ (t, $J=7.2 \mathrm{~Hz}, 6 \mathrm{H}), 0.90$ (t, $J=6.4 \mathrm{~Hz}, 3 \mathrm{H}) ;{ }^{13} \mathrm{C} \mathrm{NMR}\left(101 \mathrm{MHz}, \mathrm{CDCl}_{3}\right) \delta(\mathrm{ppm}): 147.2$, 131.6, 127.1, 111.6, 74.4, 44.3, 38.5, 31.8, 25.7, 22.6, 14.0, 12.5.

HRMS (positive ESI): m/z calculated for $\mathrm{C}_{16} \mathrm{H}_{28} \mathrm{NO}[\mathrm{M}+\mathrm{H}]^{+}:$250.2165; found: 250.2168 .<smiles>CCCCCCCC(O)c1ccc(N(C)C)cc1</smiles>

1-(4-(Dimethylamino)phenyl)octan-1-ol (1i): CAS No. 26751-77-9.

Prepared according to procedure $A$ from Grignard reagent SG1.

Pale yellow oil; yield: $510 \mathrm{mg}, 82 \%$; ${ }^{1} \mathrm{H}$ NMR $(400 \mathrm{MHz}$, $\left.\mathrm{CD}_{3} \mathrm{COCD}_{3}\right) \delta(\mathrm{ppm}): 7.17(\mathrm{dt}, J=8.8 \mathrm{~Hz}, 2.0 \mathrm{~Hz}, 2 \mathrm{H}), 6.70(\mathrm{dt}, J=8.8 \mathrm{~Hz}, 2.0 \mathrm{~Hz}, 2 \mathrm{H}$ ), $4.51(\mathrm{td}, J=6.4 \mathrm{~Hz}, 4.4 \mathrm{~Hz}, 1 \mathrm{H}), 3.76(\mathrm{~d}, J=4.0 \mathrm{~Hz}, 1 \mathrm{H}), 2.90(\mathrm{~s}, 6 \mathrm{H}), 1.74-1.66(\mathrm{~m}, 2 \mathrm{H})$, 
1.35-1.25 (m, 10H), $\left.0.88(\mathrm{t}, J=6.0 \mathrm{~Hz}, 3 \mathrm{H}) ;{ }^{13} \mathrm{C} \mathrm{NMR} \mathrm{(101} \mathrm{MHz,} \mathrm{CD}_{3} \mathrm{COCD}_{3}\right) \delta(\mathrm{ppm}):$ $150.8,135.1,127.5,113.1,74.1,40.8,40.4,32.6,30.4,30.1,26.8,23.3,14.4$.<smiles>CN(C)c1ccc(C(O)CCc2ccccc2)cc1</smiles>

(1j): CAS No. 101581-84-4.

Prepared according to procedure $A$ from Grignard reagent SG1.

White solid; m.p.: $70-71^{\circ} \mathrm{C}$ (reported $\left.69-71{ }^{\circ} \mathrm{C}\right)^{10}$; yield: 536 mg, 84\%; ${ }^{1} \mathrm{H} \mathrm{NMR}\left(400 \mathrm{MHz}, \mathrm{CDCl}_{3}\right) \delta$ (ppm): 7.34-7.21 (m, 7H), $6.77(\mathrm{~d}, J=8.4 \mathrm{~Hz}, 2 \mathrm{H})$, $4.63(\mathrm{t}, J=6.4 \mathrm{~Hz}, 1 \mathrm{H}), 3.00(\mathrm{~s}, 6 \mathrm{H}), 2.81-2.64(\mathrm{~m}, 2 \mathrm{H}), 2.23-2.02(\mathrm{~m}, 2 \mathrm{H}), 1.86$ (brs, $1 \mathrm{H})$; ${ }^{13} \mathrm{C}$ NMR $\left(101 \mathrm{MHz}, \mathrm{CDCl}_{3}\right) \delta(\mathrm{ppm}): 150.2,142.0,132.2,128.4,128.3,127.0,125.7$, $112.5,73.7,40.6,40.0,32.3$.<smiles>CC(C)=CCCC(C)CC(O)c1ccc(N(C)C)cc1</smiles>
1-(4-(Dimethylamino)phenyl)-3,7-dimethyloct-6-en-1-ol (1k): Prepared according to procedure $A$ from Grignard reagent SG1. Pale yellow oil; yield: $605 \mathrm{mg}, 88 \% ;{ }^{1} \mathrm{H} \mathrm{NMR}\left(400 \mathrm{MHz}, \mathrm{CDCl}_{3}\right) \delta$ (ppm): 7.22 (d, J = 8.8 Hz, 2H), $6.72(\mathrm{~d}, J=8.8 \mathrm{~Hz}, 2 \mathrm{H}$ ), 5.07 (t, $J$ $=7.0 \mathrm{~Hz}, 1 \mathrm{H}), 4.67(\mathrm{t}, J=7.0 \mathrm{~Hz}, 1 \mathrm{H}), 2.95(\mathrm{~s}, 6 \mathrm{H}), 2.05-1.13(\mathrm{~m}$, $14 \mathrm{H}), 0.94(\mathrm{~d}, J=6.4 \mathrm{~Hz}, 3 \mathrm{H}) ;{ }^{13} \mathrm{C} \mathrm{NMR}\left(101 \mathrm{MHz}, \mathrm{CDCl}_{3}\right) \delta(\mathrm{ppm}): 150.3,132.7,131.1$, 127.1, 124.9, 112.6, 72.6, 45.7, 40.7, 37.0, 29.5, 25.7, 25.3, 20.0, 17.7.

HRMS (positive ESI): $\mathrm{m} / \mathrm{z}$ calculated for $\mathrm{C}_{18} \mathrm{H}_{30} \mathrm{NO}[\mathrm{M}+\mathrm{H}]^{+}:$276.2322; found: 276.2322 .<smiles>CN(C)c1ccc(C(O)c2ccc3c(c2)OCO3)cc1</smiles>

\section{Benzo[d][1,3]dioxol-5-yl(4-(dimethylamino)phenyl)meth} anol (11):

Prepared according to procedure $A$ from Grignard reagent SG1.

White solid; m.p.: $60-61{ }^{\circ} \mathrm{C}$; yield: $542 \mathrm{mg}, 80 \%$; ${ }^{1} \mathrm{H}$ NMR (400 MHz, $\left.\mathrm{CDCl}_{3}\right) \delta(\mathrm{ppm}): 7.21(\mathrm{~d}, J=8.8 \mathrm{~Hz}, 2 \mathrm{H}), 6.87-6.84(\mathrm{~m}, 2 \mathrm{H}), 6.76(\mathrm{~d}, J=8.0 \mathrm{~Hz}$, $1 \mathrm{H}), 6.71(\mathrm{~d}, J=8.8 \mathrm{~Hz}, 2 \mathrm{H}), 5.91(\mathrm{~s}, 2 \mathrm{H}), 5.66(\mathrm{~s}, 1 \mathrm{H}), 2.94(\mathrm{~s}, 6 \mathrm{H}), 2.54$ (brs, $1 \mathrm{H}) ;{ }^{13} \mathrm{C}$ NMR (101 MHz, $\left.\mathrm{CDCl}_{3}\right) \delta$ (ppm): 150.0, 147.5, 146.5, 138.5, 132.0, 127.4, 119.6, 112.5, 107.8, 107.0, 100.8, 75.5, 40.5 .

HRMS (positive ESI): m/z calculated for $\mathrm{C}_{16} \mathrm{H}_{18} \mathrm{NO}_{3}[\mathrm{M}+\mathrm{H}]^{+}:$272.1281; found: 272.1284 .

\section{(4-(Butyl(methyl)amino)phenyl)(4-(dimethylamino)phenyl)methanol (1m):}

Prepared according to procedure $A$ from Grignard reagent SG1.

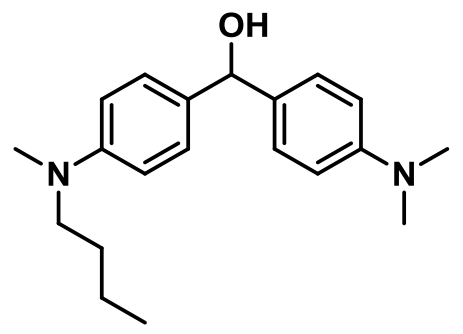

Pale yellow oil; yield: $585 \mathrm{mg}, 75 \%$; ${ }^{1} \mathrm{H}$ NMR $(400 \mathrm{MHz}$, $\left.\mathrm{CD}_{3} \mathrm{COCD}_{3}\right) \delta(\mathrm{ppm}): 7.20(\mathrm{~d}, J=8.8 \mathrm{~Hz}, 2 \mathrm{H}), 7.17(\mathrm{~d}, J=$ $8.8 \mathrm{~Hz}, 2 \mathrm{H}), 6.68(\mathrm{~d}, J=8.8 \mathrm{~Hz}, 2 \mathrm{H}), 6.64(\mathrm{~d}, J=8.8 \mathrm{~Hz}$, $2 \mathrm{H}), 5.61(\mathrm{~d}, J=4.0 \mathrm{~Hz}, 1 \mathrm{H}), 4.19(\mathrm{~d}, J=4.0 \mathrm{~Hz}, 1 \mathrm{H}), 3.31$ (t, $J=7.6 \mathrm{~Hz}, 2 \mathrm{H}$ ), 2.89-2.86 (m, 9H), 1.53 (qui, $J=7.6 \mathrm{~Hz}$, $2 \mathrm{H}), 1.39-1.30(\mathrm{~m}, 2 \mathrm{H}), 0.93(\mathrm{t}, J=7.6 \mathrm{~Hz}, 3 \mathrm{H}) ;{ }^{13} \mathrm{C}$ NMR $\left(101 \mathrm{MHz}, \mathrm{CD}_{3} \mathrm{COCD}_{3}\right) \delta(\mathrm{ppm}):$ 150.6, 149.3, 135.1, 134.3,

128.2, 128.1, 113.1, 112.5, 75.7, 53.0, 40.8, 38.5, 29.5, 20.9, 14.3.

HRMS (positive ESI): $\mathrm{m} / \mathrm{z}$ calculated for $\mathrm{C}_{20} \mathrm{H}_{29} \mathrm{~N}_{2} \mathrm{O}[\mathrm{M}+\mathrm{H}]^{+}: 313.2274$; found: 313.2274 . 
(4-(Dimethylamino)phenyl)(mesityl)methanol (1n): CAS No. 93948-30-2.

Prepared according to procedure $A$ from Grignard reagent SG1.<smiles>Cc1cc(C)c(C(O)c2ccc(N(C)C)cc2)c(C)c1</smiles>

Yellow oil; yield: $457 \mathrm{mg}, 68 \% ;{ }^{1} \mathrm{H}$ NMR $\left(400 \mathrm{MHz}, \mathrm{CDCl}_{3}\right) \delta$ (ppm): 7.16 (d, J = 8.8 Hz, 2H), 6.89 (s, 2H), 6.72 (d, $J=8.8$ $\mathrm{Hz}, 2 \mathrm{H}), 6.29$ (s, 1H), 2.95 (s, 6H), $2.33(\mathrm{~s}, 3 \mathrm{H}), 2.69$ (s, 6H);

${ }^{13} \mathrm{C} \mathrm{NMR}\left(101 \mathrm{MHz}, \mathrm{CDCl}_{3}\right) \delta$ (ppm): 149.4, 136.9, 136.8, $136.6,130.9,129.9,126.5,112.4,71.0,40.7,20.8,20.6$.<smiles>CSc1ccc(C(O)c2ccc(N(C)C)cc2)cc1</smiles>

\section{(4-(Dimethylamino)phenyl)(4-(methylthio)phenyl)metha nol (10): \\ Prepared according to procedure $A$ from Grignard reagent SG1.}

Brown solid; m.p.: $85-86{ }^{\circ} \mathrm{C}$; yield: $594 \mathrm{mg}, 87 \%$; ${ }^{1} \mathrm{H}$ NMR $\left(400 \mathrm{MHz}, \mathrm{CDCl}_{3}\right) \delta$ (ppm): 7.31 $(\mathrm{d}, J=8.0 \mathrm{~Hz}, 2 \mathrm{H}), 7.21(\mathrm{t}, J=9.2 \mathrm{~Hz}, 4 \mathrm{H}), 6.69(\mathrm{~d}, J=8.4 \mathrm{~Hz}, 2 \mathrm{H}), 5.73(\mathrm{~s}, 1 \mathrm{H}), 2.93(\mathrm{~s}$, $6 \mathrm{H}$ ), 2.47 (s, 3H), 2.15 (brs, $1 \mathrm{H}) ;{ }^{13} \mathrm{C}$ NMR (101 MHz, $\left.\mathrm{CDCl}_{3}\right) \delta$ (ppm): 150.2, 141.3, 137.0, $131.8,127.7,127.6,126.9,126.8,126.7,112.4,75.5,40.6,16.0$.

HRMS (positive ESI): $\mathrm{m} / \mathrm{z}$ calculated for $\mathrm{C}_{16} \mathrm{H}_{20} \mathrm{NOS}[\mathrm{M}+\mathrm{H}]^{+}: 274.1260$; found: 274.1268 .<smiles>COc1ccc(C(O)c2ccc(N(C)C)cc2)cc1</smiles>

\section{(4-(Dimethylamino)phenyl)(4-methoxyphenyl)methanol} (1p): CAS No. 94207-51-9.

Prepared according to procedure $A$ from Grignard reagent SG1.

white solid; m.p.: 73-74 ${ }^{\circ} \mathrm{C}$ (reported $\left.78-79{ }^{\circ} \mathrm{C}\right) ;{ }^{15}$ yield: $567 \mathrm{mg}, 88 \%$; ${ }^{1} \mathrm{H}$ NMR $(400 \mathrm{MHz}$, $\left.\mathrm{CDCl}_{3}\right) \delta(\mathrm{ppm}): 7.30(\mathrm{~d}, J=8.4 \mathrm{~Hz}, 2 \mathrm{H}), 7.22(\mathrm{~d}, J=8.4 \mathrm{~Hz}, 2 \mathrm{H}), 6.87(\mathrm{~d}, J=8.4 \mathrm{~Hz}, 2 \mathrm{H}$ ), $6.71(\mathrm{~d}, \mathrm{~J}=8.0 \mathrm{~Hz}, 2 \mathrm{H}), 5.73(\mathrm{~s}, 1 \mathrm{H}), 3.80(\mathrm{~s}, 3 \mathrm{H}), 2.93(\mathrm{~s}, 6 \mathrm{H}), 2.36$ (brs, $1 \mathrm{H}) ;{ }^{13} \mathrm{C} \mathrm{NMR}$ $\left(101 \mathrm{MHz}, \mathrm{CDCl}_{3}\right) \delta$ (ppm): 158.6, 150.0, 136.6, 132.2, 127.6, 127.5, 113.6, 112.4, 75.4, $55.2,40.6$.

(4-(Dimethylamino)phenyl)(4-tolyl)methanol (1q): CAS No. 93026-72-3.

Prepared according to procedure $A$ from Grignard reagent SG1.<smiles>Cc1ccc(C(O)c2ccc(N(C)C)cc2)cc1</smiles>
white solid; m.p.: $90-92{ }^{\circ} \mathrm{C}$ (reported $87-88{ }^{\circ} \mathrm{C}$ ); ${ }^{16}$ yield: 536 mg, 89\%; ${ }^{1} \mathrm{H}$ NMR $\left(400 \mathrm{MHz}, \mathrm{CDCl}_{3}\right) \delta$ (ppm): 7.28 (d, $J=8.4$ $\mathrm{Hz}, 2 \mathrm{H}$ ), 7.22 (d, $J=8.4 \mathrm{~Hz}, 2 \mathrm{H}), 7.14(\mathrm{~d}, J=8.0 \mathrm{~Hz}, 2 \mathrm{H}), 6.70$ (d, J = 8.4 Hz, 2H), $5.75(\mathrm{~s}, 1 \mathrm{H}), 2.93(\mathrm{~s}, 6 \mathrm{H}), 2.34(\mathrm{~s}, 3 \mathrm{H})$, 2.14 (brs, $\left.1 \mathrm{H}) ;{ }^{13} \mathrm{C} \mathrm{NMR} \mathrm{(101} \mathrm{MHz,} \mathrm{CDCl}_{3}\right) \delta$ (ppm): 150.1, 141.4, 136.7, 132.1, 128.9, 127.6, 126.3, 112.5, 75.8, 40.6, 21.1.<smiles>Cc1cccc(C(O)c2ccc(N(C)C)cc2)c1</smiles>
(4-(Dimethylamino)phenyl)(3-tolyl)methanol (1r): CAS No. 844683-28-9. Prepared according to procedure $A$ from Grignard reagent SG1. 
white solid; m.p.: 83-84 ${ }^{\circ} \mathrm{C}$; yield: $494 \mathrm{mg}, 82 \%$; ${ }^{1} \mathrm{H}$ NMR $\left(400 \mathrm{MHz}, \mathrm{CDCl}_{3}\right) \delta(\mathrm{ppm})$ : 7.17-7.10 (m, 5H), 6.99-6.93 (m, 1H), $6.62(\mathrm{~d}, J=8.8 \mathrm{~Hz}, 2 \mathrm{H}), 5.66(\mathrm{~s}, 1 \mathrm{H}), 2.35(\mathrm{~s}, 3 \mathrm{H})$, $2.94(\mathrm{~s}, 6 \mathrm{H}), 2.17-2.12(\mathrm{brm}, 1 \mathrm{H}) ;{ }^{13} \mathrm{C}$ NMR $\left(101 \mathrm{MHz}, \mathrm{CDCl}_{3}\right) \delta$ (ppm): 150.0, 144.2, $137.9,128.5,128.2,127.9,127.7,127.0,123.4,112.5,76.0,40.6,21.5$.<smiles>Cc1ccccc1C(O)c1ccc(N(C)C)cc1</smiles>

(4-(Dimethylamino)phenyl)(2-tolyl)methanol (1s): CAS No. 93026-71-2.

Prepared according to procedure $A$ from Grignard reagent SG1. white solid; m.p.: $79-81{ }^{\circ} \mathrm{C}$ (reported $82-83^{\circ} \mathrm{C}$ ) ${ }^{14}$; yield: $496 \mathrm{mg}$, $82 \% ;{ }^{1} \mathrm{H}$ NMR $\left(400 \mathrm{MHz}, \mathrm{CDCl}_{3}\right) \delta(\mathrm{ppm}): 7.66$ (d, $J=7.6 \mathrm{~Hz}$, $1 \mathrm{H}), 7.30$ (t, J=3.4 Hz, 1H), 7.24-7.14 (m, 4H), $6.71(\mathrm{~d}, J=8.4 \mathrm{~Hz}, 2 \mathrm{H}), 5.90(\mathrm{~s}, 1 \mathrm{H}), 2.95$ (s, 6H), 2.33 (brs, 1H), 2.22 (s, 3H); $\left.{ }^{13} \mathrm{C} \mathrm{NMR} \mathrm{(101} \mathrm{MHz,} \mathrm{CDCl}_{3}\right) \delta$ (ppm): 150.0, 141.9, $135.0,130.8,130.2,128.2,127.0,125.8,125.6,112.4,73.0,40.5,19.3$.<smiles>CC(C)c1ccc(C(O)c2ccc(N(C)C)cc2)cc1</smiles>

(4-(Dimethylamino)phenyl)(4-isopropylphenyl)methanol (1t): CAS No. 1225898-41-8.

Prepared according to procedure $A$ from Grignard reagent SG1.

white solid; m.p.: 88-89 ${ }^{\circ} \mathrm{C}$, yield: $570 \mathrm{mg}, 85 \%$; ${ }^{1} \mathrm{H}$ NMR (400 MHz, $\left.\mathrm{CDCl}_{3}\right) \delta$ (ppm): 7.31 (d, $J=8.0 \mathrm{~Hz}, 2 \mathrm{H}$ ), $7.24(\mathrm{~d}, J=8.4 \mathrm{~Hz}, 2 \mathrm{H}), 7.19(\mathrm{~d}, J=7.6 \mathrm{~Hz}, 2 \mathrm{H}), 6.71(\mathrm{~d}, J=8.4 \mathrm{~Hz}$, 2H), 5.76 (s, 1H), 3.05 (s, 6H), 2.89 (sep, J = 2.8 Hz, 1H), 2.13 (brs, 1H), 1.24 (d, J = 2.8 $\mathrm{Hz}, 6 \mathrm{H}) ;{ }^{13} \mathrm{C}$ NMR $\left(101 \mathrm{MHz}, \mathrm{CDCl}_{3}\right) \delta$ (ppm): 150.1, 147.75, 147.74, 132.1, 127.6, $126.35,126.32,112.4,75.8,40.6,33.8,24.0$.<smiles>CN(C)c1ccc(C(O)c2ccc(-c3ccccc3)cc2)cc1</smiles>

[1,1'-Biphenyl]-4-yl(4-(dimethylamino)phenyl)methan ol (1u) CAS No. 94964-68-8.

Prepared according to procedure $A$ from Grignard reagent SG1.

Pale yellow solid; m.p.:144-146 ${ }^{\circ} \mathrm{C}$; yield: $561 \mathrm{mg}, 74 \%$; ${ }^{1} \mathrm{H} \mathrm{NMR}\left(400 \mathrm{MHz}, \mathrm{CDCl}_{3}\right) \delta$ (ppm): 7.55 (t, $J=8.0 \mathrm{~Hz}, 4 \mathrm{H}), 7.43(\mathrm{t}, J=8.4 \mathrm{~Hz}, 4 \mathrm{H}), 7.32(\mathrm{~d}, J=7.2 \mathrm{~Hz}, 1 \mathrm{H}), 7.24(\mathrm{~d}, J$ $=8.0 \mathrm{~Hz}, 2 \mathrm{H}), 6.70(\mathrm{~d}, J=7.6 \mathrm{~Hz}, 2 \mathrm{H}), 5.79(\mathrm{~s}, 1 \mathrm{H}), 2.91(\mathrm{~s}, 6 \mathrm{H}), 2.26(\mathrm{brs}, 1 \mathrm{H}) ;{ }^{13} \mathrm{C} \mathrm{NMR}$ $\left(101 \mathrm{MHz}, \mathrm{CDCl}_{3}\right) \delta$ (ppm): 150.2, 143.4, 140.9, 140.0, 131.9, 129.9, 128.7, 127.7, 127.1, $127.0,126.7,112.5,75.7,40.6$.<smiles>CN(C)c1ccc(C(O)c2ccc(F)cc2)cc1</smiles>

(4-(Dimethylamino)phenyl)(4-fluorophenyl)methanol (1v): CAS No. 39768-80-4.

Prepared according to procedure $A$ from Grignard reagent SG1.

White solid; m.p.:70-71 ${ }^{\circ} \mathrm{C}$; yield: $544 \mathrm{mg}, 89 \%$; ${ }^{1} \mathrm{H}$ NMR $(400$ $\left.\mathrm{MHz}, \mathrm{CDCl}_{3}\right) \delta(\mathrm{ppm}): 7.36(\mathrm{dd}, J=8.4 \mathrm{~Hz}, 5.6 \mathrm{~Hz}, 2 \mathrm{H}), 7.19(\mathrm{~d}, J=8.4 \mathrm{~Hz}, 2 \mathrm{H}), 7.01(\mathrm{~d}, J$ $=8.4 \mathrm{~Hz}, 2 \mathrm{H}), 6.70(\mathrm{~d}, J=8.8 \mathrm{~Hz}, 2 \mathrm{H}), 5.74(\mathrm{~s}, 1 \mathrm{H}), 2.94(\mathrm{~s}, 6 \mathrm{H}), 2.28(\mathrm{brs}, 1 \mathrm{H}) ;{ }^{13} \mathrm{C} \mathrm{NMR}$ $\left(101 \mathrm{MHz}, \mathrm{CDCl}_{3}\right) \delta(\mathrm{ppm}): 161.9\left(J_{C-F}=245.4 \mathrm{~Hz}\right), 150.2,140.0,131.7,127.9\left(J_{C-F}=8.0\right.$ 
$\mathrm{Hz}), 127.6,115.0\left(J_{C-F}=21.2 \mathrm{~Hz}\right), 112.5,75.3,40.5$.<smiles>CN(C)c1ccc(C(O)c2ccc(Cl)cc2)cc1</smiles>

(4-Chlorophenyl)(4-(dimethylamino)phenyl)methanol (1w): CAS No. 844683-40-5 Prepared according to procedure $A$ from Grignard reagent SG1.

White solid; m.p.: $100-101^{\circ} \mathrm{C}$ (reported $\left.103-104{ }^{\circ} \mathrm{C}\right) ;{ }^{17}$ yield: $435 \mathrm{mg}, 82 \%$; ${ }^{1} \mathrm{H}$ NMR $\left(400 \mathrm{MHz}, \mathrm{CDCl}_{3}\right) \delta$ (ppm): 7.36-7.28 (m, 4H), $7.18(\mathrm{~d}, J=8.8 \mathrm{~Hz}$, $2 \mathrm{H}), 6.69(\mathrm{~d}, J=8.8 \mathrm{~Hz}, 2 \mathrm{H}), 5.73(\mathrm{~s}, 1 \mathrm{H}), 2.94(\mathrm{~s}, 6 \mathrm{H}), 2.28(\mathrm{bd}, J=2.4 \mathrm{~Hz}, 1 \mathrm{H}) ;{ }^{13} \mathrm{C} \mathrm{NMR}$ $\left(101 \mathrm{MHz}, \mathrm{CDCl}_{3}\right) \delta$ (ppm): 150.2, 142.7, 132.7, 131.4, 128.3, 127.72, 127.67, 112.5, 75.3, 40.5 .<smiles>CN(C)c1ccc(C(O)c2ccc(Br)cc2)cc1</smiles>

\section{(4-Bromophenyl)(4-(dimethylamino)phenyl)methanol}

(1x): CAS No. 860560-80-1. Prepared according to procedure $A$ from Grignard reagent SG1.

White solid; m.p.:115-116 ${ }^{\circ} \mathrm{C}$; yield: $653 \mathrm{mg}, 85 \%$; ${ }^{1} \mathrm{H}$ NMR $\left(400 \mathrm{MHz}, \mathrm{CDCl}_{3}\right) \delta(\mathrm{ppm}): 7.44(\mathrm{~d}, J=8.0 \mathrm{~Hz}, 2 \mathrm{H}), 7.27(\mathrm{~d}$, $J=8.0 \mathrm{~Hz}, 2 \mathrm{H}), 7.18(\mathrm{~d}, J=8.8 \mathrm{~Hz}, 2 \mathrm{H}), 6.69(\mathrm{~d}, J=8.4 \mathrm{~Hz}, 2 \mathrm{H}), 5.72(\mathrm{~s}, 1 \mathrm{H}), 2.94(\mathrm{~s}, 6 \mathrm{H})$, $2.16(\mathrm{bd}, J=2.8 \mathrm{~Hz}, 1 \mathrm{H}) ;{ }^{13} \mathrm{C} \mathrm{NMR}\left(101 \mathrm{MHz}, \mathrm{CDCl}_{3}\right) \delta(\mathrm{ppm}): 150.2,143.2,131.3,128.0$, 127.7, 120.9, 112.5, 104.9, 75.4, 40.6 .<smiles>CN(C)c1ccc(C(O)c2cccc(Br)c2)cc1</smiles>

\section{(3-Bromophenyl)(4-(dimethylamino)phenyl)methanol}

(1y):

Prepared according to procedure $A$ from Grignard reagent SG1.

Pale yellow solid; m.p.: $60-62{ }^{\circ} \mathrm{C}$; yield: $628 \mathrm{mg}, 82 \%$; ${ }^{1} \mathrm{H}$ $\operatorname{NMR}\left(400 \mathrm{MHz}, \mathrm{CDCl}_{3}\right) \delta(\mathrm{ppm}): 7.57(\mathrm{t}, J=2.4 \mathrm{~Hz}, 1 \mathrm{H}), 7.27(\mathrm{dt}, J=8.0 \mathrm{~Hz}, 2.4 \mathrm{~Hz}, 1 \mathrm{H})$, $7.29(\mathrm{~d}, J=7.6 \mathrm{~Hz}, 1 \mathrm{H}), 7.20-7.16(\mathrm{~m}, 3 \mathrm{H}), 6.69(\mathrm{~d}, J=8.8 \mathrm{~Hz}, 2 \mathrm{H}), 5.70(\mathrm{~d}, J=2.4 \mathrm{~Hz}$, $1 \mathrm{H}), 2.94(\mathrm{~s}, 6 \mathrm{H}), 2.36(\mathrm{bd}, J=3.2 \mathrm{~Hz}, 1 \mathrm{H}) ;{ }^{13} \mathrm{C} \mathrm{NMR}\left(101 \mathrm{MHz}, \mathrm{CDCl}_{3}\right) \delta(\mathrm{ppm}): 150.2$, 146.6, 131.2, 130.0, 129.8, 129.3, 127.7, 124.9, 122.4, 112.5, 75.3, 40.5.

HRMS (positive ESI): $\mathrm{m} / \mathrm{z}$ calculated for $\mathrm{C}_{15} \mathrm{H}_{17} \mathrm{NOBr}[\mathrm{M}+\mathrm{H}]^{+}: 306.0488$; found: 306.0493 .<smiles>CN(C)c1ccc(C(O)c2ccccc2Br)cc1</smiles>

(2-Bromophenyl)(4-(dimethylamino)phenyl)methanol (1z): CAS No. 1431794-46-5.

Prepared according to procedure $A$ from Grignard reagent SG1. Yellow solid; m.p.: $67-69^{\circ} \mathrm{C}$; yield: $650 \mathrm{mg}, 85 \%$; ${ }^{1} \mathrm{H}$ NMR $(400$ $\left.\mathrm{MHz}, \mathrm{CDCl}_{3}\right) \delta(\mathrm{ppm}): 7.70$ (dd, $\left.J=8.0 \mathrm{~Hz}, 1.2 \mathrm{~Hz}, 1 \mathrm{H}\right), 7.52$ (dd, $J=8.0 \mathrm{~Hz}, 0.8 \mathrm{~Hz}, 1 \mathrm{H}$ ), 7.36 (td, $J=7.6 \mathrm{~Hz}, 1.2 \mathrm{~Hz}, 1 \mathrm{H}$ ), 7.40 (d, $J=8.4 \mathrm{~Hz}, 2 \mathrm{H}), 7.13$ (td, $J=8.0 \mathrm{~Hz}, 1.2 \mathrm{~Hz}, 1 \mathrm{H}), 6.69(\mathrm{~d}, J=8.8 \mathrm{~Hz}, 2 \mathrm{H}), 6.07(\mathrm{~s}, 1 \mathrm{H}), 2.94(\mathrm{~s}, 6 \mathrm{H}), 2.16(\mathrm{bd}, J=$ $7.6 \mathrm{~Hz}, 1 \mathrm{H}) ;{ }^{13} \mathrm{C}$ NMR $\left(101 \mathrm{MHz}, \mathrm{CDCl}_{3}\right) \delta$ (ppm): 150.1, 142.9, 132.7, 130.0, 128.6, 128.2 , $128.0,127.5,122.6,112.3,74.7,40.5$.

(4-(Dimethylamino)phenyl)(4-iodophenyl)methanol (1aa):

Prepared according to procedure $A$ from Grignard reagent SG1. 
<smiles>CN(C)c1ccc(C(O)c2ccc(I)cc2)cc1</smiles>

Grey solid; m.p.: $108-110{ }^{\circ} \mathrm{C}$; yield: $740 \mathrm{mg}, 84 \%$; ${ }^{1} \mathrm{H}$ NMR $\left(400 \mathrm{MHz}, \mathrm{CDCl}_{3}\right) \delta$ (ppm): $7.64(\mathrm{~d}, J=8.4 \mathrm{~Hz}, 2 \mathrm{H}), 7.17$ (d, $J$ $=8.8 \mathrm{~Hz}, 2 \mathrm{H}), 7.14(\mathrm{~d}, J=8.4 \mathrm{~Hz}, 2 \mathrm{H}), 6.68(\mathrm{~d}, J=7.2 \mathrm{~Hz}, 2 \mathrm{H})$, $5.70(\mathrm{~s}, 1 \mathrm{H}), 2.93(\mathrm{~s}, 6 \mathrm{H}), 2.17(\mathrm{bd}, J=2.8 \mathrm{~Hz}, 1 \mathrm{H}) ;{ }^{13} \mathrm{C} \mathrm{NMR}$ $\left(101 \mathrm{MHz}, \mathrm{CDCl}_{3}\right) \delta(\mathrm{ppm}): 150.3,144.0,137.3,131.4,128.3$,

$127.8,112.5,92.5,75.5,40.6$.

HRMS (positive ESI): $\mathrm{m} / \mathrm{z}$ calculated for $\mathrm{C}_{15} \mathrm{H}_{17} \mathrm{NOI}[\mathrm{M}+\mathrm{H}]^{+}: 354.0349$; found: 354.0355 .<smiles>CN(C)c1ccc(C(O)c2ccc(C(F)(F)F)cc2)cc1</smiles>

(4-(Dimethylamino)phenyl)(4-(trifluoromethyl)phenyl)m ethanol (1ab): CAS No. 1409716-40-0.

Prepared according to procedure $A$ from Grignard reagent SG1.

White solid; m.p.: 90-92 ${ }^{\circ} \mathrm{C}$; yield: $649 \mathrm{mg}, 88 \%$; ${ }^{1} \mathrm{H} \mathrm{NMR}\left(400 \mathrm{MHz}, \mathrm{CDCl}_{3}\right) \delta$ (ppm): 7.58 $(\mathrm{d}, J=8.0 \mathrm{~Hz}, 2 \mathrm{H}), 7.51(\mathrm{~d}, J=8.0 \mathrm{~Hz}, 2 \mathrm{H}), 7.18(\mathrm{~d}, J=8.0 \mathrm{~Hz}, 2 \mathrm{H}), 6.69(\mathrm{~d}, J=8.0 \mathrm{~Hz}$, $2 \mathrm{H}$ ), 5.79 (s, 1H), $2.94(\mathrm{~s}, 6 \mathrm{H}), 2.33$ (brs, $1 \mathrm{H}) ;{ }^{13} \mathrm{C} \mathrm{NMR}\left(101 \mathrm{MHz}, \mathrm{CDCl}_{3}\right) \delta$ (ppm): 150.4, 148.1, 131.1, $129.2\left(\mathrm{q}, J_{C-F}=32.3 \mathrm{~Hz}\right), 127.8,126.5,125.1\left(\mathrm{q}, J_{C-F}=3.7 \mathrm{~Hz}\right), 124.2(\mathrm{q}$, $\left.J_{C-F}=272.7 \mathrm{~Hz}\right), 112.5,75.5,40.5$.<smiles>COC(=O)c1ccc(C(O)c2ccc(N(C)C)cc2)cc1</smiles>

\section{Methyl-4-((4-(dimethylamino)phenyl)(hydroxy)meth} yl)benzoate (1ac): CAS No. 867364-21-4

Prepared according to procedure $A$ from Grignard reagent SG1.

Pale yellow solid; m.p.: $72-74^{\circ} \mathrm{C}$; yield: $556 \mathrm{mg}, 78 \% ;{ }^{1} \mathrm{H}$ $\operatorname{NMR}\left(400 \mathrm{MHz}, \mathrm{CDCl}_{3}\right) \delta(\mathrm{ppm}): 7.98(\mathrm{~d}, J=8.0 \mathrm{~Hz}, 2 \mathrm{H}), 7.46(\mathrm{~d}, J=7.6 \mathrm{~Hz}, 2 \mathrm{H}), 7.17(\mathrm{~d}$, $J=7.6 \mathrm{~Hz}, 2 \mathrm{H}), 6.67(\mathrm{~d}, J=7.6 \mathrm{~Hz}, 2 \mathrm{H}), 5.79(\mathrm{~s}, 1 \mathrm{H}), 3.90(\mathrm{~s}, 3 \mathrm{H}), 2.93(\mathrm{~s}, 6 \mathrm{H}), 2.35$ (brs, $1 \mathrm{H}) ;{ }^{13} \mathrm{C}$ NMR (101 MHz, $\left.\mathrm{CDCl}_{3}\right) \delta$ (ppm): 167.0, 150.3, 149.3, 131.2, 129.6, 128.8, 127.9, $126.1,112.5,75.6,52.0,40.5$.<smiles>CN(C)c1ccc(C(O)c2ccc(C#N)cc2)cc1</smiles>

4-((4-(Dimethylamino)phenyl)(hydroxy)methyl)benzonitrile (1ad): CAS No. 867364-22-5.

Prepared according to procedure $A$ from Grignard reagent SG1. White solid; m.p.: $104-105^{\circ} \mathrm{C}$; yield: $510 \mathrm{mg}, 81 \%$; ${ }^{1} \mathrm{H}$ NMR $(400 \mathrm{MHz}$, $\left.\mathrm{CDCl}_{3}\right) \delta$ (ppm): 7.60 (d, $\left.J=7.6 \mathrm{~Hz}, 2 \mathrm{H}\right), 7.51(\mathrm{~d}, J=8.0 \mathrm{~Hz}, 2 \mathrm{H})$, $7.15(\mathrm{~d}, J=8.8 \mathrm{~Hz}, 2 \mathrm{H}), 6.68(\mathrm{~d}, J=8.8 \mathrm{~Hz}, 2 \mathrm{H}), 5.78(\mathrm{~s}, 1 \mathrm{H}), 2.94$ (s, $6 \mathrm{H}), 2.25$ (brs, $1 \mathrm{H}) ;{ }^{13} \mathrm{C}$ NMR (101 MHz, $\left.\mathrm{CDCl}_{3}\right) \delta$ (ppm): 150.5, $149.5,132.1,130.6,127.9,126.8,119.0,112.4,110.7,75.4,40.4$.<smiles>CN(C)c1ccc(C(O)c2ccc([N+](=O)[O-])cc2)cc1</smiles>

\section{(4-(Dimethylamino)phenyl)(4-nitrophenyl)methanol}

(1ae): CAS No. 2126163-21-9.

Prepared according to procedure $A$ from Grignard reagent SG1.

White solid; m.p.: $75-76^{\circ} \mathrm{C}$; yield: $618 \mathrm{mg}, 91 \%$; ${ }^{1} \mathrm{H}$ NMR $\left(400 \mathrm{MHz}, \mathrm{CDCl}_{3}\right) \delta(\mathrm{ppm}): 8.17(\mathrm{~d}, J=8.8 \mathrm{~Hz}, 2 \mathrm{H}), 7.57(\mathrm{~d}, J=8.8 \mathrm{~Hz}, 2 \mathrm{H}), 7.16(\mathrm{~d}, J=$ 
$8.4 \mathrm{~Hz}, 2 \mathrm{H}$ ), 6.68 (d, J = 8.8 Hz, 2H), 5.83 (s, 1H), 2.94 (s, 6H), 2.83 (brs, 1H); ${ }^{13} \mathrm{C} \mathrm{NMR}$ $\left(101 \mathrm{MHz}, \mathrm{CDCl}_{3}\right) \delta$ (ppm): 151.5, 150.5, 146.9, 130.4, 127.9, 126.9, 123.5, 112.5, 75.3, 40.4 .<smiles>CN(C)c1ccc(C(O)c2ccc3ccccc3c2)cc1</smiles>

\section{(4-(Dimethylamino)phenyl)(naphthalen-2-yl)methanol} (1af): CAS No. 95278-80-1.

Prepared according to procedure $A$ from Grignard reagent SG1.

White solid; m.p.: $93-94^{\circ} \mathrm{C}$; yield: $440 \mathrm{mg}, 77 \%$; ${ }^{1} \mathrm{H}$ NMR (400 MHz, $\left.\mathrm{CDCl}_{3}\right) \delta$ (ppm): $7.96(\mathrm{~s}, 1 \mathrm{H}), 7.89-7.80(\mathrm{~m}, 3 \mathrm{H}), 7.53-7.45(\mathrm{~m}, 3 \mathrm{H}), 7.29(\mathrm{~d}, J=$ $8.8 \mathrm{~Hz}, 2 \mathrm{H}), 6.73(\mathrm{~d}, J=8.8 \mathrm{~Hz}, 2 \mathrm{H}), 5.96(\mathrm{~d}, J=2.0 \mathrm{~Hz}, 1 \mathrm{H}), 2.96(\mathrm{~s}, 6 \mathrm{H}), 2.32$ (brs, $1 \mathrm{H}$ ); ${ }^{13} \mathrm{C} \mathrm{NMR}\left(101 \mathrm{MHz}, \mathrm{CDCl}_{3}\right) \delta$ (ppm): 150.2, 141.6, 133.3, 132.7, 131.8, 128.04, 127.98, 127.93, 127.6, 126.0, 125.7, 124.9, 124.5, 112.5, 76.0, 40.5.<smiles>CN(C)c1ccc(C(O)c2ccco2)cc1</smiles>

(4-(Dimethylamino)phenyl)(furan-2-yl)methanol (1ag): CAS No. 138256-14-1.

Prepared according to procedure $A$ from Grignard reagent SG1. Yellow oil; yield: $412 \mathrm{mg}, 76 \% ;{ }^{1} \mathrm{H}$ NMR (400 MHz, $\left.\mathrm{CDCl}_{3}\right) \delta$ (ppm): 7.39 (s, 1H), 7.30 (d, $J=7.6 \mathrm{~Hz}, 2 \mathrm{H}), 6.73$ (d, $J=8.4 \mathrm{~Hz}$, 2H), $6.32(\mathrm{~s}, 1 \mathrm{H}), 6.14(\mathrm{~s}, 1 \mathrm{H}), 5.74(\mathrm{~s}, 1 \mathrm{H}), 2.96(\mathrm{~s}, 6 \mathrm{H}), 2.34$ (brs, 1H); ${ }^{13} \mathrm{C}$ NMR $(101$ $\left.\mathrm{MHz}, \mathrm{CDCl}_{3}\right) \delta(\mathrm{ppm}): 155.5,150.5,142.2,128.8,127.7,112.4,110.1,106.9,70.1,40.6$.<smiles>CN(C)c1ccc(C(O)c2cccs2)cc1</smiles>

\section{(4-(Dimethylamino)phenyl)(thiophen-2-yl)methanol (1ah):} CAS No. 93187-70-3.

Prepared according to procedure $A$ from Grignard reagent SG1. Yellow solid; m.p.: $74-76^{\circ} \mathrm{C}$; yield: $392 \mathrm{mg}, 67 \%$; ${ }^{1} \mathrm{H}$ NMR $(400$ $\left.\mathrm{MHz}, \mathrm{CDCl}_{3}\right) \delta(\mathrm{ppm}): 7.31$ (d, $\left.J=8.4 \mathrm{~Hz}, 2 \mathrm{H}\right), 7.25(\mathrm{~d}, J=5.2 \mathrm{~Hz}$, $1 \mathrm{H}), 6.96-6.94(\mathrm{~m}, 1 \mathrm{H}), 6.90-6.86(\mathrm{~m}, 1 \mathrm{H}), 6.74(\mathrm{~d}, J=8.8 \mathrm{~Hz}, 2 \mathrm{H}), 5.97(\mathrm{~s}, 1 \mathrm{H}), 2.96(\mathrm{~s}$, $6 \mathrm{H}), 2.47$ (brs, $1 \mathrm{H}) ;{ }^{13} \mathrm{C} \mathrm{NMR}\left(101 \mathrm{MHz}, \mathrm{CDCl}_{3}\right) \delta(\mathrm{ppm}): 150.3,148.9,131.3,127.4$, $126.5,124.8,124.3,112.4,72.4,40.6$.<smiles>CN(C)c1ccc(C(O)c2ccncc2)cc1</smiles>

(4-(Dimethylamino)phenyl)(pyridin-4-yl)methanol (1ai): CAS No. 787515-64-4.

Prepared according to procedure $A$ from Grignard reagent SG1. Yellow solid; $107-108^{\circ} \mathrm{C}$; yield: $468 \mathrm{mg}, 82 \%$; ${ }^{1} \mathrm{H}$ NMR $(400 \mathrm{MHz}$, DMSO- $\left.d_{6}\right) \delta(\mathrm{ppm}): 8.45(\mathrm{~d}, J=5.6 \mathrm{~Hz}, 2 \mathrm{H}), 7.30(\mathrm{~d}, J=5.6 \mathrm{~Hz}$, 2H), $7.15(\mathrm{~d}, J=8.8 \mathrm{~Hz}, 2 \mathrm{H}), 6.66(\mathrm{~d}, J=8.4 \mathrm{~Hz}, 2 \mathrm{H}), 5.88(\mathrm{~d}, J=4.0 \mathrm{~Hz}, 1 \mathrm{H}), 5.59$ (brd, $J$ $=2.8 \mathrm{~Hz}, 1 \mathrm{H}), 2.84(\mathrm{~s}, 6 \mathrm{H}) ;{ }^{13} \mathrm{C}$ NMR $\left(101 \mathrm{MHz}, \mathrm{DMSO}-d_{6}\right) \delta$ (ppm): 154.8, 149.7, 149.3, $132.0,127.3,121.1,112.2,72.9,40.2$.<smiles>CN(C)c1ccc(C(O)c2ccccn2)cc1</smiles>

(4-(Dimethylamino)phenyl)(pyridin-2-yl)methanol (1aj): CAS No. 109520-25-4.

Prepared according to procedure $A$ from Grignard reagent SG1. Yellow solid; m.p.: $103-104^{\circ} \mathrm{C}$; yield: $471 \mathrm{mg}, 83 \%$; ${ }^{1} \mathrm{H}$ NMR (400 
$\left.\mathrm{MHz}, \mathrm{CDCl}_{3}\right) \delta(\mathrm{ppm}): 8.55(\mathrm{~d}, J=4.8 \mathrm{~Hz}, 1 \mathrm{H}), 7.60(\mathrm{td}, J=8.0 \mathrm{~Hz}, 1.6 \mathrm{~Hz}, 1 \mathrm{H}), 7.23-7.13$ $(\mathrm{m}, 4 \mathrm{H}), 6.70(\mathrm{~d}, J=8.8 \mathrm{~Hz}, 2 \mathrm{H}), 5.69(\mathrm{~s}, 1 \mathrm{H}), 5.09$ (brs, 1H), $2.93(\mathrm{~s}, 6 \mathrm{H}) ;{ }^{13} \mathrm{C}$ NMR $(101$ $\left.\mathrm{MHz} \mathrm{CDCl}_{3}\right) \delta(\mathrm{ppm}): 161.6,150.2,147.6,136.6,131.1,128.0,122.1,121.3,112.5,74.7$, 40.5<smiles>CN(C)c1ccc(C(O)c2ccc(-c3ccc4c(n3)CCCC4)cc2)cc1</smiles>

(4-(Dimethylamino)phenyl)(4-(5,6,7,8-tetrahydro quinolin-2-yl)phenyl)methanol (1ak): Prepared according to procedure $A$ from Grignard reagent SG1.

Yellow solid; m.p.: $130-132^{\circ} \mathrm{C}$; yield: $752 \mathrm{mg}, 84 \% ;{ }^{1} \mathrm{H}$ NMR $\left(400 \mathrm{MHz}, \mathrm{CDCl}_{3}\right) \delta(\mathrm{ppm})$ : $7.89(\mathrm{~d}, J=8.4 \mathrm{~Hz}, 2 \mathrm{H}), 7.44(\mathrm{~d}, J=8.4 \mathrm{~Hz}, 2 \mathrm{H}), 7.41(\mathrm{~d}, J=4.8 \mathrm{~Hz}, 2 \mathrm{H}), 7.21(\mathrm{~d}, J=8.4$ $\mathrm{Hz}, 2 \mathrm{H}), 6.68(\mathrm{~d}, J=8.4 \mathrm{~Hz}, 2 \mathrm{H}), 5.78(\mathrm{~s}, 1 \mathrm{H}), 3.00(\mathrm{t}, J=6.4 \mathrm{~Hz}, 2 \mathrm{H}), 2.92(\mathrm{~s}, 6 \mathrm{H}), 2.79(\mathrm{t}$ $J=6.4 \mathrm{~Hz}, 2 \mathrm{H}$ ), 2.50 (brs, $1 \mathrm{H}$ ), 1.93 (qui, $J=5.6 \mathrm{~Hz}, 2 \mathrm{H}$ ), 1.84 (t, $J=5.6 \mathrm{~Hz}, 2 \mathrm{H}$ ); ${ }^{13} \mathrm{C}$ $\operatorname{NMR}\left(101 \mathrm{MHz}, \mathrm{CDCl}_{3}\right) \delta(\mathrm{ppm}):$ 157.1, 154.5, 150.1, 144.5, 138.6, 137.3, 132.0, 130.5, 127.8, 126.7, 126.6, 117.8, 112.4, 75.6, 40.6, 32.7, 28.5, 23.1, 22.7.

HRMS (positive ESI): $\mathrm{m} / \mathrm{z}$ calculated for $\mathrm{C}_{24} \mathrm{H}_{27} \mathrm{~N}_{2} \mathrm{O}[\mathrm{M}+\mathrm{H}]^{+}: 359.2118$; found: 359.2123

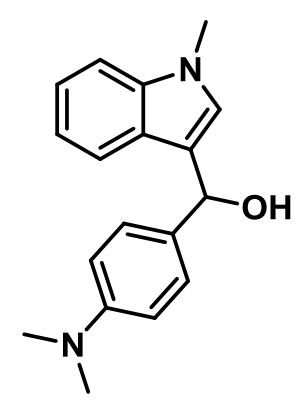

\section{(4-(Dimethylamino)phenyl)(1-methyl-1H-indol-3-yl)methanol} (1al):

Prepared according to procedure $A$ from Grignard reagent SG1.

Pink solid; m.p.: $168-170^{\circ} \mathrm{C}$; yield: $441 \mathrm{mg}, 63 \%$; ${ }^{1} \mathrm{H}$ NMR $(400 \mathrm{MHz}$, $\left.\mathrm{CDCl}_{3}\right) \delta$ (ppm): 7.41-7.36 (m, 4H), 7.23-7.20 (m, 2H), $6.89(\mathrm{~s}, 1 \mathrm{H})$, $6.73(\mathrm{~d}, J=8.8 \mathrm{~Hz}, 2 \mathrm{H}), 5.68(\mathrm{~s}, 1 \mathrm{H}), 3.67(\mathrm{~s}, 3 \mathrm{H}), 3.06(\mathrm{~d}, J=3.6 \mathrm{~Hz}$, $1 \mathrm{H}), 2.94(\mathrm{~s}, 6 \mathrm{H}) ;{ }^{13} \mathrm{C} \mathrm{NMR}\left(101 \mathrm{MHz}, \mathrm{CDCl}_{3}\right) \delta$ (ppm): 150.0, 137.2, $130.3,128.8,127.6,126.9,121.2,120.4,118.7,117.7,112.4,108.9$,

73.5, 40.7, 32.6.

HRMS (positive ESI): $\mathrm{m} / \mathrm{z}$ calculated for $\mathrm{C}_{18} \mathrm{H}_{21} \mathrm{~N}_{2} \mathrm{O}[\mathrm{M}+\mathrm{H}]^{+}:$281.1648; found: 281.1653.<smiles>CN(C)c1ccc(C(O)c2ccc(C(O)c3ccc(N(C)C)cc3)cc2)cc1</smiles>

1,4-Phenylenebis((4-(dimethylamino)phenyl) methanol) (1an): CAS No.1086267-54-0. Prepared according to procedure $A$ from Grignard reagent SG1 $(6.0 \mathrm{~mL}$ of $\mathrm{SG} 1$ was used).

White solid; $179-180^{\circ} \mathrm{C}$; yield: $402 \mathrm{mg}, 43 \%$; ${ }^{1} \mathrm{H}$ NMR $\left(400 \mathrm{MHz}, \mathrm{CD}_{3} \mathrm{COCD}_{3}\right) \delta(\mathrm{ppm})$ : 7.34-7.21 (m, 8H), 6.69 (d, J = 2.8 Hz, 4H), $5.71(\mathrm{~s}, 2 \mathrm{H}), 2.89(\mathrm{~s}, 12 \mathrm{H}) ;{ }^{13} \mathrm{C} \mathrm{NMR}(101 \mathrm{MHz}$, $\left.\mathrm{CD}_{3} \mathrm{COCD}_{3}\right) \delta(\mathrm{ppm}): 149.9,144.5,133.6,127.3,125.9,112.3,75.0,39.9$.<smiles>CSCc1ccc(C(O)c2ccc(N(C)C)cc2)cc1</smiles>

(4-(Dimethylamino)phenyl)(4-((methylthio)methyl)phe nyl)methanol (1ao):

Prepared according to procedure $A$ from Grignard reagent SG1.

Yellow solid; m.p.: $52-54{ }^{\circ} \mathrm{C}$; yield: $548 \mathrm{mg}, 76 \% ;{ }^{1} \mathrm{H} \mathrm{NMR}\left(400 \mathrm{MHz}, \mathrm{CDCl}_{3}\right) \delta$ (ppm): 7.34 
$(\mathrm{d}, J=8.0 \mathrm{~Hz}, 2 \mathrm{H}), 7.26(\mathrm{~d}, J=8.0 \mathrm{~Hz}, 2 \mathrm{H}), 7.21(\mathrm{~d}, J=8.8 \mathrm{~Hz}, 2 \mathrm{H}), 6.70(\mathrm{~d}, J=8.8 \mathrm{~Hz}$, 2H), $5.76(\mathrm{~d}, J=3.2 \mathrm{~Hz}, 1 \mathrm{H}), 3.65(\mathrm{~s}, 2 \mathrm{H}), 2.93(\mathrm{~s}, 6 \mathrm{H}), 2.18(\mathrm{~d}, J=3.6 \mathrm{~Hz}, 1 \mathrm{H}), 1.99(\mathrm{~s}$, $3 \mathrm{H}) ;{ }^{13} \mathrm{C}$ NMR $\left(101 \mathrm{MHz}, \mathrm{CDCl}_{3}\right.$ ) $\delta$ (ppm):150.1, 143.0, 137.0, 131.9, 128.8, 127.7, 126.4, 112.4 , 75.7, 40.5, 38.0, 14.9.; HRMS (positive ESI): $\mathrm{m} / \mathrm{z}$ calculated for $\mathrm{C}_{17} \mathrm{H}_{22} \mathrm{NOS}[\mathrm{M}+\mathrm{H}]^{+}$: 288.1417; found: 288.1421 .

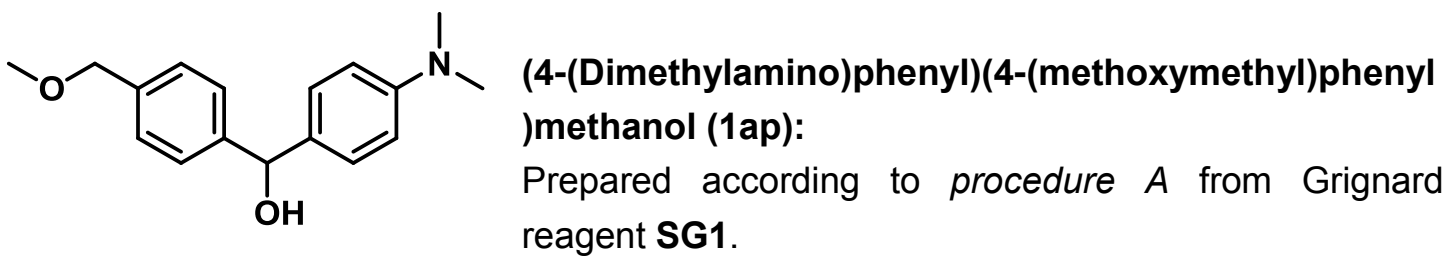

Pale yellow liquid; yield: $564 \mathrm{mg}, 83 \% ;{ }^{1} \mathrm{H}$ NMR $\left(400 \mathrm{MHz}, \mathrm{CDCl}_{3}\right) \delta(\mathrm{ppm}): 7.38(\mathrm{~d}, \mathrm{~J}=$ $8.0 \mathrm{~Hz}, 2 \mathrm{H}), 7.30(\mathrm{~d}, J=8.0 \mathrm{~Hz}, 2 \mathrm{H}), 7.21(\mathrm{~d}, J=8.8 \mathrm{~Hz}, 2 \mathrm{H}), 6.69(\mathrm{~d}, J=8.8 \mathrm{~Hz}, 2 \mathrm{H})$, $5.77(\mathrm{~d}, J=2.0 \mathrm{~Hz}, 1 \mathrm{H}), 4.44(\mathrm{~s}, 2 \mathrm{H}), 3.38(\mathrm{~s}, 3 \mathrm{H}), 2.93(\mathrm{~s}, 6 \mathrm{H}), 2.20(\mathrm{~d}, J=2.8 \mathrm{~Hz}, 1 \mathrm{H}$ ); ${ }^{13} \mathrm{C}$ NMR DEPT-135 (101 MHz, $\left.\mathrm{CDCl}_{3}\right) \delta(\mathrm{ppm}): 127.8,126.4,112.5,75.8,74.5,58.1$, 40.6 .

HRMS (positive ESI): m/z calculated for $\mathrm{C}_{17} \mathrm{H}_{22} \mathrm{NO}_{2}[\mathrm{M}+\mathrm{H}]^{+}: 272.1645$; found: 272.1646 .<smiles>CN(C)Cc1ccc(C(O)c2ccc(N(C)C)cc2)cc1</smiles>

\section{(4-((Dimethylamino)methyl)phenyl)(4-(dimethylamino} )phenyl)methanol (1aq):

Prepared according to procedure $A$ from Grignard reagent SG1.

White solid; m.p.: $85-87^{\circ} \mathrm{C}$; yield: $447 \mathrm{mg}, 63 \%$; ${ }^{1} \mathrm{H}$ NMR $\left(400 \mathrm{MHz}, \mathrm{CDCl}_{3}\right) \delta$ (ppm): 7.25 $(\mathrm{d}, J=8.0 \mathrm{~Hz}, 2 \mathrm{H}), 7.17(\mathrm{~d}, J=8.4 \mathrm{~Hz}, 2 \mathrm{H}), 7.12(\mathrm{~d}, J=8.8 \mathrm{~Hz}, 2 \mathrm{H}), 6.60(\mathrm{~d}, J=8.8 \mathrm{~Hz}$, $2 \mathrm{H}), 5.65(\mathrm{~s}, 1 \mathrm{H}), 3.33(\mathrm{~s}, 2 \mathrm{H}), 2.83(\mathrm{~s}, 6 \mathrm{H}), 2.13(\mathrm{~s}, 6 \mathrm{H}) ;{ }^{13} \mathrm{C} \mathrm{NMR}\left(101 \mathrm{MHz}, \mathrm{CDCl}_{3}\right) \delta$ (ppm): 150.0, 143.5, 136.6, 132.2, 129.2, 127.6, 126.3, 112.4, 75.6, 63.8, 45.0, 40.6.

HRMS (positive ESI): m/z calculated for $\mathrm{C}_{18} \mathrm{H}_{25} \mathrm{~N}_{2} \mathrm{O}[\mathrm{M}+\mathrm{H}]^{+}: 285.1961$; found: 285.1965 .<smiles>CN(C)c1ccc(C(O)C#Cc2ccccc2)cc1</smiles>

1-(4-(Dimethylamino)phenyl)-3-phenylprop-2-yn-1-ol (1ar): CAS No. 477330-47-5.

Prepared according to procedure $A$ from Grignard reagent SG1.

Yellow oil; yield: $332 \mathrm{mg}, 53 \% ;{ }^{1} \mathrm{H}$ NMR $\left(400 \mathrm{MHz}, \mathrm{CDCl}_{3}\right) \delta$ (ppm): 7.51-7.49 (m, 4H), 7.34-7.32 (m, 3H), $6.76(\mathrm{~d}, J=8.8 \mathrm{~Hz}, 2 \mathrm{H}), 5.62(\mathrm{~s}, 1 \mathrm{H}), 2.97(\mathrm{~s}$, $6 \mathrm{H}), 2.50$ (brs, $1 \mathrm{H}) ;{ }^{13} \mathrm{C}$ NMR $\left(101 \mathrm{MHz}, \mathrm{CDCl}_{3}\right) \delta$ (ppm): 150.6, 131.6, 128.7, 128.3, $128.2,127.8,122.7,112.4,89.4,86.0,64.8,40.5$.<smiles>CN(C)c1ccc(C(O)/C=C/c2ccccc2)cc1</smiles>

(E)-1-(4-(Dimethylamino)phenyl)-3-phenylprop-2-en-1-ol (1as): CAS No. 35552-46-6.

Prepared according to procedure $A$ from Grignard reagent SG1.

Yellow oil; yield: $305 \mathrm{mg}, 48 \%$; ${ }^{1} \mathrm{H}$ NMR $\left(400 \mathrm{MHz}, \mathrm{CD}_{3} \mathrm{COCD}_{3}\right) \delta$ (ppm): 7.44-7.41 (m, 
2H), 7.32-7.21 (m, 5H), 6.74-6.71 (m, 2H), 6.70-6.64 (m, 1H), $6.43(\mathrm{dd}, J=16.0 \mathrm{~Hz}, 6.0$ $\mathrm{Hz}, 1 \mathrm{H}), 5.25(\mathrm{t}, J=4.8 \mathrm{~Hz}, 1 \mathrm{H}), 4.26(\mathrm{~d}, J=4.0 \mathrm{~Hz}, 1 \mathrm{H}), 2.90(\mathrm{~s}, 6 \mathrm{H}) ;{ }^{13} \mathrm{C} \mathrm{NMR}(101 \mathrm{MHz}$, $\left.\mathrm{CD}_{3} \mathrm{COCD}_{3}\right) \delta(\mathrm{ppm}): 151.0,138.3,134.8,133.0,129.4,128.9,128.04,128.02,127.2$, 113.2, 74.8, 40.8.<smiles>CN(C)c1ccc(C(O)c2ccc(O)cc2)cc1</smiles>

\title{
4-((4-(Dimethylamino)phenyl)(hydroxy)methyl)phenol
} (1at): CAS No. 159335-06-5 Prepared according to procedure $C$.

White solid; m.p.:150-152 ${ }^{\circ} \mathrm{C}$ (reported $162^{\circ} \mathrm{C}$ ); $;^{18}$ yield: 480 mg, 79\%; ${ }^{1} \mathrm{H}$ NMR $\left(400 \mathrm{MHz}, \mathrm{DMSO}-d_{6}\right) \delta(\mathrm{ppm}): 9.12$ (s, 1H), 7.10 (dd, $J=8.4 \mathrm{~Hz}, 6.4 \mathrm{~Hz}, 4 \mathrm{H}), 6.65$ (t, $J=8.4 \mathrm{~Hz}, 4 \mathrm{H}), 5.48$ (d, J=4.0 Hz, 1H), $5.42(\mathrm{~d}, J=4.0 \mathrm{~Hz}, 1 \mathrm{H}), 2.83(\mathrm{~s}, 6 \mathrm{H}) ;{ }^{13} \mathrm{C}$ NMR $\left(101 \mathrm{MHz}, \mathrm{DMSO}-d_{6}\right) \delta(\mathrm{ppm}): 155.9$, $149.3,136.8,134.0,127.3,126.9,114.6,112.1,73.7,40.3$.<smiles>CN(C)c1ccc(C(O)c2ccc(CO)cc2)cc1</smiles>

\author{
(4-(Dimethylamino)phenyl)(4-(hydroxymethyl)phenyl) \\ methanol (1au): \\ Prepared according to procedure $C$. \\ White solid; m.p.:126-128 ${ }^{\circ} \mathrm{C}$; yield: $573 \mathrm{mg}, 89 \%$; ${ }^{1} \mathrm{H}$
} NMR (400 MHz, DMSO-d $\left.d_{6}\right) \delta(p p m): 7.27(\mathrm{~d}, J=8.0 \mathrm{~Hz}, 2 \mathrm{H}), 7.21(\mathrm{~d}, J=8.4 \mathrm{~Hz}, 2 \mathrm{H}), 7.12$ (d, $J=8.4 \mathrm{~Hz}, 2 \mathrm{H}), 6.64(\mathrm{~d}, J=8.8 \mathrm{~Hz}, 2 \mathrm{H}), 5.56(\mathrm{~s}, 1 \mathrm{H}), 5.08$ (brs, 1H), $4.44(\mathrm{~s}, 2 \mathrm{H}), 2.83$ $(\mathrm{s}, 6 \mathrm{H}) ;{ }^{13} \mathrm{C}$ NMR $(101 \mathrm{MHz}$, DMSO-d 6 ) $\delta$ (ppm): 149.4, 144.8, 140.5, 133.7, 127.1, 126.1, 125.8, 112.1, 73.9, 62.8, 40.3 .

HRMS (positive ESI): $\mathrm{m} / \mathrm{z}$ calculated for $\mathrm{C}_{16} \mathrm{H}_{20} \mathrm{NO}_{2}[\mathrm{M}+\mathrm{H}]^{+}: 258.1489$; found: 258.1494.

2-(4-(Dimethylamino)phenyl)propan-2-ol (3a): CAS No. 83026-55-5.

Prepared according to procedure $A$ from Grignard reagent SG1.<smiles>CN(C)c1ccc(C(C)(C)O)cc1</smiles>

Colorless liquid; yield: $398 \mathrm{mg}, 89 \%$; ${ }^{1} \mathrm{H}$ NMR (400 MHz, DMSO- $d_{6}$ )

$\delta$ (ppm): 7.28 (d, $J=8.8 \mathrm{~Hz}, 2 \mathrm{H}$ ), 6.67 (d, $J=8.8 \mathrm{~Hz}, 2 \mathrm{H}$ ), 4.76 (s,

$1 \mathrm{H}), 2.84(\mathrm{~s}, 6 \mathrm{H}), 1.40(\mathrm{~s}, 6 \mathrm{H}) ;{ }^{13} \mathrm{C}$ NMR $\left(101 \mathrm{MHz}\right.$, DMSO- $\left.d_{6}\right) \delta$ (ppm): 148.8, 138.5, 125.1, 112.0, 70.2, 54.9, 40.4, 32.1.<smiles>CN(C)c1ccc(C(C)(O)c2ccccc2)cc1</smiles>

1-(4-(Dimethylamino)phenyl)-1-phenylethan-1-ol (3b): CAS No. 768295-58-5.

Prepared according to procedure $A$ from Grignard reagent SG1 . Pale yellow oil; yield: $434 \mathrm{mg}, 72 \%$; ${ }^{1} \mathrm{H}$ NMR $\left(400 \mathrm{MHz}, \mathrm{CDCl}_{3}\right) \delta$ (ppm): $7.43(\mathrm{~d}, J=8.4 \mathrm{~Hz}, 2 \mathrm{H}), 7.33-7.22(\mathrm{~m}, 5 \mathrm{H}), 6.69(\mathrm{~d}, J=$ $8.8 \mathrm{~Hz}, 2 \mathrm{H}), 2.93(\mathrm{~s}, 6 \mathrm{H}), 2.22$ (brs, $1 \mathrm{H}), 1.92(\mathrm{~s}, 3 \mathrm{H}) ;{ }^{13} \mathrm{C} \mathrm{NMR}\left(101 \mathrm{MHz}, \mathrm{CDCl}_{3}\right) \delta(\mathrm{ppm})$ : $149.4,148.6,136.0,127.9,126.8,126.5,125.7,112.1,75.9,40.6,30.9$.

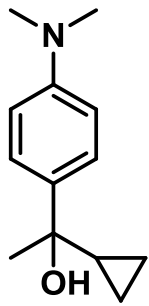

1-Cyclopropyl-1-(4-(dimethylamino)phenyl)ethan-1-ol (3c): CAS No. 1824056-09-8.

Prepared according to procedure $A$ from Grignard reagent SG1.

Yellow oil; yield: $440 \mathrm{mg}, 86 \% ;{ }^{1} \mathrm{H}$ NMR $\left(400 \mathrm{MHz}, \mathrm{CD}_{3} \mathrm{COCD}_{3}\right) \delta$ (ppm): 7.38 
(dt, $J=8.8 \mathrm{~Hz}, 2.4 \mathrm{~Hz}, 2 \mathrm{H}$ ), 6.69 (dt, J = 8.8 Hz, $2.4 \mathrm{~Hz}, 2 \mathrm{H}$ ), 3.40 (s, 1H), 2.90 (s, 6H), $1.47(\mathrm{~s}, 3 \mathrm{H}), 1.23-1.16(\mathrm{~m}, 1 \mathrm{H}), 0.48-0.23(\mathrm{~m}, 4 \mathrm{H}) ;{ }^{13} \mathrm{C}$ NMR $\left(101 \mathrm{MHz}, \mathrm{CD}_{3} \mathrm{COCD}_{3}\right) \delta$ (ppm): 150.2, 138.2, 126.7, 112.8, 71.9, 40.8, 30.0, 23.7, 1.7, 1.4 .<smiles>CN(C)c1ccc(C(C)(O)C(C)(C)C)cc1</smiles>

2-(4-(Dimethylamino)phenyl)-3,3-dimethylbutan-2-ol (3d):

Prepared according to procedure $A$ from Grignard reagent SG1.

White solid; m.p.: $84-85{ }^{\circ} \mathrm{C}$; yield: $376 \mathrm{mg}, 68 \%$; ${ }^{1} \mathrm{H}$ NMR $(400 \mathrm{MHz}$, $\left.\mathrm{CDCl}_{3}\right) \delta(\mathrm{ppm}): 7.31$ (d, J = $\left.8.4 \mathrm{~Hz}, 2 \mathrm{H}\right), 6.69(\mathrm{~d}, J=8.8 \mathrm{~Hz}, 2 \mathrm{H})$, $2.94(\mathrm{~s}, 6 \mathrm{H}), 1.58(\mathrm{~s}, 3 \mathrm{H}), 1.54(\mathrm{brs}, 1 \mathrm{H}), 0.93(\mathrm{~s}, 6 \mathrm{H}) ;{ }^{13} \mathrm{C}$ NMR $(101$

$\left.\mathrm{MHz}, \mathrm{CDCl}_{3}\right) \delta$ (ppm): 149.0, 134.3, 127.8, 111.3, 78.3, 40.6, 38.2, 25.8, 25.3.

HRMS (positive ESI): $\mathrm{m} / \mathrm{z}$ calculated for $\mathrm{C}_{14} \mathrm{H}_{24} \mathrm{NO}[\mathrm{M}+\mathrm{H}]^{+}: 222.1852$; found: 222.1858 .

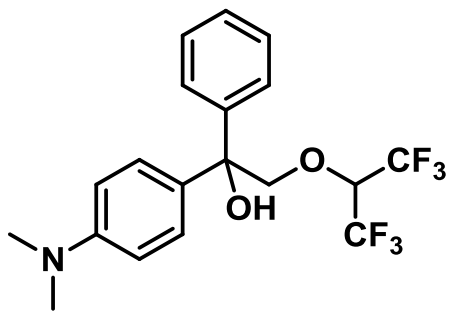

1-(4-(Dimethylamino)phenyl)-2-((1,1,1,3,3,3-hexafluorop ropan-2-yl)oxy)-1-phenylethan-1-ol (3e):

Prepared according to procedure $A$ from Grignard reagent SG1.

Yellow liquid; yield: $834 \mathrm{mg}, 82 \%$; ${ }^{1} \mathrm{H}$ NMR $(400 \mathrm{MHz}$, $\left.\mathrm{CDCl}_{3}\right) \delta(\mathrm{ppm}):$ 7.32-7.28 (m, 2H), 7.24-7.19 (m, 2H), 7.17-7.11 (m, 3H), 6.69 (dt, $J=9.6 \mathrm{~Hz}, 2.8 \mathrm{~Hz}, 2 \mathrm{H}), 4.24-4.16(\mathrm{~m}, 2 \mathrm{H}), 4.12$ (sep, $J=6.0$ $\mathrm{Hz}, 1 \mathrm{H}), 2.90$ (brs, 1H), 2.82 (s, 6H); ${ }^{13} \mathrm{C} \mathrm{NMR} \mathrm{(101} \mathrm{MHz,} \mathrm{CDCl} 3$ ) $\delta$ (ppm): 149.8, 143.5, $130.6,128.1,127.5,127.3,126.5,121.4\left(J_{C-F}=284.0 \mathrm{~Hz}\right), 112.0,80.7,77.8,76.7\left(J_{C-F}=\right.$ $32.0 \mathrm{~Hz}), 40.3$;

HRMS (positive ESI): $\mathrm{m} / \mathrm{z}$ calculated for $\mathrm{C}_{19} \mathrm{H}_{20} \mathrm{NO}_{2} \mathrm{~F}_{6}[\mathrm{M}+\mathrm{H}]^{+}:$408.1393; found: 408.1410 .

1-(4-(Dimethylamino)phenyl)-2-phenoxy-1-phenylethan-1-ol (3f):

Prepared according to procedure $A$ from Grignard reagent SG1.

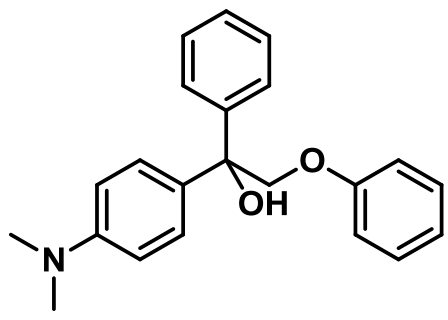

White solid; m.p.: $106-108{ }^{\circ} \mathrm{C}$; yield: $733 \mathrm{mg}, 88 \%$; ${ }^{1} \mathrm{H}$ NMR (400 MHz, $\left.\mathrm{CDCl}_{3}\right) \delta(\mathrm{ppm}):$ 7.59-7.55 (m, 2H), 7.44-7.33 (m, $7 \mathrm{H}), 7.06(\mathrm{t}, J=7.2 \mathrm{~Hz}, 1 \mathrm{H}), 7.00(\mathrm{~d}, J=8.0 \mathrm{~Hz}, 2 \mathrm{H}), 6.78(\mathrm{~d}$ $J=8.8 \mathrm{~Hz}, 2 \mathrm{H}), 4.55-4.49(\mathrm{~m}, 2 \mathrm{H}), 3.46$ (brs, $1 \mathrm{H}), 3.01(\mathrm{~s}$, $6 \mathrm{H}) ;{ }^{13} \mathrm{C}$ NMR $\left(101 \mathrm{MHz}, \mathrm{CDCl}_{3}\right) \delta(\mathrm{ppm}): 158.4,149.7$, $144.5,131.7,129.4,128.0,127.6,127.1,126.7,121.2$,

114.7, 112.0, 77.4, 73.8, 40.4.

HRMS (positive ESI): m/z calculated for $\mathrm{C}_{22} \mathrm{H}_{24} \mathrm{NO}_{2}[\mathrm{M}+\mathrm{H}]^{+}: 334.1802$; found: 334.1812 .

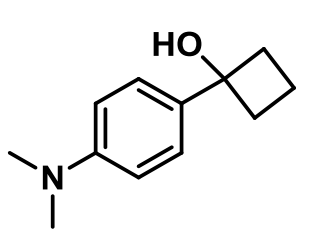

1-(4-(Dimethylamino)phenyl)cyclobutan-1-ol (3g):

Prepared according to procedure $A$ from Grignard reagent SG1..

White solid; m.p.: $46-48{ }^{\circ} \mathrm{C}$; yield: $349 \mathrm{mg}, 73 \%$; ${ }^{1} \mathrm{H}$ NMR $(400 \mathrm{MHz}$, $\left.\mathrm{CDCl}_{3}\right) \delta$ (ppm): $7.26(\mathrm{~d}, J=8.8 \mathrm{~Hz}, 2 \mathrm{H}), 6.63(\mathrm{~d}, J=8.8 \mathrm{~Hz}, 2 \mathrm{H})$, $2.83(\mathrm{~s}, 6 \mathrm{H}), 2.45-2.19(\mathrm{~m}, 5 \mathrm{H}), 1.88-1.75(\mathrm{~m}, 1 \mathrm{H}), 1.55-1.44(\mathrm{~m}, 1 \mathrm{H})$;

${ }^{13} \mathrm{C}$ NMR (101 MHz, CDCl 3 ) $\delta$ (ppm): 149.7, 134.1, 125.9, 112.4, 76.4, 40.6, 36.5, 12.7. HRMS (positive ESI): $\mathrm{m} / \mathrm{z}$ calculated for $\mathrm{C}_{12} \mathrm{H}_{18} \mathrm{NO}[\mathrm{M}+\mathrm{H}]^{+}:$192.1383; found: 192.1385 .

1-(4-(Dimethylamino)phenyl)cyclopentan-1-ol (3h): CAS No. 1518326-84-5. 
Prepared according to procedure A from Grignard reagent SG1.<smiles>CN(C)c1ccc(C2(O)CCCC2)cc1</smiles><smiles>CN(C)c1ccc(C2(O)CCCCC2)cc1</smiles>

White solid; m.p.: $105-106{ }^{\circ} \mathrm{C}$; yield: $434 \mathrm{mg}, 85 \%$; ${ }^{1} \mathrm{H}$ NMR $(400$ $\left.\mathrm{MHz}, \mathrm{DMSO}-\mathrm{d}_{6}\right) \delta$ (ppm): $7.26(\mathrm{~d}, J=8.8 \mathrm{~Hz}, 2 \mathrm{H}), 6.66(\mathrm{~d}, J=8.4 \mathrm{~Hz}$, $2 \mathrm{H}), 4.50(\mathrm{~s}, 1 \mathrm{H}), 2.84(\mathrm{~s}, 6 \mathrm{H}), 1.85-1.60(\mathrm{~m}, 8 \mathrm{H}) ;{ }^{13} \mathrm{C} \mathrm{NMR}(101 \mathrm{MHz}$, DMSO- $\left.d_{6}\right) \delta(\mathrm{ppm}): 149.0,136.2,125.8,112.0,81.1,41.1,40.4$, 23.5.

1-(4-(Dimethylamino)phenyl)cyclohexan-1-ol (3i): CAS No. 13299-39-3.

Prepared according to procedure $A$ from Grignard reagent SG1.

White solid; m.p.: 66-67 ${ }^{\circ} \mathrm{C}$; yield: $480 \mathrm{mg}, 88 \%$; ${ }^{1} \mathrm{H}$ NMR $(400 \mathrm{MHz}$, DMSO- $\left.d_{6}\right) \delta(\mathrm{ppm}): 7.27(\mathrm{~d}, J=8.8 \mathrm{~Hz}, 2 \mathrm{H}), 6.66(\mathrm{~d}, J=8.8 \mathrm{~Hz}$, $2 \mathrm{H}), 4.40(\mathrm{~s}, 1 \mathrm{H}), 2.84(\mathrm{~s}, 6 \mathrm{H}), 1.77-1.15(\mathrm{~m}, 10 \mathrm{H}) ;{ }^{13} \mathrm{C} \mathrm{NMR}\left(101 \mathrm{MHz}, \mathrm{DMSO}-d_{6}\right) \delta$ (ppm): 148.9, 139.0, 125.3, 112.0, 70.8, 40.4, 38.6, 25.3, 21.9.<smiles>CN(C)c1ccc(C2(O)CCCCCC2)cc1</smiles>

\section{1-(4-(Dimethylamino)phenyl)cycloheptan-1-ol (3j):}

Prepared according to procedure $A$ from Grignard reagent SG1.

Yellow liquid; yield: $495 \mathrm{mg}, 85 \%$; ${ }^{1} \mathrm{H}$ NMR $\left(400 \mathrm{MHz}\right.$, DMSO- $\left.d_{6}\right) \delta$ (ppm): 7.27 (d, $J=8.8 \mathrm{~Hz}, 2 \mathrm{H}), 6.65(\mathrm{~d}, J=8.8 \mathrm{~Hz}, 2 \mathrm{H}), 4.50$ (s, $1 \mathrm{H}), 2.84(\mathrm{~s}, 6 \mathrm{H}), 1.89-1.40(\mathrm{~m}, 12 \mathrm{H}) ;{ }^{13} \mathrm{C}$ NMR $(101 \mathrm{MHz}$, DMSO- $\left.d_{6}\right) \delta(\mathrm{ppm}): 148.7,140.1,125.2,112.0,74.5,42.9,40.4,28.6,22.0$. HRMS (positive ESI): $\mathrm{m} / \mathrm{z}$ calculated for $\mathrm{C}_{15} \mathrm{H}_{24} \mathrm{NO}[\mathrm{M}+\mathrm{H}]^{+}:$234.1852; found: 234.1848 .<smiles>CN(C)c1ccc(C2(O)CCCCCCCCCC2)cc1</smiles>

\section{1-(4-(Dimethylamino)phenyl)cyclododecan-1-ol (3k):}

Prepared according to procedure $D$.

White solid; m.p.: $112-113{ }^{\circ} \mathrm{C}$; yield: $1.44 \mathrm{~g}, 95 \%$; ${ }^{1} \mathrm{H}$ NMR (400 $\left.\mathrm{MHz}, \mathrm{CD}_{3} \mathrm{COCD}_{3}\right) \delta$ (ppm): $7.32(\mathrm{~d}, J=8.8 \mathrm{~Hz}, 2 \mathrm{H}), 6.68(\mathrm{~d}, J$ $=8.8 \mathrm{~Hz}, 2 \mathrm{H}), 3.33(\mathrm{brs}, 1 \mathrm{H}), 2.89(\mathrm{~s}, 6 \mathrm{H}), 1.84-1.18(\mathrm{~m}, 22 \mathrm{H})$; ${ }^{13} \mathrm{C}$ NMR $\left(101 \mathrm{MHz}, \mathrm{CD}_{3} \mathrm{COCD}_{3}\right) \delta(\mathrm{ppm}): 150.2,138.5,126.8,112.8,75.6,40.8,36.5$, 27.3, 26.9, 23.3, 23.1, 20.8.

HRMS (positive ESI): m/z calculated for $\mathrm{C}_{20} \mathrm{H}_{34} \mathrm{NO}[\mathrm{M}+\mathrm{H}]^{+}:$304.2635; found: 304.2651 .<smiles>CN(C)c1ccc(C2(O)c3ccccc3Oc3ccccc32)cc1</smiles>

9-(4-(Dimethylamino)phenyl)-9H-xanthen-9-ol (3I): CAS No. 113493-33-7.

Prepared according to procedure $A$ from Grignard reagent SG1. White solid; m.p.: $179-180{ }^{\circ} \mathrm{C}$; yield: $650 \mathrm{mg}, 82 \%$; ${ }^{1} \mathrm{H} \mathrm{NMR}(400 \mathrm{MHz}$, DMSO- $\left.d_{6}\right) \delta$ (ppm): $7.44(\mathrm{~d}, J=7.2 \mathrm{~Hz}, 2 \mathrm{H}), 7.29(\mathrm{t}, J=7.2 \mathrm{~Hz}, 2 \mathrm{H})$, $7.20(\mathrm{t}, J=7.6 \mathrm{~Hz}, 2 \mathrm{H}), 7.08(\mathrm{~d}, J=7.6 \mathrm{~Hz}, 4 \mathrm{H}), 6.60(\mathrm{~d}, J=8.0 \mathrm{~Hz}$, $2 \mathrm{H}), 6.55$ (brs, $1 \mathrm{H}), 2.81(\mathrm{~s}, 6 \mathrm{H}) ;{ }^{13} \mathrm{C}$ NMR $\left(101 \mathrm{MHz}, \mathrm{DMSO}-d_{6}\right) \delta$ (ppm): 149.2, 148.8, 137.8, 129.2, 128.8, 128.2, 126.3, 123.1, 115.7, 111.8, 68.5, 40.1.

\section{1-(4-(Dimethylamino)phenyl)-1,2,3,4-tetrahydronaphthalen-1-ol (3m):}

Prepared according to procedure $A$ from Grignard reagent SG1.

Yellow solid; m.p.: $62-63{ }^{\circ} \mathrm{C}$; yield: $566 \mathrm{mg}, 85 \%$; ${ }^{1} \mathrm{H} \mathrm{NMR}\left(400 \mathrm{MHz}, \mathrm{CD}_{3} \mathrm{COCD}_{3}\right) \delta(\mathrm{ppm})$ : 


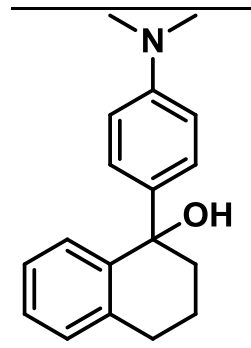

7.15-7.04 (m, 6H), $6.65(\mathrm{~d}, J=8.8 \mathrm{~Hz}, 2 \mathrm{H}), 4.12(\mathrm{~s}, 1 \mathrm{H}), 2.89(\mathrm{~s}, 6 \mathrm{H})$, 2.84-2.82 (m, 2H), 2.09-1.63 (m, 4H); ${ }^{13} \mathrm{C}$ NMR (101 MHz, $\left.\mathrm{CD}_{3} \mathrm{COCD}_{3}\right) \delta$ (ppm): 150.2, 144.3, 138.9, 138.0, 130.1, 129.0, 128.0, 127.4, 126.4, 112.5, 74.9, 42.3, 40.8, 30.5, 20.4.

HRMS (positive ESI): $\mathrm{m} / \mathrm{z}$ calculated for $\mathrm{C}_{18} \mathrm{H}_{22} \mathrm{NO}[\mathrm{M}+\mathrm{H}]^{+}:$268.1696; found: 268.1634 .

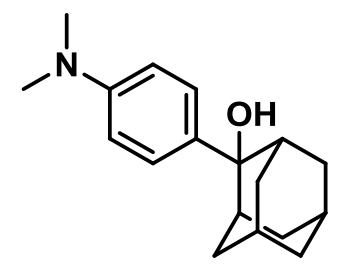

2-(4-(Dimethylamino)phenyl)adamantan-2-ol (3na): CAS No. 116437-95-7.

Prepared according to procedure $A$ from Grignard reagent SG1.

White solid; m.p.: $148-150^{\circ} \mathrm{C}$ (reported $149-150^{\circ} \mathrm{C}$ ); ${ }^{19}$ yield: $536 \mathrm{mg}$, $79 \% ;{ }^{1} \mathrm{H}$ NMR $\left(400 \mathrm{MHz}, \mathrm{CDCl}_{3}\right) \delta$ (ppm): 7.41 (d, $\left.J=8.8 \mathrm{~Hz}, 2 \mathrm{H}\right)$, $6.74(\mathrm{~d}, J=8.4 \mathrm{~Hz}, 2 \mathrm{H}), 2.96(\mathrm{~s}, 6 \mathrm{H}), 2.53(\mathrm{~s}, 2 \mathrm{H}), 2.40$ (d, J = $12.4 \mathrm{~Hz}, 2 \mathrm{H}), 1.89(\mathrm{~s}, 1 \mathrm{H})$, 1.72 (s, 9H), 1.58 (brs, $1 \mathrm{H}) ;{ }^{13} \mathrm{C}$ NMR $\left(101 \mathrm{MHz}, \mathrm{CDCl}_{3}\right) \delta$ (ppm): 149.5, 133.3, 126.3, $112.5,75.4,40.5,37.8,35.8,35.0,33.0,27.6,27.0$.

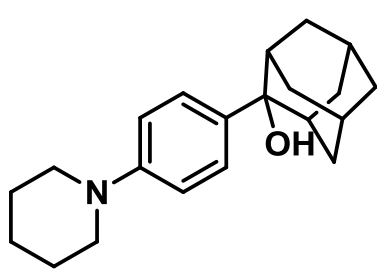

2-(4-(Piperidin-1-yl)phenyl)adamantan-2-ol (3nb): CAS No. 1783817-08-2.

Prepared according to procedure $A$ from Grignard reagent SG6. White solid; m.p.: $162-163{ }^{\circ} \mathrm{C}$; yield: $560 \mathrm{mg}, 72 \%$; ${ }^{1} \mathrm{H}$ NMR $\left(400 \mathrm{MHz}, \mathrm{CDCl}_{3}\right) \delta$ (ppm): 7.41 (d, $\left.J=8.4 \mathrm{~Hz}, 2 \mathrm{H}\right), 6.92$ (d, $J=$ $8.4 \mathrm{~Hz}, 2 \mathrm{H}), 3.17(\mathrm{t}, J=4.6 \mathrm{~Hz}, 4 \mathrm{H}), 2.52(\mathrm{~s}, 2 \mathrm{H}), 2.40(\mathrm{~d}, J=$ $12.0 \mathrm{~Hz}, 2 \mathrm{H}), 1.89$ (s, 1H), $1.71(\mathrm{~s}, 14 \mathrm{H}), 1.59$ (brm, 2H); ${ }^{13} \mathrm{C} \mathrm{NMR}\left(101 \mathrm{MHz}, \mathrm{CDCl}_{3}\right) \delta$ (ppm): 151.0, 135.7, 126.2, 116.0, 75.4, 50.2, 37.8, 35.8, 35.0, 33.0, 27.6, 27.0, 25.9, 24.3.

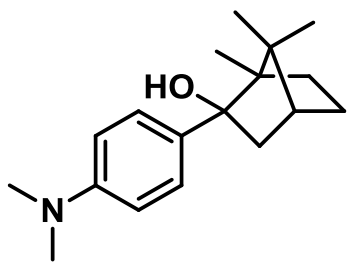

\section{2-(4-(Dimethylamino)phenyl)-1,7,7-trimethylbicyclo[2.2.1]hep} tan-2-ol (3o):

Prepared according to procedure $D$.

White solid; m.p.: $98-99{ }^{\circ} \mathrm{C}$; yield: $1.26 \mathrm{~g}, 92 \% ;{ }^{1} \mathrm{H}$ NMR $(400 \mathrm{MHz}$, $\left.\mathrm{CD}_{3} \mathrm{COCD}_{3}\right) \delta(\mathrm{ppm}): 7.38(\mathrm{~d}, J=8.8 \mathrm{~Hz}, 2 \mathrm{H}), 6.69(\mathrm{~d}, J=8.8 \mathrm{~Hz}$, 2H), 3.41 (brs, $1 \mathrm{H}), 2.91(\mathrm{~s}, 6 \mathrm{H}), 2.26-2.12(\mathrm{~m}, 2 \mathrm{H}), 1.81(\mathrm{t}, J=4.0 \mathrm{~Hz}, 1 \mathrm{H}), 1.74-1.64(\mathrm{~m}$, $1 \mathrm{H}), 1.30(\mathrm{~s}, 3 \mathrm{H}), 1.27-1.04(\mathrm{~m}, 2 \mathrm{H}), 0.92-0.86(\mathrm{~m}, 7 \mathrm{H}) ;{ }^{13} \mathrm{C} \mathrm{NMR}\left(101 \mathrm{MHz}, \mathrm{CD}_{3} \mathrm{COCD}_{3}\right)$ $\delta$ (ppm): 150.3, 135.5, 128.4, 112.3, 82.9, 54.0, 50.9, 46.7, 45.9, 40.7, 31.9, 27.2, 22.4, 22.2, 10.5 .

HRMS (positive ESI): m/z calculated for $\mathrm{C}_{18} \mathrm{H}_{28} \mathrm{NO}[\mathrm{M}+\mathrm{H}]^{+}:$274.2165; found: 274.2170 .<smiles>C=C(C)[C@@H]1CC=C(C)C(O)(c2ccc(N3CCCCC3)cc2)C1</smiles>

(3S)-6-Methyl-4'-(piperidin-1-yl)-3-(prop-1-en-2-yl)-3,4-di hydro-[1,1'-biphenyl]-1(2H)-ol (3p):

Prepared according to procedure $A$ from Grignard reagent SG6.

White solid; m.p.: $108-110{ }^{\circ} \mathrm{C}$; yield: $540 \mathrm{mg}, 69 \%$; ${ }^{1} \mathrm{H}$ NMR 
(400 MHz, $\left.\mathrm{CD}_{3} \mathrm{COCD}_{3}\right) \delta(\mathrm{ppm}): 7.32(\mathrm{~d}, J=8.8 \mathrm{~Hz}, 2 \mathrm{H}), 6.88(\mathrm{~d}, J=8.8 \mathrm{~Hz}, 2 \mathrm{H})$, 5.70-5.68 (brm, 1H), 4.62-4.57 (m, 2H), $3.85(\mathrm{~s}, 1 \mathrm{H}), 3.13(\mathrm{t}, J=5.6 \mathrm{~Hz}, 4 \mathrm{H}), 2.24-2.12(\mathrm{~m}$, $2 \mathrm{H}), 1.97-1.87(\mathrm{~m}, 3 \mathrm{H}), 1.69-1.53(\mathrm{~m}, 12 \mathrm{H}) ;{ }^{13} \mathrm{C} \mathrm{NMR}\left(101 \mathrm{MHz}, \mathrm{CD}_{3} \mathrm{COCD}_{3}\right) \delta(\mathrm{ppm}):$ 151.9, 149.9, 138.6, 137.6, 127.9, 124.9, 116.2, 109.0, 76.7, 51.1, 46.8, 39.1, 32.1, 26.7, 25.1, 20.8, 18.6.

HRMS (positive ESI): $\mathrm{m} / \mathrm{z}$ calculated for $\mathrm{C}_{21} \mathrm{H}_{30} \mathrm{NO}[\mathrm{M}+\mathrm{H}]^{+}: 312.2322$; found: 312.2325

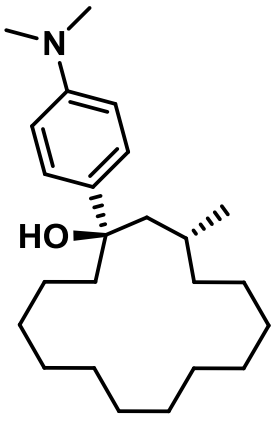

Trans-1-(4-(Dimethylamino)phenyl)-3-methylcyclopentadecan-1-o
I (3qa):
Prepared according to procedure $D$.
Pale yellow oil; yield: $1.45 \mathrm{~g}, 81 \% ;{ }^{1} \mathrm{H}$ NMR $\left(400 \mathrm{MHz}, \mathrm{CD}_{3} \mathrm{COCD}_{3}\right) \delta$
(ppm): $7.30(\mathrm{~d}, \mathrm{~J}=8.8 \mathrm{~Hz}, 2 \mathrm{H}), 6.68(\mathrm{~d}, \mathrm{~J}=9.2 \mathrm{~Hz}, 2 \mathrm{H}), 3.31(\mathrm{brs}, 1 \mathrm{H})$,
$2.89(\mathrm{~s}, 6 \mathrm{H}), 2.03-1.13(\mathrm{~m}, 23 \mathrm{H}), 0.98(\mathrm{~d}, \mathrm{~J}=6.4 \mathrm{~Hz}, 3 \mathrm{H}) ;{ }^{13} \mathrm{C} \mathrm{NMR}$
$\left(101 \mathrm{MHz}, \mathrm{CD}_{3} \mathrm{COCD}_{3}\right) \delta(\mathrm{ppm}): 149.9,138.5,126.5,112.8,76.5,54.9$,
$49.4,41.5,40.8,38.4,28.3,28.1,27.4,27.4,27.2,26.9,26.6,26.1$,

23.4, 23.4 .

HRMS (positive ESI): m/z calculated for $\mathrm{C}_{24} \mathrm{H}_{42} \mathrm{NO}[\mathrm{M}+\mathrm{H}]^{+}: 360.3261$; found: 360.3272 .

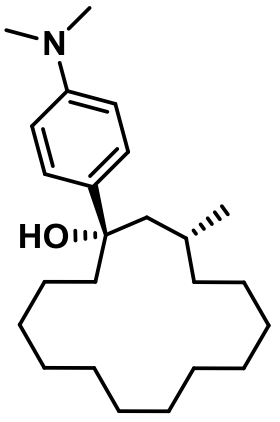
Cis-1-(4-(Dimethylamino)phenyl)-3-methylcyclopentadecan-1-ol
(3qb):
Prepared according to procedure $D$.
Pale yellow oil; yield: $160 \mathrm{mg}, 9 \%$; ${ }^{1} \mathrm{H}$ NMR $\left(400 \mathrm{MHz}, \mathrm{CD}_{3} \mathrm{COCD}_{3}\right) \delta$ (ppm): 7.32 (d, $J=9.2 \mathrm{~Hz}, 2 \mathrm{H}), 6.69(\mathrm{~d}, J=9.2 \mathrm{~Hz}, 2 \mathrm{H}$ ), 3.30 (brs, $1 \mathrm{H}$ ), $2.90(\mathrm{~s}, 6 \mathrm{H}), 1.87-0.86(\mathrm{~m}, 23 \mathrm{H}), 0.42(\mathrm{~d}, J=6.8 \mathrm{~Hz}, 3 \mathrm{H}) ;{ }^{13} \mathrm{C}$ NMR $\left(101 \mathrm{MHz}, \mathrm{CD}_{3} \mathrm{COCD}_{3}\right) \delta(\mathrm{ppm}): 150.0,137.2,127.3,112.7,76.0,51.2$ $40.8,39.0,37.5,29.2,28.3,28.1,27.5,27.5,27.2,26.8,26.8,26.0$,

22.2, 22.0.

HRMS (positive ESI): m/z calculated for $\mathrm{C}_{24} \mathrm{H}_{42} \mathrm{NO}[\mathrm{M}+\mathrm{H}]^{+}: 360.3261$; found: 360.3274 .

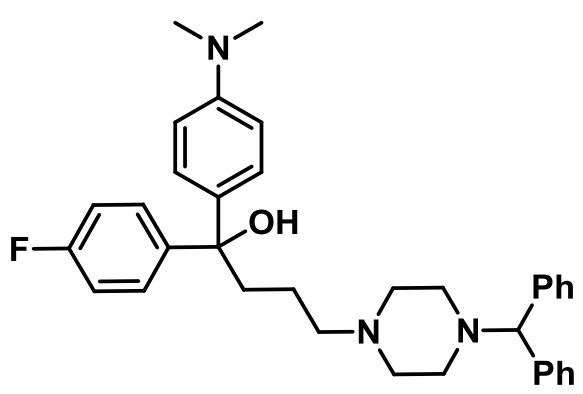

4-(4-Benzhydrylpiperazin-1-yl)-1-(4-(dimethylam ino)phenyl)-1-(4-fluorophenyl)butan-1-ol (3r):

Prepared according to procedure $D$.

Brown solid; m.p.: $135-137^{\circ} \mathrm{C}$; yield: $2.43 \mathrm{~g}, 91 \%$; ${ }^{1} \mathrm{H}$ NMR $\left(400 \mathrm{MHz}, \mathrm{CDCl}_{3}\right) \delta(\mathrm{ppm}):$ 7.50-7.48 (m, $5 \mathrm{H}), 7.36-7.31(\mathrm{~m}, 5 \mathrm{H}), 7.24(\mathrm{t}, J=7.4 \mathrm{~Hz}, 2 \mathrm{H})$, $6.99(\mathrm{t}, J=8.6 \mathrm{~Hz}, 2 \mathrm{H}), 6.80-6.70(\mathrm{~m}, 4 \mathrm{H}), 4.32(\mathrm{~s}$, $1 \mathrm{H}), 2.93(\mathrm{~s}, 6 \mathrm{H}), 2.89(\mathrm{~s}, 2 \mathrm{H}), 2.56-2.39(\mathrm{~m}, 11 \mathrm{H})$, 1.70-1.55 (m, 2H); ${ }^{13} \mathrm{C}$ NMR (101 MHz, $\left.\mathrm{CDCl}_{3}\right) \delta(\mathrm{ppm}): 161.1\left(\mathrm{~J}_{\mathrm{C}-\mathrm{F}}=244.0 \mathrm{~Hz}\right), 148.9$, $144.8,142.7\left(J_{C-F}=5.2 \mathrm{~Hz}\right), 135.8,128.4,127.7,126.8,116.1,115.5,114.3\left(J_{C-F}=20.9\right.$ $\mathrm{Hz}), 112.1,76.1,75.9,59.0,52.9,51.2,42.8,40.5,21.5$.

HRMS (positive ESI): m/z calculated for $\mathrm{C}_{35} \mathrm{H}_{41} \mathrm{~N}_{3} \mathrm{OF}[\mathrm{M}+\mathrm{H}]^{+}: 538.3228$; found: 538.3240 .

1-(4-(Dimethylamino)phenyl)-1-phenylethane-1,2-diol (3s): CAS No.1805822-33-6. Prepared according to procedure $C$. 
<smiles>CN(C)c1ccc(C(O)(CO)c2ccccc2)cc1</smiles>

White solid; m.p.: 98-100 ${ }^{\circ} \mathrm{C}$; yield: $478 \mathrm{mg}, 74 \%$; ${ }^{1} \mathrm{H}$ NMR $(400$ $\left.\mathrm{MHz}, \mathrm{CD}_{3} \mathrm{COCD}_{3}\right) \delta(\mathrm{ppm}):$ 7.48--7.44 (m, 2H), 7.27-7.14 (m, 5H), $6.69(\mathrm{~d}, J=8.8 \mathrm{~Hz}, 2 \mathrm{H}), 4.22(\mathrm{brs}, 1 \mathrm{H}), 4.05-4.00(\mathrm{~m}, 2 \mathrm{H}), 4.22$ (brs, $1 \mathrm{H}), 3.90-3,85$ (brm, 1H), $2.86(\mathrm{~s}, 6 \mathrm{H}) ;{ }^{13} \mathrm{C} \mathrm{NMR}(101 \mathrm{MHz}$, $\left.\mathrm{CD}_{3} \mathrm{COCD}_{3}\right) \delta(\mathrm{ppm}): 150.4,147.3,134.3,128.4,128.2,127.5$, 127.1, 112.8, 78.6, 69.8, 40.7 .

1-(4-(Dimethylamino)phenyl)-1-phenylethane-1,2-diol (3t):<smiles>CN(C)c1ccc(C2(O)CCC(O)CC2)cc1</smiles>

Prepared according to procedure $C$.

White solid; yield: $464 \mathrm{mg}, 79 \%$; mixture of 2 stereomers, $d . r$. $=$ $70: 30$, characterization of the major stereomer: ${ }^{1} \mathrm{H}$ NMR $(400$ $\left.\mathrm{MHz}, \mathrm{DMSO}-\mathrm{d}_{6}\right) \delta$ (ppm): $7.26(\mathrm{~d}, J=8.8 \mathrm{~Hz}, 2 \mathrm{H}), 6.65$ (d, $J=$ $8.8 \mathrm{~Hz}, 2 \mathrm{H}), 4.46-4.43(\mathrm{~m}, 1 \mathrm{H}), 3.44$ (brs, 1H), $2.84(\mathrm{~s}, 6 \mathrm{H})$, 1.75-1.50 (m, 8H); ${ }^{13} \mathrm{C}$ NMR (101 MHz, DMSO-d $) \delta$ (ppm): 148.9, 138.5, 125.2, 112.0, 70.1, 68.7, 40.4, 37.1, 31.3. HRMS (positive ESI): $\mathrm{m} / \mathrm{z}$ calculated for $\mathrm{C}_{14} \mathrm{H}_{22} \mathrm{NO}_{2}[\mathrm{M}+\mathrm{H}]^{+}: 236.1645$; found: 236.1654.<smiles>CN(C)c1ccc(C(C)(O)CC(C)(C)O)cc1</smiles>

\section{2-(4-(Dimethylamino)phenyl)-4-methylpentane-2,4-diol}

(3u):

Prepared according to procedure $C$.

White solid, $450 \mathrm{mg}$ crude product, unstable, $45 \mathrm{mg}$ of which was used in the next step immediately; ${ }^{1} \mathrm{H}$ NMR $(400 \mathrm{MHz}$, $\left.\mathrm{CD}_{3} \mathrm{COCD}_{3}\right) \delta(\mathrm{ppm}): 7.36(\mathrm{~d}, J=8.8 \mathrm{~Hz}, 2 \mathrm{H}), 6.72(\mathrm{~d}, J=8.8 \mathrm{~Hz}, 2 \mathrm{H}), 5.43(\mathrm{~s}, 1 \mathrm{H}), 4.47$ (s, 1H), $2.83(\mathrm{~s}, 6 \mathrm{H}), 2.16-2.06(\mathrm{~m}, 2 \mathrm{H}), 1.47(\mathrm{~s}, 3 \mathrm{H}), 1.22(\mathrm{~s}, 3 \mathrm{H}), 0.82(\mathrm{~s}, 3 \mathrm{H}) ;{ }^{13} \mathrm{C}$ NMR $\left(101 \mathrm{MHz}, \mathrm{CD}_{3} \mathrm{COCD}_{3}\right) \delta$ (ppm): 149.8, 138.5, 126.3, 112.8, 75.1, 72.7, 53.5, 40.8, 34.9, 33.4, 30.0.

HRMS (positive ESI): m/z calculated for $\mathrm{C}_{14} \mathrm{H}_{24} \mathrm{NO}_{2}[\mathrm{M}+\mathrm{H}]^{+}:$238.1802; found: 238.1810 .<smiles>CC(=O)C(C)(O)c1ccc(N(C)C)cc1</smiles>

\section{3-(4-(Dimethylamino)phenyl)-3-hydroxybutan-2-one (3v):}

Prepared according to procedure $A$ from Grignard reagent SG1. and biacetyl.

Colorless oil; yield: $150 \mathrm{mg}, 29 \%$; ${ }^{1} \mathrm{H}$ NMR $\left(400 \mathrm{MHz}, \mathrm{DMSO}-d_{6}\right) \delta$ (ppm): 7.23 (d, J = 8.4 Hz, 2H), 6.69 (d, J = 8.8 Hz, 2H), $5.78(\mathrm{~s}, 1 \mathrm{H})$ $2.87(\mathrm{~s}, 6 \mathrm{H}), 2.00(\mathrm{~s}, 3 \mathrm{H}), 1.48(\mathrm{~s}, 3 \mathrm{H}) ;{ }^{13} \mathrm{C}$ NMR (101 MHz, DMSO-d $) \delta(\mathrm{ppm}):$ 149.5, 130.5, 125.7, 112.1, 79.3, 40.1, 25.8, 24.0.

HRMS (positive ESI): m/z calculated for $\mathrm{C}_{12} \mathrm{H}_{18} \mathrm{NO}_{2}[\mathrm{M}+\mathrm{H}]^{+}:$208.1332; found: 208.1334.

\subsection{Data of deoxygenation products}<smiles>CC(C)Cc1ccc(N(C)C)cc1</smiles>

4-Isobutyl-N,N-dimethylaniline (2a): CAS No. 30090-19-8. $\mathbf{S} / \mathbf{C}=$ 100,000 in water. 
Colorless liquid; yield: $41 \mathrm{mg}, 92 \%{ }^{1} \mathrm{H}$ NMR $\left(400 \mathrm{MHz}, \mathrm{CDCl}_{3}\right) \delta$ (ppm): 7.06 (d, $J=8.0 \mathrm{~Hz}$, 2H), 6.76 (d, J = 8.4 Hz, 2H), $2.95(\mathrm{~s}, 6 \mathrm{H}), 2.42(\mathrm{~d}, J=7.2 \mathrm{~Hz}, 2 \mathrm{H}), 1.83(\operatorname{sep}, J=6.8 \mathrm{~Hz}$, $1 \mathrm{H}), 0.92(\mathrm{~d}, J=6.8 \mathrm{~Hz}, 6 \mathrm{H}) ;{ }^{13} \mathrm{C}$ NMR $\left(101 \mathrm{MHz}, \mathrm{CDCl}_{3}\right) \delta(\mathrm{ppm}): 148.6,130.5,129.7$, $113.1,44.4,41.1,30.4,22.3$.<smiles>CN(C)c1ccc(Cc2ccccc2)cc1</smiles>

4-Benzyl-N,N-dimethylaniline (2b): CAS No. 46734-13-8. S/C= 100,000 in water.

Colorless liquid; yield: $49 \mathrm{mg}, 92 \% ;{ }^{1} \mathrm{H}$ NMR $\left(400 \mathrm{MHz}, \mathrm{CDCl}_{3}\right) \delta$ (ppm): 7.29-7.18 (m, 5H), 7.08-7.05 (m, 2H), 6.71-6.67 (m, 2H), 3.90-3.89 (m, 2H), 2.91-2.89 (m, 6H); $\left.{ }^{13} \mathrm{C} \mathrm{NMR} \mathrm{(101} \mathrm{MHz,} \mathrm{CDCl}_{3}\right) \delta(\mathrm{ppm}): 149.1,142.0$, $129.5,129.2,128.8,128.3,125.7,112.9,40.9,40.8$.

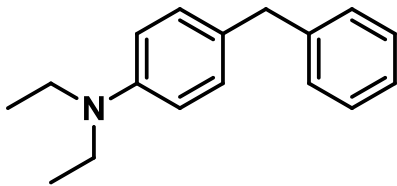

4-Benzyl-N,N-diethylaniline (2b-3): CAS No. 46906-42-7. $S / C=100,000$ in water.

Colorless liquid; yield: $53 \mathrm{mg}, 89 \%$; ${ }^{1} \mathrm{H}$ NMR $\left(400 \mathrm{MHz}, \mathrm{CDCl}_{3}\right)$ $\delta$ (ppm): 7.20-6.94 (m, 5H), 6.95 (d, J = $8.0 \mathrm{~Hz}, 2 \mathrm{H}), 6.54(\mathrm{~d}, J$ $=6.4 \mathrm{~Hz}, 2 \mathrm{H}), 3.80(\mathrm{~s}, 2 \mathrm{H}), 3.23(\mathrm{q}, J=6.8 \mathrm{~Hz}, 4 \mathrm{H}), 1.05(\mathrm{t}, J=7.0 \mathrm{~Hz}, 6 \mathrm{H}) ;{ }^{13} \mathrm{C} \mathrm{NMR}(101$ $\left.\mathrm{MHz}, \mathrm{CDCl}_{3}\right) \delta(\mathrm{ppm}): 146.2,142.1,129.7,128.8,128.3,127.9,125.7,112.7,44.4,40.9$, 12.6.<smiles>c1ccc(Cc2ccc(N3CCCC3)cc2)cc1</smiles>

1-(4-Benzylphenyl)pyrrolidine (2b-4): $S / C=100,000$ in water.

Pale yellow liquid; yield: $50 \mathrm{mg}, 85 \%$; ${ }^{1} \mathrm{H}$ NMR $(400 \mathrm{MHz}$, $\left.\mathrm{CDCl}_{3}\right) \delta(\mathrm{ppm}):$ 7.27-7.14 (m, 5H), $7.04(\mathrm{~d}, J=8.4 \mathrm{~Hz}, 2 \mathrm{H})$, $6.51(\mathrm{~d}, J=8.4 \mathrm{~Hz}, 2 \mathrm{H}), 3.88(\mathrm{~s}, 2 \mathrm{H}), 3.25(\mathrm{t}, J=6.2 \mathrm{~Hz}, 4 \mathrm{H}), 1.97(\mathrm{t}, J=6.2 \mathrm{~Hz}, 4 \mathrm{H}) ;{ }^{13} \mathrm{C}$ $\operatorname{NMR}\left(101 \mathrm{MHz}, \mathrm{CDCl}_{3}\right) \delta(\mathrm{ppm}):$ 146.4, 142.3, 129.6, 128.7, 128.3, 127.8, 125.7, 111.7, 47.7, 41.0, 25.4.

HRMS (positive ESI): m/z calculated for $\mathrm{C}_{17} \mathrm{H}_{20} \mathrm{~N}[\mathrm{M}+\mathrm{H}]^{+}$: 238.1590; found: 238.1592 .<smiles>c1ccc(Cc2ccc(N3CCCCC3)cc2)cc1</smiles>

1-(4-Benzylphenyl)piperidine $(2 b-5): S / C=100,000$ in water.

Pale yellow liquid; yield: $56 \mathrm{mg}, 90 \%$; ${ }^{1} \mathrm{H}$ NMR $(400 \mathrm{MHz}$, $\left.\mathrm{CDCl}_{3}\right) \delta(\mathrm{ppm}): 7.27-7.14(\mathrm{~m}, 5 \mathrm{H}), 7.05(\mathrm{~d}, J=8.8 \mathrm{~Hz}, 2 \mathrm{H})$, $6.86(\mathrm{~d}, J=8.4 \mathrm{~Hz}, 2 \mathrm{H}$ ), $3.89(\mathrm{~s}, 2 \mathrm{H}$ ), $3.09(\mathrm{t}, J=5.4 \mathrm{~Hz}, 4 \mathrm{H}$ ), 1.69 (qui, $J=5.6 \mathrm{~Hz}, 4 \mathrm{H}$ ), 1.54 (qui, $J=5.6 \mathrm{~Hz}, 4 \mathrm{H}$ ); ${ }^{13} \mathrm{C} \mathrm{NMR}\left(101 \mathrm{MHz}, \mathrm{CDCl}_{3}\right) \delta$ (ppm): 150.6, 141.8, 131.8, 129.4 , 128.8, 128.3, 125.8, 116.7, 50.9, 41.0, 25.9, 24.3.

HRMS (positive ESI): $\mathrm{m} / \mathrm{z}$ calculated for $\mathrm{C}_{18} \mathrm{H}_{22} \mathrm{~N}[\mathrm{M}+\mathrm{H}]^{+}:$252.1747; found: 252.1753 .

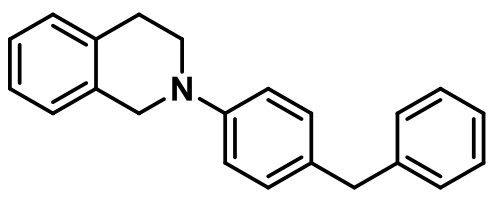

\section{2-(4-Benzylphenyl)-1,2,3,4-tetrahydroisoquinoline} $(2 b-6): S / C=100,000$ in water.

Pale yellow liquid; yield: $63 \mathrm{mg}, 84 \%$; ${ }^{1} \mathrm{H}$ NMR $(400 \mathrm{MHz}$, $\left.\mathrm{CDCl}_{3}\right) \delta(\mathrm{ppm}):$ 7.31-7.13 (m, 11H), 6.96-6.94 (m, 2H), $4.40(\mathrm{~s}, 2 \mathrm{H}), 3.95(\mathrm{~s}, 2 \mathrm{H}), 3.55(\mathrm{t}, J=5.8 \mathrm{~Hz}, 2 \mathrm{H}), 3.00(\mathrm{t}, J=5.6 \mathrm{~Hz}, 2 \mathrm{H}) ;{ }^{13} \mathrm{C}$ NMR $(101$ $\left.\mathrm{MHz}, \mathrm{CDCl}_{3}\right) \delta(\mathrm{ppm}): 148.9,141.8,134.7,134.5,131.4,129.6,128.8,128.5,128.4$, 
126.5, 126.2, 125.9, 125.8, 115.5, 51.1, 46.8, 41.0, 29.1.

HRMS (positive ESI): m/z calculated for $\mathrm{C}_{22} \mathrm{H}_{22} \mathrm{~N}[\mathrm{M}+\mathrm{H}]^{+}: 300.1747$; found: 300.1750 .<smiles>CNc1ccc(Cc2ccccc2)cc1</smiles>

4-Benzyl-N-methylaniline (2b-8): CAS No. 6851-78-1. $\mathrm{S} / \mathrm{C}=$ 100,000 in water.

Colorless liquid; yield: $40 \mathrm{mg}, 82 \%$; ${ }^{1} \mathrm{H}$ NMR $\left(400 \mathrm{MHz}, \mathrm{CDCl}_{3}\right) \delta$ (ppm): 7.28-7.15 (m, 5H), 7.02-7.00 (m, 2H), 6.57-6.53 (m, 2H), 3.88 (s, 2H), 3.59 (brs, $1 \mathrm{H}), 2.80$ (s, 3H); $\left.{ }^{13} \mathrm{C} \mathrm{NMR} \mathrm{(101} \mathrm{MHz,} \mathrm{CDCl}_{3}\right) \delta$ (ppm): 147.6, 142.1, 129.8, 129.7, 128.8, 128.3, 125.8, 112.6, 41.0, 30.9.<smiles>CC(=O)Nc1ccc(Cc2ccccc2)cc1</smiles>

4-Benzyl-N-methylaniline (2b-9): CAS No. 76472-81-6. S/C = 1000, water: $\mathrm{HPIP}=1: 1$.

White solid; m.p.: $113-115^{\circ} \mathrm{C}$ (reported $\left.128-129^{\circ} \mathrm{C}\right) ;{ }^{20}$ yield: 48 mg, 85\%; ${ }^{1} \mathrm{H}$ NMR (400 MHz, $\left.\mathrm{CDCl}_{3}\right) \delta$ (ppm): 7.54 (brs, $\left.1 \mathrm{H}\right)$, $7.37(\mathrm{~d}, J=8.4 \mathrm{~Hz}, 2 \mathrm{H}), 7.24-7.12(\mathrm{~m}, 5 \mathrm{H}), 7.08(\mathrm{~d}, J=8.4 \mathrm{~Hz}, 2 \mathrm{H}), 3.90(\mathrm{~s}, 2 \mathrm{H}), 2.10(\mathrm{~s}$, $3 \mathrm{H}) ;{ }^{13} \mathrm{C} \mathrm{NMR}\left(101 \mathrm{MHz}, \mathrm{CDCl}_{3}\right) \delta$ (ppm): 168.4, 141.0, 137.1, 135.9, 129.3, 128.8, 128.4, $126.0,120.2,41.3,24.4$.<smiles>Cc1ccc(N(C)C)cc1</smiles>

20.2 .

N,N,4-trimethylaniline (2c): CAS No. 99-97-8. $S / C=100,000$ in water. Colorless liquid; yield: $33 \mathrm{mg}, 98 \% ;{ }^{1} \mathrm{H}$ NMR $\left(400 \mathrm{MHz}, \mathrm{CDCl}_{3}\right) \delta(\mathrm{ppm})$ : $7.08(\mathrm{~d}, J=8.4 \mathrm{~Hz}, 2 \mathrm{H}), 6.73(\mathrm{~J}=8.4 \mathrm{~Hz}, 2 \mathrm{H}), 2.93(\mathrm{~s}, 6 \mathrm{H}), 2.29(\mathrm{~m}, 3 \mathrm{H})$; ${ }^{13} \mathrm{C}$ NMR $\left(101 \mathrm{MHz}, \mathrm{CDCl}_{3}\right) \delta$ (ppm): 148.8, 129.6, 126.2, 113.3, 41.1,<smiles>CCN(CC)c1ccc(C)cc1</smiles>

$N, N$-diethyl-4-methylaniline (2d): $C A S$ No. $613-48-9 . S / C=100,000$ in water.

Colorless liquid; yield: $38 \mathrm{mg}, 94 \% ;{ }^{1} \mathrm{H} \mathrm{NMR}\left(400 \mathrm{MHz}, \mathrm{CDCl}_{3}\right) \delta$ (ppm): 7.06 (d, $J=8.4 \mathrm{~Hz}, 2 \mathrm{H}), 6.66(J=8.4 \mathrm{~Hz}, 2 \mathrm{H}), 3.35$ (q, $J=6.8$ $\mathrm{Hz}, 4 \mathrm{H}), 2.28(\mathrm{~m}, 3 \mathrm{H}) ; 1.17(\mathrm{t}, J=7.2 \mathrm{~Hz}, 6 \mathrm{H}) ;{ }^{13} \mathrm{C} \mathrm{NMR}\left(101 \mathrm{MHz}, \mathrm{CDCl}_{3}\right) \delta(\mathrm{ppm}): 145.8$, 129.7, 124.8, 112.6, 44.5, 20.1, 12.5 .<smiles>CNc1ccc(C)cc1</smiles>

N,4-Dimethylaniline (2e): CAS No. 623-08-5. $S / C=100,000$ in water. Colorless liquid; yield: $24 \mathrm{mg}, 81 \%$; ${ }^{1} \mathrm{H}$ NMR $\left(400 \mathrm{MHz}, \mathrm{CDCl}_{3}\right) \delta$ (ppm): $7.03(\mathrm{~d}, \mathrm{~J}=8.0 \mathrm{~Hz}, 2 \mathrm{H}), 6.57(\mathrm{~J}=8.4 \mathrm{~Hz}, 2 \mathrm{H}), 3.56$ (brs, 1H), $2.84(\mathrm{~s}, 3 \mathrm{H})$, $2.27(\mathrm{~m}, 3 \mathrm{H}) ;{ }^{13} \mathrm{C}$ NMR $\left(101 \mathrm{MHz}, \mathrm{CDCl}_{3}\right) \delta$ (ppm): 147.1, 129.6, 126.4,

$112.5,31.0,20.3$<smiles>CCCCN(C)c1ccc(C)cc1</smiles>

$N$-Butyl- $N$,4-dimethylaniline (2f): CAS No. 57049-30-6. $S / C=$ 100,000 in water

Colorless liquid; yield: $42 \mathrm{mg}, 94 \% ;{ }^{1} \mathrm{H} \mathrm{NMR}\left(400 \mathrm{MHz}, \mathrm{CDCl}_{3}\right) \delta$ (ppm): 7.06 (d, $J=8.0 \mathrm{~Hz}, 2 \mathrm{H}), 6.66(\mathrm{~J}=8.8 \mathrm{~Hz}, 2 \mathrm{H}), 3.29$ (t, $J=7.4$ $\mathrm{Hz}, 2 \mathrm{H}), 2.91(\mathrm{~s}, 3 \mathrm{H}), 2.28(\mathrm{~s}, 3 \mathrm{H}), 1.41-1.32(\mathrm{~m}, 2 \mathrm{H}), 1.60-1.53(\mathrm{~m}$, $2 \mathrm{H}), 0.96$ (t, $J=7.4 \mathrm{~Hz}, 3 \mathrm{H}$ ); ${ }^{13} \mathrm{C}$ NMR $\left(101 \mathrm{MHz}, \mathrm{CDCl}_{3}\right) \delta$ (ppm): 147.4, 129.6, 125.1, $112.5,52.9,38.4,28.7,20.4,20.2,14.0$. 
<smiles>CN(C)c1ccc(CC2CCCCC2)cc1</smiles>

4-(Cyclohexylmethyl)- $N, N$-dimethylaniline $(2 \mathrm{~g}): S / C=20,000$, water : TFE = 1:1.

Pale yellow liquid; yield: $48 \mathrm{mg}, 89 \%$; ${ }^{1} \mathrm{H} \mathrm{NMR}\left(400 \mathrm{MHz}, \mathrm{CDCl}_{3}\right)$ $\delta$ (ppm): 7.03 (d, J = 8.4 Hz, 2H), $6.72(J=8.4 \mathrm{~Hz}, 2 \mathrm{H}), 2.92(\mathrm{~s}$, $6 \mathrm{H}), 2.40(\mathrm{~d}, J=7.2 \mathrm{~Hz}, 2 \mathrm{H}), 1.71-1.64(\mathrm{~m}, 5 \mathrm{H}), 1.21-1.16(\mathrm{~m}, 3 \mathrm{H}), 0.97-0.87(\mathrm{~m}, 3 \mathrm{H}) ;{ }^{13} \mathrm{C}$ NMR (101 MHz, $\left.\mathrm{CDCl}_{3}\right) \delta(\mathrm{ppm}):$ 148.7, 129.7, 128.2, 112.8, 43.1, 41.0, 40.0, 33.2, 26.6, 26.4 .

HRMS (positive ESI): m/z calculated for $\mathrm{C}_{15} \mathrm{H}_{24} \mathrm{~N}[\mathrm{M}+\mathrm{H}]^{+}:$218.1903; found: 218.1906 .

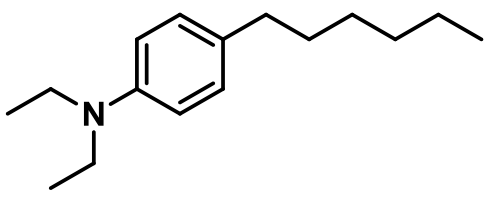

$\mathrm{N}, \mathrm{N}$-Diethyl-4-hexylaniline (2h): CAS No. 1691206-56-0. $\mathbf{S} / \mathbf{C}=\mathbf{2 0 , 0 0 0}$, water : TFE $=\mathbf{1}: \mathbf{1}$.

Colorless liquid; yield: $57 \mathrm{mg}, 97 \%$; ${ }^{1} \mathrm{H}$ NMR $(400 \mathrm{MHz}$, $\left.\mathrm{CDCl}_{3}\right) \delta(\mathrm{ppm}): 7.05(\mathrm{~d}, J=8.4 \mathrm{~Hz}, 2 \mathrm{H}), 6.65(J=6.8 \mathrm{~Hz}$ 2H), 3.33 (q, $J=7.2 \mathrm{~Hz}, 4 \mathrm{H}$ ), 2.51 (t, $J=7.8 \mathrm{~Hz}, 2 \mathrm{H}$ ), 1.58 (qui, $J=6.8 \mathrm{~Hz}, 2 \mathrm{H}$ ), 1.35-1.29 $(\mathrm{m}, 6 \mathrm{H}), 1.16(\mathrm{t}, J=7.2 \mathrm{~Hz}, 6 \mathrm{H}), 0.90(\mathrm{t}, \mathrm{J}=6.2 \mathrm{~Hz}, 3 \mathrm{H}) ;{ }^{13} \mathrm{C} \mathrm{NMR}\left(101 \mathrm{MHz}, \mathrm{CDCl}_{3}\right) \delta$ (ppm): 145.9, 130.0, 129.1, 112.2, 44.4, 34.9, 31.84, 31.81, 29.1, 22.7, 14.1, 12.6.

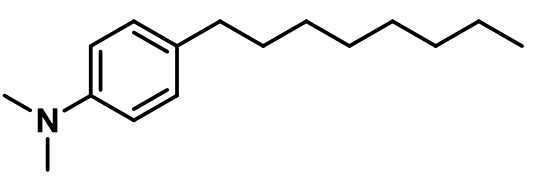

$\mathbf{N}, \mathbf{N}$-dimethyl-4-octylaniline (2i): CAS No. 1104602-56-3. $S / C=\mathbf{2 0 , 0 0 0}$, water : TFE $=\mathbf{1 : 1}$.

Colorless liquid; yield: $48 \mathrm{mg}, 82 \%$; ${ }^{1} \mathrm{H}$ NMR $(400 \mathrm{MHz}$, $\left.\mathrm{CDCl}_{3}\right) \delta(\mathrm{ppm}): 7.08(\mathrm{~d}, J=8.4 \mathrm{~Hz}, 2 \mathrm{H}), 6.72(J=8.4$

$\mathrm{Hz}, 2 \mathrm{H}$ ), $2.93(\mathrm{~s}, 6 \mathrm{H}), 2.53(\mathrm{t}, J=7.6 \mathrm{~Hz}, 2 \mathrm{H}), 1.59$ (qui, $J=7.2 \mathrm{~Hz}, 2 \mathrm{H}$ ), 1.32-1.29 (m, $10 \mathrm{H}), 0.90(\mathrm{t}, \mathrm{J}=6.2 \mathrm{~Hz}, 3 \mathrm{H}) ;{ }^{13} \mathrm{C}$ NMR $\left(101 \mathrm{MHz}, \mathrm{CDCl}_{3}\right) \delta(\mathrm{ppm}): 148.9,131.3,128.9$, $113.0,41.0,34.9,31.9,31.8,29.5,29.4,29.3,22.7,14.1$.<smiles>CN(C)c1ccc(CCCc2ccccc2)cc1</smiles>

N,N-dimethyl-4-(3-phenylpropyl)aniline (2j): CAS No. 861610-14-2. $S / C=1000$, water $: H F I P=1: 1$.

Colorless liquid; yield: $57 \mathrm{mg}, 95 \%$; ${ }^{1} \mathrm{H}$ NMR $(400 \mathrm{MHz}$, $\left.\mathrm{CDCl}_{3}\right) \delta(\mathrm{ppm}):$ 7.31-7.18 (m, 5H), $7.09(\mathrm{~d}, J=8.0 \mathrm{~Hz}, 2 \mathrm{H})$, $6.72(J=8.0 \mathrm{~Hz}, 2 \mathrm{H}), 2.93(\mathrm{~s}, 6 \mathrm{H}), 2.66(\mathrm{t}, J=7.6 \mathrm{~Hz}, 2 \mathrm{H}), 2.58(\mathrm{t}, J=7.6 \mathrm{~Hz}, 2 \mathrm{H}), 1.94$ (qui, $J=7.6 \mathrm{~Hz}, 2 \mathrm{H}$ ); ${ }^{13} \mathrm{C}$ NMR $\left(101 \mathrm{MHz}, \mathrm{CDCl}_{3}\right) \delta$ (ppm): 149.0, 142.5, 130.5, 129.0, $128.4,128.2,125.6,113.0,40.9,35.4,34.4,33.2$.<smiles>CC(C)=CCCC(C)CCc1ccc(N(C)C)cc1</smiles>

4-(3,7-Dimethyloct-6-en-1-yl)-N,N-dimethylaniline (2k): $S / C=$ 1000, water : HFIP = 1:1.

Pale yellow liquid; yield: $62 \mathrm{mg}, 96 \% ;{ }^{1} \mathrm{H}$ NMR $\left(400 \mathrm{MHz}, \mathrm{CDCl}_{3}\right)$ $\delta(\mathrm{ppm}): 7.08(\mathrm{~d}, J=8.4 \mathrm{~Hz}, 2 \mathrm{H}), 6.72(J=8.4 \mathrm{~Hz}, 2 \mathrm{H}), 5.13(\mathrm{t}, J$ $=6.8 \mathrm{~Hz}, 1 \mathrm{H}), 2.93(\mathrm{~s}, 6 \mathrm{H}), 2.62-2.46(\mathrm{~m}, 2 \mathrm{H}), 2.04-1.96(\mathrm{~m}, 2 \mathrm{H})$, $1.71(\mathrm{~s}, 3 \mathrm{H}), 1.63(\mathrm{~s}, 3 \mathrm{H}), 1.63-1.37(\mathrm{~m}, 4 \mathrm{H}), 1.23-1.18(\mathrm{~m}, 1 \mathrm{H}), 0.95(\mathrm{~d}, J=6.0 \mathrm{~Hz}, 3 \mathrm{H})$; ${ }^{13} \mathrm{C} \mathrm{NMR}\left(101 \mathrm{MHz}, \mathrm{CDCl}_{3}\right) \delta(\mathrm{ppm}): 148.9,131.4,131.0,128.9,125.0,113.1,41.0,39.2$, 37.0, 32.3, 32.1, 25.7, 25.5, 19.5, 17.6 .

HRMS (positive ESI): $\mathrm{m} / \mathrm{z}$ calculated for $\mathrm{C}_{18} \mathrm{H}_{30} \mathrm{~N}[\mathrm{M}+\mathrm{H}]^{+}: 260.2373$; found: 260.2385 . 
4-(Benzo[d][1,3]dioxol-5-ylmethyl)- $N, N$-dimethylaniline (2I): $S / C=100,000$ in water.<smiles>CN(C)c1ccc(Cc2ccc3c(c2)OCO3)cc1</smiles>
Pale yellow liquid; yield: $61 \mathrm{mg}, 95 \% ;{ }^{1} \mathrm{H}$ NMR $(400 \mathrm{MHz}$, $\left.\mathrm{CDCl}_{3}\right) \delta(\mathrm{ppm}): 7.06(\mathrm{~d}, J=8.8 \mathrm{~Hz}, 2 \mathrm{H}), 6.74-6.65(\mathrm{~m}, 5 \mathrm{H})$, $5.90(\mathrm{~s}, 2 \mathrm{H}), 3.81(\mathrm{~s}, 2 \mathrm{H}), 2.92(\mathrm{~s}, 6 \mathrm{H}) ;{ }^{13} \mathrm{C}$ NMR $(101 \mathrm{MHz}$, $\left.\mathrm{CDCl}_{3}\right) \delta(\mathrm{ppm}): 149.0,147.6,145.6,136.0,129.4,121.5$,

113.1, 109.3, 108.0, 100.7, 77.2, 40.9, 40.6.

HRMS (positive ESI): m/z calculated for $\mathrm{C}_{16} \mathrm{H}_{18} \mathrm{O}_{2} \mathrm{~N}[\mathrm{M}+\mathrm{H}]^{+}:$256.1332; found: 256.1342 .<smiles>CCCCN(C)c1ccc(Cc2ccc(N(C)C)cc2)cc1</smiles>

$\mathrm{N}$-Butyl-4-(4-(dimethylamino)benzyl)- $\mathrm{N}$-methyla niline $(2 \mathrm{~m}): S / C=20,000$, water : TFE $=1: 1$. Colorless liquid; yield: $70 \mathrm{mg}, 94 \% ;{ }^{1} \mathrm{H}$ NMR $(400$ $\left.\mathrm{MHz}, \mathrm{CDCl}_{3}\right) \delta(\mathrm{ppm}): 7.11-7.06(\mathrm{~m}, 4 \mathrm{H}), 6.72(\mathrm{~d}, J$ $=8.4 \mathrm{~Hz}, 2 \mathrm{H}), 6.66(\mathrm{~d}, J=8.8 \mathrm{~Hz}, 2 \mathrm{H}), 3.84(\mathrm{~s}, 2 \mathrm{H}), 3.30(\mathrm{t}, J=7.4 \mathrm{~Hz}, 2 \mathrm{H}), 2.93(\mathrm{~s}, 6 \mathrm{H})$, $2.92(\mathrm{~s}, 3 \mathrm{H}), 1.61-1.53(\mathrm{~m}, 2 \mathrm{H}), 1.42-1.34(\mathrm{~m}, 2 \mathrm{H}), 0.97(\mathrm{t}, J=7.4 \mathrm{~Hz}, 3 \mathrm{H}) ;{ }^{13} \mathrm{C}$ NMR $(101$ $\left.\mathrm{MHz}, \mathrm{CDCl}_{3}\right) \delta(\mathrm{ppm}): 149.0,147.7,130.4,129.41,129.37,129.36,113.0,112.3,52.7$, 40.9, 39.8, 38.4, 28.8, 20.4, 14.0.

HRMS (positive ESI): $\mathrm{m} / \mathrm{z}$ calculated for $\mathrm{C}_{20} \mathrm{H}_{29} \mathrm{~N}_{2}[\mathrm{M}+\mathrm{H}]^{+}:$: 297.2325; found: 297.2340 .<smiles>Cc1cc(C)c(Cc2ccc(N(C)C)cc2)c(C)c1</smiles>

$N, N$-dimethyl-4-(2,4,6-trimethylbenzyl)aniline $(2 n): S / C=$ 20,000, water : TFE $=1: 1$.

Colorless oil; yield: $63 \mathrm{mg}, 99 \% ;{ }^{1} \mathrm{H}$ NMR $\left(400 \mathrm{MHz}, \mathrm{CDCl}_{3}\right) \delta$ (ppm): 6.94-6.92 (m, 4H), 6.69 (dd, $J=8.6 \mathrm{~Hz}, 2.2 \mathrm{~Hz}, 2 \mathrm{H})$, $3.96(\mathrm{~s}, 2 \mathrm{H}), 2.92(\mathrm{~d}, J=2.0 \mathrm{~Hz}, 6 \mathrm{H}), 2.33(\mathrm{~d}, J=2.4 \mathrm{~Hz}, 3 \mathrm{H})$, $2.26(\mathrm{~d}, J=2.0 \mathrm{~Hz}, 6 \mathrm{H}) ;{ }^{13} \mathrm{C} \mathrm{NMR}\left(101 \mathrm{MHz}, \mathrm{CDCl}_{3}\right) \delta(\mathrm{ppm}): 148.9,137.0,135.4,134.6$, 128.8, 128.5, 128.2, 113.1, 40.9, 33.7, 21.0, 20.2.

HRMS (positive ESI): m/z calculated for $\mathrm{C}_{18} \mathrm{H}_{24} \mathrm{~N}$ [M+H] $:$ : 254.1903; found: 254.1907.<smiles>CSc1ccc(Cc2ccc(N(C)C)cc2)cc1</smiles>

N,N-Dimethyl-4-(4-(methylthio)benzyl)aniline (2o): $S / C=$ 20,000, water: TFE $=1: 1$.

Pale yellow solid; m.p.: $66-68{ }^{\circ} \mathrm{C}$; yield: $51 \mathrm{mg}, 80 \% ;{ }^{1} \mathrm{H}$ NMR (400 MHz, $\left.\mathrm{CDCl}_{3}\right) \delta(\mathrm{ppm}): 7.19(\mathrm{~d}, J=8.0 \mathrm{~Hz}, 2 \mathrm{H})$,

$7.10(\mathrm{~d}, J=8.0 \mathrm{~Hz}, 2 \mathrm{H}), 7.04(\mathrm{~d}, J=8.4 \mathrm{~Hz}, 2 \mathrm{H}), 6.69$ (d, J = 8.8 Hz, 2H), $3.85(\mathrm{~s}, 2 \mathrm{H})$, $2.91(\mathrm{~s}, 6 \mathrm{H}), 2.46(\mathrm{~s}, 3 \mathrm{H}) ;{ }^{13} \mathrm{C} \mathrm{NMR}\left(101 \mathrm{MHz}, \mathrm{CDCl}_{3}\right) \delta$ (ppm): 149.2, 139.3, 135.3, 129.5, 129.3, 129.1, 127.2, 113.0, 40.8, 40.4, 16.4 .

HRMS (positive ESI): m/z calculated for $\mathrm{C}_{16} \mathrm{H}_{20} \mathrm{NS}[\mathrm{M}+\mathrm{H}]^{+}: 258.1311$; found: 258.1400 .<smiles>COc1ccc(Cc2ccc(N(C)C)cc2)cc1</smiles>

4-(4-Methoxybenzyl)- $N, N$-dimethylaniline (2p): CAS No. 53039-60-4. $S / C=100,000$ in water.

Colorless liquid; yield: $57 \mathrm{mg}, 94 \%$; ${ }^{1} \mathrm{H}$ NMR $(400 \mathrm{MHz}$, $\left.\mathrm{CDCl}_{3}\right) \delta(\mathrm{ppm}): 7.10(\mathrm{~d}, J=8.4 \mathrm{~Hz}, 2 \mathrm{H}), 7.07(\mathrm{~d}, J=8.4 \mathrm{~Hz}$, 2H), $6.82(\mathrm{~d}, J=8.4 \mathrm{~Hz}, 2 \mathrm{H}), 6.73(\mathrm{~d}, J=8.0 \mathrm{~Hz}, 2 \mathrm{H}$ ), $3.85(\mathrm{~s}, 2 \mathrm{H}), 3.78(\mathrm{~s}, 3 \mathrm{H}), 2.92(\mathrm{~s}$, $6 \mathrm{H}) ;{ }^{13} \mathrm{C} \mathrm{NMR}\left(101 \mathrm{MHz}, \mathrm{CDCl}_{3}\right) \delta$ (ppm): 157.8, 149.1, 134.2, 129.8, 129.7, 129.4, 113.7, $113.0,55.2,40.9,40.0$. 
<smiles>Cc1ccc(Cc2ccc(N(C)C)cc2)cc1</smiles>

N,N-dimethyl-4-(4-methylbenzyl)aniline (2q): CAS No. 54158-98-4. $S / C=100,000$ in water.

White solid; m.p.: $62-64{ }^{\circ} \mathrm{C}$; yield: $49 \mathrm{mg}, 87 \% ;{ }^{1} \mathrm{H}$ NMR $(400$ $\left.\mathrm{MHz}, \mathrm{CDCl}_{3}\right) \delta(\mathrm{ppm}): 7.10-7.07(\mathrm{~m}, 6 \mathrm{H}), 6.71(\mathrm{~d}, J=8.0 \mathrm{~Hz}$, 2H), 3.87 (s, 2H), $2.92(\mathrm{~s}, 6 \mathrm{H}), 2.33$ (s, 3H); ${ }^{13} \mathrm{C} \mathrm{NMR}\left(101 \mathrm{MHz}, \mathrm{CDCl}_{3}\right) \delta$ (ppm): 149.1, 139.0, 135.2, 129.6, 129.4, 129.0, 128.6, 113.0, 40.9, 40.4, 21.0.<smiles>Cc1cccc(Cc2ccc(N(C)C)cc2)c1</smiles>

$N, N$-dimethyl-4-(3-methylbenzyl)aniline (2r): $S / C=100,000$ in water.

Colorless liquid; yield: $51 \mathrm{mg}, 90 \% ;{ }^{1} \mathrm{H} \mathrm{NMR}\left(400 \mathrm{MHz}, \mathrm{CDCl}_{3}\right)$ $\delta$ (ppm): 7.17 (t, $J=7.4 \mathrm{~Hz}, 1 \mathrm{H}), 7.09(\mathrm{~d}, J=8.8 \mathrm{~Hz}, 2 \mathrm{H})$,

7.02-6.99 (m, 3H), $6.73(\mathrm{~d}, J=8.4 \mathrm{~Hz}, 2 \mathrm{H}), 3.87(\mathrm{~s}, 2 \mathrm{H}), 2.93(\mathrm{~s}, 6 \mathrm{H}), 2.32(\mathrm{~s}, 3 \mathrm{H}) ;{ }^{13} \mathrm{C}$ $\operatorname{NMR}\left(101 \mathrm{MHz}, \mathrm{CDCl}_{3}\right) \delta$ (ppm): 141.9, 137.9, 129.6, 129.5, 128.2, 126.5, 125.8, 113.1, 40.95, 40.86, 21.4.

HRMS (positive ESI): m/z calculated for $\mathrm{C}_{16} \mathrm{H}_{20} \mathrm{~N}[\mathrm{M}+\mathrm{H}]^{+}:$226.1590; found: 226.1585 .<smiles>Cc1ccccc1Cc1ccc(N(C)C)cc1</smiles>

$\mathrm{N}, \mathrm{N}$-dimethyl-4-(2-methylbenzyl)aniline (2s): CAS No. 857007-80-8. $S / C=100,000$ in water.

Colorless liquid; yield: $52 \mathrm{mg}, 93 \% ;{ }^{1} \mathrm{H}$ NMR $\left(400 \mathrm{MHz}, \mathrm{CDCl}_{3}\right) \delta$ (ppm): 7.15-7.10 (m, 4H), 7.01 (d, $J=8.4 \mathrm{~Hz}, 2 \mathrm{H}), 6.70$ (d, $J=$ $8.4 \mathrm{~Hz}, 2 \mathrm{H}), 3.90(\mathrm{~s}, 2 \mathrm{H}), 2.92(\mathrm{~s}, 6 \mathrm{H}), 2.27(\mathrm{~s}, 3 \mathrm{H}) ;{ }^{13} \mathrm{C}$ NMR $\left(101 \mathrm{MHz}, \mathrm{CDCl}_{3}\right) \delta(\mathrm{ppm}): 139.9,136.7,130.3,129.9,129.5,126.3,126.0,113.2,41.0$, $38.5,19.8$.<smiles>CC(C)c1ccc(Cc2ccc(N(C)C)cc2)cc1</smiles>

\section{4-(4-Isopropylbenzyl)- $N, N$-dimethylaniline (2t): $S / C=$ 100,000 in water.}

Pale yellow oil; yield: $60 \mathrm{mg}, 95 \%$; ${ }^{1} \mathrm{H}$ NMR $(400 \mathrm{MHz}$,

$\left.\mathrm{CDCl}_{3}\right) \delta(\mathrm{ppm}): 7.15-7.09(\mathrm{~m}, 6 \mathrm{H}), 6.73(\mathrm{~d}, J=8.4 \mathrm{~Hz}, 2 \mathrm{H})$, $3.89(\mathrm{~s}, 2 \mathrm{H}), 2.93(\mathrm{~s}, 6 \mathrm{H}), 2.89(\mathrm{sep}, J=5.1 \mathrm{~Hz}, 1 \mathrm{H}), 1.26(\mathrm{~d}, J=5.1 \mathrm{~Hz}, 6 \mathrm{H}) ;{ }^{13} \mathrm{C}$ NMR $\left(101 \mathrm{MHz}, \mathrm{CDCl}_{3}\right) \delta(\mathrm{ppm}): 149.1,146.2,139.4,129.6,129.5,128.6,126.3,113.0,40.9$, 40.5, 33.7, 24.0.

HRMS (positive ESI): m/z calculated for $\mathrm{C}_{18} \mathrm{H}_{24} \mathrm{~N}[\mathrm{M}+\mathrm{H}]^{+}:$254.1903; found: 254.1906.<smiles>CN(C)c1ccc(Cc2ccc(-c3ccccc3)cc2)cc1</smiles>

\section{4-([1,1'-Biphenyl]-4-ylmethyl)- $\mathrm{N}, \mathrm{N}$-dimethylaniline} $(2 u): S / C=20,000$ in water : TFE $=1: 1$.

White solid; m.p.: $60-62{ }^{\circ} \mathrm{C}$; yield: $62 \mathrm{mg}, 87 \%$; ${ }^{1} \mathrm{H}$ NMR $\left(400 \mathrm{MHz}, \mathrm{CDCl}_{3}\right) \delta(\mathrm{ppm}): 7.56(\mathrm{~d}, J=7.6 \mathrm{~Hz}, 2 \mathrm{H}), 7.49$ (d, J = 7.2 Hz, 2H), 7.40 (t, J = 7.4 Hz, 2H), 7.34 (t, J = 7.2 Hz, 1H), 7.28 (d, J = 7.6 Hz, 2H), 7.13 (d, $J=7.6 \mathrm{~Hz}, 2 \mathrm{H}), 6.73(\mathrm{~d}, J=7.6 \mathrm{~Hz}, 2 \mathrm{H}), 3.96(\mathrm{~s}, 2 \mathrm{H}), 2.94(\mathrm{~s}, 6 \mathrm{H}) ;{ }^{13} \mathrm{C}$ NMR $\left(101 \mathrm{MHz}, \mathrm{CDCl}_{3}\right) \delta$ (ppm): 149.2, 141.2, 141.1, 138.7, 129.5, 129.2, 129.1, 128.7, 127.1 , 127.0, 126.9, 113.0, 40.8, 40.6.

HRMS (positive ESI): m/z calculated for $\mathrm{C}_{21} \mathrm{H}_{22} \mathrm{~N}[\mathrm{M}+\mathrm{H}]^{+}: 288.1747$; found: 288.1745 . 
<smiles>CN(C)c1ccc(Cc2ccc(F)cc2)cc1</smiles>

4-(4-Fluorobenzyl)-N,N-dimethylaniline (2v): CAS No. 38695-24-8. $S / C=100,000$ in water.

Colorless liquid; yield: $56 \mathrm{mg}, 98 \% ;{ }^{1} \mathrm{H}$ NMR $\left(400 \mathrm{MHz}, \mathrm{CDCl}_{3}\right)$ $\delta(\mathrm{ppm}):$ 7.18-7.15 (m, 2H), $7.08(\mathrm{~d}, J=8.4 \mathrm{~Hz}, 2 \mathrm{H}), 6.98(\mathrm{t}, J$ $=8.6 \mathrm{~Hz}, 2 \mathrm{H}), 6.74(\mathrm{~d}, J=8.0 \mathrm{~Hz}, 2 \mathrm{H}), 3.89(\mathrm{~s}, 2 \mathrm{H}), 2.95(\mathrm{~s}, 6 \mathrm{H}) ;{ }^{13} \mathrm{C}$ NMR $(101 \mathrm{MHz}$, $\left.\mathrm{CDCl}_{3}\right) \delta(\mathrm{ppm}): 161.3\left(J_{C-F}=243.4 \mathrm{~Hz}\right), 149.2,137.7\left(J_{C-F}=3.2 \mathrm{~Hz}\right), 130.1\left(J_{C-F}=7.8 \mathrm{~Hz}\right)$, $129.4,129.0,115.0\left(J_{C-F}=21.0 \mathrm{~Hz}\right), 112.9,40.8,40.0$.<smiles>CN(C)c1ccc(Cc2ccc(Cl)cc2)cc1</smiles>

4-(4-Chlorobenzyl)-N,N-dimethylaniline (2w): CAS No. $54159-00-1 S / C=100,000$ in water.

White solid; m.p.: $65-67{ }^{\circ} \mathrm{C}$; yield: $55 \mathrm{mg}, 89 \%$; ${ }^{1} \mathrm{H}$ NMR $(400$ $\left.\mathrm{MHz}, \mathrm{CDCl}_{3}\right) \delta$ (ppm): $7.24(\mathrm{~d}, J=8.0 \mathrm{~Hz}, 2 \mathrm{H}), 7.12(\mathrm{~d}, J=$ $8.4 \mathrm{~Hz}, 2 \mathrm{H}$ ), 7.05 (t, J=8.4 Hz, 2H), $6.72(\mathrm{~d}, J=8.4 \mathrm{~Hz}, 2 \mathrm{H}), 3.87$ (s, 2H), $2.93(\mathrm{~s}, 6 \mathrm{H})$; ${ }^{13} \mathrm{C} \mathrm{NMR}\left(101 \mathrm{MHz}, \mathrm{CDCl}_{3}\right) \delta$ (ppm): 149.1, 140.5, 131.5, 130.1, 129.5, 128.7, 128.4, 113.0. $40.8,40.2$.<smiles>CN(C)c1ccc(Cc2ccc(Br)cc2)cc1</smiles>

\section{4-(4-Bromobenzyl)- $N, N$-dimethylaniline (2x): $S / C=$ 100,000 in water.}

White solid; m.p.: 59-61 ${ }^{\circ} \mathrm{C}$; yield: $65 \mathrm{mg}, 90 \% ;{ }^{1} \mathrm{H}$ NMR $(400$ $\left.\mathrm{MHz}, \mathrm{CDCl}_{3}\right) \delta(\mathrm{ppm}): 7.39(\mathrm{~d}, J=8.4 \mathrm{~Hz}, 2 \mathrm{H}), 7.07-7.04(\mathrm{~m}$, $4 \mathrm{H}), 6.71(\mathrm{~d}, J=8.8 \mathrm{~Hz}, 2 \mathrm{H}), 3.85(\mathrm{~s}, 2 \mathrm{H}), 2.93(\mathrm{~s}, 6 \mathrm{H}) ;{ }^{13} \mathrm{C} \mathrm{NMR}\left(101 \mathrm{MHz}, \mathrm{CDCl}_{3}\right) \delta$ (ppm): 149.1, 141.0, 131.3, 130.5, 129.5, 128.6, 119.6, 113.0. 40.8, 40.3.

HRMS (positive ESI): $\mathrm{m} / \mathrm{z}$ calculated for $\mathrm{C}_{15} \mathrm{H}_{17} \mathrm{NBr}[\mathrm{M}+\mathrm{H}]^{+}: 290.0539$; found: 290.0508 .<smiles>CN(C)c1ccc(Cc2cccc(Br)c2)cc1</smiles>

\section{4-(3-Bromobenzyl)- $N, N$-dimethylaniline (2y): $S / C=$} 100,000 in water.

Pale yellow liquid; yield: $69 \mathrm{mg}, 95 \%$; ${ }^{1} \mathrm{H}$ NMR $(400 \mathrm{MHz}$, $\left.\mathrm{CDCl}_{3}\right) \delta(\mathrm{ppm}):$ 7.35-7.31 (m, 2H), 7.15-7.13 (m, 2H), 7.06

$(\mathrm{d}, J=8.8 \mathrm{~Hz}, 2 \mathrm{H}), 6.72(\mathrm{~d}, J=8.4 \mathrm{~Hz}, 2 \mathrm{H}), 3.87(\mathrm{~s}, 2 \mathrm{H}), 2.94(\mathrm{~s}, 6 \mathrm{H}) ;{ }^{13} \mathrm{C}$ NMR $(101 \mathrm{MHz}$, $\left.\mathrm{CDCl}_{3}\right) \delta(\mathrm{ppm}): 149.2,144.4,131.7,129.9,129.5,128.9,128.3,127.4,122.4,113.0 .40 .8$, 40.6 .

HRMS (positive ESI): $\mathrm{m} / \mathrm{z}$ calculated for $\mathrm{C}_{15} \mathrm{H}_{17} \mathrm{NBr}[\mathrm{M}+\mathrm{H}]^{+}: 290.0539$; found: 290.0526 .<smiles>CN(C)c1ccc(Cc2ccccc2Br)cc1</smiles>

4-(2-Bromobenzyl)-N,N-dimethylaniline (2z): $S / C=100,000$ in water.

Pale yellow solid; m.p.: $55-56{ }^{\circ} \mathrm{C}$; yield: $64 \mathrm{mg}, 88 \%$; ${ }^{1} \mathrm{H}$ NMR $\left(400 \mathrm{MHz}, \mathrm{CDCl}_{3}\right) \delta$ (ppm): $7.55(\mathrm{~d}, J=8.0 \mathrm{~Hz}, 2 \mathrm{H}), 7.23-7.04(\mathrm{~m}$, $5 \mathrm{H}), 6.74(\mathrm{~d}, J=8.0 \mathrm{~Hz}, 2 \mathrm{H}), 4.03(\mathrm{~s}, 2 \mathrm{H}), 2.93(\mathrm{~s}, 6 \mathrm{H}) ;{ }^{13} \mathrm{C}$ NMR $\left(101 \mathrm{MHz}, \mathrm{CDCl}_{3}\right) \delta$ (ppm): 149.1, 141.3, 132.7, 130.9, 129.7, 127.6, 127.4, 124.8, 113.0, 105.0, 40.9, 40.8.

HRMS (positive ESI): m/z calculated for $\mathrm{C}_{15} \mathrm{H}_{17} \mathrm{NBr}[\mathrm{M}+\mathrm{H}]^{+}: 290.0539$; found: 290.0546 .<smiles>CN(C)c1ccc(Cc2ccc(I)cc2)cc1</smiles>

4-(4-lodobenzyl)-N,N-dimethylaniline (2aa): CAS No. 143128-33-0. $S / C=100,000$ in water. 
White solid; m.p.: $79-80{ }^{\circ} \mathrm{C}$; $0.125 \mathrm{mmol}$ scale, yield: $40 \mathrm{mg}, 94 \% ;{ }^{1} \mathrm{H}$ NMR $(400 \mathrm{MHz}$, $\left.\mathrm{CDCl}_{3}\right) \delta(\mathrm{ppm}): 7.58(\mathrm{~d}, J=8.0 \mathrm{~Hz}, 2 \mathrm{H}), 7.03(\mathrm{~d}, J=8.4 \mathrm{~Hz}, 2 \mathrm{H}), 6.94(\mathrm{~d}, J=7.6 \mathrm{~Hz}, 2 \mathrm{H})$, $6.69(\mathrm{~d}, J=8.4 \mathrm{~Hz}, 2 \mathrm{H}), 3.83(\mathrm{~s}, 2 \mathrm{H}), 2.92(\mathrm{~s}, 6 \mathrm{H}) ;{ }^{13} \mathrm{C} \mathrm{NMR}\left(101 \mathrm{MHz}, \mathrm{CDCl}_{3}\right) \delta(\mathrm{ppm})$ : $149.2,141.8,137.3,130.9,129.5,128.4,112.9,90.9,40.8,40.4$.<smiles>CN(C)c1ccc(Cc2ccc(C(F)(F)F)cc2)cc1</smiles>

$N, N$-Dimethyl-4-(4-(trifluoromethyl)benzyl)aniline (2ab): $S / C=20,000$, water $:$ TFE $=1: 1$.

White solid; m.p.: $62-64{ }^{\circ} \mathrm{C}$; yield: $64 \mathrm{mg}, 95 \%$; ${ }^{1} \mathrm{H}$ NMR $\left(400 \mathrm{MHz}, \mathrm{CDCl}_{3}\right) \delta(\mathrm{ppm}): 7.55(\mathrm{~d}, J=7.6 \mathrm{~Hz}, 2 \mathrm{H}), 7.31(\mathrm{~d}$, $J=7.6 \mathrm{~Hz}, 2 \mathrm{H}), 7.10(\mathrm{~d}, J=7.6 \mathrm{~Hz}, 2 \mathrm{H}), 6.84(\mathrm{~d}, J=8.0 \mathrm{~Hz}, 2 \mathrm{H}), 3.97(\mathrm{~s}, 2 \mathrm{H}), 2.96(\mathrm{~s}, 6 \mathrm{H})$; ${ }^{13} \mathrm{C} \mathrm{NMR}\left(101 \mathrm{MHz}, \mathrm{CDCl}_{3}\right) \delta(\mathrm{ppm}): 148.4,145.9,129.7,129.0,128.2$ (q, JC-F $=32.3 \mathrm{~Hz}$ ), 126.8, $125.3\left(\mathrm{q}, J_{C-F}=3.8 \mathrm{~Hz}\right), 124.3\left(\mathrm{q}, J_{C-F}=272.7 \mathrm{~Hz}\right.$ ), 113.8, 41.3, 40.7.

HRMS (positive ESI): $\mathrm{m} / \mathrm{z}$ calculated for $\mathrm{C}_{16} \mathrm{H}_{17} \mathrm{~F}_{3} \mathrm{~N}[\mathrm{M}+\mathrm{H}]^{+}: 280.1308$; found: 280.1195 .<smiles>COC(=O)c1ccc(Cc2ccc(N(C)C)cc2)cc1</smiles>

Methyl 4-(4-(dimethylamino)benzyl)benzoate (2ac):

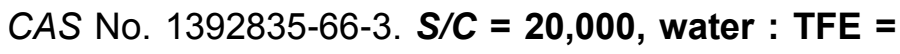
$1: 1$.

White solid; m.p.: $52-53^{\circ} \mathrm{C}$; yield: $61 \mathrm{mg}, 90 \%$; ${ }^{1} \mathrm{H}$ NMR $\left(400 \mathrm{MHz}, \mathrm{CDCl}_{3}\right) \delta(\mathrm{ppm}): 7.96(\mathrm{~d}, J=7.6 \mathrm{~Hz}, 2 \mathrm{H}), 7.26(\mathrm{~d}, J=7.6 \mathrm{~Hz}, 2 \mathrm{H}), 7.06(\mathrm{~d}, J=$ $7.6 \mathrm{~Hz}, 2 \mathrm{H}), 6.71(\mathrm{~d}, J=8.8 \mathrm{~Hz}, 2 \mathrm{H}), 3.95(\mathrm{~s}, 2 \mathrm{H}), 3.91(\mathrm{~s}, 3 \mathrm{H}), 2.93(\mathrm{~s}, 6 \mathrm{H}) ;{ }^{13} \mathrm{C}$ NMR $\left(101 \mathrm{MHz}, \mathrm{CDCl}_{3}\right) \delta$ (ppm): 167.1, 149.3, 147.5, 129.7, 129.5, 128.8, 128.0, 127.8, 112.9, $51.9,40.9,40.7$.

4-(4-(Dimethylamino)benzyl)benzonitrile (2ad): CAS No. 67155-37-7. $S / C=100,000$ in water.<smiles>CN(C)c1ccc(Cc2ccc(C#N)cc2)cc1</smiles>
White solid; m.p.: $113-115{ }^{\circ} \mathrm{C}$; yield: $57 \mathrm{mg}, 96 \% ;{ }^{1} \mathrm{H}$ NMR $(400 \mathrm{MHz}$, $\left.\mathrm{CDCl}_{3}\right) \delta(\mathrm{ppm}): 7.54(\mathrm{~d}, J=8.0 \mathrm{~Hz}, 2 \mathrm{H}), 7.27(\mathrm{~d}, J=8.0 \mathrm{~Hz}, 2 \mathrm{H})$, $7.02(\mathrm{~d}, J=8.4 \mathrm{~Hz}, 2 \mathrm{H}), 6.68(\mathrm{~d}, J=8.4 \mathrm{~Hz}, 2 \mathrm{H}), 3.93(\mathrm{~s}, 2 \mathrm{H}), 2.92(\mathrm{~s}$, $6 \mathrm{H}) ;{ }^{13} \mathrm{C}$ NMR $\left(101 \mathrm{MHz}, \mathrm{CDCl}_{3}\right) \delta$ (ppm): 149.4, 147.8, 132.2, 129.6, $129.5,127.1,119.1,112.9,109.6,41.0,40.6$.

$\mathbf{N}, \mathbf{N}$-dimethyl-4-(4-nitrobenzyl)aniline (2ae): CAS No. 35065-75-9. $S / C=\mathbf{1 0 0 , 0 0 0 ~ i n ~}$ water.<smiles>CN(C)c1ccc(Cc2ccc([N+](=O)[O-])cc2)cc1</smiles>

Yellow solid; m.p.: $186-187^{\circ} \mathrm{C}$ (reported $189^{\circ} \mathrm{C}$ ); yield: 61 mg, 96\%; ${ }^{1} \mathrm{H}$ NMR (400 MHz, $\left.\mathrm{CDCl}_{3}\right) \delta$ (ppm): 8.12 (d, $J=$ $8.4 \mathrm{~Hz}, 2 \mathrm{H}), 7.33(\mathrm{~d}, J=8.4 \mathrm{~Hz}, 2 \mathrm{H}), 7.04(\mathrm{~d}, J=8.4 \mathrm{~Hz}$, $2 \mathrm{H}), 6.71(\mathrm{~d}, J=8.4 \mathrm{~Hz}, 2 \mathrm{H}), 3.98(\mathrm{~s}, 2 \mathrm{H}), 2.93(\mathrm{~s}, 6 \mathrm{H}) ;{ }^{13} \mathrm{C}$ $\operatorname{NMR}\left(101 \mathrm{MHz}, \mathrm{CDCl}_{3}\right) \delta$ (ppm): 150.0, 149.4, 146.3, 129.6, 129.5, 128.7, 123.7, 113.0, $40.8,40.7$.<smiles>CN(C)c1ccc(Cc2ccc3ccccc3c2)cc1</smiles>

$\mathrm{N}, \mathrm{N}$-dimethyl-4-(naphthalen-2-ylmethyl)aniline (2af): CAS No. $1395463-41-8 . S / C=100,000$ in water. White solid; m.p.: $55-56{ }^{\circ} \mathrm{C}$; yield: $60 \mathrm{mg}, 92 \%$; ${ }^{1} \mathrm{H}$ NMR 
(400 MHz, $\left.\mathrm{CDCl}_{3}\right) \delta(\mathrm{ppm}): 7.82-7.76(\mathrm{~m}, 3 \mathrm{H}), 7.65(\mathrm{~s}, 1 \mathrm{H}), 7.48-7.41(\mathrm{~m}, 2 \mathrm{H}), 7.35(\mathrm{~d}, J=$ $8.4 \mathrm{~Hz}, 1 \mathrm{H}$ ), $7.13(\mathrm{~d}, J=8.4 \mathrm{~Hz}, 2 \mathrm{H}), 6.73(\mathrm{~d}, J=8.4 \mathrm{~Hz}, 2 \mathrm{H}), 4.08(\mathrm{~s}, 2 \mathrm{H}), 2.94(\mathrm{~s}, 6 \mathrm{H})$; ${ }^{13} \mathrm{C}$ NMR $\left(101 \mathrm{MHz}, \mathrm{CDCl}_{3}\right) \delta(\mathrm{ppm}): 149.2,139.5,133.6,132.0,129.6,129.1,127.9$, $127.7,127.6,127.5,126.8,125.8,125.1,112.9,41.1,40.8$.

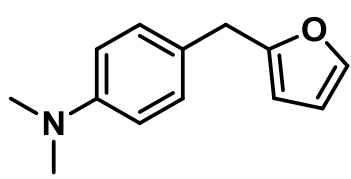

4-(Furan-2-ylmethyl)-N, $\mathrm{N}$-dimethylaniline (2ag): CAS No. 100371-88-8. $S / C=100,000$ in water.

Pale yellow liquid; yield: $37 \mathrm{mg}, 74 \% ;{ }^{1} \mathrm{H}$ NMR $\left(400 \mathrm{MHz}, \mathrm{CDCl}_{3}\right)$ $\delta$ (ppm): $7.31(\mathrm{~s}, 1 \mathrm{H}), 7.11(\mathrm{~d}, J=8.4 \mathrm{~Hz}, 2 \mathrm{H}), 6.70(\mathrm{~d}, J=8.0 \mathrm{~Hz}$, 2H), $6.27(\mathrm{~s}, 1 \mathrm{H}), 5.97(\mathrm{~s}, 1 \mathrm{H}), 3.88(\mathrm{~s}, 2 \mathrm{H}), 2.92(\mathrm{~s}, 6 \mathrm{H}) ;{ }^{13} \mathrm{C} \mathrm{NMR}\left(101 \mathrm{MHz}, \mathrm{CDCl}_{3}\right) \delta$ (ppm): 155.6, 149.4, 141.2, 129.3, 126.1, 112.9, 110.1, 105.7, 40.8, 33.5 .<smiles>CN(C)c1ccc(Cc2cccs2)cc1</smiles>

$\mathrm{N}, \mathrm{N}$-dimethyl-4-(thiophen-2-ylmethyl)aniline (2ah): $\mathrm{S} / \mathrm{C}=$ 20,000, water : TFE $=1: 1$.

Yellow liquid; yield: $46 \mathrm{mg}, 85 \%$; ${ }^{1} \mathrm{H} \mathrm{NMR}\left(400 \mathrm{MHz}, \mathrm{CDCl}_{3}\right) \delta$ (ppm): 7.16-7.13 (m, 3H), 6.94-6.92 (m, 1H), 6.80 (d, $J=2.8 \mathrm{~Hz}$, $1 \mathrm{H}), 6.73(\mathrm{~d}, \mathrm{~J}=8.4 \mathrm{~Hz}, 2 \mathrm{H}), 4.09(\mathrm{~s}, 2 \mathrm{H}), 2.94(\mathrm{~s}, 6 \mathrm{H}) ;{ }^{13} \mathrm{C} \mathrm{NMR}\left(101 \mathrm{MHz}, \mathrm{CDCl}_{3}\right) \delta$ (ppm): 149.3, 145.4, 129.2, 128.6, 126.7, 124.6, 123.6, 112.9, 40.8, 35.1. HRMS (positive ESI): m/z calculated for $\mathrm{C}_{13} \mathrm{H}_{16} \mathrm{NS}[\mathrm{M}+\mathrm{H}]^{+}:$218.0998; found: 280.1002.<smiles>CN(C)c1ccc(Cc2ccncc2)cc1</smiles>

N,N-dimethyl-4-(pyridin-4-ylmethyl)aniline (2ai): CAS No. 131416-55-2. $S / C=100,000$ in water.

Pale yellow liquid; yield: $52 \mathrm{mg}, 99 \%$; ${ }^{1} \mathrm{H}$ NMR $\left(400 \mathrm{MHz}, \mathrm{CDCl}_{3}\right)$ $\delta$ (ppm): 8.48 (d, $J=4.4 \mathrm{~Hz}, 2 \mathrm{H}), 7.10$ (d, $J=5.6 \mathrm{~Hz}, 2 \mathrm{H}), 7.04$ $(\mathrm{d}, J=8.8 \mathrm{~Hz}, 2 \mathrm{H}), 6.70(\mathrm{~d}, \mathrm{~J}=8.8 \mathrm{~Hz}, 2 \mathrm{H}), 3.87(\mathrm{~s}, 2 \mathrm{H}), 2.93(\mathrm{~s}, 6 \mathrm{H}) ;{ }^{13} \mathrm{C}$ NMR $(101 \mathrm{MHz}$, $\left.\mathrm{CDCl}_{3}\right) \delta(\mathrm{ppm}): 151.1,149.6,149.4,129.6,126.6,124.1,112.8,40.6,40.2$.<smiles>CN(C)c1ccc(Cc2ccccn2)cc1</smiles>

N,N-dimethyl-4-(pyridin-2-ylmethyl)aniline (2aj): CAS No. 857436-34-1. $S / C=100,000$ in water.

Pale yellow liquid; yield: $52 \mathrm{mg}, 99 \%$; ${ }^{1} \mathrm{H}$ NMR $\left(400 \mathrm{MHz}, \mathrm{CDCl}_{3}\right)$ $\delta$ (ppm): 8.54 (d, $J=4.4 \mathrm{~Hz}, 1 \mathrm{H}), 7.54$ (dt, $J=4.4 \mathrm{~Hz}, 1.6 \mathrm{~Hz}, 1 \mathrm{H})$, $7.15(\mathrm{~d}, J=8.8 \mathrm{~Hz}, 2 \mathrm{H}), 7.11-7.08(\mathrm{~m}, 2 \mathrm{H}), 6.71(\mathrm{~d}, J=8.4 \mathrm{~Hz}, 2 \mathrm{H}), 4.08(\mathrm{~s}, 2 \mathrm{H}), 2.91(\mathrm{~s}$, $6 \mathrm{H}) ;{ }^{13} \mathrm{C} \mathrm{NMR}\left(101 \mathrm{MHz}, \mathrm{CDCl}_{3}\right) \delta$ (ppm): 161.8, 149.3, 149.0, 136.4, 129.7, 127.4, 122.9, $120.9,112.9,43.7,40.7$.<smiles>CN(C)c1ccc(Cc2ccc(-c3ccc4c(n3)CCCC4)cc2)cc1</smiles>

N,N-dimethyl-4-(4-(5,6,7,8-tetrahydroquinolin-2yl)benzyl)aniline (2ak): $S / C=20,000$ in water : TFE $=1: 1$

White solid; m.p.: $89-91^{\circ} \mathrm{C} ; 0.125 \mathrm{mmol}$ scale; yield: $37 \mathrm{mg}, 87 \%$; ${ }^{1} \mathrm{H}$ NMR $\left(400 \mathrm{MHz}, \mathrm{CDCl}_{3}\right) \delta$ (ppm): 7.84 (d, J=8.0 Hz, 2H), 7.38 (q, J = 8.0 $\mathrm{Hz}, 2 \mathrm{H}), 7.25(\mathrm{~d}, J=7.6 \mathrm{~Hz}, 2 \mathrm{H}), 7.06(\mathrm{~d}, J=8.4 \mathrm{~Hz}, 2 \mathrm{H}), 6.68(\mathrm{~d}, J=8.8 \mathrm{~Hz}, 2 \mathrm{H}), 3.93(\mathrm{~s}$, 2H), $2.98(\mathrm{t}, J=6.2 \mathrm{~Hz}, 2 \mathrm{H}$ ), $2.90(\mathrm{~s}, 6 \mathrm{H}), 2.77(\mathrm{t}, J=6.2 \mathrm{~Hz}, 2 \mathrm{H}$ ), 1.92 (qui, $J=6.2 \mathrm{~Hz}$, 2H), 1.82 (qui, $J=6.2 \mathrm{~Hz}, 2 \mathrm{H}$ ); ${ }^{13} \mathrm{C}$ NMR $\left(101 \mathrm{MHz}, \mathrm{CDCl}_{3}\right) \delta$ (ppm): 157.1, 154.8, 149.2, 142.3, 137.6, 137.4, 130.4, 129.5, 129.3, 129.2, 126.9, 117.7, 113.0, 40.9, 40.7, 32.9, 
28.6, 23.3, 22.9.

HRMS (positive ESI): $\mathrm{m} / \mathrm{z}$ calculated for $\mathrm{C}_{24} \mathrm{H}_{27} \mathrm{~N}_{2}[\mathrm{M}+\mathrm{H}]^{+}$: 343.2169 ; found: 343.2174 .

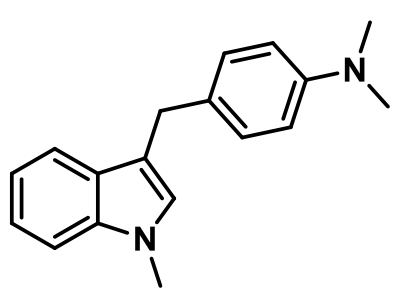

\section{$\mathrm{N}, \mathrm{N}$-dimethyl-4-((1-methyl-1H-indol-3-yl)methyl)aniline} (2al): CAS No. 134627-73-9

$S / C=100,000$ in water.

Yellow liquid; yield: $58 \mathrm{mg}, 88 \% ;{ }^{1} \mathrm{H} \mathrm{NMR}\left(400 \mathrm{MHz}, \mathrm{CDCl}_{3}\right) \delta$ (ppm): $7.54(\mathrm{~d}, J=7.6 \mathrm{~Hz}, 1 \mathrm{H}), 7.26-7.04(\mathrm{~m}, 5 \mathrm{H}), 6.72-6.68(\mathrm{~m}$, $3 \mathrm{H}), 4.00$ (s, 2H), $3.71(\mathrm{~s}, 3 \mathrm{H}), 2.90(\mathrm{~s}, 6 \mathrm{H}) ;{ }^{13} \mathrm{C} \mathrm{NMR}(101 \mathrm{MHz}$, $\left.\mathrm{CDCl}_{3}\right) \delta(\mathrm{ppm}): 149.1,137.2,129.6,129.3,127.9,127.0,121.4,119.3,118.6,115.3$, $113.0,109.0,41.0,32.6,30.5$.<smiles>CN(C)c1ccc(Cc2c[nH]c3ccccc23)cc1</smiles>

4-((1H-indol-3-yl)methyl)- $\mathbf{N}, \mathbf{N}$-dimethylaniline (2am): $C A S$ No. 31859-50-4. $S / C=20,000$, water : TFE $=1: 1$.

White solid; m.p.: $118-120^{\circ} \mathrm{C}$ (reported $\left.143-145^{\circ} \mathrm{C}\right) ;{ }^{21}$ yield: $58 \mathrm{mg}, 92 \% ;{ }^{1} \mathrm{H}$ NMR $\left(400 \mathrm{MHz}, \mathrm{CDCl}_{3}\right) \delta$ (ppm): 7.91 (brs, $1 \mathrm{H}), 7.55(\mathrm{~d}, J=8.0 \mathrm{~Hz}, 1 \mathrm{H}), 7.35(\mathrm{~d}, J=8.0 \mathrm{~Hz}, 1 \mathrm{H})$ 7.20-7.06 (m, 4H), $6.90(\mathrm{~s}, 1 \mathrm{H}), 6.70(\mathrm{~d}, J=8.4 \mathrm{~Hz}, 2 \mathrm{H}), 4.03(\mathrm{~s}, 2 \mathrm{H}), 2.91(\mathrm{~s}, 6 \mathrm{H}) ;{ }^{13} \mathrm{C}$ $\operatorname{NMR}\left(101 \mathrm{MHz}, \mathrm{CDCl}_{3}\right) \delta(\mathrm{ppm}):$ 149.1, 136.5, 129.4, 129.2, 127.6, 122.1, 121.9, 119.2 , $116.8,113.0,110.9,105.0,40.9,30.5$.<smiles>CN(C)c1ccc(Cc2ccc(Cc3ccc(N(C)C)cc3)cc2)cc1</smiles>

4,4'-(1,4-Phenylenebis(methylene))bis( $N, N$-di methylaniline) (2an): CAS No. 1086262-00-1. $S / C=100,000$ in water.

White solid; m.p.: $149-151^{\circ} \mathrm{C} ; 0.125 \mathrm{mmol}$ scale; yield: $39 \mathrm{mg}, 90 \% ;{ }^{1} \mathrm{H}$ NMR $\left(400 \mathrm{MHz}, \mathrm{CDCl}_{3}\right) \delta$

(ppm): 7.09-7.05 (m, 8H), 6.70-6.68 (m, $4 \mathrm{H}), 3.86(\mathrm{~s}, 4 \mathrm{H}), 2.91$ (s, 12H); ${ }^{13} \mathrm{C}$ NMR $(101$ $\left.\mathrm{MHz}, \mathrm{CDCl}_{3}\right) \delta(\mathrm{ppm}): 149.1,139.4,129.7,129.5,128.7,113.0,40.8,40.5$.<smiles>CSCc1ccc(Cc2ccc(N(C)C)cc2)cc1</smiles>

\section{$\mathrm{N}, \mathrm{N}$-dimethyl-4-(4-((methylthio)methyl)benzyl)aniline} (2ao): $S / C=100,000$ in water.

Pale yellow liquid; yield: $62 \mathrm{mg}, 92 \%$; ${ }^{1} \mathrm{H}$ NMR $(400 \mathrm{MHz}$, $\left.\mathrm{CDCl}_{3}\right) \delta(\mathrm{ppm}): 7.22(\mathrm{~d}, J=8.0 \mathrm{~Hz}, 2 \mathrm{H}), 7.15(\mathrm{~d}, J=8.0$ $\mathrm{Hz}, 2 \mathrm{H}), 7.08$ (d, J = 8.4 Hz, 2H), $6.71(\mathrm{~d}, J=8.0 \mathrm{~Hz}, 2 \mathrm{H}), 3.89(\mathrm{~s}, 2 \mathrm{H}), 3.65(\mathrm{~s}, 2 \mathrm{H}), 2.93$ (s, 6H), 2.00 (s, 3H); $\left.{ }^{13} \mathrm{C} \mathrm{NMR} \mathrm{(101} \mathrm{MHz,} \mathrm{CDCl}_{3}\right) \delta$ (ppm): 149.1, 146.8, 140.8, 135.6, 129.5, 129.2, 128.8, 112.9, 40.8, 40.5, 38.0, 14.9.

HRMS (positive ESI): m/z calculated for $\mathrm{C}_{17} \mathrm{H}_{22} \mathrm{NS}[\mathrm{M}+\mathrm{H}]^{+}: 272.1467$; found: 272.1472 .<smiles>COCc1ccc(Cc2ccc(N(C)C)cc2)cc1</smiles>

\section{4-(4-(Methoxymethyl)benzyl)-N,N-dimethylaniline} (2ap): $S / C=100,000$ in water.

Colorless liquid; yield: $57 \mathrm{mg}, 90 \%$; ${ }^{1} \mathrm{H}$ NMR $(400 \mathrm{MHz}$, $\left.\mathrm{CDCl}_{3}\right) \delta(\mathrm{ppm}): 7.16(\mathrm{~d}, J=8.4 \mathrm{~Hz}, 2 \mathrm{H}), 7.09(\mathrm{~d}, J=8.0$ $\mathrm{Hz}, 2 \mathrm{H}), 6.97(\mathrm{~d}, J=8.4 \mathrm{~Hz}, 2 \mathrm{H}), 6.61(\mathrm{~d}, J=8.8 \mathrm{~Hz}, 2 \mathrm{H}), 4.34(\mathrm{~s}, 2 \mathrm{H}), 3.81(\mathrm{~s}, 2 \mathrm{H}), 3.29$ 
(s, 3H), $2.83(\mathrm{~s}, 6 \mathrm{H}) ;{ }^{13} \mathrm{C} \mathrm{NMR}\left(101 \mathrm{MHz}, \mathrm{CDCl}_{3}\right) \delta(\mathrm{ppm}): 149.1,141.5,135.6,129.5$, 129.2, 128.8, 127.9, 112.9, 74.6, 58.0, 40.8, 40.6, 29.7.

HRMS (positive ESI): m/z calculated for $\mathrm{C}_{17} \mathrm{H}_{22} \mathrm{NO}[\mathrm{M}+\mathrm{H}]^{+}: 256.1696$; found: 256.1720 .<smiles>CN(C)Cc1ccc(Cc2ccc(N(C)C)cc2)cc1</smiles>

4-(4-((Dimethylamino)methyl)benzyl)-N,N-dimethylani line (2aq): $S / C=100,000$ in water.

Pale yellow liquid; yield: $60 \mathrm{mg}, 90 \%$; ${ }^{1} \mathrm{H}$ NMR $(400 \mathrm{MHz}$, $\left.\mathrm{CDCl}_{3}\right) \delta(\mathrm{ppm}): 7.13(\mathrm{~d}, J=8.0 \mathrm{~Hz}, 2 \mathrm{H}), 7.06(\mathrm{~d}, J=8.0$ $\mathrm{Hz}, 2 \mathrm{H}), 6.97(\mathrm{~d}, J=8.8 \mathrm{~Hz}, 2 \mathrm{H}), 6.60(\mathrm{~d}, J=8.4 \mathrm{~Hz}, 2 \mathrm{H}), 3.79(\mathrm{~s}, 2 \mathrm{H}), 3.34(\mathrm{~s}, 2 \mathrm{H}), 2.82$ $(\mathrm{s}, 6 \mathrm{H}), 2.17(\mathrm{~s}, 6 \mathrm{H}) ;{ }^{13} \mathrm{C} \mathrm{NMR}\left(101 \mathrm{MHz}, \mathrm{CDCl}_{3}\right) \delta(\mathrm{ppm}): 149.2,141.2,135.4,129.6$, 129.4, 129.3, 128.8, 113.0, 63.9, 45.1, 40.9, 40.6.

HRMS (positive ESI): $\mathrm{m} / \mathrm{z}$ calculated for $\mathrm{C}_{18} \mathrm{H}_{25} \mathrm{~N}_{2}[\mathrm{M}+\mathrm{H}]^{+}:$:269.2012; found: 269.2020 .<smiles>CN(C)c1ccc(CC#Cc2ccccc2)cc1</smiles>

\section{N,N-dimethyl-4-(3-phenylprop-2-yn-1-yl)aniline (2ar):} $S / C=1000$, water $: H F I P=1: 1$

Yellow liquid; yield: $46 \mathrm{mg}, 79 \%$; ${ }^{1} \mathrm{H}$ NMR $\left(400 \mathrm{MHz}, \mathrm{CDCl}_{3}\right)$ $\delta$ (ppm): 7.45-7.43 (m, 2H), 7.29-7.27 (m, 5H), $6.74(\mathrm{~d}, J=$ $8.8 \mathrm{~Hz}, 2 \mathrm{H}), 3.74(\mathrm{~s}, 2 \mathrm{H}), 2.94(\mathrm{~s}, 6 \mathrm{H}) ;{ }^{13} \mathrm{C} \mathrm{NMR}\left(101 \mathrm{MHz}, \mathrm{CDCl}_{3}\right) \delta(\mathrm{ppm}): 149.6,131.6$, 128.6, 128.1, 127.6, 124.7, 123.9, 113.0, 88.5, 82.0, 40.8, 24.7.

HRMS (positive ESI): $\mathrm{m} / \mathrm{z}$ calculated for $\mathrm{C}_{17} \mathrm{H}_{18} \mathrm{~N}[\mathrm{M}+\mathrm{H}]^{+}: 236.1434$; found: 236.1439 .<smiles>CN(C)c1ccc(C/C=C/c2ccccc2)cc1</smiles>

4-Cinnamyl-N,N-dimethylaniline (2as): CAS No.

62056-42-2. $S / C=1000$, water : HFIP = 1:1.

Colorless liquid; mixture of two compounds, 2 as : $\mathbf{2 t}=\mathbf{2 0}$ :

20, calculated yield: $56 \mathrm{mg}, 76 \%$; characterization of the crude product: ${ }^{1} \mathrm{H}$ NMR $\left(400 \mathrm{MHz}, \mathrm{CDCl}_{3}\right) \delta(\mathrm{ppm}): 7.39(\mathrm{~d}, J=7.6 \mathrm{~Hz}, 2 \mathrm{H}), 7.35-7.20(\mathrm{~m}$, $3 \mathrm{H}), 7.16(\mathrm{~d}, J=8.0 \mathrm{~Hz}, 2 \mathrm{H}), 6.76(\mathrm{~d}, J=8.4 \mathrm{~Hz}, 2 \mathrm{H}), 3.50(\mathrm{~d}, J=6.4 \mathrm{~Hz}, 2 \mathrm{H}), 2.96(\mathrm{~s}, 6 \mathrm{H})$; ${ }^{13} \mathrm{C} \mathrm{NMR}\left(101 \mathrm{MHz}, \mathrm{CDCl}_{3}\right) \delta$ (ppm): 149.3, 137.7, 130.3, 130.2, 129.2, 128.4, 128.2, $126.9,126.1,113.0,40.9,38.4$.<smiles>CN(C)c1ccc(Cc2ccc(O)cc2)cc1</smiles>

4-(4-(Dimethylamino)benzyl)phenol (2at): CAS No. 54158-97-3. $S / C=100,000$ in water.

White solid; m.p.: $104-105^{\circ} \mathrm{C}$ (reported $\left.107-108^{\circ} \mathrm{C}\right) ;{ }^{22}$ yield: $46 \mathrm{mg}, 82 \%$; ${ }^{1} \mathrm{H}$ NMR $\left(400 \mathrm{MHz}, \mathrm{CDCl}_{3}\right) \delta$ (ppm): 7.06 (d, J $=8.4 \mathrm{~Hz}, 2 \mathrm{H}), 7.03(\mathrm{~d}, J=8.4 \mathrm{~Hz}, 2 \mathrm{H}), 6.72(\mathrm{~d}, J=8.8 \mathrm{~Hz}, 2 \mathrm{H}), 6.70(\mathrm{~d}, J=8.4 \mathrm{~Hz}, 2 \mathrm{H})$, 5.09 (brs, $1 \mathrm{H}$ ), 3.83 (s, 2H), 2.91 (s, 6H); $\left.{ }^{13} \mathrm{C} \mathrm{NMR} \mathrm{(101} \mathrm{MHz,} \mathrm{CDCl}_{3}\right) \delta$ (ppm): 153.7, $149.1,134.1,130.1,129.8,129.5,115.1,113.4,41.0,40.0$.

(4-(4-(Dimethylamino)benzyl)phenyl)methanol (2au): $S / C=20,000$, water : TFE = $1: 1$.<smiles>CN(C)c1ccc(Cc2ccc(CO)cc2)cc1</smiles>
White solid; m.p.: $66-67{ }^{\circ} \mathrm{C}$; yield: $51 \mathrm{mg}, 85 \%$; ${ }^{1} \mathrm{H}$ NMR $\left(400 \mathrm{MHz}, \mathrm{CDCl}_{3}\right) \delta$ (ppm): $7.28(\mathrm{~d}, J=8.4 \mathrm{~Hz}, 2 \mathrm{H}), 7.19$ (d, J = 8.4 Hz, 2H), $7.06(\mathrm{~d}, J=8.8 \mathrm{~Hz}, 2 \mathrm{H}), 6.70(\mathrm{~d}, J=$ $8.8 \mathrm{~Hz}, 2 \mathrm{H}), 4.65$ (s, 2H), $3.90(\mathrm{~s}, 2 \mathrm{H}), 2.92(\mathrm{~s}, 6 \mathrm{H}), 1.68$ 
(brs, $1 \mathrm{H}) ;{ }^{13} \mathrm{C} \mathrm{NMR}\left(101 \mathrm{MHz}, \mathrm{CDCl}_{3}\right) \delta$ (ppm): 149.1, 141.6, 138.3, 129.5, 129.1, 129.0, 127.2, 113.0, 65.2, 40.8, 40.6.

HRMS (positive ESI): $\mathrm{m} / \mathrm{z}$ calculated for $\mathrm{C}_{16} \mathrm{H}_{20} \mathrm{NO}[\mathrm{M}+\mathrm{H}]^{+}: 242.1539$; found: 242.1543.<smiles>CC(C)c1ccc(N(C)C)cc1</smiles>

4-Isopropyl-N,N-dimethylaniline (4a): CAS No. 4139-78-0. $S / C=$ 1000, water: TFE $=1: 1$.

Colorless liquid; yield: $38 \mathrm{mg}, 94 \%{ }^{1} \mathrm{H} \mathrm{NMR}\left(400 \mathrm{MHz}, \mathrm{CDCl}_{3}\right) \delta(\mathrm{ppm})$ : $7.13(\mathrm{~d}, J=8.4 \mathrm{~Hz}, 2 \mathrm{H}), 6.74$ (d, $J=8.4 \mathrm{~Hz}, 2 \mathrm{H}), 2.92(\mathrm{~s}, 6 \mathrm{H}), 2.84$ (sep, $J=6.8 \mathrm{~Hz}, 1 \mathrm{H}), 1.23(\mathrm{~d}, J=6.8 \mathrm{~Hz}, 6 \mathrm{H}) ;{ }^{13} \mathrm{C}$ NMR $(101 \mathrm{MHz}$, $\left.\mathrm{CDCl}_{3}\right) \delta(\mathrm{ppm}): 148.8,137.8,126.9,113.1,41.0,33.0,24.2$.<smiles>CC(c1ccccc1)c1ccc(N(C)C)cc1</smiles>

N,N-dimethyl-4-(1-phenylethyl)aniline (4b): CAS No. 26675-61-6. $S / C=1000$ in water

Colorless liquid; yield: $54 \mathrm{mg}, 96 \% ;{ }^{1} \mathrm{H}$ NMR $\left(400 \mathrm{MHz}, \mathrm{CDCl}_{3}\right) \delta$ (ppm): 7.31-7.16 (m, 4H), $7.18(\mathrm{t}, J=7.0 \mathrm{~Hz}, 1 \mathrm{H}), 7.12(\mathrm{~d}, J=8.4$ $\mathrm{Hz}, 2 \mathrm{H}), 6.72(\mathrm{~d}, J=8.4 \mathrm{~Hz}, 2 \mathrm{H}), 4.09$ (q, $J=7.2 \mathrm{~Hz}, 1 \mathrm{H}), 2.93(\mathrm{~s}$, $6 \mathrm{H}), 1.63$ (d, $J=7.2 \mathrm{~Hz}, 3 \mathrm{H}) ;{ }^{13} \mathrm{C}$ NMR (101 MHz, $\left.\mathrm{CDCl}_{3}\right) \delta$ (ppm): 148.9, 147.2, 134.7, $128.22,128.16,127.5,125.7,112.8,43.8,40.8,22.0$.<smiles>CC(c1ccc(N(C)C)cc1)C1CC1</smiles>

4-(1-Cyclopropylethyl)- $N, N$-dimethylaniline (4c): $S / C=1000$ in water.

Colorless liquid; yield: $46 \mathrm{mg}, 98 \% ;{ }^{1} \mathrm{H}$ NMR $\left(400 \mathrm{MHz}, \mathrm{CDCl}_{3}\right) \delta$ (ppm): $7.18(\mathrm{~d}, J=8.4 \mathrm{~Hz}, 2 \mathrm{H}), 6.76(\mathrm{~d}, J=8.8 \mathrm{~Hz}, 2 \mathrm{H}), 2.95$ (s, $6 \mathrm{H}), 1.95(\mathrm{dq}, J=8.8 \mathrm{~Hz}, 7.2 \mathrm{~Hz}, 1 \mathrm{H}), 1.33(\mathrm{~d}, J=7.2 \mathrm{~Hz}, 3 \mathrm{H})$, 0.98-0.89 (m, 1H), 0.46-0.41 (m, 1H), 0.58-0.50 (m, 1H), 0.23-0.14 (m, 2H); ${ }^{13} \mathrm{C}$ NMR $(101$ $\left.\mathrm{MHz}, \mathrm{CDCl}_{3}\right) \delta(\mathrm{ppm}): 149.0,135.7,127.5,112.9,43.5,40.9,21.6,18.7,4.4,4.3$; HRMS (positive ESI): $\mathrm{m} / \mathrm{z}$ calculated for $\mathrm{C}_{13} \mathrm{H}_{20} \mathrm{~N}[\mathrm{M}+\mathrm{H}]^{+}$: 190.1590; found: 190.1588 .<smiles>CC(c1ccc(N(C)C)cc1)C(C)(C)C</smiles>

4-(3,3-Dimethylbutan-2-yl)-N,N-dimethylaniline (4d): $S / C=1000$, water : TFE $=1: 1$.

Colorless liquid; yield: $49 \mathrm{mg}, 96 \% ;{ }^{1} \mathrm{H}$ NMR $\left(400 \mathrm{MHz}, \mathrm{CDCl}_{3}\right) \delta$ (ppm): 7.05 (d, J = 8.8 Hz, 2H), $6.72(\mathrm{~d}, J=8.0 \mathrm{~Hz}, 2 \mathrm{H}), 2.93(\mathrm{~s}, 6 \mathrm{H})$, 2.48 (q, J = 7.2 Hz, 1H), $1.23(\mathrm{~d}, J=7.2 \mathrm{~Hz}, 3 \mathrm{H}), 0.86(\mathrm{~s}, 9 \mathrm{H}) ;{ }^{13} \mathrm{C}$ NMR (101 MHz, $\left.\mathrm{CDCl}_{3}\right) \delta$ (ppm): 129.6, 112.3, 48.9, 41.1, 33.9, 27.8, 15.9.

HRMS (positive ESI): $\mathrm{m} / \mathrm{z}$ calculated for $\mathrm{C}_{14} \mathrm{H}_{24} \mathrm{~N}[\mathrm{M}+\mathrm{H}]^{+}: 206.1903$; found: 206.1913 .

\section{4-(2-((1,1,1,3,3,3-Hexafluoropropan-2-yl)oxy)-1-phenylethyl)- $N, N$-dimethylaniline} $(4 e): S / C=1000$, water $:$ TFE $=1: 1$.<smiles>CN(C)c1ccc(C(COC(C(F)(F)F)C(F)(F)F)c2ccccc2)cc1</smiles>

White solid; m.p.: $66-68^{\circ} \mathrm{C} ; 0125 \mathrm{mmol}$ scale; yield: 48 mg, 98\%; ${ }^{1} \mathrm{H}$ NMR (400 MHz, $\mathrm{CDCl}_{3}$ ) $\delta$ (ppm): 7.33-7.20 $(\mathrm{m}, 5 \mathrm{H}), 7.09(\mathrm{~d}, J=8.8 \mathrm{~Hz}, 2 \mathrm{H}), 6.69(\mathrm{~d}, J=8.8 \mathrm{~Hz}, 2 \mathrm{H})$, 4.36-4.26 (m, 3H), $4.05(\mathrm{sep}, J=6.0 \mathrm{~Hz}, 1 \mathrm{H}), 2.93(\mathrm{~s}, 6 \mathrm{H})$; ${ }^{13} \mathrm{C}$ NMR (101 MHz, $\left.\mathrm{CDCl}_{3}\right) \delta(\mathrm{ppm}): 149.5,141.4,128.7$, $128.4,128.1,127.5,126.6,121.4\left(J_{C-F}=283.4 \mathrm{~Hz}\right), 112.7$, 
78.2, $76.7\left(J_{C-F}=32.5 \mathrm{~Hz}\right), 50.0,40.6$.

HRMS (positive ESI): $\mathrm{m} / \mathrm{z}$ calculated for $\mathrm{C}_{19} \mathrm{H}_{20} \mathrm{~F}_{6} \mathrm{NO}[\mathrm{M}+\mathrm{H}]^{+}: 392.1444$; found: 392.1453 .<smiles>CN(C)c1ccc(C(COc2ccccc2)c2ccccc2)cc1</smiles>

$N, N$-dimethyl-4-(2-phenoxy-1-phenylethyl)aniline (4f): $S / C=1000$, water $:$ TFE $=1: 1$.

White solid; m.p.: $79-81{ }^{\circ} \mathrm{C}$; yield: $75 \mathrm{mg}, 95 \%$; ${ }^{1} \mathrm{H}$ NMR $\left(400 \mathrm{MHz}, \mathrm{CDCl}_{3}\right) \delta(\mathrm{ppm}): 7.26-7.18(\mathrm{~m}, 7 \mathrm{H}), 7.10(\mathrm{~d}, J=$ $8.8 \mathrm{~Hz}, 2 \mathrm{H}), 6.89-6.85(\mathrm{~m}, 3 \mathrm{H}), 6.65(\mathrm{~d}, J=8.8 \mathrm{~Hz}, 2 \mathrm{H})$, 4.42-4.37 (m, 3H), $2.87(\mathrm{~s}, 6 \mathrm{H}) ;{ }^{13} \mathrm{C} \mathrm{NMR}\left(101 \mathrm{MHz}, \mathrm{CDCl}_{3}\right)$

$\delta$ (ppm): 158.8, 149.4, 142.4, 129.6, 129.3, 128.9, 128.4, 128.3, 126.3, 120.7, 114.7, 112.7, 71.0, 49.5, 40.6 .

HRMS (positive ESI): m/z calculated for $\mathrm{C}_{22} \mathrm{H}_{24} \mathrm{NO}[\mathrm{M}+\mathrm{H}]^{+}: 318.1852$; found: 318.1867.<smiles>CN(C)c1ccc(C2CCC2)cc1</smiles>

4-Cyclobutyl- $N, N$-dimethylaniline (4g): $S / C=100,000$ in water. Colorless liquid; yield: $43 \mathrm{mg}, 99 \% ;{ }^{1} \mathrm{H}$ NMR $\left(400 \mathrm{MHz}, \mathrm{CDCl}_{3}\right) \delta$ (ppm): 7.14 (d, $J=8.8 \mathrm{~Hz}, 2 \mathrm{H}$ ), 6.75 (d, $J=8.4 \mathrm{~Hz}, 2 \mathrm{H}$ ), 3.47 (qui, $J=$ $7.2 \mathrm{~Hz}, 1 \mathrm{H}$ ), 2.93 (s, 6H), 2.31 (qt, $J=8.4 \mathrm{~Hz}, 2.8 \mathrm{~Hz}, 2 \mathrm{H}$ ), 2.17-1.80 $(\mathrm{m}, 4 \mathrm{H}) ;{ }^{13} \mathrm{C}$ NMR $\left(101 \mathrm{MHz}, \mathrm{CDCl}_{3}\right) \delta$ (ppm): 149.0, 134.8, 127.0,

113.0, 41.0, 39.8, 30.1, 18.2.

HRMS (positive ESI): m/z calculated for $\mathrm{C}_{12} \mathrm{H}_{18} \mathrm{~N}$ [M+H] $]^{+}: 176.1434$; found: 176.1440 .<smiles>CN(C)c1ccc(C2CCCC2)cc1</smiles>

4-Cyclopentyl-N,N-dimethylaniline (4h): CAS No. 860735-93-9. $S / C=1000$, water : TFE $=1: 1$.

Colorless liquid; yield: $46 \mathrm{mg}, 98 \% ;{ }^{1} \mathrm{H}$ NMR $\left(400 \mathrm{MHz}, \mathrm{CDCl}_{3}\right) \delta$ (ppm): 7.15 (d, $J=8.0 \mathrm{~Hz}, 2 \mathrm{H}), 6.76(\mathrm{~d}, J=7.6 \mathrm{~Hz}, 2 \mathrm{H}), 2.96-2.88$ $(\mathrm{m}, 7 \mathrm{H}), 2.03(\mathrm{~m}, 2 \mathrm{H}), 1.79-1.55(\mathrm{~m}, 6 \mathrm{H}) ;{ }^{13} \mathrm{C} \mathrm{NMR}\left(101 \mathrm{MHz}, \mathrm{CDCl}_{3}\right)$ $\delta$ (ppm): 126.6, 112.3, 44.0, 40.2, 33.6, 24.4 .<smiles>CN(C)c1ccc(C2CCCCC2)cc1</smiles>

4-Cyclohexyl-N,N-dimethylaniline (4i): CAS No. 151563-09-6. $S / C=1000$, water $:$ TFE $=1: 1$.

Colorless liquid; yield: $50 \mathrm{mg}, 99 \% ;{ }^{1} \mathrm{H}$ NMR $\left(400 \mathrm{MHz}, \mathrm{CDCl}_{3}\right) \delta$ (ppm): 7.12 (d, J = 8.4 Hz, 2H), 6.76 (d, J = 7.6 Hz, 2H), $2.93(\mathrm{~s}, 6 \mathrm{H})$, $2.43(\mathrm{~m}, 1 \mathrm{H}), 1.87-1.73(\mathrm{~m}, 5 \mathrm{H}), 1.45-1.23(\mathrm{~m}, 5 \mathrm{H}) ;{ }^{13} \mathrm{C}$ NMR $(101$ $\left.\mathrm{MHz}, \mathrm{CDCl}_{3}\right) \delta(\mathrm{ppm}): 149.0,136.5,127.3,112.9,43.5,40.9,34.7,27.0,26.2$.

4-Cycloheptyl-N,N-dimethylaniline (4j): CAS No. 5085-71-2. $S / C=1000$, water : TFE = $1: 1$.<smiles>CN(C)c1ccc(C2CCCCCC2)cc1</smiles>

Colorless liquid; yield: $53 \mathrm{mg}, 98 \% ;{ }^{1} \mathrm{H}$ NMR $\left(400 \mathrm{MHz}, \mathrm{CDCl}_{3}\right) \delta$ (ppm): 7.12 (d, $J=8.4 \mathrm{~Hz}, 2 \mathrm{H}), 6.75(\mathrm{~d}, J=8.4 \mathrm{~Hz}, 2 \mathrm{H}), 2.94(\mathrm{~s}, 6 \mathrm{H})$, 2.66-2.59 (m, 1H), 2.00-1.96 (m, 2H), 1.96-1.54 (m, 10H); ${ }^{13} \mathrm{C}$ NMR $\left(101 \mathrm{MHz}, \mathrm{CDCl}_{3}\right) \delta(\mathrm{ppm}): 148.6,138.7,127.2,113.1,46.0,41.0$, $37.0,28.0,27.1$. 
<smiles>CN(C)c1ccc(C2CCCCCCCCCC2)cc1</smiles>

4-Cyclododecyl-N,N-dimethylaniline (4k): $S / C=1000$, water : HFIP = 1:1.

White solid; m.p.: $82-83{ }^{\circ} \mathrm{C}$; yield: $71 \mathrm{mg}, 99 \% ;{ }^{1} \mathrm{H}$ NMR $(400$ $\left.\mathrm{MHz}, \mathrm{CDCl}_{3}\right) \delta(\mathrm{ppm}): 7.10(\mathrm{~d}, J=8.8 \mathrm{~Hz}, 2 \mathrm{H}), 6.73(\mathrm{~d}, J=8.8$ $\mathrm{Hz}, 2 \mathrm{H}$ ), $2.94(\mathrm{~s}, 6 \mathrm{H}), 2.69$ (qui, $J=6.0 \mathrm{~Hz}, 1 \mathrm{H}), 1.81-1.76(\mathrm{~m}$, $2 \mathrm{H}), 1.52-1.35(\mathrm{~m}, 2 \mathrm{H}) ;{ }^{13} \mathrm{C} \mathrm{NMR}\left(101 \mathrm{MHz}, \mathrm{CDCl}_{3}\right) \delta$ (ppm): 148.9, 136.0, 128.1, 112.8, 40.9, 38.5, 31.8, 24.0, 23.9, 23.5, 23.2, 22.8.

HRMS (positive ESI): $\mathrm{m} / \mathrm{z}$ calculated for $\mathrm{C}_{20} \mathrm{H}_{34} \mathrm{~N}[\mathrm{M}+\mathrm{H}]^{+}:$288.2686; found: 288.2689 .<smiles>CN(C)c1ccc(C2c3ccccc3Oc3ccccc32)cc1</smiles>

43.4, 40.6.

N,N-dimethyl-4-(9H-xanthen-9-yl)aniline (4I): CAS No. 15128-49-1. $S / C=100,000$, water $:$ TFE $=1: 1$.

White solid; m.p.: $152-153^{\circ} \mathrm{C}$ (reported $157^{\circ} \mathrm{C}$ ); ${ }^{23}$ yield: $71 \mathrm{mg}, 95 \%$; ${ }^{1} \mathrm{H} \mathrm{NMR}\left(400 \mathrm{MHz}, \mathrm{CDCl}_{3}\right) \delta(\mathrm{ppm}): 7.19(\mathrm{td}, J=8.0 \mathrm{~Hz}, 1.2 \mathrm{~Hz}, 2 \mathrm{H})$, 7.13-7.06 (m, 6H), $6.98(\mathrm{td}, J=8.0 \mathrm{~Hz}, 1.2 \mathrm{~Hz}, 2 \mathrm{H}), 6.66(\mathrm{~d}, J=8.4 \mathrm{~Hz}$, $2 \mathrm{H}), 5.17(\mathrm{~s}, 1 \mathrm{H}) 2.94(\mathrm{~s}, 6 \mathrm{H}) ;{ }^{13} \mathrm{C} \mathrm{NMR}\left(101 \mathrm{MHz}, \mathrm{CDCl}_{3}\right) \delta(\mathrm{ppm})$ : 151.1, 149.3, 134.7, 129.7, 129.1, 127.6, 125.2, 123.1, 116.4, 112.7,<smiles>CN(C)c1ccc(C2CCCC3C=CC=CC32)cc1</smiles>

N,N-dimethyl-4-(1,2,3,4-tetrahydronaphthalen-1-yl)aniline (4m): CAS No. 132777-32-3. $S / C=1000$, water $:$ TFE $=1: 1$.

Yellow liquid; yield: $60 \mathrm{mg}, 95 \% ;{ }^{1} \mathrm{H}$ NMR $\left(400 \mathrm{MHz}, \mathrm{CDCl}_{3}\right) \delta(\mathrm{ppm})$ : 7.16-6.93 (m, 6H), $6.74(\mathrm{~d}, J=8.8 \mathrm{~Hz}, 2 \mathrm{H}), 4.08(\mathrm{t}, J=6.4 \mathrm{~Hz}, 1 \mathrm{H}), 2.97(\mathrm{~s}$, $6 \mathrm{H}), 2.94-2.88(\mathrm{~m}, 2 \mathrm{H}), 2.20-2.14(\mathrm{~m}, 1 \mathrm{H}), 1.98-1.77(\mathrm{~m}, 3 \mathrm{H}) ;{ }^{13} \mathrm{C}$ NMR $(101$ $\left.\mathrm{MHz}, \mathrm{CDCl}_{3}\right) \delta(\mathrm{ppm}): 148.9,140.1,137.5,130.2,129.39,129.35,128.8$, 125.6, 125.5, 112.6, 44.6, 40.8, 33.3, 29.8, 21.0.<smiles>CN(C)c1ccc(C2=C3CC4CC(C3)CC2C4)cc1</smiles>

4-(Adamantan-2-yl)-N,N-dimethylaniline (4na): $S / C=100,000$, water : TFE $=1: 1$.

White solid; m.p.: $134-135{ }^{\circ} \mathrm{C}$; yield: $63 \mathrm{mg}, 99 \%$; ${ }^{1} \mathrm{H}$ NMR $(400$ $\left.\mathrm{MHz}, \mathrm{CDCl}_{3}\right) \delta(\mathrm{ppm}): 7.23(\mathrm{~d}, J=8.4 \mathrm{~Hz}, 2 \mathrm{H}), 6.76(\mathrm{~d}, J=8.4 \mathrm{~Hz}$, $2 \mathrm{H}), 4.08(\mathrm{t}, J=6.4 \mathrm{~Hz}, 1 \mathrm{H}), 2.93(\mathrm{~m}, 7 \mathrm{H}), 2.42(\mathrm{~s}, 2 \mathrm{H}), 2.01-1.87$ $(\mathrm{m}, 7 \mathrm{H}), 1.77(\mathrm{~s}, 3 \mathrm{H}), 1.56-1.53(\mathrm{~m}, 2 \mathrm{H}) ;{ }^{13} \mathrm{C} \mathrm{NMR}\left(101 \mathrm{MHz}, \mathrm{CDCl}_{3}\right) \delta(\mathrm{ppm}): 148.4$, 132.6, 127.4, 112.8, 46.0, 40.9, 39.2, 38.0, 31.9, 31.0, 28.2, 27.9.

HRMS (positive ESI): m/z calculated for $\mathrm{C}_{18} \mathrm{H}_{26} \mathrm{~N}[\mathrm{M}+\mathrm{H}]^{+}:$256.2060; found: 256.2065.

1-(4-(Adamantan-2-yl)phenyl)piperidine (4nb): CAS No. 1783817-29-7. $S / C=\mathbf{1 0 0 , 0 0 0 ,}$ water : TFE $=1: 1$.<smiles>c1cc(N2CCCCC2)ccc1C1C2CC3CC(C2)CC1C3</smiles>

White solid; m.p.: 220-221 ${ }^{\circ} \mathrm{C}$; yield: $73 \mathrm{mg}, 99 \%$; ${ }^{1} \mathrm{H}$ NMR (400 $\left.\mathrm{MHz}, \mathrm{CDCl}_{3}\right) \delta(\mathrm{ppm}): 7.23(\mathrm{~d}, J=8.4 \mathrm{~Hz}, 2 \mathrm{H}), 6.92(\mathrm{~d}, J=8.8$ $\mathrm{Hz}, 2 \mathrm{H}), 3.13(\mathrm{t}, J=5.4 \mathrm{~Hz}, 4 \mathrm{H}), 2.94(\mathrm{~s}, 1 \mathrm{H}), 2.42(\mathrm{~s}, 2 \mathrm{H})$, 2.00-1.52 (m, 18H); ${ }^{13} \mathrm{C}$ NMR (101 MHz, $\left.\mathrm{CDCl}_{3}\right) \delta$ (ppm): 149.7, $135.1,127.3,116.4,50.9,46.1,39.1,38.0,31.9,31.0,28.1$, $27.8,26.0,24.3$. 


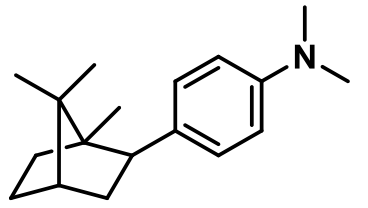

$N, N$-dimethyl-4-(1,7,7-trimethylbicyclo[2.2.1]heptan-2-yl)anili

ne (40): $S / C=1000$, water : HFIP = 1:1.

White solid; m.p.: $45-46{ }^{\circ} \mathrm{C}$; yield: $64 \mathrm{mg}, 99 \%$, mixture of 2

stereomers, d.r. $=80: 20$; characterization of the major stereomer:

${ }^{1} \mathrm{H}$ NMR $\left(400 \mathrm{MHz}, \mathrm{CDCl}_{3}\right) \delta(\mathrm{ppm}): 7.16(\mathrm{~d}, J=8.8 \mathrm{~Hz}, 2 \mathrm{H}), 6.69(\mathrm{~d}, J=8.8 \mathrm{~Hz}, 2 \mathrm{H}), 2.93$ (s, 6H), $2.84(\mathrm{t}, J=8.6 \mathrm{~Hz}, 1 \mathrm{H}), 2.31-2.22(\mathrm{~m}, 1 \mathrm{H}), 1.35-0.90(\mathrm{~m}, 6 \mathrm{H}), 0.84(\mathrm{~s}, 6 \mathrm{H}), 0.77(\mathrm{~s}$, $3 \mathrm{H}) ;{ }^{13} \mathrm{C} \mathrm{NMR}\left(101 \mathrm{MHz}, \mathrm{CDCl}_{3}\right) \delta$ (ppm): 148.5, 131.4, 129.8, 112.1, 51.6, 49.4, 47.7, 45.7, 40.8, 40.5, 33.6, 27.6, 21.4, 20.1, 14.6.

HRMS (positive ESI): m/z calculated for $\mathrm{C}_{18} \mathrm{H}_{28} \mathrm{~N}[\mathrm{M}+\mathrm{H}]^{+}:$258.2216; found: 258.2235 .<smiles>C=C(C)[C@@H]1CC[C@@H](C)[C@H](c2ccc(N3CCCCC3)cc2)C1</smiles>

1-(4-((5S)-2-methyl-5-(prop-1-en-2-yl)cyclohexyl)phenyl) piperidine (4p): $S / C=1000$, water : HFIP $=1: 1$.

White solid.; mixture of 4 stereomers; yield: $71 \mathrm{mg}, 96 \%$; HRMS (positive ESI): $\mathrm{m} / \mathrm{z}$ calculated for $\mathrm{C}_{18} \mathrm{H}_{28} \mathrm{~N}[\mathrm{M}+\mathrm{H}]^{+}$: 296.2373; found: 296.2384 .

$N, N$-dimethyl-4-(3-methylcyclopentadecyl)aniline (4q): $S / C=1000$, water : HFIP $=$ $1: 1$.

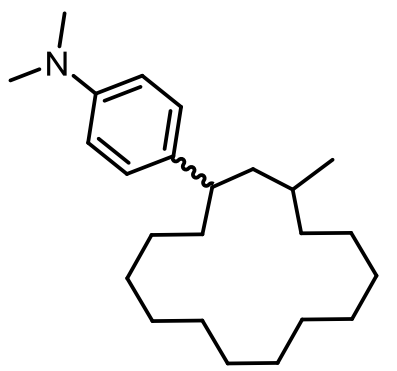

Colorless oil; $0.125 \mathrm{mmol}$ scale; yield: $42 \mathrm{mg}, 99 \%$, mixture of 2 stereomers, d.r. $=80: 20$; characterization of the major stereomer: ${ }^{1} \mathrm{H}$ NMR $\left(400 \mathrm{MHz}, \mathrm{CDCl}_{3}\right) \delta(\mathrm{ppm}): 7.08$ (d, $J=8.4$ $\mathrm{Hz}, 2 \mathrm{H}), 6.73(\mathrm{~d}, J=8.4 \mathrm{~Hz}, 2 \mathrm{H}), 2.94(\mathrm{~s}, 6 \mathrm{H}), 2.65-2.50(\mathrm{~m}, 1 \mathrm{H})$, 1.85-1.15 (m, 26H), 1.02-0.90 (m, 1H), $0.87(\mathrm{~d}, J=6.4 \mathrm{~Hz}, 3 \mathrm{H})$;

${ }^{13} \mathrm{C} \mathrm{NMR}\left(101 \mathrm{MHz}, \mathrm{CDCl}_{3}\right) \delta(\mathrm{ppm}): 148.5,131.4,129.8,112.1$, 51.6, 49.4, 47.7, 45.7, 40.8, 40.5, 33.6, 27.6, 21.4, 20.1, 14.6.;

HRMS (positive ESI): m/z calculated for $\mathrm{C}_{24} \mathrm{H}_{42} \mathrm{~N}[\mathrm{M}+\mathrm{H}]^{+}: 344.3312$; found: 344.3318 .

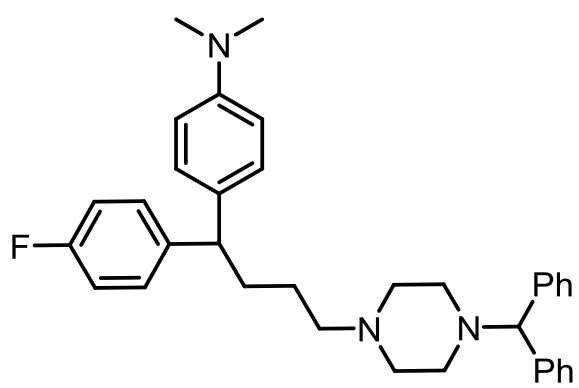

4-(4-(4-Benzhydrylpiperazin-1-yl)-1-(4-fluorophe nyl)butyl)- $N, N$-dimethylaniline (4r): $S / C=1000$, water : HFIP = 1:1.

Brown oil; $0.125 \mathrm{mmol}$ scale; yield: $63 \mathrm{mg}, 97 \%$; ${ }^{1} \mathrm{H}$ $\operatorname{NMR}\left(400 \mathrm{MHz}, \mathrm{CDCl}_{3}\right) \delta(\mathrm{ppm}):$ 7.47-7.45 (m, 4H), $7.31(\mathrm{td}, J=7.6 \mathrm{~Hz}, 2.0 \mathrm{~Hz}, 4 \mathrm{H}), 7.23-7.19(\mathrm{~m}, 4 \mathrm{H})$, $7.12(\mathrm{dd}, J=8.6 \mathrm{~Hz}, 3.2 \mathrm{~Hz}, 2 \mathrm{H}), 6.97(\mathrm{td}, J=8.6$ $\mathrm{Hz}, 2.4 \mathrm{~Hz}, 2 \mathrm{H}), 6.71(\mathrm{dd}, J=8.6 \mathrm{~Hz}, 2.6 \mathrm{~Hz}, 2 \mathrm{H})$, $4.26(\mathrm{~d}, J=2.4 \mathrm{~Hz}, 1 \mathrm{H}), 3.83(\mathrm{td}, J=7.6 \mathrm{~Hz}, 2.4 \mathrm{~Hz}, 1 \mathrm{H}), 2.97-2.90(\mathrm{~m}, 6 \mathrm{H}), 2.48-2.40(\mathrm{~m}$, $10 \mathrm{H}), 2.08-1.95(\mathrm{~m}, 2 \mathrm{H}), 1.58-1.40(\mathrm{~m}, 2 \mathrm{H}) ;{ }^{13} \mathrm{C} \mathrm{NMR}$ (101 MHz, $\left.\mathrm{CDCl}_{3}\right) \delta(\mathrm{ppm}): 161.0$ $\left(J_{C-F}=244.4 \mathrm{~Hz}\right), 149.0,142.7,141.5,132.8,129.0\left(J_{C-F}=7.8 \mathrm{~Hz}\right), 128.4,128.2,127.8$, $126.9,114.9\left(J_{C-F}=21.1 \mathrm{~Hz}\right), 112.7,76.2,58.5,53.4,51.7,49.4,40.6,33.9,25.2$. HRMS (positive ESI): $\mathrm{m} / \mathrm{z}$ calculated for $\mathrm{C}_{35} \mathrm{H}_{41} \mathrm{FN}_{3}[\mathrm{M}+\mathrm{H}]^{+}: 522.3279$; found: 522.3268 .<smiles>CN(C)c1ccc(C(CO)c2ccccc2)cc1</smiles>

2-(4-(Dimethylamino)phenyl)-2-phenylethan-1-ol (4s): $S / C=$ 1000, water : HFIP = 1:1. 
White solid; m.p.: $74-75{ }^{\circ} \mathrm{C}$; yield: $57 \mathrm{mg}, 94 \%$; ${ }^{1} \mathrm{H}$ NMR $\left(400 \mathrm{MHz}, \mathrm{CDCl}_{3}\right) \delta(\mathrm{ppm})$ : 7.31-7.21 (m, 5H), $7.14(\mathrm{~d}, J=8.4 \mathrm{~Hz}, 2 \mathrm{H}), 6.71(\mathrm{~d}, J=8.8 \mathrm{~Hz}, 2 \mathrm{H}), 4.13-4.12(\mathrm{~m}, 3 \mathrm{H})$, $2.93(\mathrm{~s}, 6 \mathrm{H}) ;{ }^{13} \mathrm{C}$ NMR $\left(101 \mathrm{MHz}, \mathrm{CDCl}_{3}\right) \delta$ (ppm): 149.5, 142.1, 128.9, 128.6, 128.2, $126.5,112.9,66.3,52.7,40.6$.

HRMS (positive ESI): m/z calculated for $\mathrm{C}_{16} \mathrm{H}_{20} \mathrm{NO}[\mathrm{M}+\mathrm{H}]^{+}:$242.1539; found: 242.1959.<smiles>CN(C)c1ccc([C@H]2CC[C@H](O)CC2)cc1</smiles>

\section{trans-4-(4-(Dimethylamino)phenyl)cyclohexan-1-ol} (trans-4t): $S / C=1000$, water : HFIP = 1:1.

White solid; m.p.: $70-72{ }^{\circ} \mathrm{C}$; yield: $45 \mathrm{mg}, 81 \%$; ${ }^{1} \mathrm{H}$ NMR $(400$ $\left.\mathrm{MHz}, \mathrm{CDCl}_{3}\right) \delta(\mathrm{ppm}): 7.10(\mathrm{~d}, J=8.4 \mathrm{~Hz}, 2 \mathrm{H}), 6.72(\mathrm{~d}, J=8.8$ $\mathrm{Hz}, 2 \mathrm{H}), 3.71-3.64(\mathrm{~m}, 1 \mathrm{H}), 2.93(\mathrm{~s}, 6 \mathrm{H}), 2.42(\mathrm{tt}, J=16.0 \mathrm{~Hz}$, $3.2 \mathrm{~Hz}, 1 \mathrm{H}), 2.09-2.07(\mathrm{~m}, 2 \mathrm{H}), 1.93-1.90(\mathrm{~m}, 2 \mathrm{H}), 1.64$ (brs, $1 \mathrm{H}), 1.55-1.37(\mathrm{~m}, 4 \mathrm{H}) ;{ }^{13} \mathrm{C}$ NMR (101 MHz, $\left.\mathrm{CDCl}_{3}\right) \delta(\mathrm{ppm}):$ 149.1, 134.8, 127.2, 112.9, 70.7, 42.3, 40.8, 36.0, 32.6. HRMS (positive ESI): m/z calculated for $\mathrm{C}_{14} \mathrm{H}_{22} \mathrm{NO}[\mathrm{M}+\mathrm{H}]^{+}: 220.1696$; found: 220.2091 .

cis-4-(4-(Dimethylamino)phenyl)cyclohexan-1-ol (cis-4t): $S / C=1000$, water : HFIP = $1: 1$.<smiles>CN(C)c1ccc(C2CCC(O)CC2)cc1</smiles>

Colorless oil; yield: $8 \mathrm{mg}, 15 \%$; ${ }^{1} \mathrm{H}$ NMR $\left(400 \mathrm{MHz}, \mathrm{CDCl}_{3}\right) \delta$ (ppm): 7.14 (d, $J=8.4 \mathrm{~Hz}, 2 \mathrm{H}), 6.73(\mathrm{~d}, J=8.4 \mathrm{~Hz}, 2 \mathrm{H})$, 4.14-4.09 (m, 1H), $2.92(\mathrm{~s}, 6 \mathrm{H}), 2.46(\mathrm{tt}, J=16.0 \mathrm{~Hz}, 3.2 \mathrm{~Hz}$, $1 \mathrm{H}), 1.90-1.41(\mathrm{~m}, 9 \mathrm{H}) ;{ }^{13} \mathrm{C} \mathrm{NMR}\left(101 \mathrm{MHz}, \mathrm{CDCl}_{3}\right) \delta$ (ppm): 149.1, 135.7, 127.3, 112.9, 65.8, 42.7, 40.9, 33.1, 28.0.; HRMS (positive ESI): $\mathrm{m} / \mathrm{z}$ calculated for $\mathrm{C}_{14} \mathrm{H}_{22} \mathrm{NO}[\mathrm{M}+\mathrm{H}]^{+}:$: 220.1696; found: 220.2093 .<smiles>CC(CC(C)(C)O)c1ccc(N(C)C)cc1</smiles>

3-(4-(Dimethylamino)phenyl)butan-2-one (4u): $S / C=1000$ in water: HFIP = 1:1.

Colorless liquid; two-step yield: $33 \mathrm{mg}, 59 \% ;{ }^{1} \mathrm{H}$ NMR (400 $\left.\mathrm{MHz}, \mathrm{CDCl}_{3}\right) \delta(\mathrm{ppm}): 7.12(\mathrm{~d}, J=8.8 \mathrm{~Hz}, 2 \mathrm{H}), 6.71(\mathrm{~d}, J=8.8$ $\mathrm{Hz}, 2 \mathrm{H}), 2.91(\mathrm{~s}, 6 \mathrm{H}), 2.92-2.85(\mathrm{~m}, 1 \mathrm{H}), 1.94(\mathrm{dd}, J=14.4 \mathrm{~Hz}$, $9.2 \mathrm{~Hz}, 1 \mathrm{H}$ ), 1.74 (dd, $J=14.2 \mathrm{~Hz}, 4.4 \mathrm{~Hz}, 1 \mathrm{H}), 1.24$ (d, J=7.2 Hz, 3H), 1.24 (brs, $1 \mathrm{H}$ ), 1.17 (s, 3H), 1.14 (s, 3H); $\left.{ }^{13} \mathrm{C} \mathrm{NMR} \mathrm{(101} \mathrm{MHz,} \mathrm{CDCl}_{3}\right) \delta$ (ppm): 149.2, 135.4, 127.6, 113.2, 71.4, 51.2, 40.8, 35.4, 30.2, 29.6, 25.3.

HRMS (positive ESI): $\mathrm{m} / \mathrm{z}$ calculated for $\mathrm{C}_{14} \mathrm{H}_{24} \mathrm{NO}[\mathrm{M}+\mathrm{H}]^{+}: 222.1852$; found: 222.2251 .<smiles>CC(=O)C(C)c1ccc(N(C)C)cc1</smiles>

3-(4-(Dimethylamino)phenyl)butan-2-one (4v): CAS No. 79341-87-0. $S / C=1000$, water $:$ HFIP $=1: 1$.

Colorless liquid; yield: $42 \mathrm{mg}, 88 \%$; ${ }^{1} \mathrm{H}$ NMR $\left(400 \mathrm{MHz}, \mathrm{CDCl}_{3}\right) \delta$ (ppm): 7.08 (d, J = 8.8 Hz, 2H), $6.70(\mathrm{~d}, J=8.4 \mathrm{~Hz}, 2 \mathrm{H}), 3.64$ (q, $J=$ $7.2 \mathrm{~Hz}, 1 \mathrm{H}), 2.94(\mathrm{~s}, 6 \mathrm{H}), 2.04(\mathrm{~s}, 3 \mathrm{H}), 1.35$ (d, J = 7.6 Hz, 3H); ${ }^{13} \mathrm{C} \mathrm{NMR}\left(101 \mathrm{MHz}, \mathrm{CDCl}_{3}\right)$ $\delta$ (ppm): 209.5, 149.7, 128.5, 128.3, 112.9, 52.8, 40.6, 28.1, 17.4 . 


\subsection{Data of steroid derivatives}

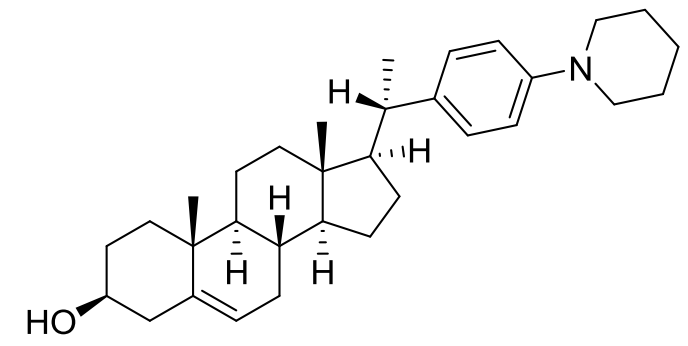

$(3 S, 8 S, 9 S, 10 R, 13 S, 14 S, 17 R)-10,13-$ dimethy I-17-((S)-1-(4-(piperidin-1-yl)phenyl)ethyl)-2 ,3,4,7,8,9,10,11,12,13,14,15,16,17-tetradeca hydro-1H-cyclopenta[a]phenanthren-3-ol (8a):

$S / C=1000$, water $: H F I P=1: 1,2 \mathrm{~mL}$ in

total.

White solid; m.p.: $152-154{ }^{\circ} \mathrm{C}$; two-step yield: $70 \mathrm{mg}, 61 \%$; ${ }^{1} \mathrm{H}$ NMR $\left(400 \mathrm{MHz}, \mathrm{CDCl}_{3}\right) \delta$ (ppm): 7.03 (d, $J=8.8 \mathrm{~Hz}, 2 \mathrm{H}), 6.84(\mathrm{~d}, J=8.4 \mathrm{~Hz}, 2 \mathrm{H}), 5.36-5.33(\mathrm{~m}, 1 \mathrm{H}), 3.52(\mathrm{tt}, J=$ $10.8 \mathrm{~Hz}, 4.6 \mathrm{~Hz}, 1 \mathrm{H}), 3.10$ (t, J = 5.4 Hz, 4H), 2.54-2.47 (m, 1H), 2.28-0.91 (m, 29H), 0.78 (s, 3H); ${ }^{13} \mathrm{C} \mathrm{NMR}\left(101 \mathrm{MHz}, \mathrm{CDCl}_{3}\right) \delta$ (ppm): 150.2, 140.7, 139.7, 127.6, 121.7, 116.4, $71.8,57.0,56.8,51.0,50.1,42.3,42.2$, 39.8, 37.3, 36.5, 31.93, 31.87, 31.7, 29.0, 26.0, 24.3, 24.1, 22.2, 21.1, 19.4, 11.9 .

HRMS (positive ESI): $\mathrm{m} / \mathrm{z}$ calculated for $\mathrm{C}_{30} \mathrm{H}_{44} \mathrm{NO}[\mathrm{M}+\mathrm{H}]^{+}: 462.34730$; found: 462.3744 .

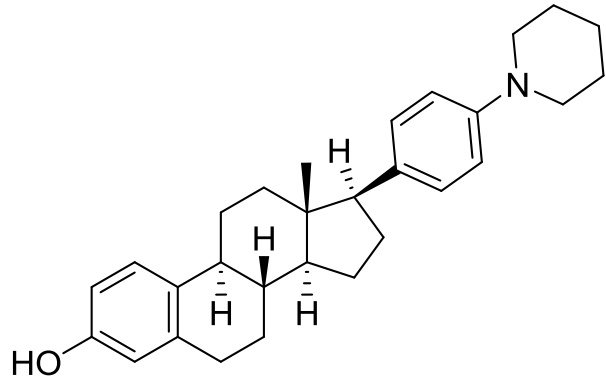

(8S,9S,13S,14S,17S)-13-Methyl-17-(4-(piperidi n-1-yl)phenyl)-7,8,9,11,12,13,14,15,16,17-deca hydro-6H-cyclopenta[a]phenanthren-3-ol (8b): $S / C=1000$, water : HFIP $=1: 1,2 \mathrm{~mL}$ in total. White solid; m.p.: $206-208^{\circ} \mathrm{C}$; two-step yield: 65 mg, 63\%; ${ }^{1} \mathrm{H} \mathrm{NMR}\left(400 \mathrm{MHz}, \mathrm{CDCl}_{3}\right) \delta$ (ppm): $7.13(\mathrm{~d}, J=8.4 \mathrm{~Hz}, 2 \mathrm{H}), 7.06(\mathrm{~d}, J=8.4 \mathrm{~Hz}, 1 \mathrm{H})$, $6.91(\mathrm{~d}, J=8.4 \mathrm{~Hz}, 2 \mathrm{H}), 6.54(\mathrm{~d}, J=2.8 \mathrm{~Hz}, 1 \mathrm{H}), 6.39(\mathrm{dd}, J=8.4 \mathrm{~Hz}, 2.8 \mathrm{~Hz}, 1 \mathrm{H}), 5.24$ (brs, 1H), 3.14 (td, $J=5.2 \mathrm{~Hz}, 3.2 \mathrm{~Hz}, 4 \mathrm{H}), 2.85-2.81(\mathrm{~m}, 2 \mathrm{H}), 2.69(\mathrm{t}, J=10.0 \mathrm{~Hz}, 1 \mathrm{H})$, 2.24-1.35 (m, 19H), 0.48 (s, 3H); $\left.{ }^{13} \mathrm{C} \mathrm{NMR} \mathrm{(101} \mathrm{MHz,} \mathrm{CDCl}_{3}\right) \delta$ (ppm): 153.4, 150.4, 138.3, $132.8,132.2,129.2,126.3,116.3,115.2,112.5,56.4,55.1,51.1,44.4,43.9,39.2,37.7$, 29.7, 27.8, 26.4, 25.8, 24.2, 12.8.

HRMS (positive ESI): m/z calculated for $\mathrm{C}_{29} \mathrm{H}_{38} \mathrm{NO}[\mathrm{M}+\mathrm{H}]^{+}: 416.2948$; found: 416.2957 .

(3S,8R,9S,10S,13S,14S,17S)-10,13-Dimethyl-17-(4-(piperidin-1-yl)phenyl)hexadecah ydro-1H-cyclopenta[a]phenanthren-3-ol (8c):

$\mathrm{S} / \mathrm{C}=1000$, water $: \mathrm{HFIP}=1: 1,2 \mathrm{~mL}$ in total.

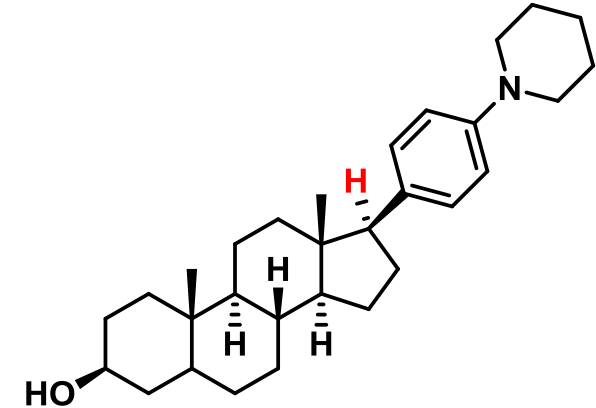

White solid; m.p.: $261-262^{\circ} \mathrm{C}$; two-step yield: 78

mg, 72\%; ${ }^{1} \mathrm{H}$ NMR $\left(400 \mathrm{MHz}, \mathrm{CDCl}_{3}\right) \delta$ (ppm): 7.08 (d, $J=8.4 \mathrm{~Hz}, 2 \mathrm{H}), 6.86(\mathrm{~d}, J=8.8 \mathrm{~Hz}, 2 \mathrm{H}), 3.59$ (tt, $J=11.2 \mathrm{~Hz}, 4.8 \mathrm{~Hz}, 1 \mathrm{H}), 3.11(\mathrm{t}, J=5.4 \mathrm{~Hz}, 4 \mathrm{H})$, $2.57(\mathrm{t}, J=10.0 \mathrm{~Hz}, 1 \mathrm{H}), 2.03-0.88(\mathrm{~m}, 28 \mathrm{H}), 0.80$ (s, 3H), 0.71-0.66 (m, 1H), $0.44(\mathrm{~s}, 3 \mathrm{H}) ;{ }^{13} \mathrm{C}$ NMR 
$\left(101 \mathrm{MHz}, \mathrm{CDCl}_{3}\right) \delta(\mathrm{ppm}): 150.5,131.9,129.1,115.9,71.3,56.3,56.2,54.7,50.9,45.0$, 44.2, 38.2, 37.8, 37.0, 36.0, 35.6, 32.2, 31.5, 28.7, 26.2, 26.0, 24.4, 24.3, 21.0, 12.7, 12.4. HRMS (positive ESI): $\mathrm{m} / \mathrm{z}$ calculated for $\mathrm{C}_{30} \mathrm{H}_{46} \mathrm{NO}[\mathrm{M}+\mathrm{H}]^{+}:$436.3574; found: 436.3589 .

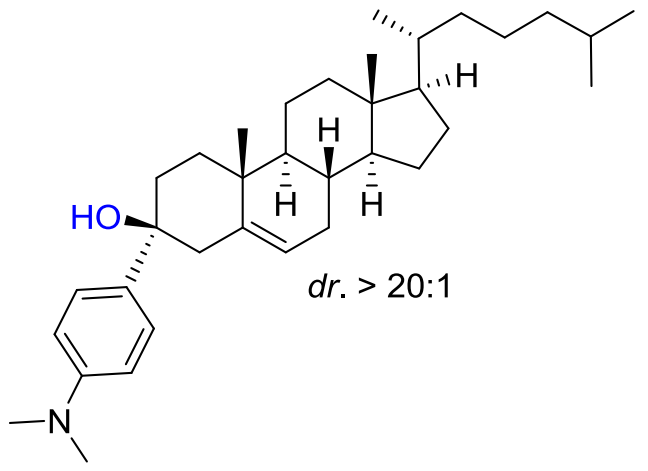

$(8 S, 9 S, 10 R, 13 R, 14 S, 17 R)-3-(4-(d i m e t h y l a m i n$ o)phenyl)-10,13-dimethyl-17-((R)-6-methylhe ptan-2-yl)-2,3,4,7,8,9,10,11,12,13,14,15,16,17tetradecahydro-1H-cyclopenta[a]phenanthre n-3-ol (10):

Pale yellow solid; m.p.: $176-178{ }^{\circ} \mathrm{C}$; yield: 348 $\mathrm{mg}, 69 \% ;{ }^{1} \mathrm{H}$ NMR $\left(400 \mathrm{MHz}, \mathrm{C}_{6} \mathrm{D}_{6}\right) \delta(\mathrm{ppm})$ : $7.61(\mathrm{~d}, J=8.8 \mathrm{~Hz}, 2 \mathrm{H}), 6.74(\mathrm{~d}, J=8.8 \mathrm{~Hz}, 2 \mathrm{H})$ 5.40-5.39 (m, 1H), $2.93(\mathrm{dq}, J=14.4 \mathrm{~Hz}, 2.2 \mathrm{~Hz}, 1 \mathrm{H}), 2.60(\mathrm{~s}, 6 \mathrm{H}), 2.23-0.93(\mathrm{~m}, 40 \mathrm{H}), 0$. 72 (s, 3H); ${ }^{13} \mathrm{C}$ NMR (101 MHz, $\left.\mathrm{C}_{6} \mathrm{D}_{6}\right) \delta$ (ppm): 148.7, 139.4, 136.4, 124.7, 122.8, 111.7, 72.2, 55.8, 55.5, 49.4, 46.0, 41.5, 39.4, 39.0, 38.8, 35.8, 35.5, 35.1, 34.9, 34.7, 34.6, 31.2, 31.1, 27.5, 27.3, 23.5, 23.3, 21.9, 21.6, 20.2, 17.9, 17.8, 11.0.; HRMS (positive ESI): m/z calculated for $\mathrm{C}_{35} \mathrm{H}_{56} \mathrm{NO}[\mathrm{M}+\mathrm{H}]^{+}: 506.4356$; found: 506.4581 .

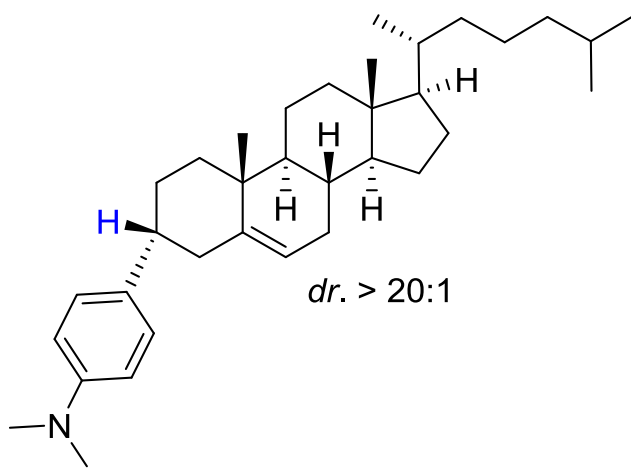

4-((8S,9S,10R,13R,14S,17R)-10,13-Dimethyl17-((R)-6-methylheptan-2-yl)-2,3,4,7,8,9,10,11 ,12,13,14,15,16,17-tetradecahydro-1H-cyclop enta[a]phenanthren-3-yl)- $N, N$-dimethylanilin e (11): $S / C=1000$, water : HFIP $=1: 1,2 \mathrm{~mL}$ in total. White solid; m.p.: $149-151^{\circ} \mathrm{C}$; yield: $111 \mathrm{mg}$, 91\%; ${ }^{1} \mathrm{H}$ NMR (400 MHz, $\left.\mathrm{CDCl}_{3}\right) \delta$ (ppm): 7.08 $(\mathrm{d}, J=8.4 \mathrm{~Hz}, 2 \mathrm{H}), 6.78(\mathrm{~d}, J=8.4 \mathrm{~Hz}, 2 \mathrm{H})$,

$5.28(\mathrm{~s}, 1 \mathrm{H}), 3.21-3.18(\mathrm{~m}, 1 \mathrm{H}), 2.92(\mathrm{~s}, 6 \mathrm{H}), 2.32-2.20(\mathrm{~m}, 1 \mathrm{H}), 1.85-0.87(\mathrm{~m}, 39 \mathrm{H}), 0.71$ (s, 3H); ${ }^{13} \mathrm{C}$ NMR $\left(101 \mathrm{MHz}, \mathrm{CDCl}_{3}\right) \delta$ (ppm): 149.1, 145.3, 135.8, 127.9, 123.7, 113.0, 56.3, 56.2, 54.7, 42.5, 42.3, 40.9, 40.0, 39.5, 38.0, 37.0, 36.2, 36.1, 35.8, 33.3, 32.7, 29.8, 28.2, 28.0, 24.3, 23.9, 22.8, 22.6, 21.4, 19.6, 18.7, 12.0 .

HRMS (positive ESI): $\mathrm{m} / \mathrm{z}$ calculated for $\mathrm{C}_{39} \mathrm{H}_{56} \mathrm{NO}[\mathrm{M}+\mathrm{H}]^{+}: 490.4407$; found: 490.4428 .

\subsection{Data for derivatives of directing group and carbocation}

\section{probing}




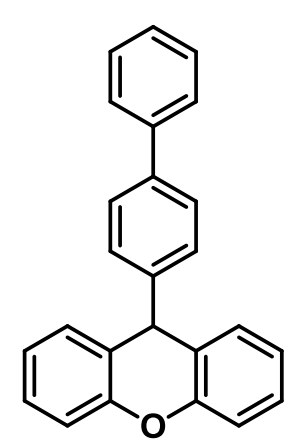

9-([1,1'-Biphenyl]-4-yl)-9H-xanthene (14): CAS No. 854661-12-4. White solid; m.p.: $220-221^{\circ} \mathrm{C}$ (reported 206-207 ${ }^{\circ} \mathrm{C}$ ); yield: $94 \mathrm{mg}$, 94\%; ${ }^{1} \mathrm{H}$ NMR (400 MHz, $\left.\mathrm{CDCl}_{3}\right) \delta$ (ppm): 7.55 (d, J = 7.6 Hz, 2H), $7.51(\mathrm{~d}, J=8.0 \mathrm{~Hz}, 2 \mathrm{H}), 7.42(\mathrm{t}, J=7.6 \mathrm{~Hz}, 2 \mathrm{H}), 7.35-7.27(\mathrm{~m}, 5 \mathrm{H})$, 7.18-7.12 (m, 4H), 7.02 (t, $J=7.6 \mathrm{~Hz}, 2 \mathrm{H}), 5.32(\mathrm{~s}, 1 \mathrm{H}) ;{ }^{13} \mathrm{C}$ NMR $(101$ $\left.\mathrm{MHz}, \mathrm{CDCl}_{3}\right) \delta(\mathrm{ppm}): 151.1,145.5,140.7,139.5,129.7,128.7,127.9$, $127.5,127.2,127.0,124.3,123.3,116.6,44.1$.<smiles>c1ccc2c(c1)Oc1ccccc1C2c1ccc(-c2cccc3c2oc2ccccc23)cc1</smiles>

\section{9-(4-(Dibenzo[b,d]furan-4-yl)phenyl)-9H-xanthene (16):}

White solid; m.p.: $142-144{ }^{\circ} \mathrm{C}$; yield: $106 \mathrm{mg}, 83 \% ;{ }^{1} \mathrm{H}$ NMR $(400$ $\left.\mathrm{MHz} \mathrm{CDCl}_{3}\right) \delta(\mathrm{ppm}): 7.98(\mathrm{~d}, J=7.6 \mathrm{~Hz}, 1 \mathrm{H}), 7.91(\mathrm{dd}, J=7.6 \mathrm{~Hz}$, $1.2 \mathrm{~Hz}, 1 \mathrm{H}), 7.85(\mathrm{~d}, J=8.0 \mathrm{~Hz}, 2 \mathrm{H}), 7.59(\mathrm{~d}, \quad J=8.0 \mathrm{~Hz}, 1 \mathrm{H})$, $7.56(\mathrm{dd}, J=7.6 \mathrm{~Hz}, 1.2 \mathrm{~Hz}, 1 \mathrm{H}), 7.47(\mathrm{td}, J=7.6 \mathrm{~Hz}, 1.2 \mathrm{~Hz}, 1 \mathrm{H})$, 7.42-7.34 (m, 4H), 7.25 (td, $J=7.6 \mathrm{~Hz}, 1.2 \mathrm{~Hz}, 2 \mathrm{H}), 7.18$ (dd, $J=$ $8.0 \mathrm{~Hz}, 1.2 \mathrm{~Hz}, 4 \mathrm{H}$ ), 7.04 (td, J = 7.6 Hz, $1.2 \mathrm{~Hz}, 2 \mathrm{H}$ ), 5.37 (s, 1H); ${ }^{13} \mathrm{C}$ NMR $\left(101 \mathrm{MHz}, \mathrm{CDCl}_{3}\right) \delta(\mathrm{ppm}): 156.1,153.3,151.1,146.0$, $134.7,129.8,129.1,128.6,128.0,127.2,126.7,125.4,124.9$, 124.3, 124.2, 123.3, 123.2, 122.7, 120.6, 119.6, 116.6, 111.8, 44.3. HRMS (positive ESI): $\mathrm{m} / \mathrm{z}$ calculated for $\mathrm{C}_{31} \mathrm{H}_{21} \mathrm{O}_{2}[\mathrm{M}+\mathrm{H}]^{+}:$: 425.1536; found: 425.1405 .<smiles>CN(C)c1ccc(C2=CCCCC2)cc1</smiles>

N,N-dimethyl-2',3',4',5'-tetrahydro-[1,1'-biphenyl]-4-amine (5): CAS No. 13314-74-4.

Colorless liquid; yield: $50 \mathrm{mg}, 99 \% ;{ }^{1} \mathrm{H}$ NMR $\left(400 \mathrm{MHz}, \mathrm{CDCl}_{3}\right) \delta$ (ppm): 7.28 (d, $J=8.8 \mathrm{~Hz}, 2 \mathrm{H}), 6.69(\mathrm{~d}, J=8.8 \mathrm{~Hz}, 2 \mathrm{H}), 6.00$ (tt, $J$ $=4.0 \mathrm{~Hz}, 1.6 \mathrm{~Hz}, 1 \mathrm{H}), 2.92(\mathrm{~s}, 6 \mathrm{H}), 2.41-1.58(\mathrm{~m}, 8 \mathrm{H}) ;{ }^{13} \mathrm{C} \mathrm{NMR}$ $\left(101 \mathrm{MHz}, \mathrm{CDCl}_{3}\right) \delta(\mathrm{ppm}): 149.5,136.0,131.2,125.5,121.5,112.5,40.7,27.3,25.8$, 23.2, 22.3.<smiles>CN(C)c1ccc(C(Sc2ccc(Br)cc2)c2ccccc2)cc1</smiles>

$56.8,40.4$.
4-(((4-Bromophenyl)thio)(phenyl)methyl)-N,N-dimethylanilin e (6):

White solid; m.p.: $114-116{ }^{\circ} \mathrm{C}$; yield: $95 \mathrm{mg}, 96 \% ;{ }^{1} \mathrm{H}$ NMR $(400$ $\left.\mathrm{MHz}, \mathrm{CDCl}_{3}\right) \delta$ (ppm): $7.40(\mathrm{~d}, J=7.6 \mathrm{~Hz}, 2 \mathrm{H}), 7.30-7.15(\mathrm{~m}, 7 \mathrm{H})$, $7.05(\mathrm{~d}, J=8.4 \mathrm{~Hz}, 2 \mathrm{H}), 6.63(\mathrm{~d}, J=8.4 \mathrm{~Hz}, 2 \mathrm{H}), 5.46(\mathrm{~s}, 1 \mathrm{H})$, 2.88 (s, 6H); $\left.{ }^{13} \mathrm{C} \mathrm{NMR} \mathrm{(101} \mathrm{MHz,} \mathrm{CDCl}_{3}\right) \delta(\mathrm{ppm}): 149.7,141.1$, 135.9, 131.6, 129.0, 128.4, 128.3, 127.9, 127.1, 120.1, 112.4,

HRMS (positive ESI): m/z calculated for $\mathrm{C}_{21} \mathrm{H}_{21} \mathrm{BrNS}[\mathrm{M}+\mathrm{H}]^{+}:$398.0753; found: 398.0764 . 


\section{Copies of ${ }^{1} \mathrm{H}$ NMR and ${ }^{13} \mathrm{C}$ NMR spectra of catalysts and}

\section{products}

Complex LC-1

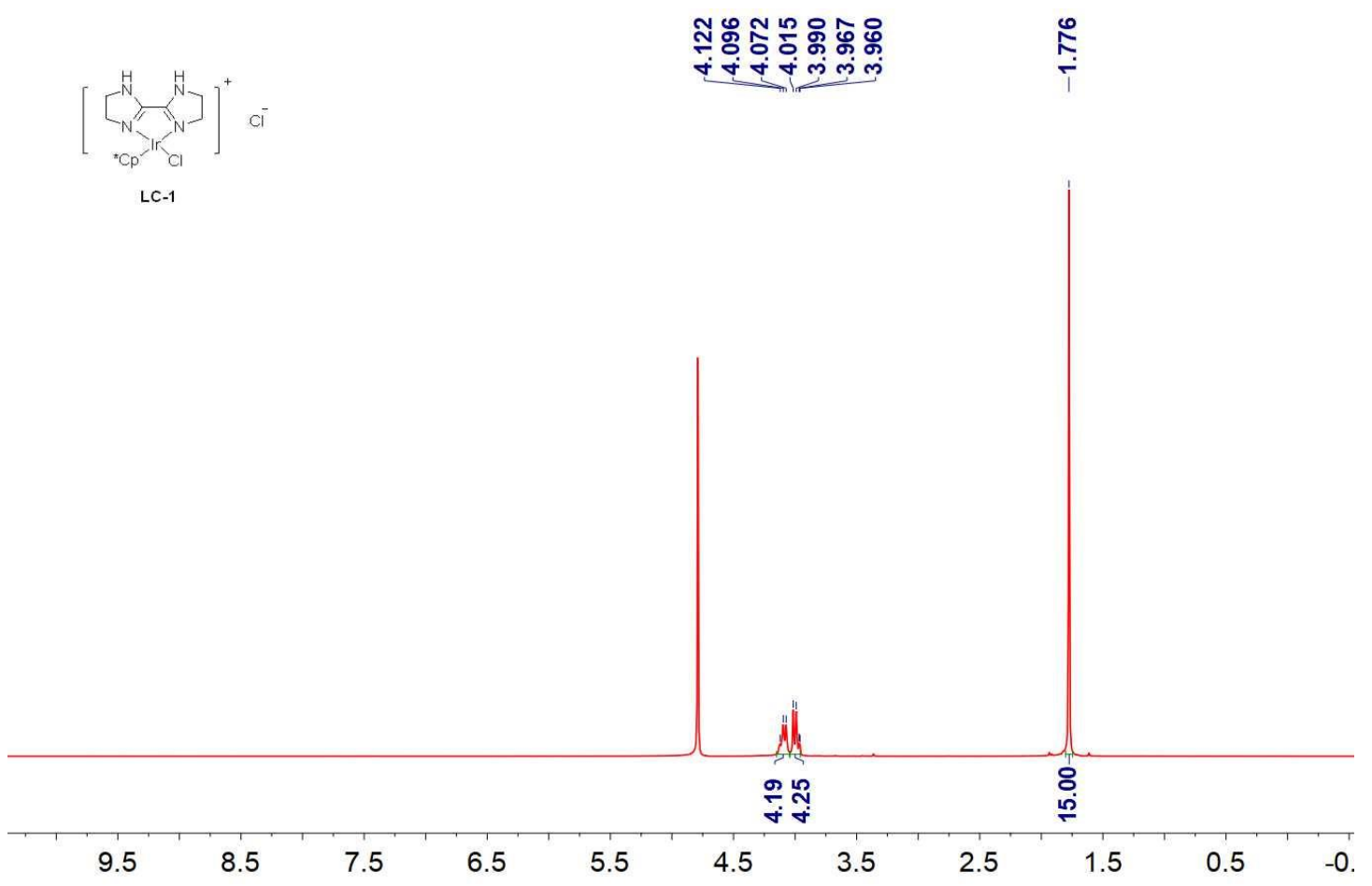

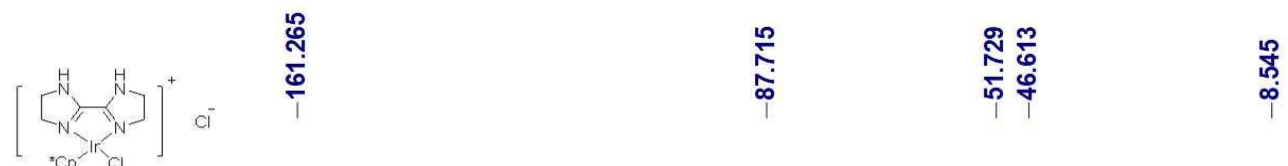

LC-1 


\section{Complex LC-2}
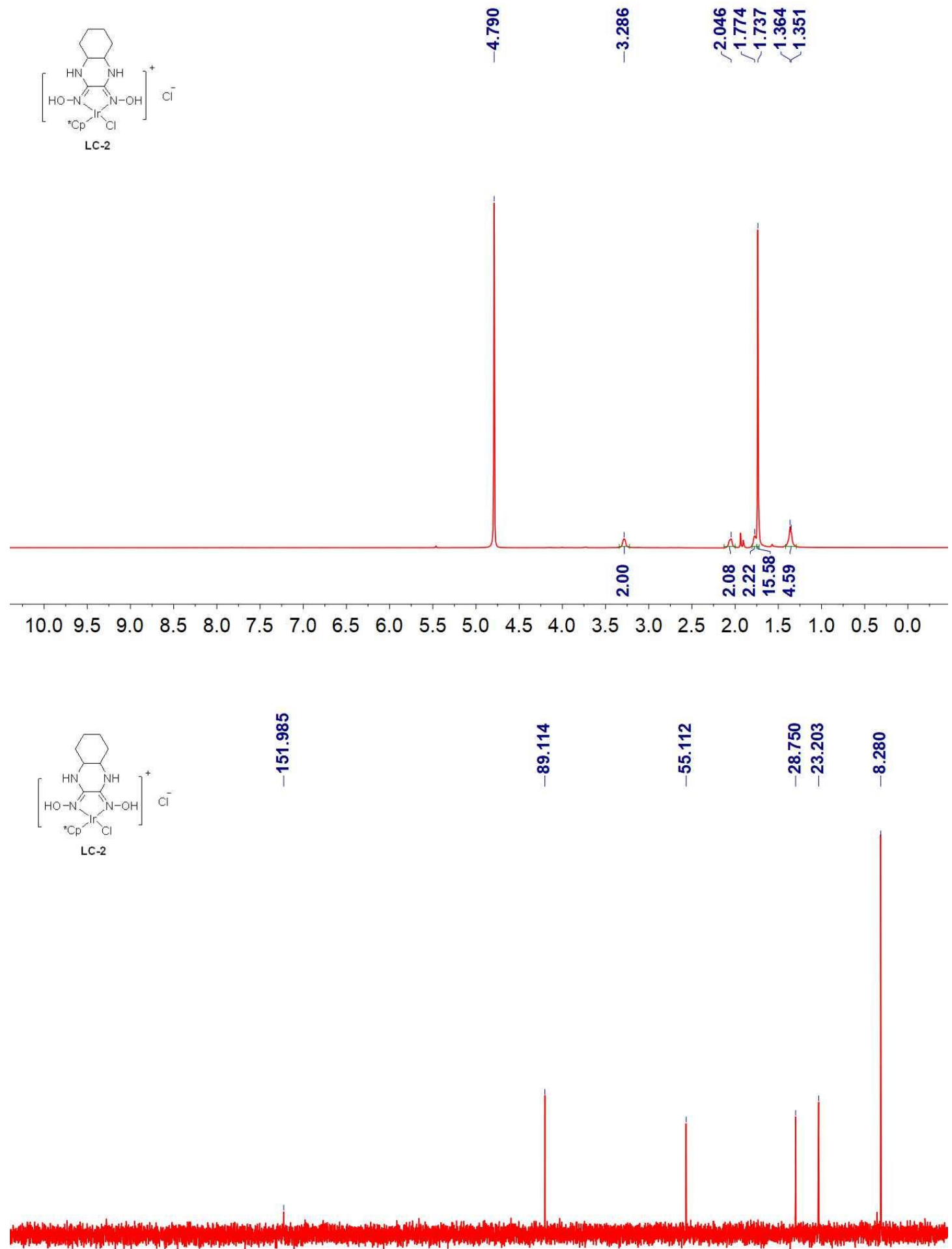

$210200190180170160150140130120110100 \quad 90 \quad 80 \quad 70 \quad 60 \quad 50 \quad 40 \quad 30 \quad 20 \quad 10 \quad 0$ 


\section{Complex LC-3}
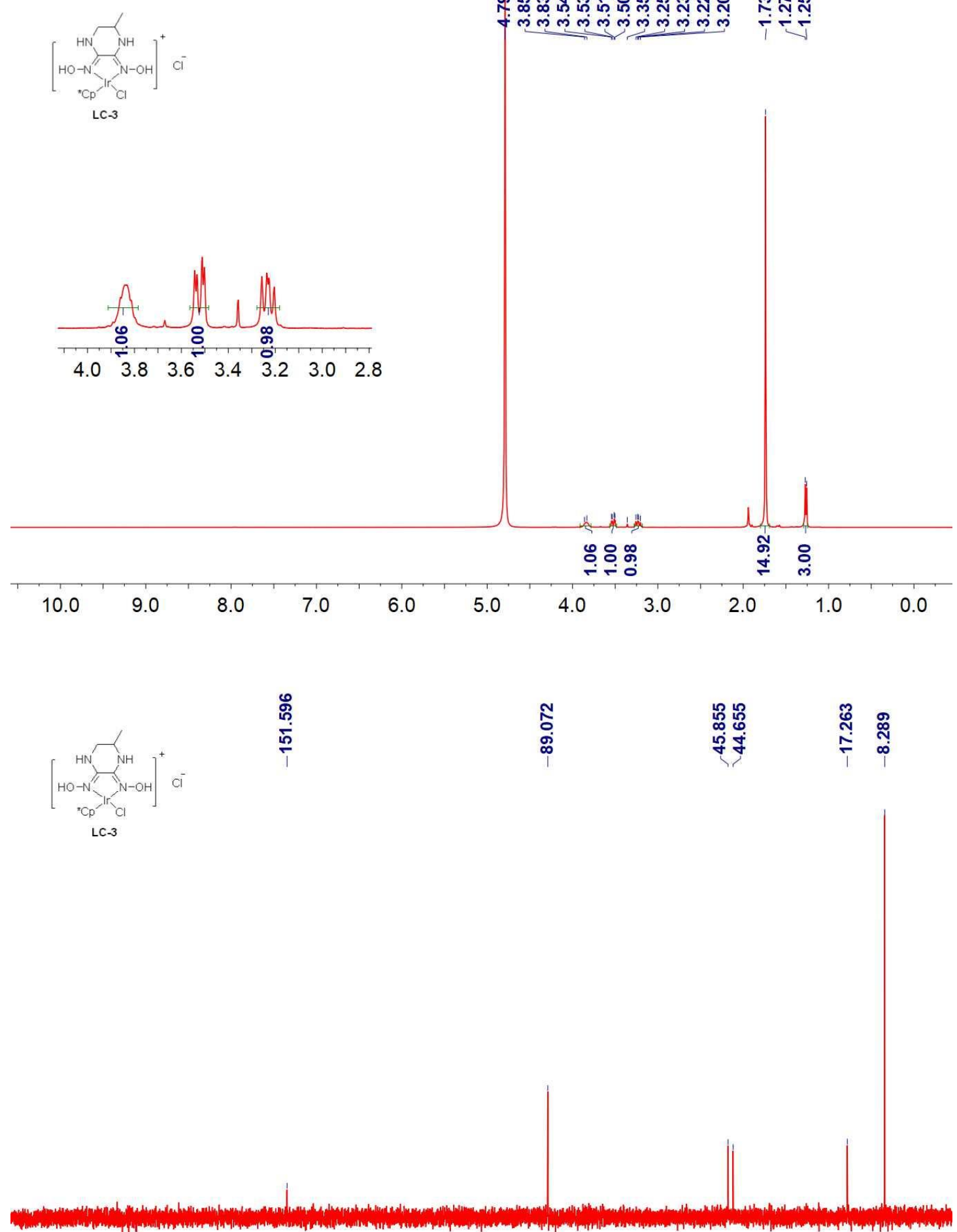

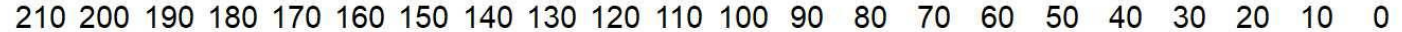


(4-Methoxyphenyl)(phenyl)methanol (S1a)

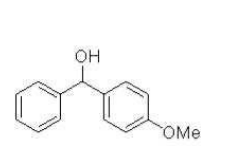

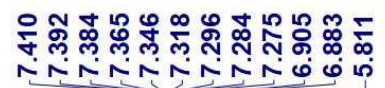

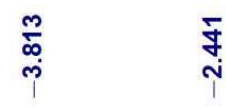

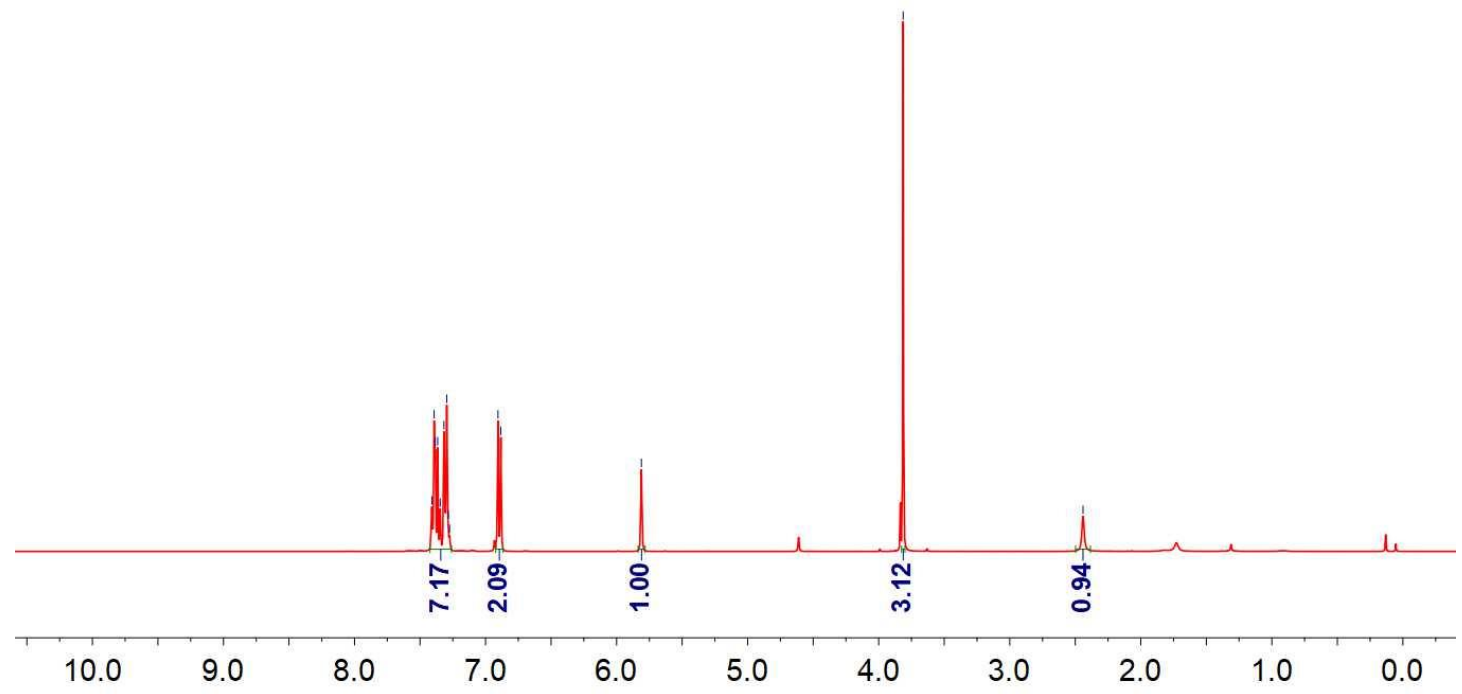

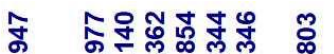

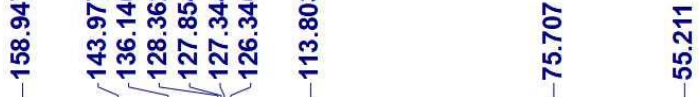

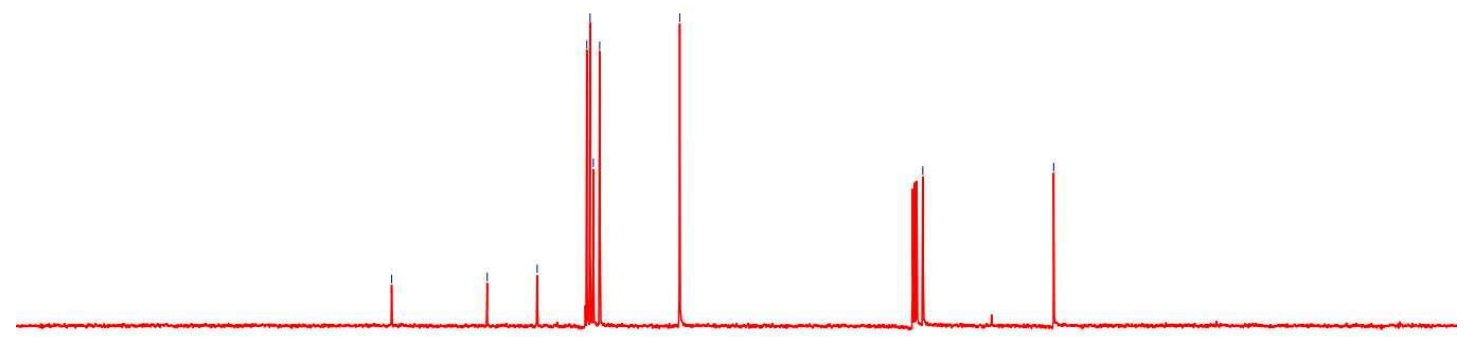

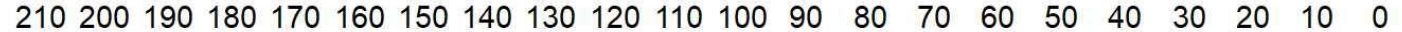




\section{Benzo[d][1,3]dioxol-5-yl(phenyl)methanol (S1b)}

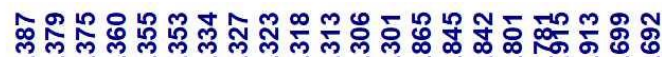

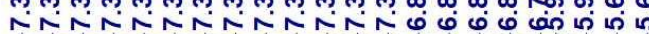

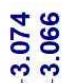
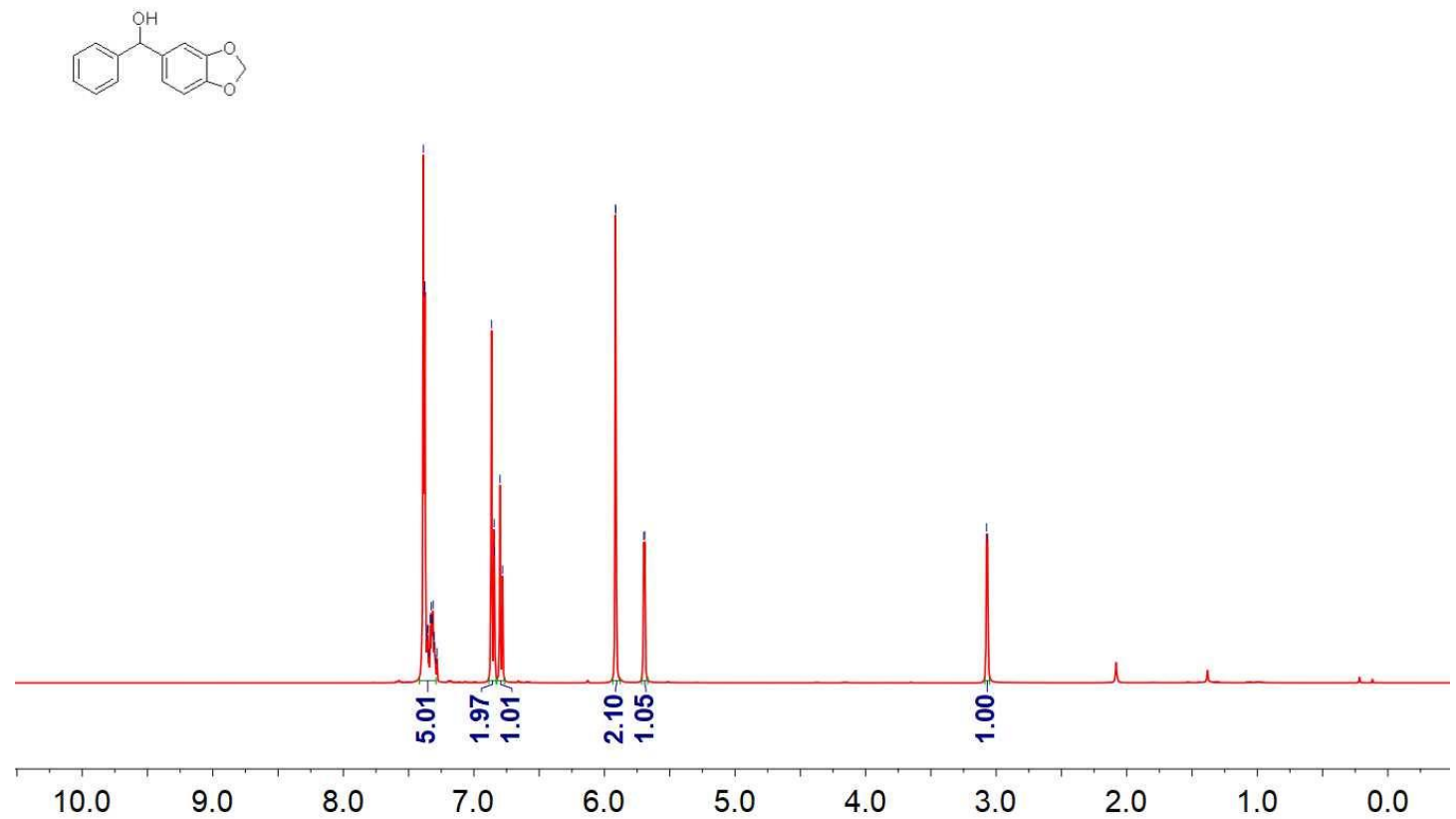

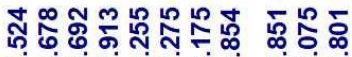

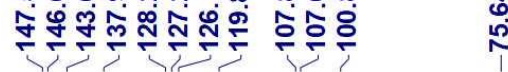
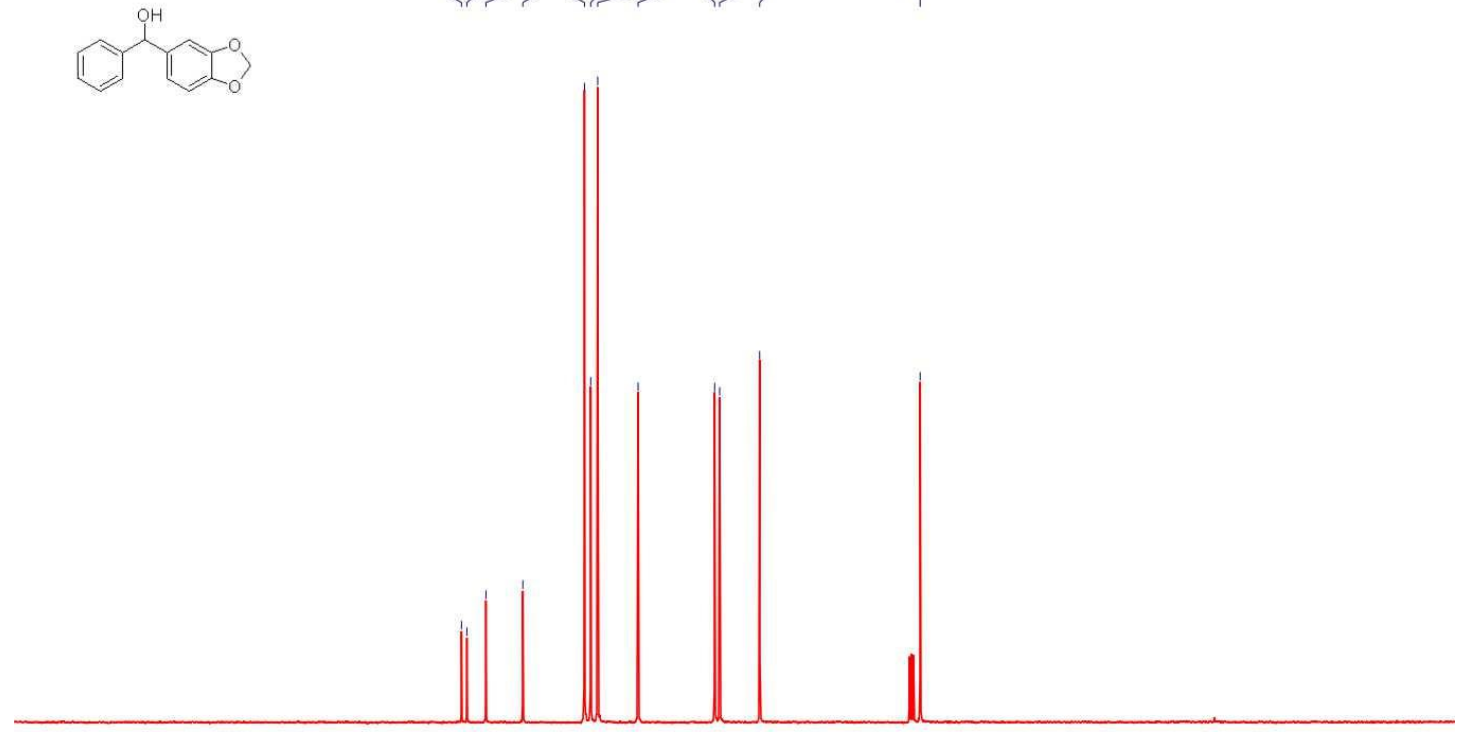

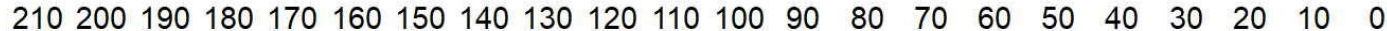




\section{4-(Hydroxy(phenyl)methyl)phenol (S1c)}
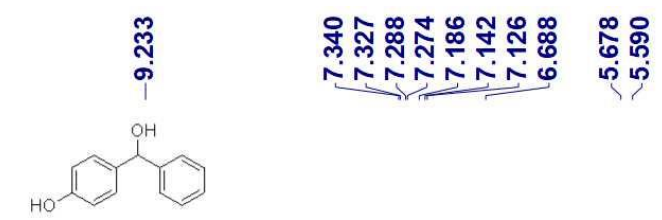

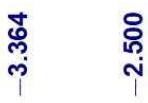
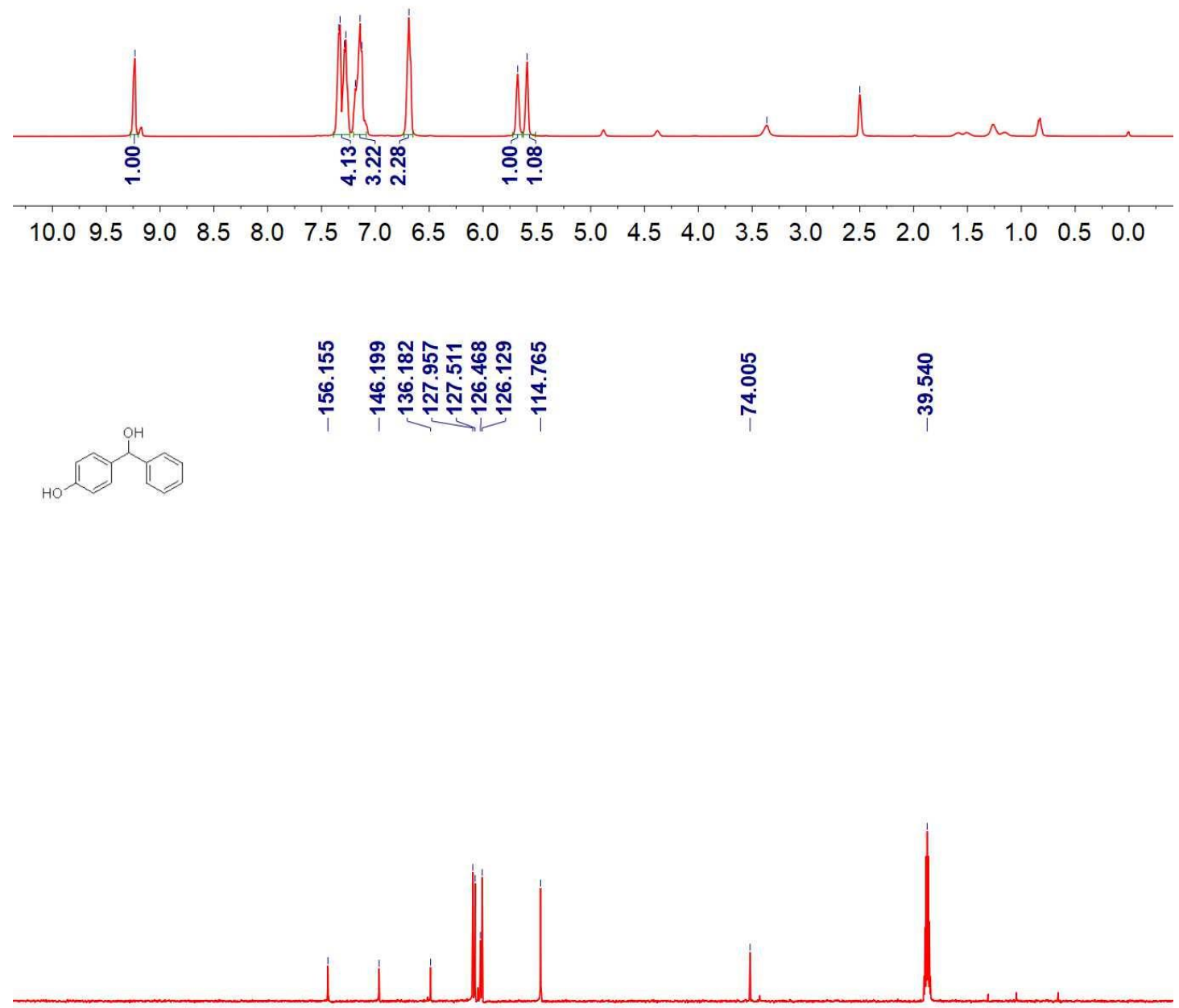

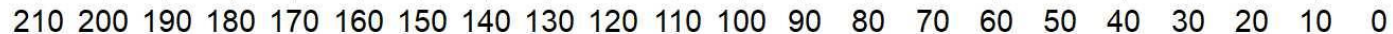




\section{Furan-2-yl(phenyl)methanol (S1d)}

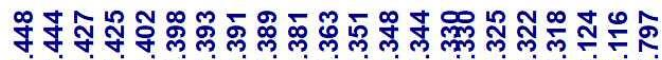

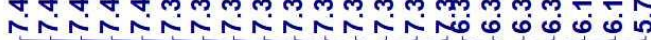

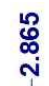
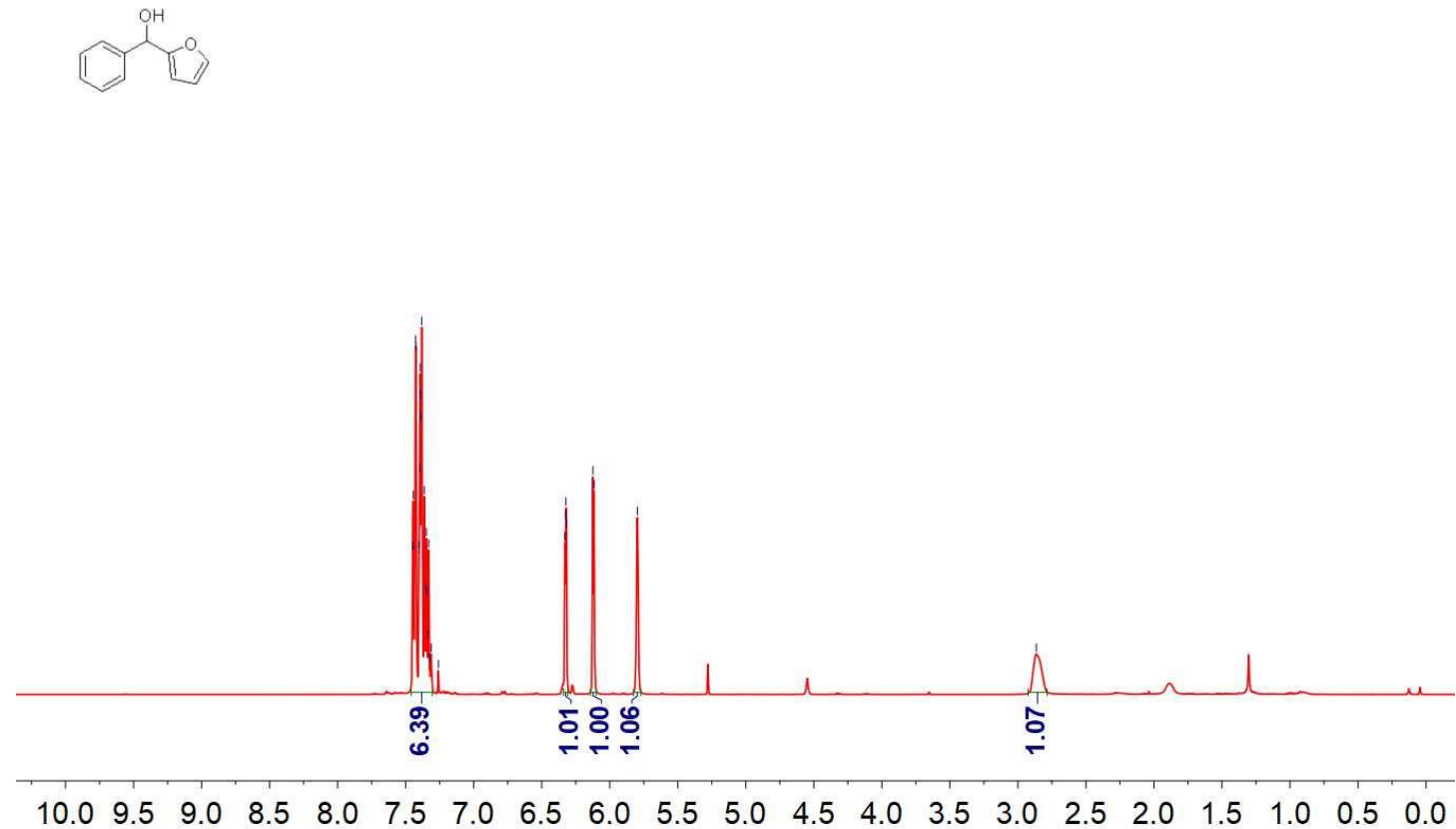

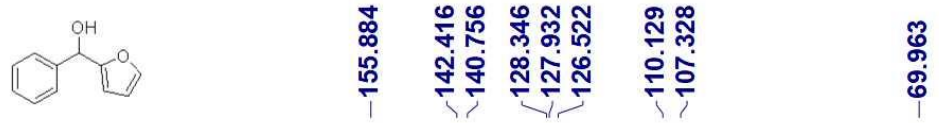

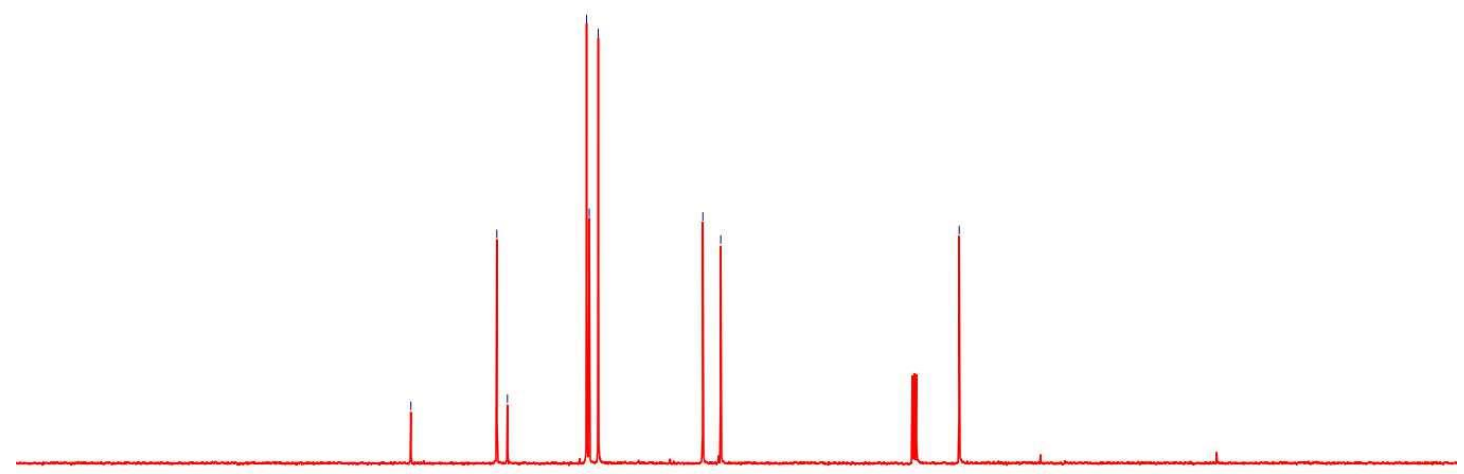

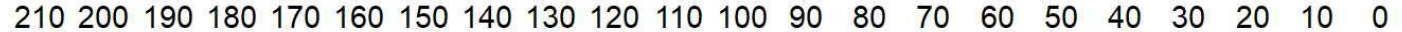




\section{Phenyl(thiophen-2-yl)methanol (S1e)}

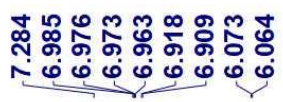

ำ ำ

붑

ข่
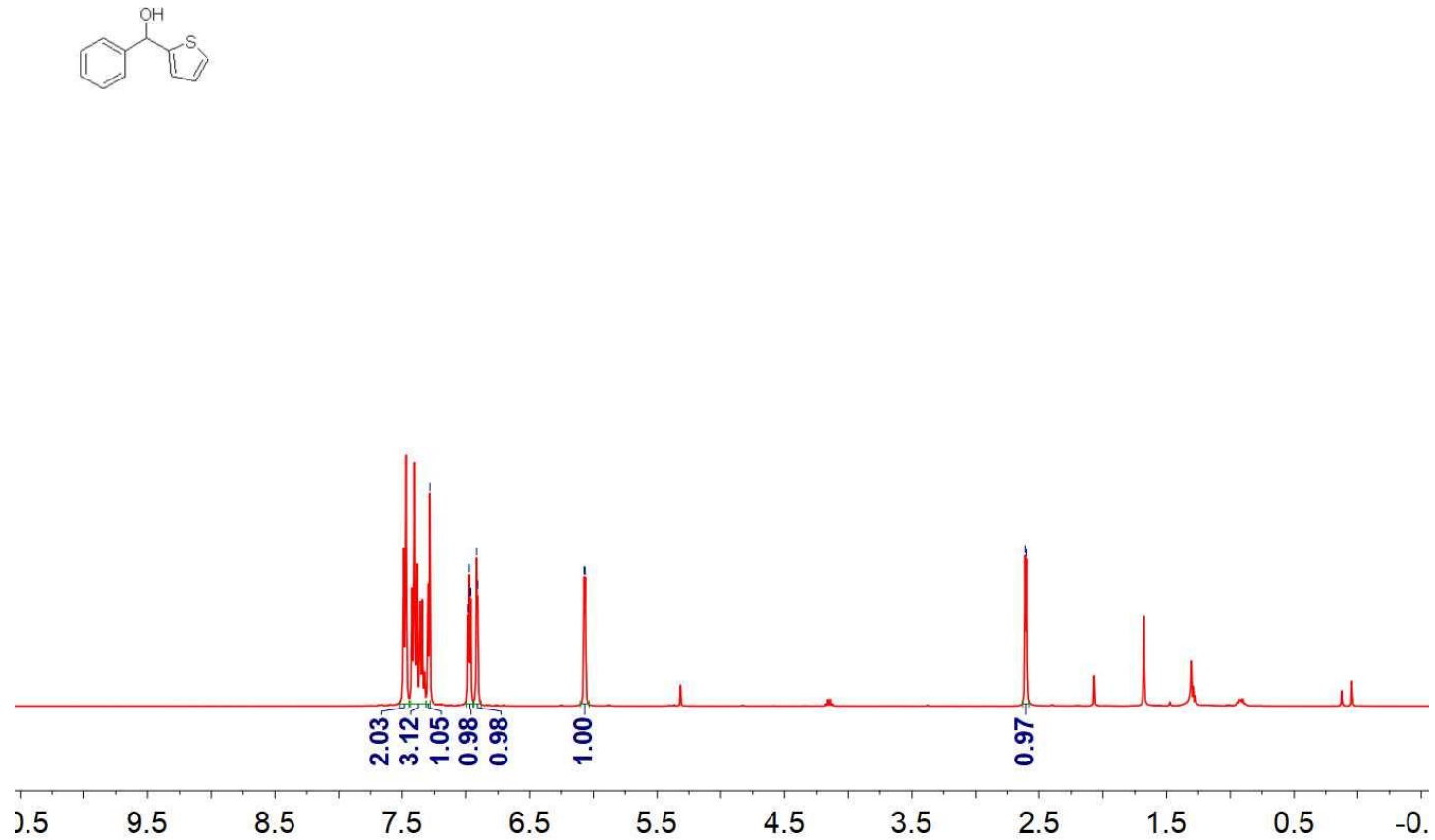

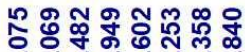

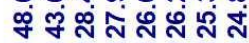

กึ ָָ

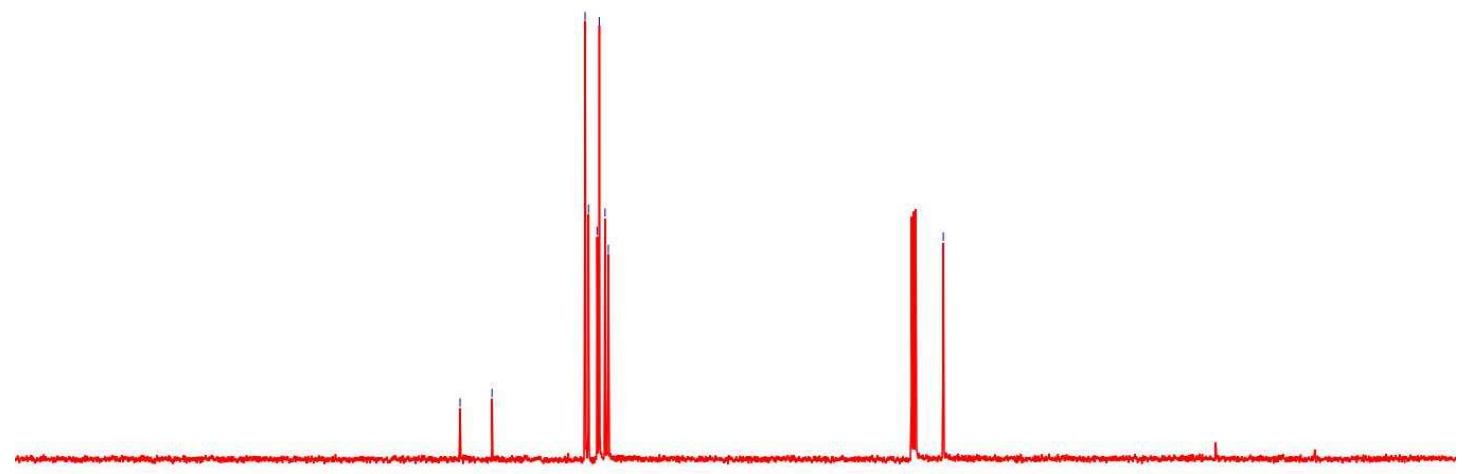

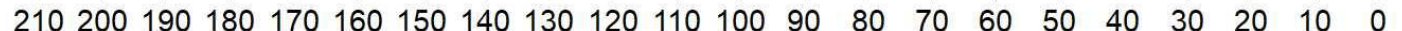


1-(4-(Dimethylamino)phenyl)-2-methylpropan-1-ol (1a)

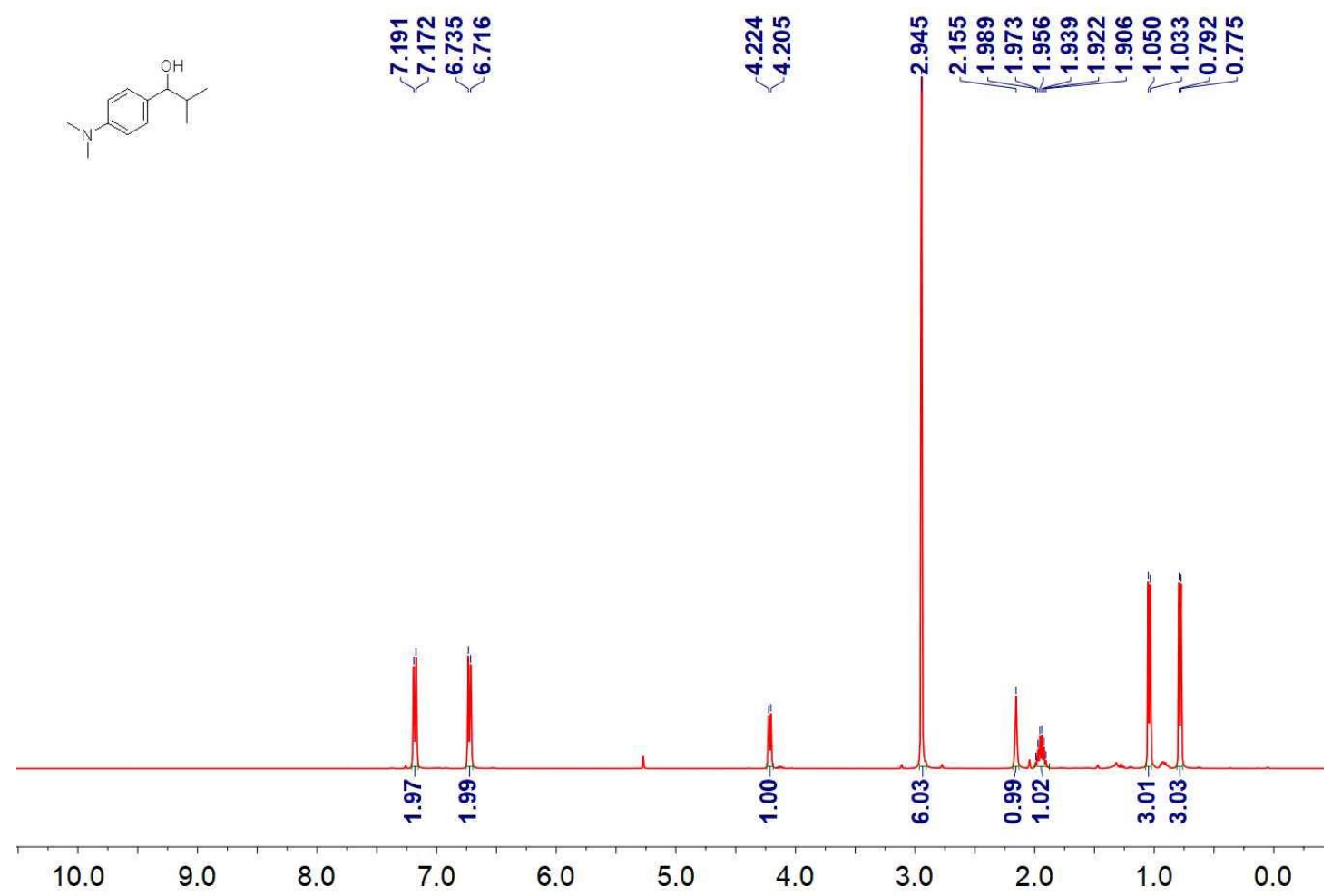

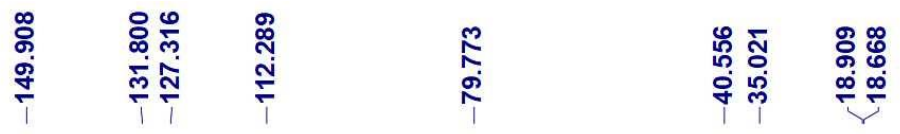

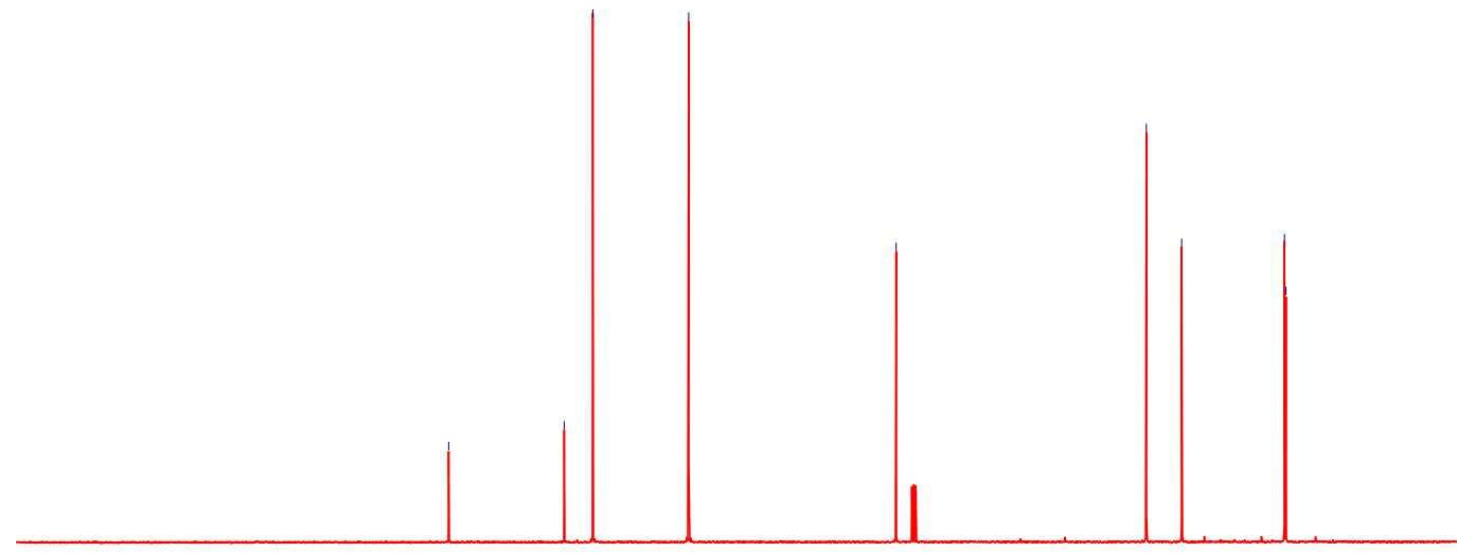

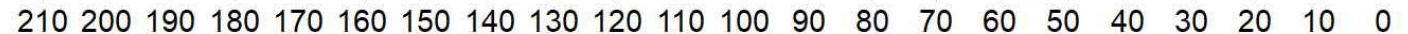




\section{(4-(dimethylamino)phenyl)(phenyl)methanol (1b)}

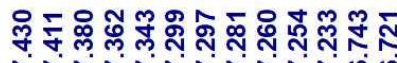

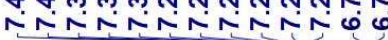

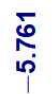

กู้

กั่ ก
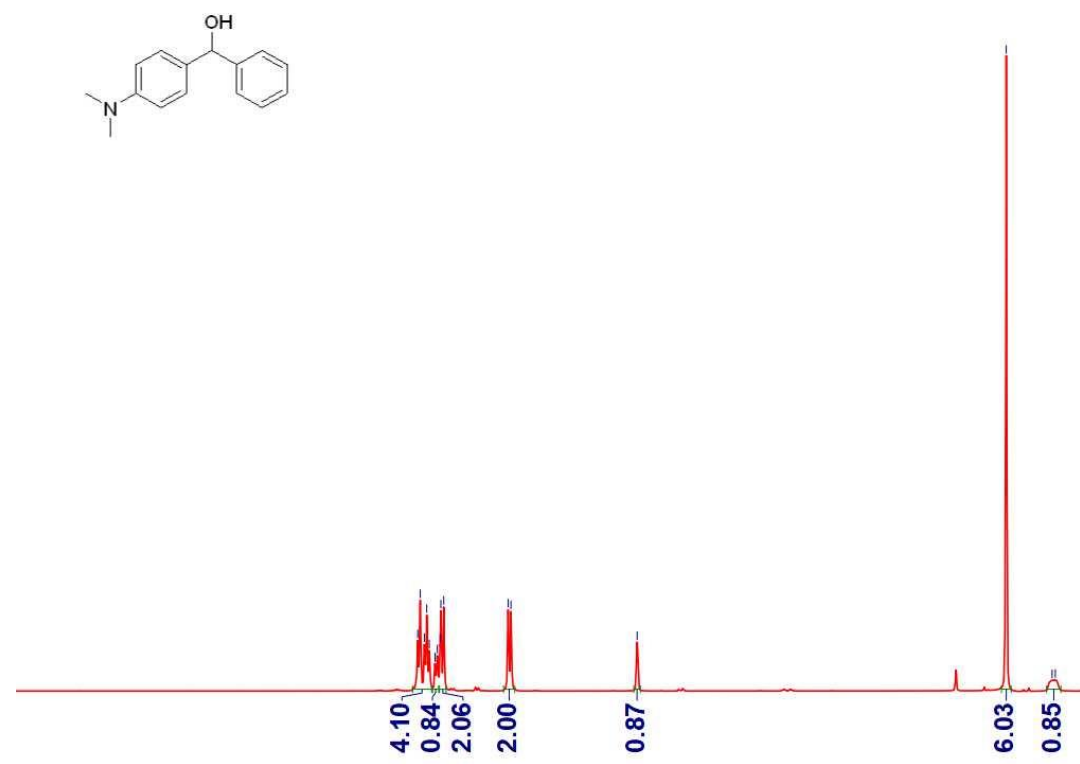

$\begin{array}{lllllllllllllllllllll}10.0 & 9.5 & 9.0 & 8.5 & 8.0 & 7.5 & 7.0 & 6.5 & 6.0 & 5.5 & 5.0 & 4.5 & 4.0 & 3.5 & 3.0 & 2.5 & 2.0 & 1.5 & 1.0 & 0.5 & 0.0\end{array}$

กิ

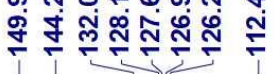
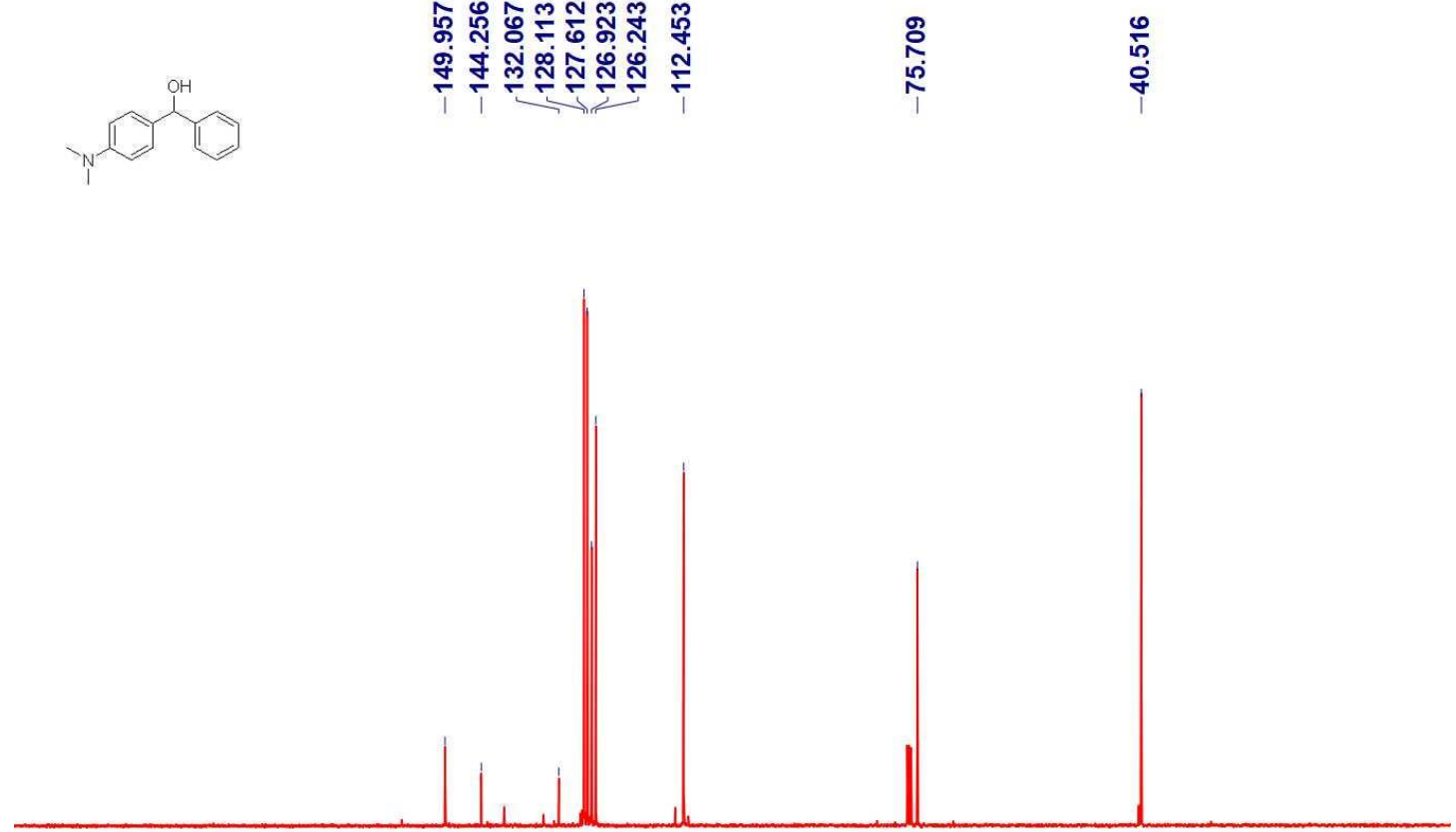

$210200190180170160150140130120110100 \quad 90 \quad 80 \quad 70 \quad 60 \quad 50 \quad 40 \quad 30 \quad 20 \quad 10 \quad 0$ 


\section{(2-(dimethylamino)phenyl)(phenyl)methanol (1b-1)}

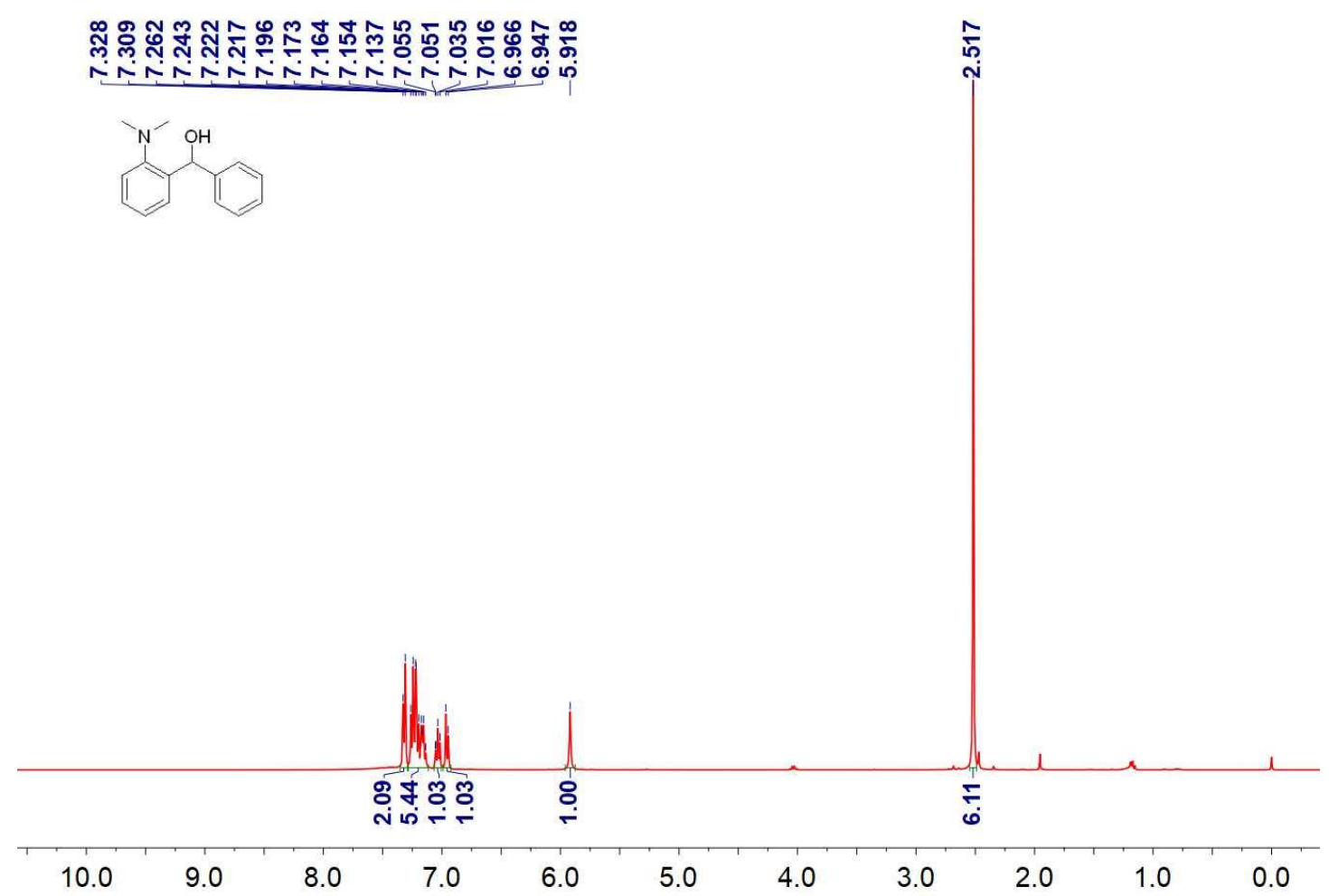

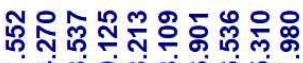

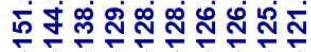
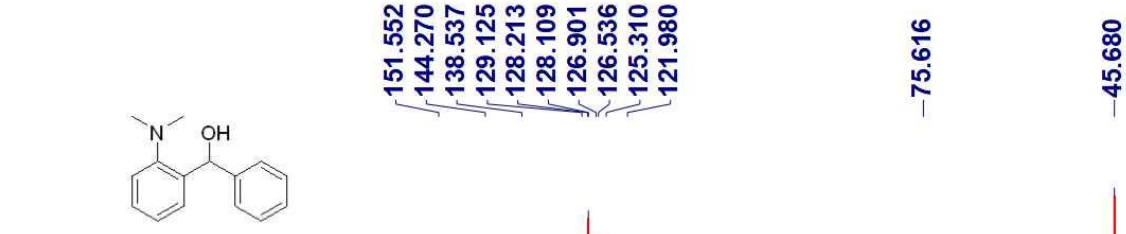

$210200190180170160150140130120110100 \quad 90 \quad 80 \quad 70 \quad 60 \quad 50 \quad 40 \quad 30 \quad 20 \quad 10 \quad 0$ 


\section{(3-(dimethylamino)phenyl)(phenyl)methanol (1b-2)}
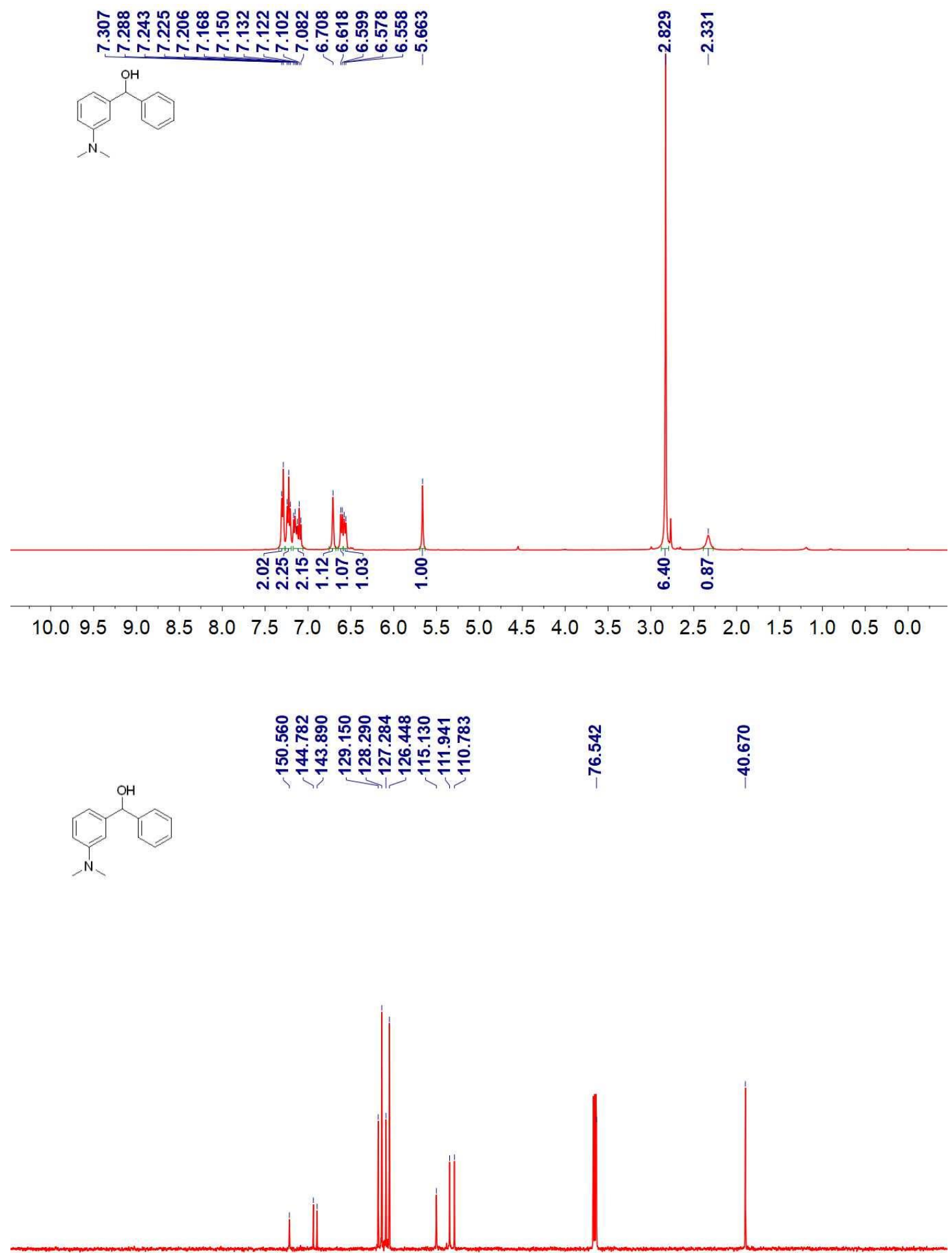

$210200190180170160150140130120110 \quad 100 \quad 90 \quad 80 \quad 70 \quad 60 \quad 50 \quad 40 \quad 30 \quad 20 \quad 10 \quad 0$ 
(4-(diethylamino)phenyl)(phenyl)methanol (1b-3)
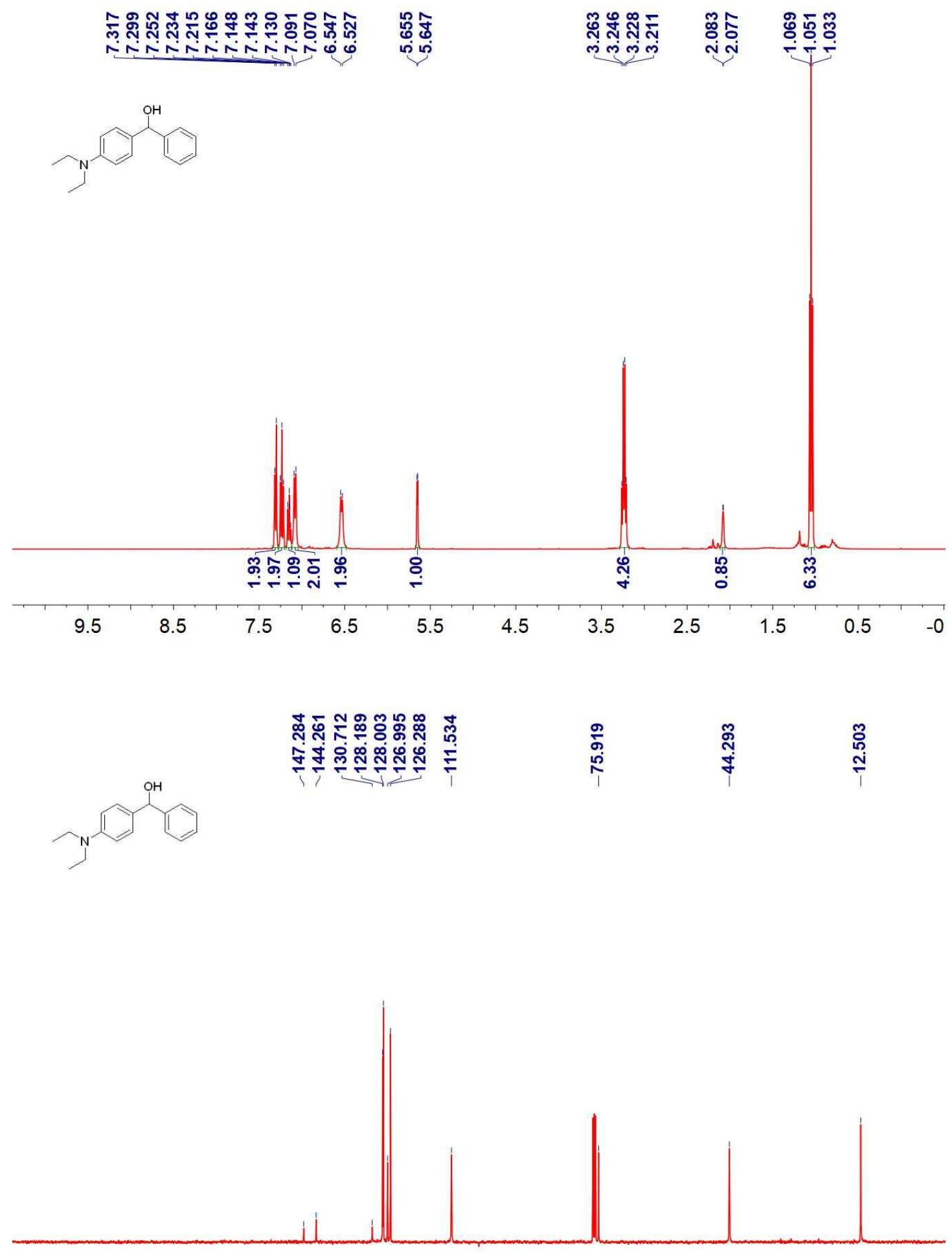

$210200190180170160150140130120110100 \quad 90 \quad 80 \quad 70 \quad 60 \quad 50 \quad 40 \quad 30 \quad 20 \quad 10 \quad 0$ 
Phenyl(4-(pyrrolidin-1-yl)phenyl)methanol (1b-4)
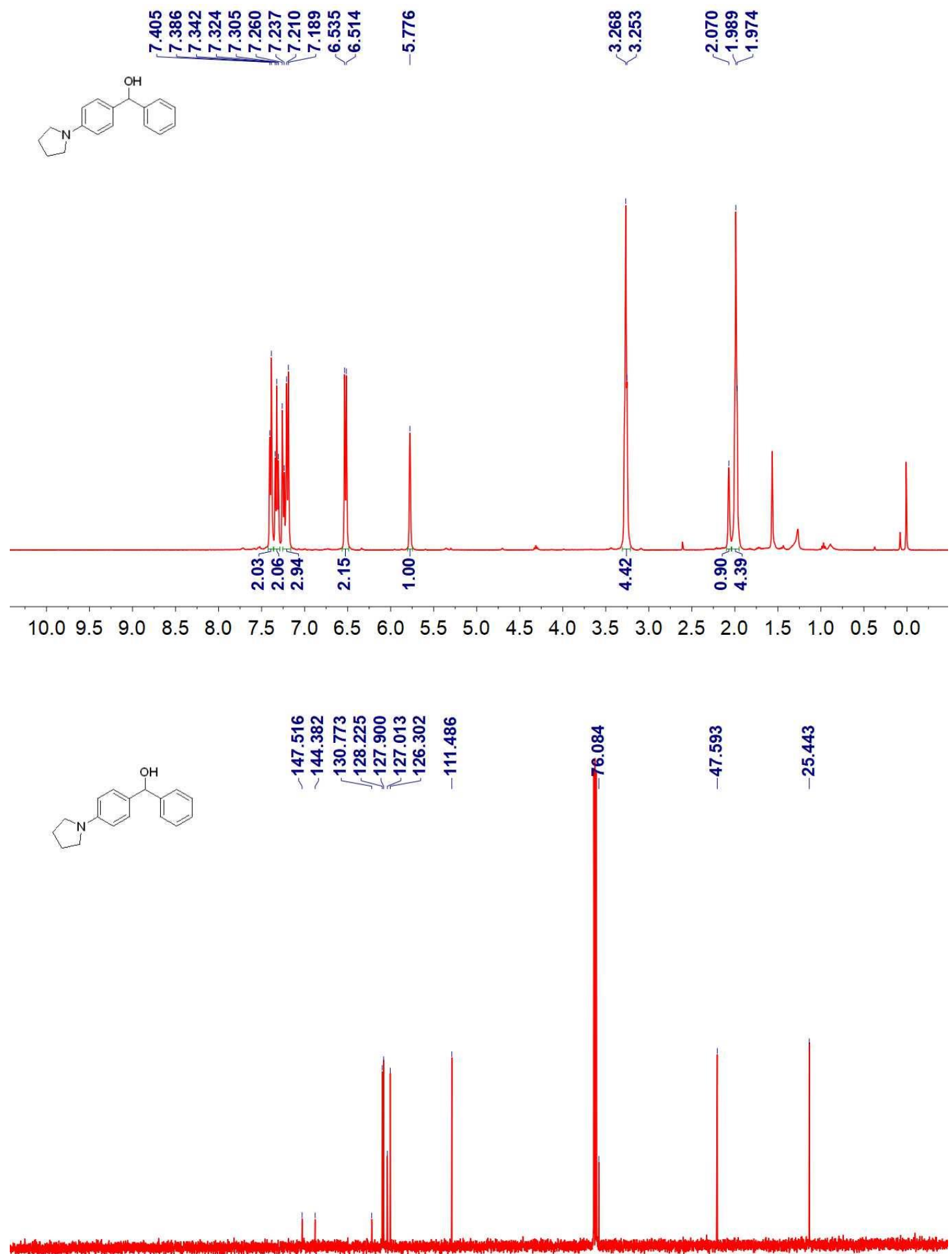

$210200190180170160150140130120110 \quad 100 \quad 90 \quad 80 \quad 70 \quad 60 \quad 50 \quad 40 \quad 30 \quad 20 \quad 10 \quad 0$ 
Phenyl(4-(piperidin-1-yl)phenyl)methanol (1b-5)

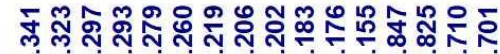

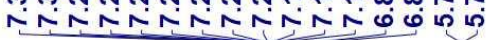

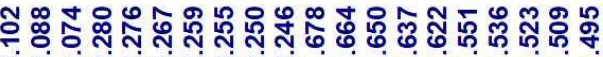

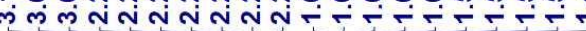
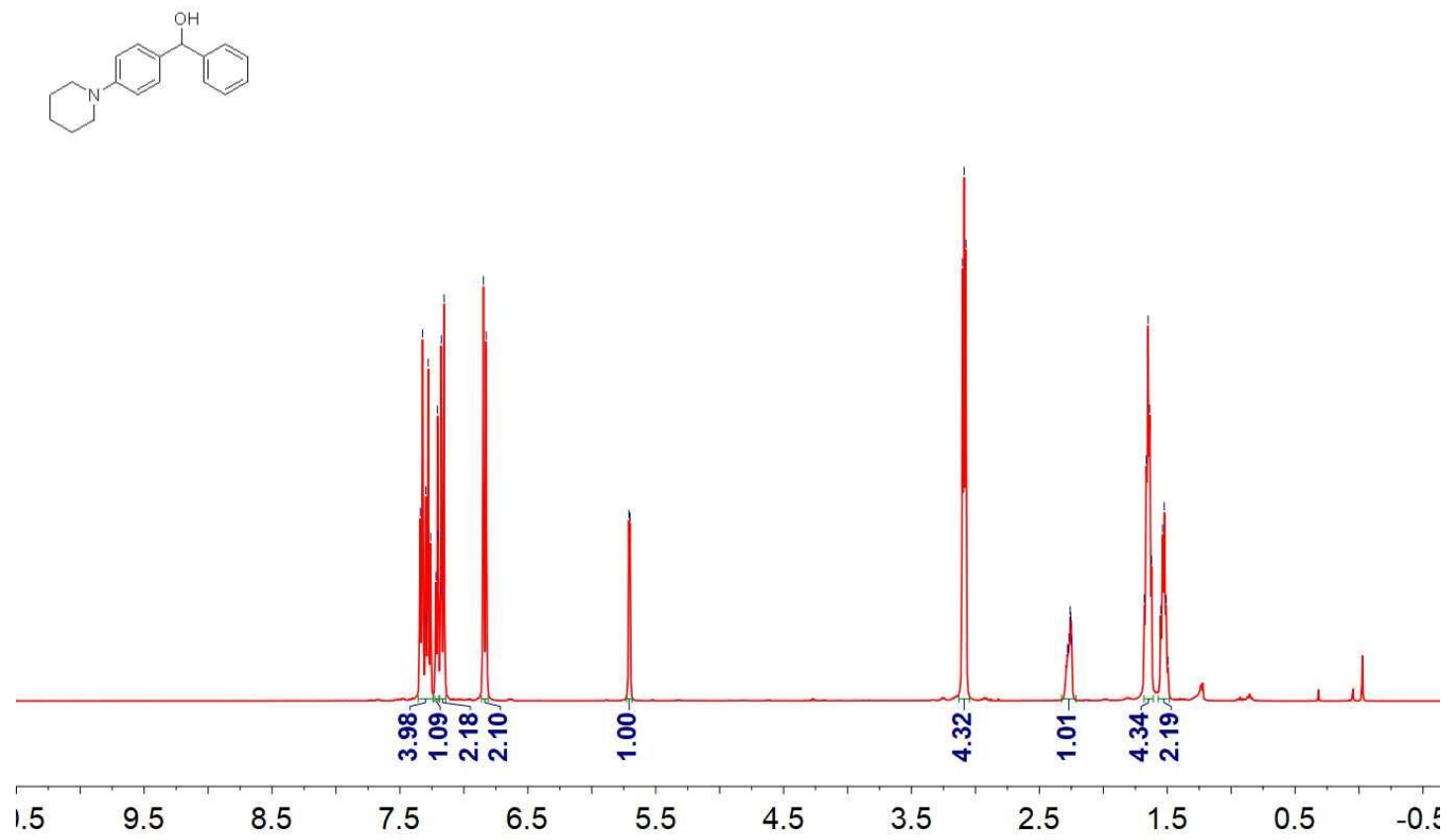

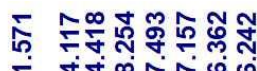

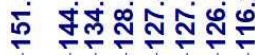

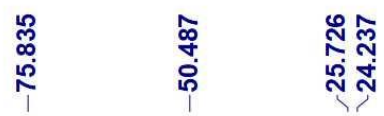

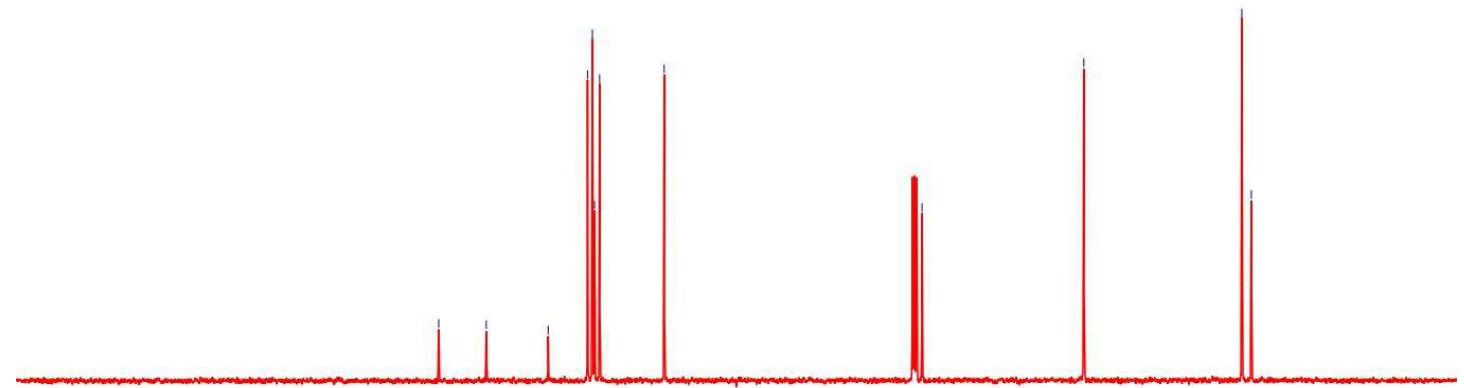

$210200190180170160150140130120110 \quad 100 \quad 90 \quad 80 \quad 70 \quad 60 \quad 50 \quad 40 \quad 30 \quad 20 \quad 10 \quad 0$ 
(4-(3,4-Dihydroisoquinolin-2(1H)-yl)phenyl)(phenyl)methanol (1b-6)

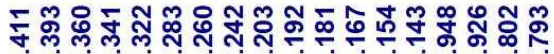

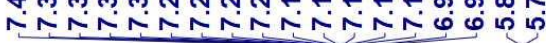

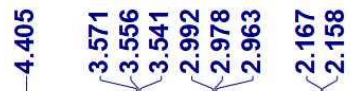

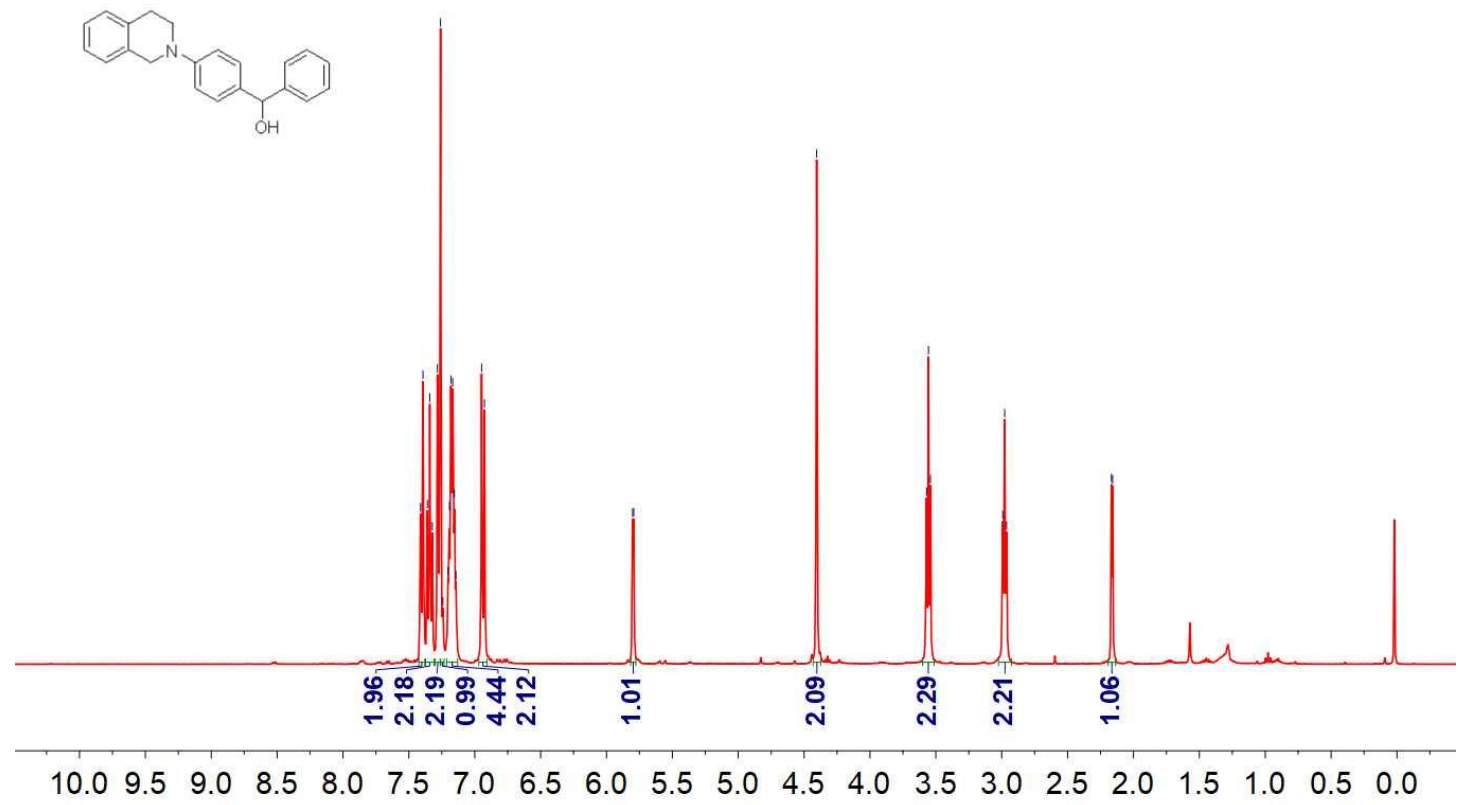

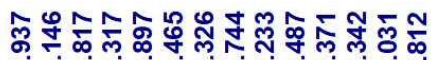

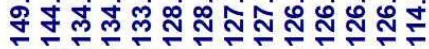

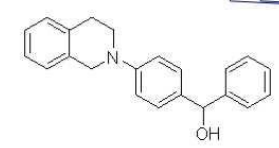

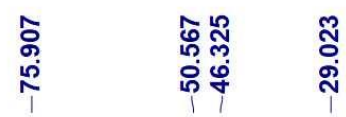

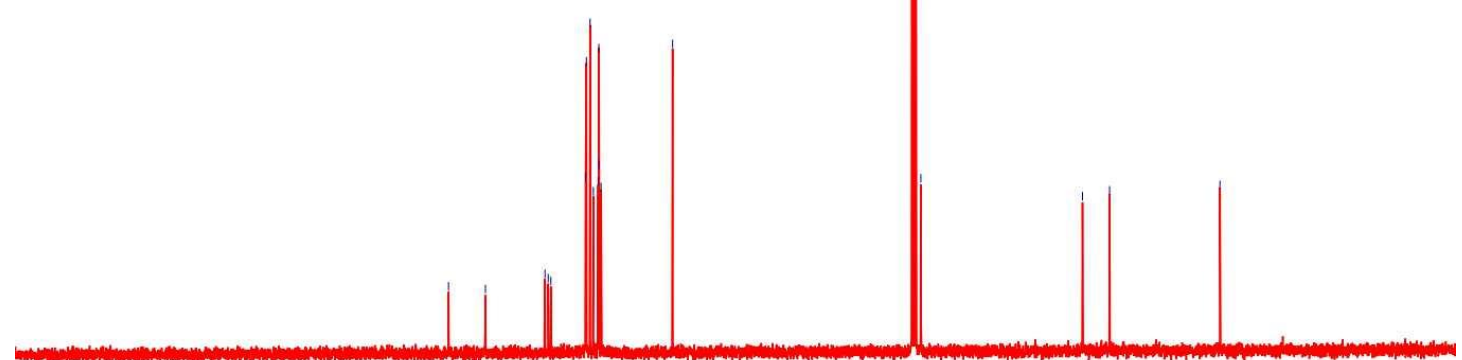

$210200190180170160150140130120110100 \quad 90 \quad 80 \quad 70 \quad 60 \quad 50 \quad 40 \quad 30 \quad 20 \quad 10 \quad 0$ 
(4-(3,4-Dihydroquinolin-1(2H)-yl)phenyl)(phenyl)methanol (1b-7)

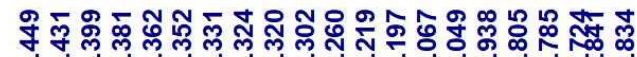

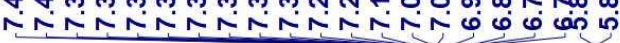

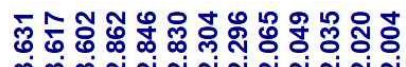

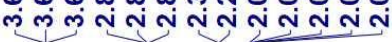

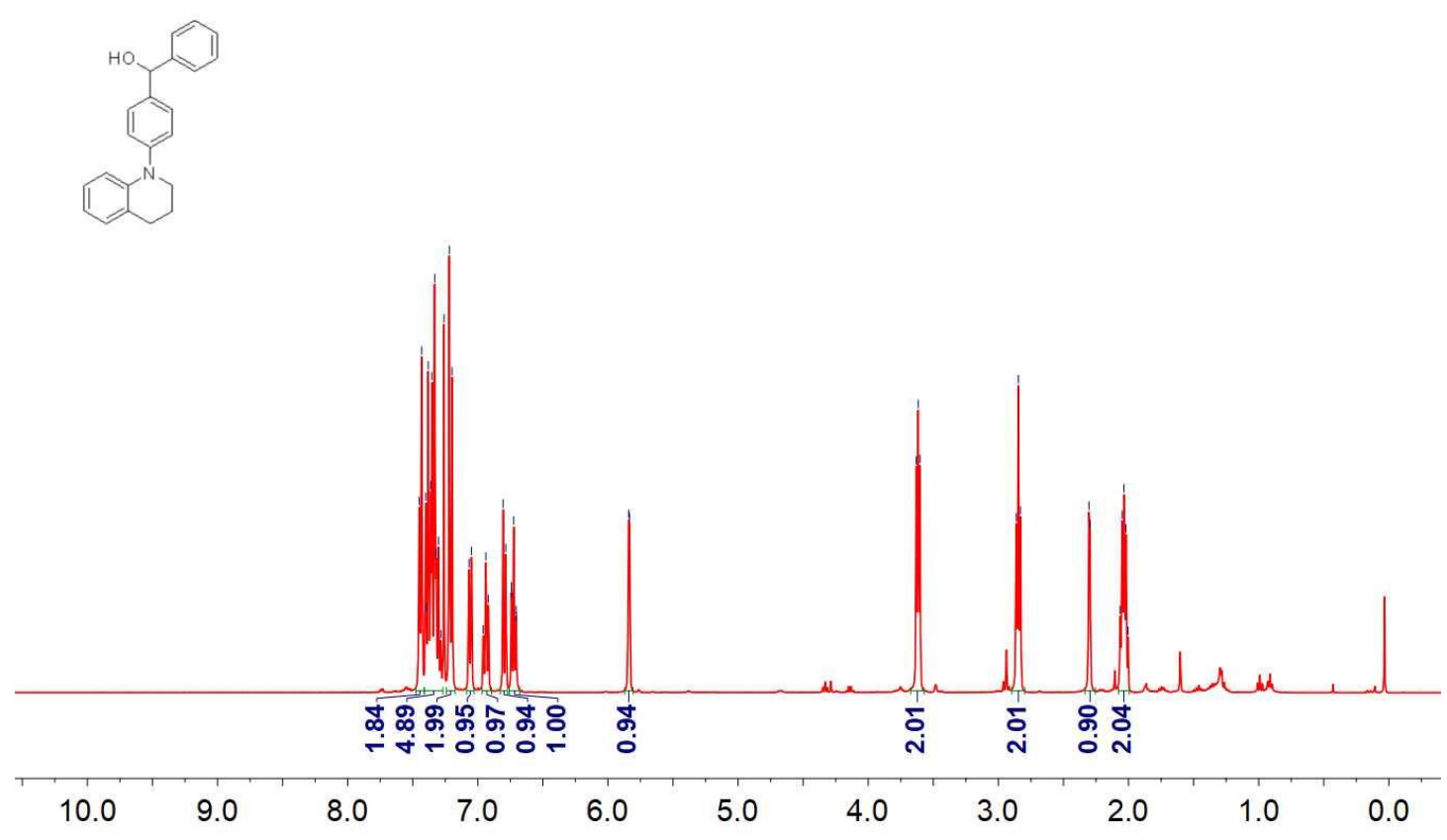

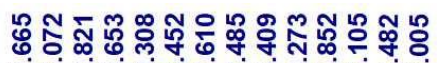

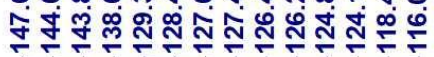

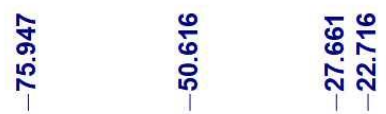

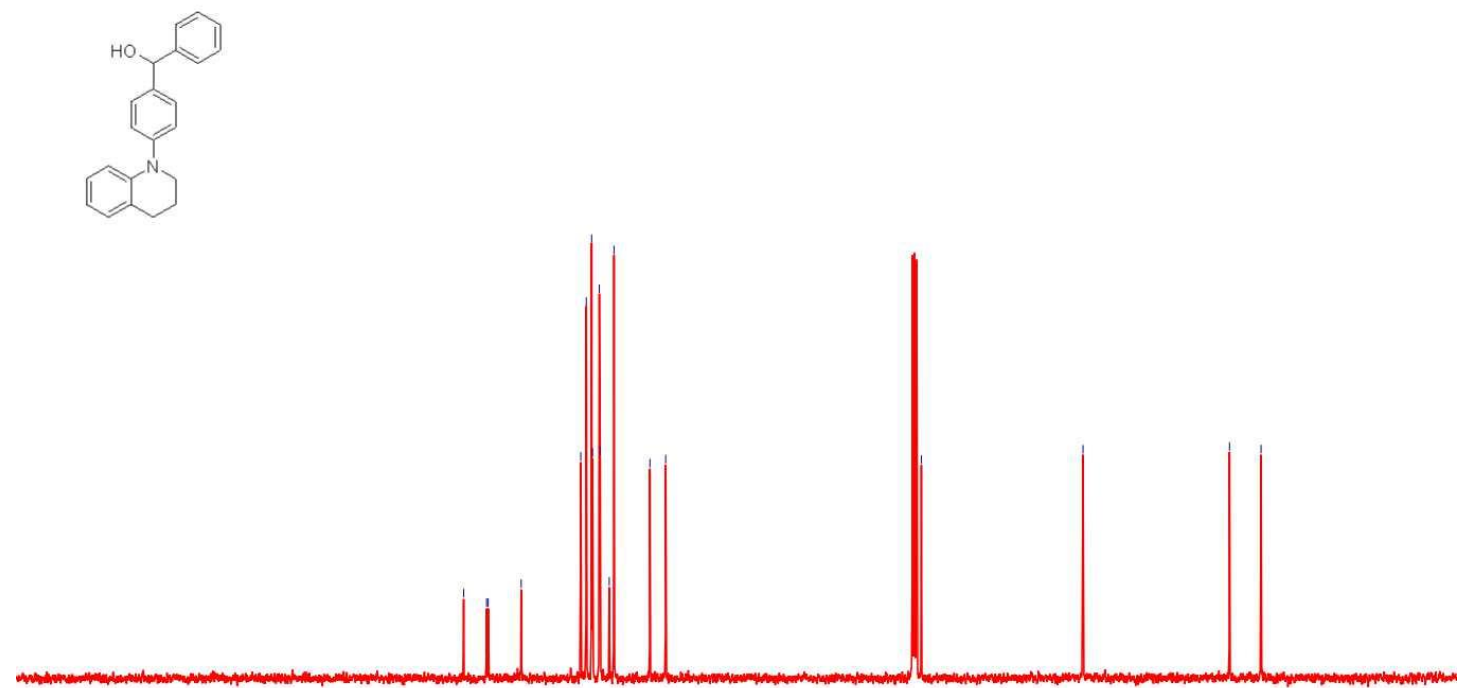

$210200190180170160150140130120110100 \quad 90 \quad 80 \quad 70 \quad 60 \quad 50 \quad 40 \quad 30 \quad 20 \quad 10 \quad 0$ 
(4-(Methylamino)phenyl)(phenyl)methanol (1b-8)

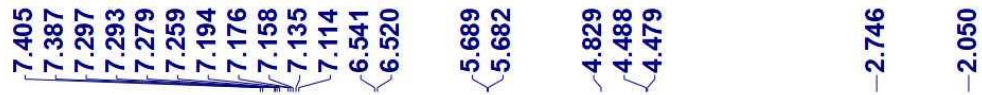
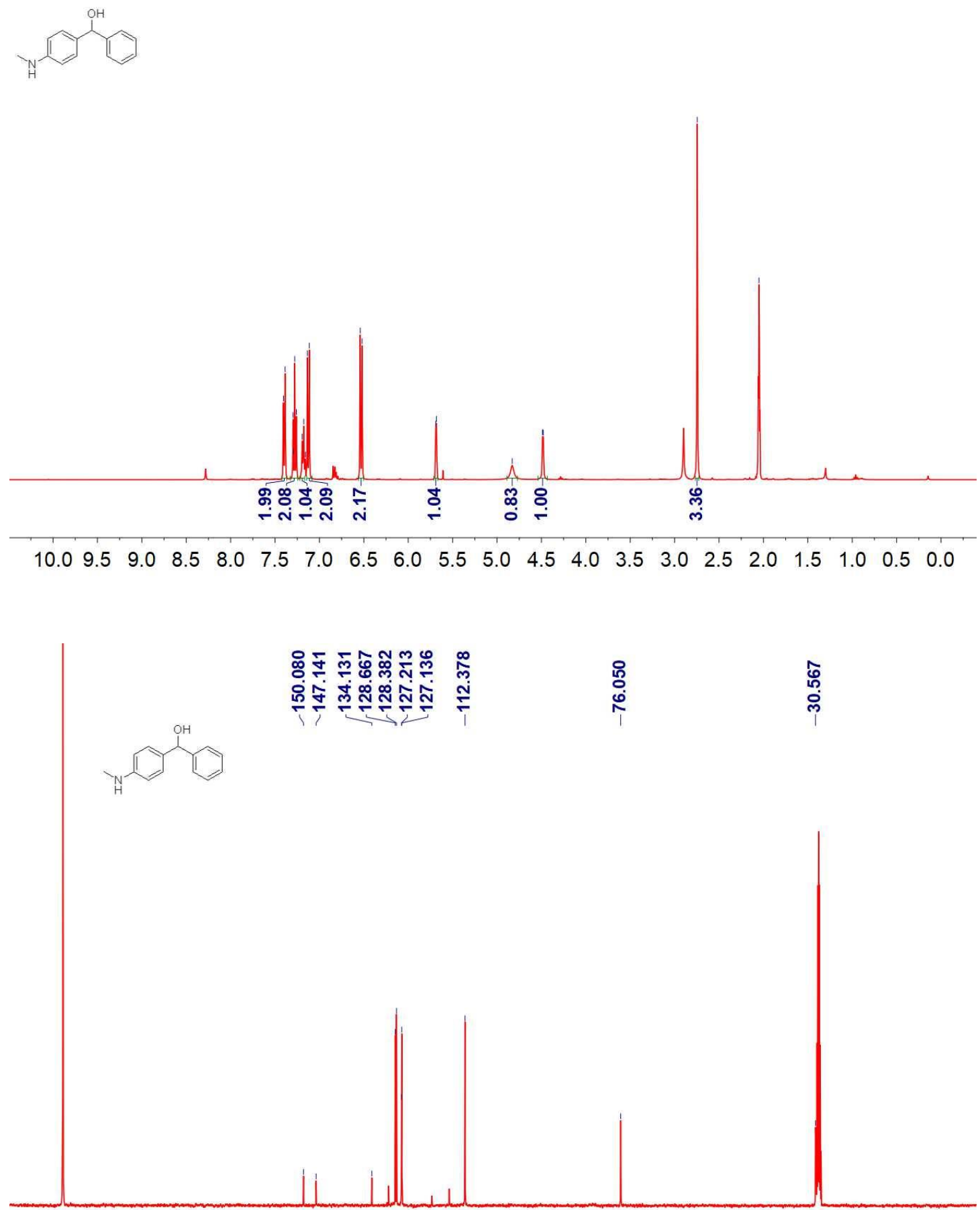

$210200190180170160150140130120110100 \quad 90 \quad 80 \quad 70 \quad 60 \quad 50 \quad 40 \quad 30 \quad 20 \quad 10 \quad 0$ 
$N$-(4-(hydroxy(phenyl)methyl)phenyl)acetamide (1b-9)

象

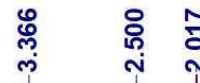
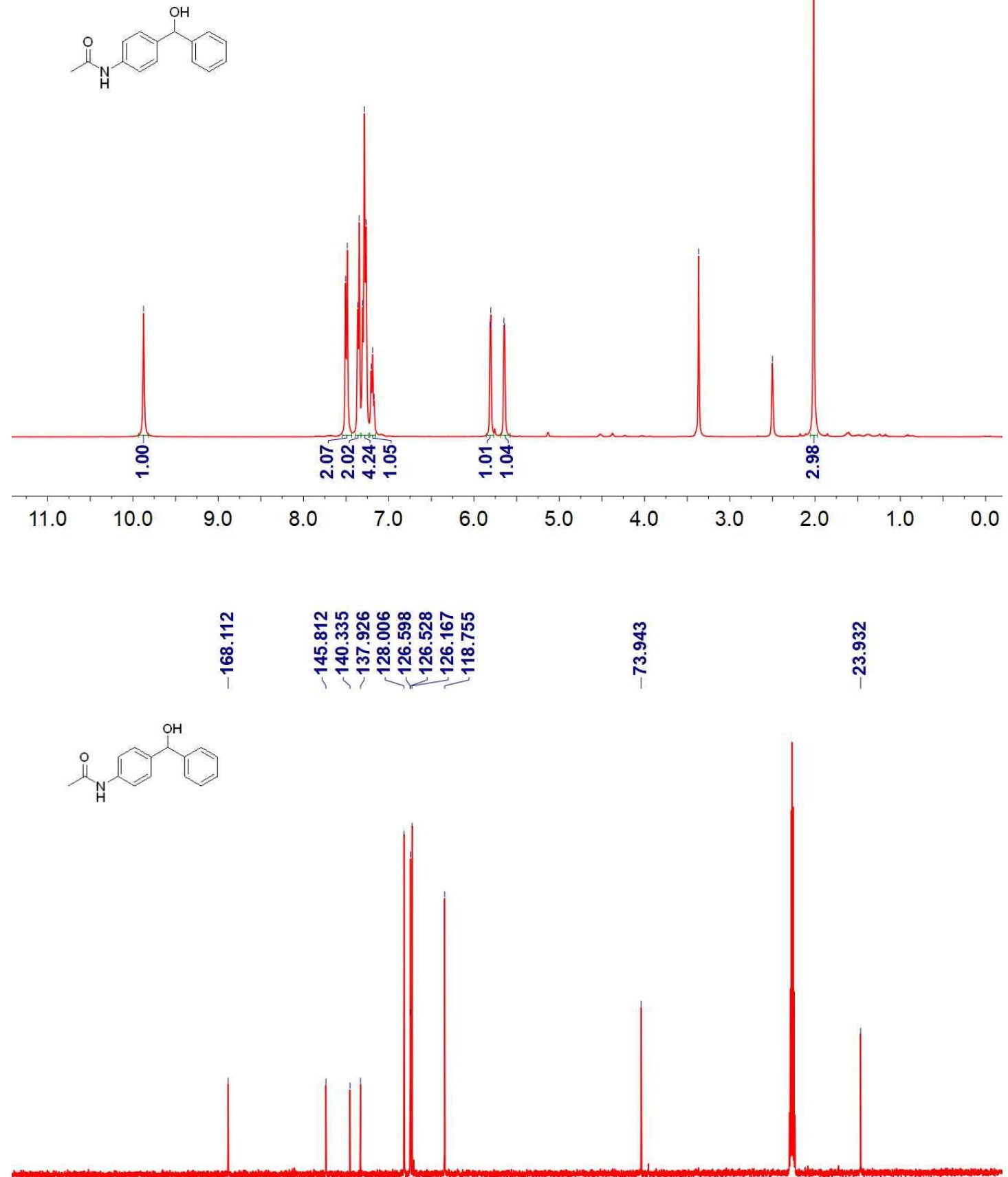

$210200190180170160150140130120110 \quad 100 \quad 90 \quad 80 \quad 70 \quad 60 \quad 50 \quad 40 \quad 30 \quad 20 \quad 10 \quad 0$ 
Cyclohexyl(4-(dimethylamino)phenyl)methanol (1g)

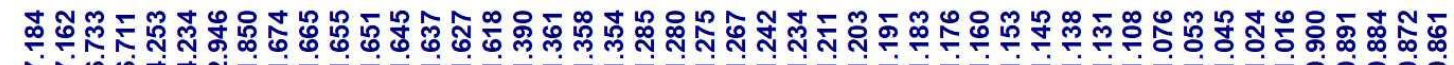
rí
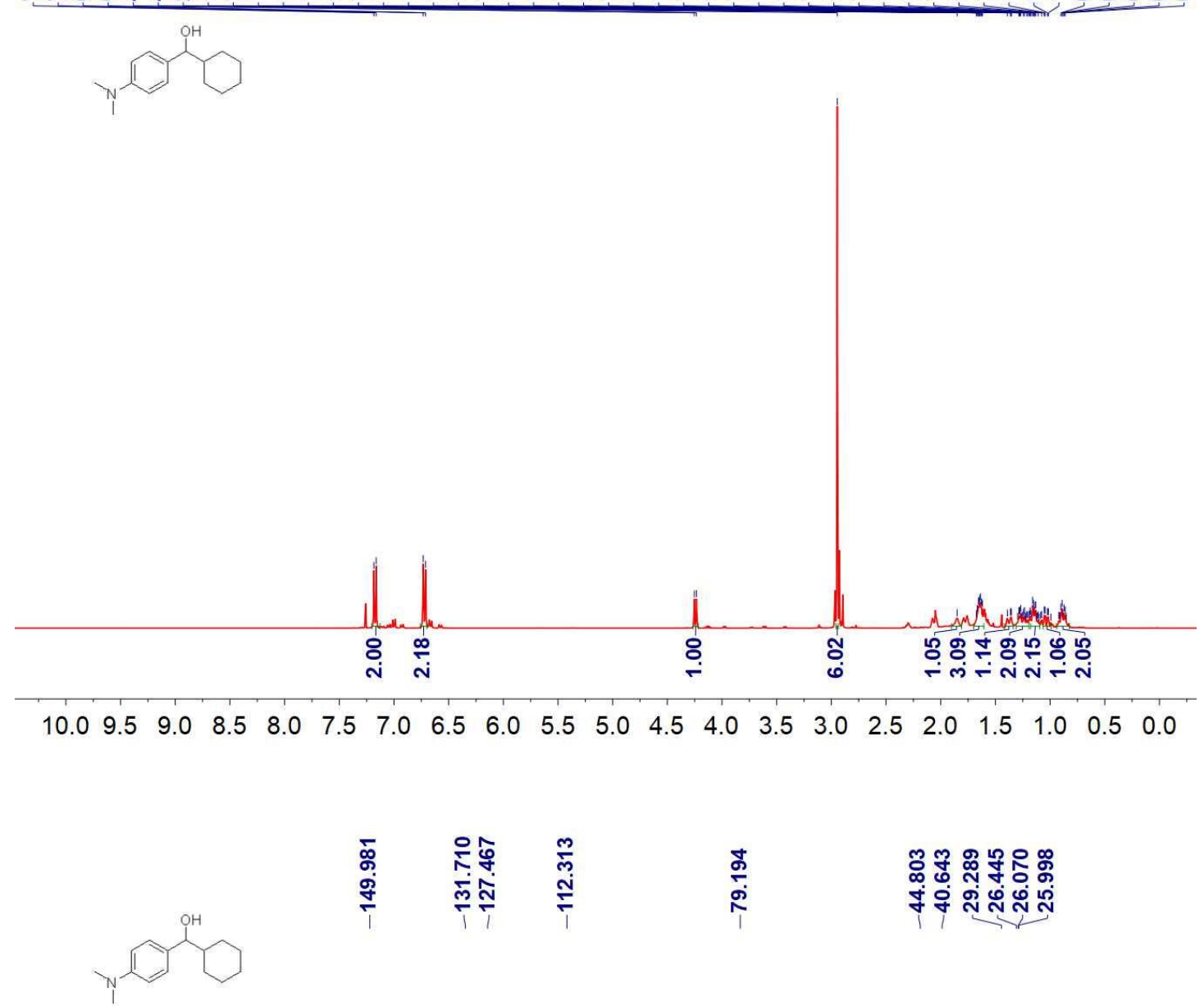

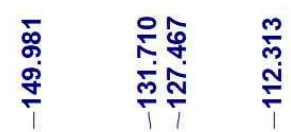

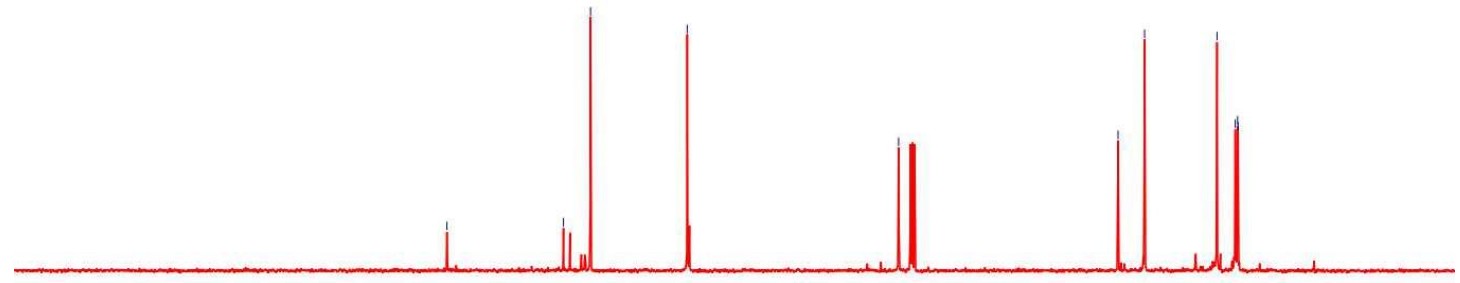

$210200190180170160150140130120110100 \quad 90 \quad 80 \quad 70 \quad 60 \quad 50 \quad 40 \quad 30 \quad 20 \quad 10 \quad 0$ 
1-(4-(Diethylamino)phenyl)hexan-1-ol (1h)
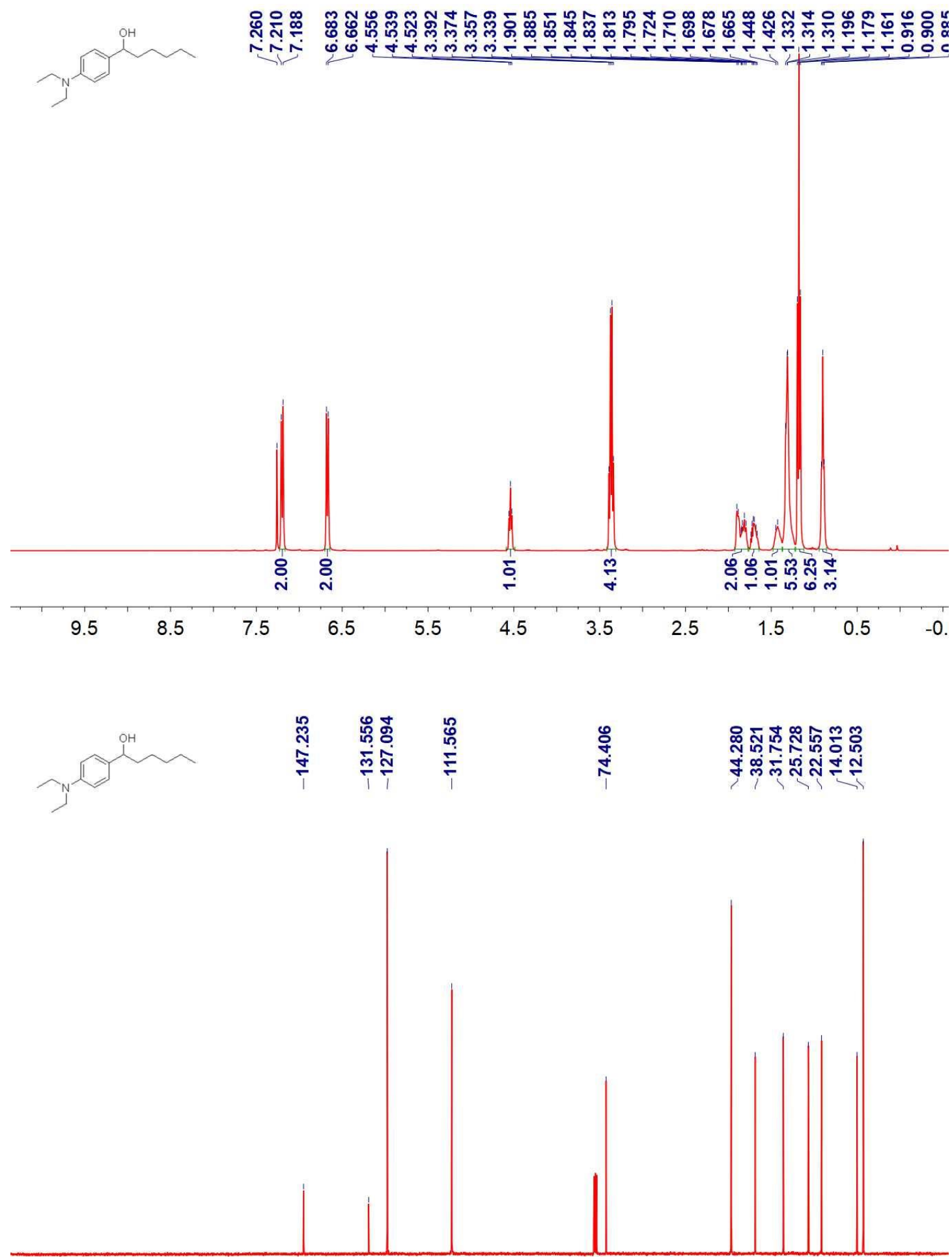

$210200190180170160150140130120110100 \quad 90 \quad 80 \quad 70 \quad 60 \quad 50 \quad 40 \quad 30 \quad 20 \quad 10 \quad 0$ 
1-(4-(Dimethylamino)phenyl)octan-1-ol (1i)

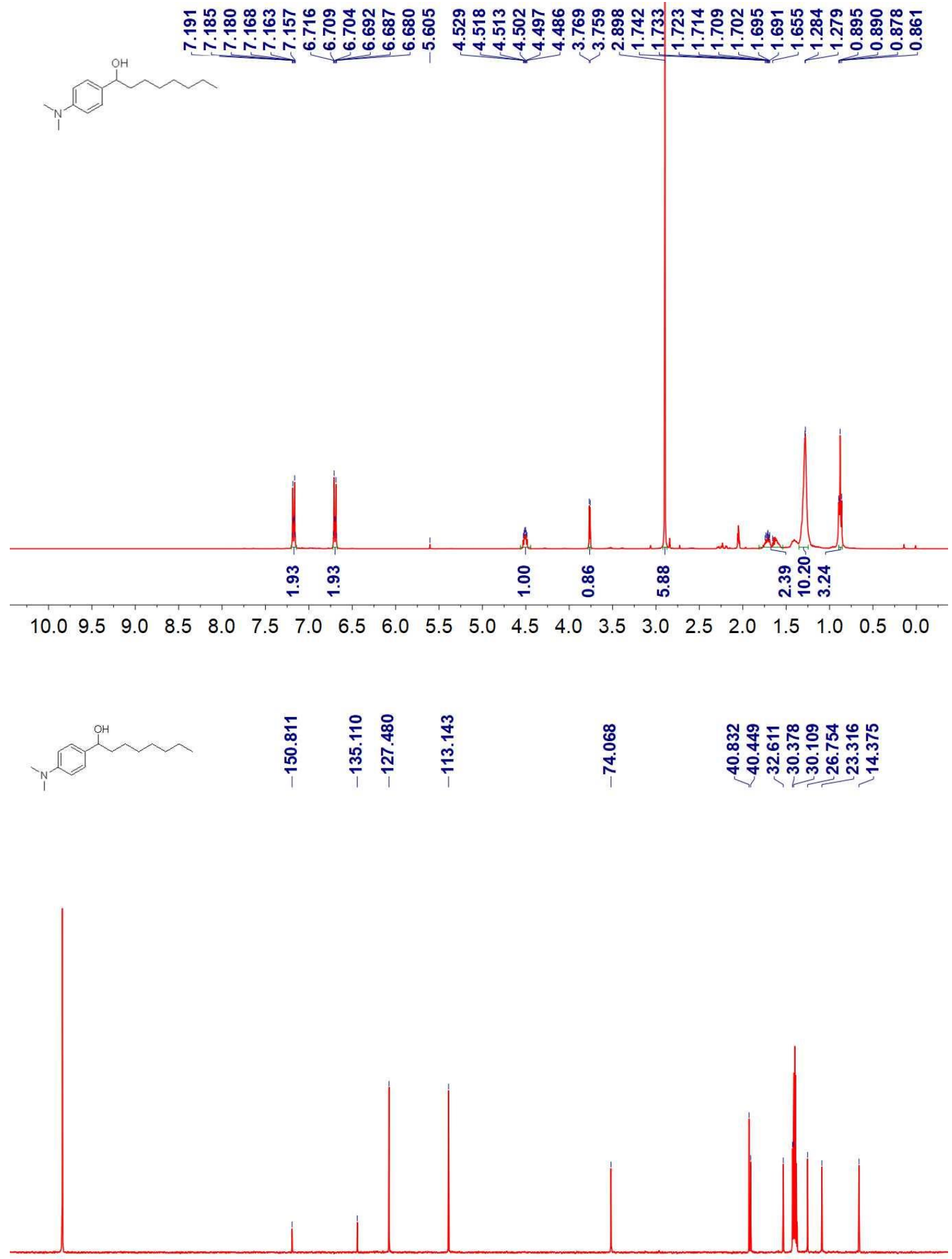

$210200190180170160150140130120110 \quad 100 \quad 90 \quad 80 \quad 70 \quad 60 \quad 50 \quad 40 \quad 30 \quad 20 \quad 10 \quad 0$ 
1-(4-(Dimethylamino)phenyl)-3-phenylpropan-1-ol (1j)

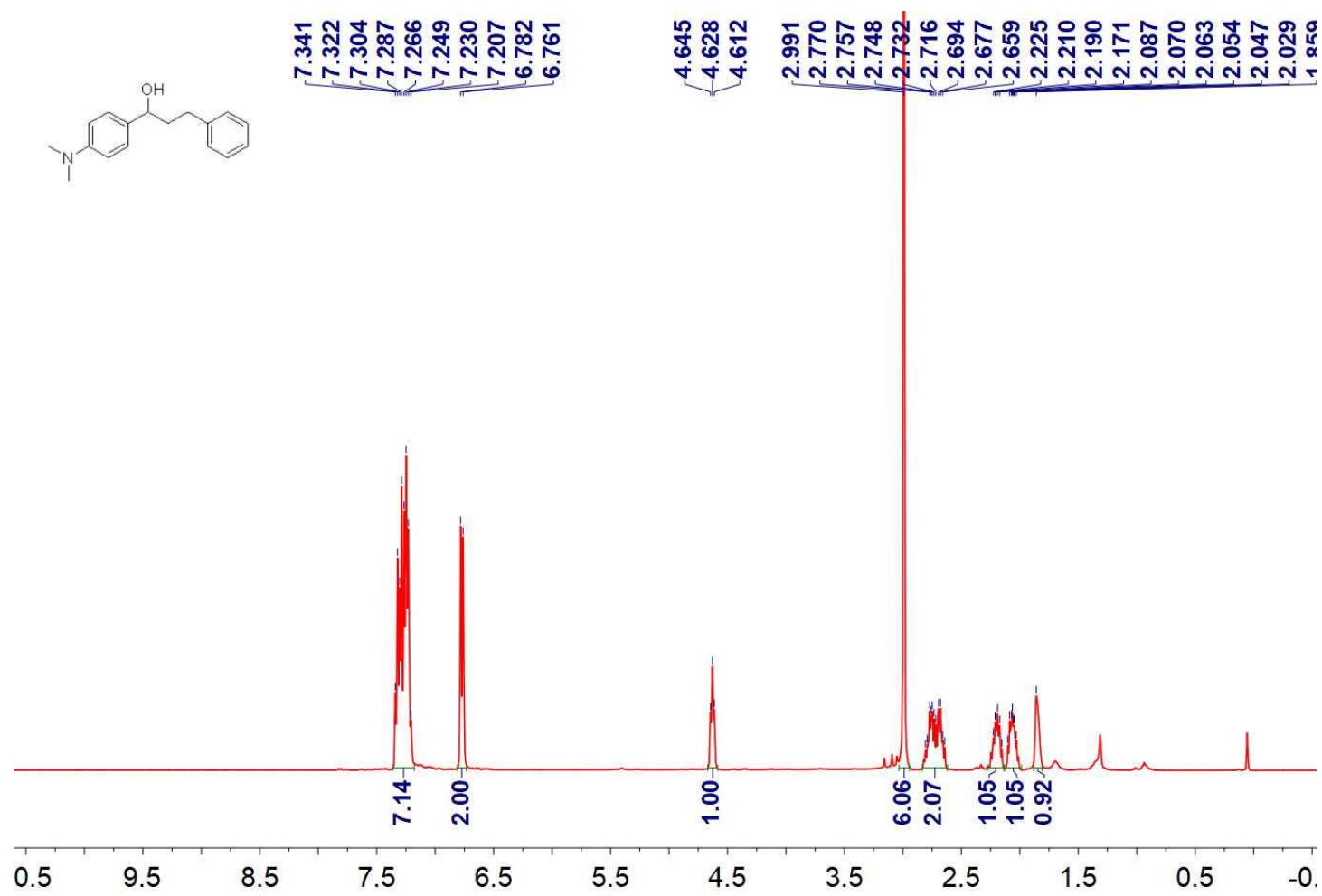

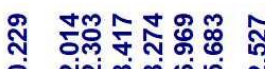

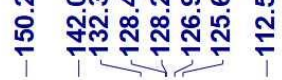

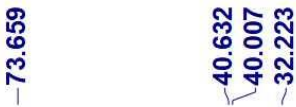
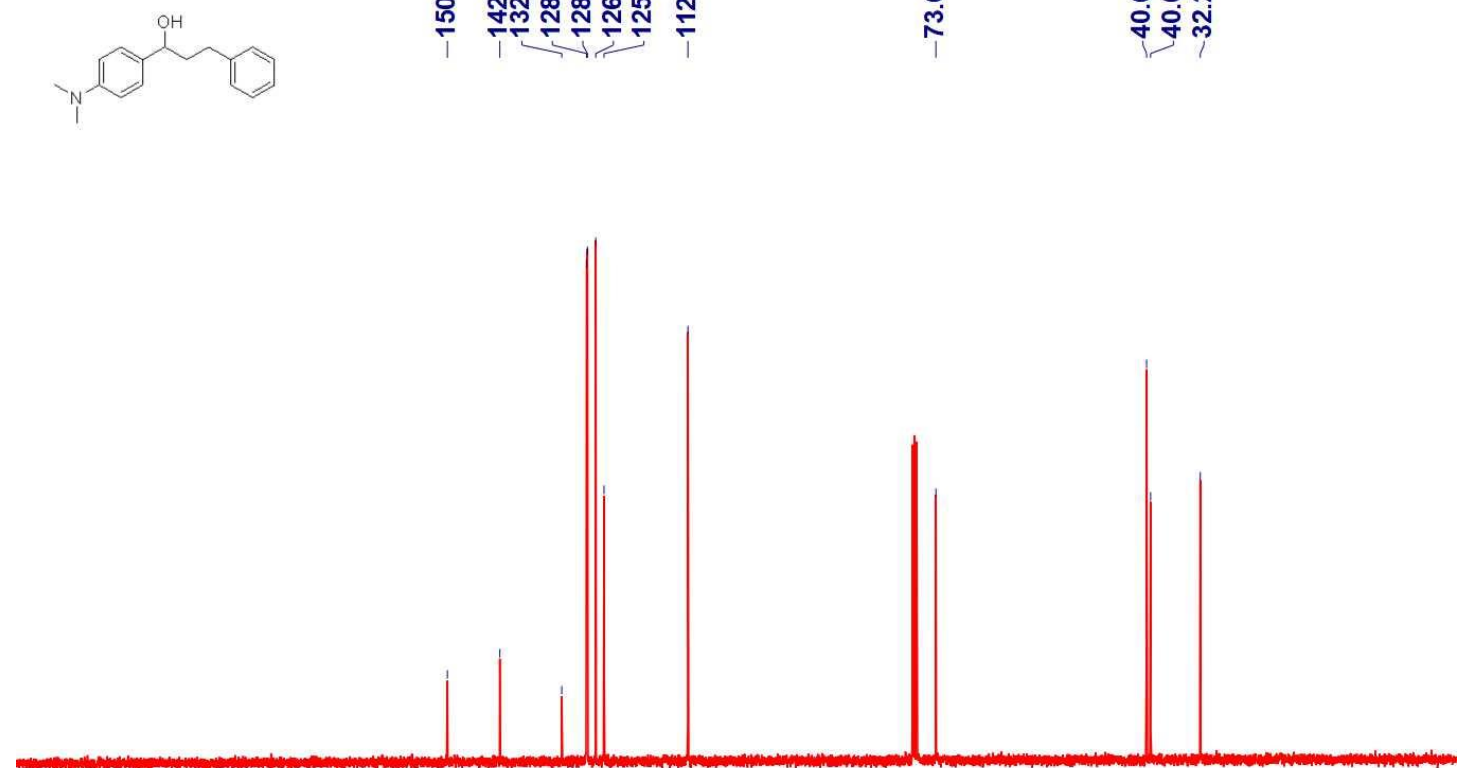

$210200190180170160150140130120110100 \quad 90 \quad 80 \quad 70 \quad 60 \quad 50 \quad 40 \quad 30 \quad 20 \quad 10 \quad 0$ 
1-(4-(Dimethylamino)phenyl)-3,7-dimethyloct-6-en-1-ol (1k)
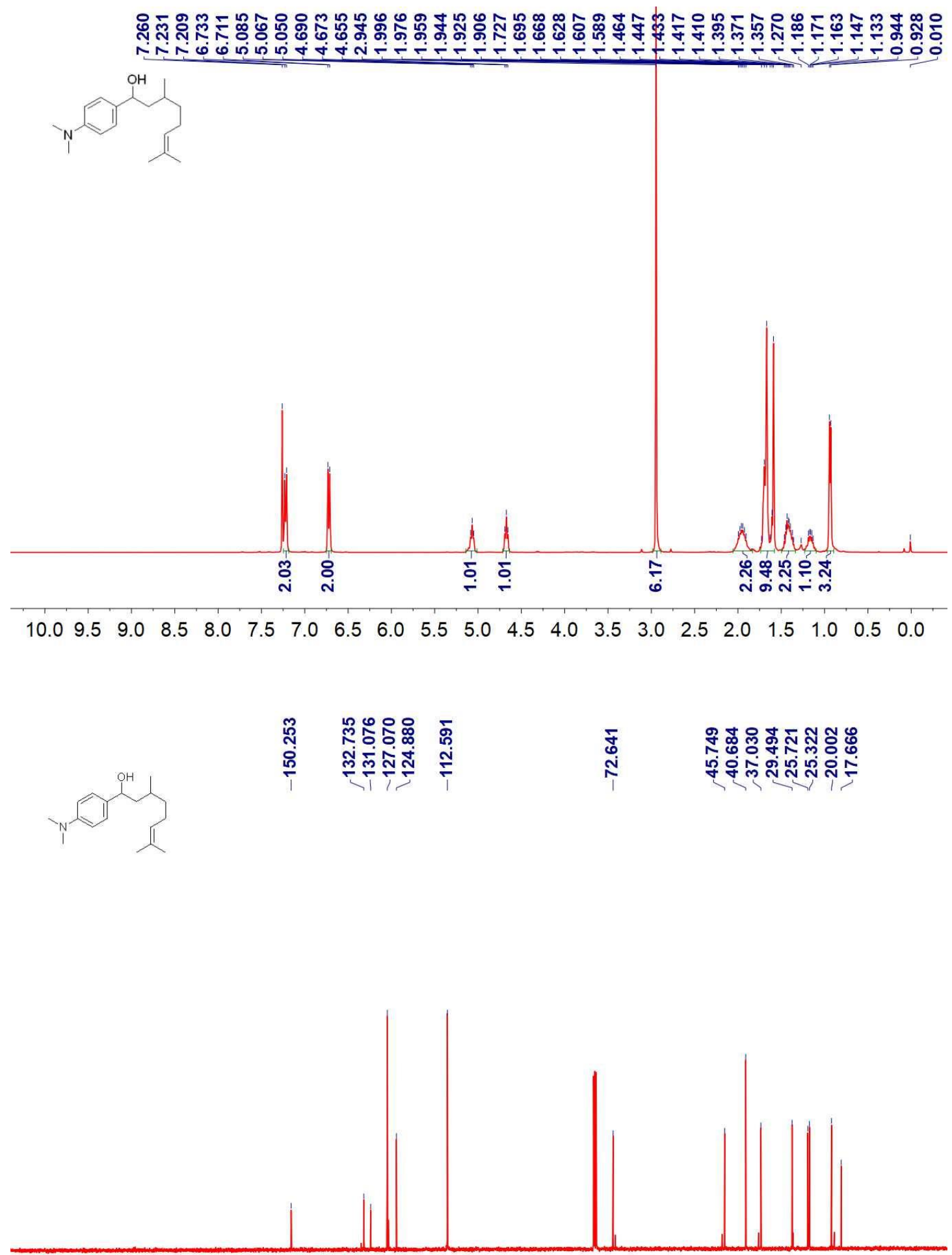

$210200190180170160150140130120110100 \quad 90 \quad 80 \quad 70 \quad 60 \quad 50 \quad 40 \quad 30 \quad 20 \quad 10 \quad 0$ 

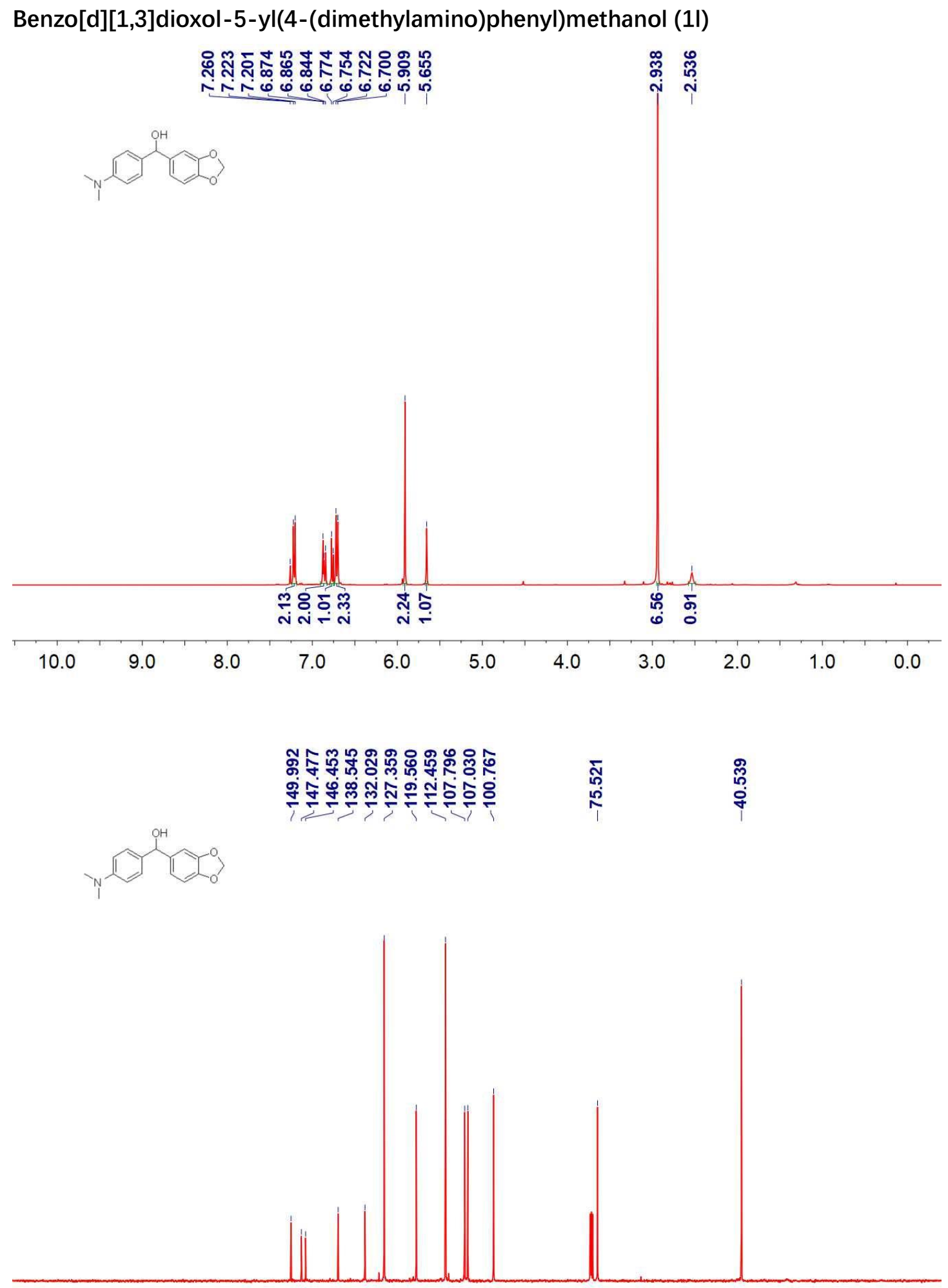

$210200190180170160150140130120110 \quad 100 \quad 90 \quad 80 \quad 70 \quad 60 \quad 50 \quad 40 \quad 30 \quad 20 \quad 10 \quad 0$ 
(4-(Butyl(methyl)amino)phenyl)(4-(dimethylamino)phenyl)methanol (1m)

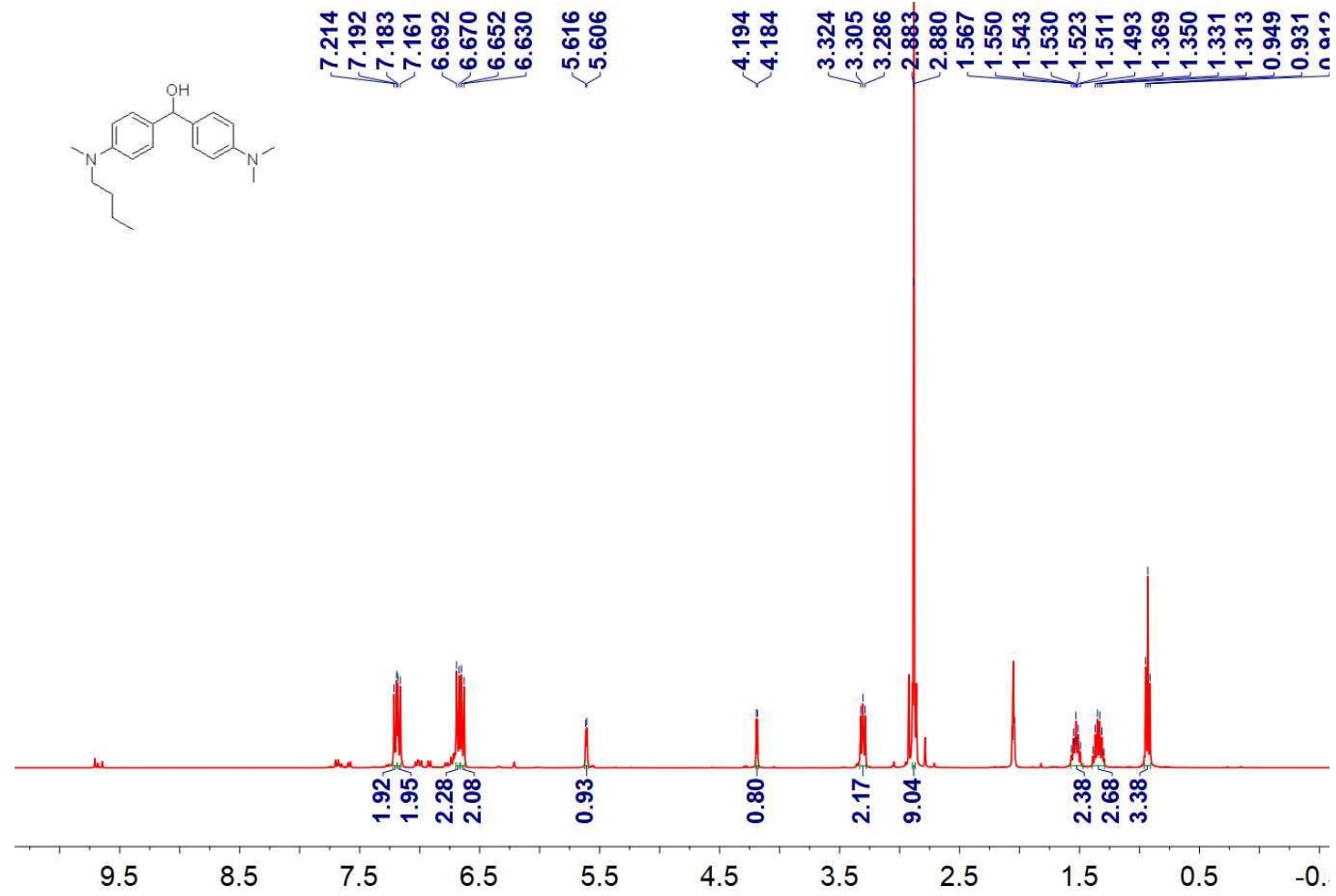

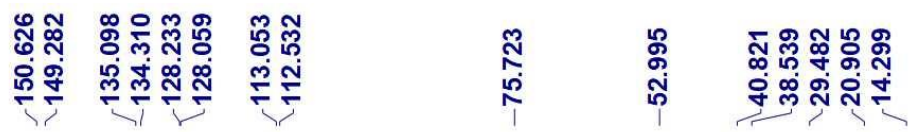
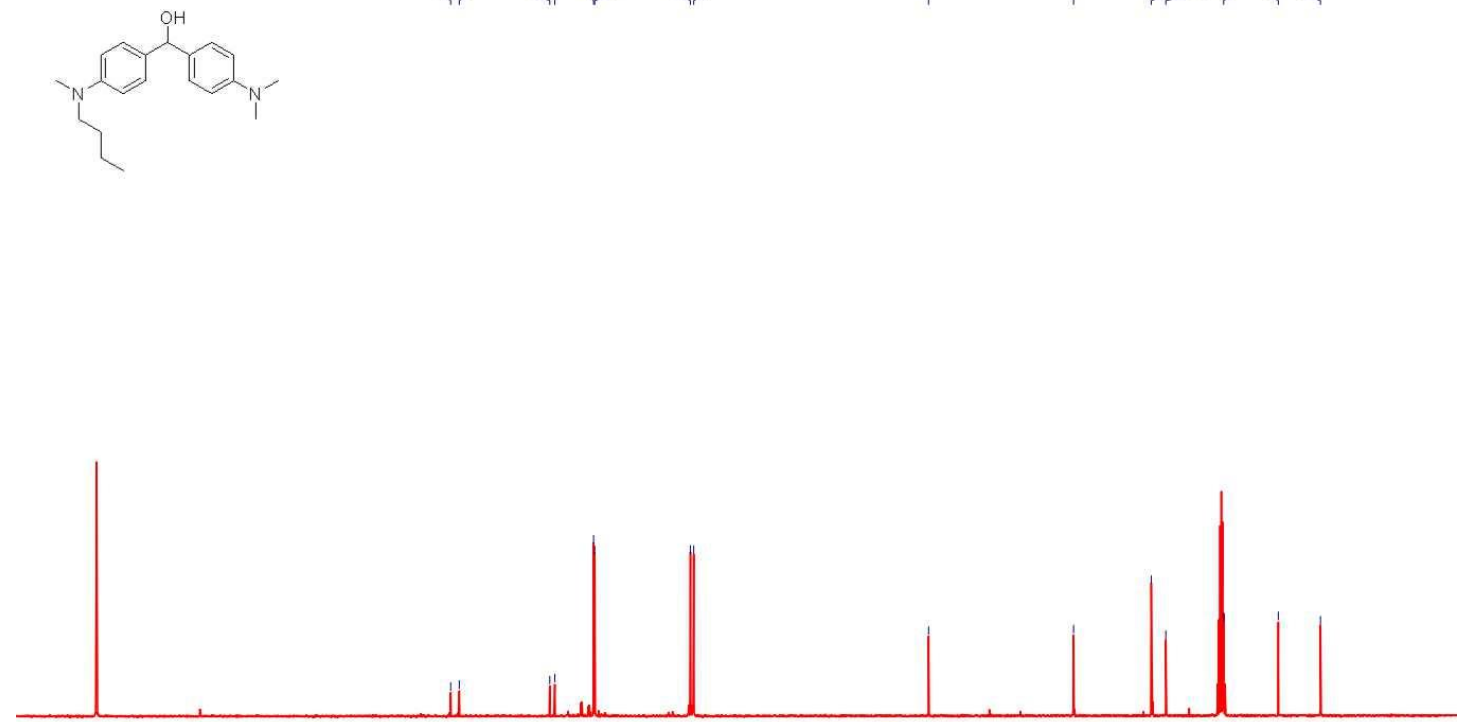

$210200190180170160150140130120110 \quad 100 \quad 90 \quad 80 \quad 70 \quad 60 \quad 50 \quad 40 \quad 30 \quad 20 \quad 10 \quad 0$ 
(4-(Dimethylamino)phenyl)(mesityl)methanol (1n)

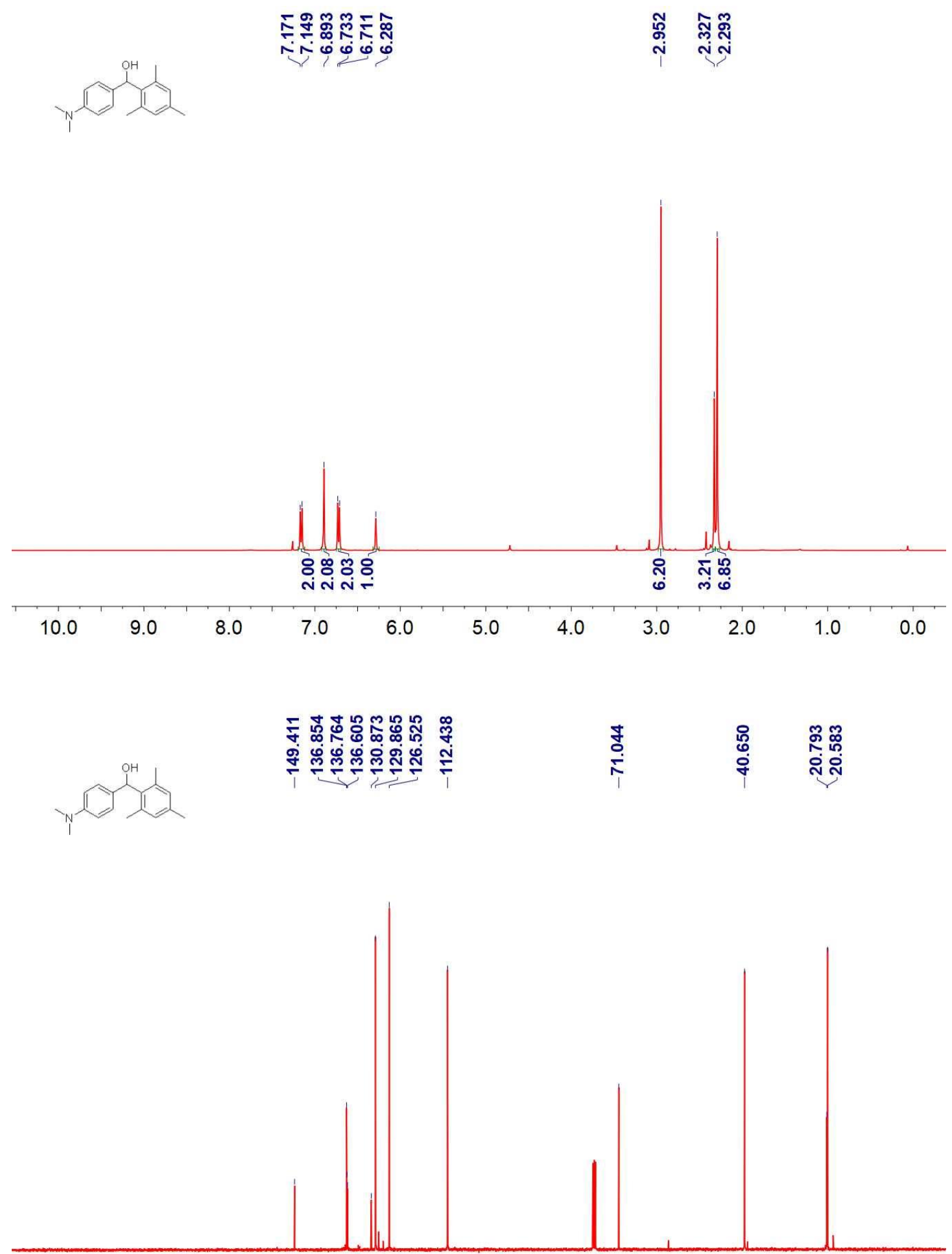

$210200190180170160150140130120110 \quad 100 \quad 90 \quad 80 \quad 70 \quad 60 \quad 50 \quad 40 \quad 30 \quad 20 \quad 10 \quad 0$ 
(4-(Dimethylamino)phenyl)(4-(methylthio)phenyl)methanol (10)

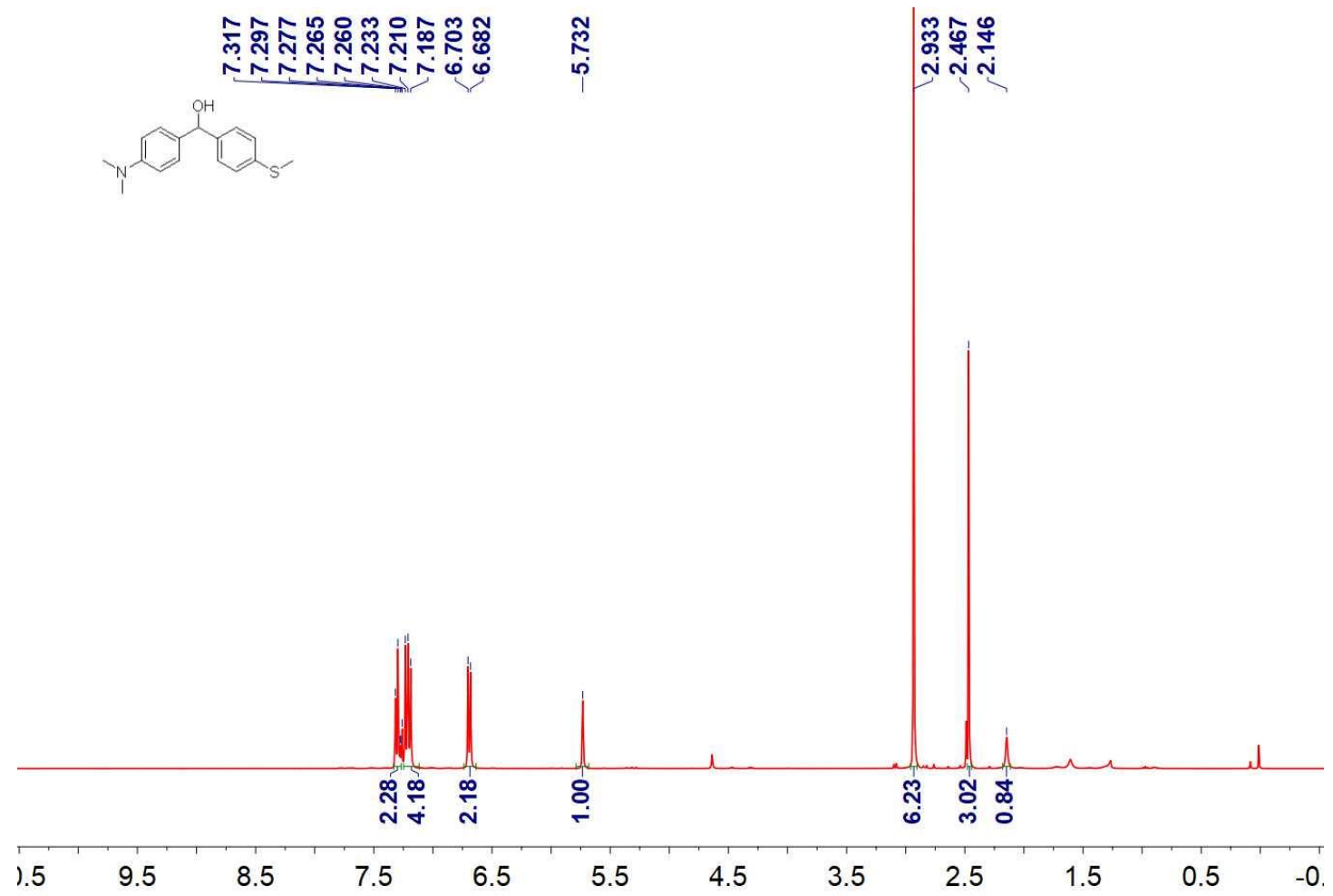

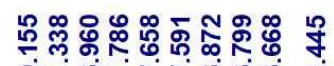

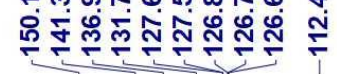

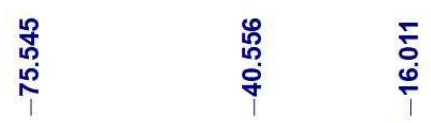

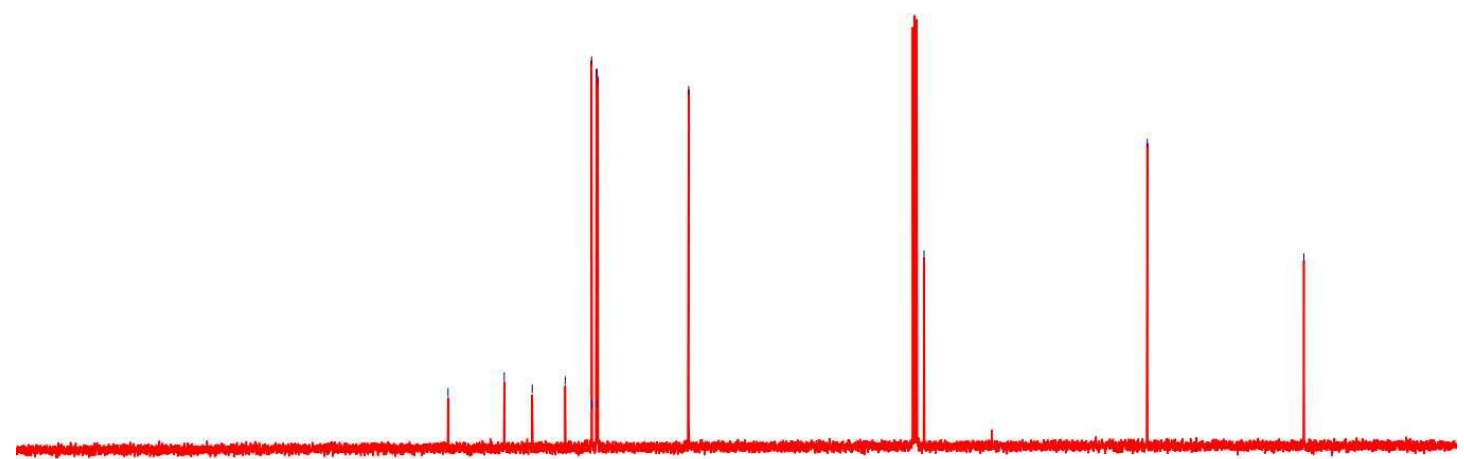

$210200190180170160150140130120110100 \quad 90 \quad 80 \quad 70 \quad 60 \quad 50 \quad 40 \quad 30 \quad 20 \quad 10 \quad 0$ 


\section{(4-(dimethylamino)phenyl)(4-methoxyphenyl)methanol (1p)}

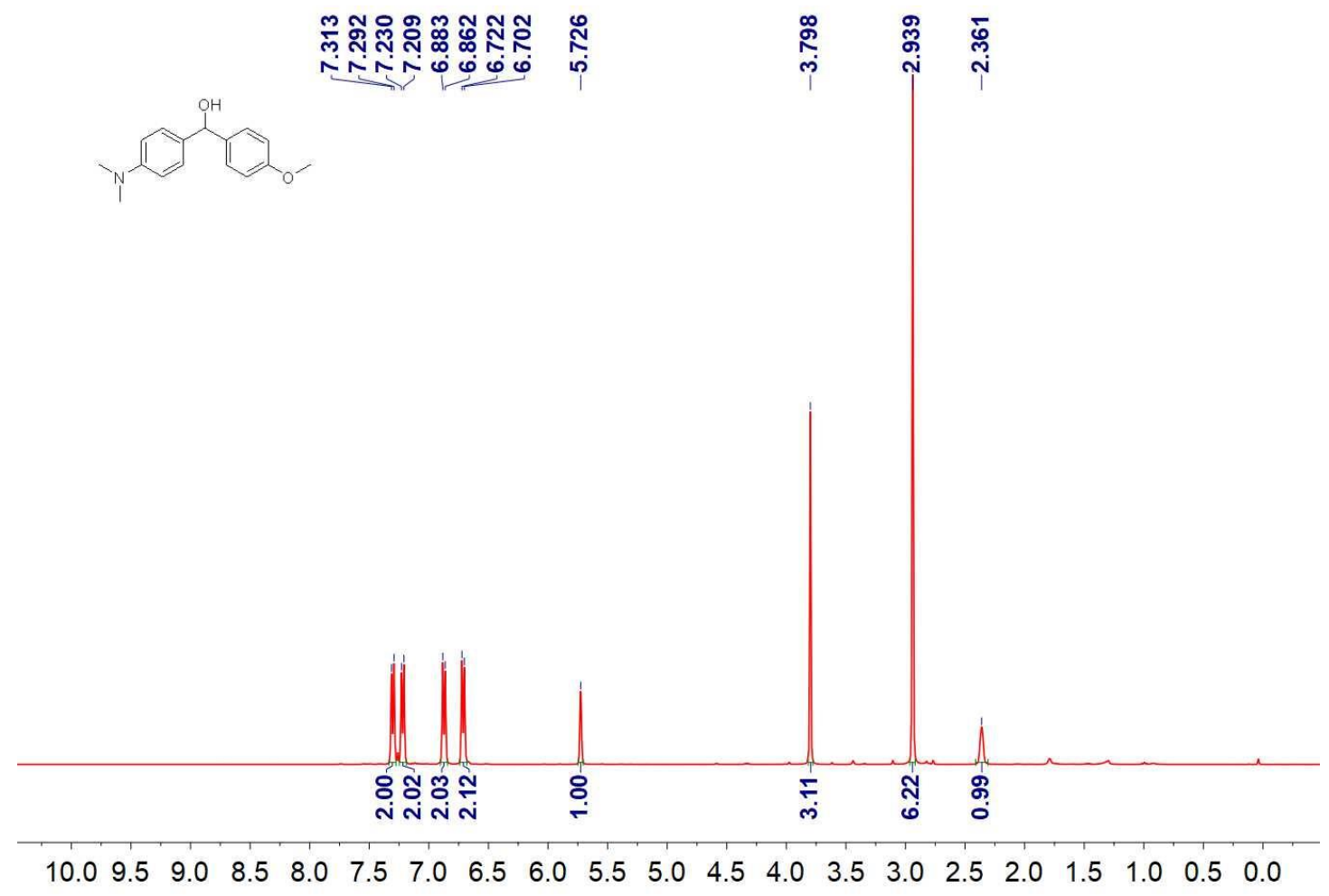

\%ัم

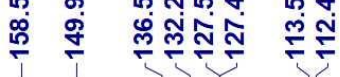

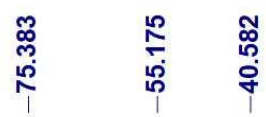

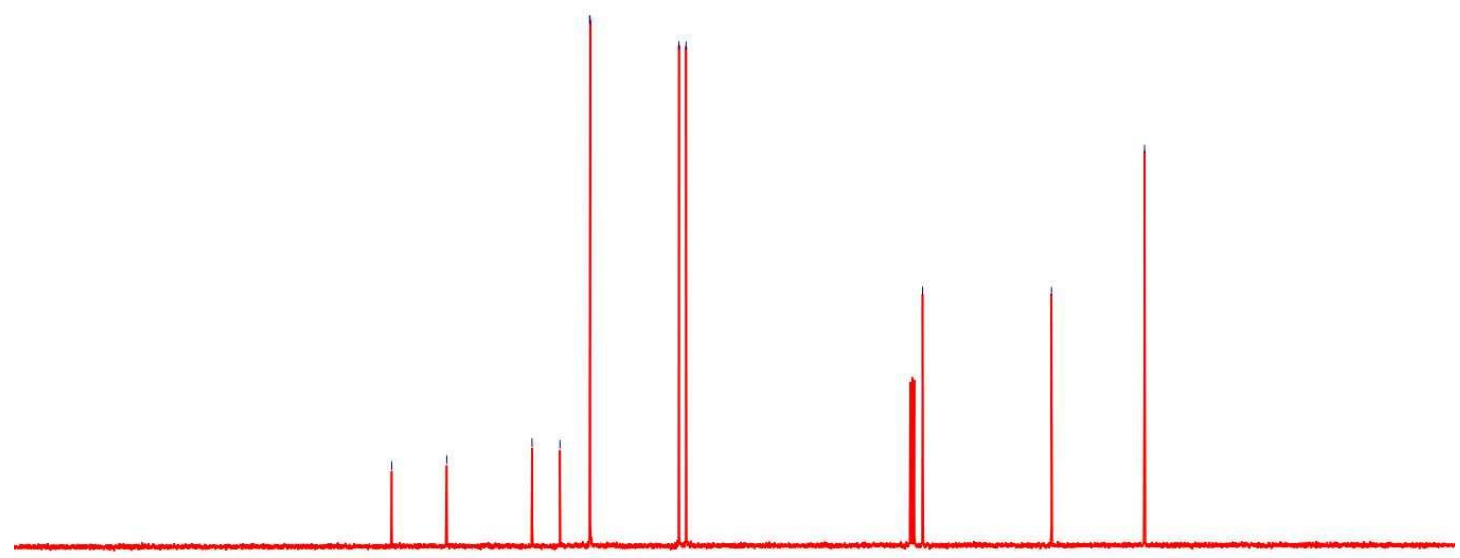

$210200190180170160150140130120110100 \quad 90 \quad 80 \quad 70 \quad 60 \quad 50 \quad 40 \quad 30 \quad 20 \quad 10 \quad 0$ 


\section{(4-(Dimethylamino)phenyl)(4-tolyl)methanol (1q)}
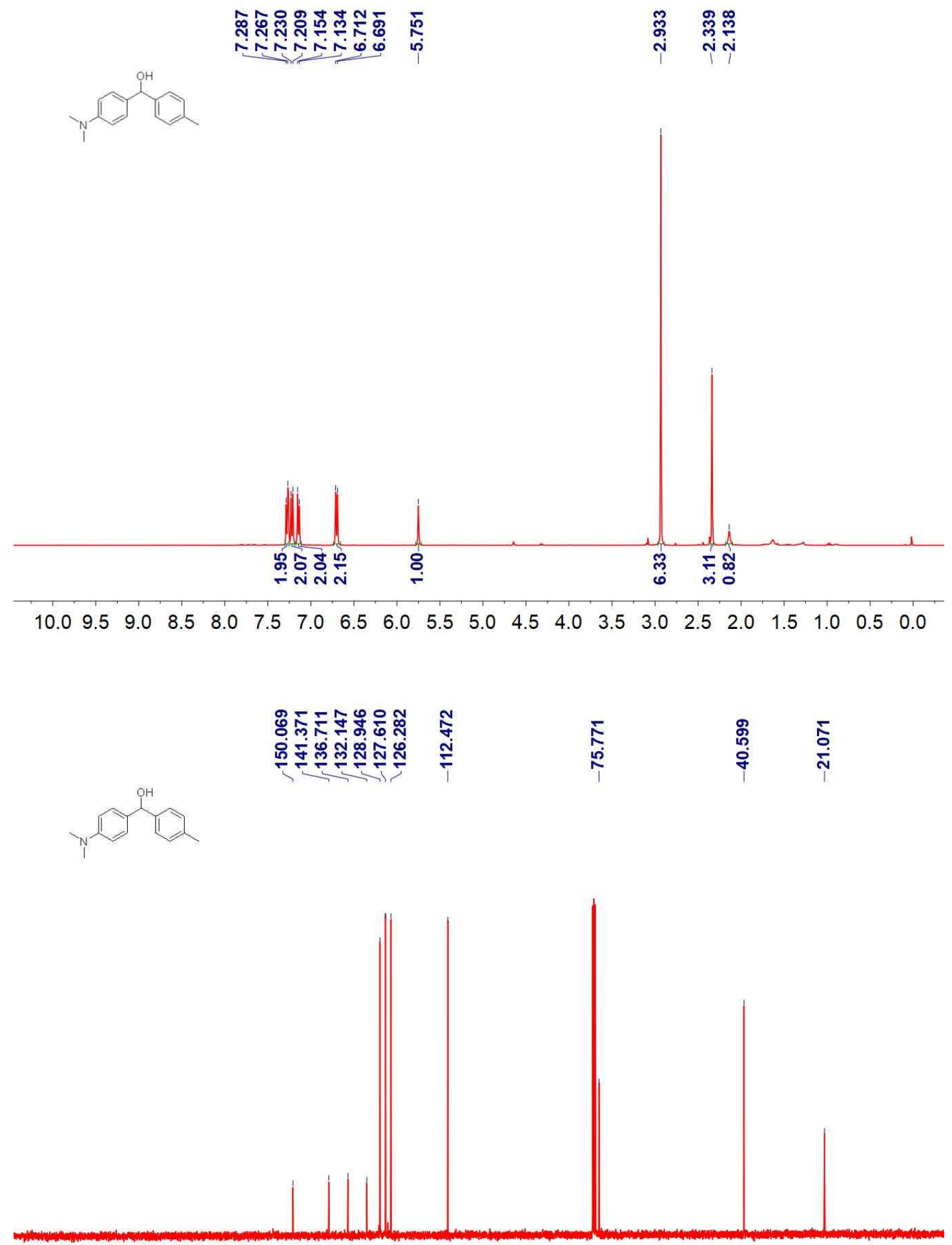

$210200190180170160150140130120110100 \quad 90 \quad 80 \quad 70 \quad 60 \quad 50 \quad 40 \quad 30 \quad 20 \quad 10 \quad 0$ 
(4-(Dimethylamino)phenyl)(3-tolyl)methanol (1r)

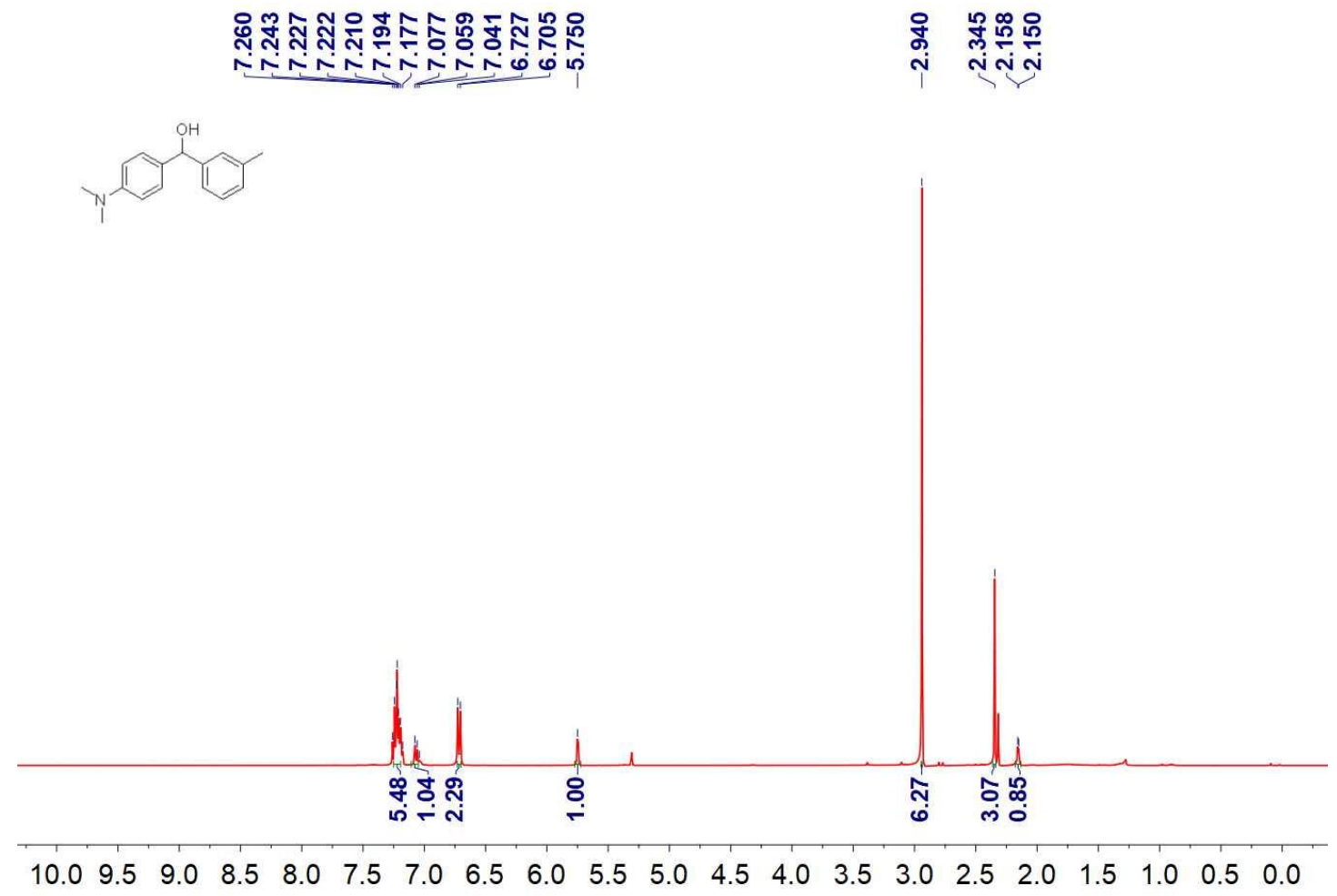

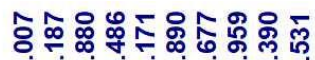

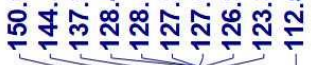

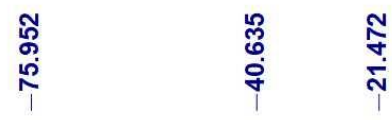

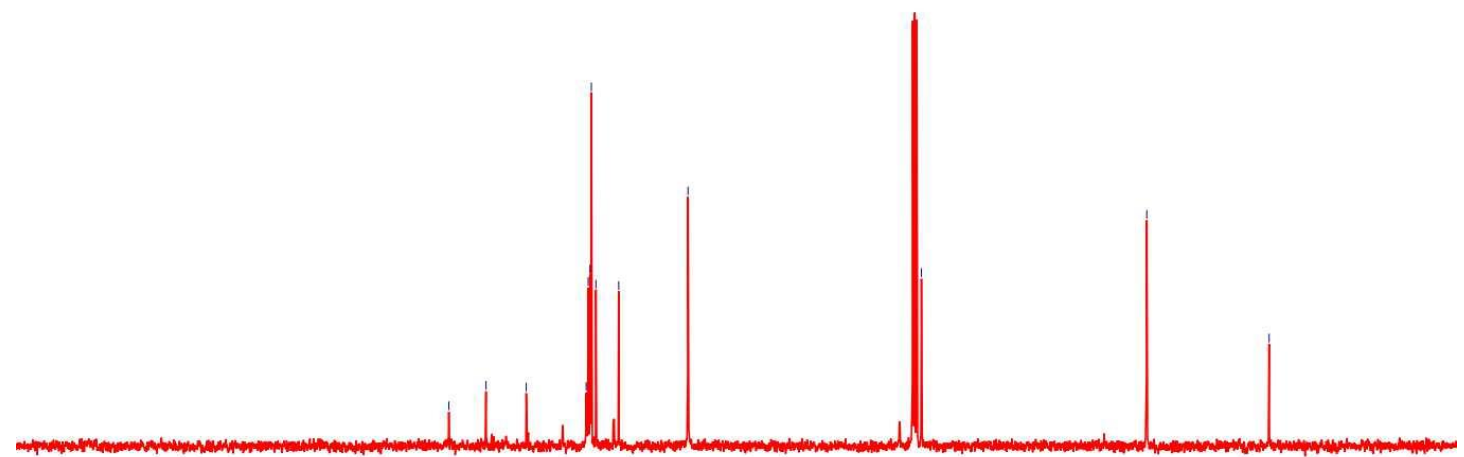

$210200190180170160150140130120110100 \quad 90 \quad 80 \quad 70 \quad 60 \quad 50 \quad 40 \quad 30 \quad 20 \quad 10 \quad 0$ 
(4-(Dimethylamino)phenyl)(2-tolyl)methanol (1s)

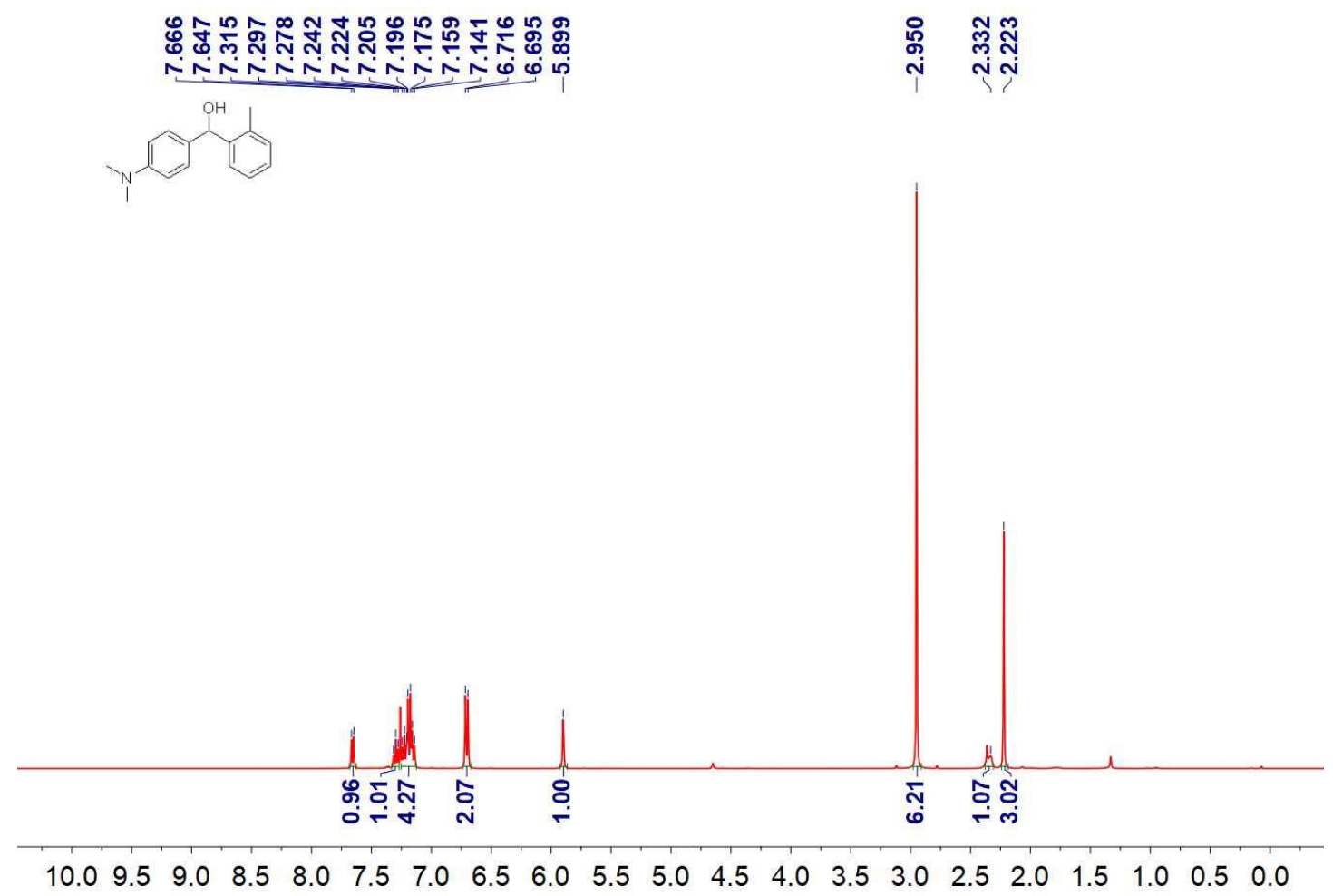

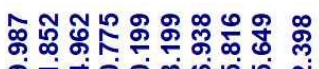

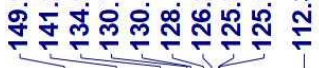

กั

昂

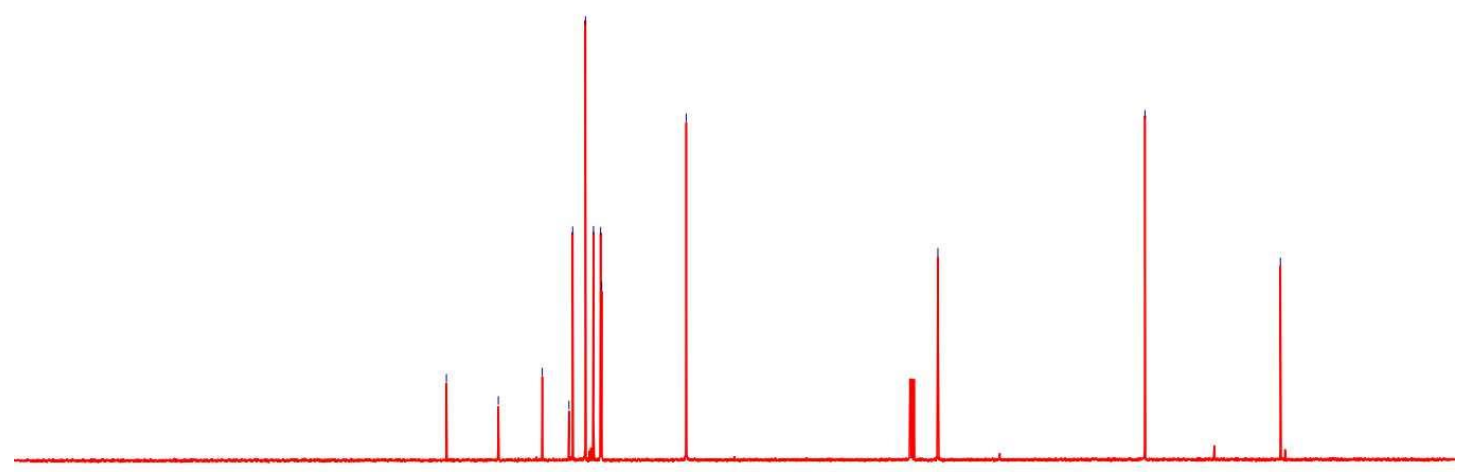

$210200190180170160150140130120110100 \quad 90 \quad 80 \quad 70 \quad 60 \quad 50 \quad 40 \quad 30 \quad 20 \quad 10 \quad 0$ 
(4-(Dimethylamino)phenyl)(4-isopropylphenyl)methanol (1t)

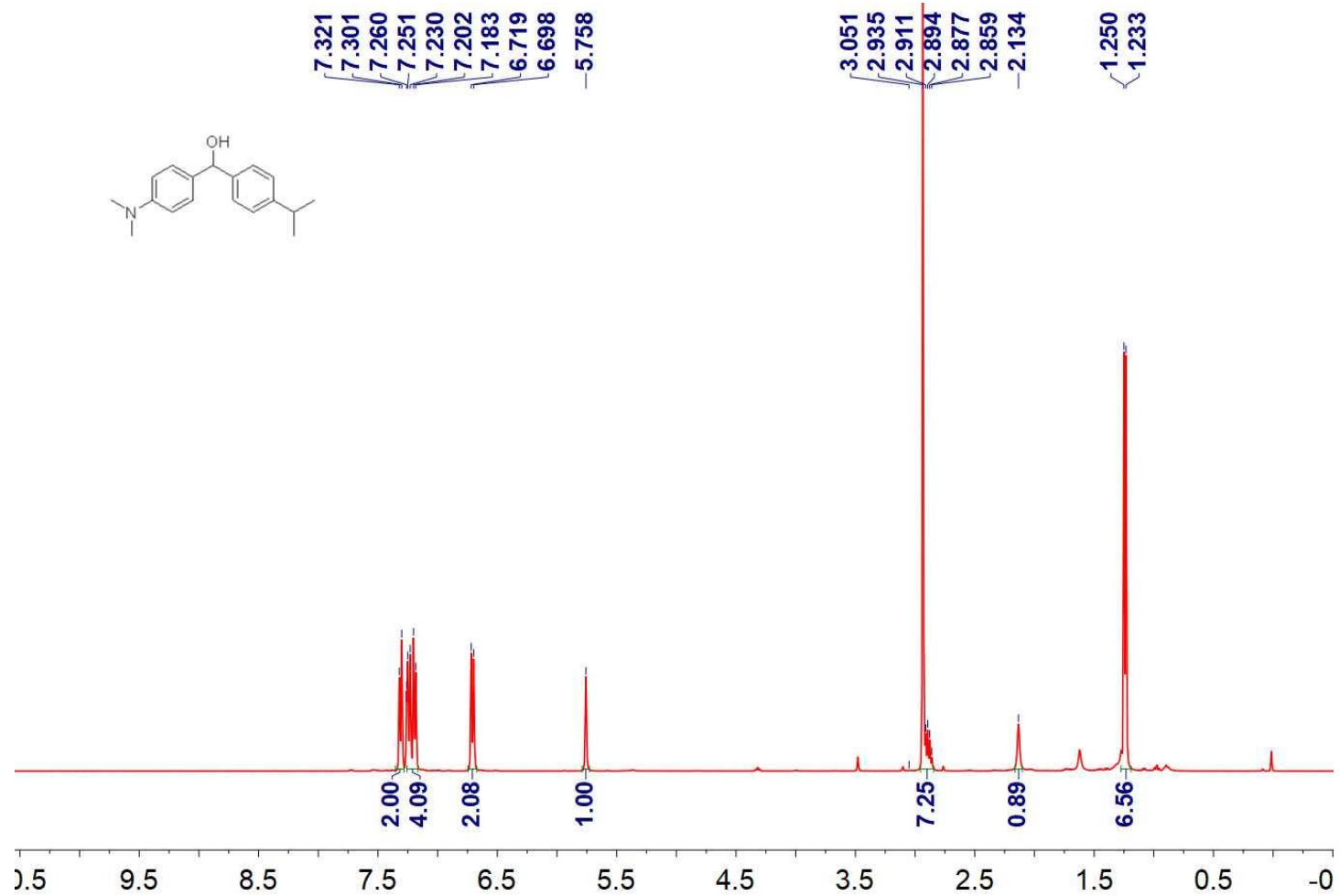

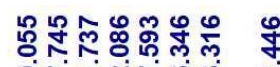

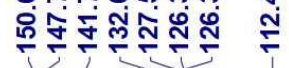

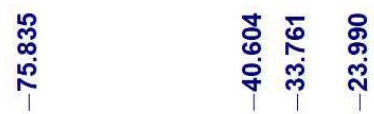
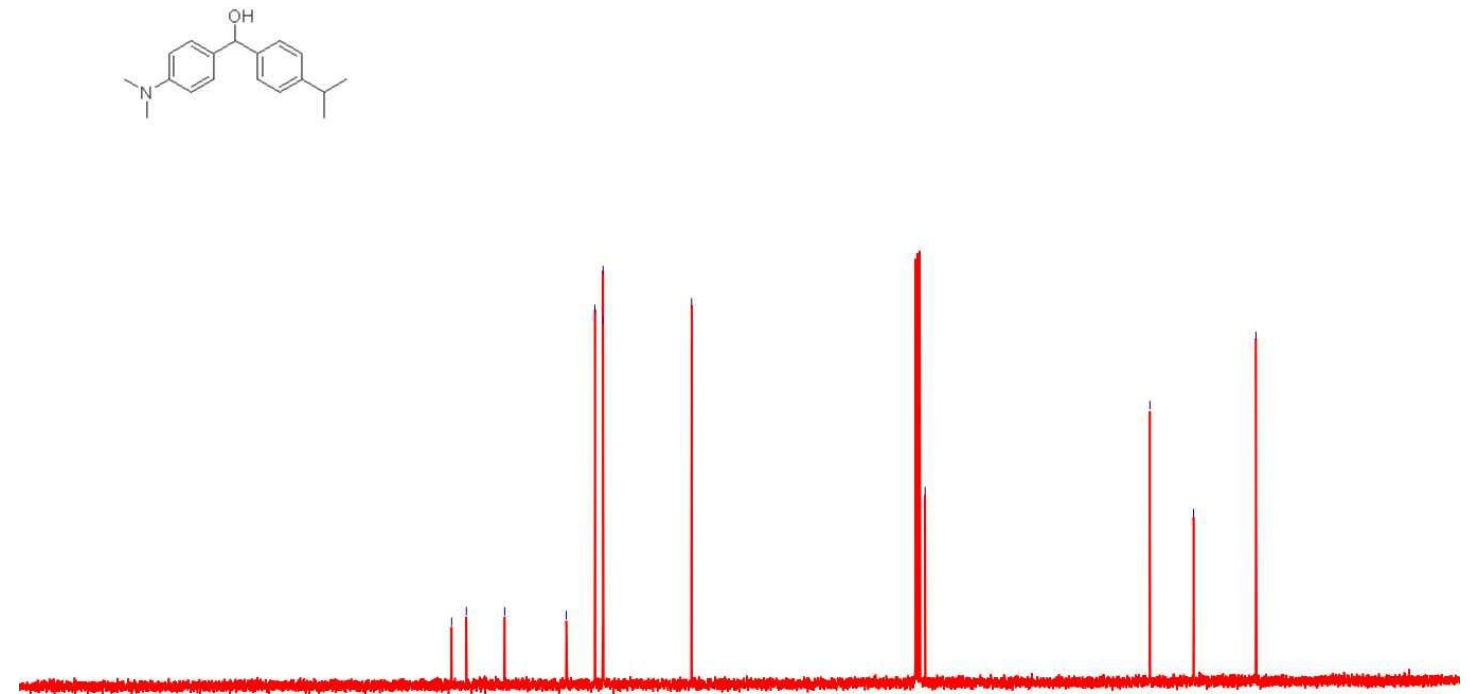

$210200190180170160150140130120110100 \quad 90 \quad 80 \quad 70 \quad 60 \quad 50 \quad 40 \quad 30 \quad 20 \quad 10 \quad 0$ 


\section{[1,1'-Biphenyl]-4-yl(4-(dimethylamino)phenyl)methanol (1u)}

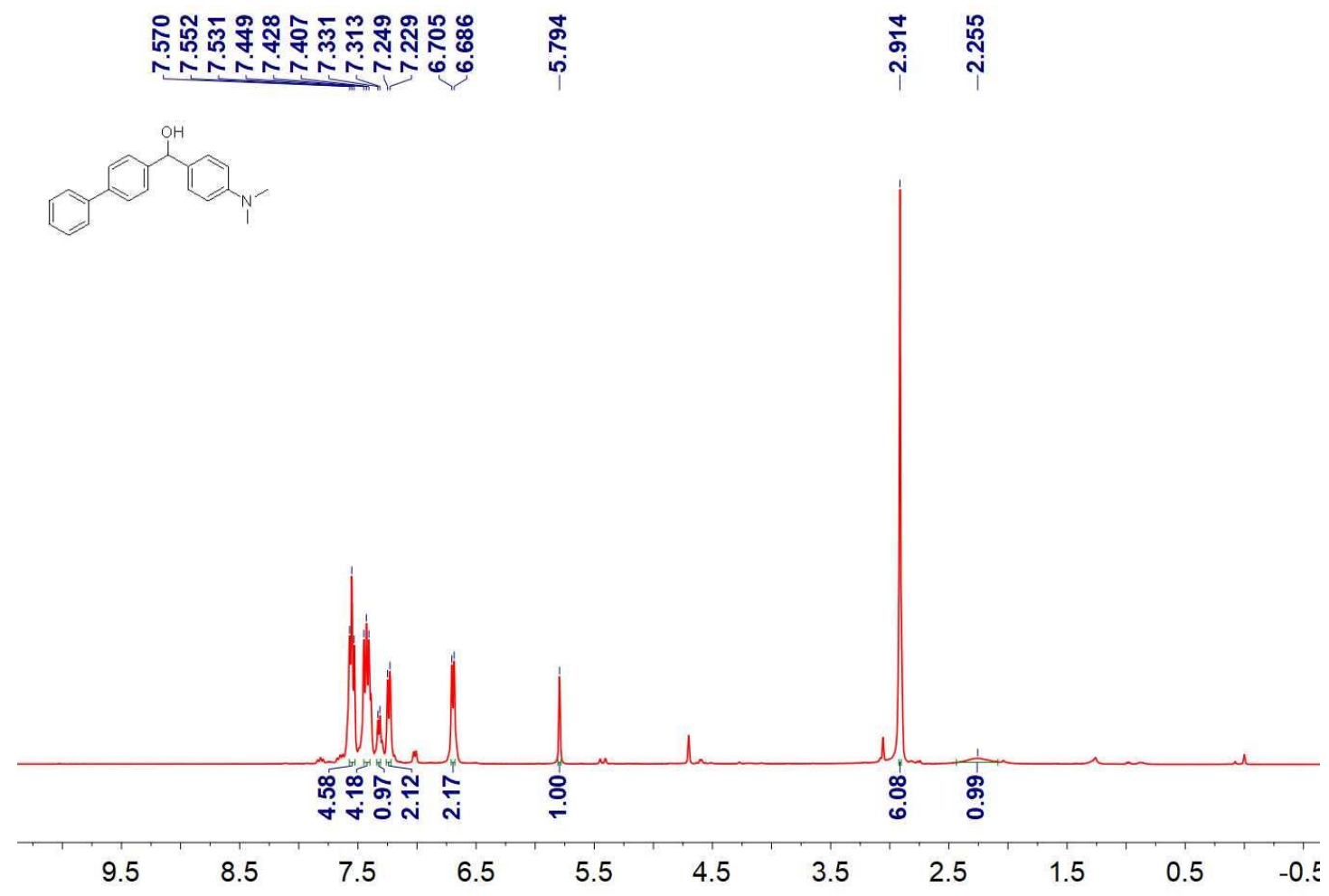

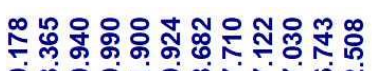

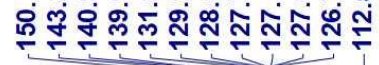
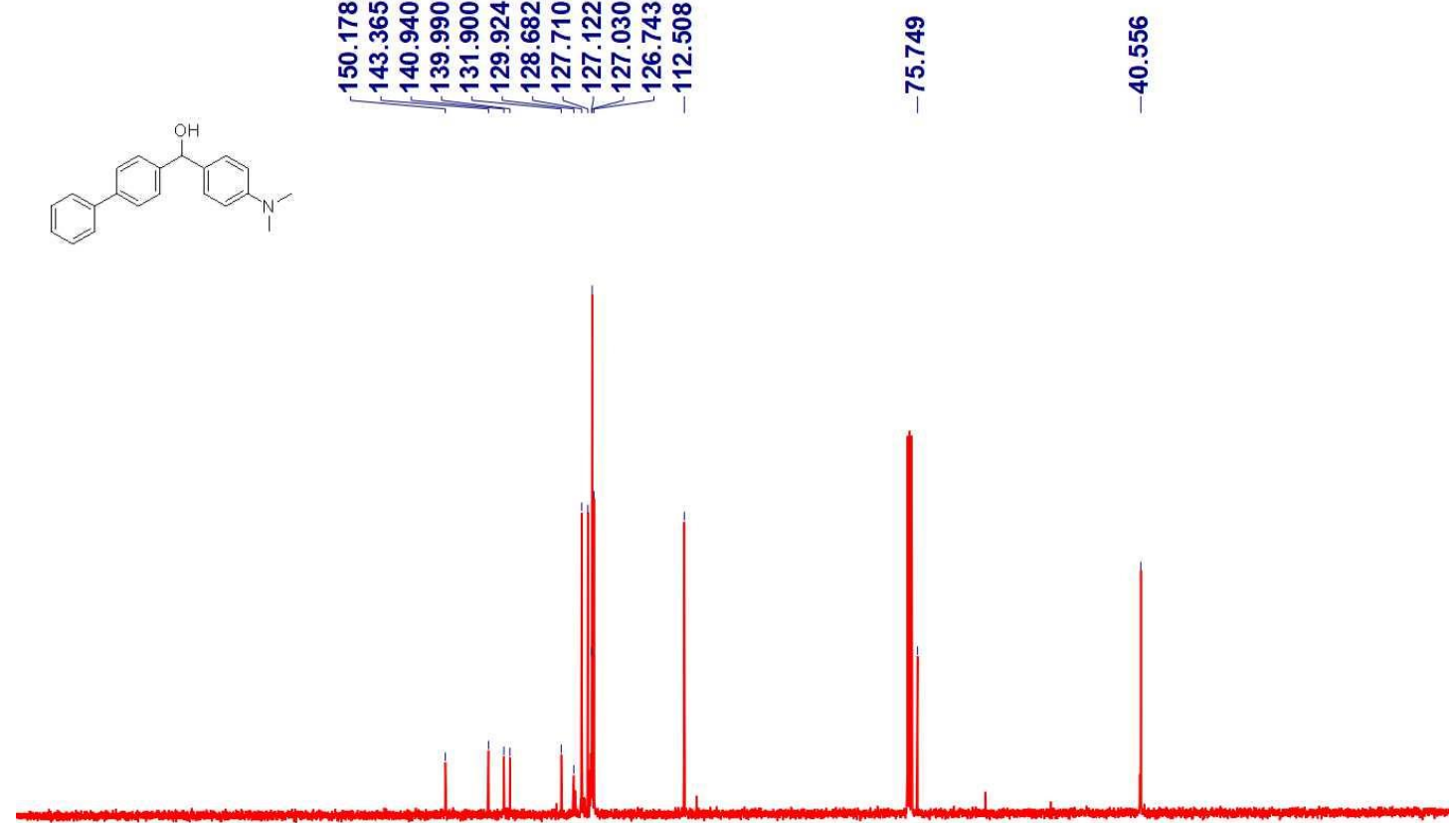

$210200190180170160150140130120110100 \quad 90 \quad 80 \quad 70 \quad 60 \quad 50 \quad 40 \quad 30 \quad 20 \quad 10 \quad 0$ 
(4-(Dimethylamino)phenyl)(4-fluorophenyl)methanol (1v)

杘

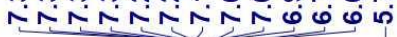

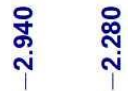
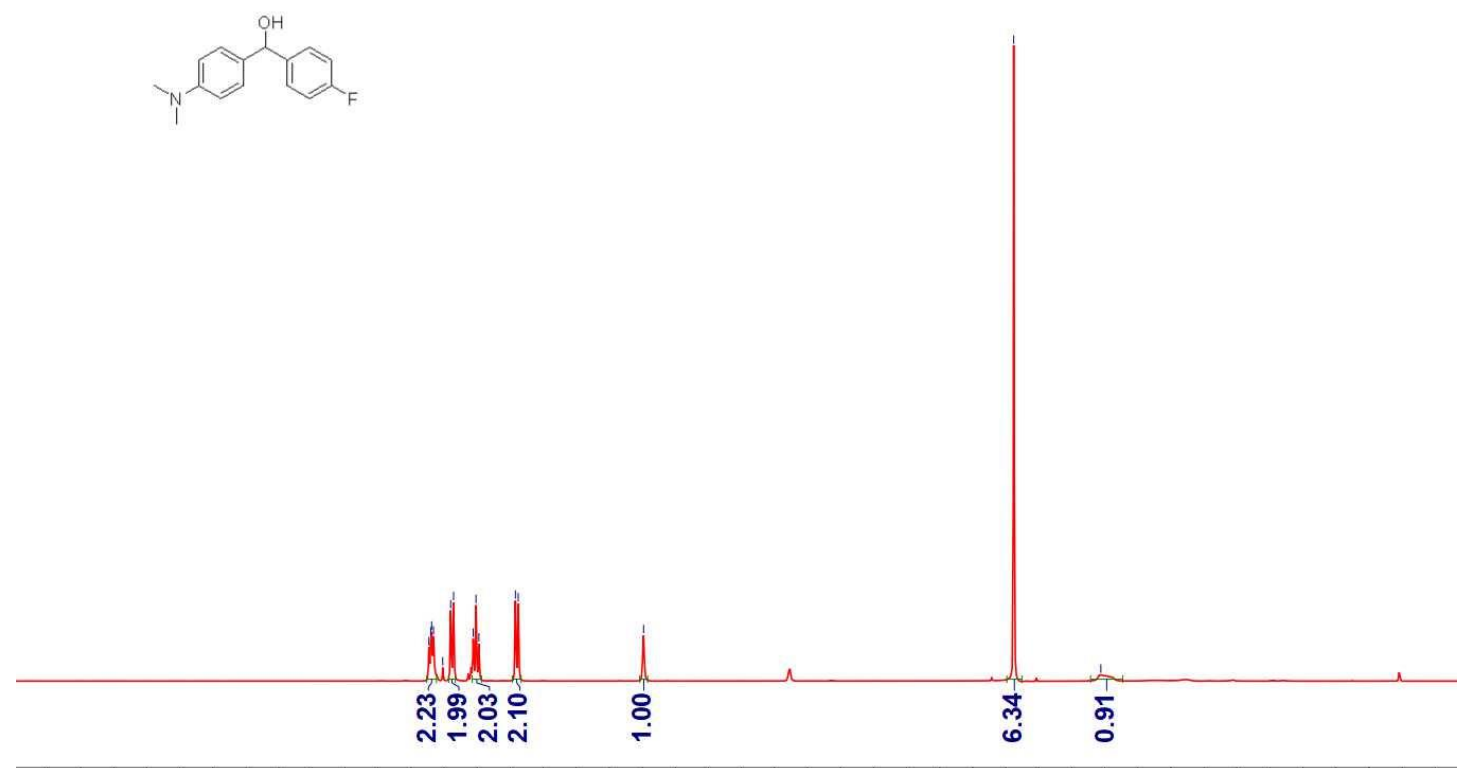

$\begin{array}{lllllllllllllllllllll}10.0 & 9.5 & 9.0 & 8.5 & 8.0 & 7.5 & 7.0 & 6.5 & 6.0 & 5.5 & 5.0 & 4.5 & 4.0 & 3.5 & 3.0 & 2.5 & 2.0 & 1.5 & 1.0 & 0.5 & 0.0\end{array}$

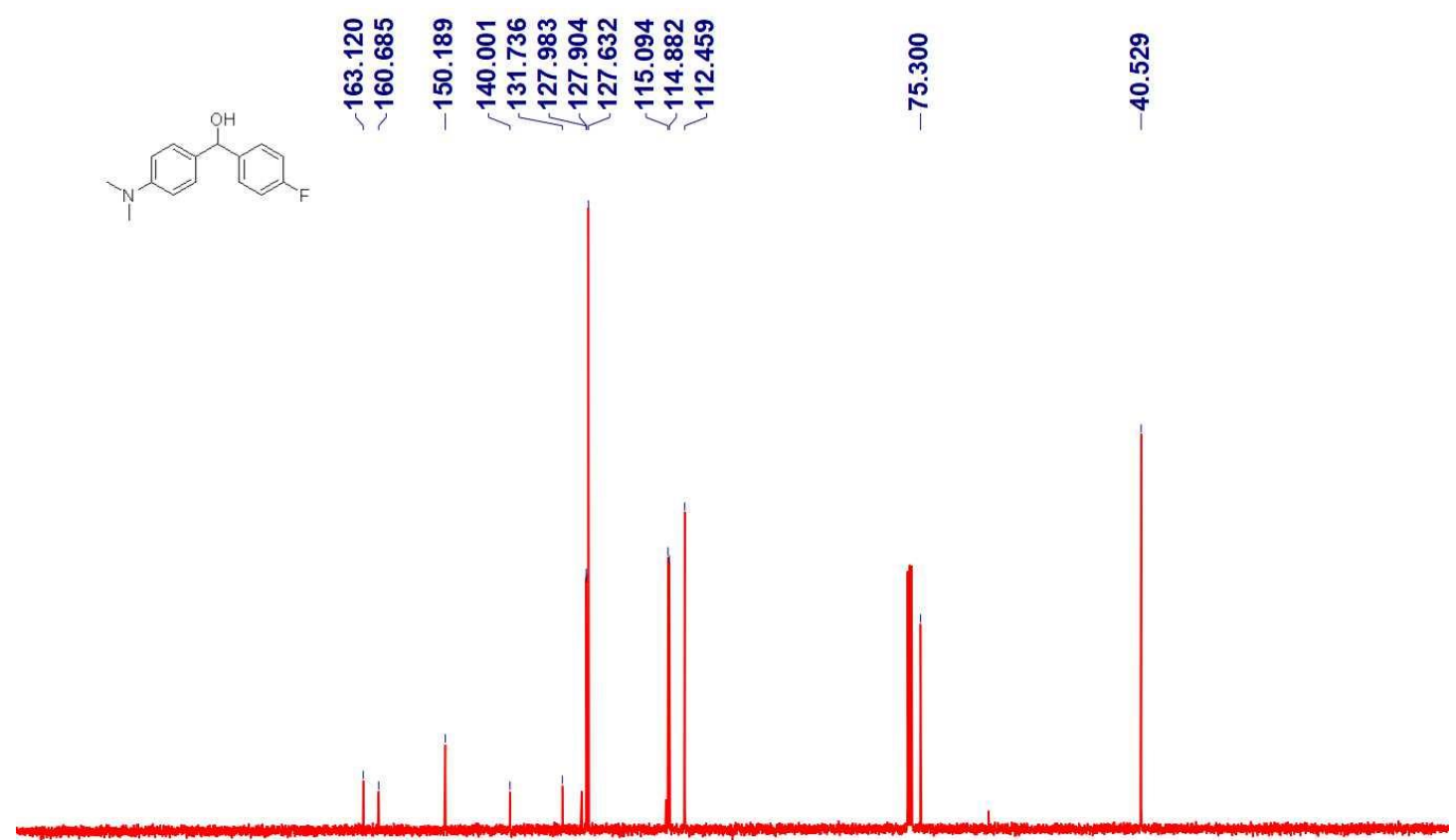

$210200190180170160150140130120110100 \quad 90 \quad 80 \quad 70 \quad 60 \quad 50 \quad 40 \quad 30 \quad 20 \quad 10 \quad 0$ 
(4-Chlorophenyl)(4-(dimethylamino)phenyl)methanol (1w)

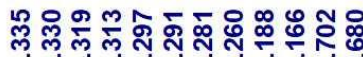

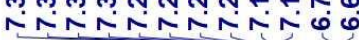

总

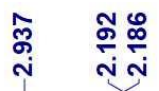

集

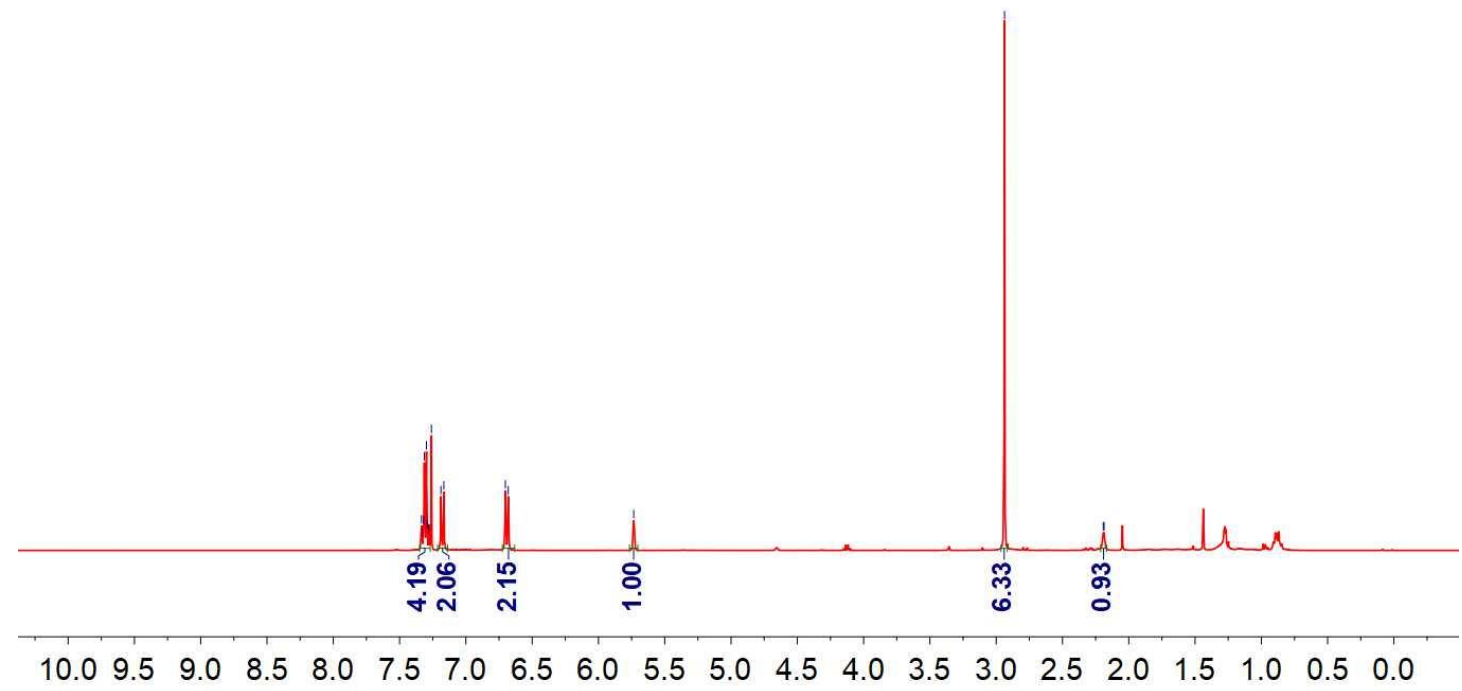

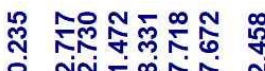

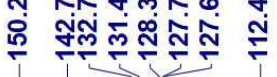

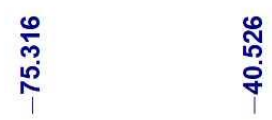
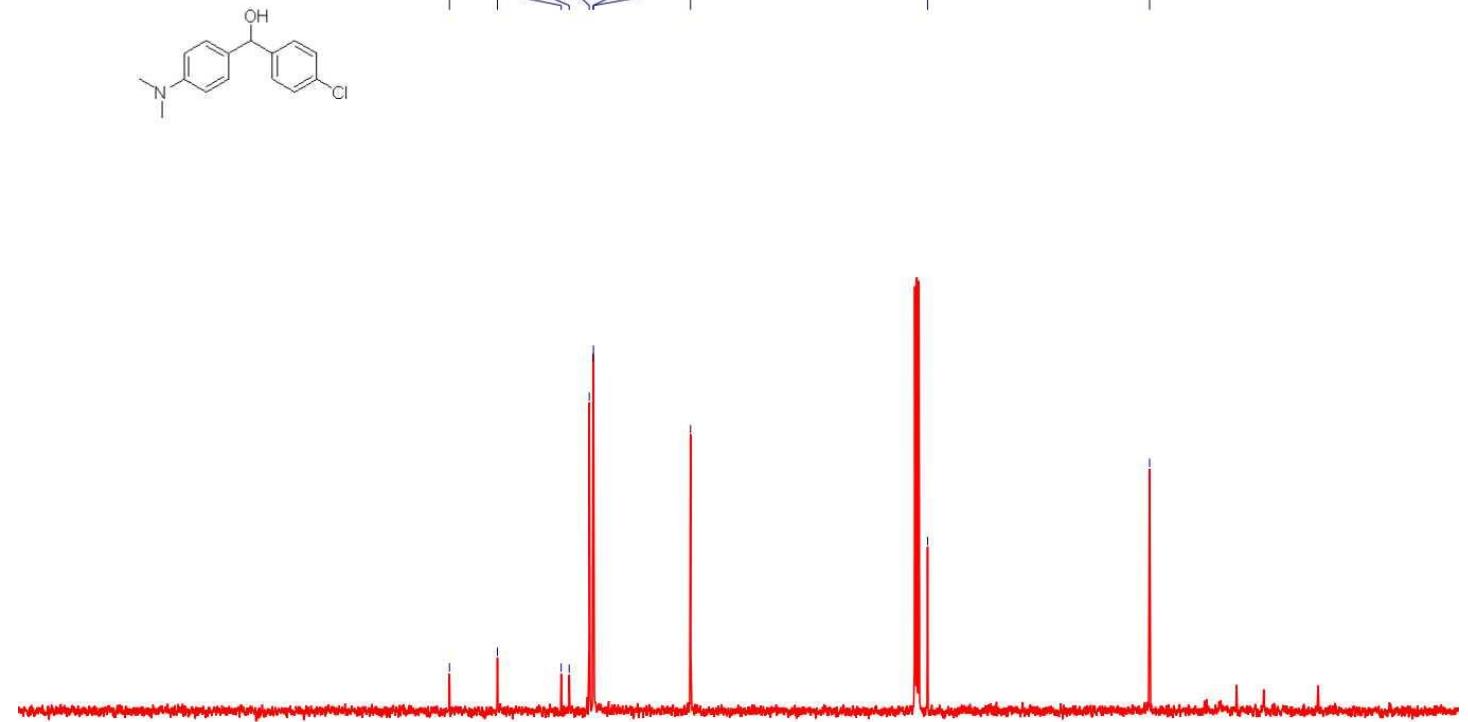

$210200190180170160150140130120110100 \quad 90 \quad 80 \quad 70 \quad 60 \begin{array}{llllllll}50 & 40 & 30 & 20 & 10 & 0\end{array}$ 
(4-Bromophenyl)(4-(dimethylamino)phenyl)methanol (1x)

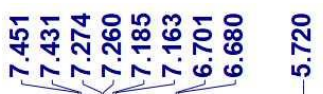

岕
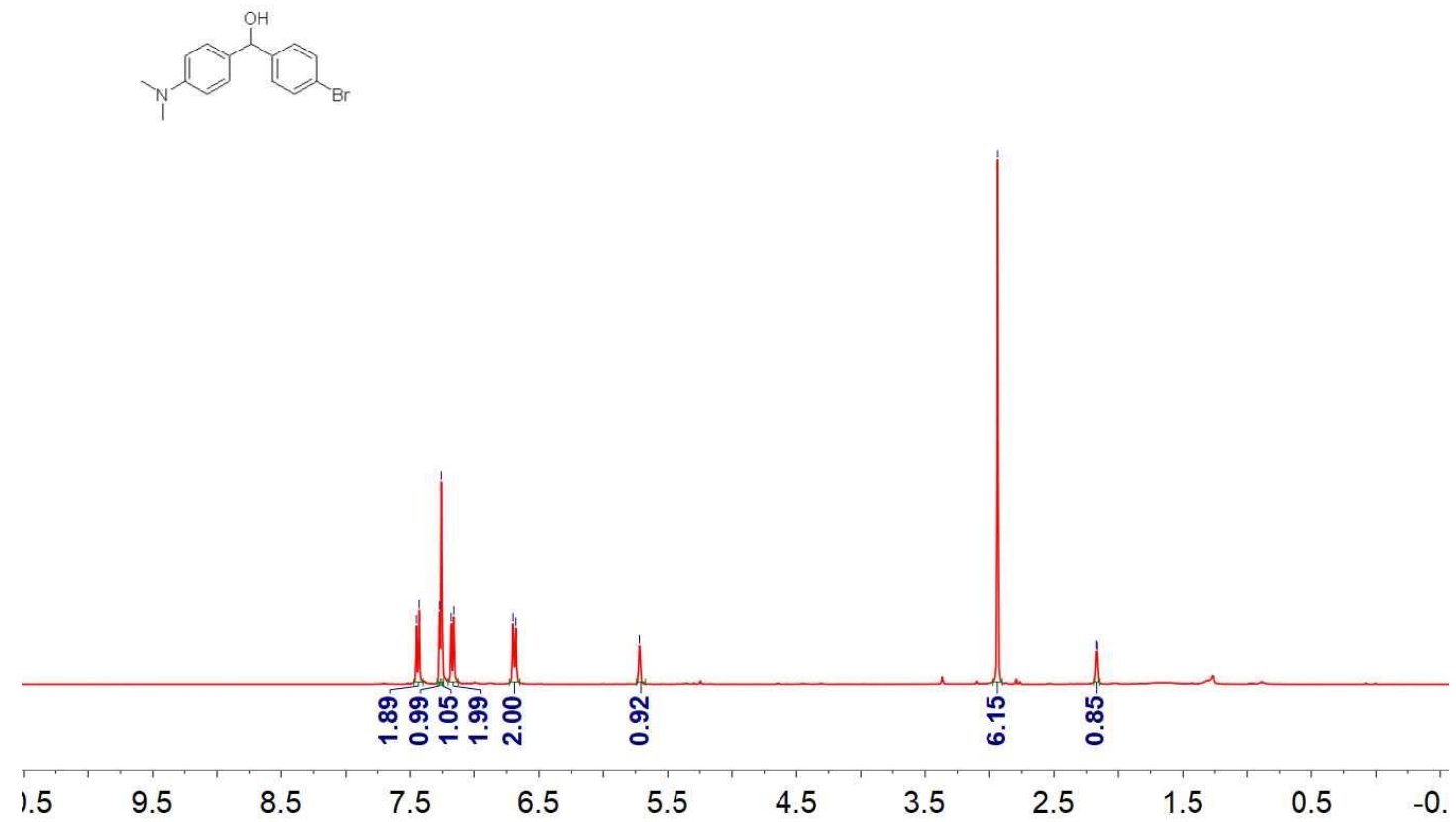

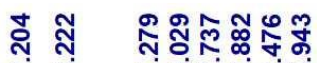

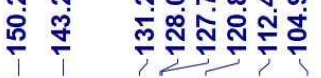
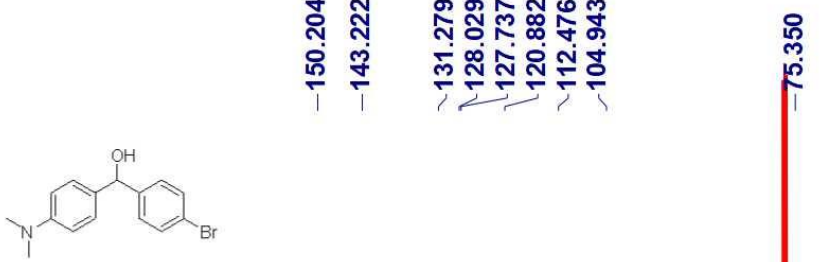

동

$210200190180170160150140130120110100 \quad 90 \quad 80 \quad 70 \quad 60 \quad 50 \quad 40 \quad 30 \quad 20 \quad 10 \quad 0$ 
(3-Bromophenyl)(4-(dimethylamino)phenyl)methanol (1y)
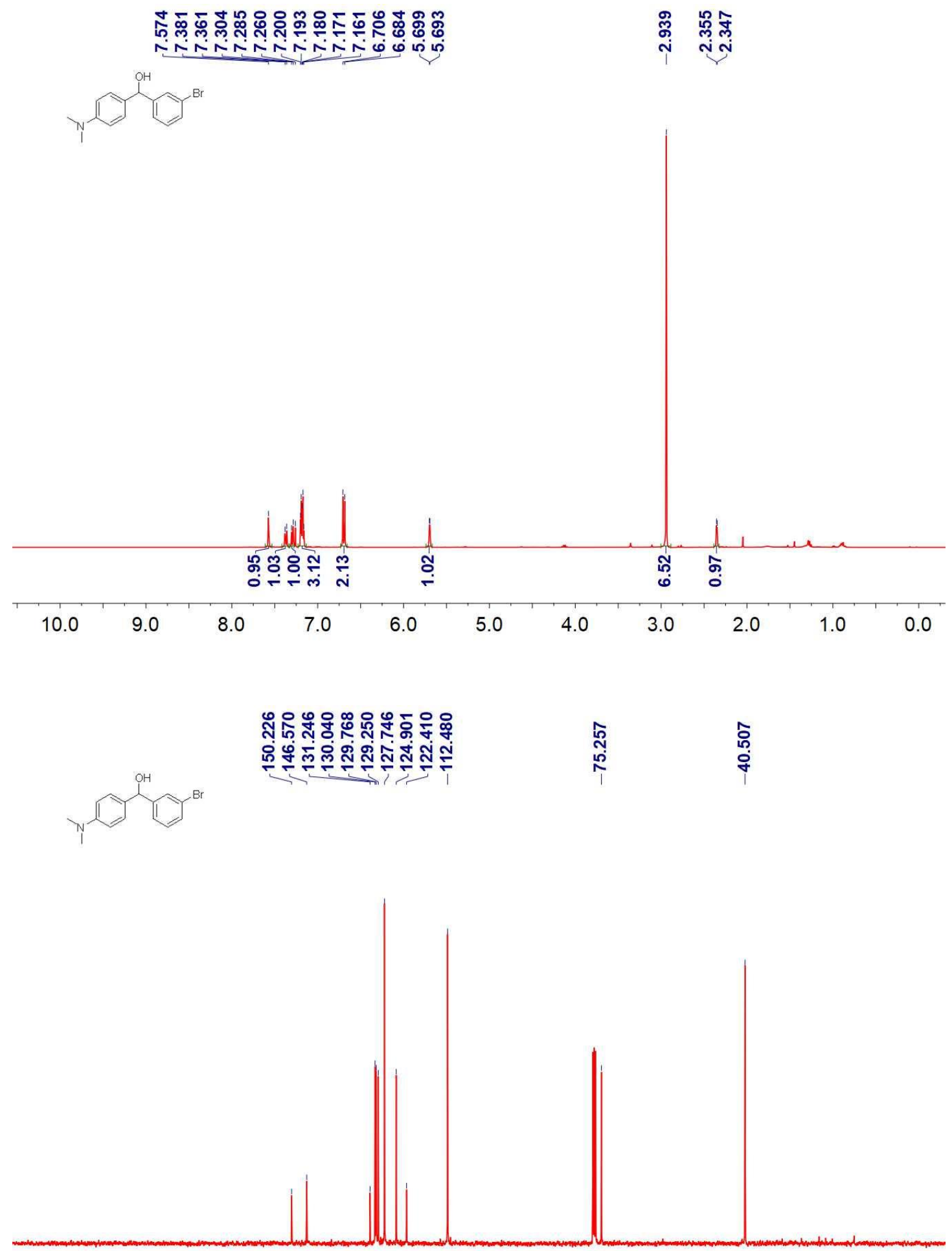

$210200190180170160150140130120110100 \quad 90 \quad 80 \quad 70 \quad 60 \quad 50 \quad 40 \quad 30 \quad 20 \quad 10 \quad 0$ 


\section{(2-Bromophenyl)(4-(dimethylamino)phenyl)methanol (1z)}
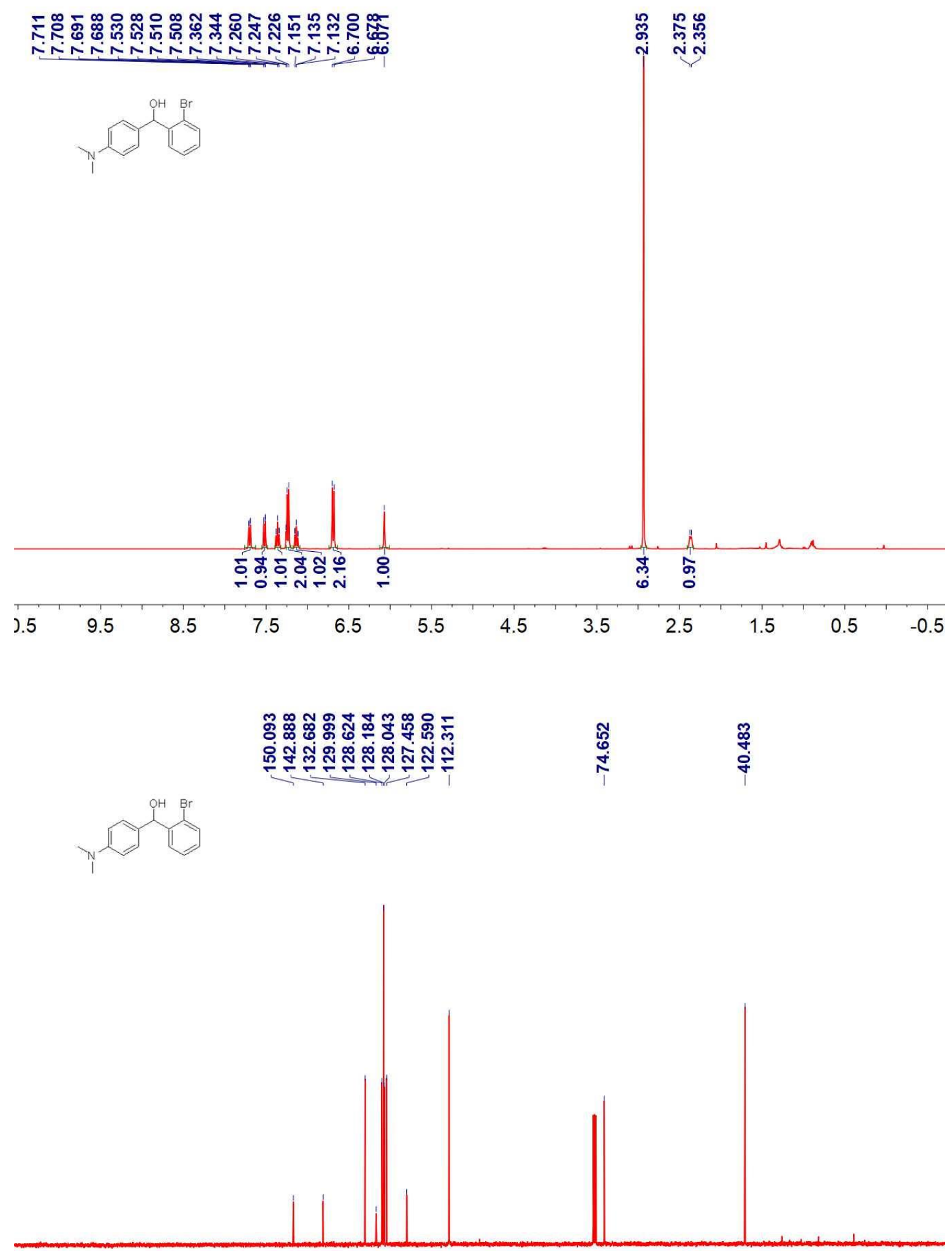

$210200190180170160150140130120110100 \quad 90 \quad 80 \quad 70 \quad 60 \quad 50 \quad 40 \quad 30 \quad 20 \quad 10 \quad 0$ 
(4-(Dimethylamino)phenyl)(4-iodophenyl)methanol (1aa)

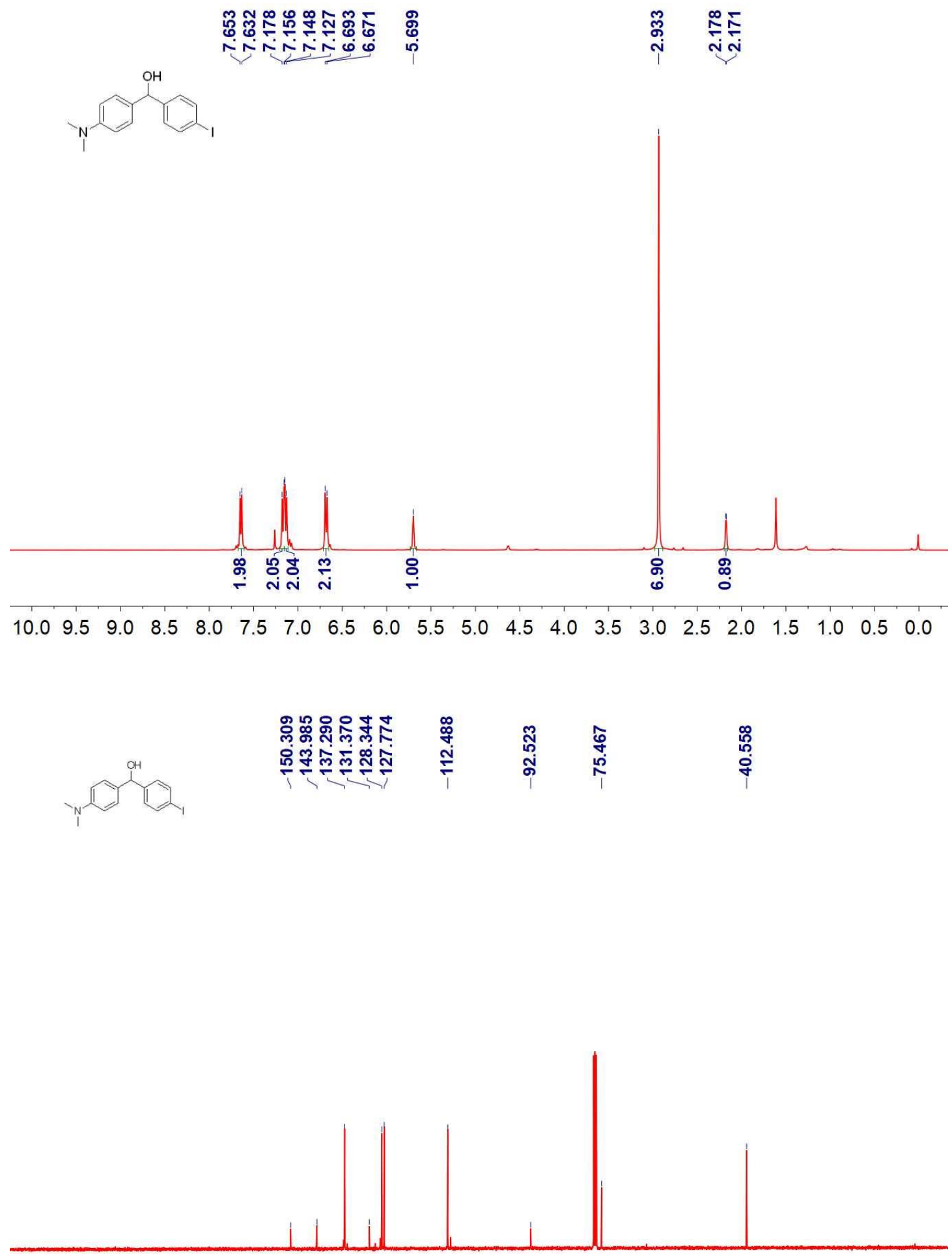

$210200190180170160150140130120110100 \quad 90 \quad 80 \quad 70 \quad 60 \quad 50 \quad 40 \quad 30 \quad 20 \quad 10 \quad 0$ 
(4-(Dimethylamino)phenyl)(4-(trifluoromethyl)phenyl)methanol (1ab)
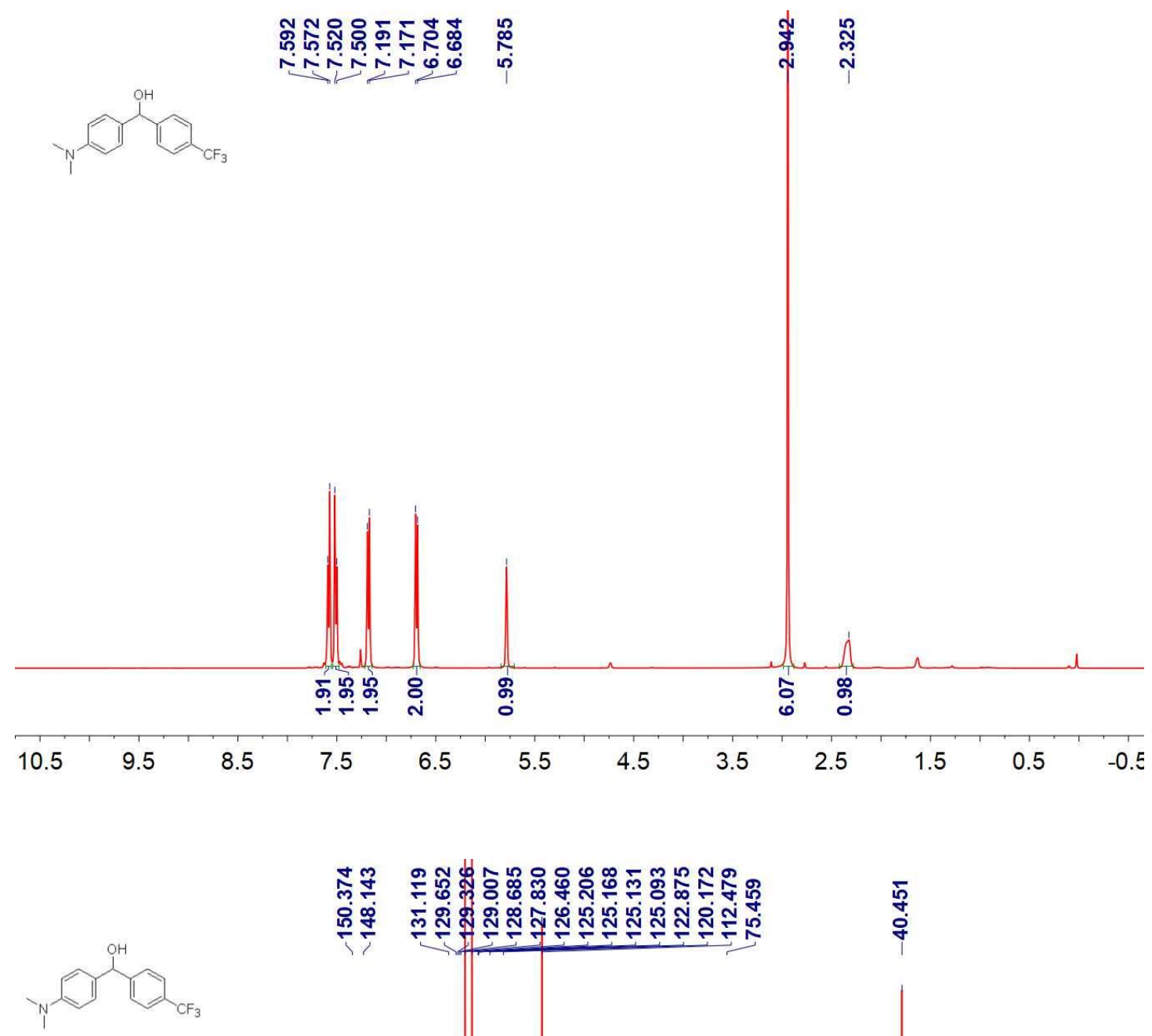

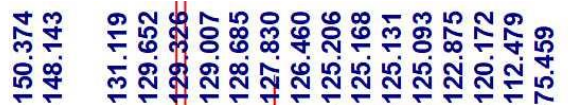

以ำ

$210200190180170160150140130120110100 \quad 90 \quad 80 \quad 70 \quad 60 \quad 50 \quad 40 \quad 30 \quad 20 \quad 10 \quad 0$ 

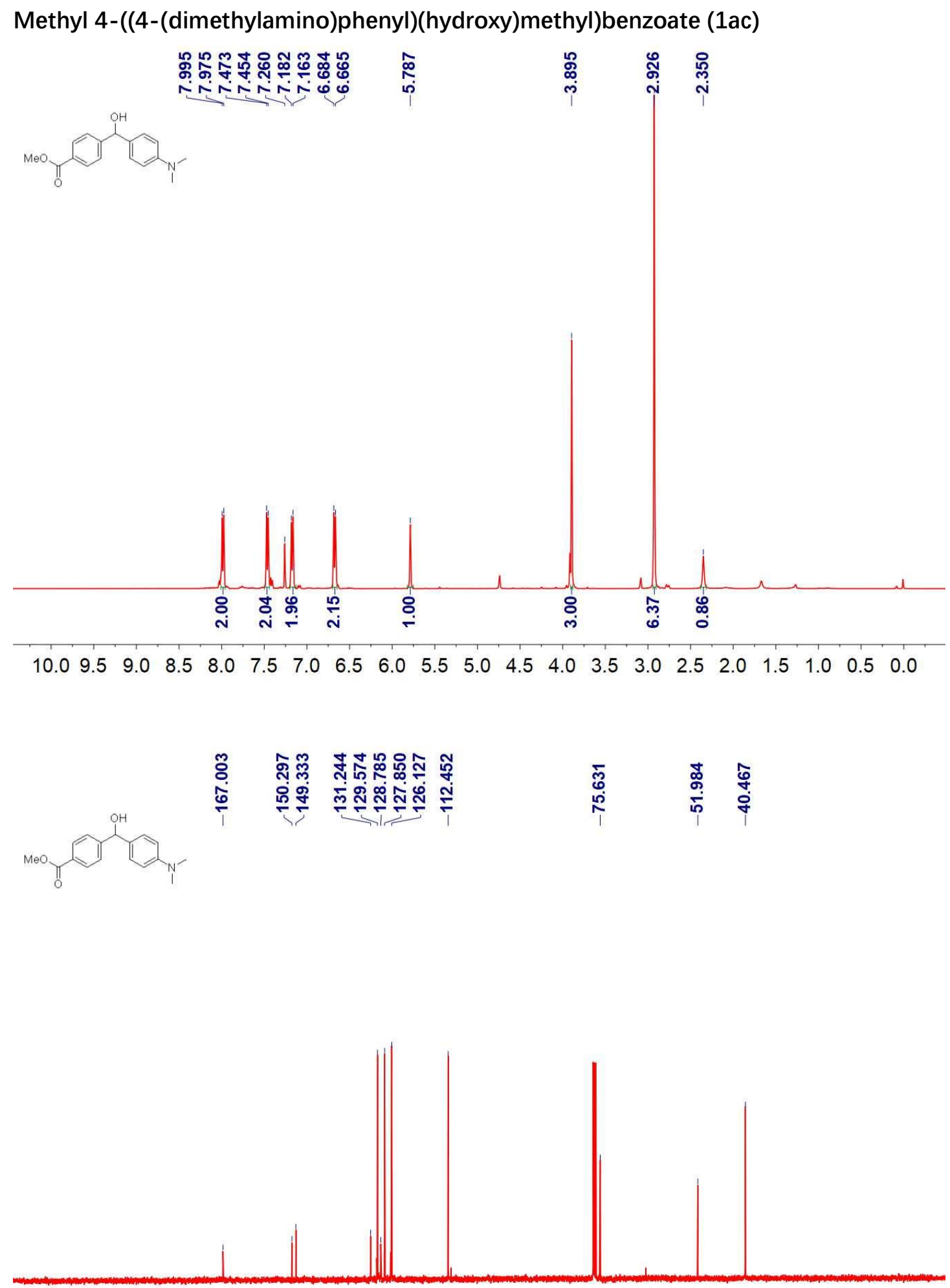

$210200190180170160150140130120110100 \quad 90 \quad 80 \quad 70 \quad 60 \quad 50 \quad 40 \quad 30 \quad 20 \quad 10 \quad 0$ 
4-((4-(Dimethylamino)phenyl)(hydroxy)methyl)benzonitrile (1ad)
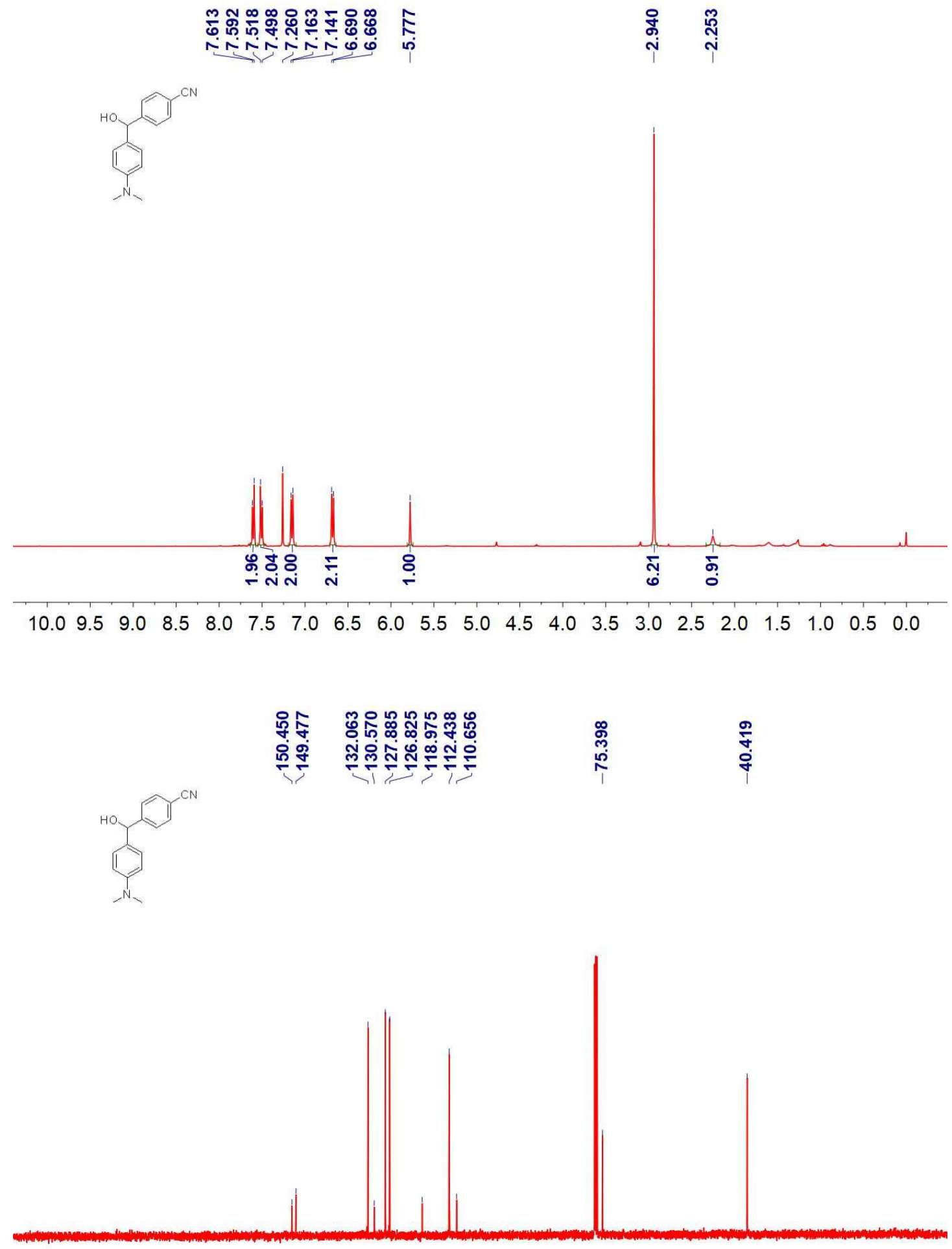

$210200190180170160150140130120110100 \quad 90 \quad 80 \quad 70 \quad 60 \quad 50 \quad 40 \quad 30 \quad 20 \quad 10 \quad 0$ 
(4-(Dimethylamino)phenyl)(4-nitrophenyl)methanol (1ae)

它占

岕

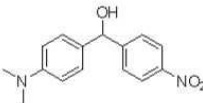

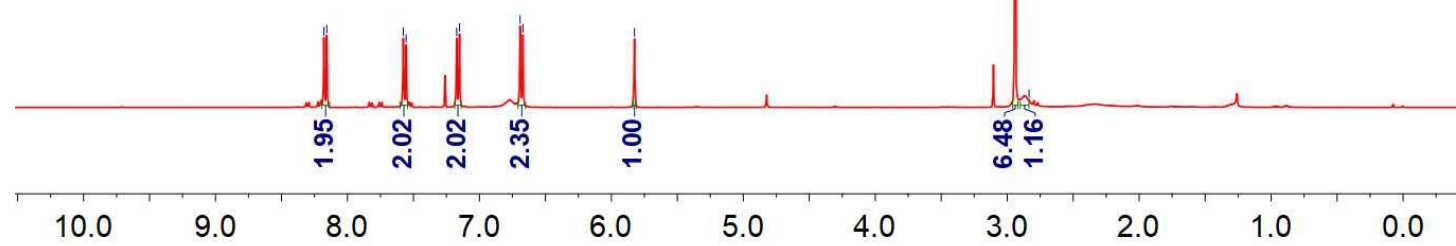

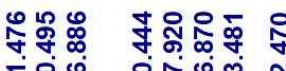

กำ

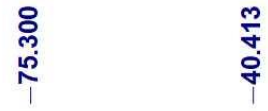

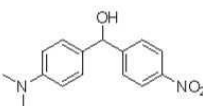

$210200190180170160150140130120110100 \quad 90 \quad 80 \quad 70 \quad 60 \quad 50 \quad 40 \quad 30 \quad 20 \quad 10 \quad 0$ 

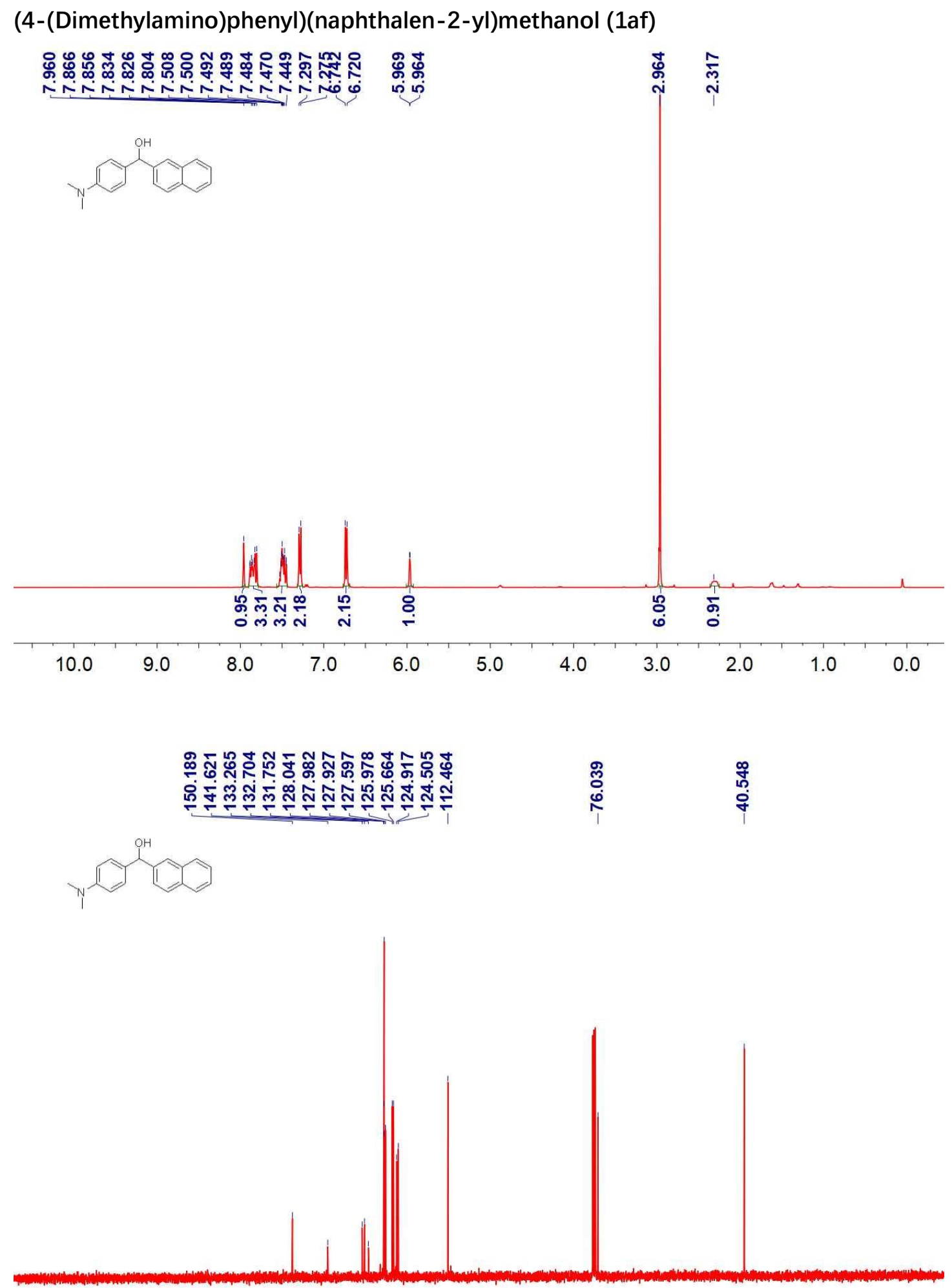

$210200190180170160150140130120110 \quad 100 \quad 90 \quad 80 \quad 70 \quad 60 \quad 50 \quad 40 \quad 30 \quad 20 \quad 10 \quad 0$ 


\section{(4-(Dimethylamino)phenyl)(furan-2-yl)methanol (1ag)}

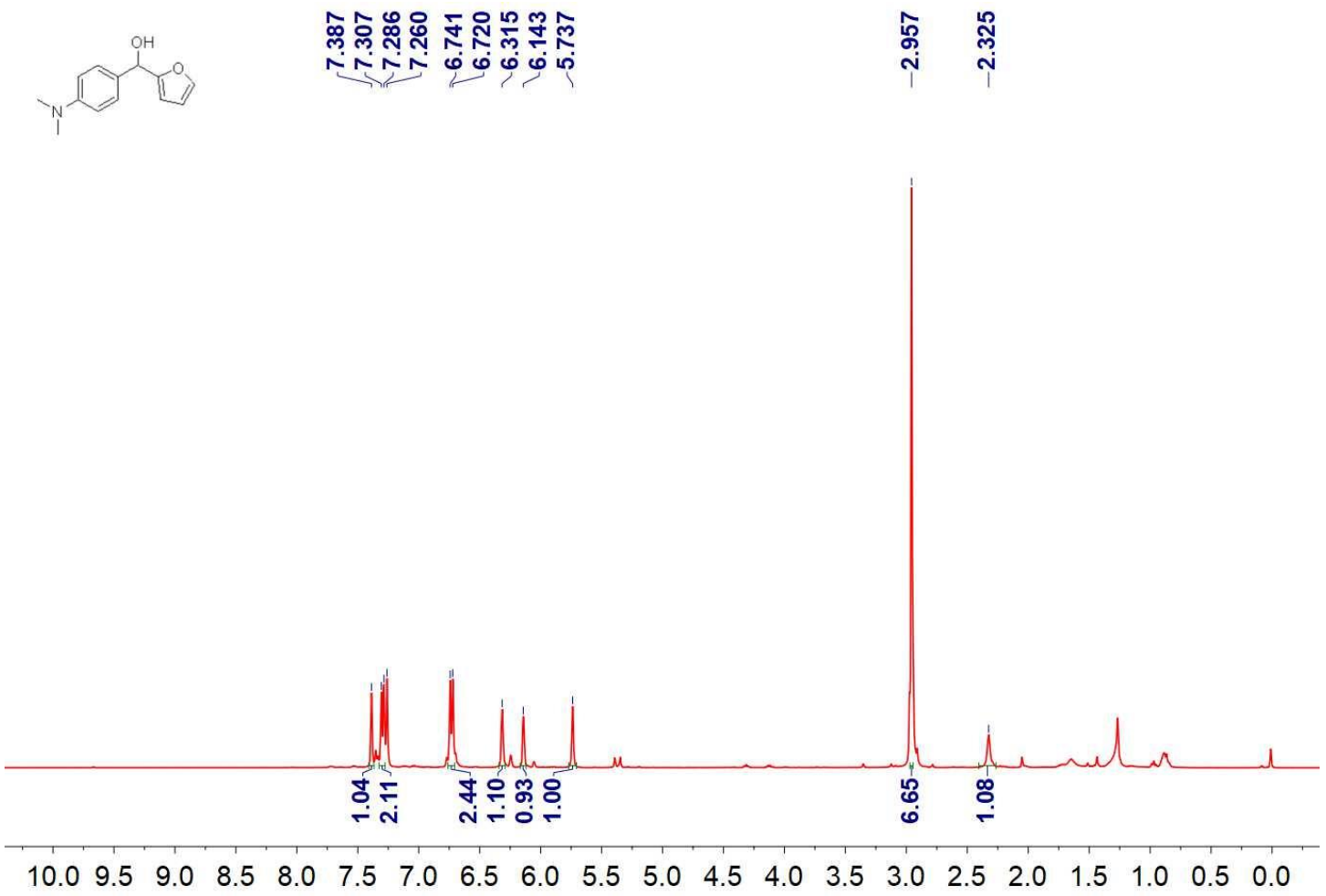

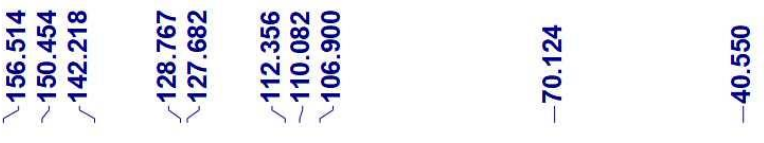

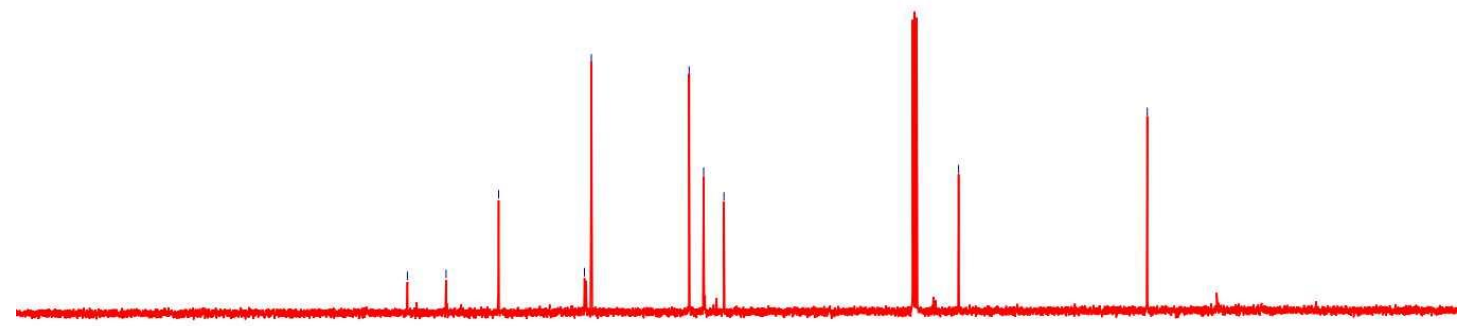

$210200190180170160150140130120110100 \quad 90 \quad 80 \quad 70 \quad 60 \quad 50 \quad 40 \quad 30 \quad 20 \quad 10 \quad 0$ 
(4-(Dimethylamino)phenyl)(thiophen-2-yl)methanol (1ah)

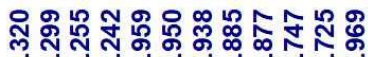

ヘヘNヘ

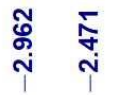

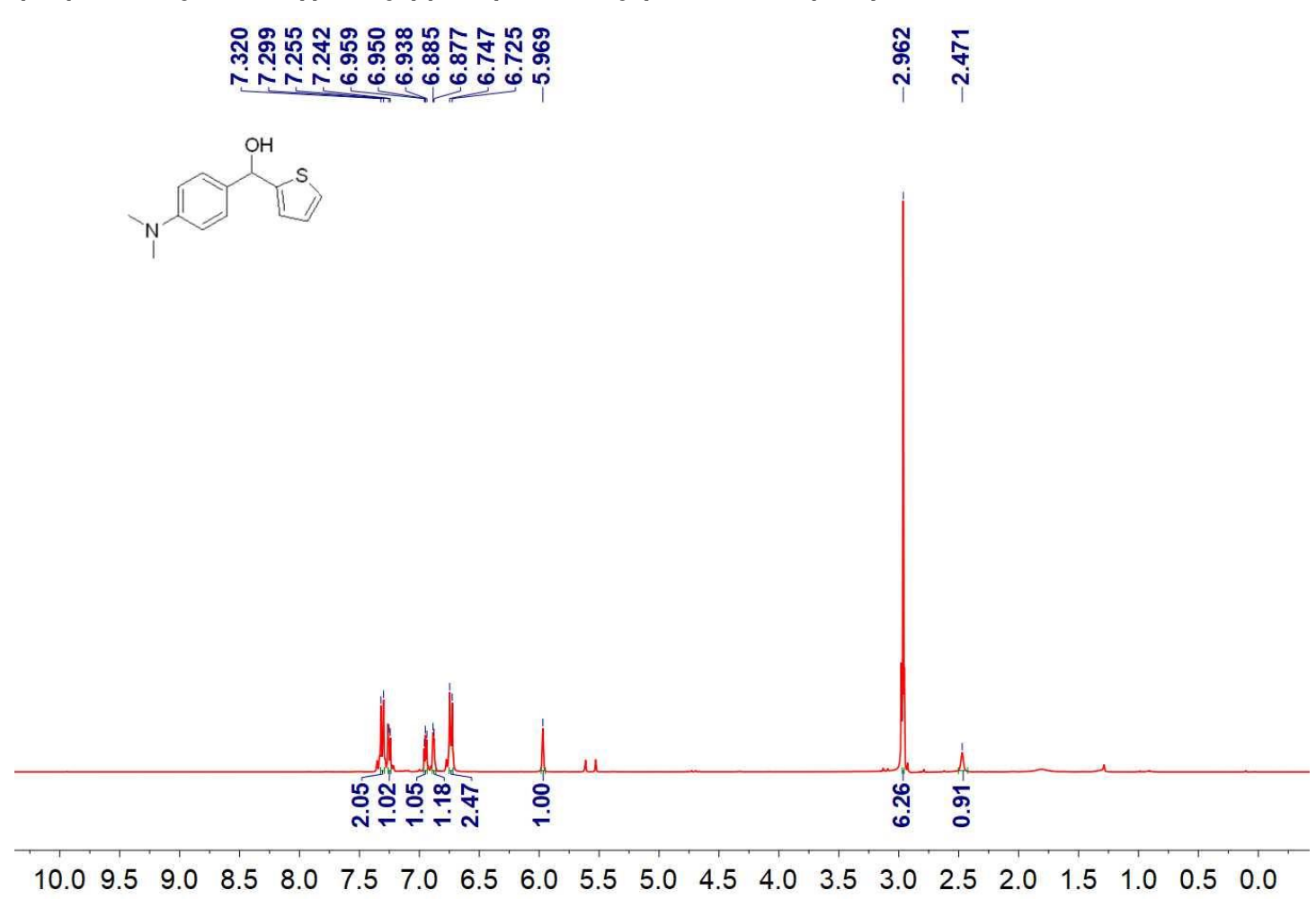

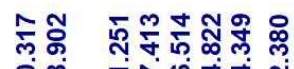

ถึำ

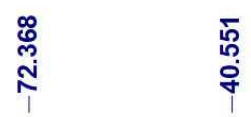
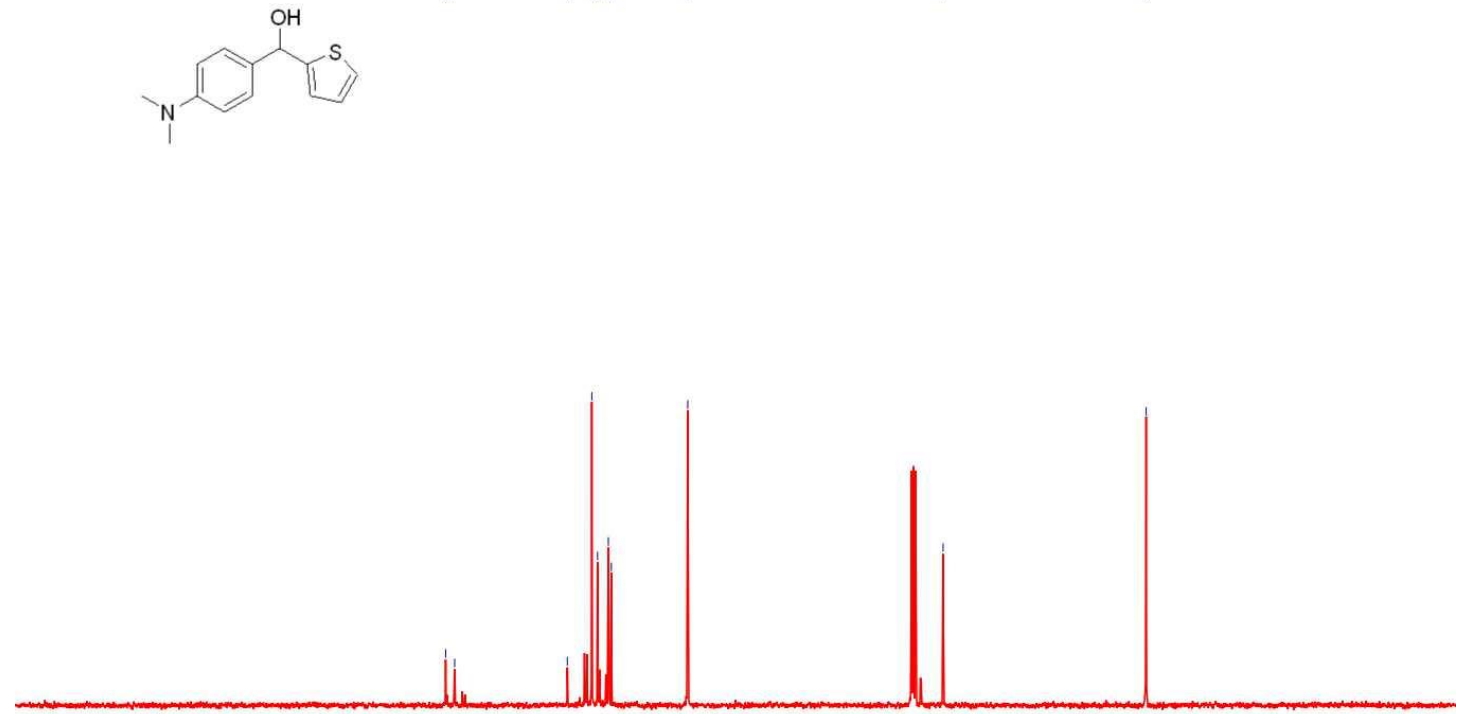

$210200190180170160150140130120110100 \quad 90 \quad 80 \quad 70 \quad 60 \quad 50 \quad 40 \quad 30 \quad 20 \quad 10 \quad 0$ 
(4-(Dimethylamino)phenyl)(pyridin-4-yl)methanol (1ai)

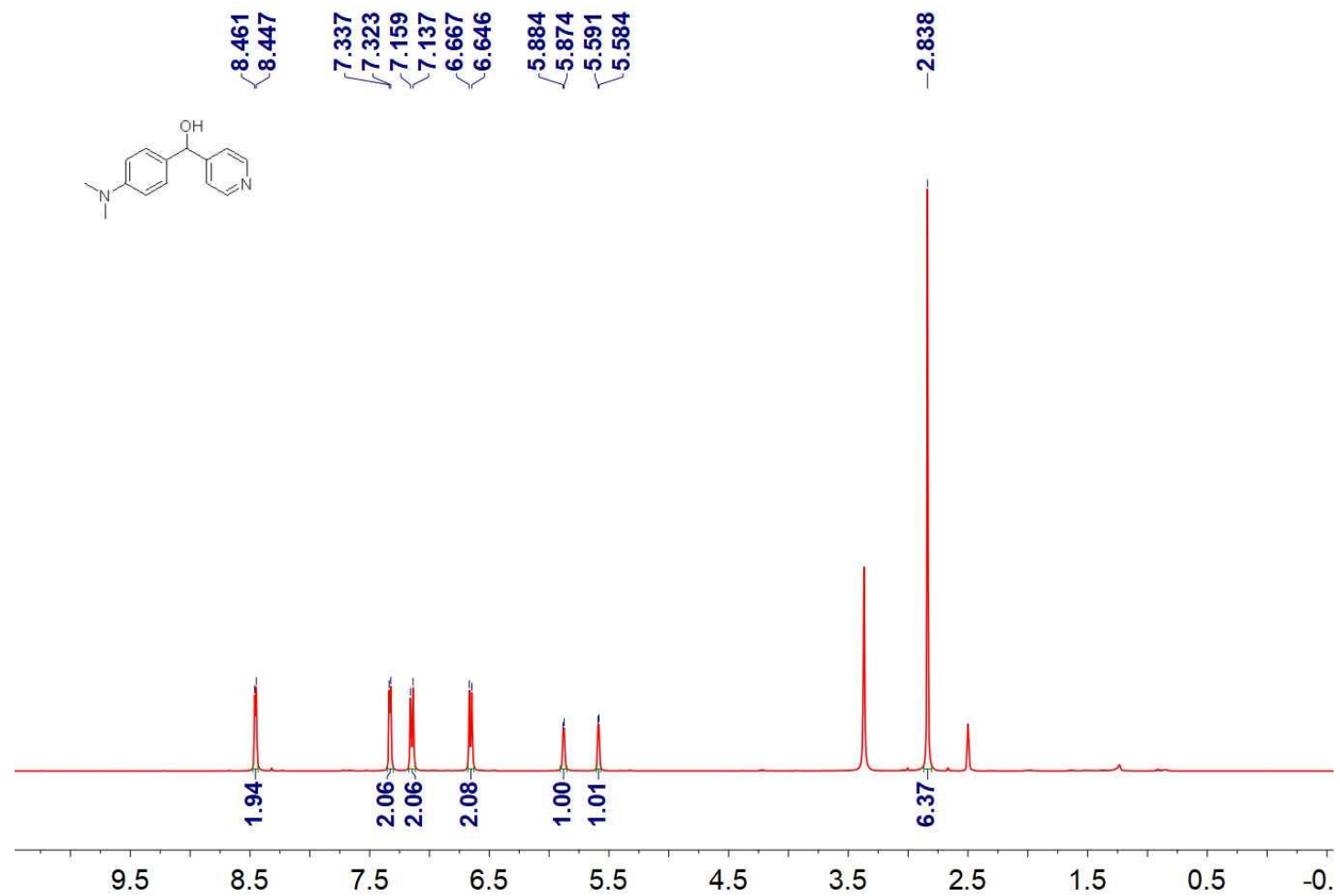

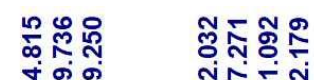

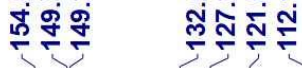

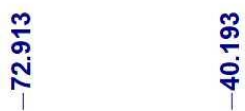

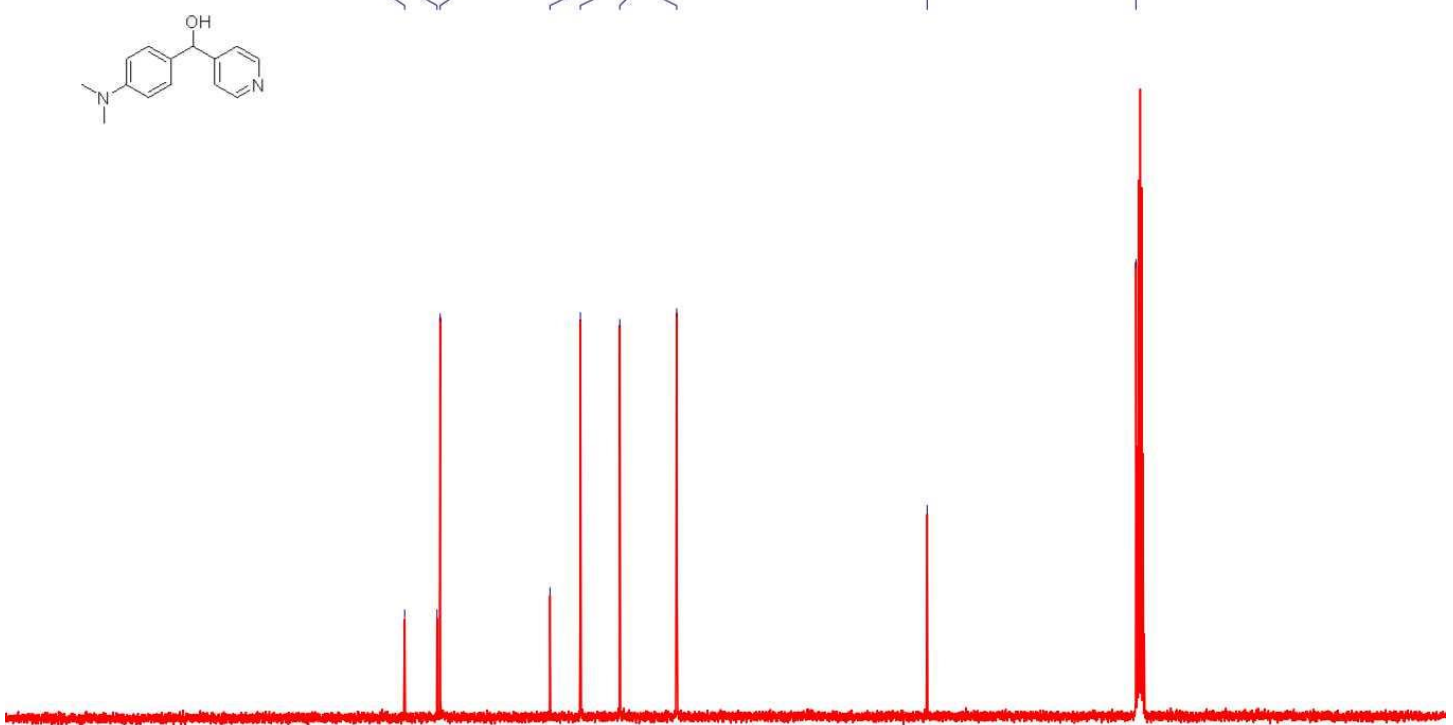

$210200190180170160150140130120110 \quad 100 \quad 90 \quad 80 \quad 70 \quad 60 \quad 50 \quad 40 \quad 30 \quad 20 \quad 10 \quad 0$ 

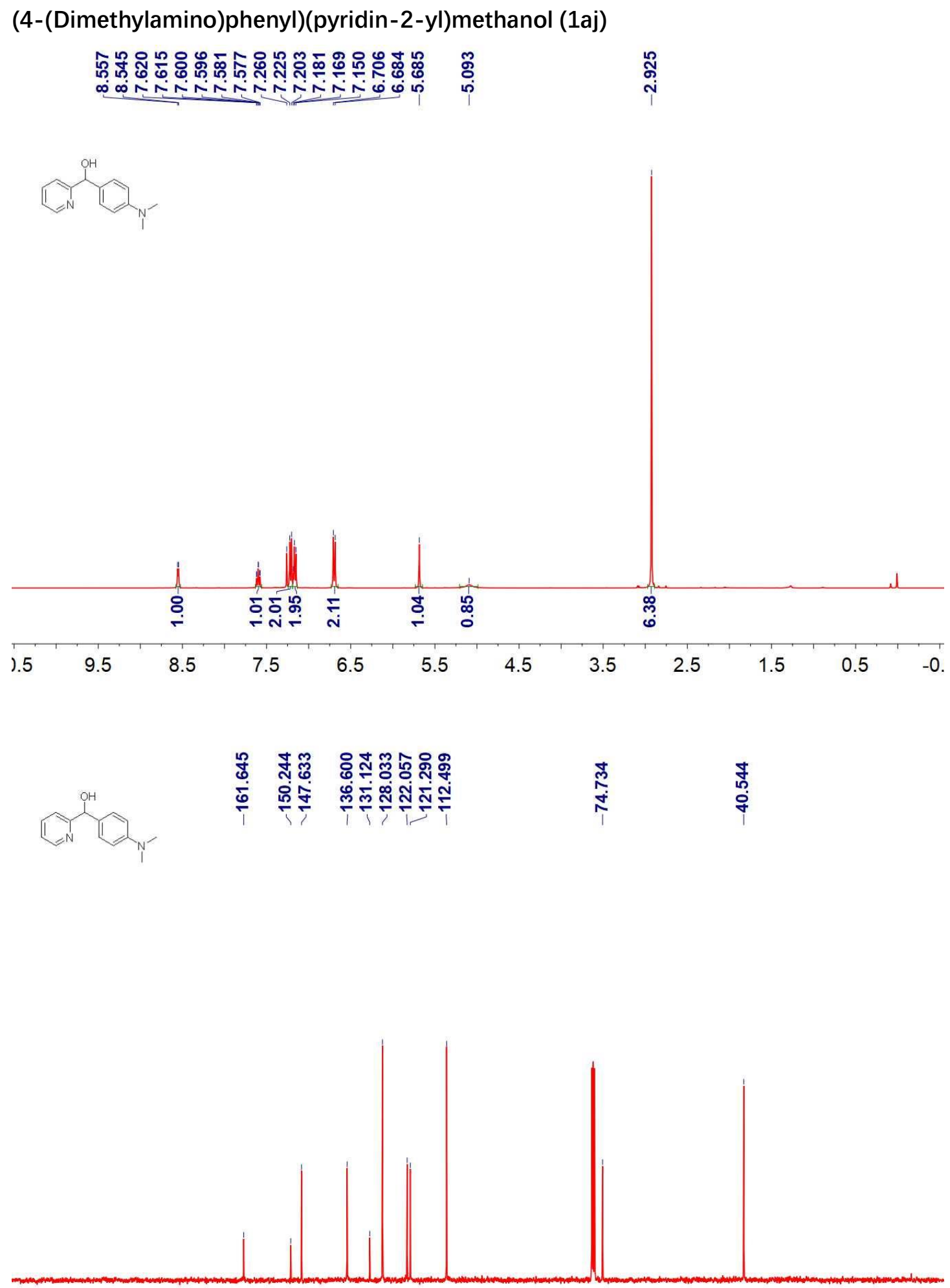

$210200190180170160150140130120110100 \quad 90 \quad 80 \quad 70 \quad 60 \quad 50 \quad 40 \quad 30 \quad 20 \quad 10 \quad 0$ 
(4-(dimethylamino)phenyl)(4-(5,6,7,8-tetrahydroquinolin-2-yl)phenyl)methanol (1ak)
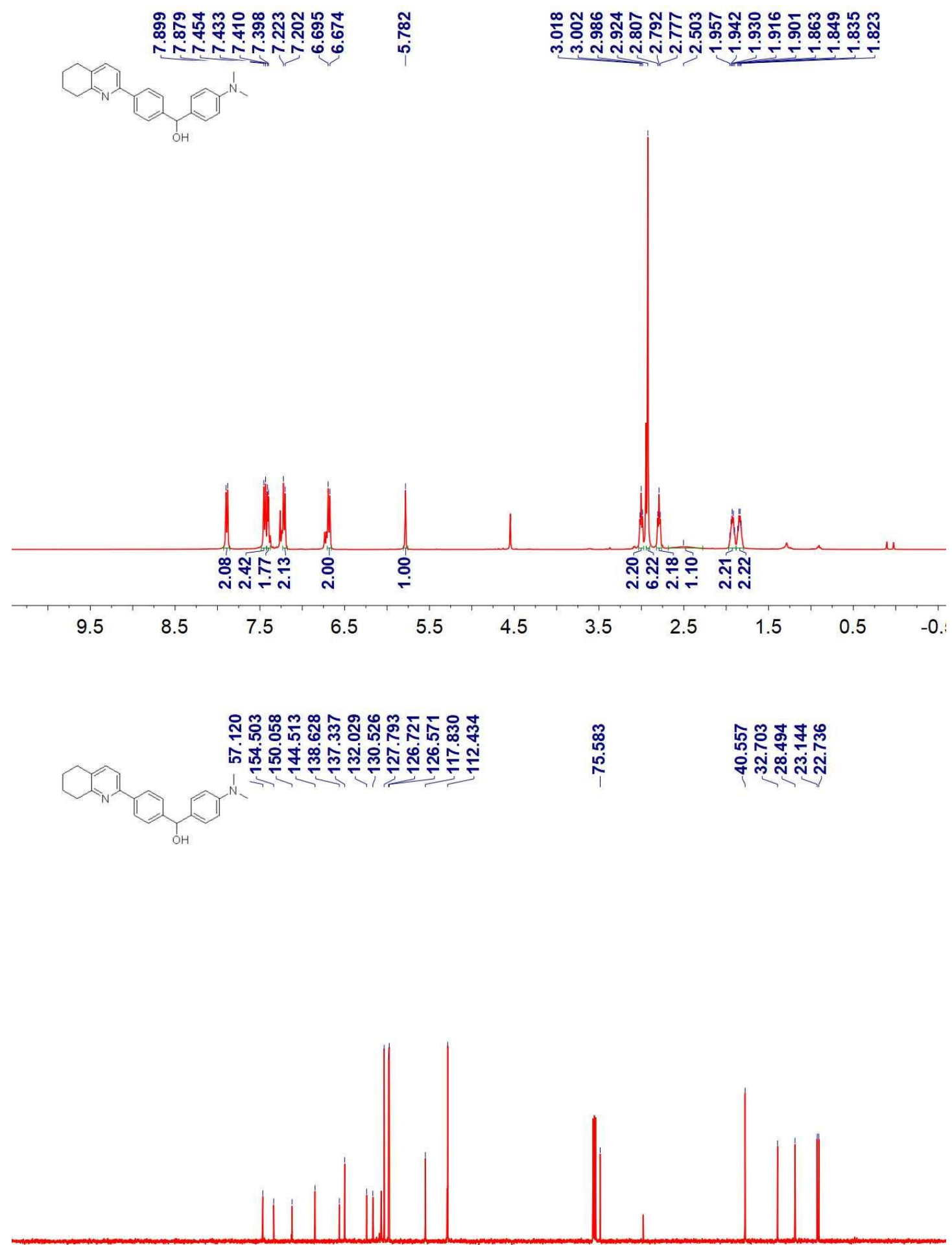

$210200190180170160150140130120110 \quad 100 \quad 90 \quad 80 \quad 70 \quad 60 \quad 50 \quad 40 \quad 30 \quad 20 \quad 10 \quad 0$ 
(4-(Dimethylamino)phenyl)(1-methyl-1H-indol-3-yl)methanol (1al)

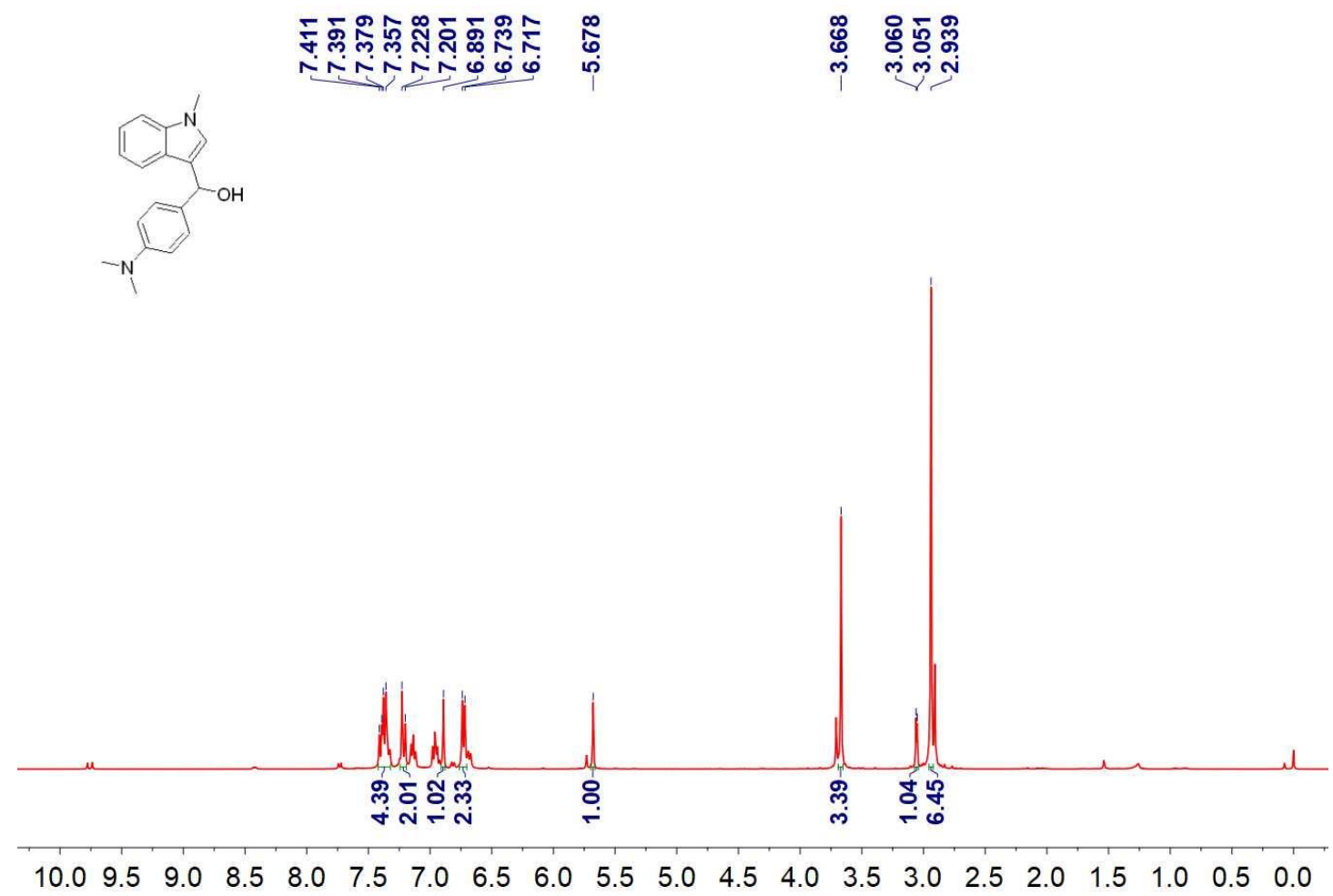

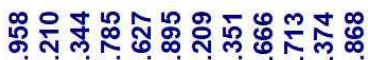

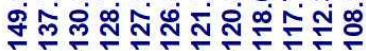

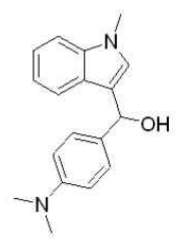

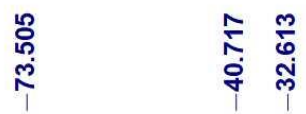

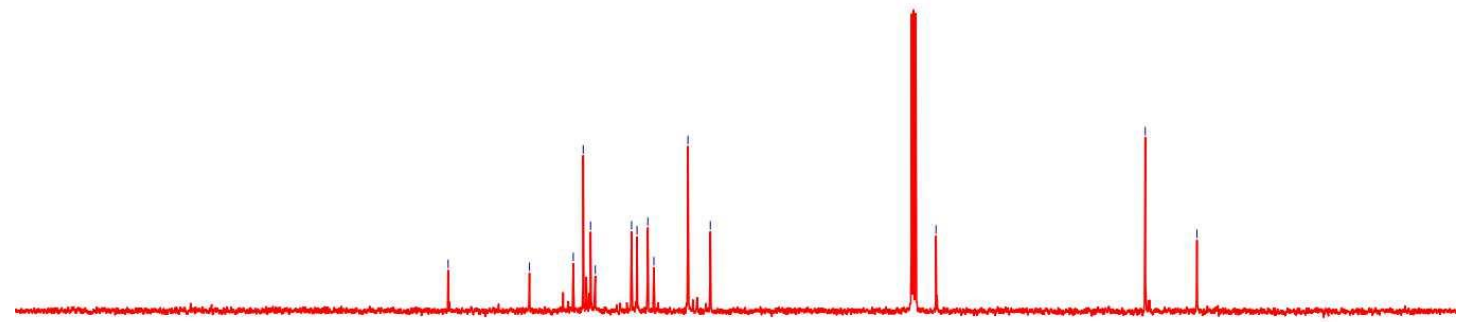

$210200190180170160150140130120110100 \quad 90 \quad 80 \quad 70 \quad 60 \quad 50 \quad 40 \quad 30 \quad 20 \quad 10 \quad 0$ 
1,4-Phenylenebis((4-(dimethylamino)phenyl)methanol) (1an)

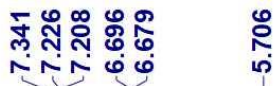
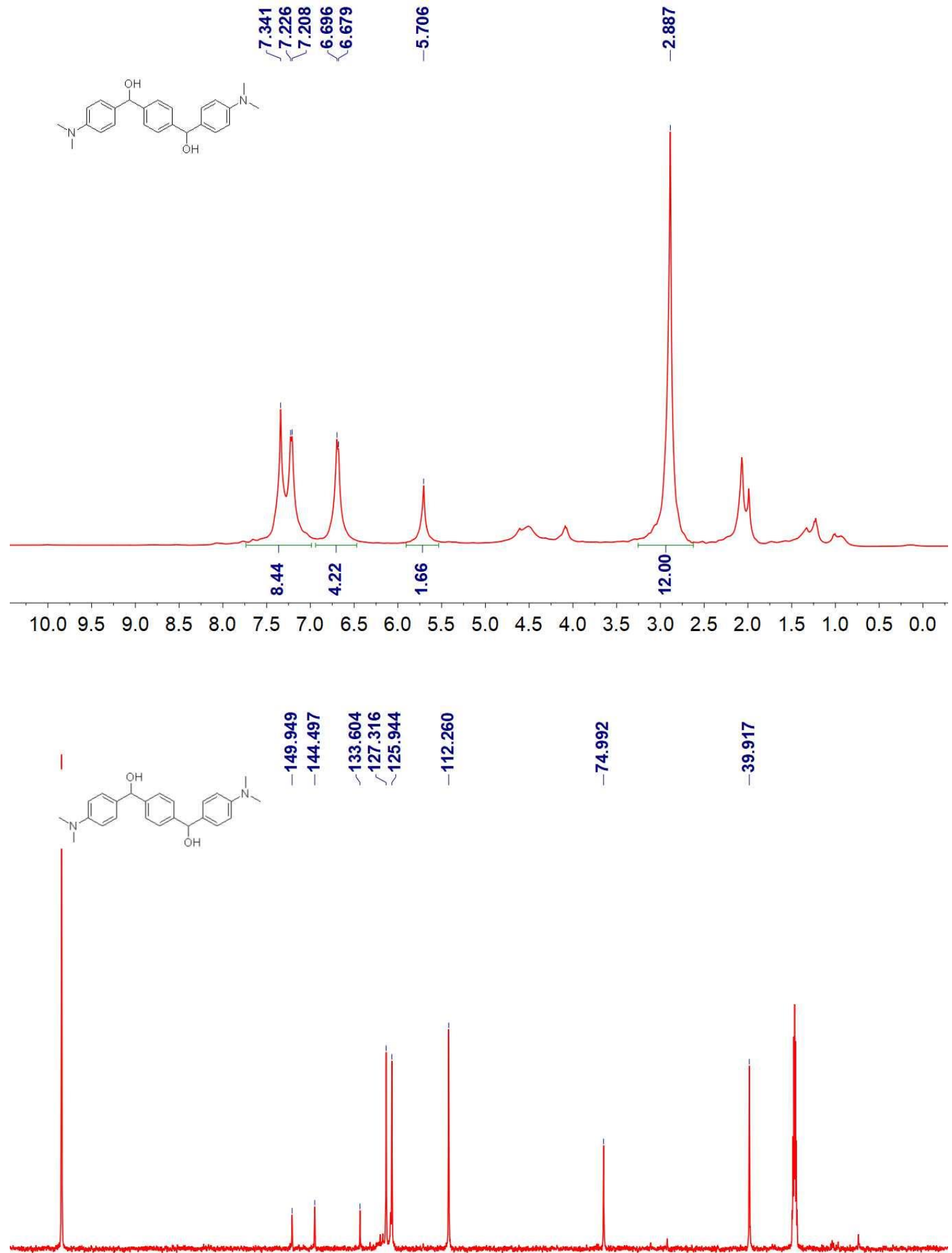

$210200190180170160150140130120110100 \quad 90 \quad 80 \quad 70 \quad 60 \quad 50 \quad 40 \quad 30 \quad 20 \quad 10 \quad 0$ 
(4-(Dimethylamino)phenyl)(4-((methylthio)methyl)phenyl)methanol (1ao)

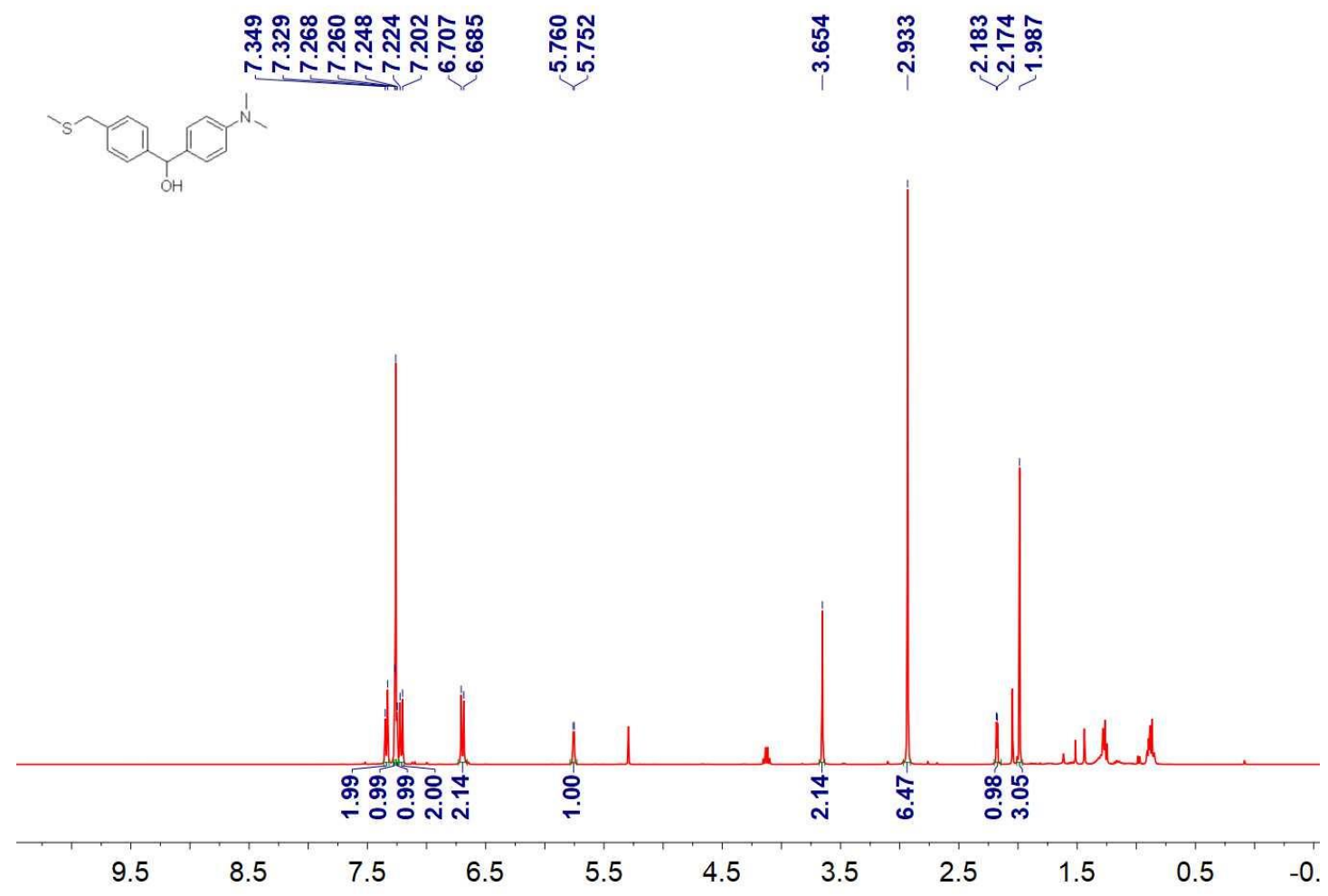

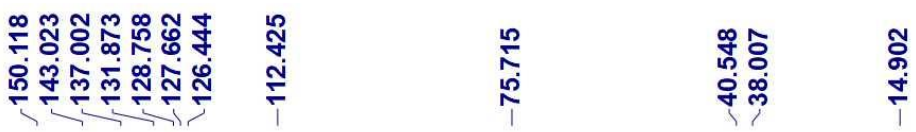

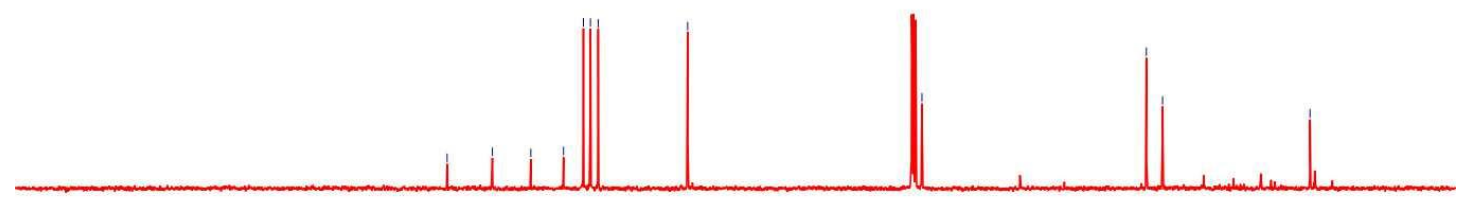

$210200190180170160150140130120110100 \quad 90 \quad 80 \quad 70 \quad 60 \quad 50 \quad 40 \quad 30 \quad 20 \quad 10 \quad 0$ 
(4-(Dimethylamino)phenyl)(4-(methoxymethyl)phenyl)methanol (1ap)
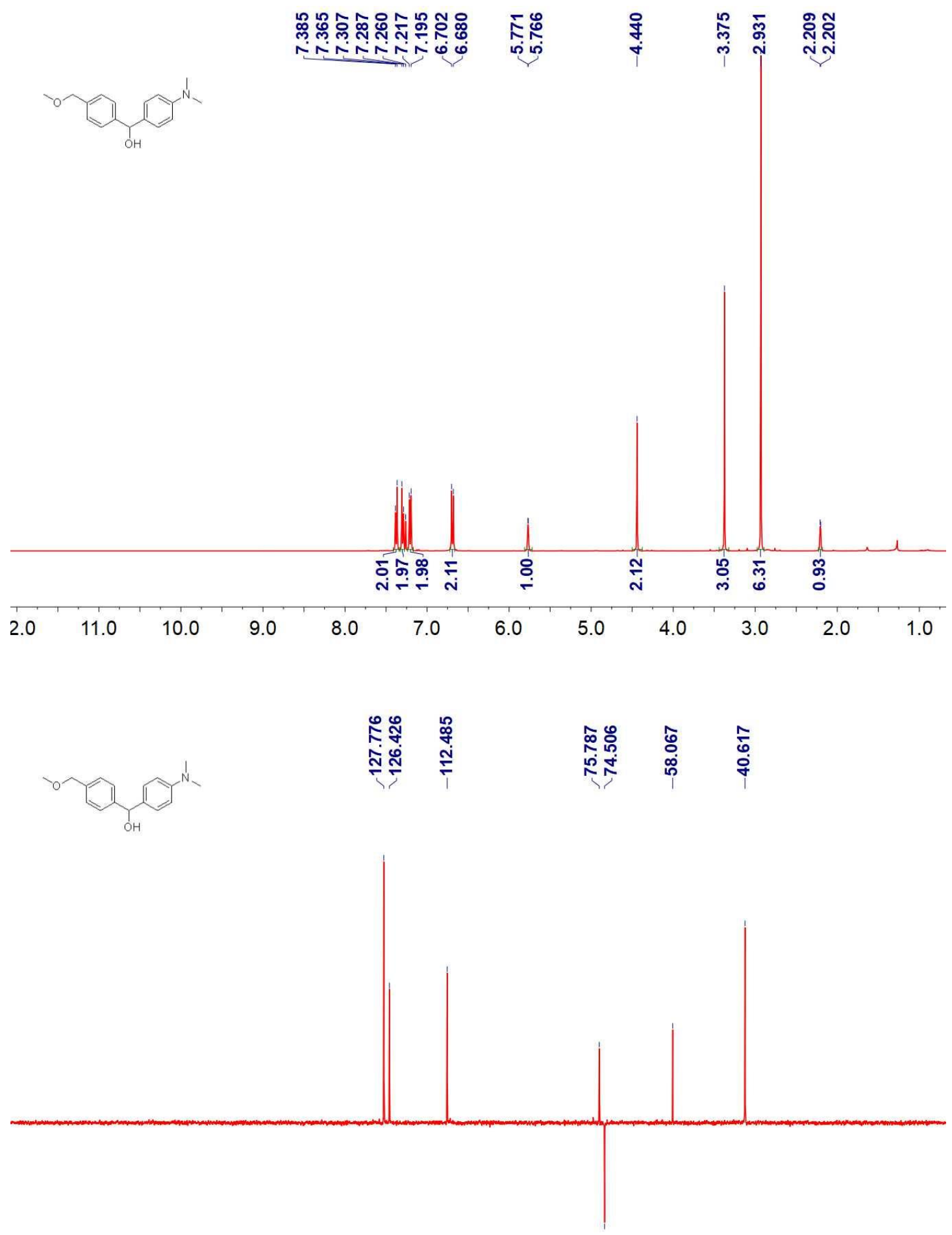

$210200190180170160150140130120110100 \quad 90 \quad 80 \quad 70 \quad 60 \quad 50 \quad 40 \quad 30 \quad 20 \quad 10 \quad 0$ 
(4-((Dimethylamino)methyl)phenyl)(4-(dimethylamino)phenyl)methanol (1aq)
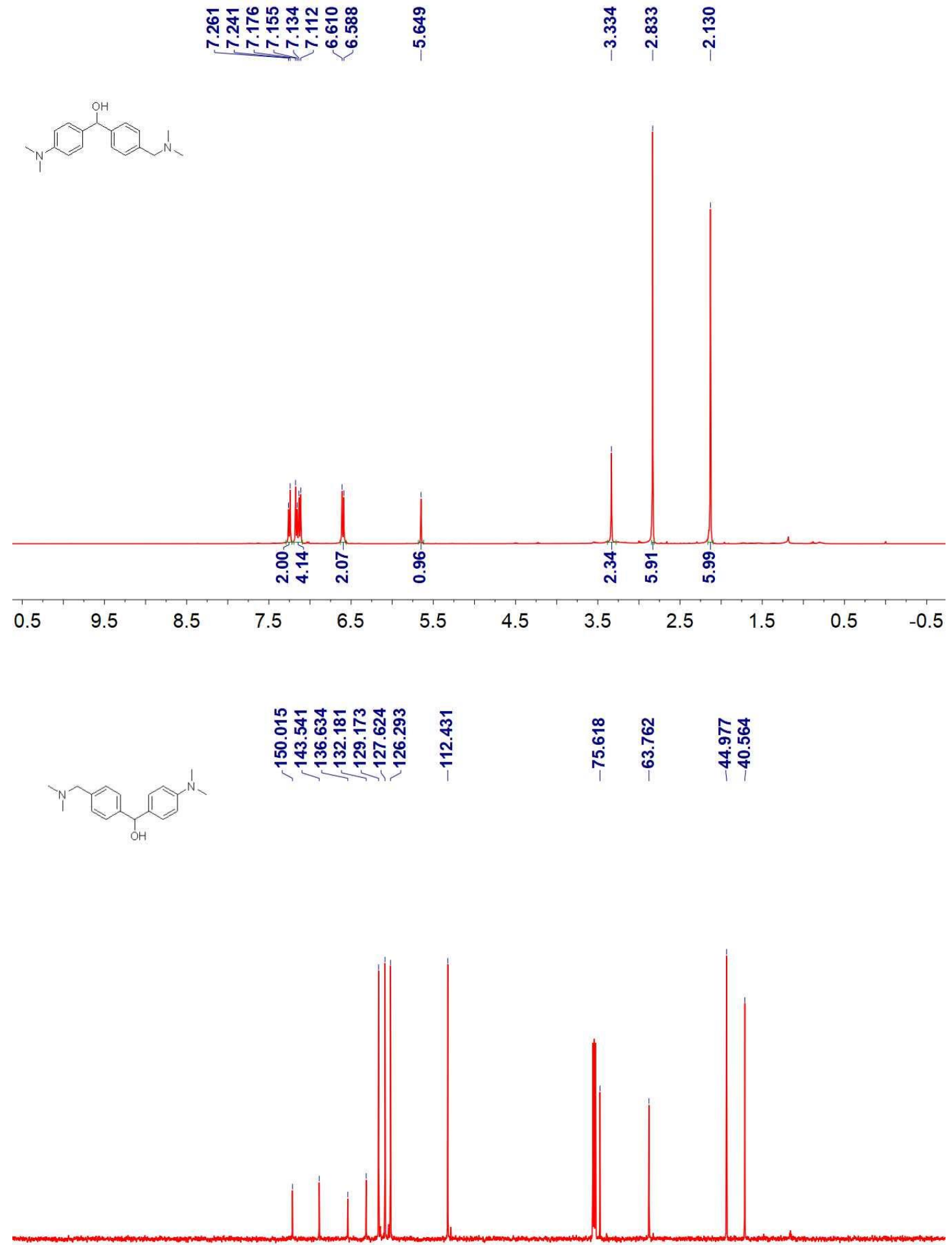

$210200190180170160150140130120110100 \quad 90 \quad 80 \quad 70 \quad 60 \quad 50 \quad 40 \quad 30 \quad 20 \quad 10 \quad 0$ 
1-(4-(Dimethylamino)phenyl)-3-phenylprop-2-yn-1-ol (1ar)

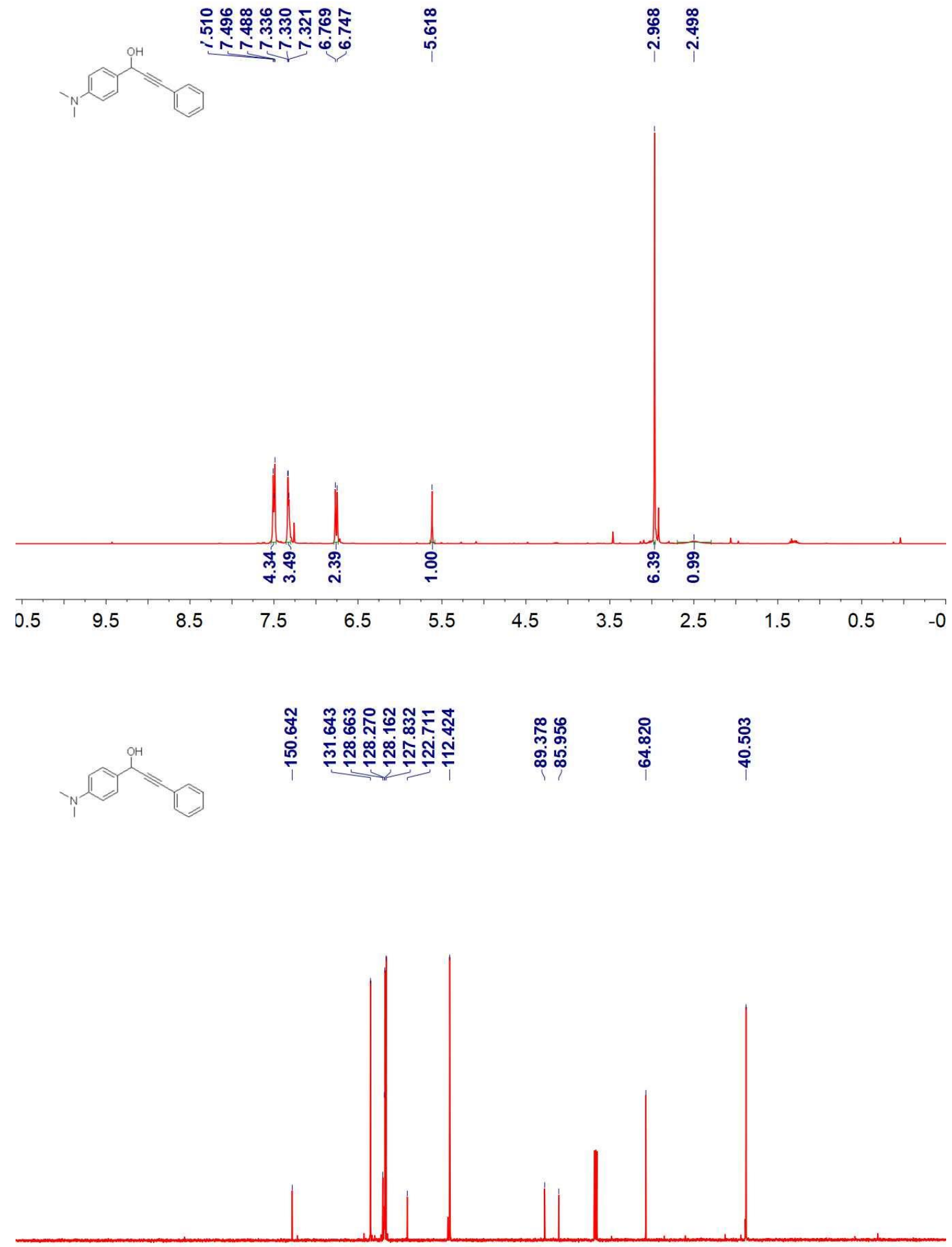

$210200190180170160150140130120110100 \quad 90 \quad 80 \quad 70 \quad 60 \quad 50 \quad 40 \quad 30 \quad 20 \quad 10 \quad 0$ 
(E)-1-(4-(Dimethylamino)phenyl)-3-phenylprop-2-en-1-ol (1as)
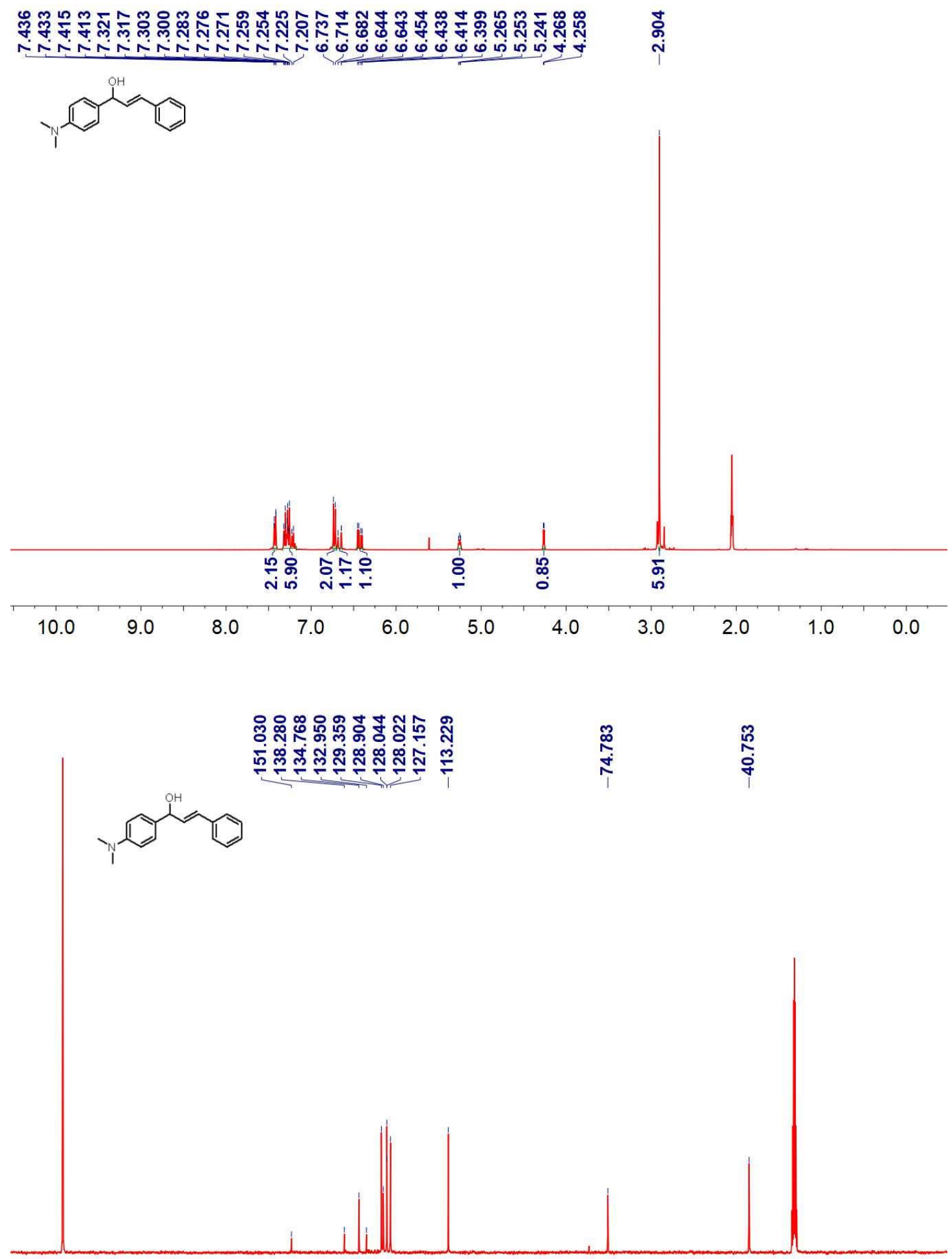

$210200190180170160150140130120110100 \quad 90 \quad 80 \quad 70 \quad 60 \quad 50 \quad 40 \quad 30 \quad 20 \quad 10 \quad 0$ 


\section{4-((4-(Dimethylamino)phenyl)(hydroxy)methyl)phenol (1at)}

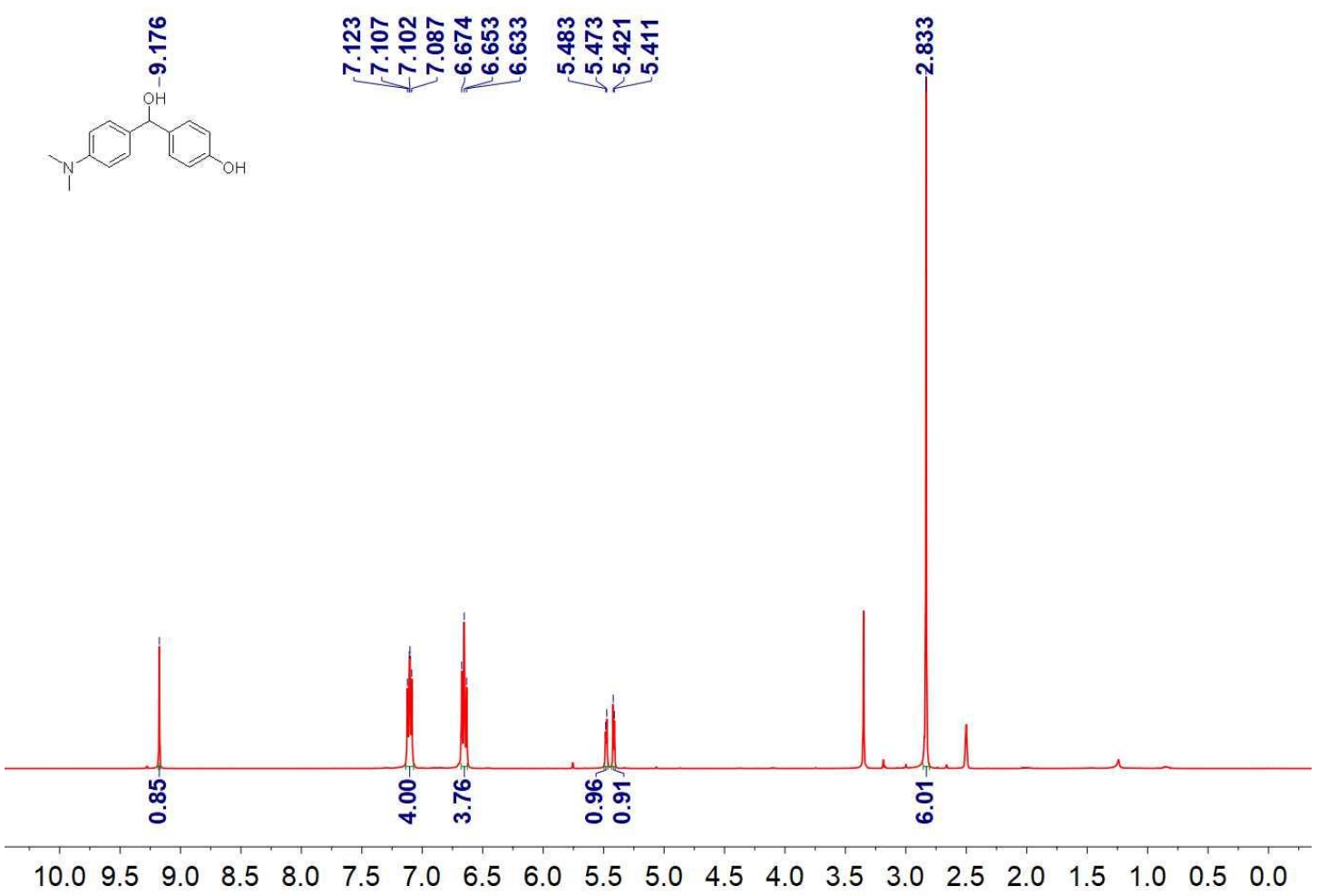

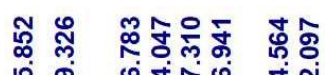

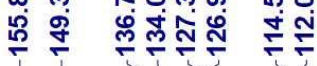

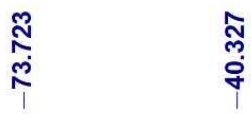

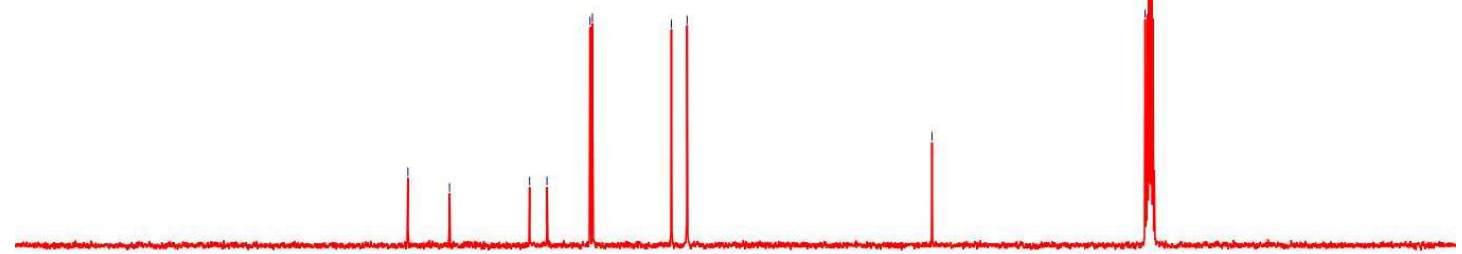

$210200190180170160150140130120110100 \quad 90 \quad 80 \quad 70 \quad 60 \quad 50 \quad 40 \quad 30 \quad 20 \quad 10 \quad 0$ 
(4-(Dimethylamino)phenyl)(4-(hydroxymethyl)phenyl)methanol (1au)
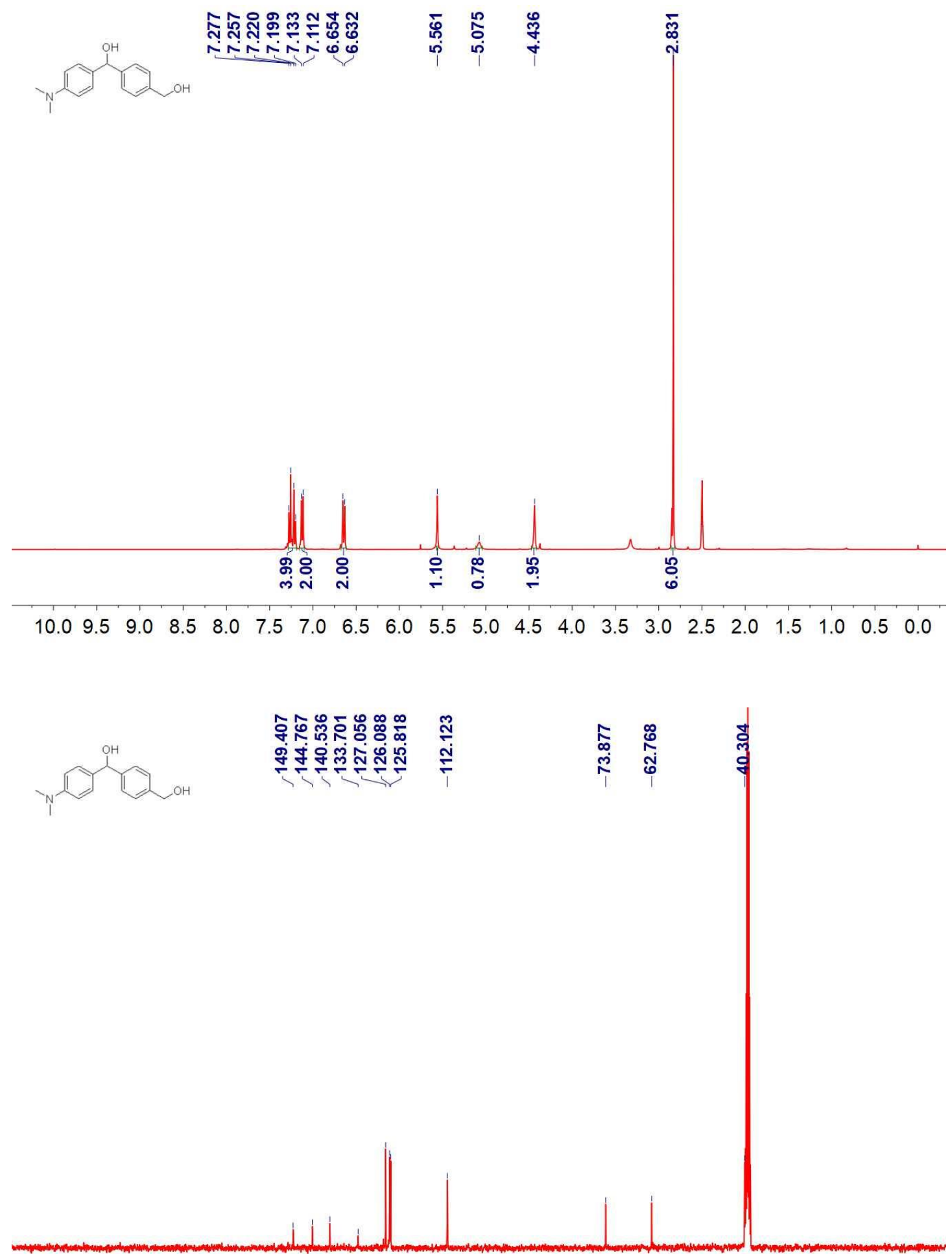

$210200190180170160150140130120110 \quad 100 \quad 90 \quad 80 \quad 70 \quad 60 \quad 50 \quad 40 \quad 30 \quad 20 \quad 10 \quad 0$ 
2-(4-(Dimethylamino)phenyl)propan-2-ol (3a)

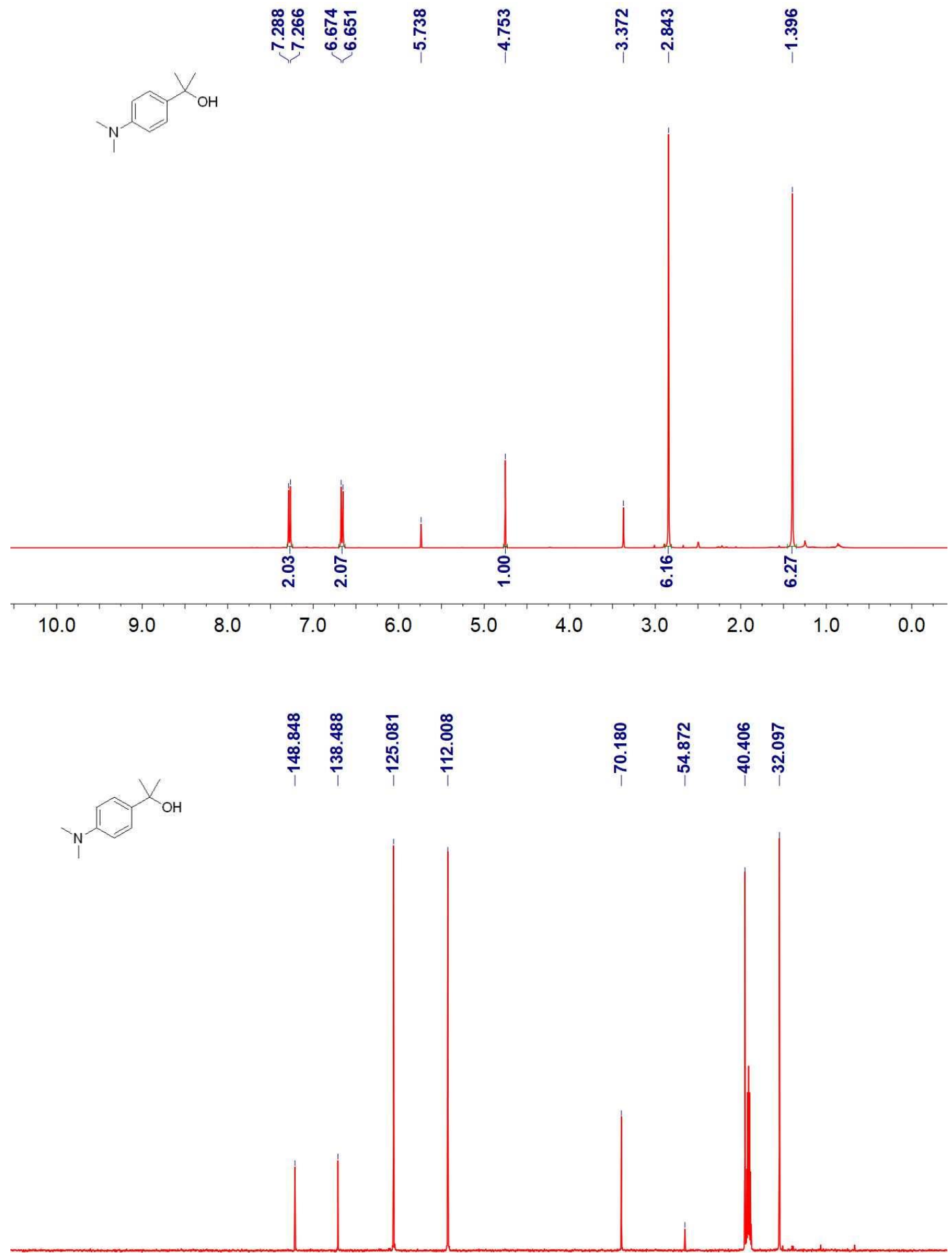

$21020019018017016015014013012011010090 \quad 80 \quad 70 \quad 60 \quad 50 \quad 40 \quad 30 \quad 20 \quad 10 \quad 0$ 
1-(4-(Dimethylamino)phenyl)-1-phenylethan-1-ol (3b)
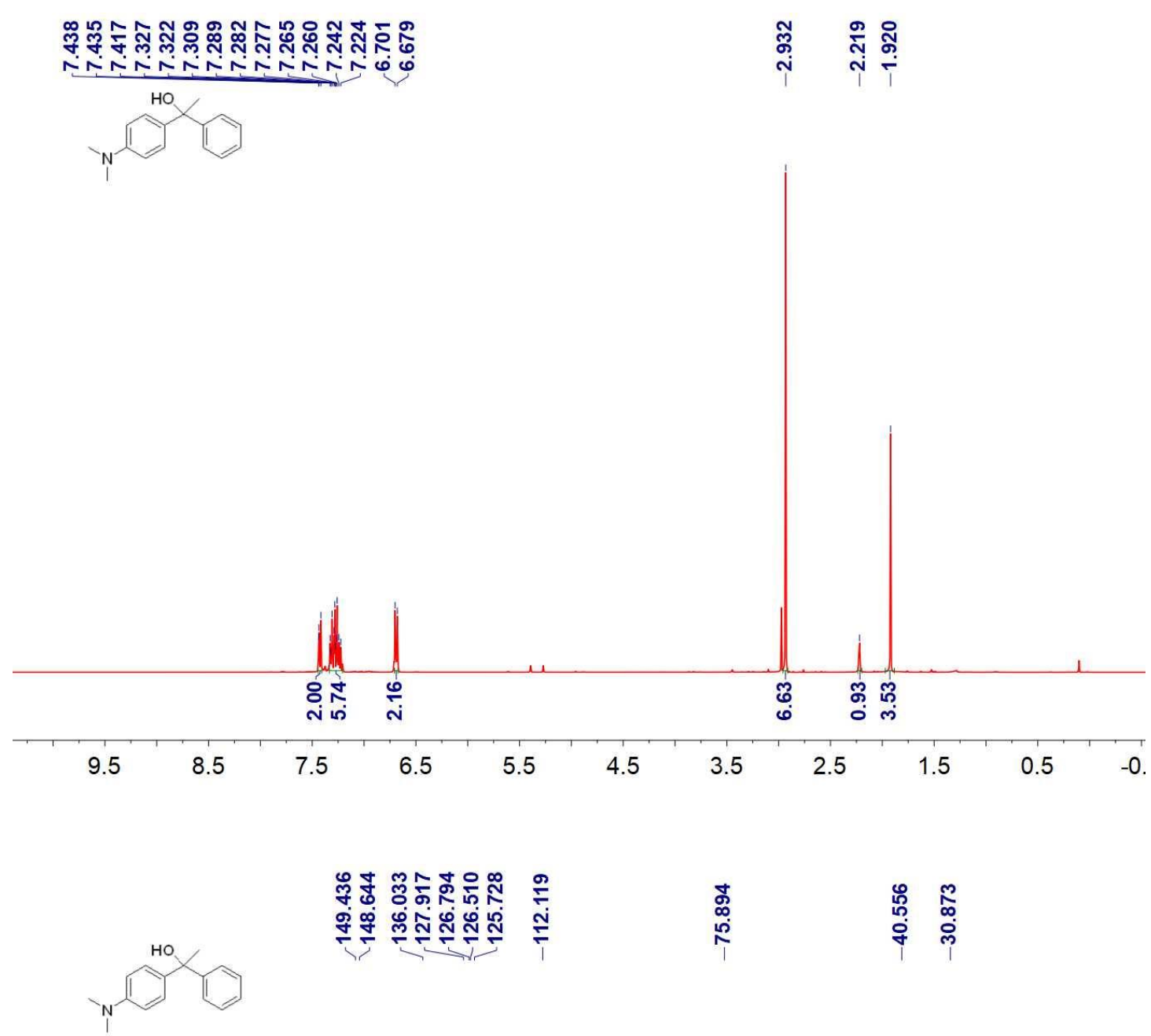

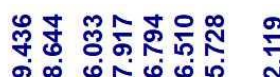

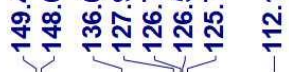

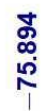

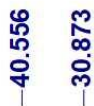

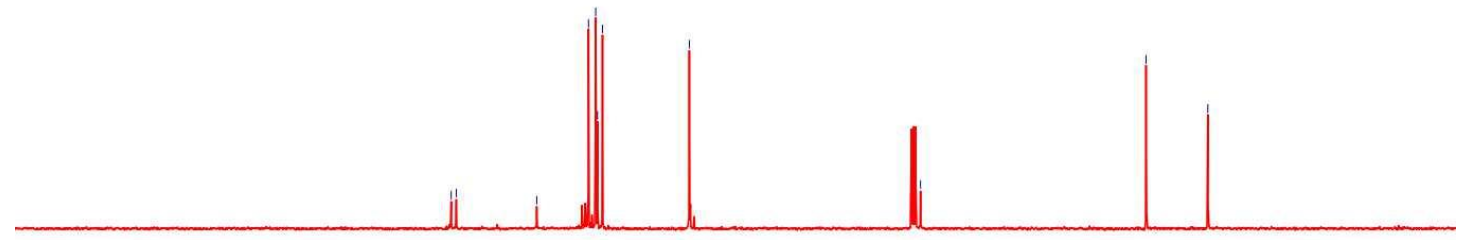

$210200190180170160150140130120110100 \quad 90 \quad 80 \quad 70 \quad 60 \quad 50 \quad 40 \quad 30 \quad 20 \quad 10 \quad 0$ 
1-Cyclopropyl-1-(4-(dimethylamino)phenyl)ethan-1-ol (3c)

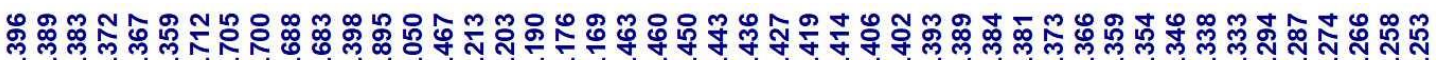

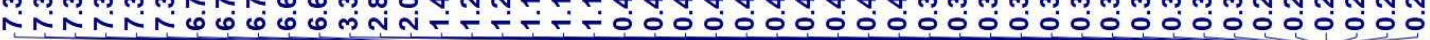<smiles>CN(C)c1ccc(C(C)(C)O)cc1</smiles>
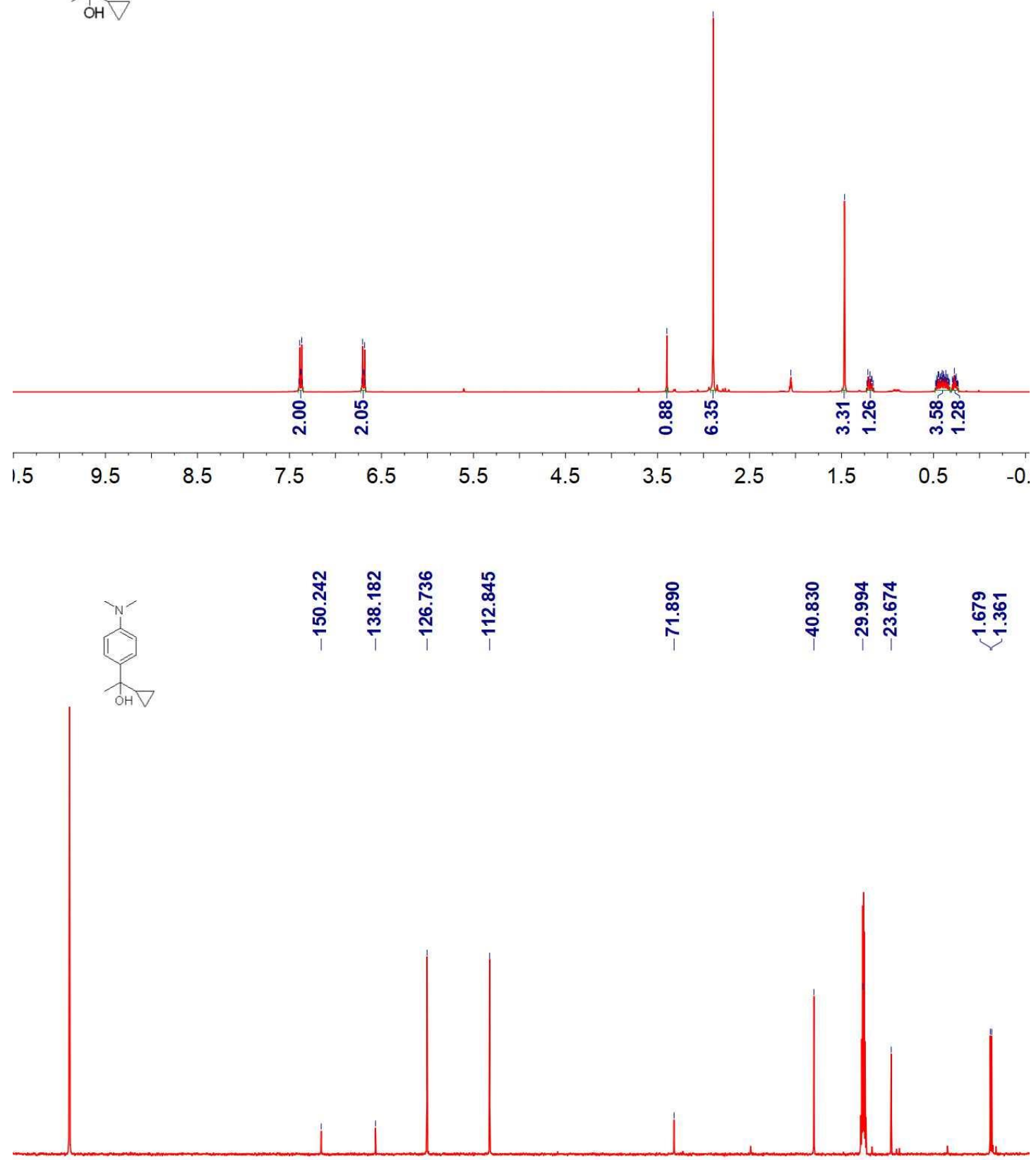

$210200190180170160150140130120110100 \quad 90 \quad 80 \quad 70 \quad 60 \quad 50 \quad 40 \quad 30 \quad 20 \quad 10 \quad 0$ 
2-(4-(Dimethylamino)phenyl)-3,3-dimethylbutan-2-ol (3d)
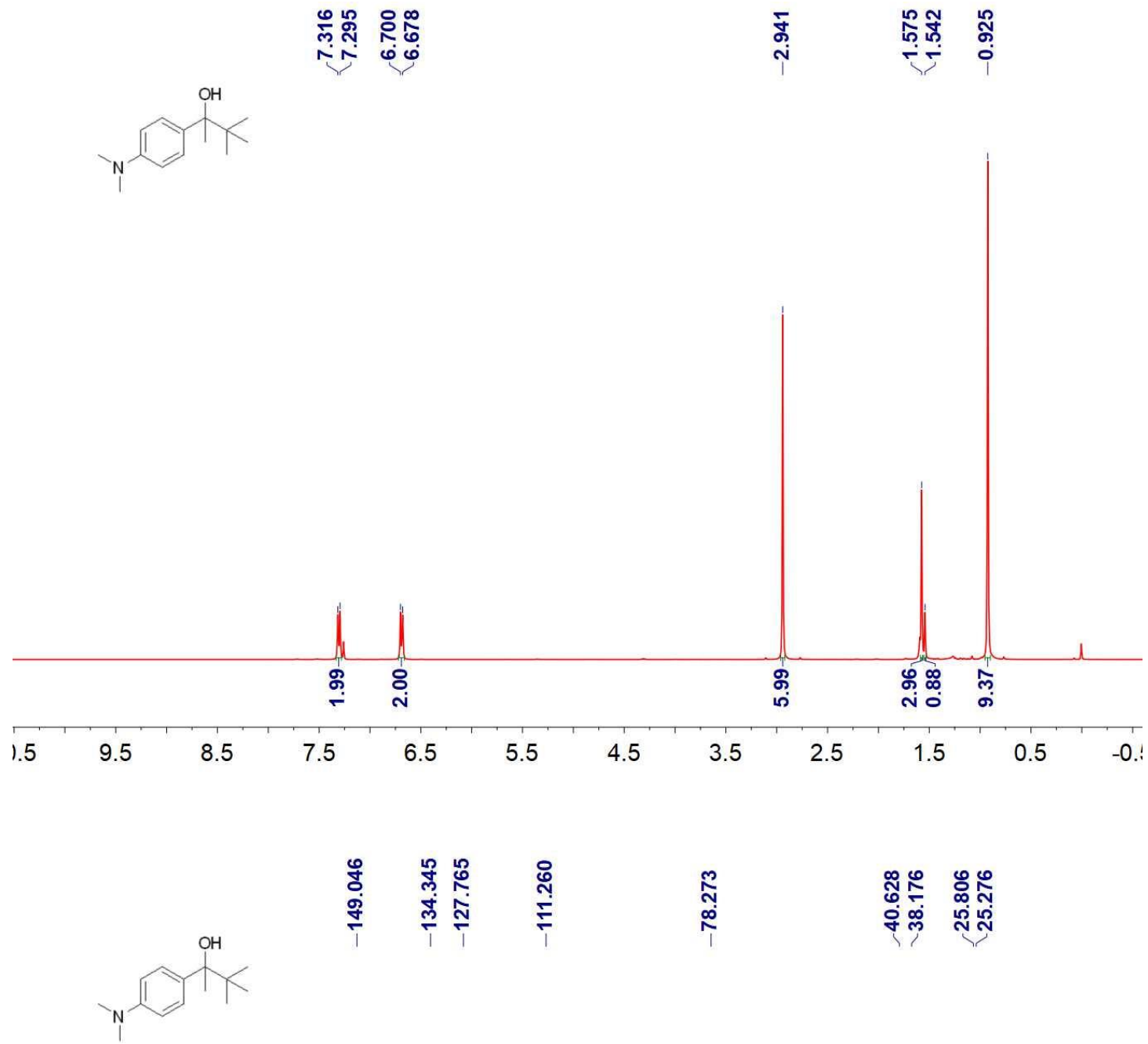

\begin{tabular}{|c|c|c|c|}
\hline 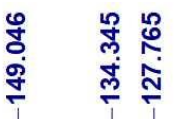 & 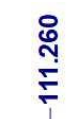 & 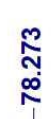 & 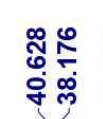 \\
\hline
\end{tabular}

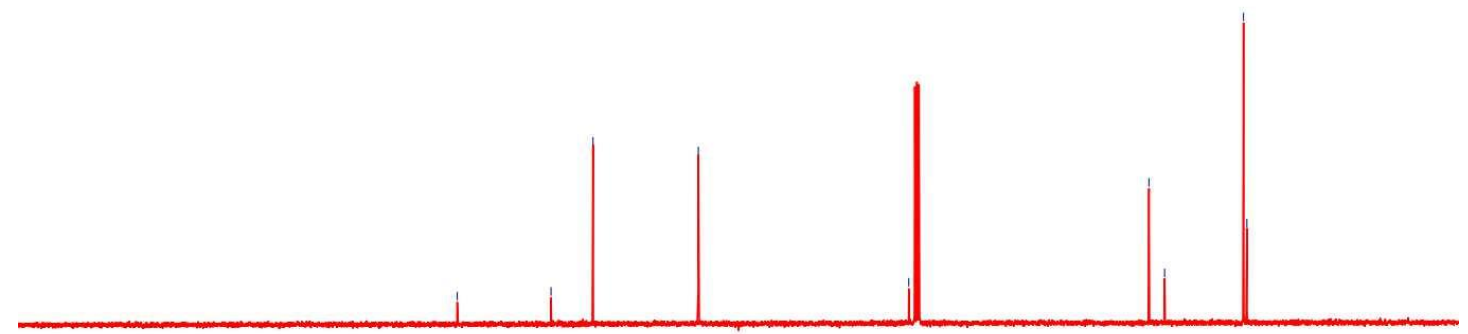

$210200190180170160150140130120110100 \quad 90 \quad 80 \quad 70 \quad 60 \quad 50 \quad 40 \quad 30 \quad 20 \quad 10 \quad 0$ 
1-(4-(Dimethylamino)phenyl)-2-((1,1,1,3,3,3-hexafluoropropan-2-yl)oxy)-1-phenylet han-1-ol (3e)
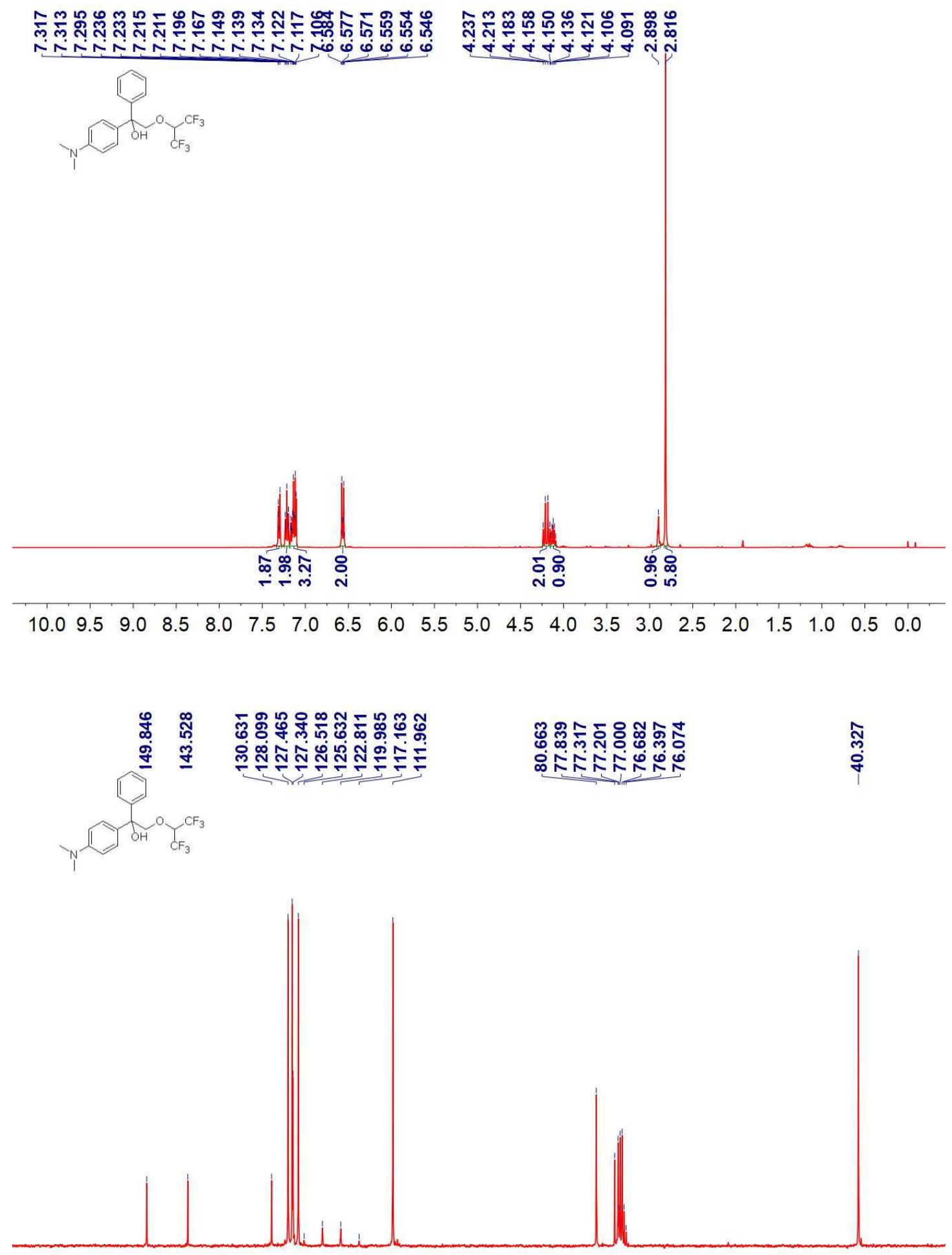

$\begin{array}{lllllllllllllllllll}70 & 160 & 150 & 140 & 130 & 120 & 110 & 100 & 90 & 80 & 70 & 60 & 50 & 40 & 30\end{array}$ 
1-(4-(Dimethylamino)phenyl)-2-phenoxy-1-phenylethan-1-ol (3f)

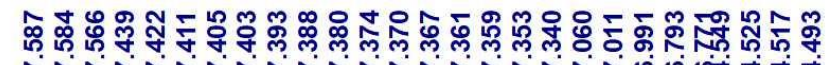

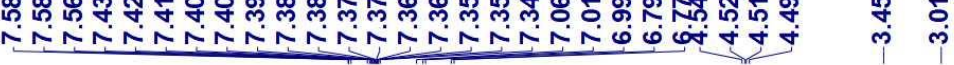

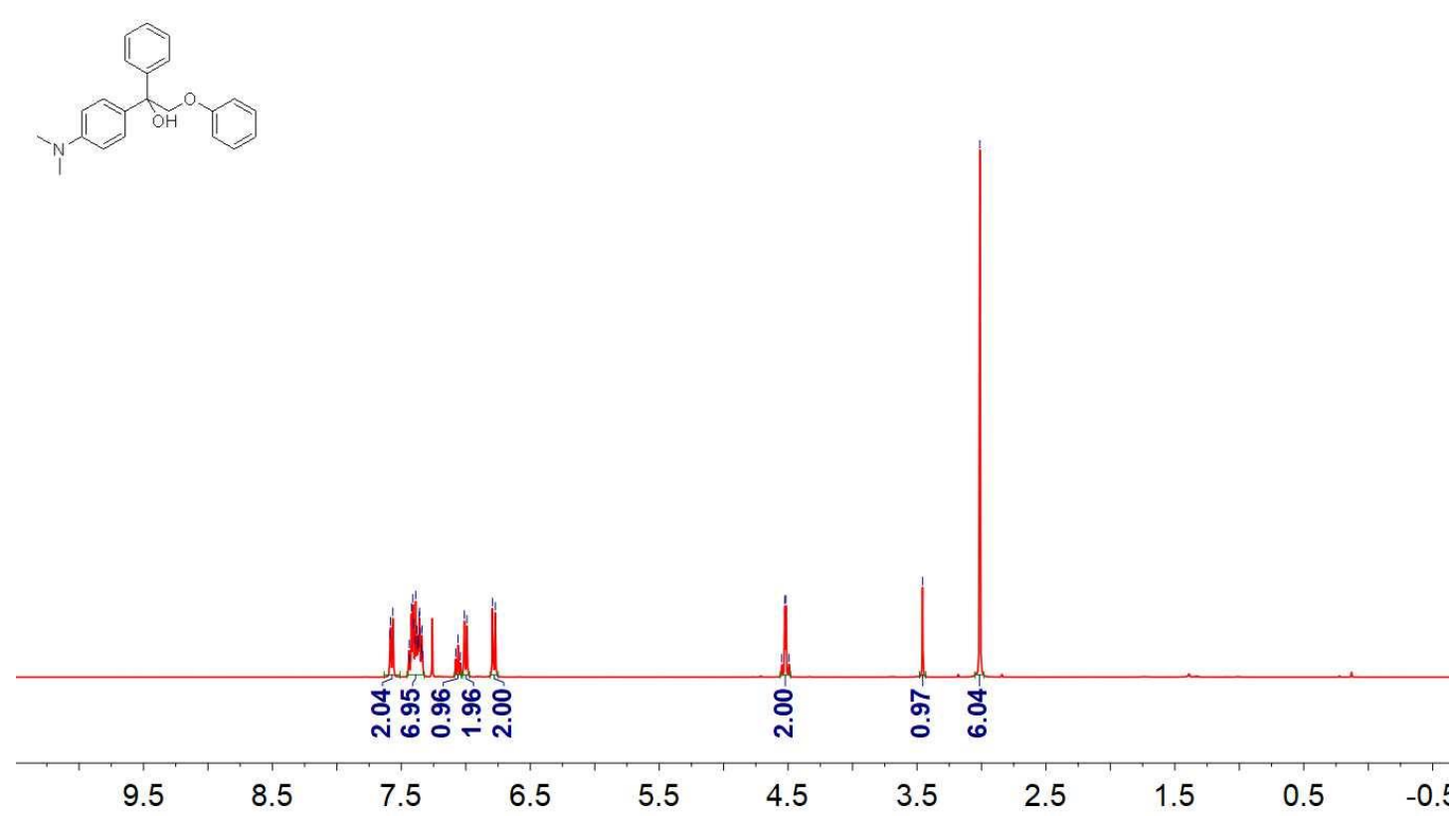

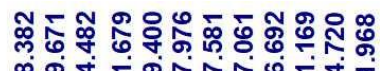

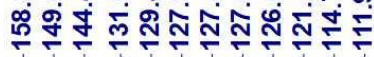

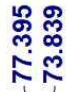

\%
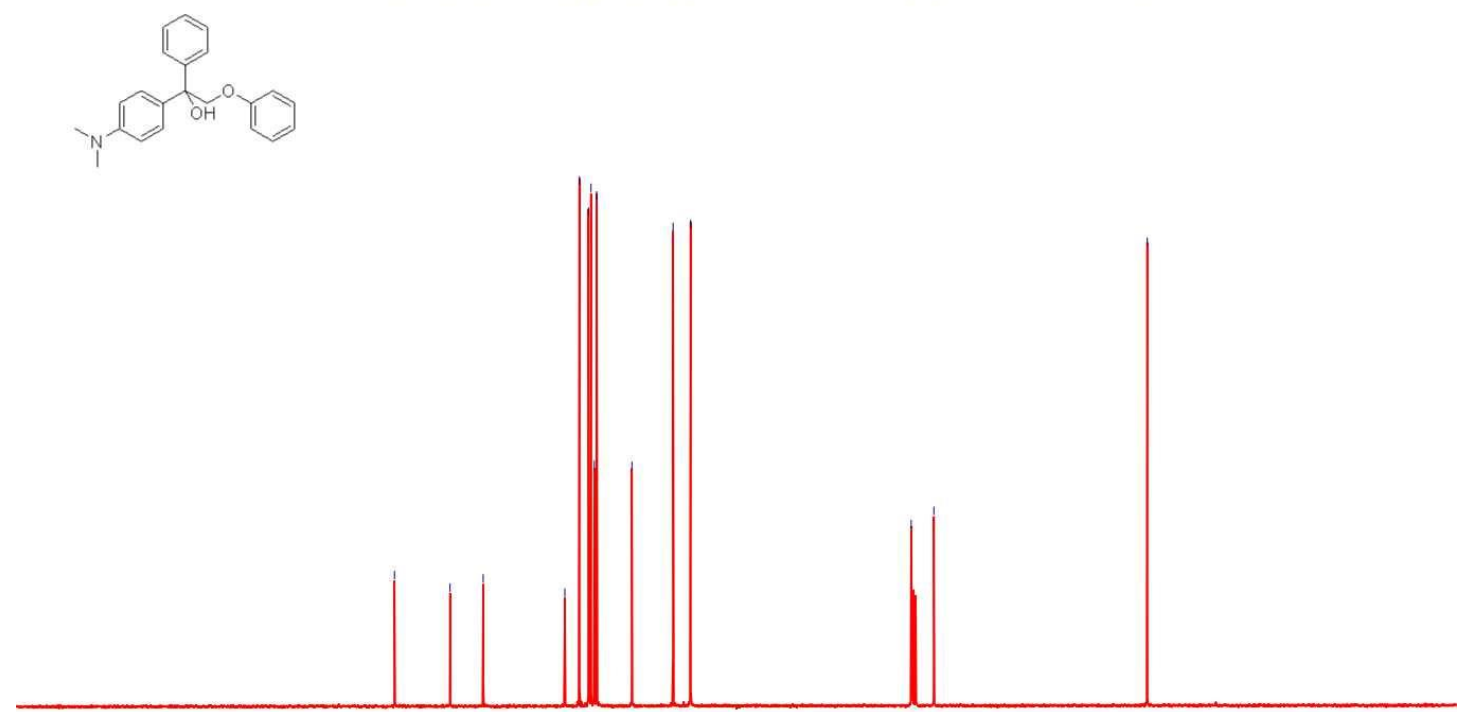

$210200190180170160150140130120110100 \quad 90 \quad 80 \quad 70 \quad 60 \quad 50 \quad 40 \quad 30 \quad 20 \quad 10 \quad 0$ 
1-(4-(Dimethylamino)phenyl)cyclobutan-1-ol (3g)

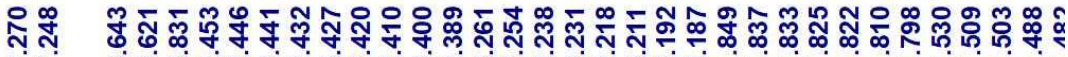
$\overbrace{}^{\text {HO }}$

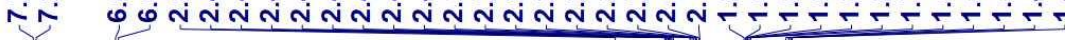
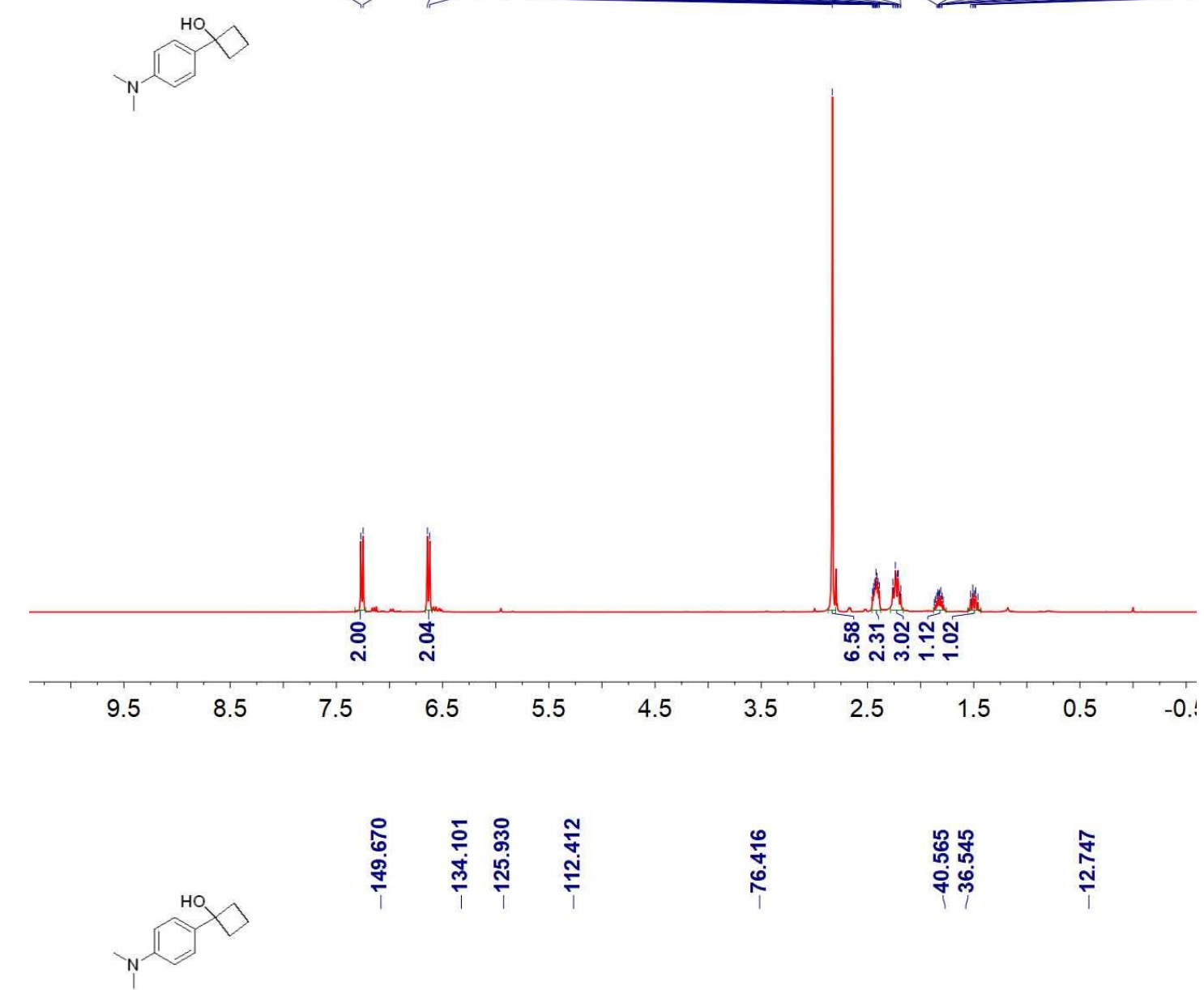

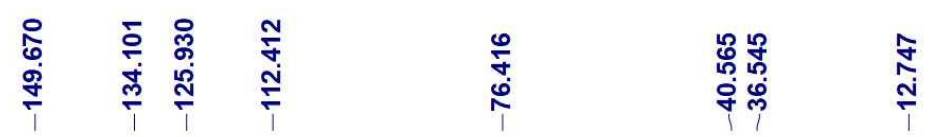

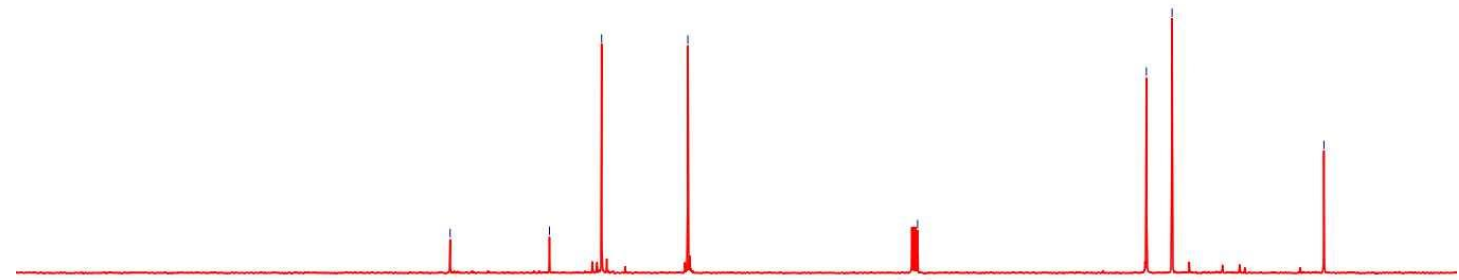

$210200190180170160150140130120110100 \quad 90 \quad 80 \quad 70 \quad 60 \quad 50 \quad 40 \quad 30 \quad 20 \quad 10 \quad 0$ 
1-(4-(Dimethylamino)phenyl)cyclopentan-1-ol (3h)

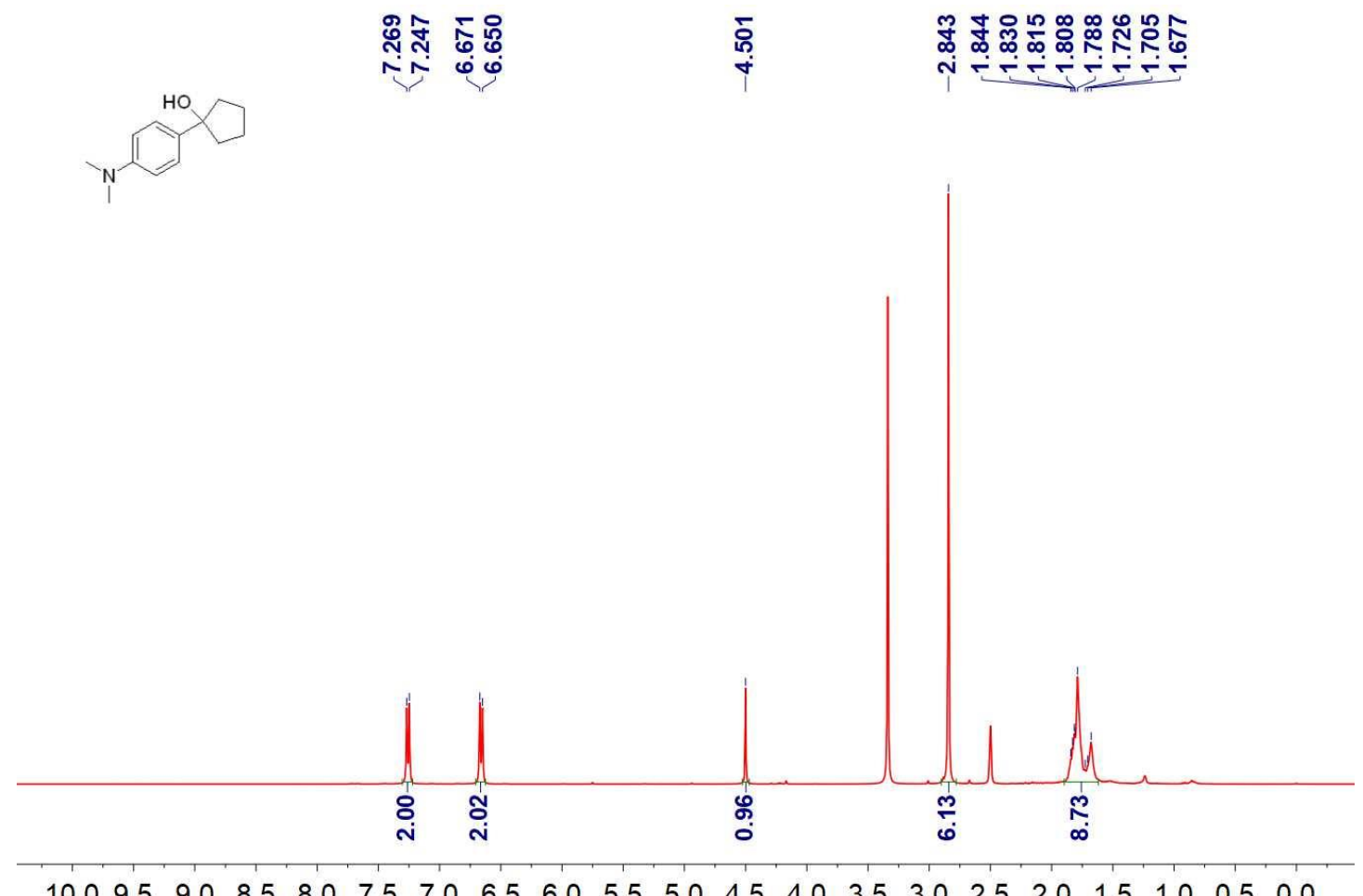

$\begin{array}{lllllllllllllllllllll}10.0 & 9.5 & 9.0 & 8.5 & 8.0 & 7.5 & 7.0 & 6.5 & 6.0 & 5.5 & 5.0 & 4.5 & 4.0 & 3.5 & 3.0 & 2.5 & 2.0 & 1.5 & 1.0 & 0.5 & 0.0\end{array}$

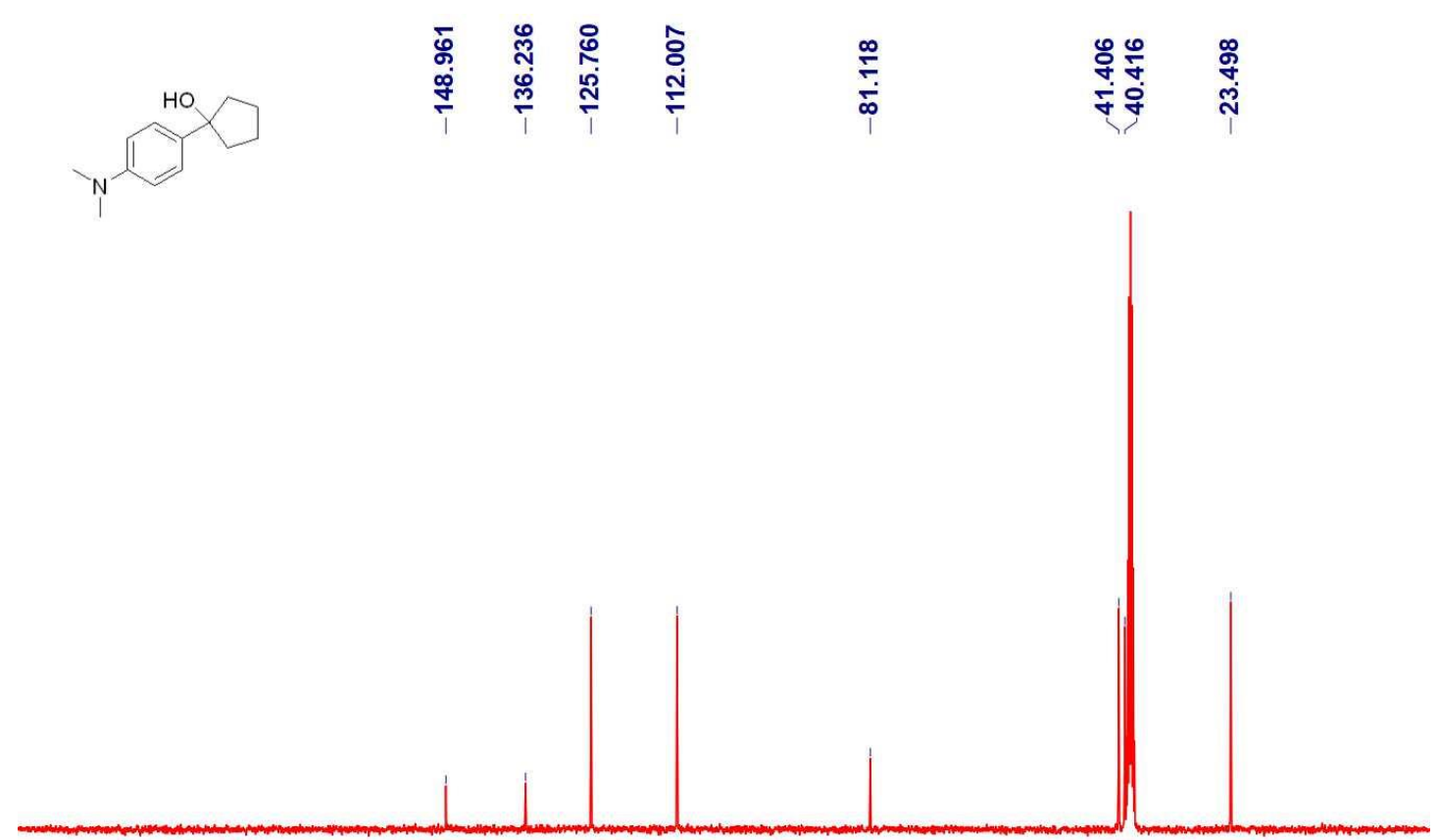

$21020019018017016015014013012011010090 \quad 80 \quad 70 \quad 60 \begin{array}{lllllll}40 & 40 & 30 & 20 & 10 & 0\end{array}$ 
1-(4-(Dimethylamino)phenyl)cyclohexan-1-ol (3i)

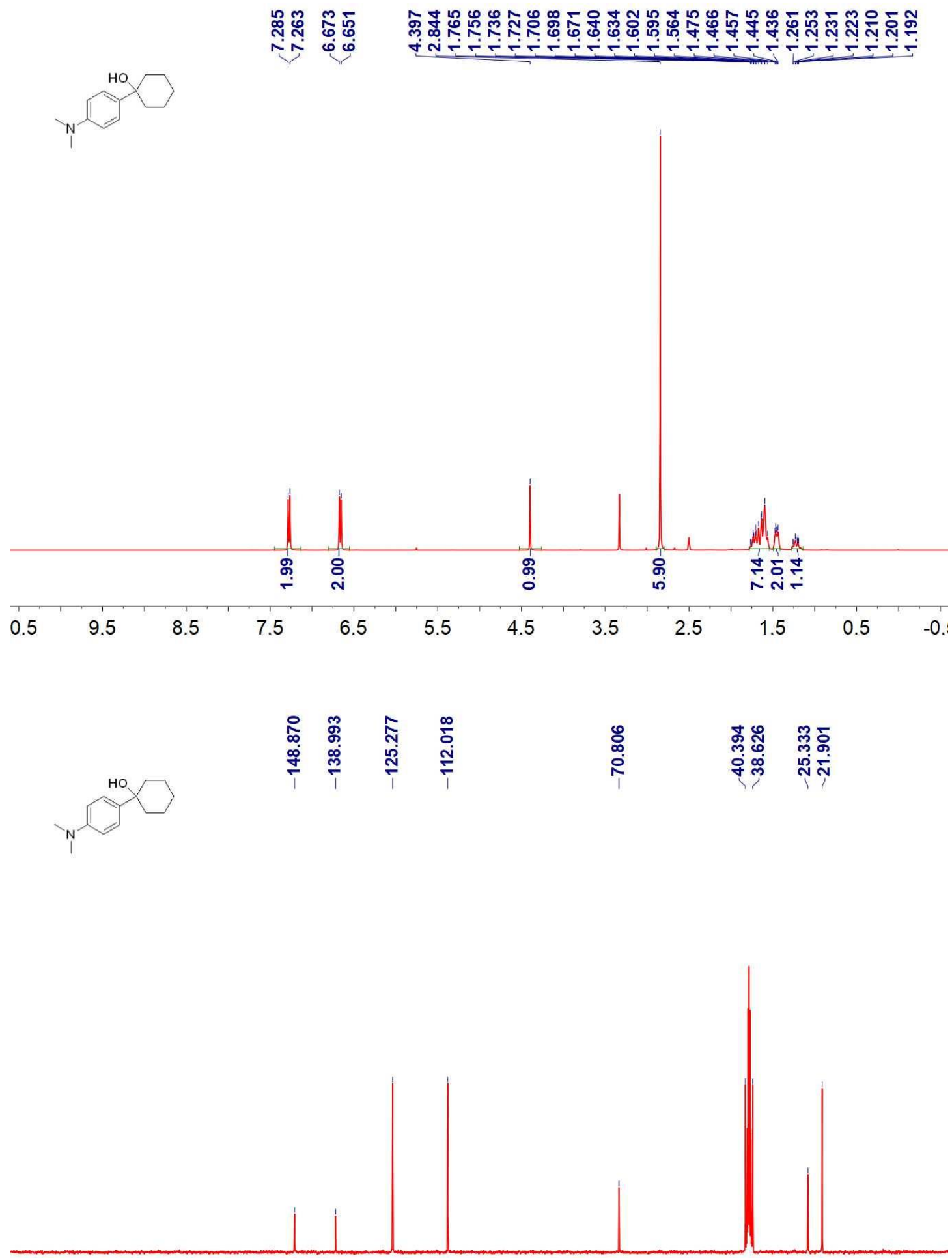

$210200190180170160150140130120110100 \quad 90 \quad 80 \quad 70 \quad 60 \quad 50 \quad 40 \quad 30 \quad 20 \quad 10 \quad 0$ 
1-(4-(Dimethylamino)phenyl)cycloheptan-1-ol (3j)
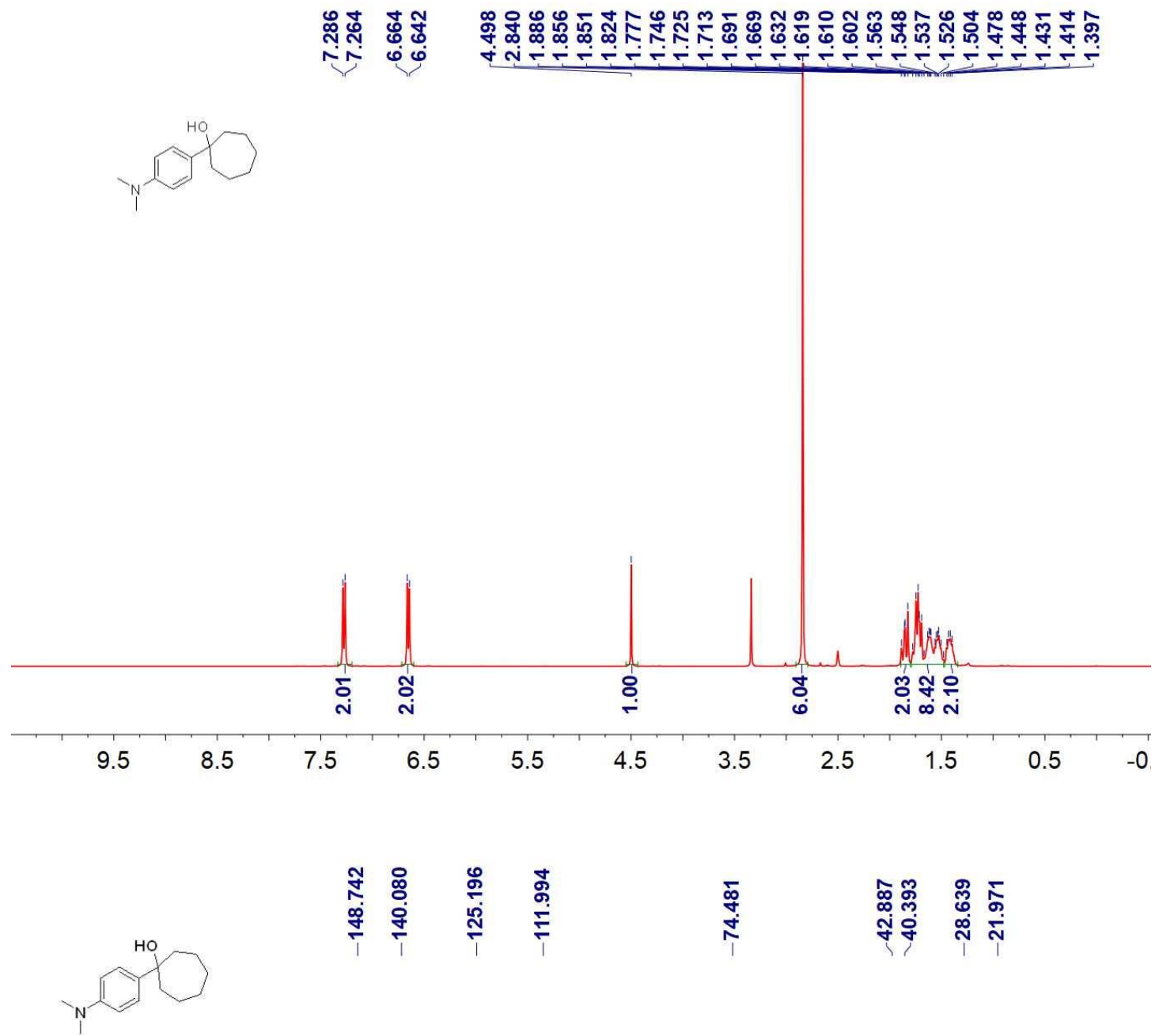

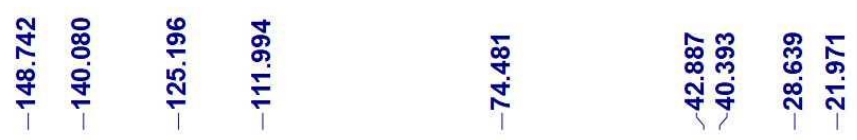

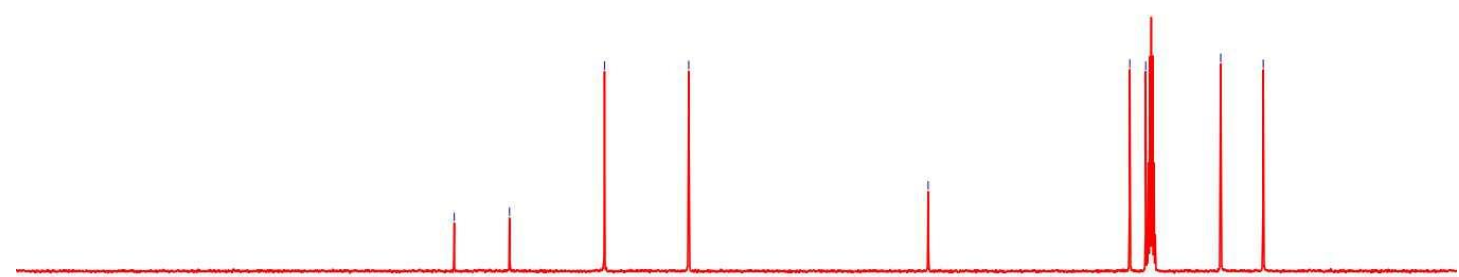

$210200190180170160150140130120110 \quad 100 \quad 90 \quad 80 \quad 70 \quad 60 \quad 50 \quad 40 \quad 30 \quad 20 \quad 10 \quad 0$ 
1-(4-(Dimethylamino)phenyl)cyclododecan-1-ol (3k)
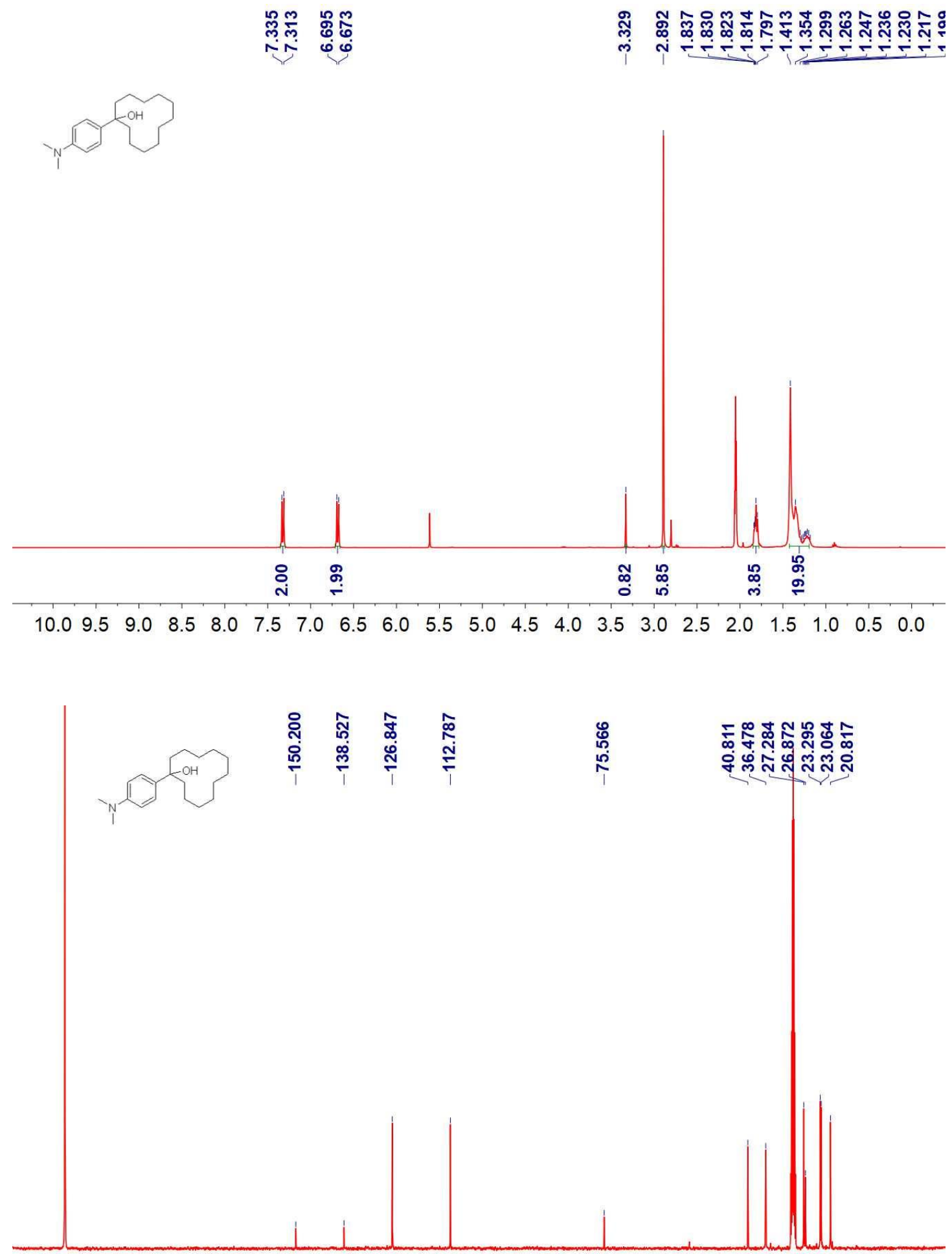

$210200190180170160150140130120110100 \quad 90 \quad 80 \quad 70 \quad 60 \quad 50 \quad 40 \quad 30 \quad 20 \quad 10 \quad 0$ 
9-(4-(Dimethylamino)phenyl)-9H-xanthen-9-ol (3l)

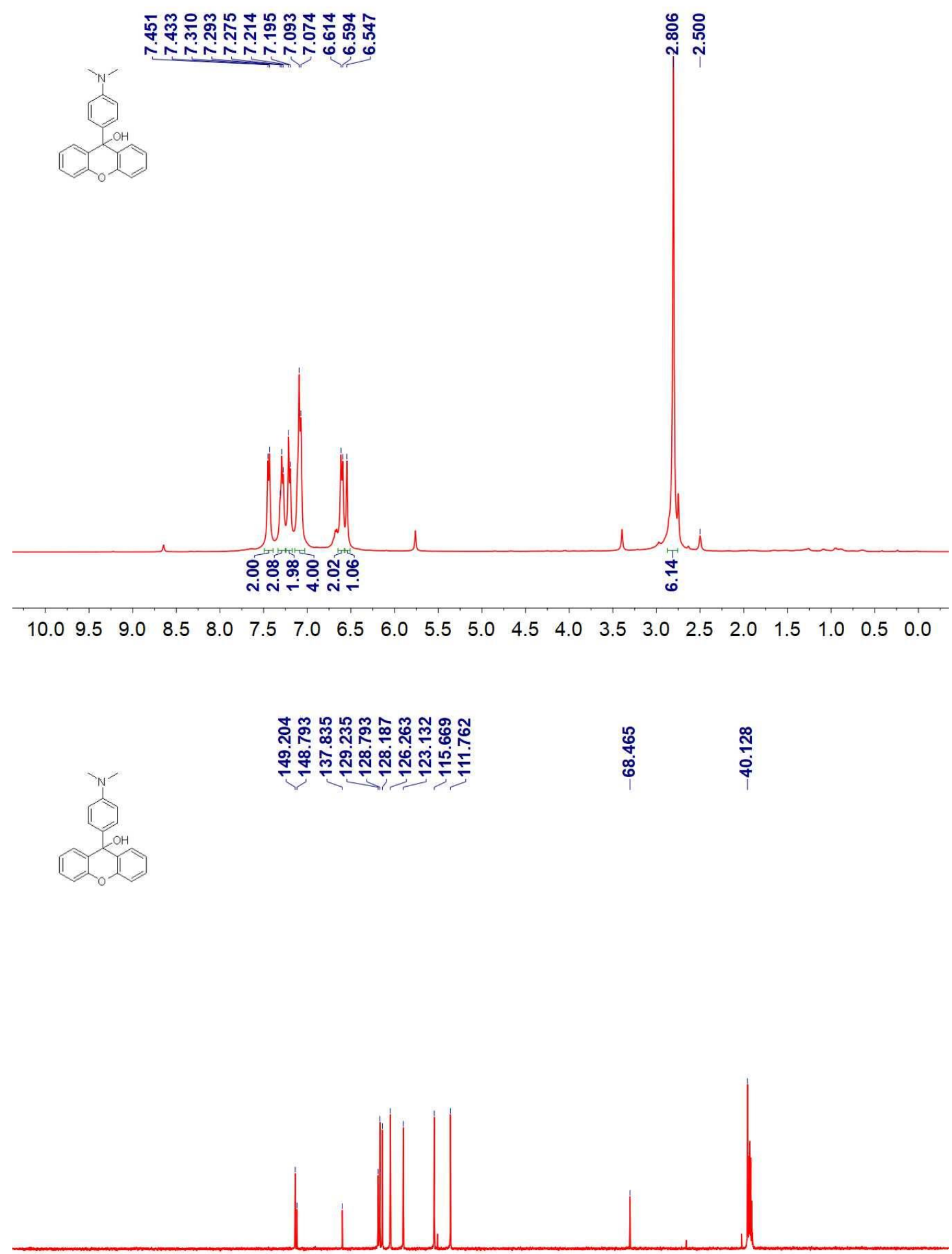

$21020019018017016015014013012011010090 \quad 80 \quad 70 \quad 60 \quad 50 \quad 40 \quad 30 \quad 20 \quad 10 \quad 0$ 
1-(4-(Dimethylamino)phenyl)-1,2,3,4-tetrahydronaphthalen-1-ol (3m)
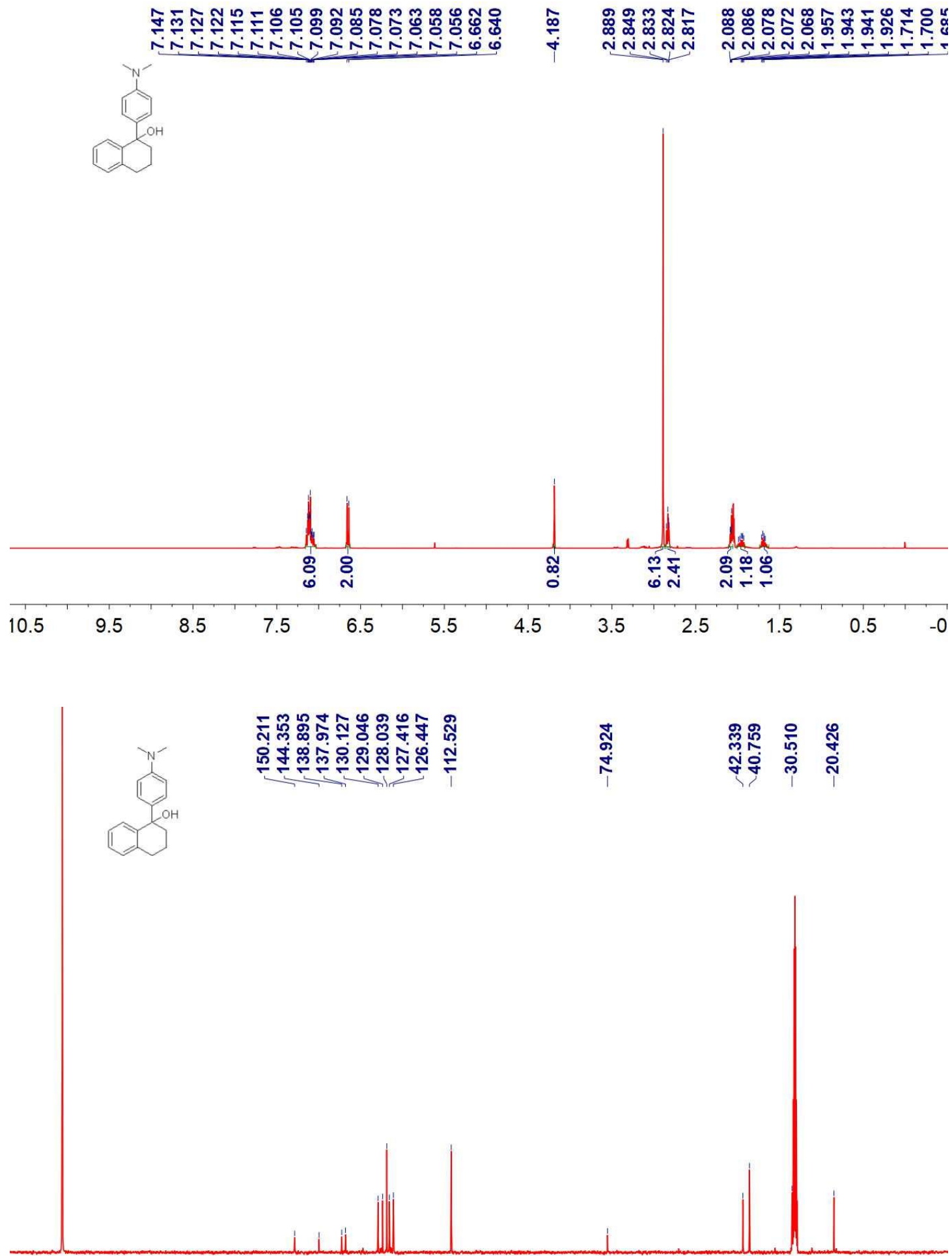

$210200190180170160150140130120110100 \quad 90 \quad 80 \quad 70 \quad 60 \quad 50 \quad 40 \quad 30 \quad 20 \quad 10 \quad 0$ 
2-(4-(Dimethylamino)phenyl)adamantan-2-ol (3na)
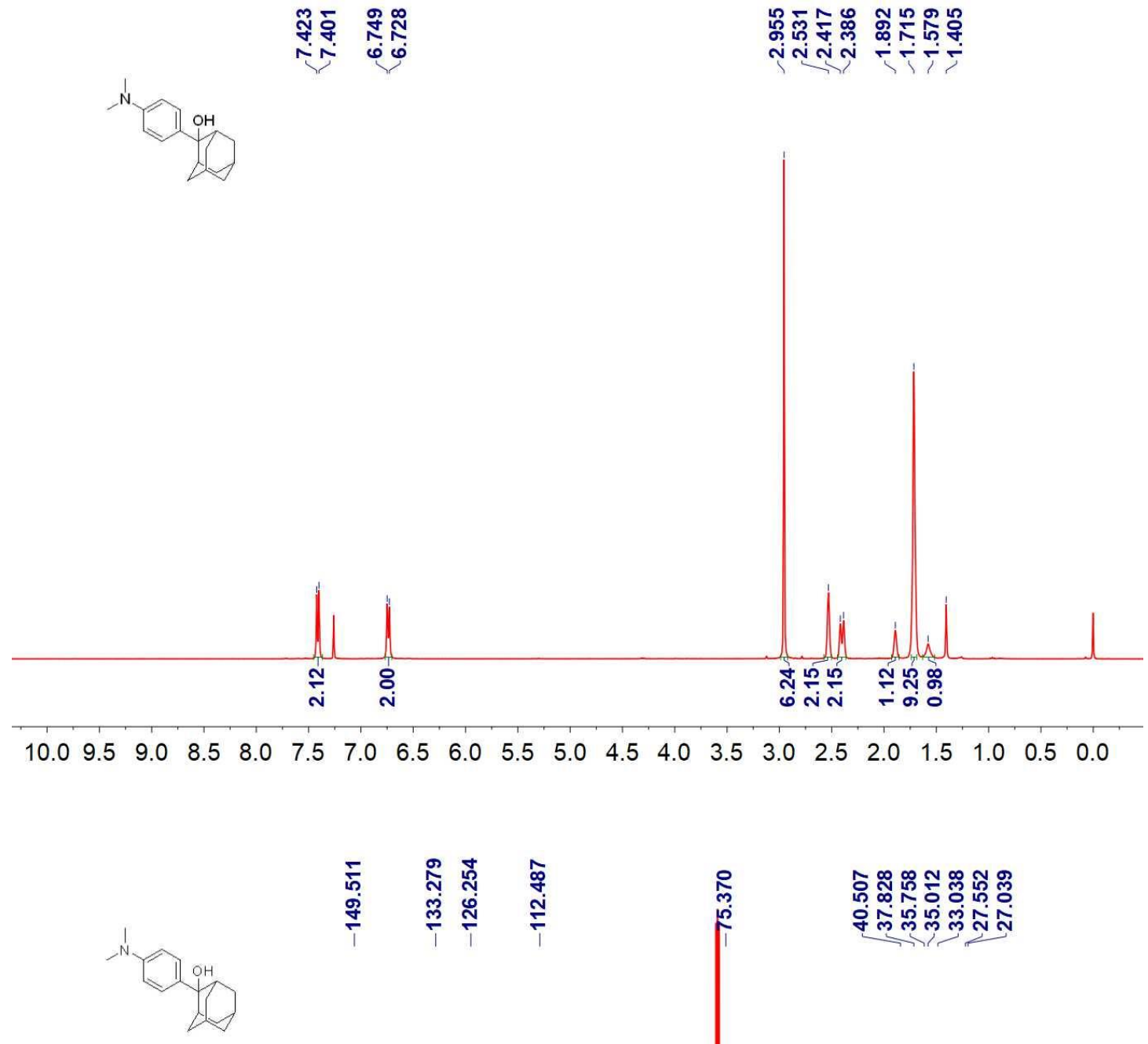

$210200190180170160150140130120110100 \quad 90 \quad 80 \quad 70 \quad 60 \quad 50 \quad 40 \quad 30 \quad 20 \quad 10 \quad 0$ 
2-(4-(Piperidin-1-yl)phenyl)adamantan-2-ol (3nb)

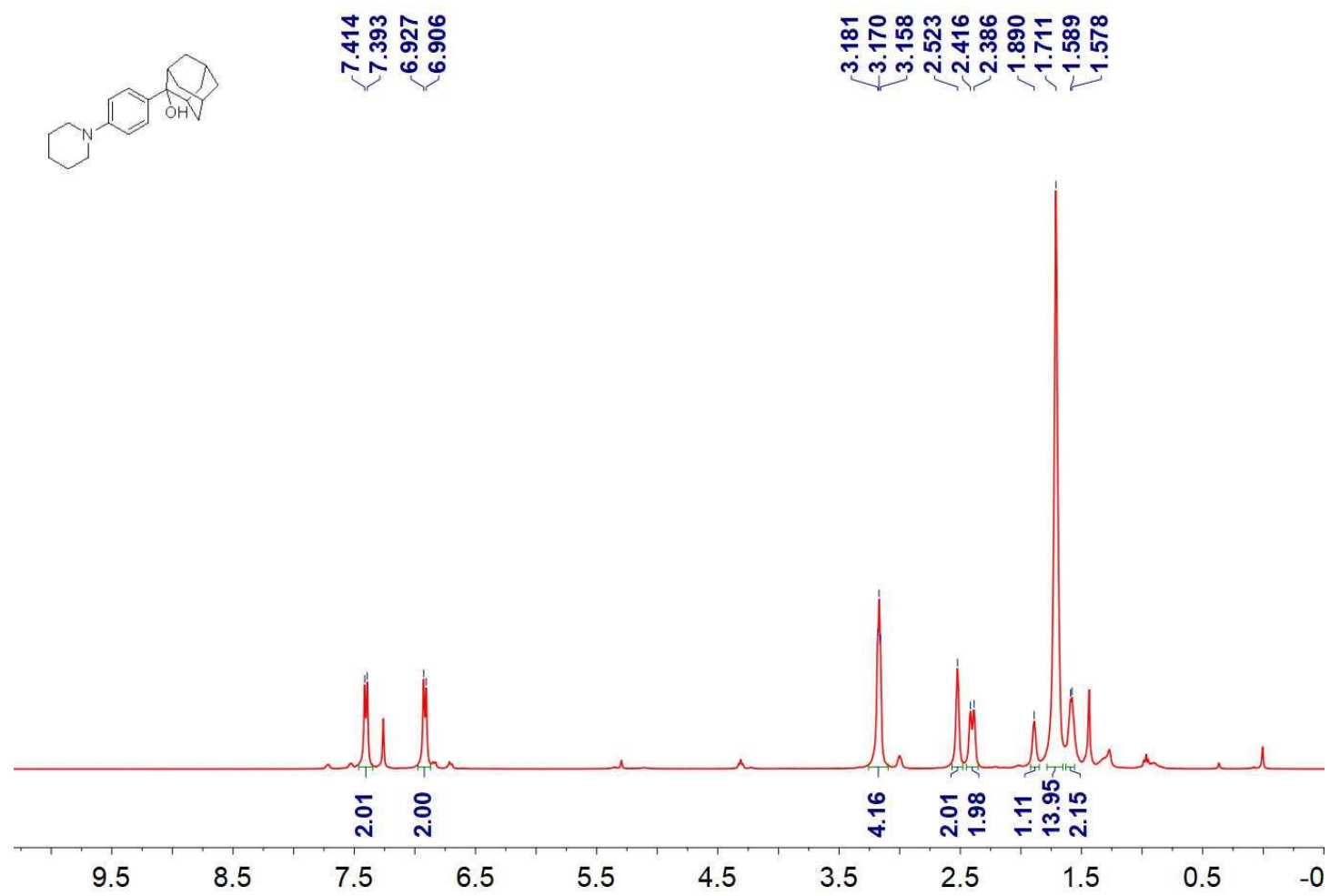

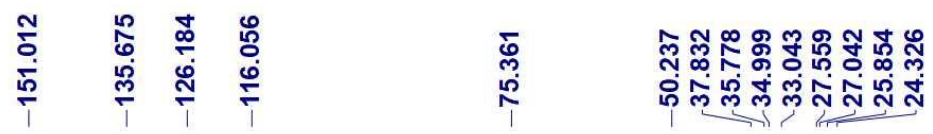

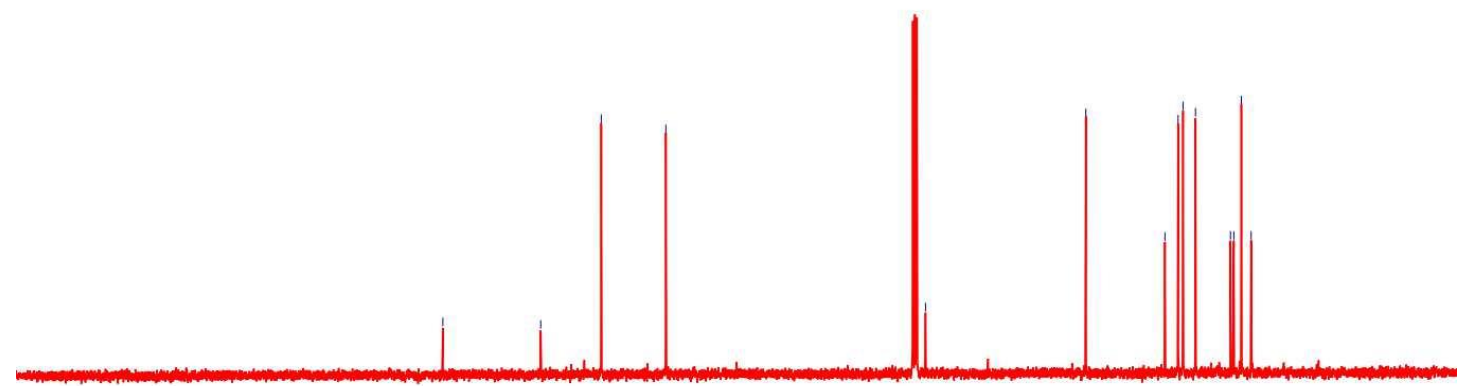

$210200190180170160150140130120110 \quad 100 \quad 90 \quad 80 \quad 70 \quad 60 \quad 50 \quad 40 \quad 30 \quad 20 \quad 10 \quad 0$ 
2-(4-(Dimethylamino)phenyl)-1,7,7-trimethylbicyclo[2.2.1] heptan-2-ol (3o)

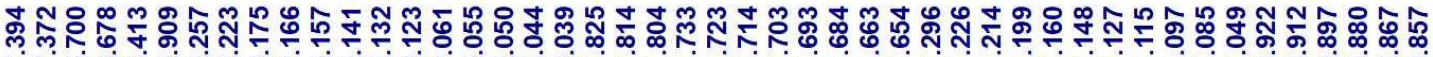

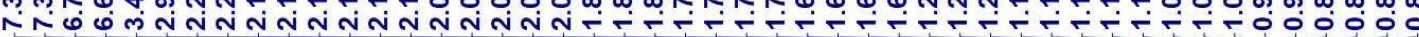

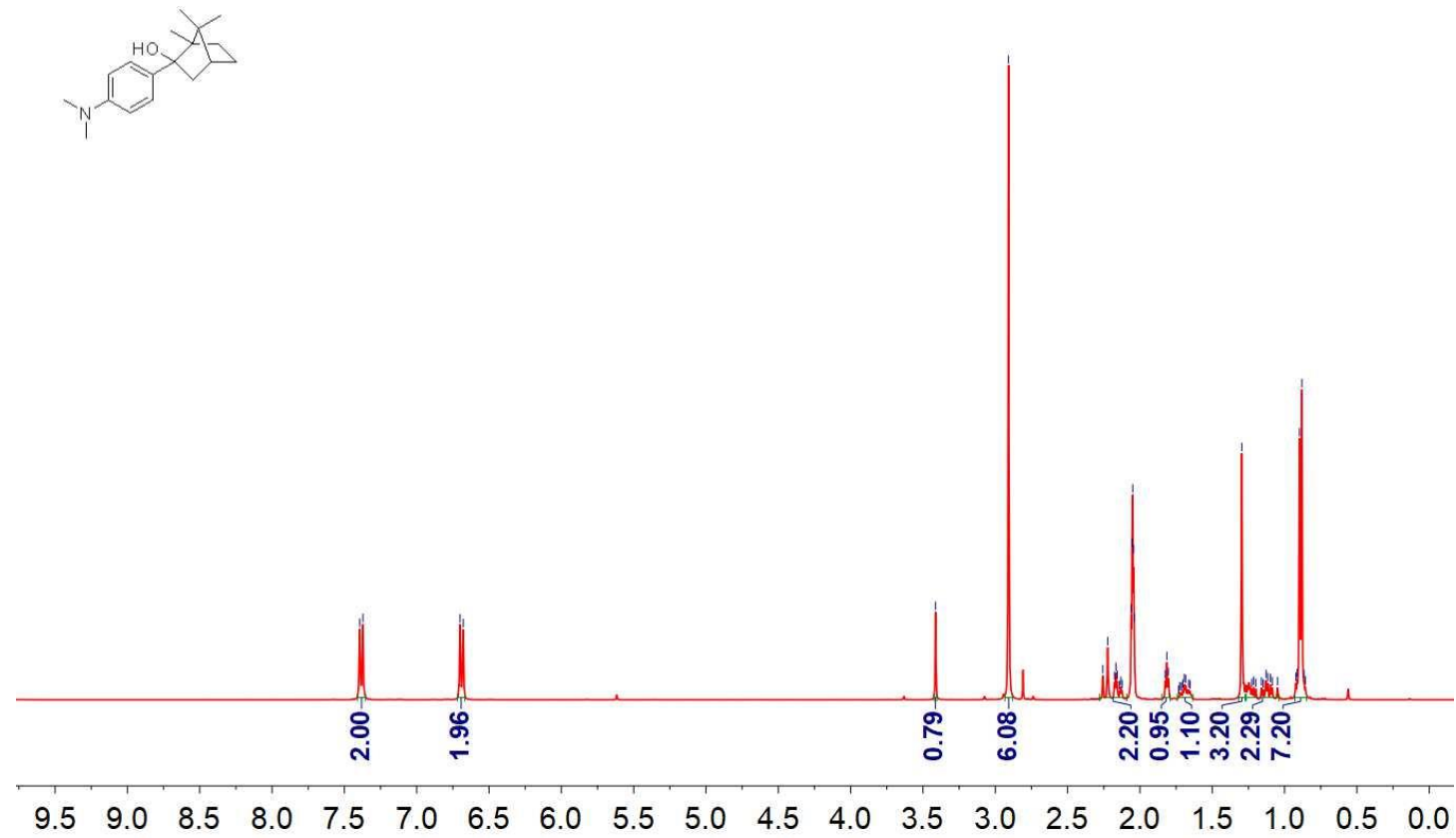

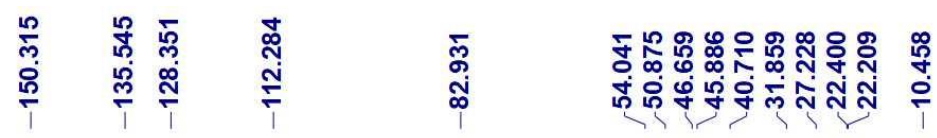

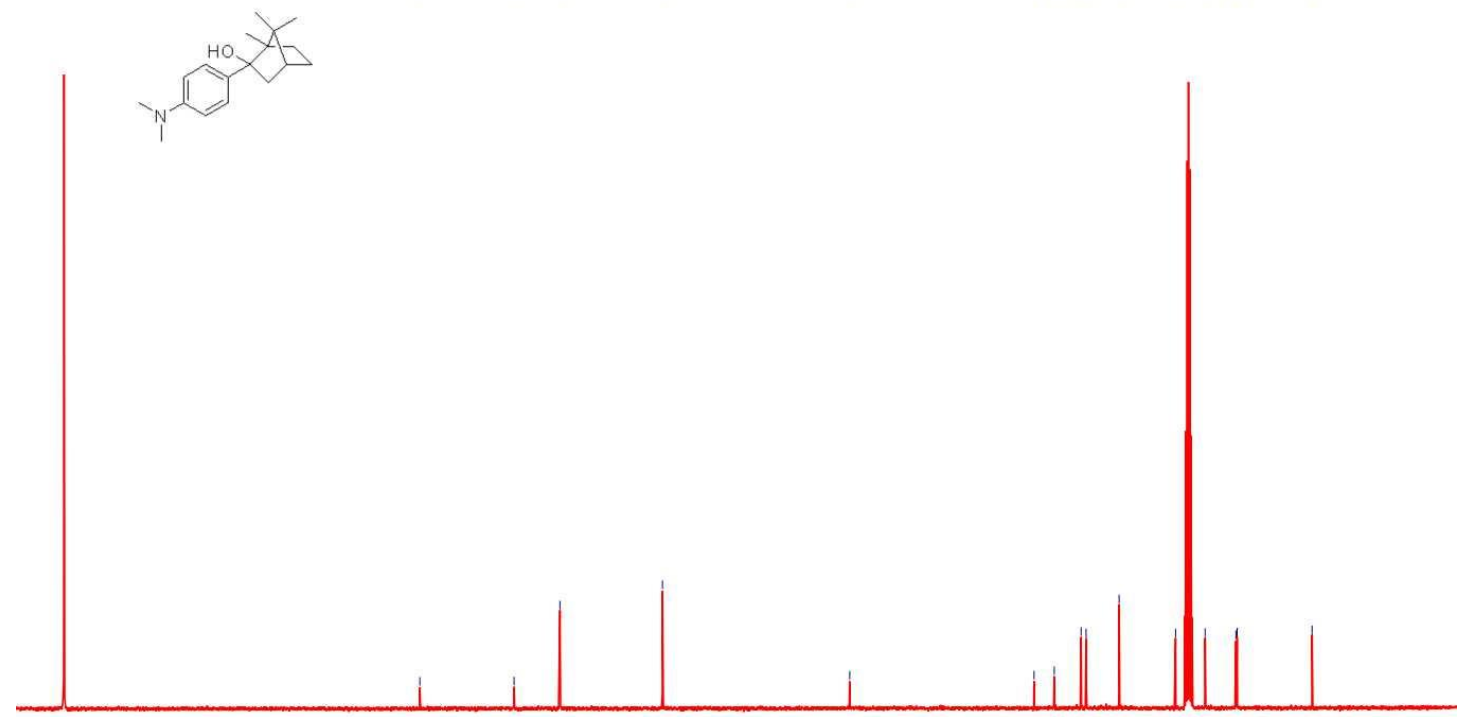

$210200190180170160150140130120110100 \quad 90 \quad 80 \quad 70 \quad 60 \quad 50 \quad 40 \quad 30 \quad 20 \quad 10 \quad 0 \quad-16$ 
6-Methyl-4' -(piperidin-1-yl)-3-(prop-1-en-2-yl)-3,4-dihydro-[1,1' - biphenyl]-1(2H)ol (3p)

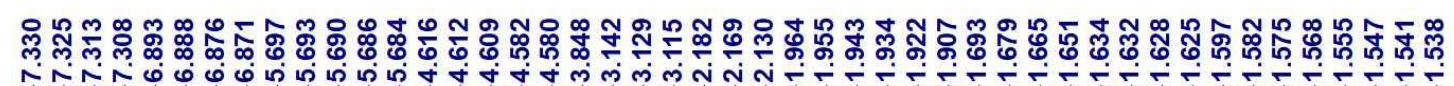
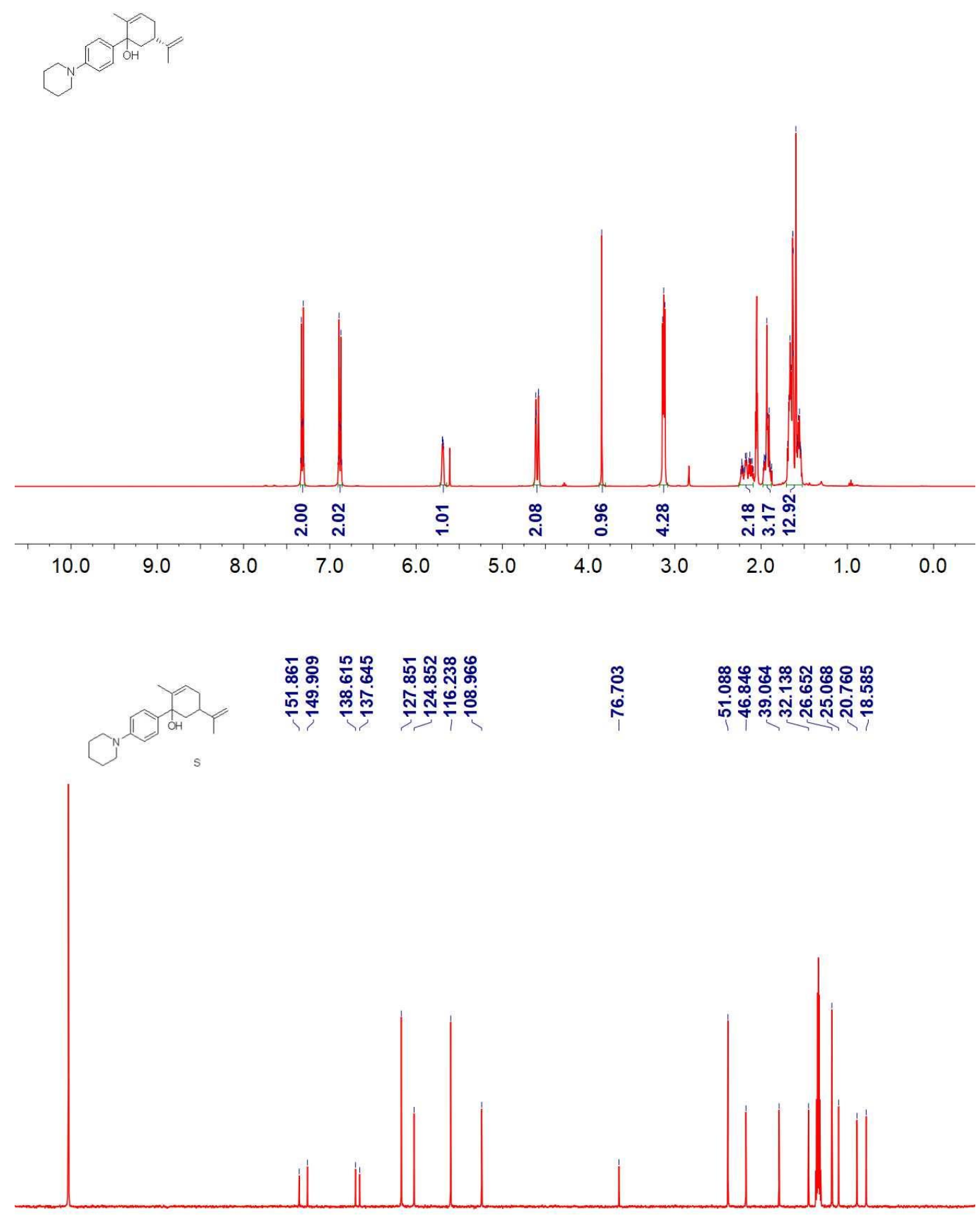

$210200190180170160150140130120110 \quad 100 \quad 90 \quad 80 \quad 70 \quad 60 \quad 50 \quad 40 \quad 30 \quad 20 \quad 10 \quad 0$ 
Trans-1-(4-(Dimethylamino)phenyl)-3-methylcyclopentadecan-1-ol (3qa) (relative stereochemistry)

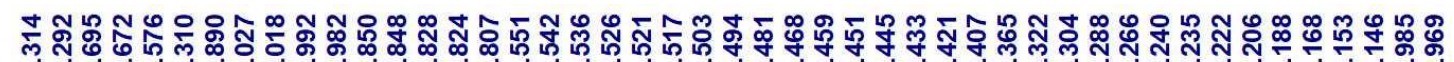
ヘ̃
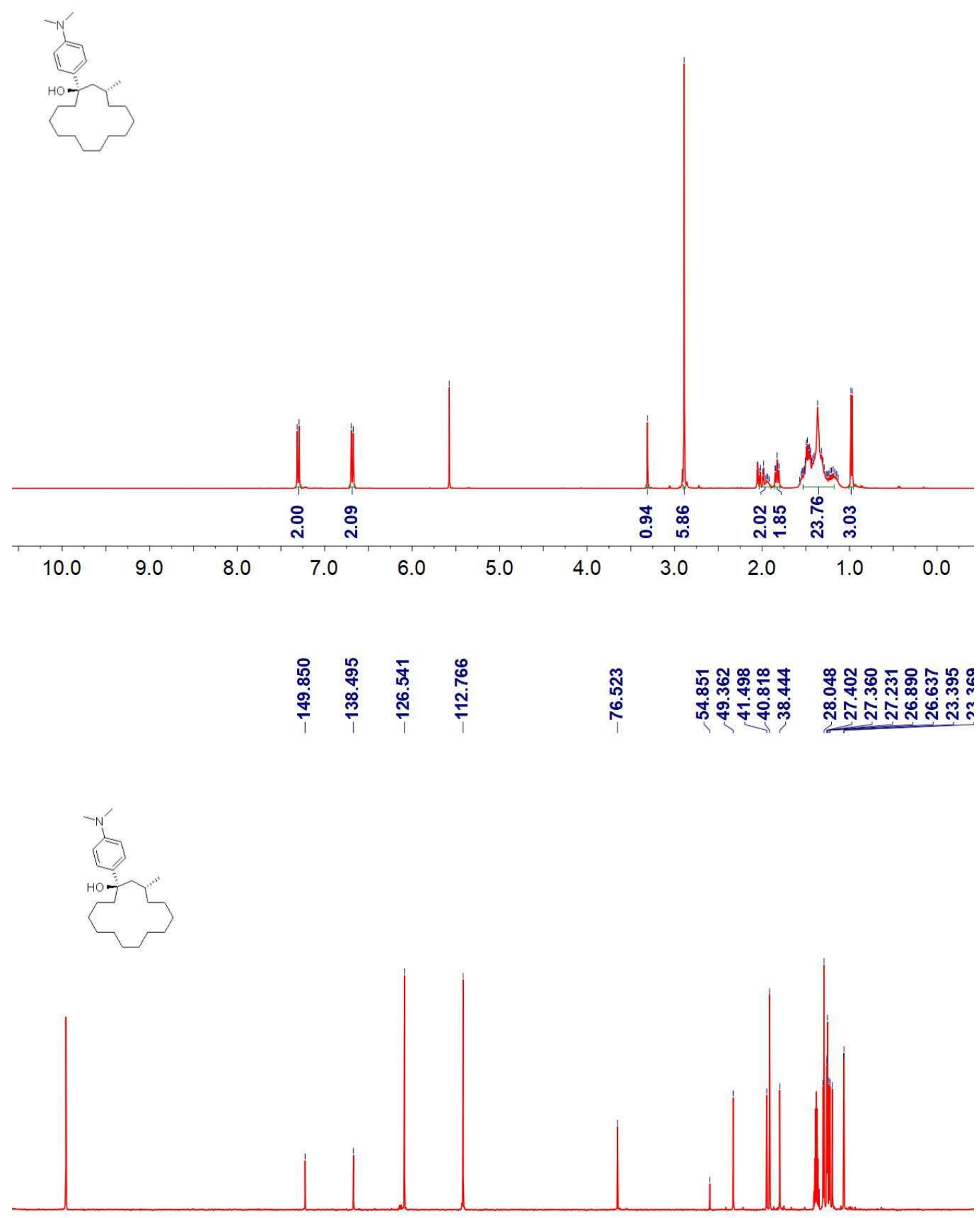

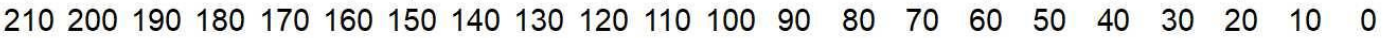


Cis-1-(4-(Dimethylamino)phenyl)-3-methylcyclopentadecan-1-ol (3qb) (relative stereochemistry)
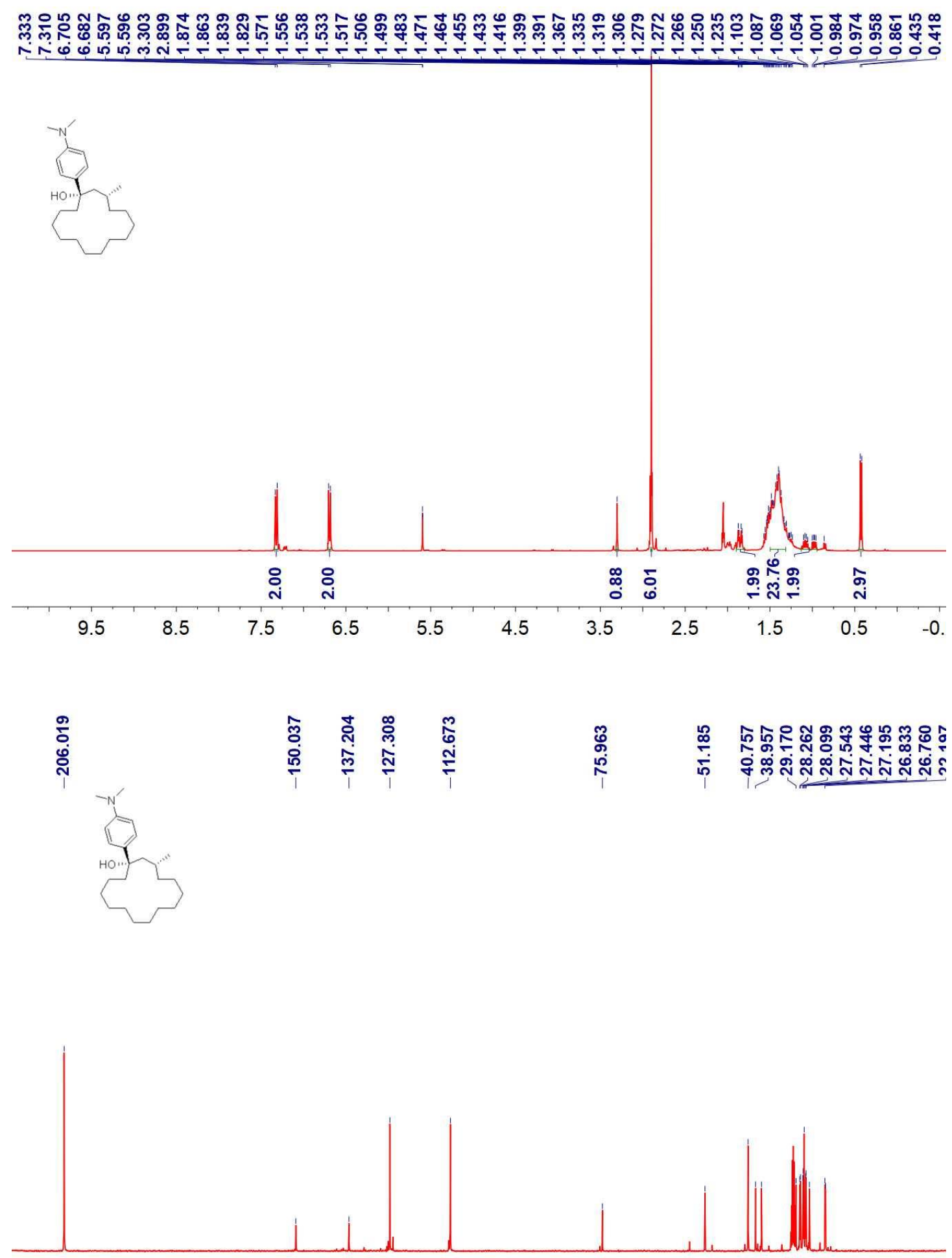

$210200190180170160150140130120110100 \quad 90 \quad 80 \quad 70 \quad 60 \quad 50 \quad 40 \quad 30 \quad 20 \quad 10 \quad 0$ 
4-(4-Benzhydrylpiperazin-1-yl)-1-(4-(dimethylamino)phenyl)-1-(4-fluorophenyl)buta n-1-ol (3r)

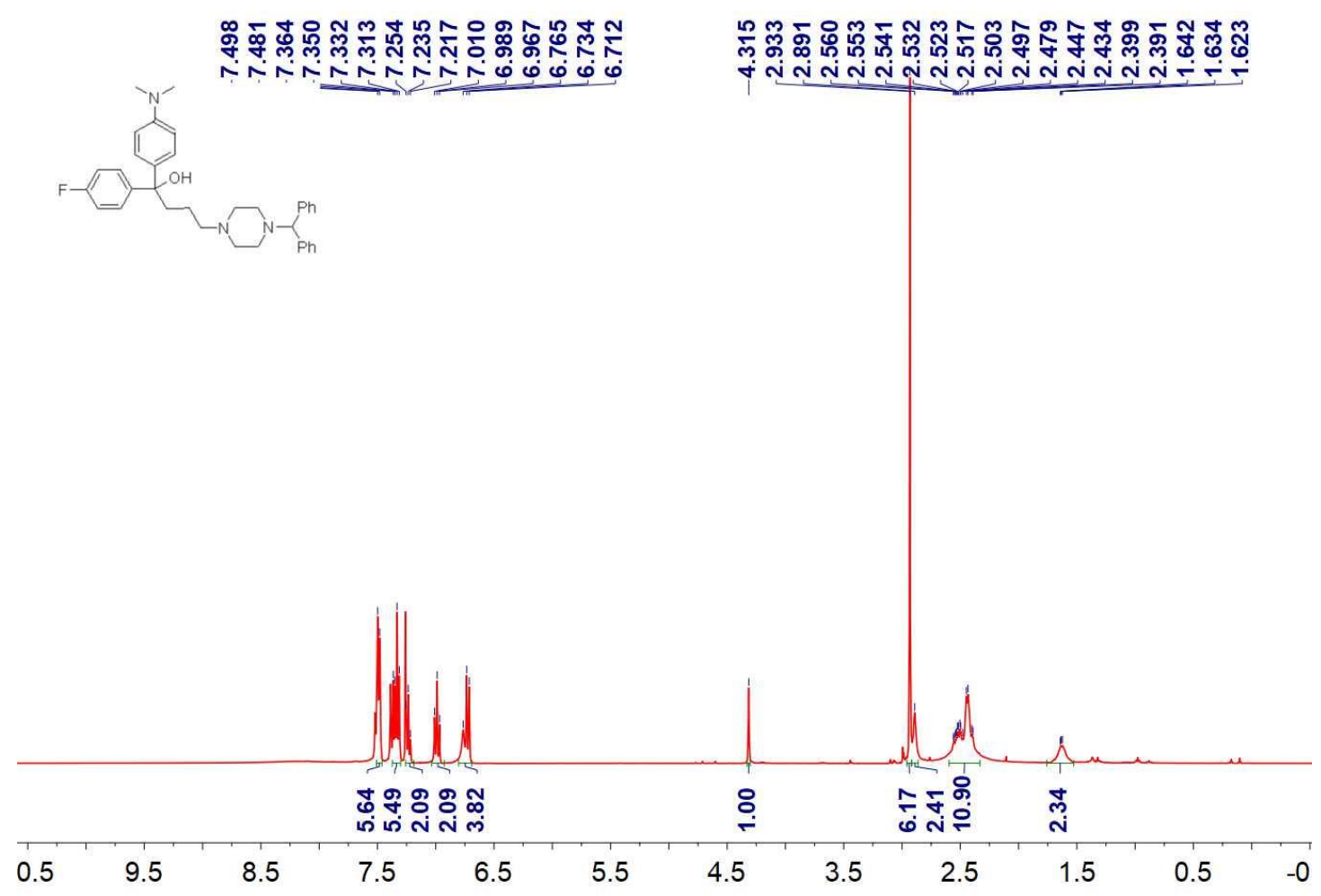

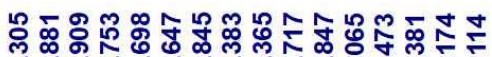

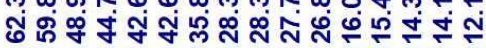
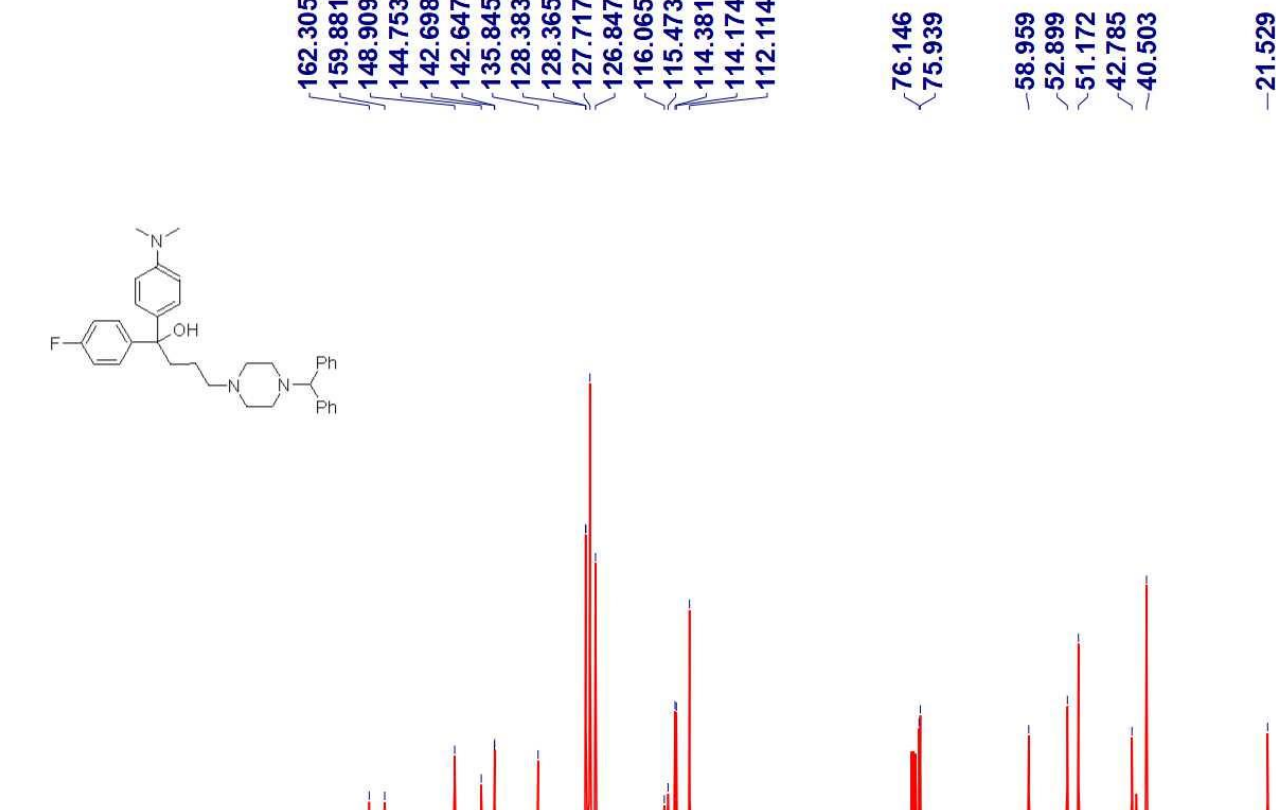

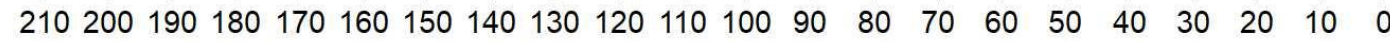


1-(4-(Dimethylamino)phenyl)-1-phenylethane-1,2-diol (3s)

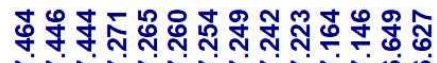

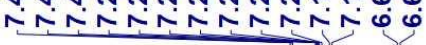

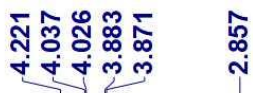
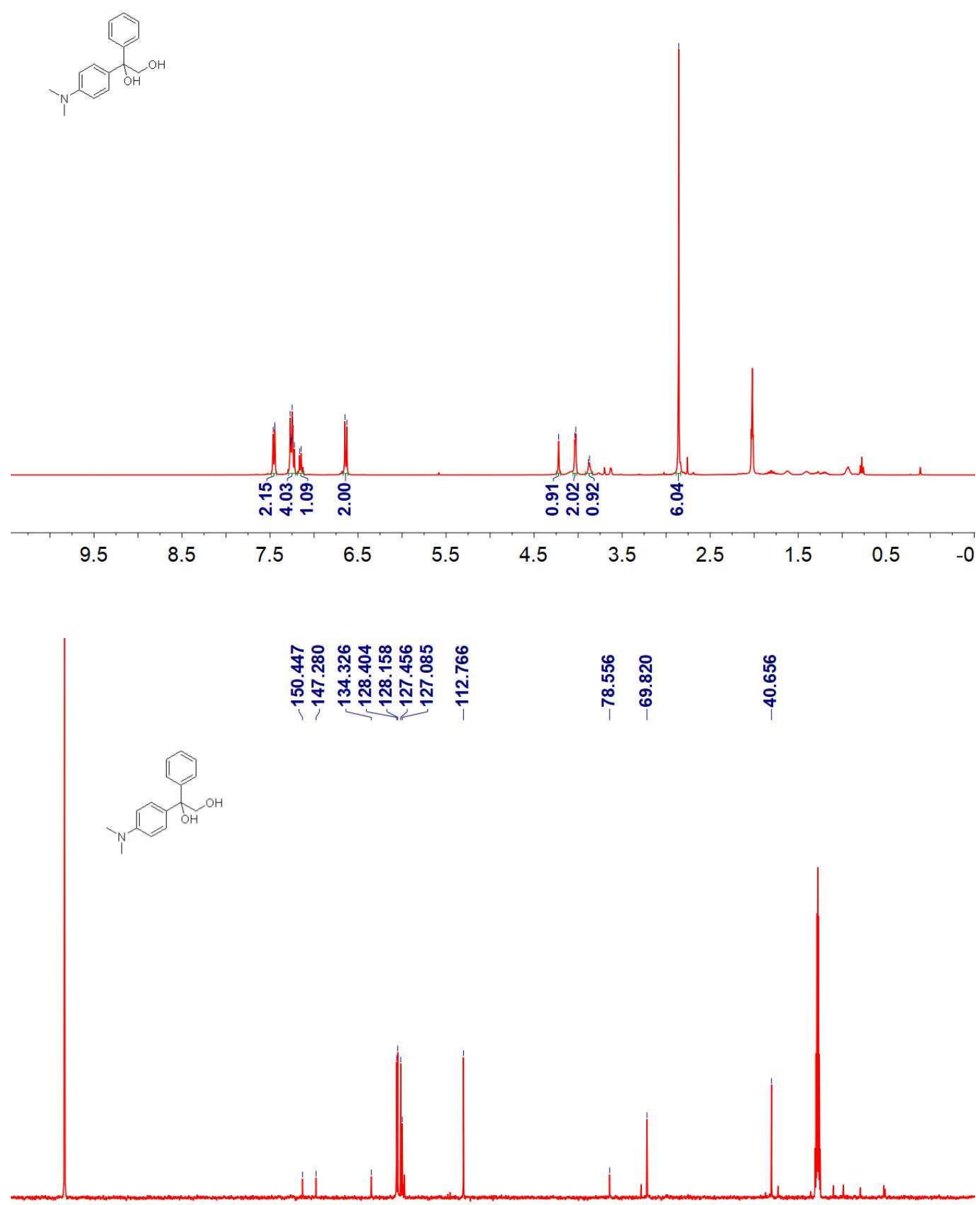

$210200190180170160150140130120110100 \quad 90 \quad 80 \quad 70 \quad 60 \quad 50 \quad 40 \quad 30 \quad 20 \quad 10 \quad 0$ 
1-(4-(Dimethylamino)phenyl)-1-phenylethane-1,2-diol (3t)

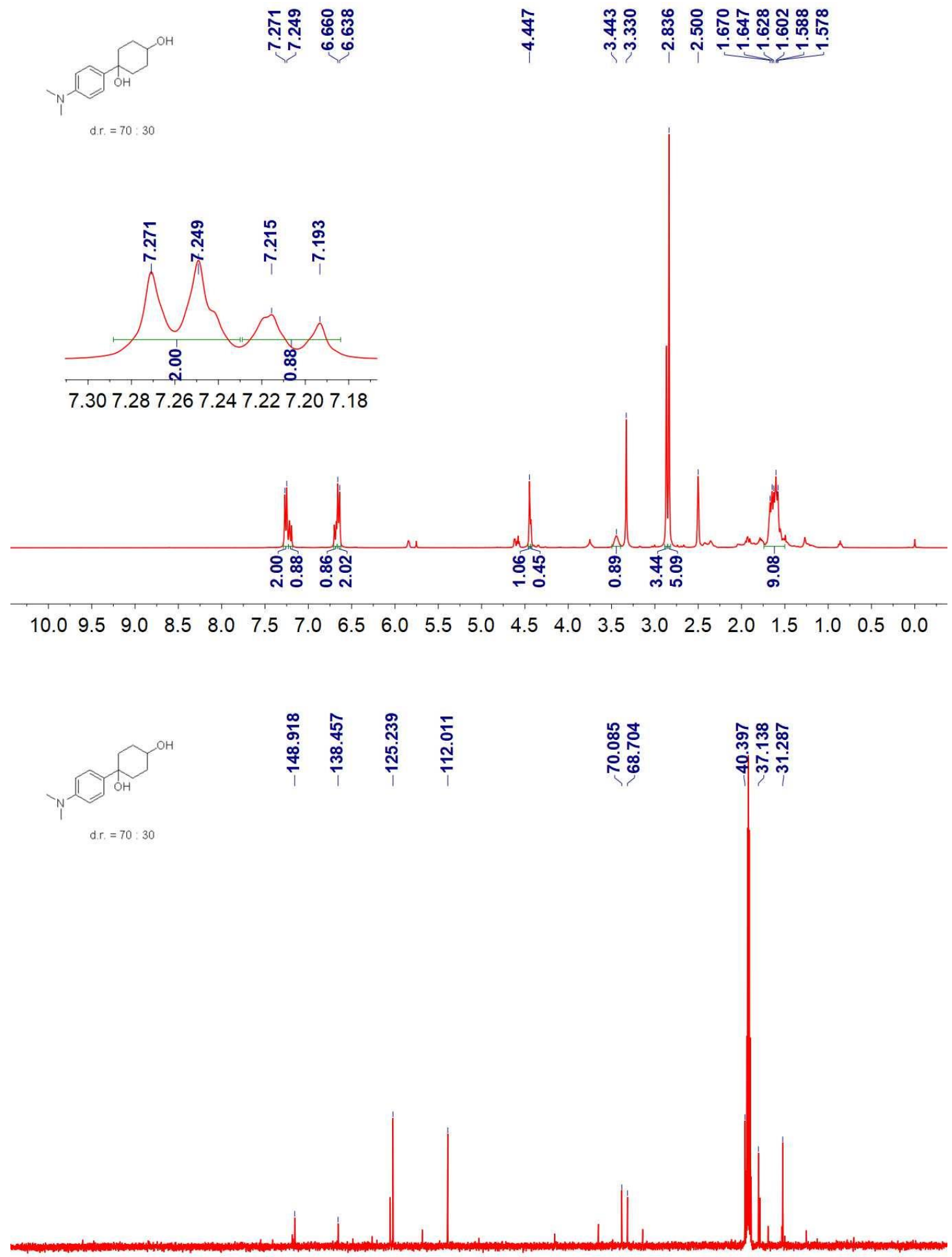

$210200190180170160150140130120110 \quad 100 \quad 90 \quad 80 \quad 70 \quad 60 \quad 50 \quad 40 \quad 30 \quad 20 \quad 10 \quad 0$ 
2-(4-(Dimethylamino)phenyl)-4-methylpentane-2,4-diol (3u) (crude product)

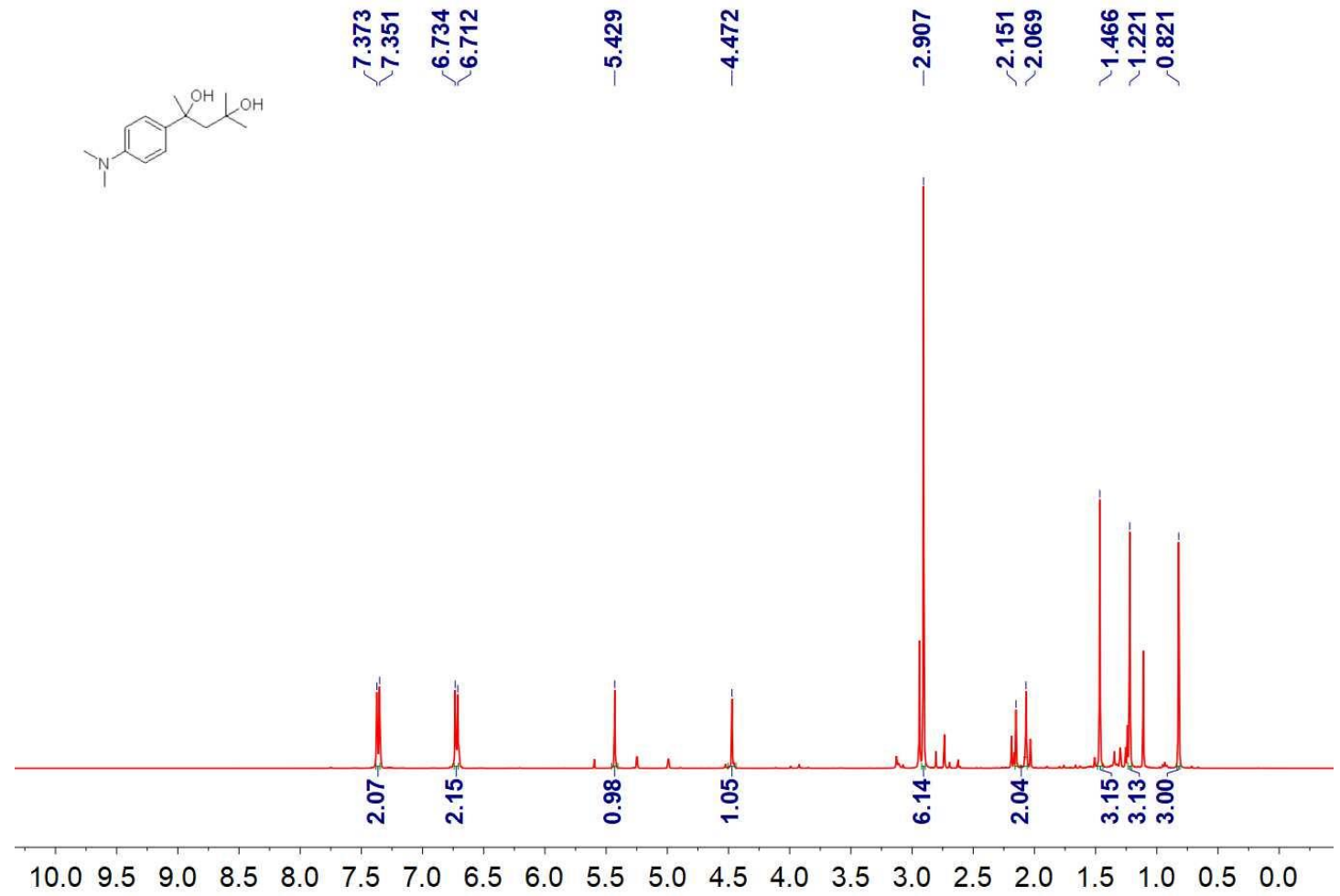

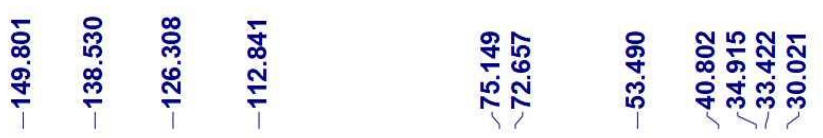

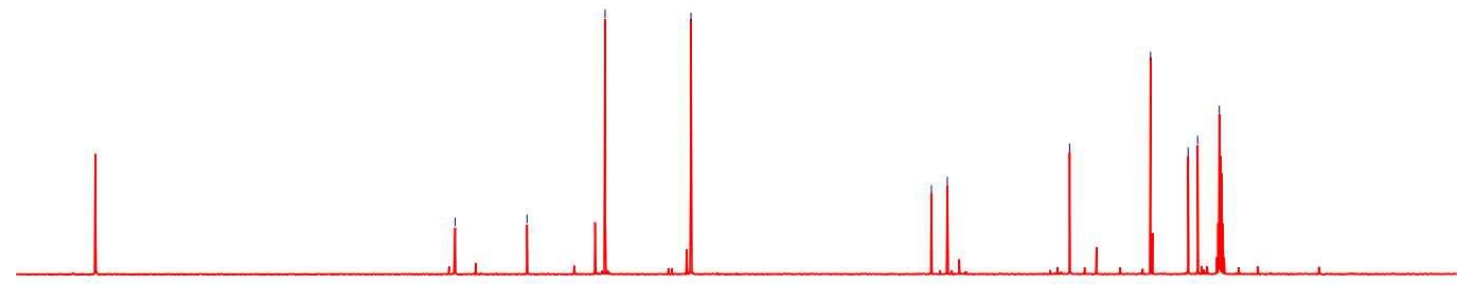

$210200190180170160150140130120 \quad 110 \quad 100 \quad 90 \quad 80 \quad 70 \quad 60 \quad 50 \quad 40 \quad 30 \quad 20 \quad 10 \quad 0$ 
3-(4-(Dimethylamino)phenyl)-3-hydroxybutan-2-one (3v)

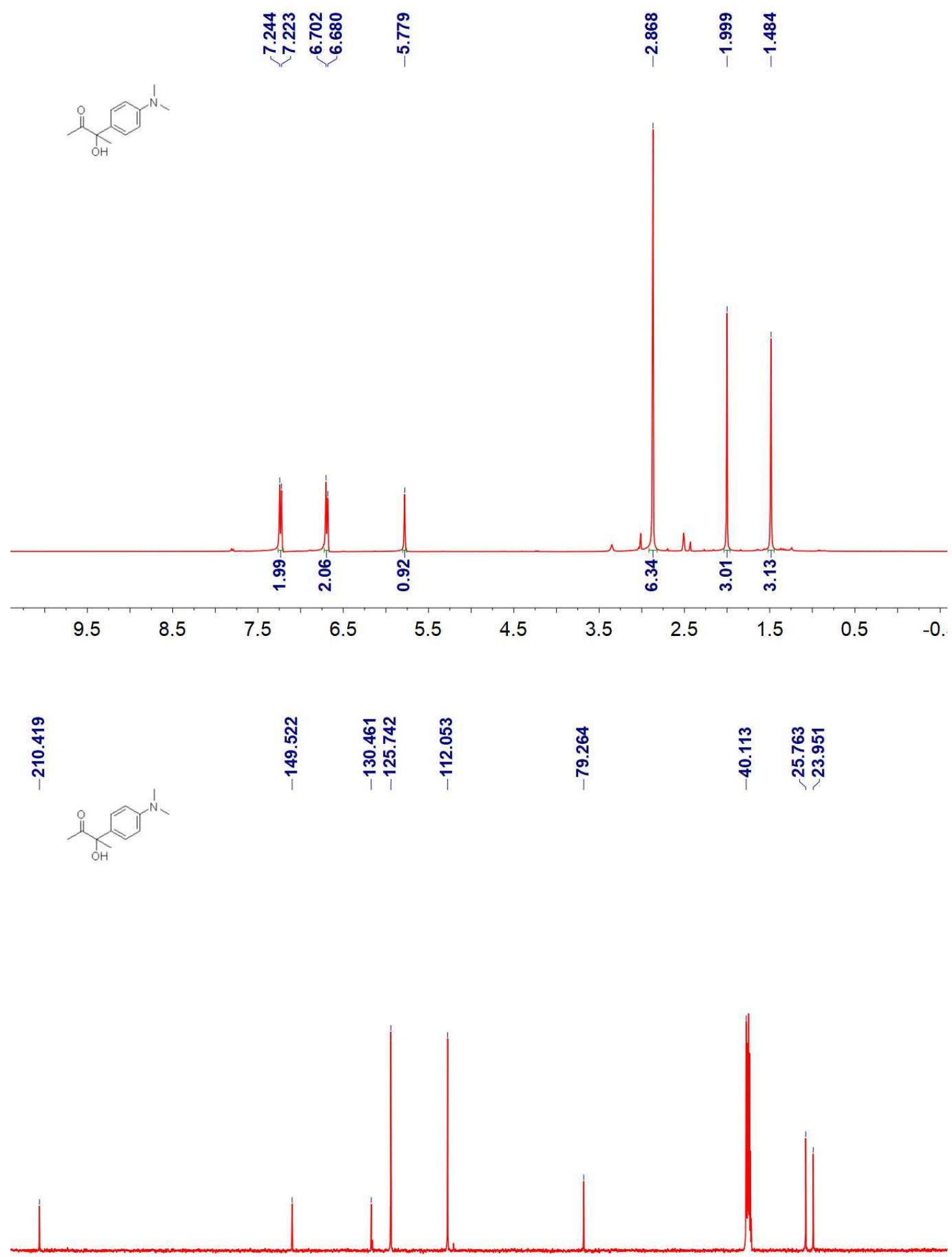

$210200190180170160150140130120110100 \quad 90 \quad 80 \quad 70 \quad 60 \quad 50 \quad 40 \quad 30 \quad 20 \quad 10 \quad 0$ 
4-Isobutyl- $N, N$-dimethylaniline (2a)
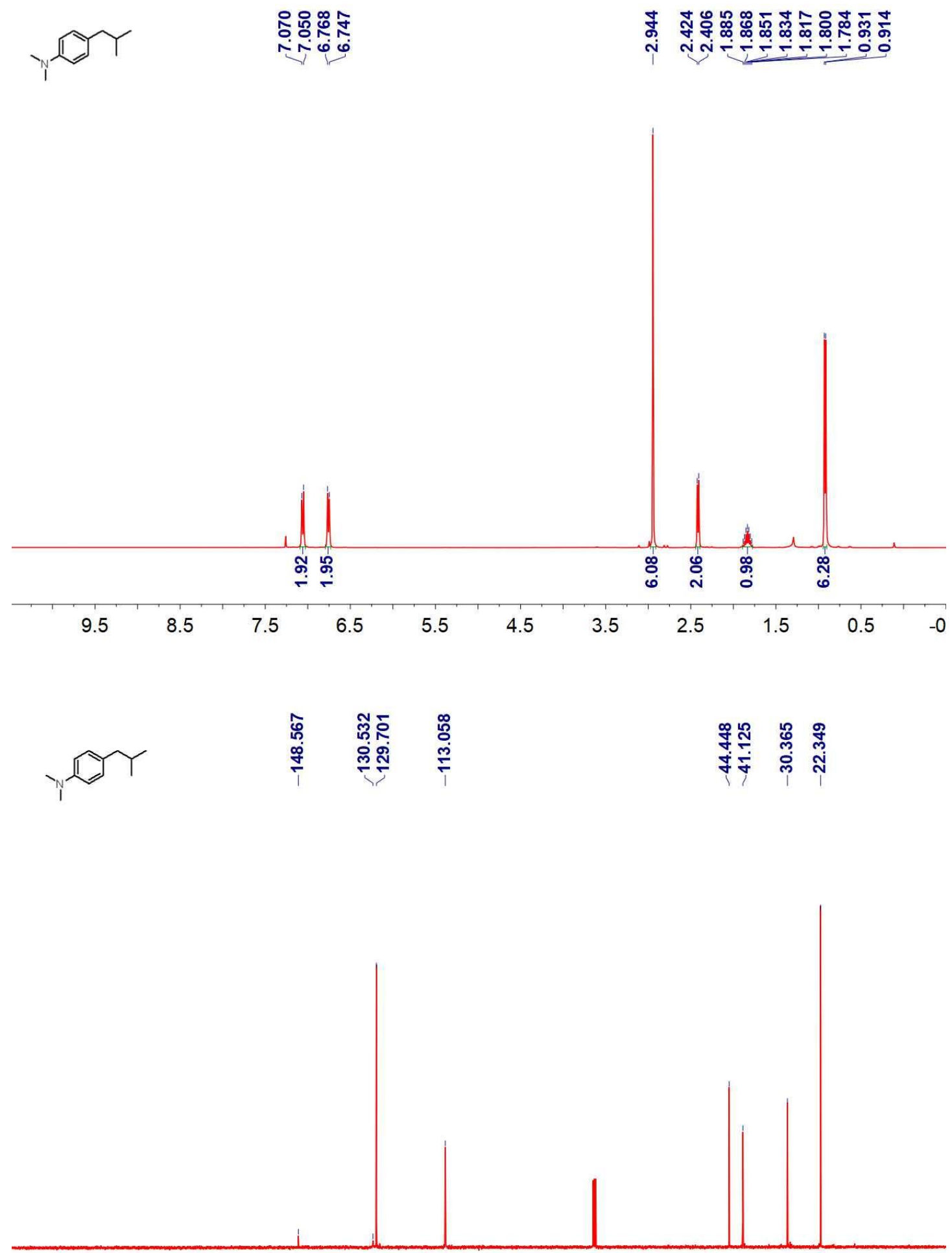

$210200190180170160150140130120110100 \quad 90 \quad 80 \quad 70 \quad 60 \quad 50 \quad 40 \quad 30 \quad 20 \quad 10 \quad 0$ 


\section{4-Benzyl-N,N-dimethylaniline (2b)}

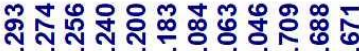

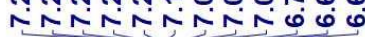

†ัฒ
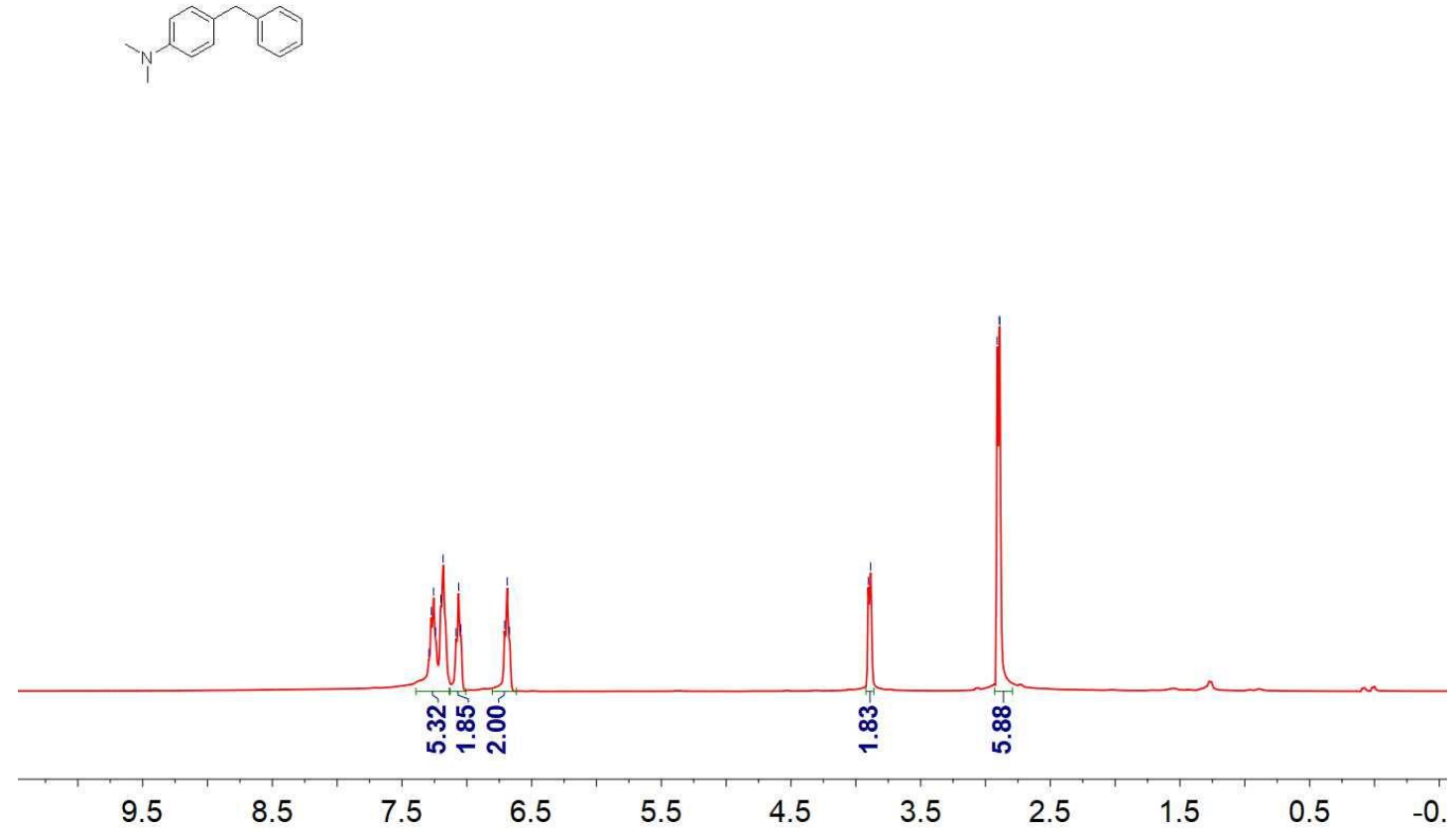

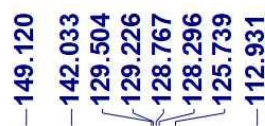
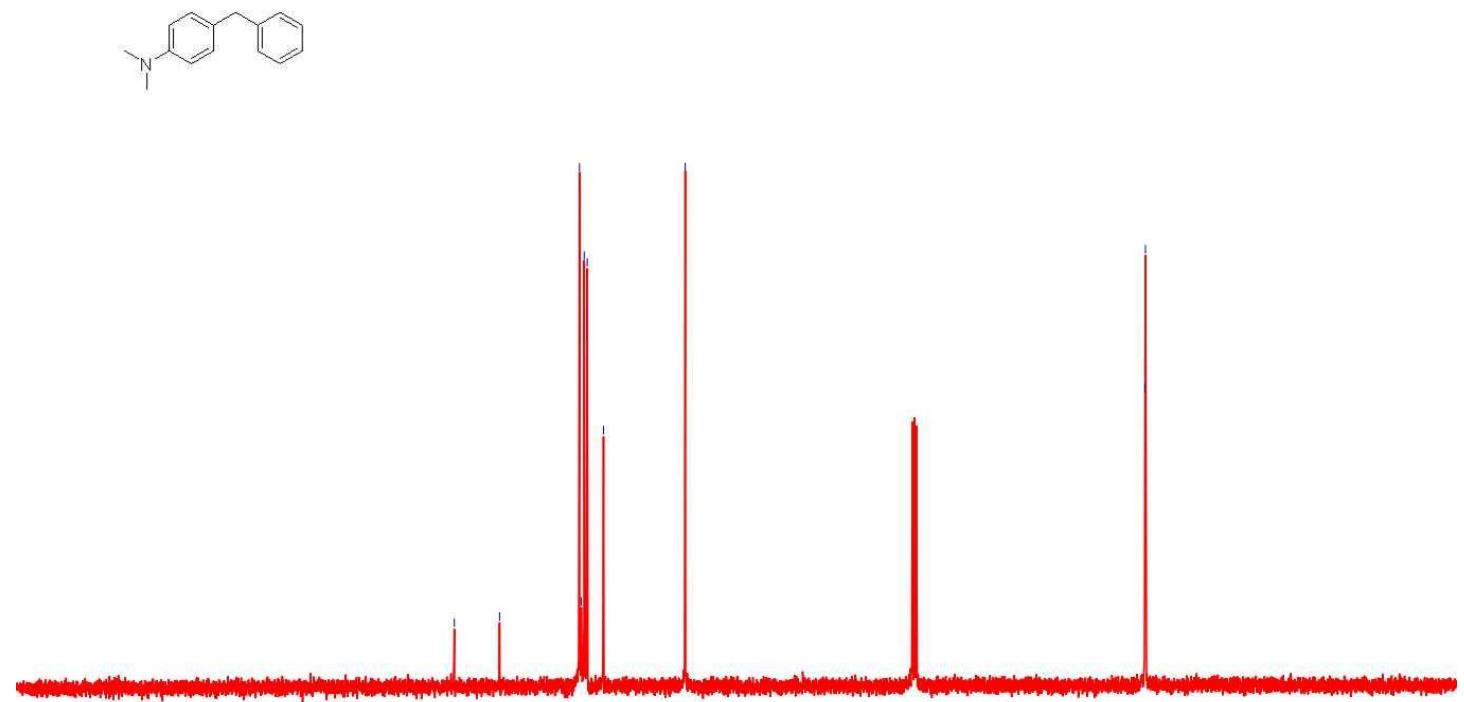

$210200190180170160150140130120110100 \quad 90 \quad 80 \quad 70 \quad 60 \quad 50 \quad 40 \quad 30 \quad 20 \quad 10 \quad 0$ 
4-Benzyl- $N, N$-diethylaniline $(2 b-3)$
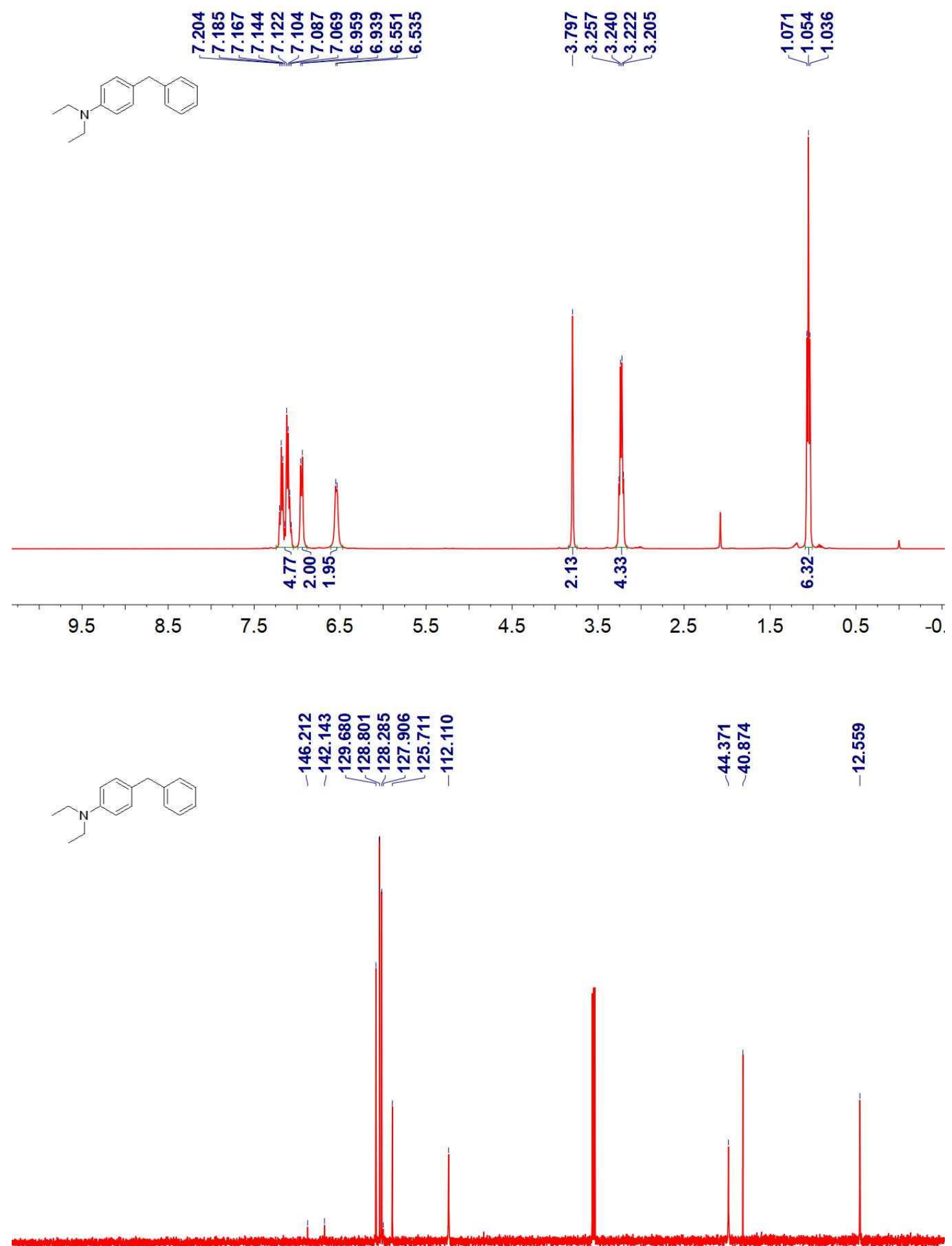

$210200190180170160150140130120110100 \quad 90 \quad 80 \quad 70 \quad 60 \quad 50 \quad 40 \quad 30 \quad 20 \quad 10 \quad 0$ 
1-(4-Benzylphenyl)pyrrolidine (2b-4)

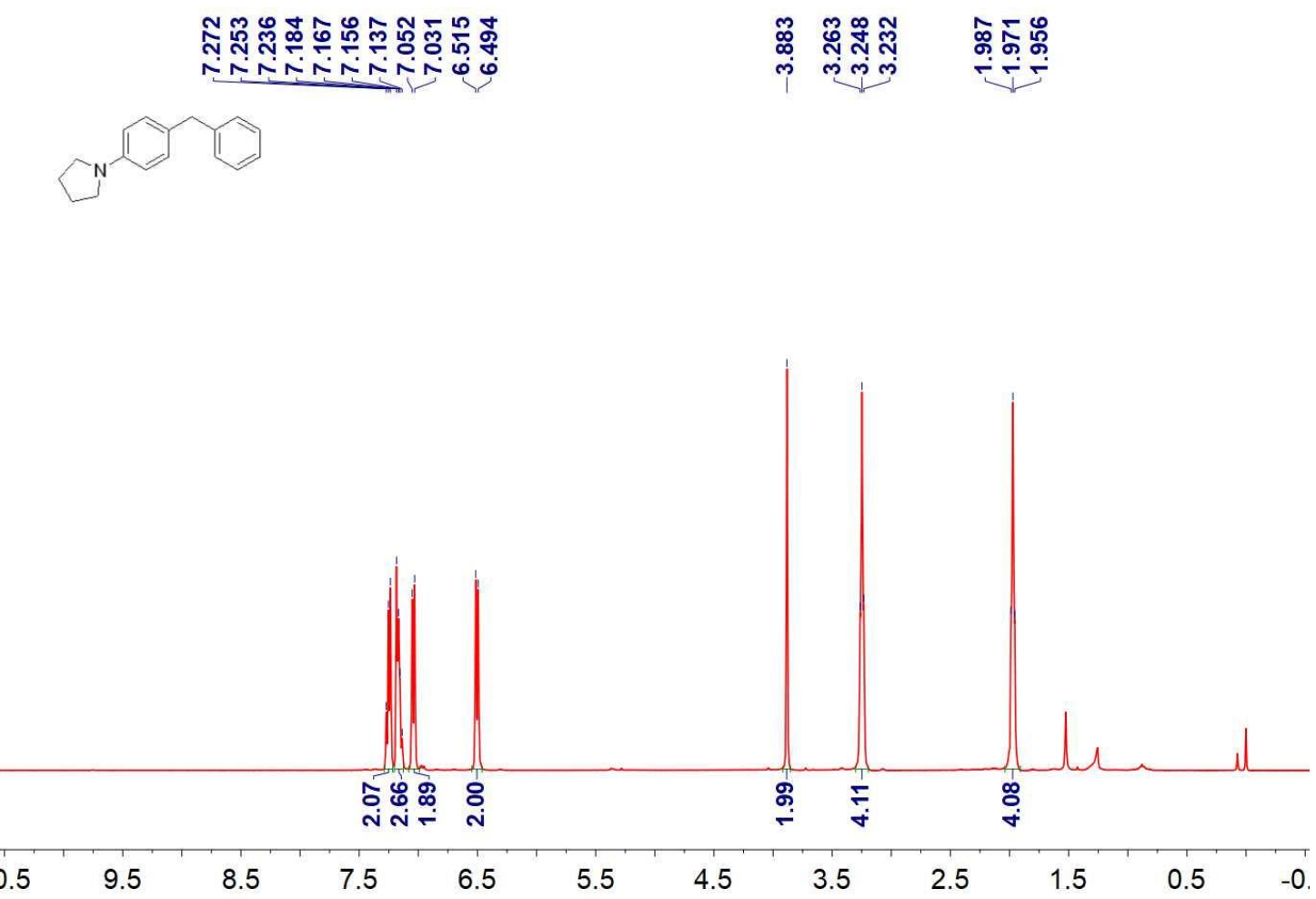

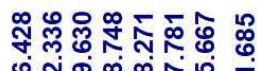

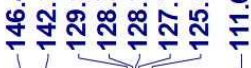

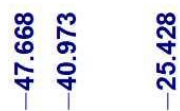

$210200190180170160150140130120110100 \quad 90 \quad 80 \quad 70 \quad 60 \quad 50 \quad 40 \quad 30 \quad 20 \quad 10 \quad 0$ 
1-(4-Benzylphenyl)piperidine (2b-5)
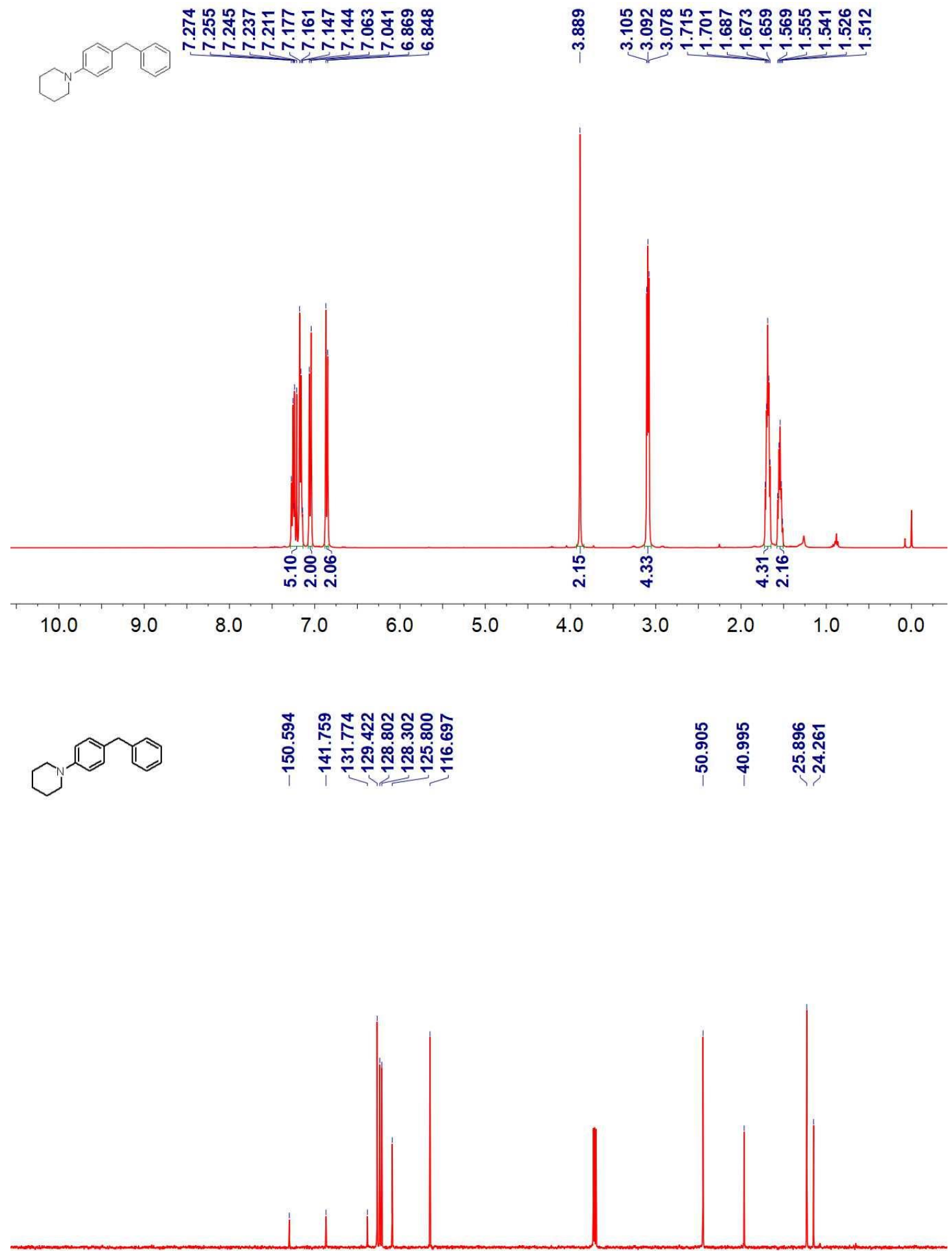

$210200190180170160150140130120110100 \quad 90 \quad 80 \quad 70 \quad 60 \quad 50 \quad 40 \quad 30 \quad 20 \quad 10 \quad 0$ 
2-(4-Benzylphenyl)-1,2,3,4-tetrahydroisoquinoline (2b-6)

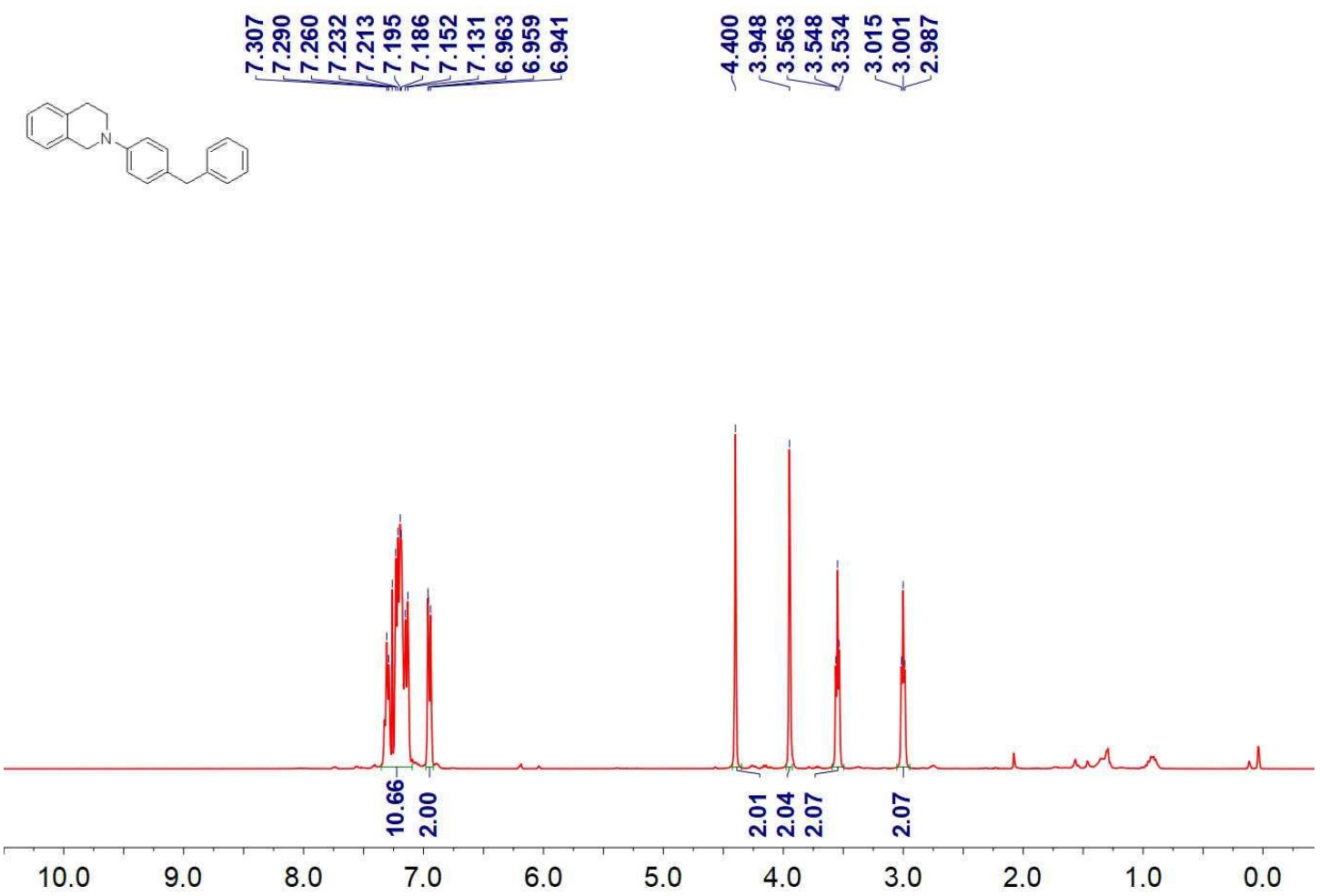

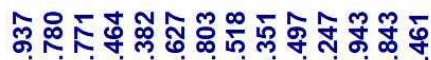

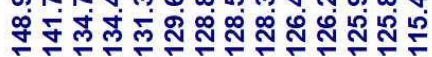

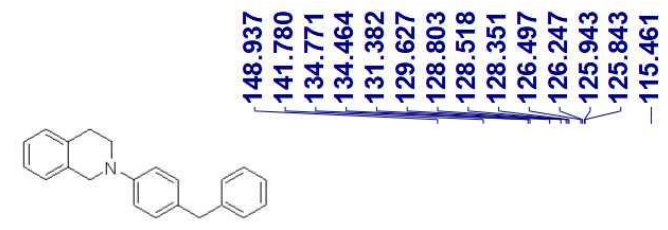

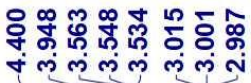

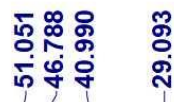

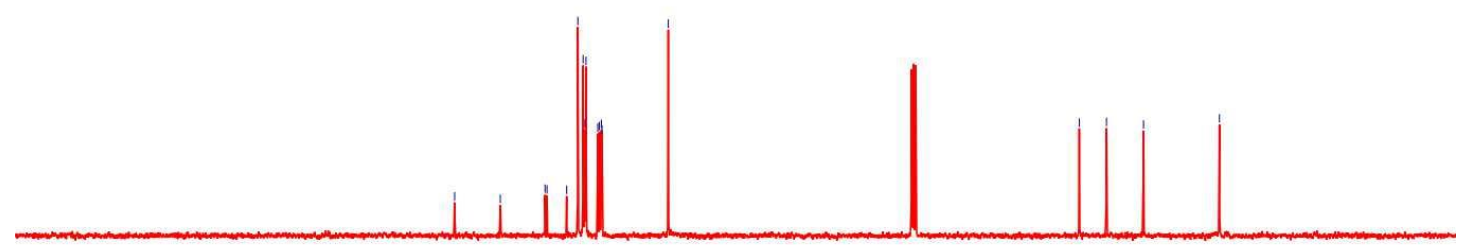

$210200190180170160150140130120110100 \quad 90 \quad 80 \quad 70 \quad 60 \quad 50 \quad 40 \quad 30 \quad 20 \quad 10 \quad 0$ 


\section{4-Benzyl- $N$-methylaniline $(2 b-8)$}

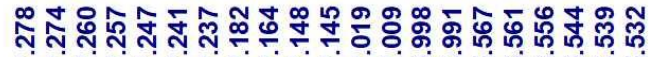

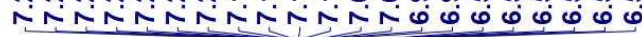
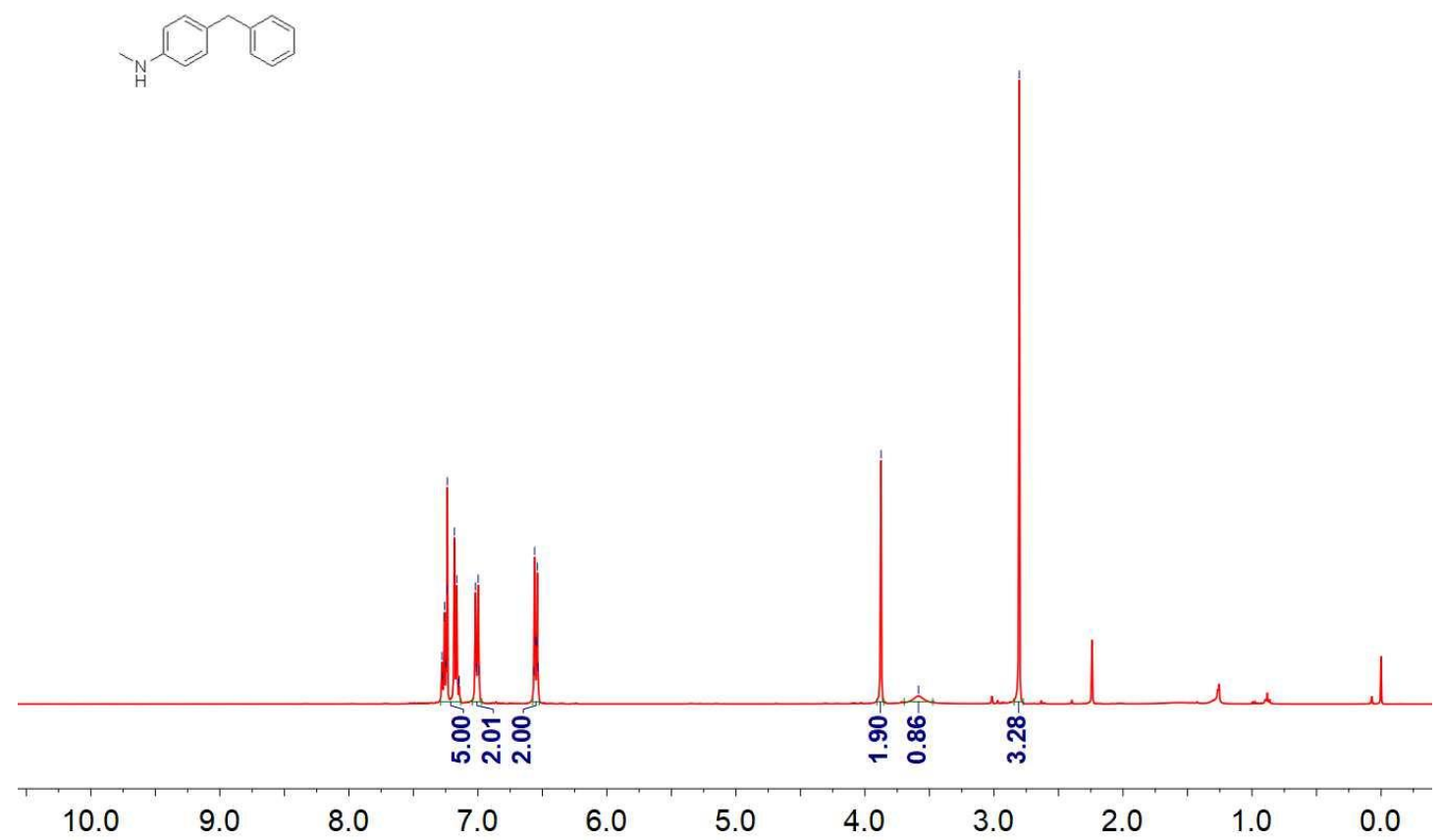
4-Benzyl- $N$-methylaniline $(2 b-9)$
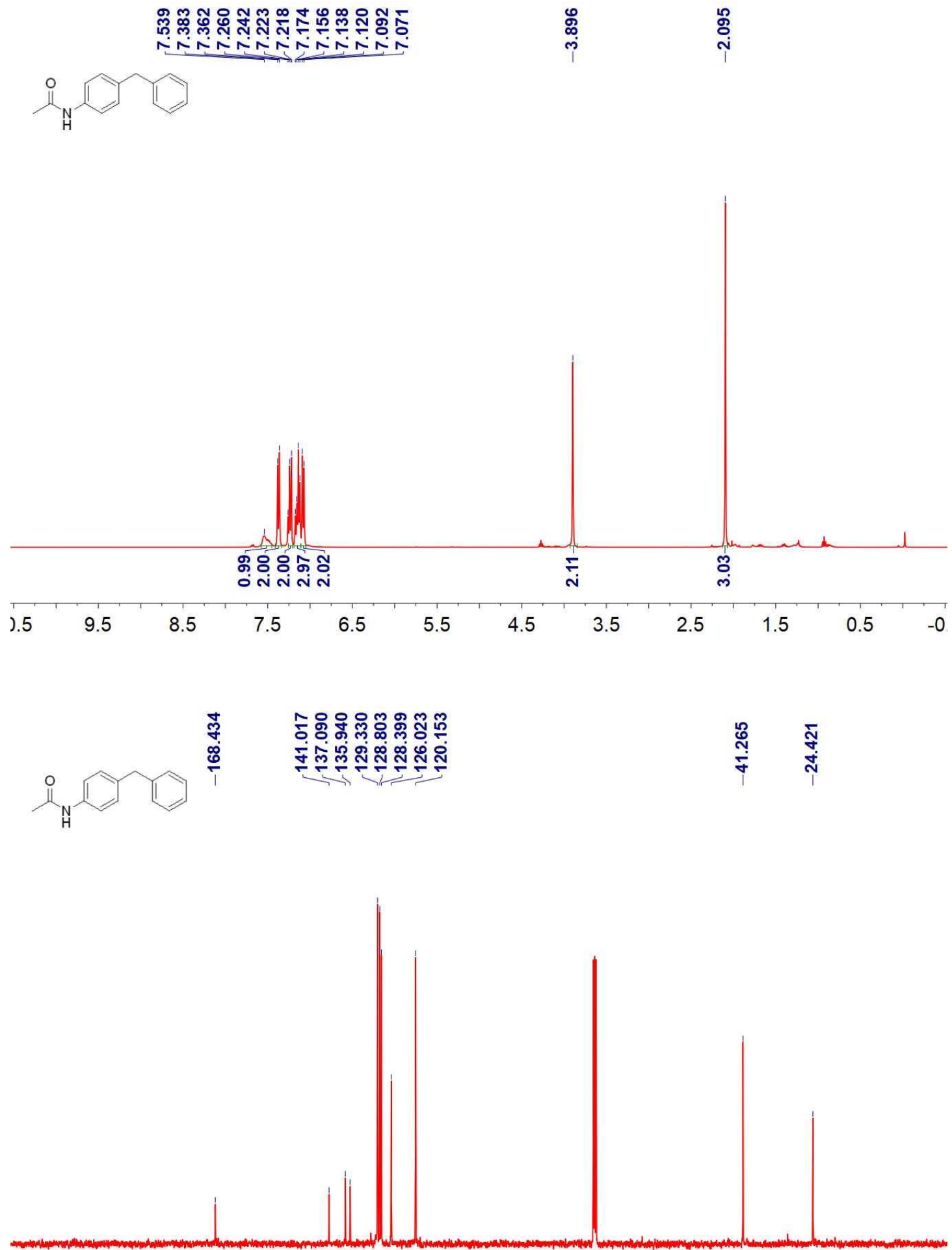

$210200190180170160150140130120110100 \quad 90 \quad 80 \quad 70 \quad 60 \quad 50 \quad 40 \quad 30 \quad 20 \quad 10 \quad 0$ 
$N, N, 4$-trimethylaniline (2c)

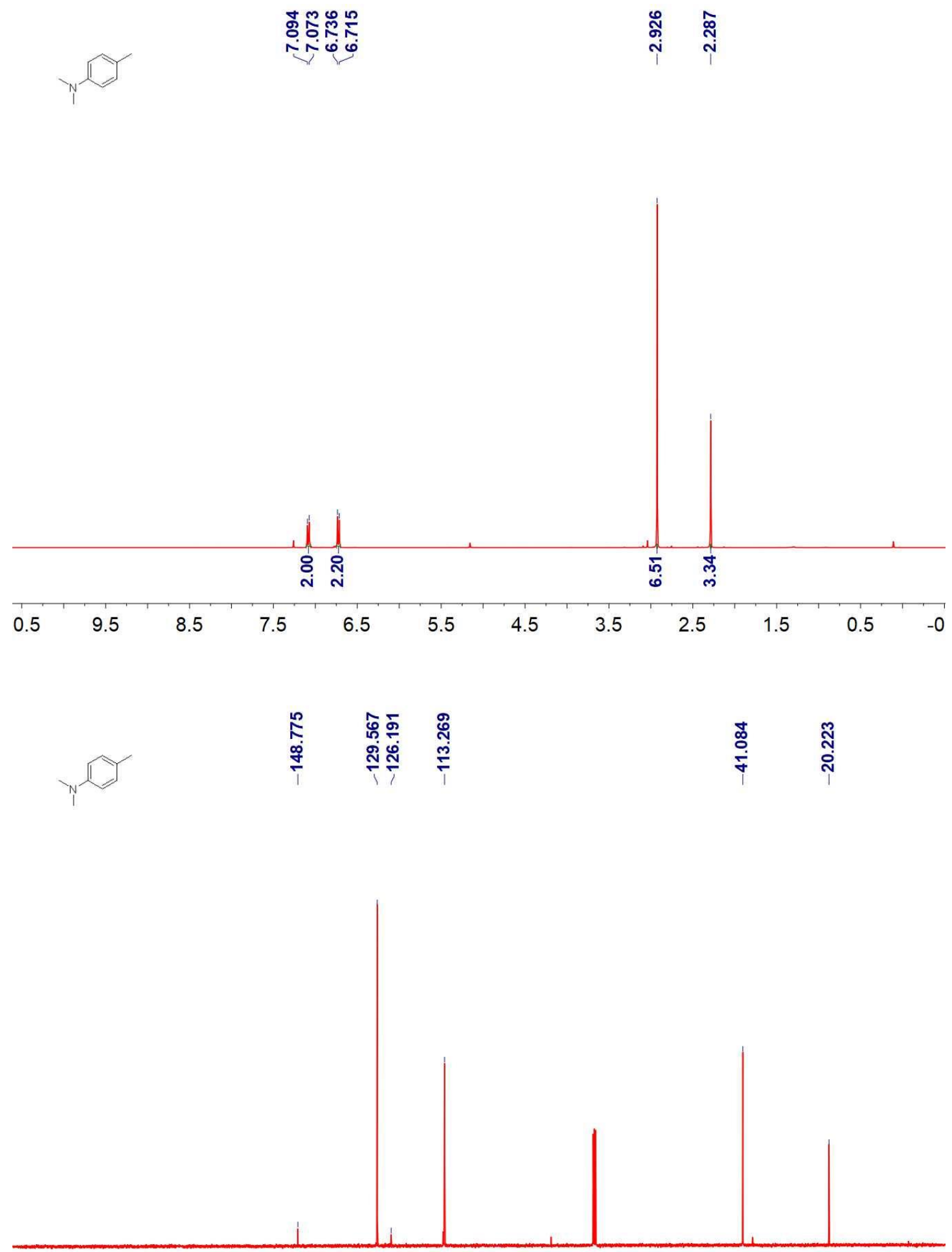

$210200190180170160150140130120110100 \quad 90 \quad 80 \quad 70 \quad 60 \quad 50 \quad 40 \quad 30 \quad 20 \quad 10 \quad 0$ 
$N, N$-diethyl-4-methylaniline (2d)

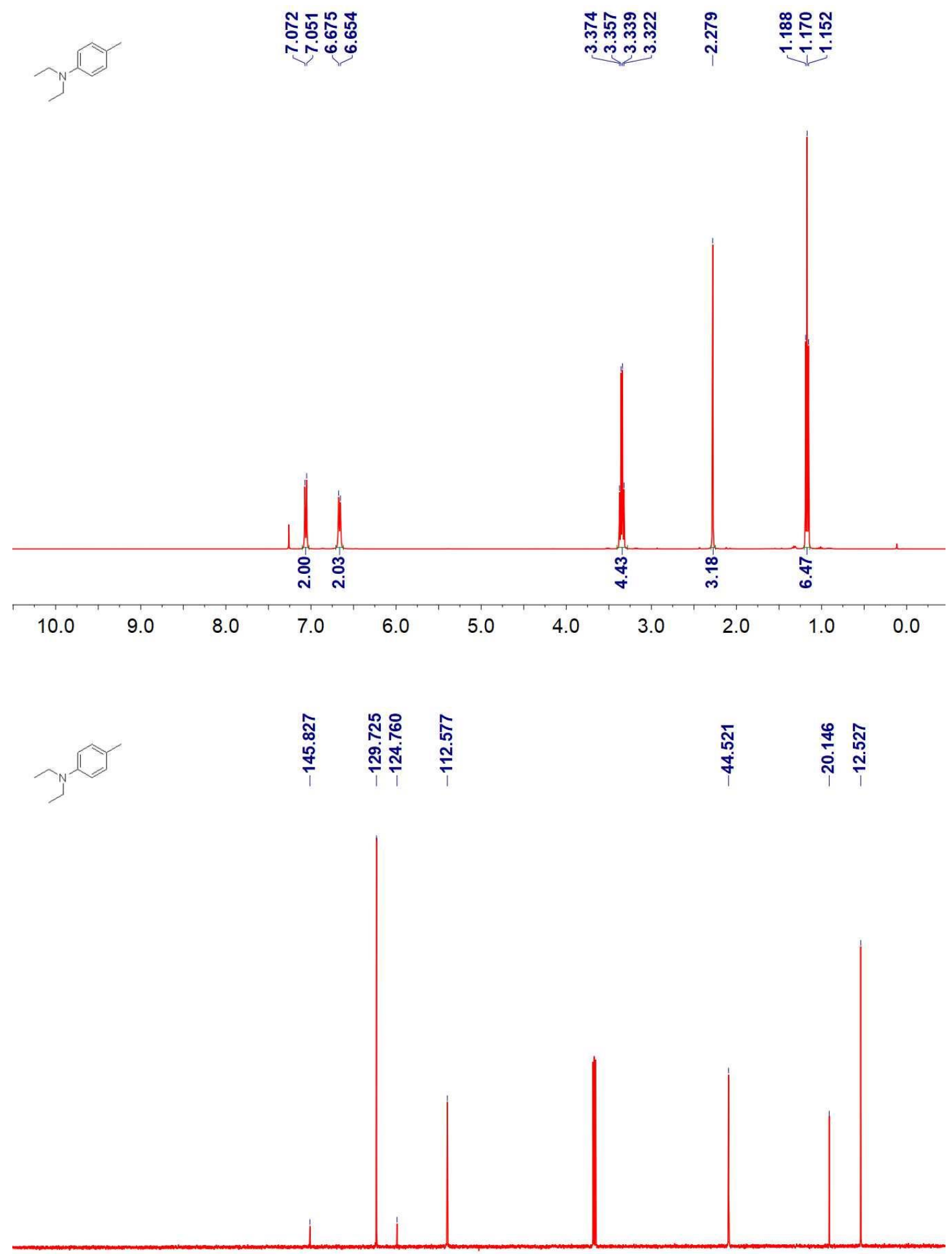

$210200190180170160150140130120110100 \quad 90 \quad 80 \quad 70 \quad 60 \quad 50 \quad 40 \quad 30 \quad 20 \quad 10 \quad 0$ 
N,4-Dimethylaniline (2e)
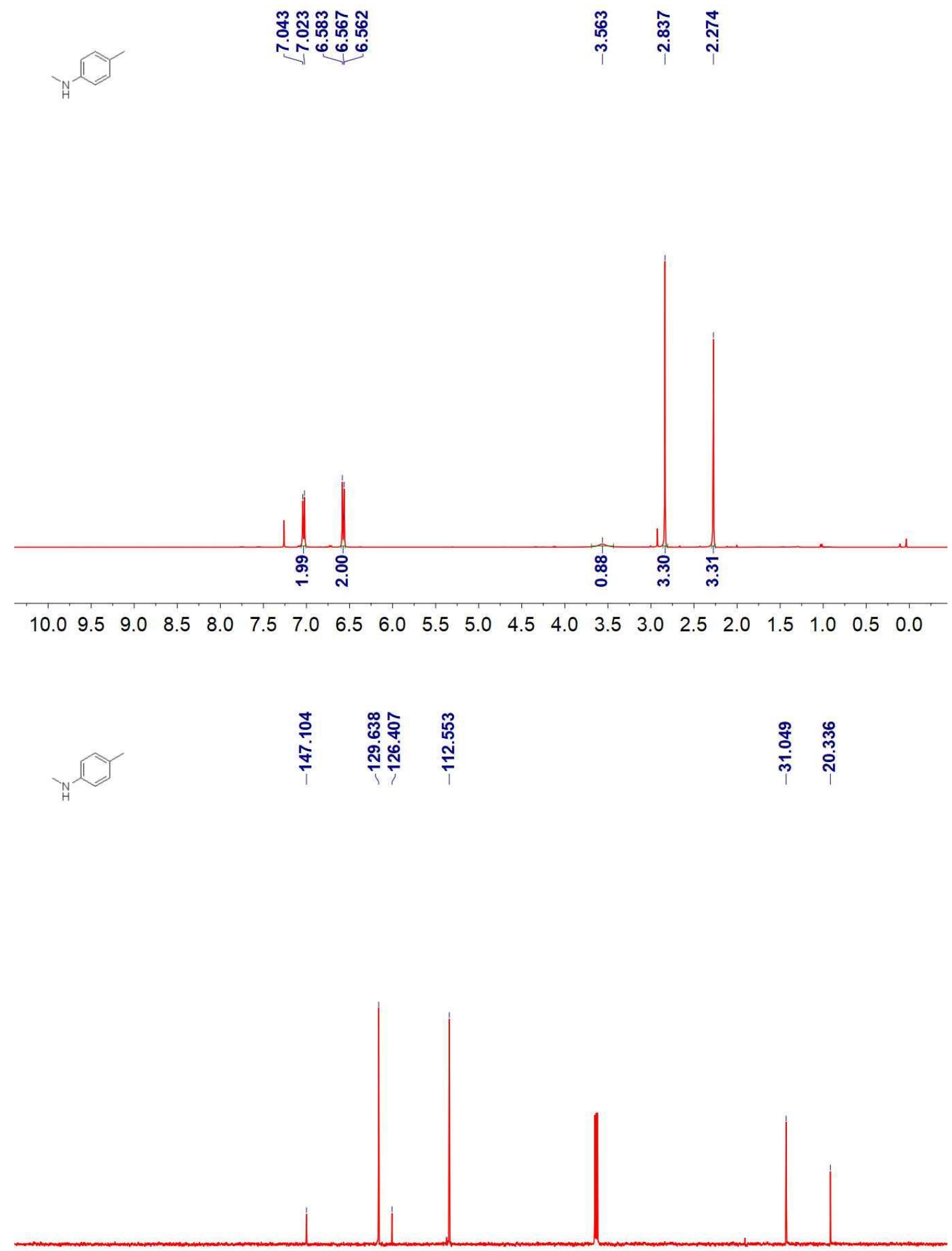

$210200190180170160150140130120110100 \quad 90 \quad 80 \quad 70 \quad 60 \begin{array}{lllllll}40 & 40 & 30 & 20 & 10 & 0\end{array}$ 
$N$-Butyl-N,4-dimethylaniline (2f)

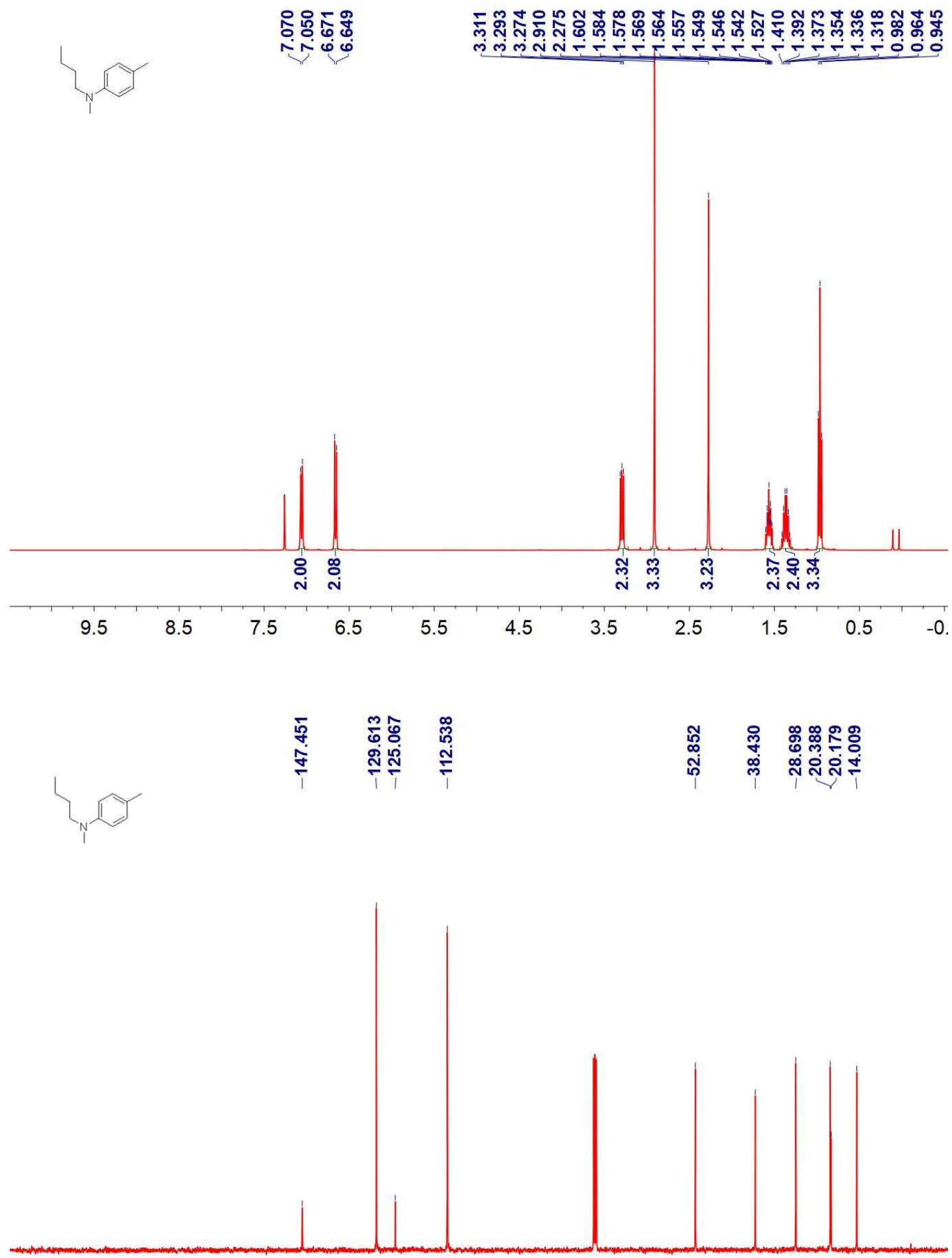

$210200190180170160150140130120110100 \quad 90 \quad 80 \quad 70 \quad 60 \quad 50 \quad 40 \quad 30 \quad 20 \quad 10 \quad 0$ 
4-(Cyclohexylmethyl)- $N, N$-dimethylaniline (2g)

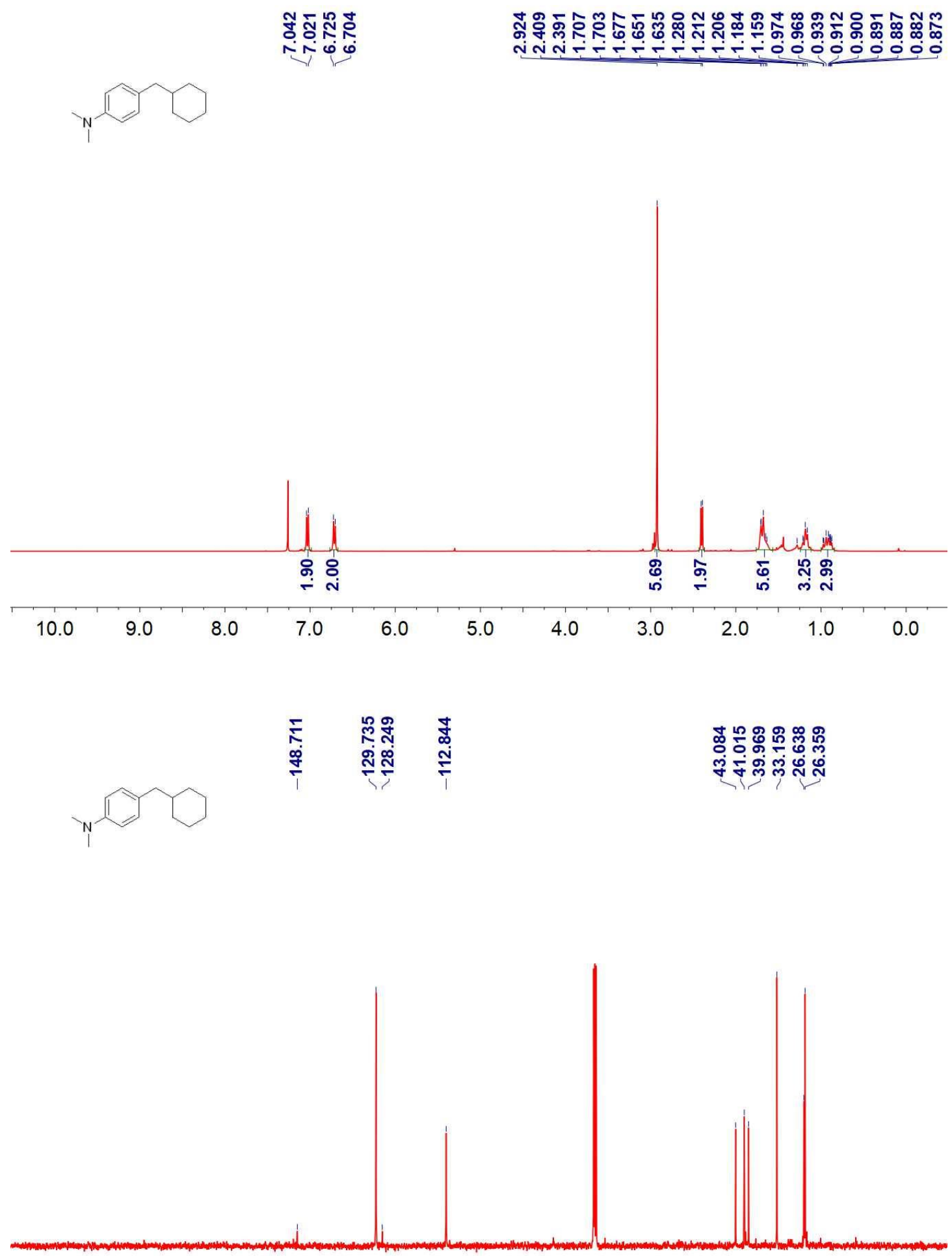

$210200190180170160150140130120110100 \quad 90 \quad 80 \quad 70 \quad 60 \quad 50 \quad 40 \quad 30 \quad 20 \quad 10 \quad 0$ 
$\mathrm{N}, \mathrm{N}$-Diethyl-4-hexylaniline $(2 \mathrm{~h})$

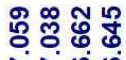

Nंต

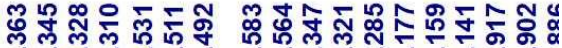

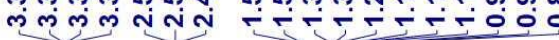

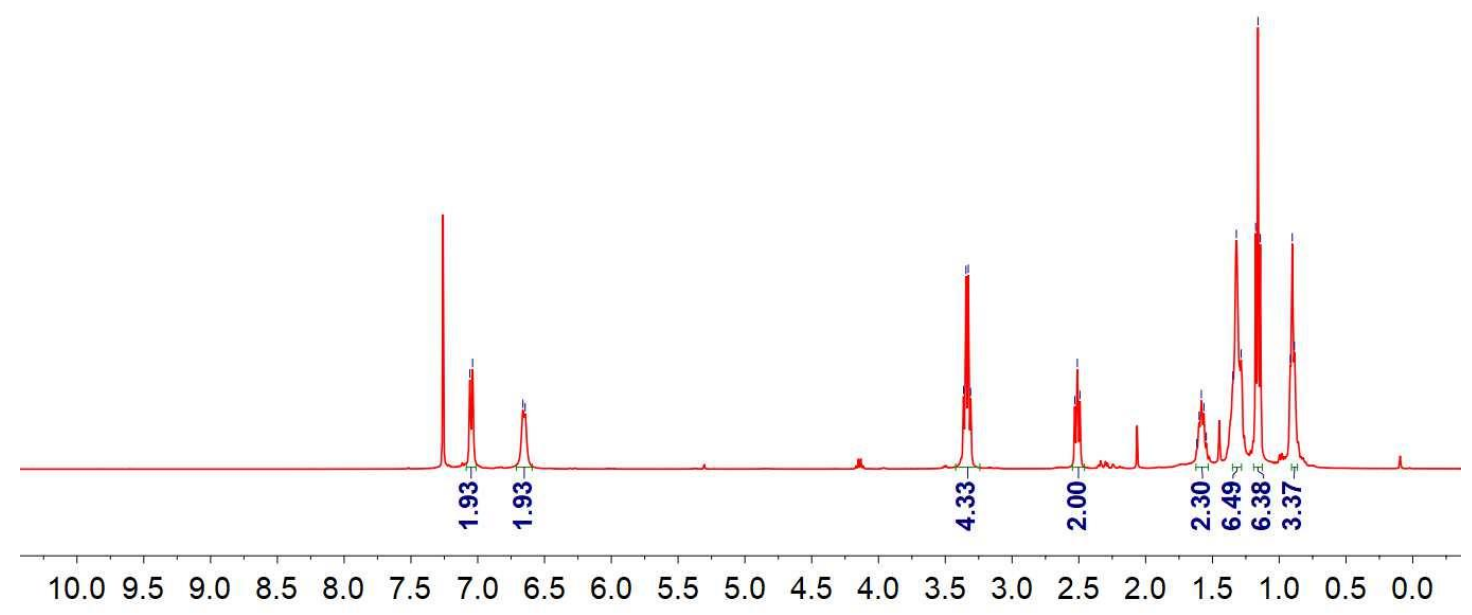

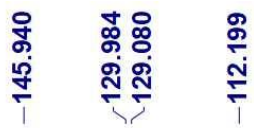

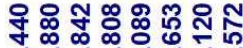

मำ

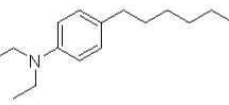

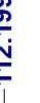

$210200190180170160150140130120110100 \quad 90 \quad 80 \quad 70 \quad 60 \quad 50 \quad 40 \quad 30 \quad 20 \quad 10 \quad 0$ 
$\mathrm{N}, \mathrm{N}$-dimethyl-4-octylaniline (2i)

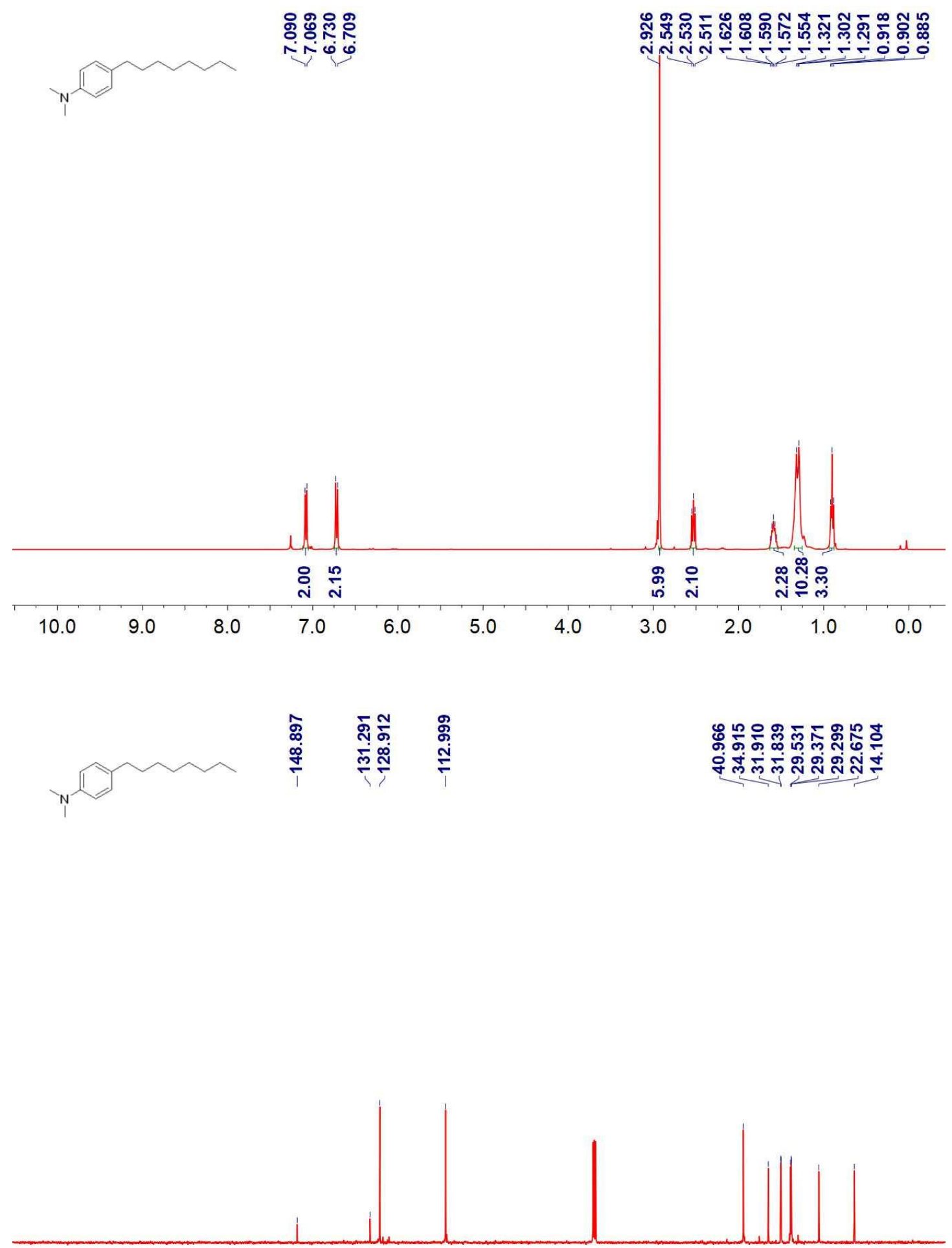

$210200190180170160150140130120110100 \quad 90 \quad 80 \quad 70 \quad 60 \quad 50 \quad 40 \quad 30 \quad 20 \quad 10 \quad 0$ 
$N, N$-dimethyl-4-(3-phenylpropyl)aniline (2j)

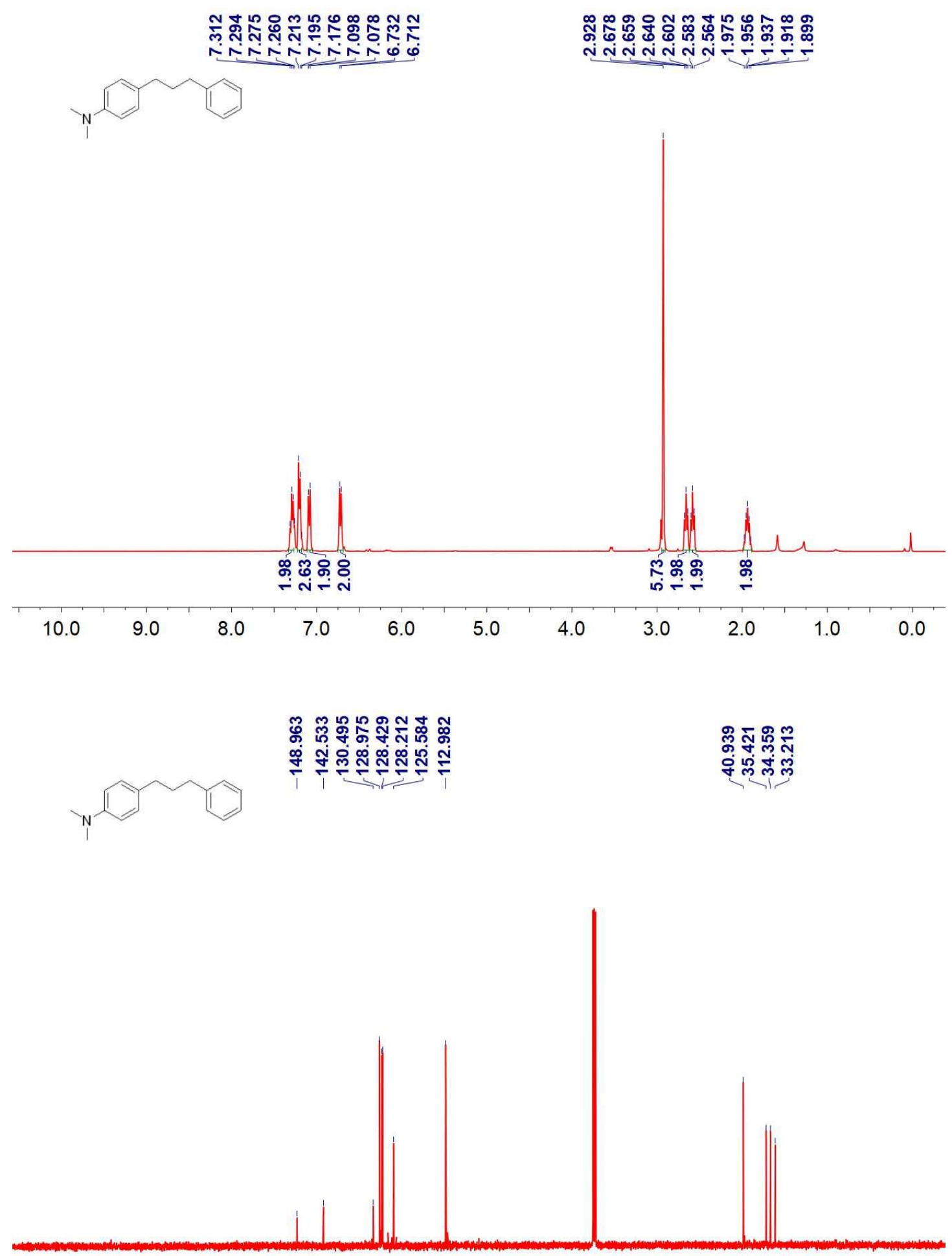

$210200190180170160150140130120110100 \quad 90 \quad 80 \quad 70 \quad 60 \quad 50 \quad 40 \quad 30 \quad 20 \quad 10 \quad 0$ 
4-(3,7-Dimethyloct-6-en-1-yl)-N,N-dimethylaniline (2k)

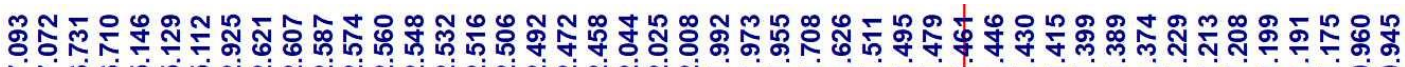
-
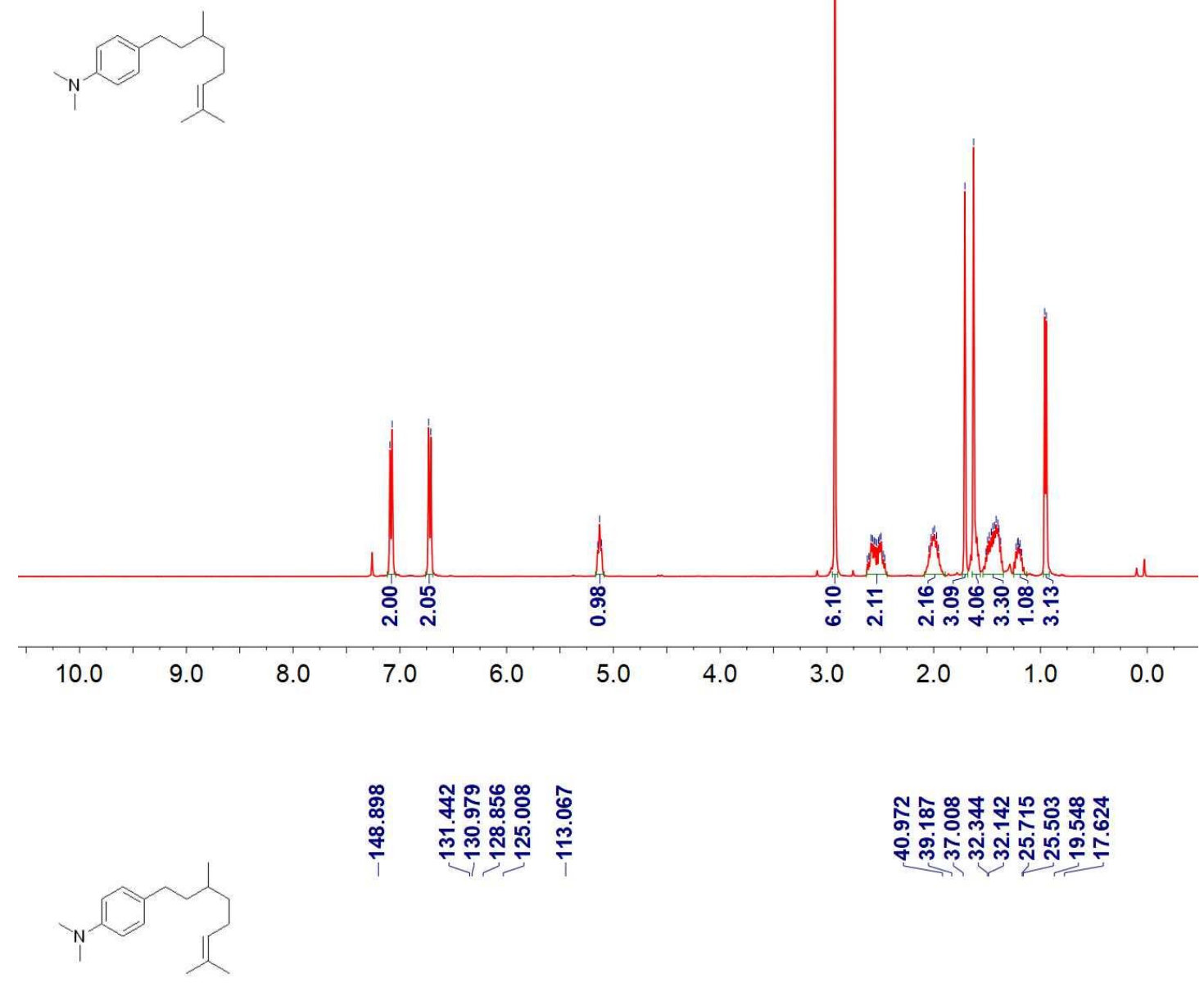

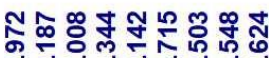

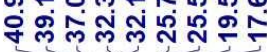

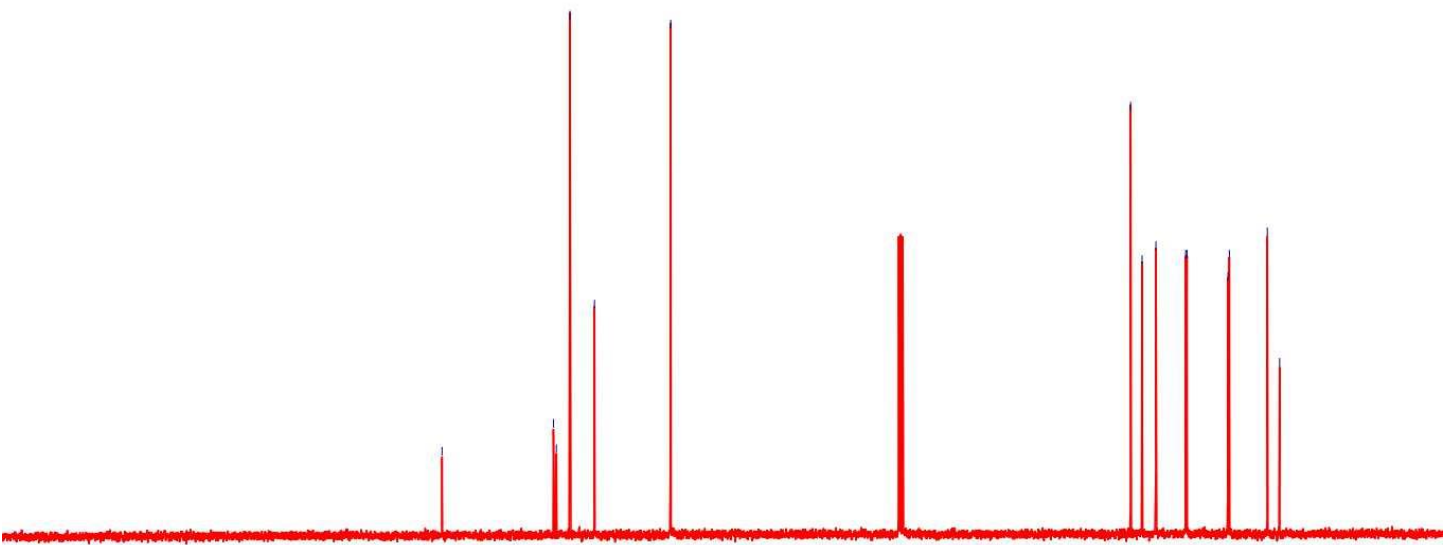

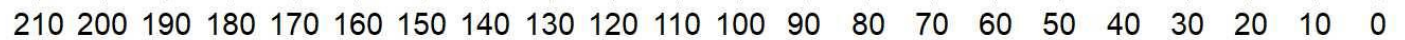


4-(Benzo[d][1,3]dioxol-5-ylmethyl)-N,N-dimethylaniline (2l)

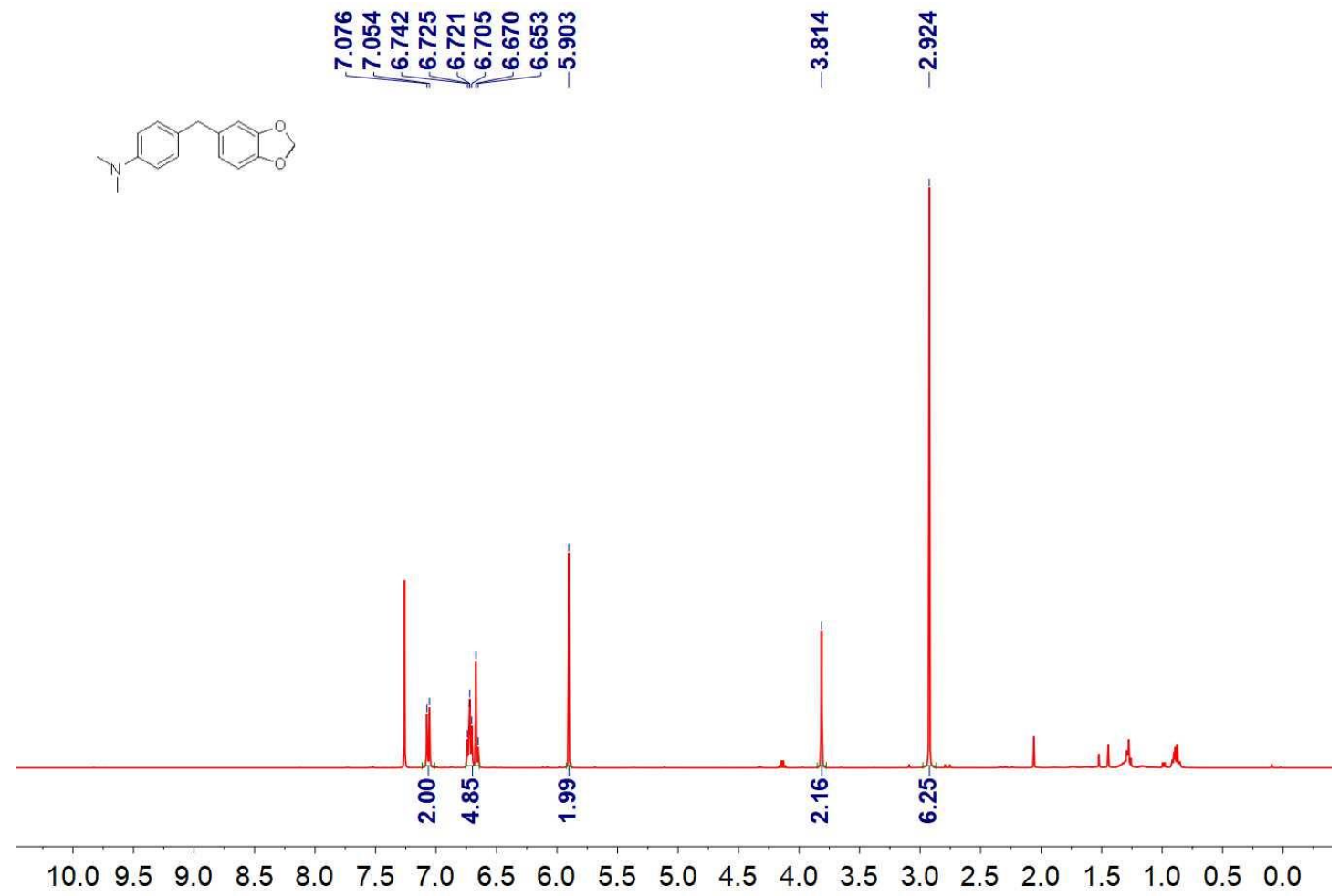

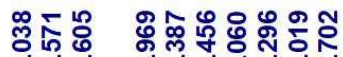

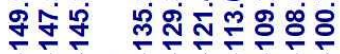

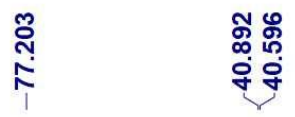
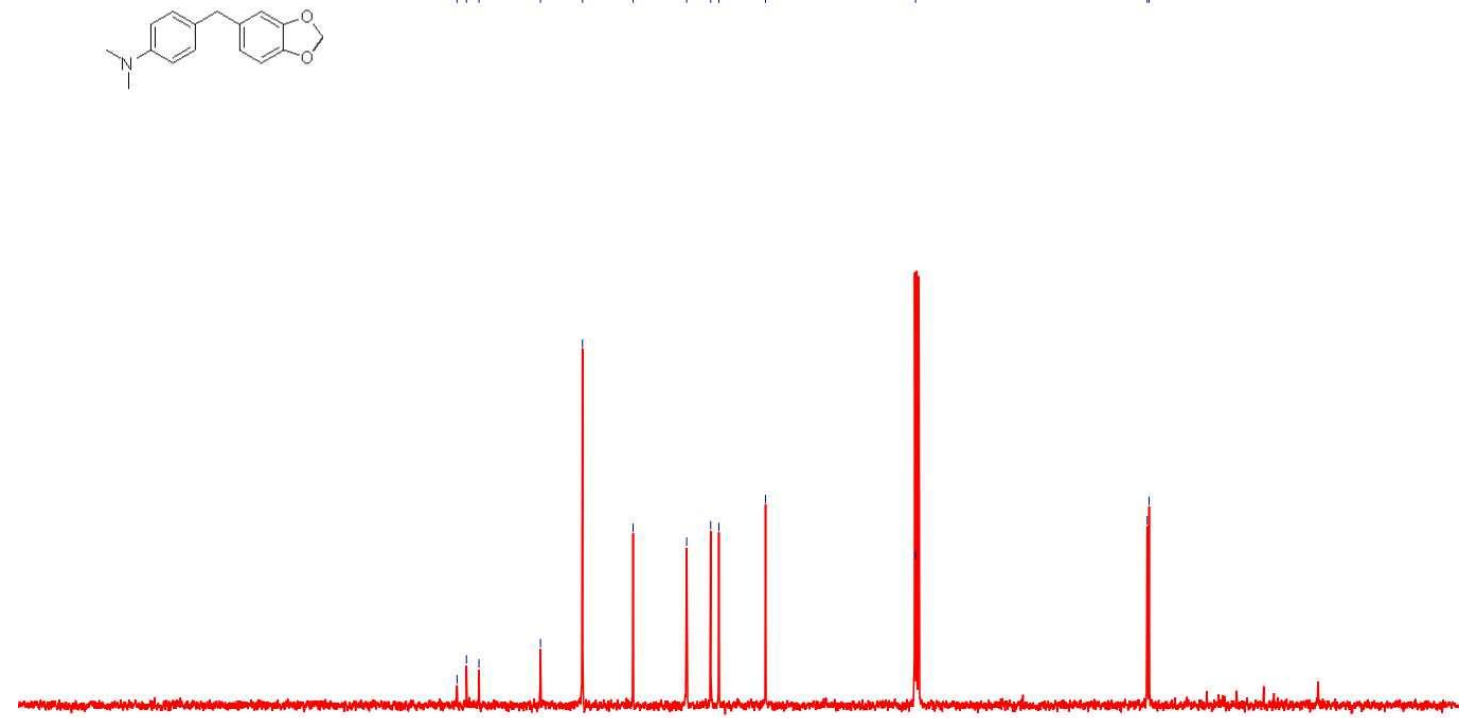

$210200190180170160150140130120110100 \quad 90 \quad 80 \quad 70 \quad 60 \quad 50 \quad 40 \quad 30 \quad 20 \quad 10 \quad 0$ 
$N$-Butyl-4-(4-(dimethylamino)benzyl)- $N$-methylaniline (2m)
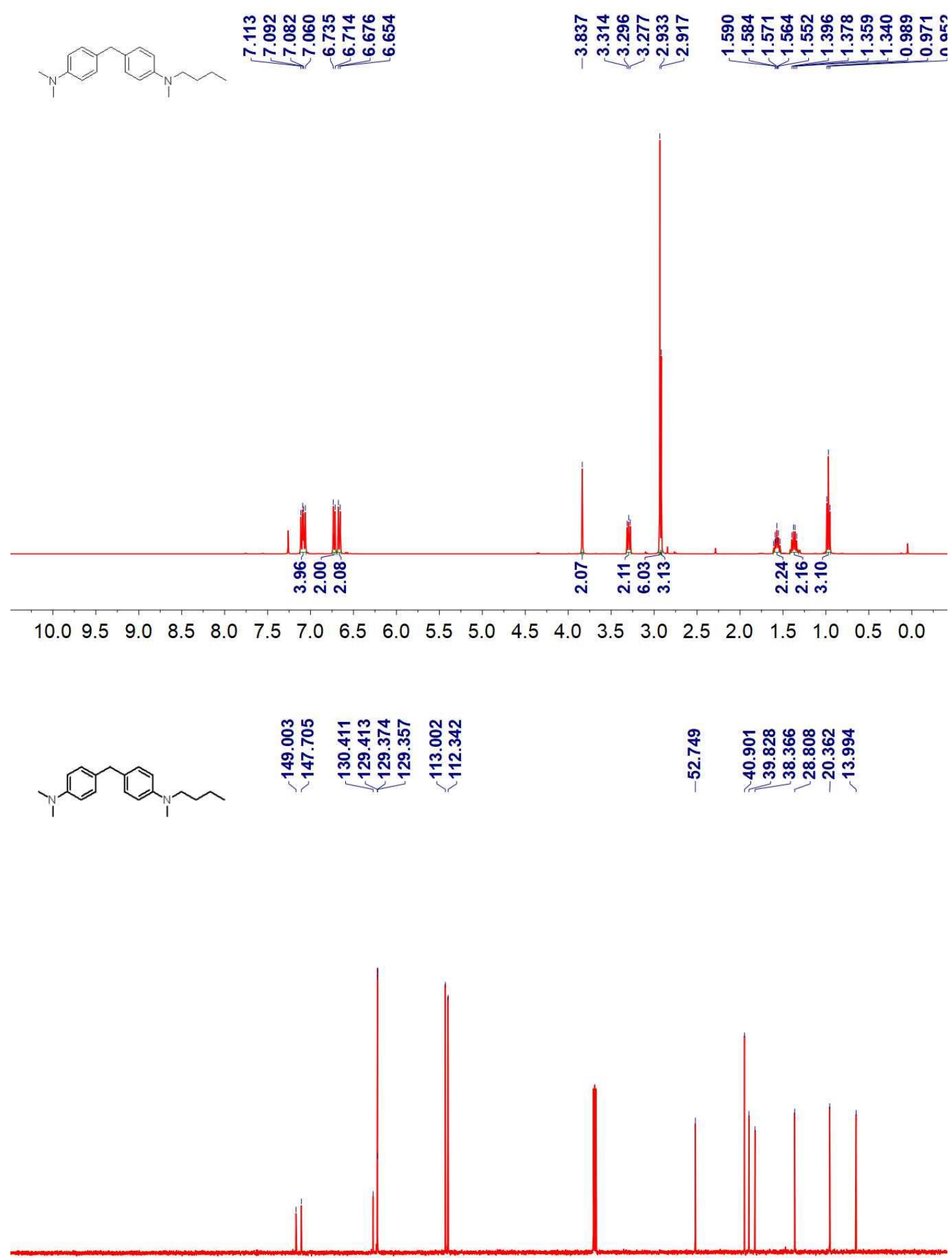

$210200190180170160150140130120110 \quad 100 \quad 90 \quad 80 \quad 70 \quad 60 \quad 50 \quad 40 \quad 30 \quad 20 \quad 10 \quad 0$ 
$N, N$-dimethyl-4-(2,4,6-trimethylbenzyl)aniline (2n) ริํำ

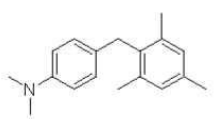
ஸ்

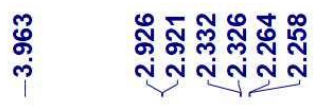

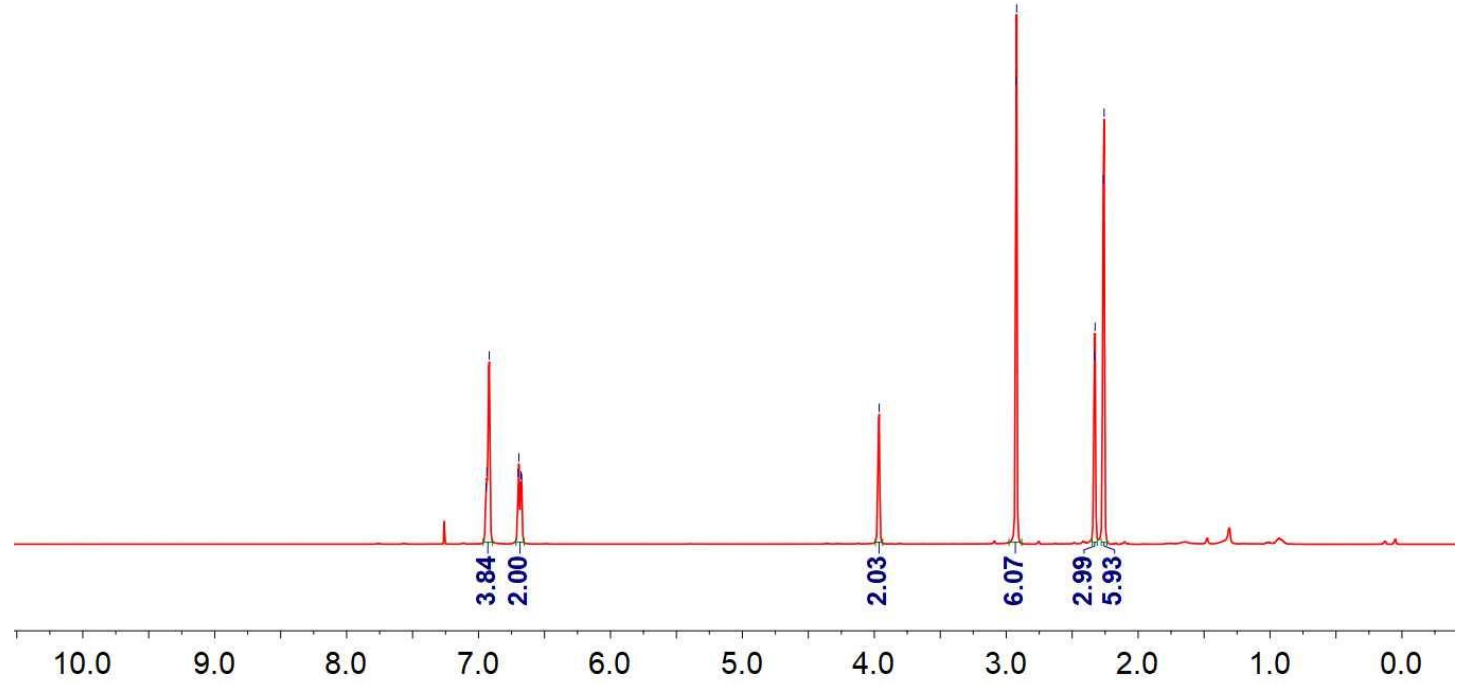

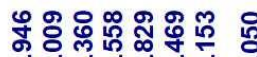

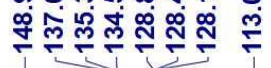

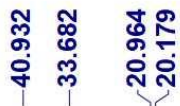

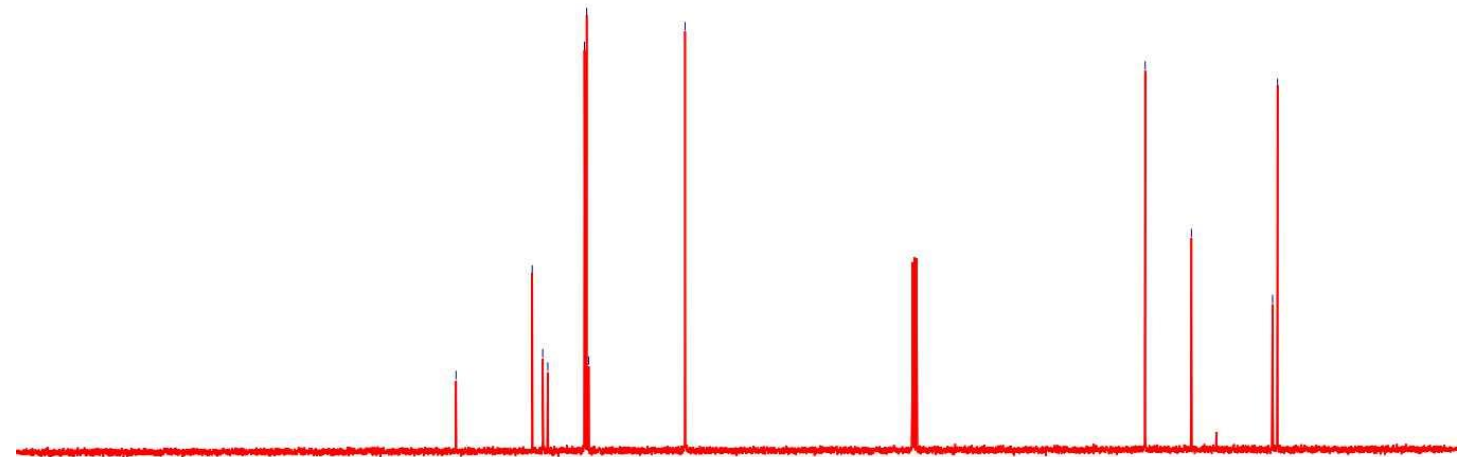

$210200190180170160150140130120110 \quad 100 \quad 90 \quad 80 \quad 70 \quad 60 \quad 50 \quad 40 \quad 30 \quad 20 \quad 10 \quad 0$ 
N,N-Dimethyl-4-(4-(methylthio)benzyl)aniline (20)
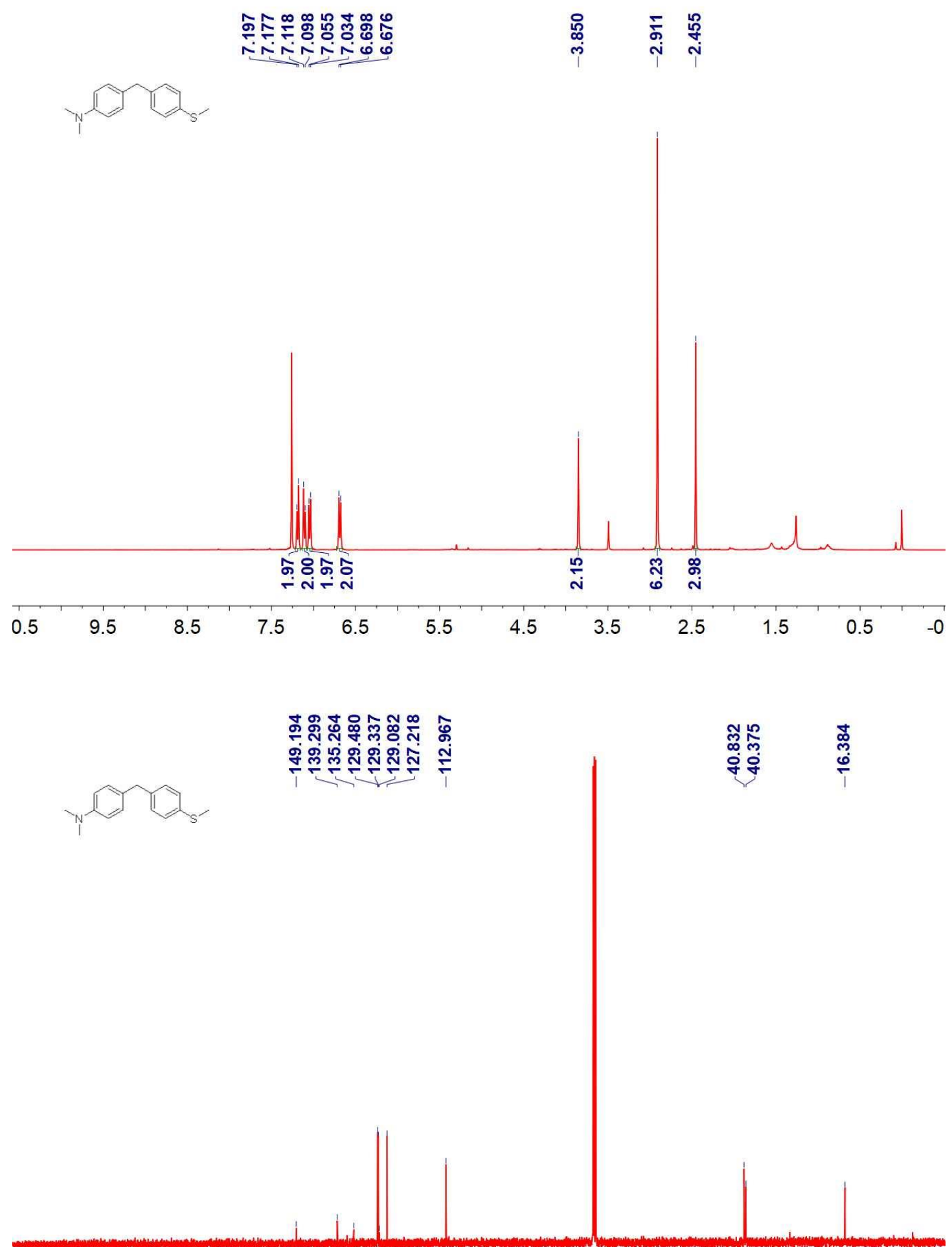

$210200190180170160150140130120110100 \quad 90 \quad 80 \quad 70 \quad 60 \quad 50 \quad 40 \quad 30 \quad 20 \quad 10 \quad 0$ 
4-(4-Methoxybenzyl)- N,N-dimethylaniline (2p)

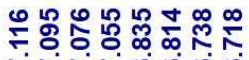

NNN

包离

N

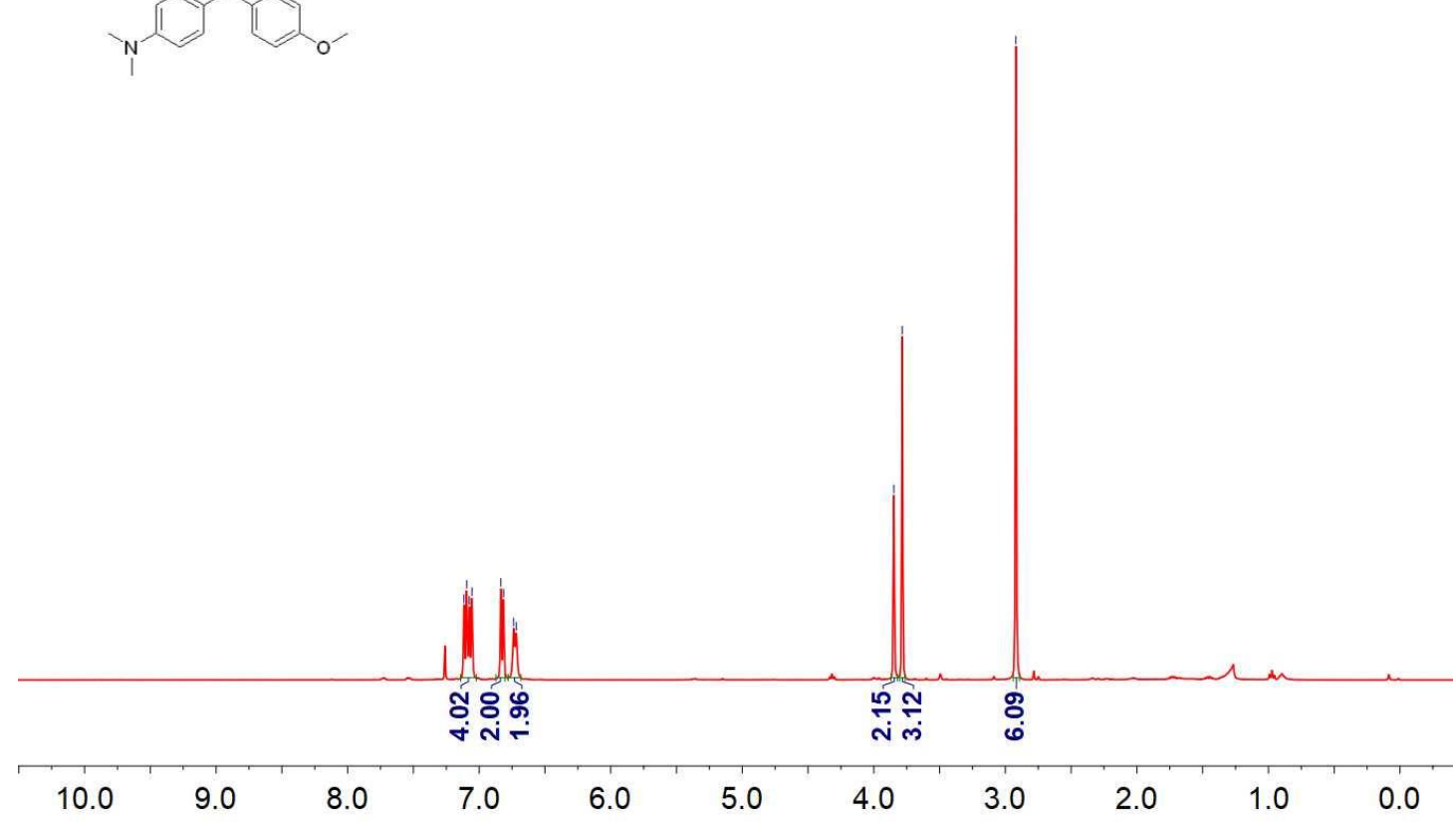

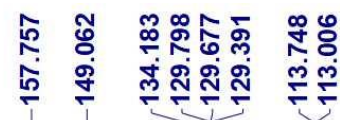

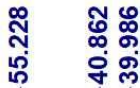

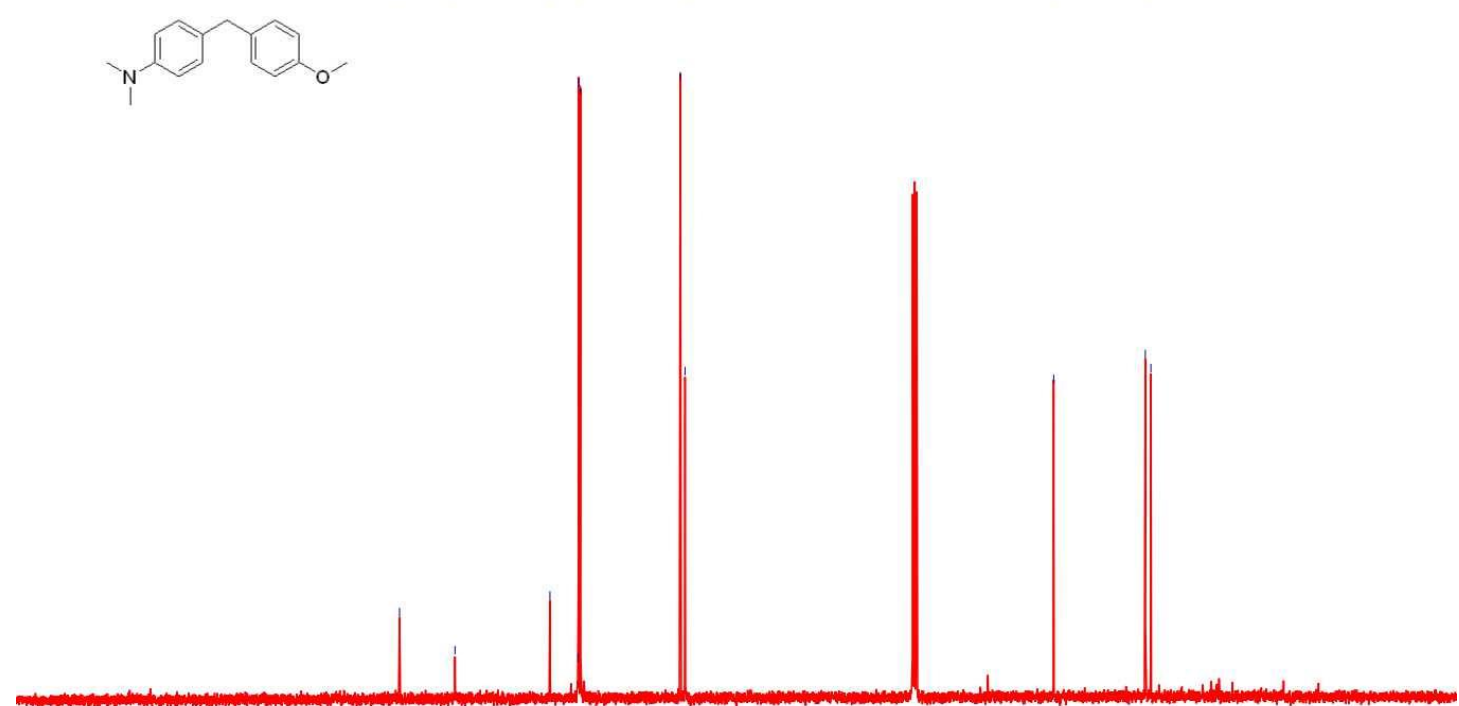

$210200190180170160150140130120110 \quad 100 \quad 90 \quad 80 \quad 70 \quad 60 \quad 50 \quad 40 \quad 30 \quad 20 \quad 10 \quad 0$ 
$N, N$-dimethyl-4-(4-methylbenzyl)aniline (2q)

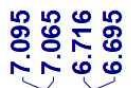

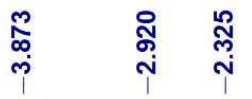
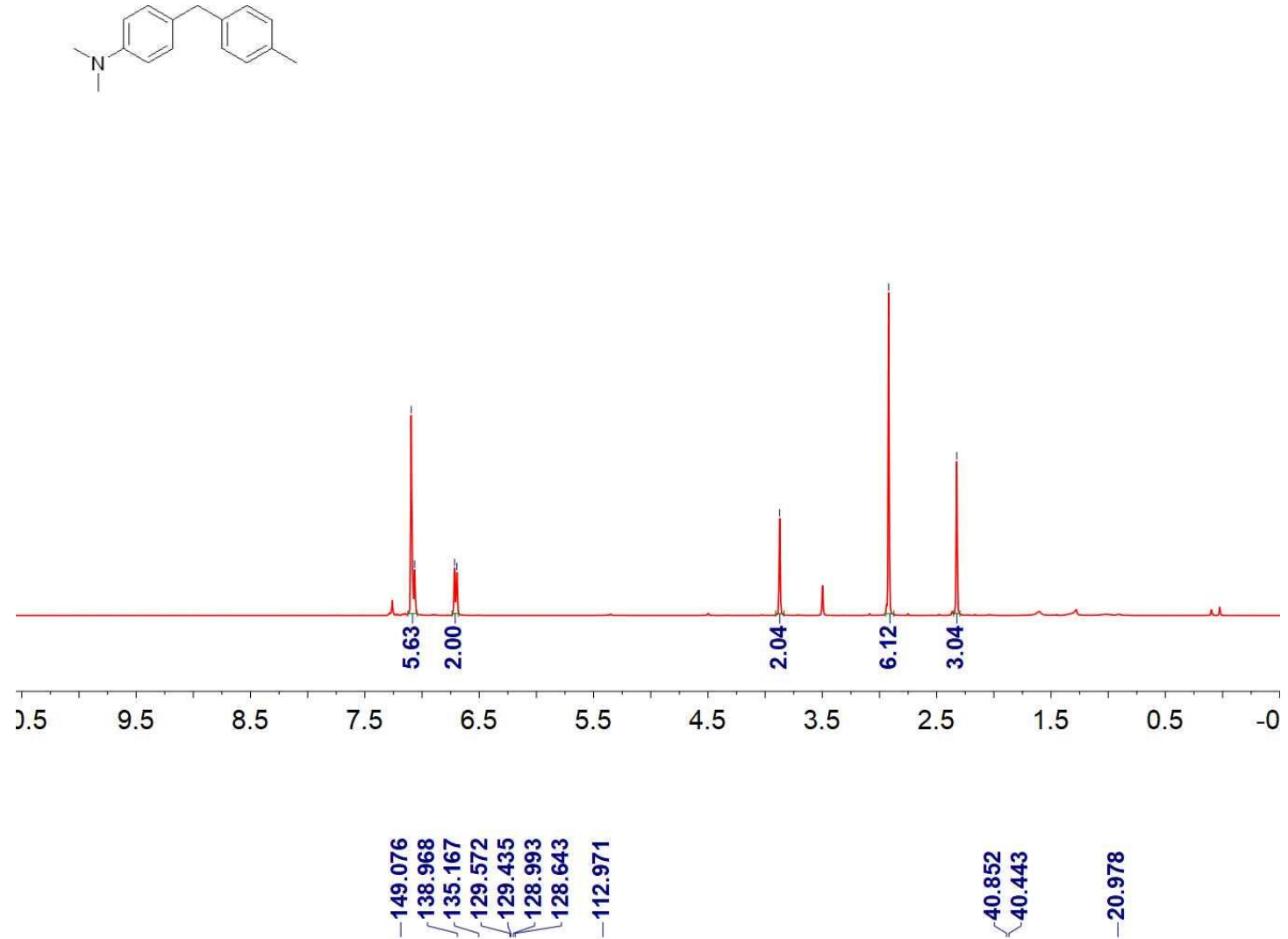

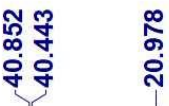
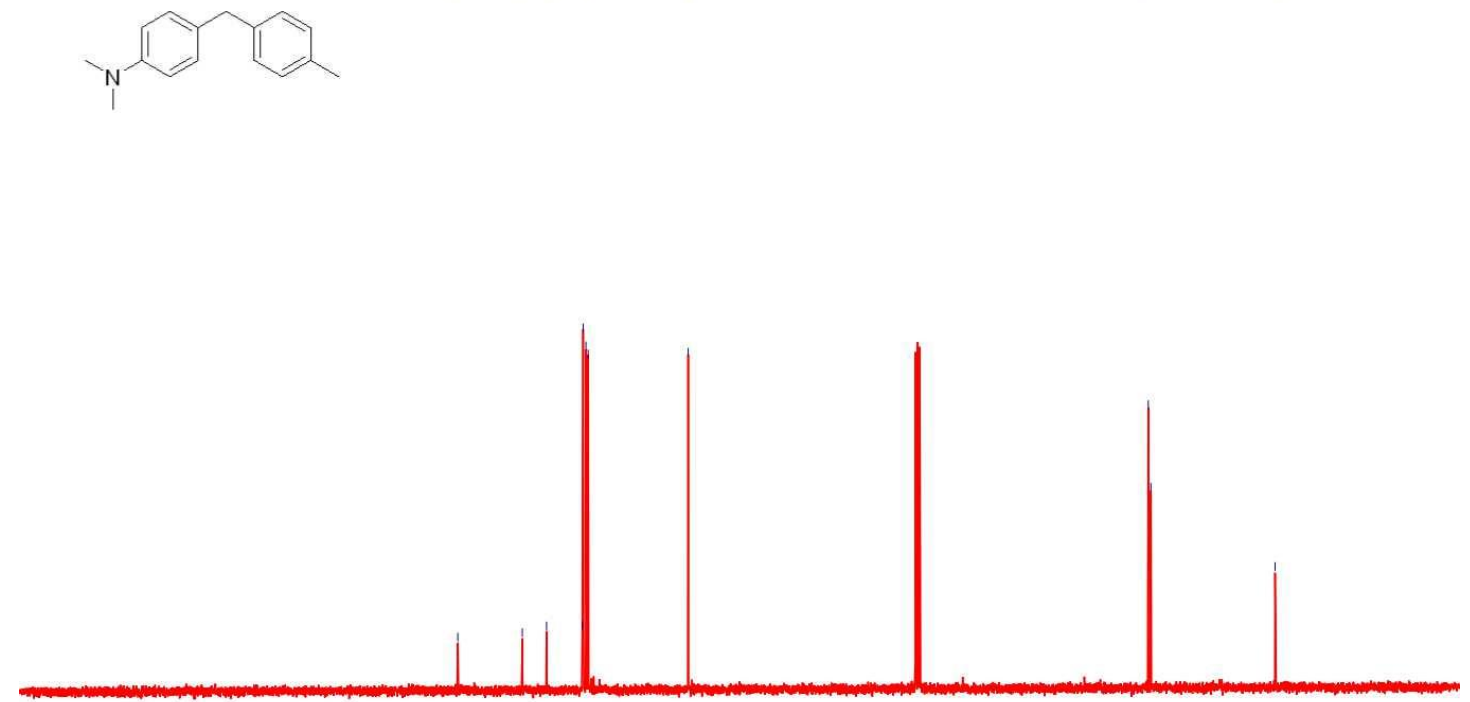

$210200190180170160150140130120110100 \quad 90 \quad 80 \quad 70 \quad 60 \quad 50 \quad 40 \quad 30 \quad 20 \quad 10 \quad 0$ 
N,N-dimethyl-4-(3-methylbenzyl)aniline (2r)

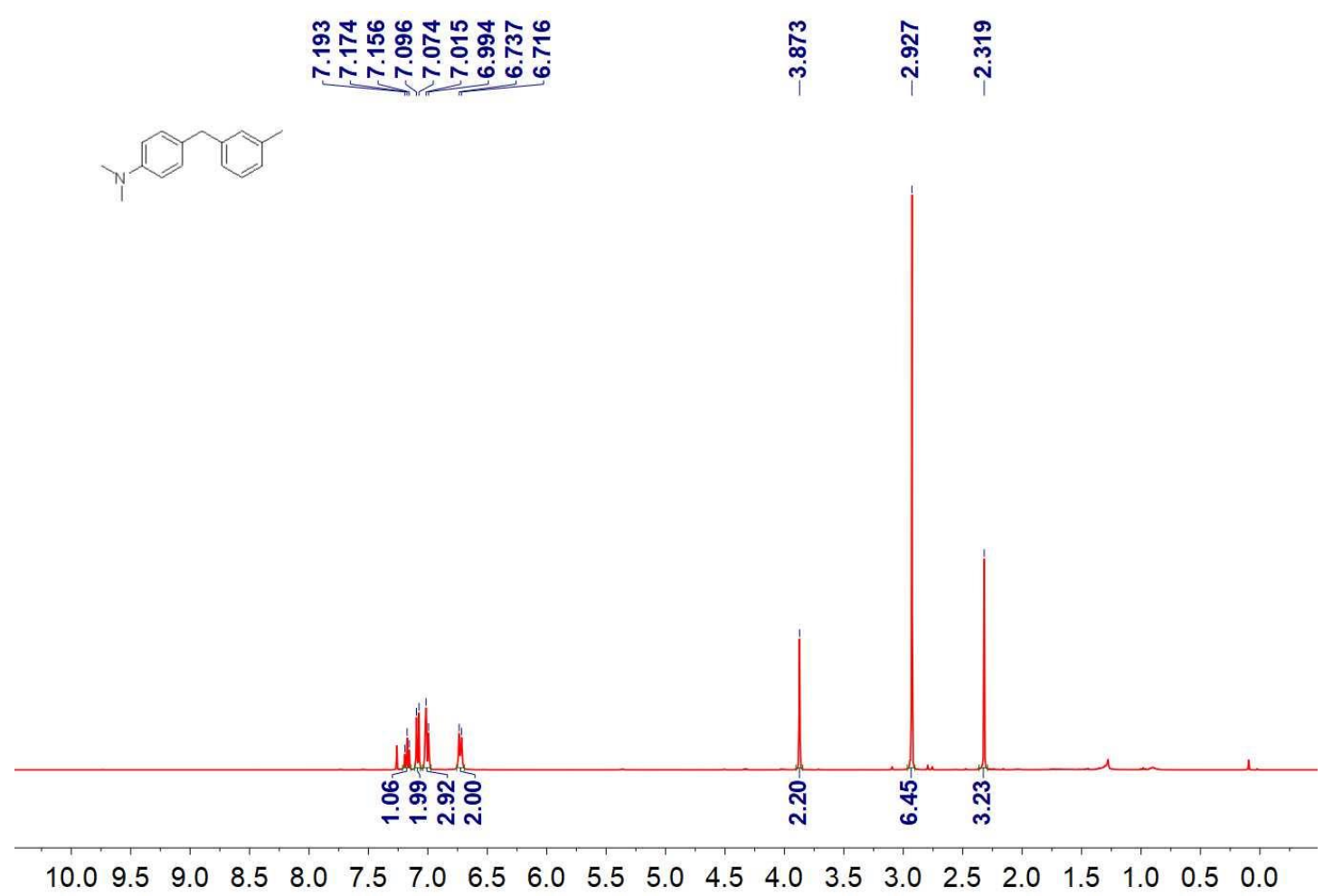

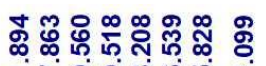

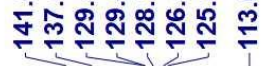

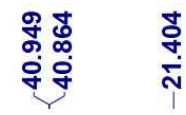

$210200190180170160150140130120110100 \quad 90 \quad 80 \quad 70 \quad 60 \begin{array}{lllllll}40 & 40 & 30 & 20 & 10 & 0\end{array}$ 
$N, N$-dimethyl-4-(2-methylbenzyl)aniline (2s)

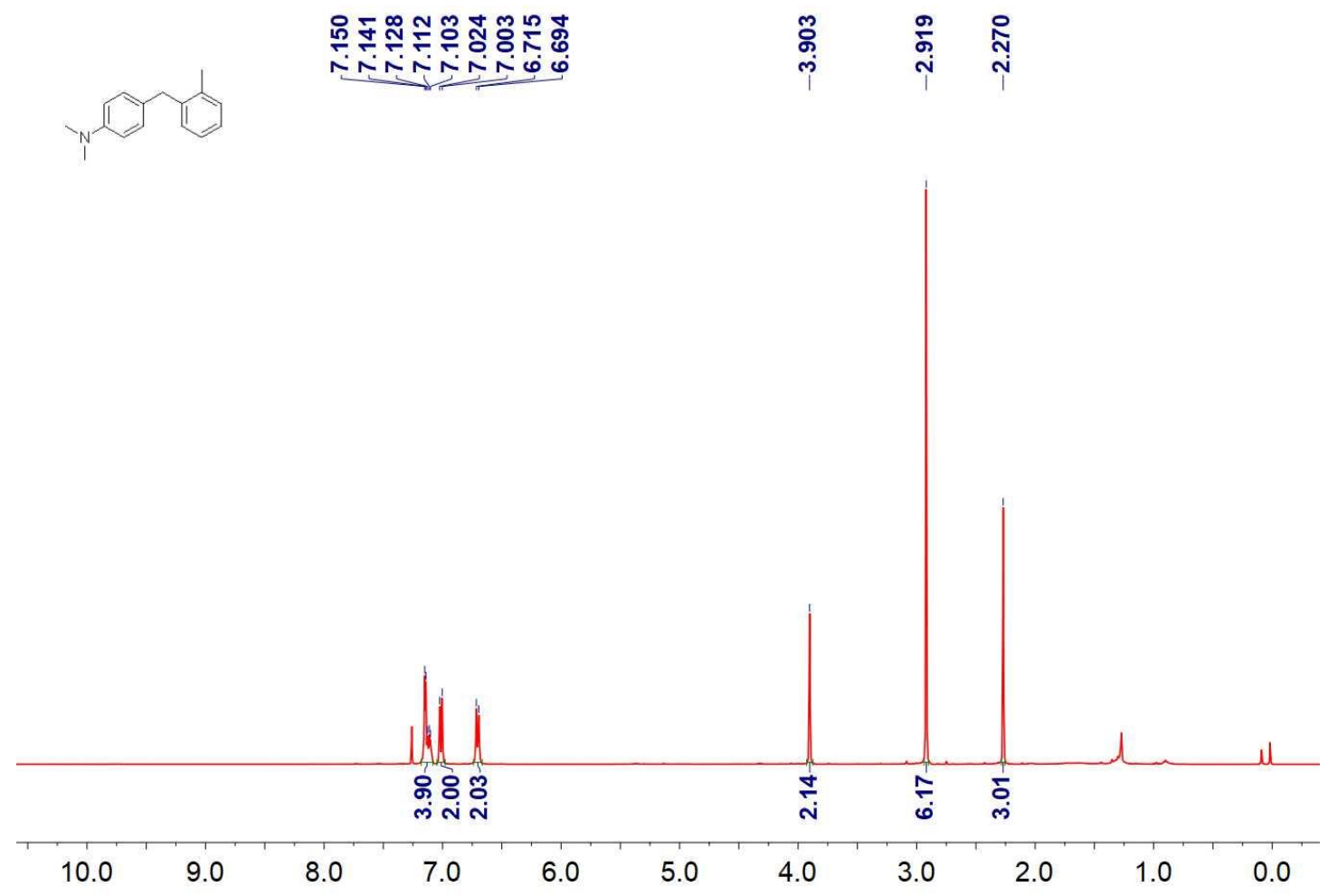

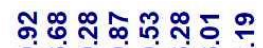

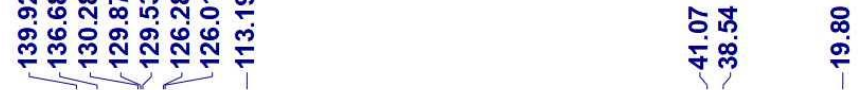
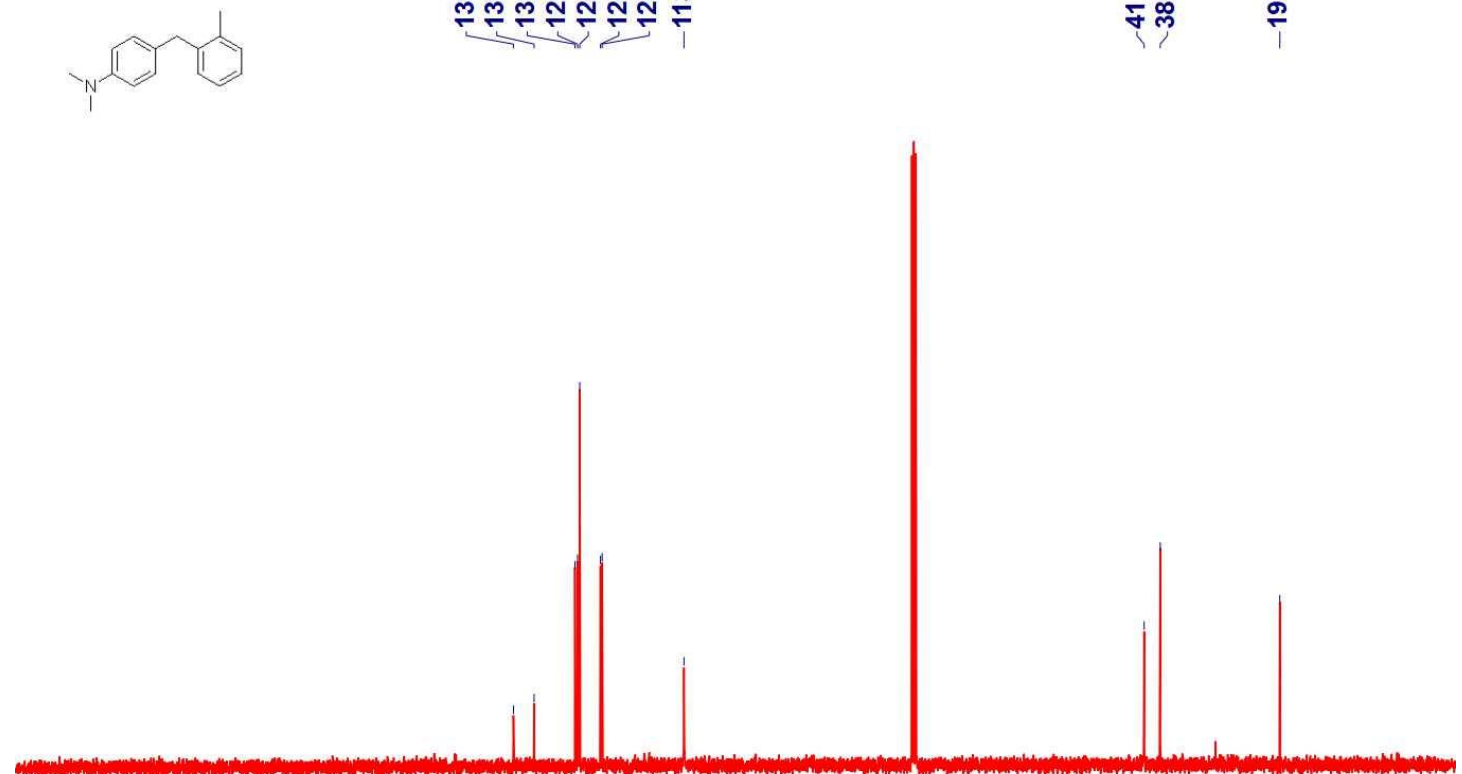

$210200190180170160150140130120110100 \quad 90 \quad 80 \quad 70 \quad 60 \quad 50 \quad 40 \quad 30 \quad 20 \quad 10 \quad 0$ 
4-(4-Isopropylbenzyl)-N,N-dimethylaniline (2t)

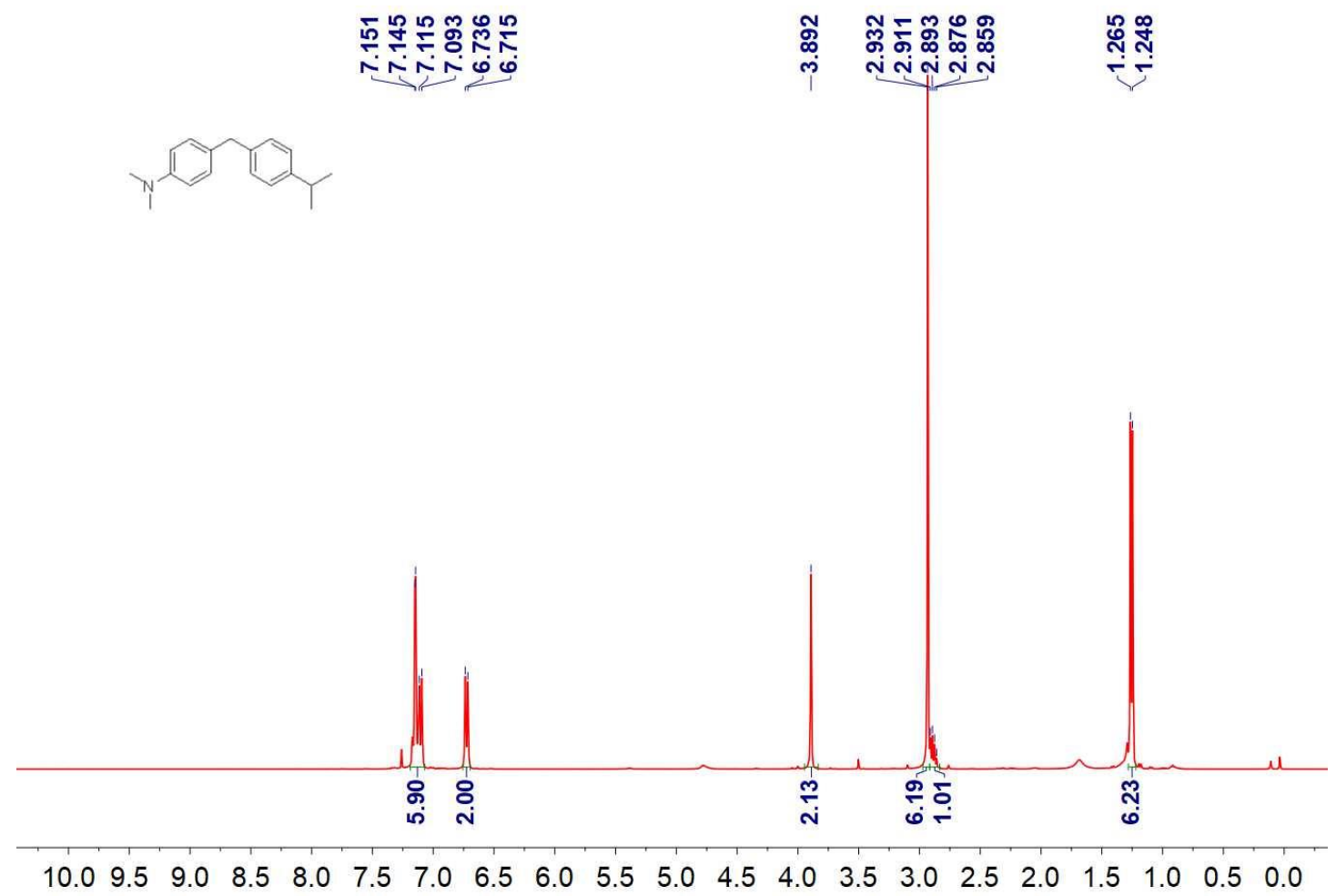

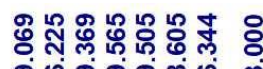

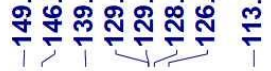

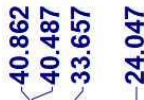
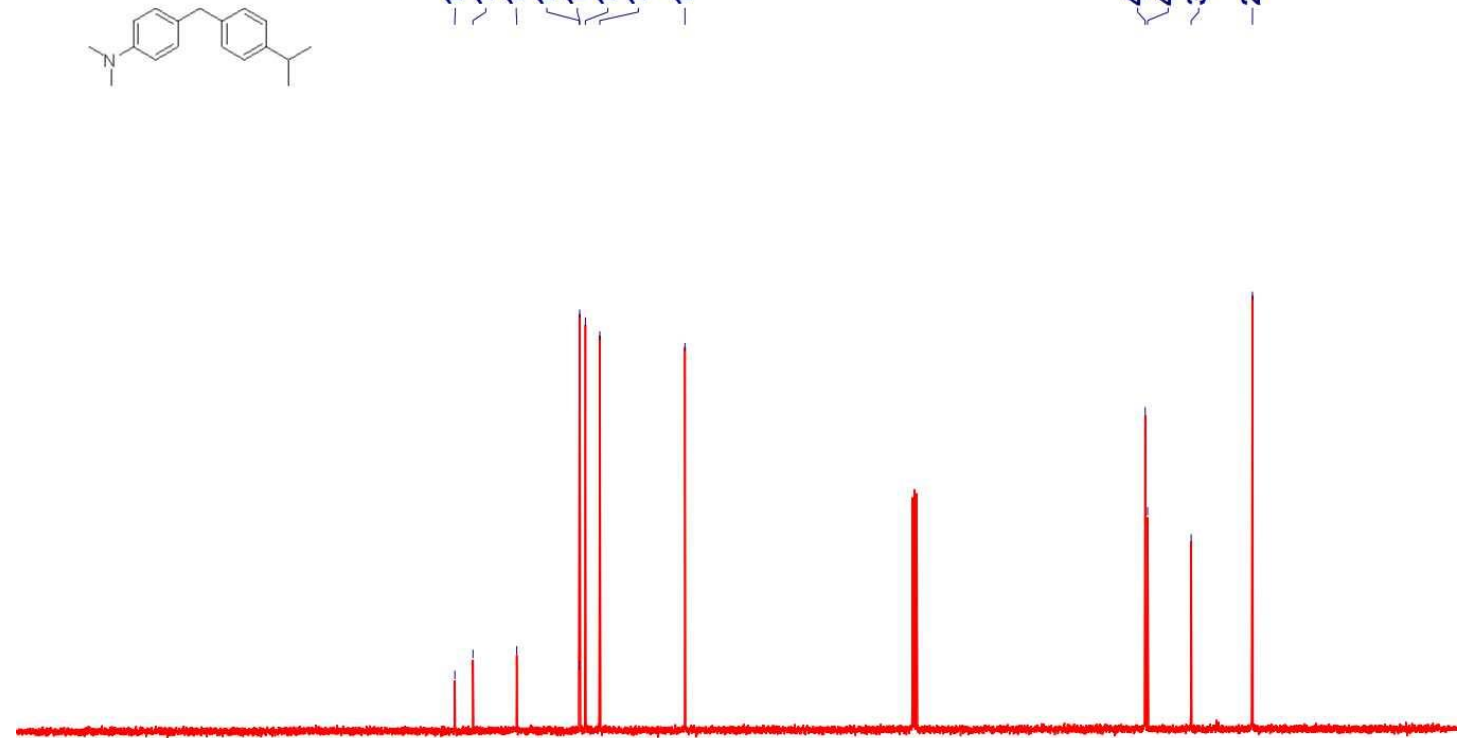

$210200190180170160150140130120110 \quad 100 \quad 90 \quad 80 \quad 70 \quad 60 \quad 50 \quad 40 \quad 30 \quad 20 \quad 10 \quad 0$ 


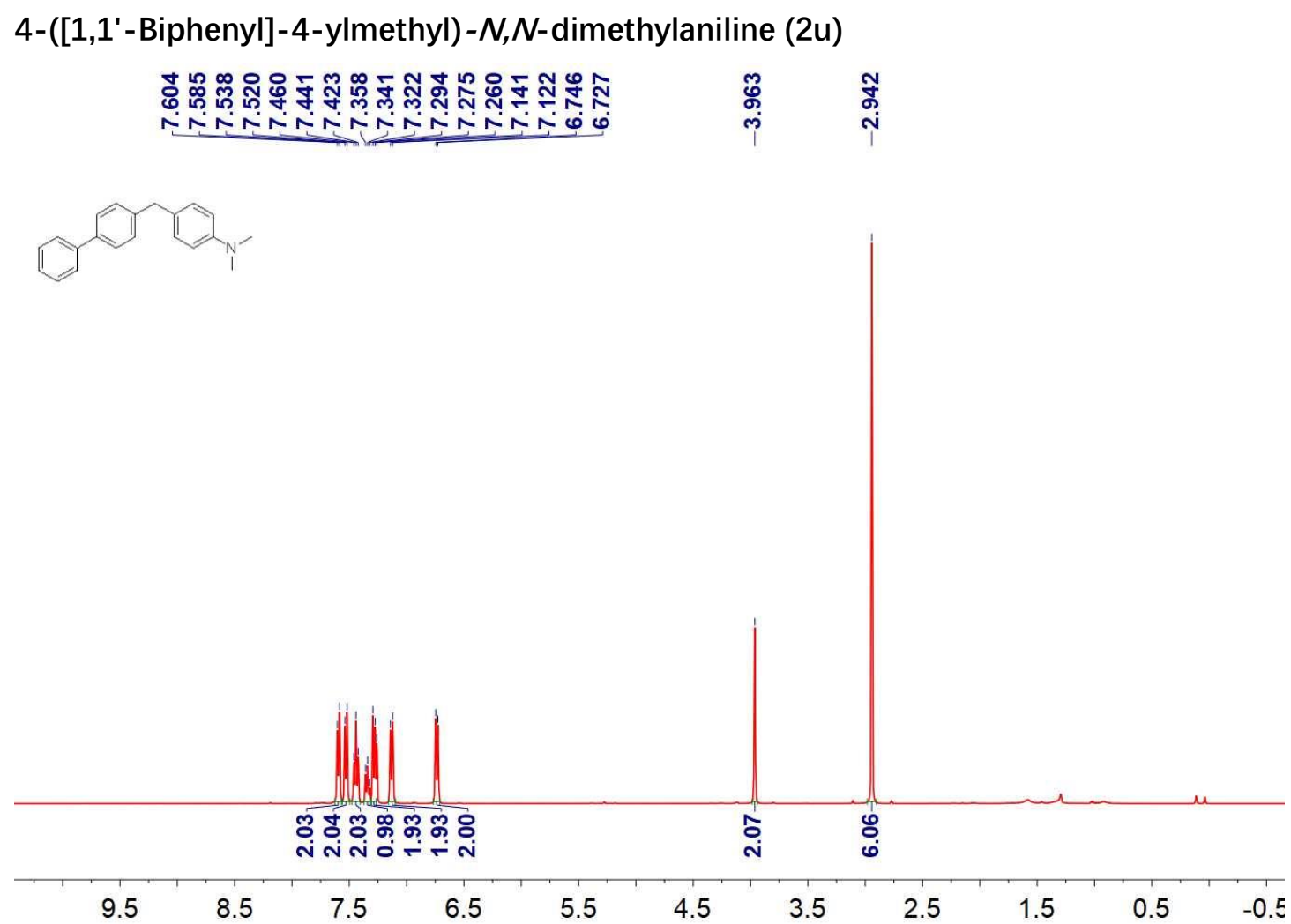

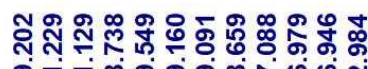

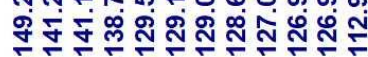

$\bar{\delta}_{\substack{0 \\ \infty}}^{\infty}$

कq
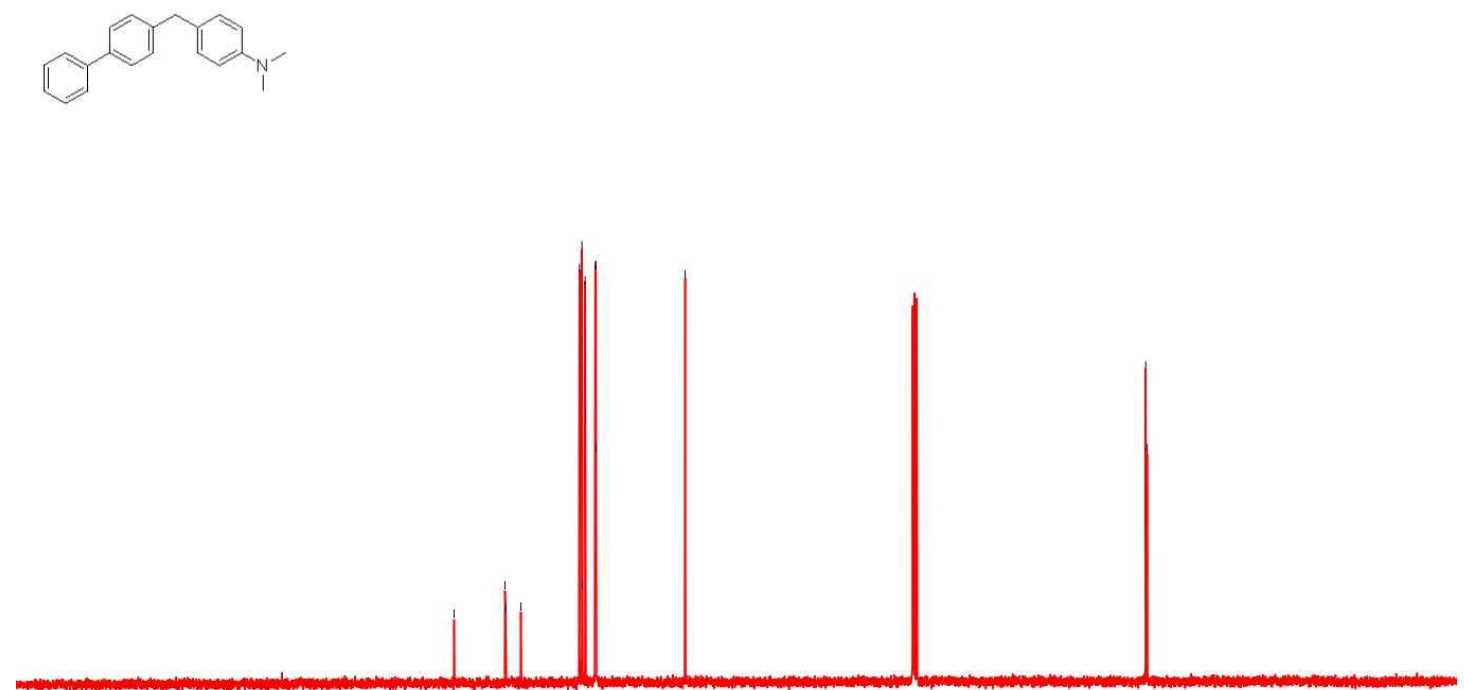

$210200190180170160150140130120110100 \quad 90 \quad 80 \quad 70 \quad 60 \quad 50 \quad 40 \quad 30 \quad 20 \quad 10 \quad 0$ 


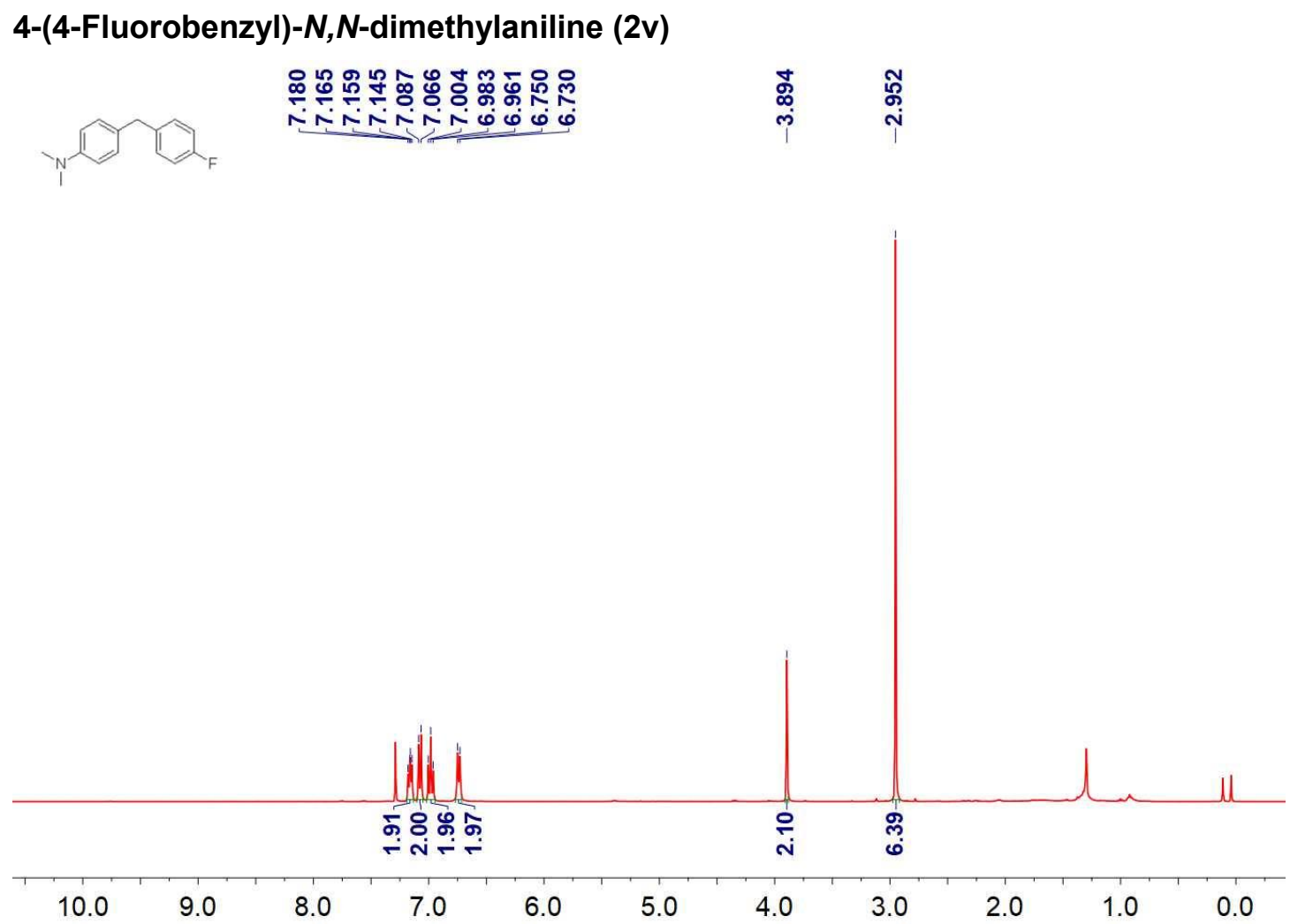

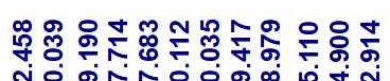

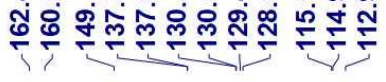

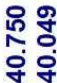
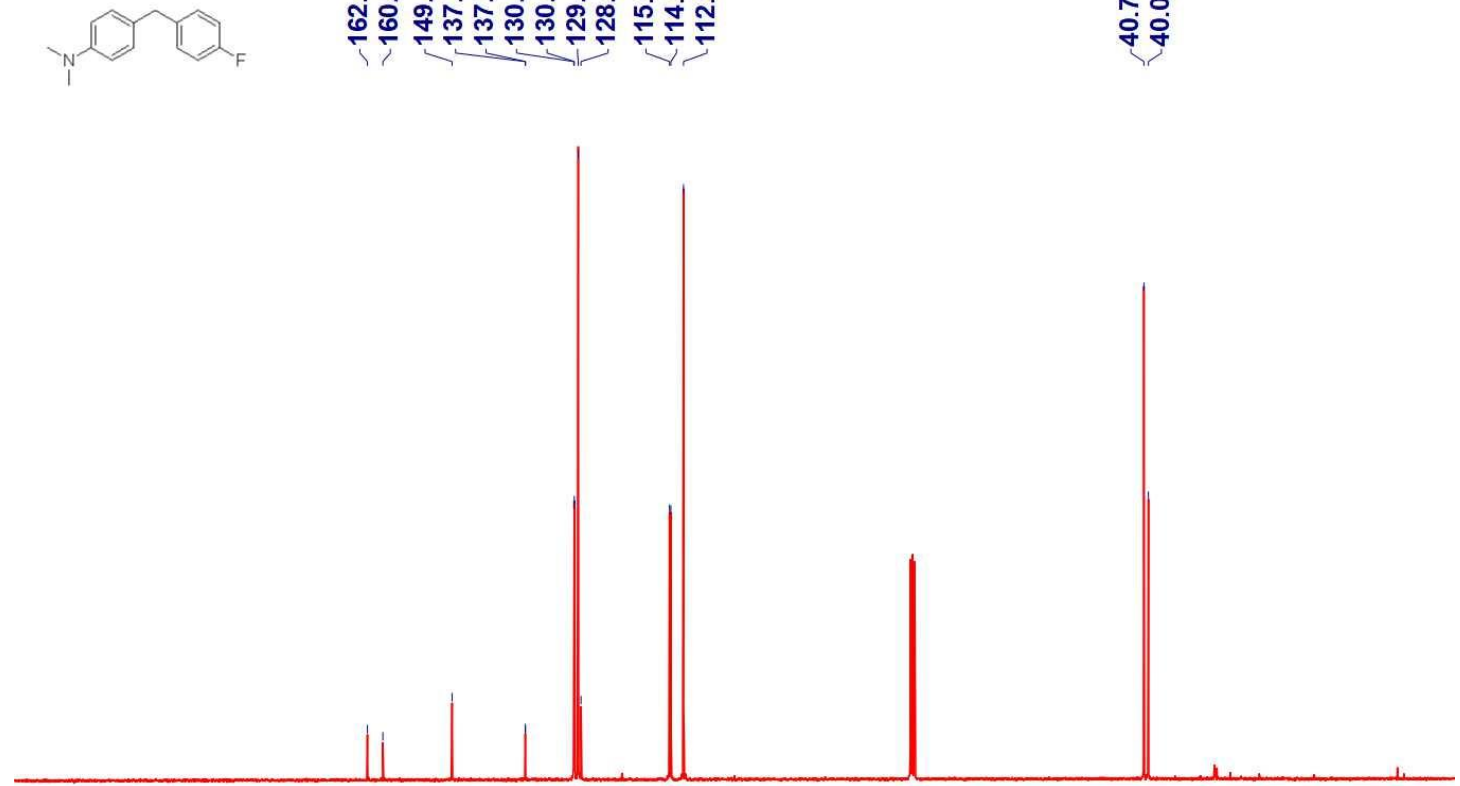

$210200190180170160150140130120110100 \quad 90 \quad 80 \quad 70 \quad 60 \quad 50 \quad 40 \quad 30 \quad 20 \quad 10 \quad 0$ 
4-(4-Chlorobenzyl)- $N, N$-dimethylaniline (2w)
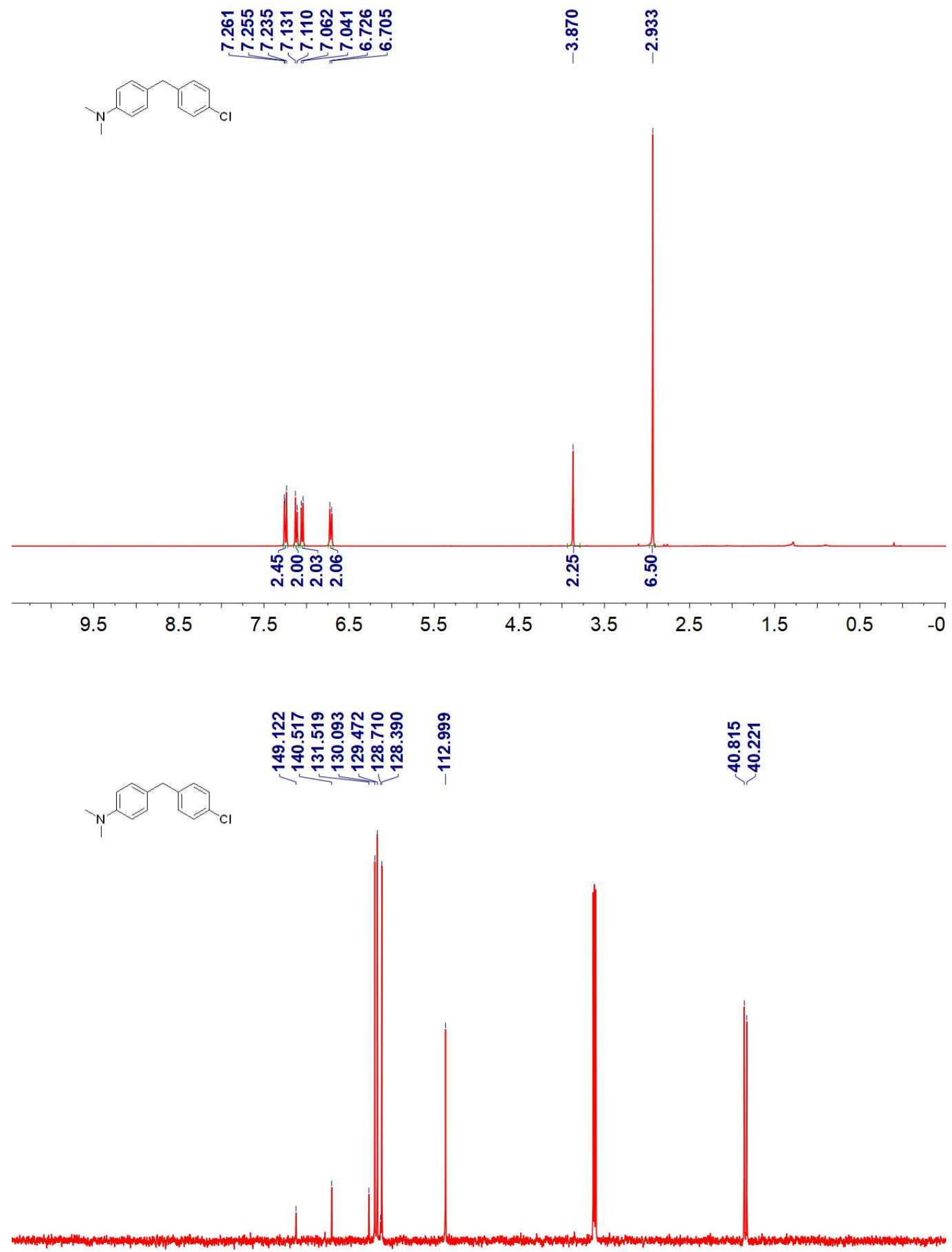

$210200190180170160150140130120110100 \quad 90 \quad 80 \quad 70 \quad 60 \quad 50 \quad 40 \quad 30 \quad 20 \quad 10 \quad 0$ 
4-(4-Bromobenzyl)- $N, N$-dimethylaniline (2x)

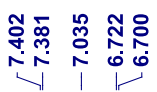

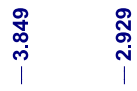

I

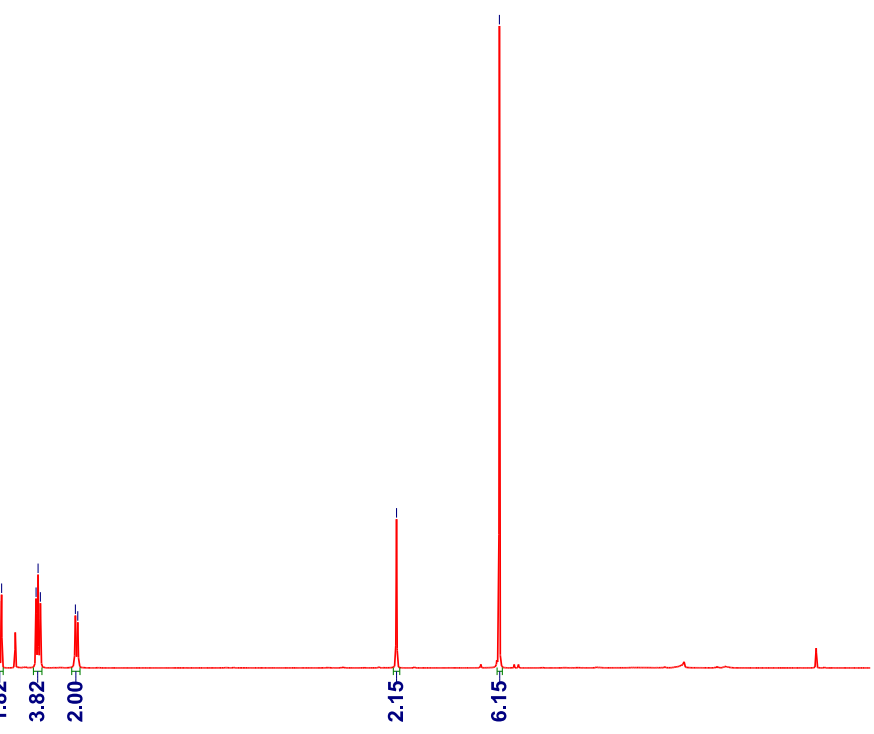

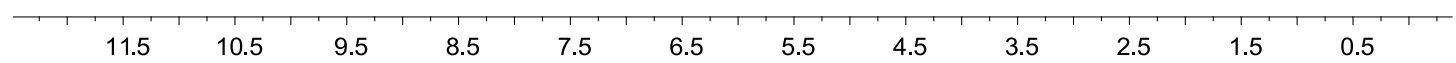

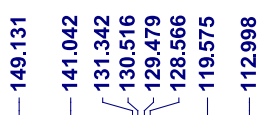

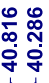
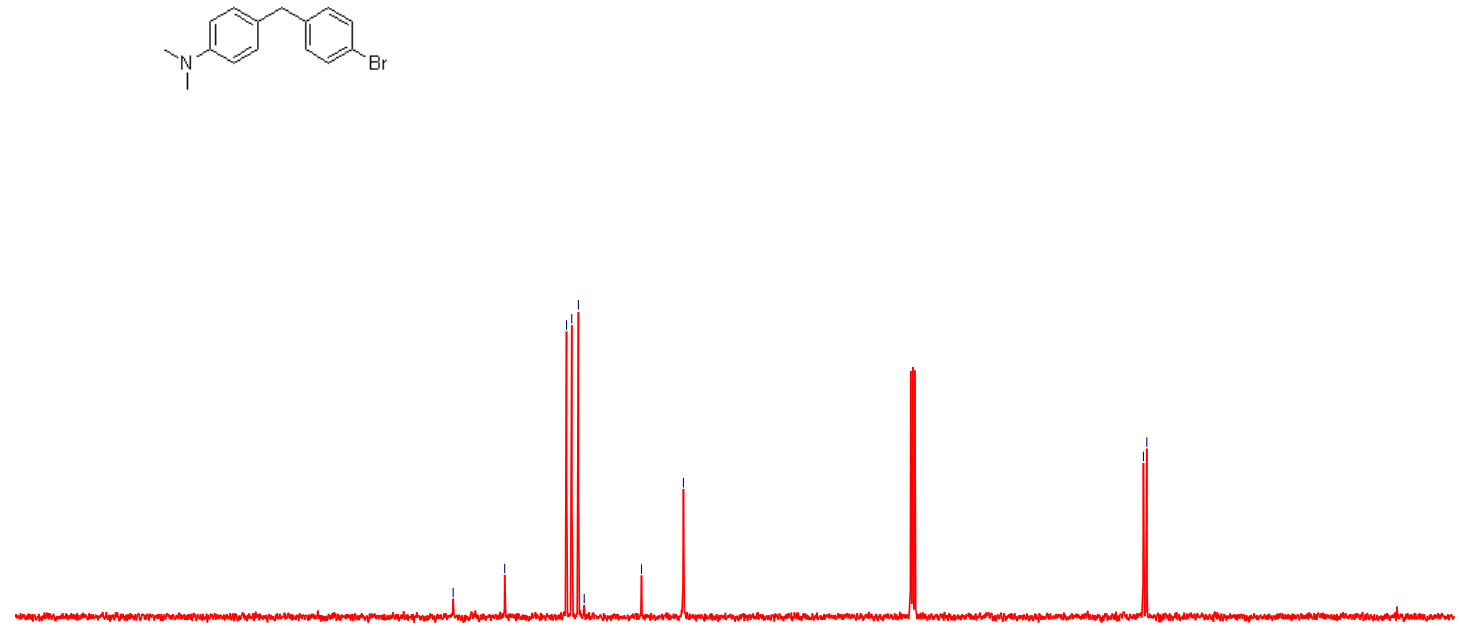

210

190

170

150

130

110

$\begin{array}{llllllllll}90 & 80 & 70 & 60 & 50 & 40 & 30 & 20 & 10 & 0\end{array}$ 
4-(3-Bromobenzyl)- $N, N$-dimethylaniline (2y)

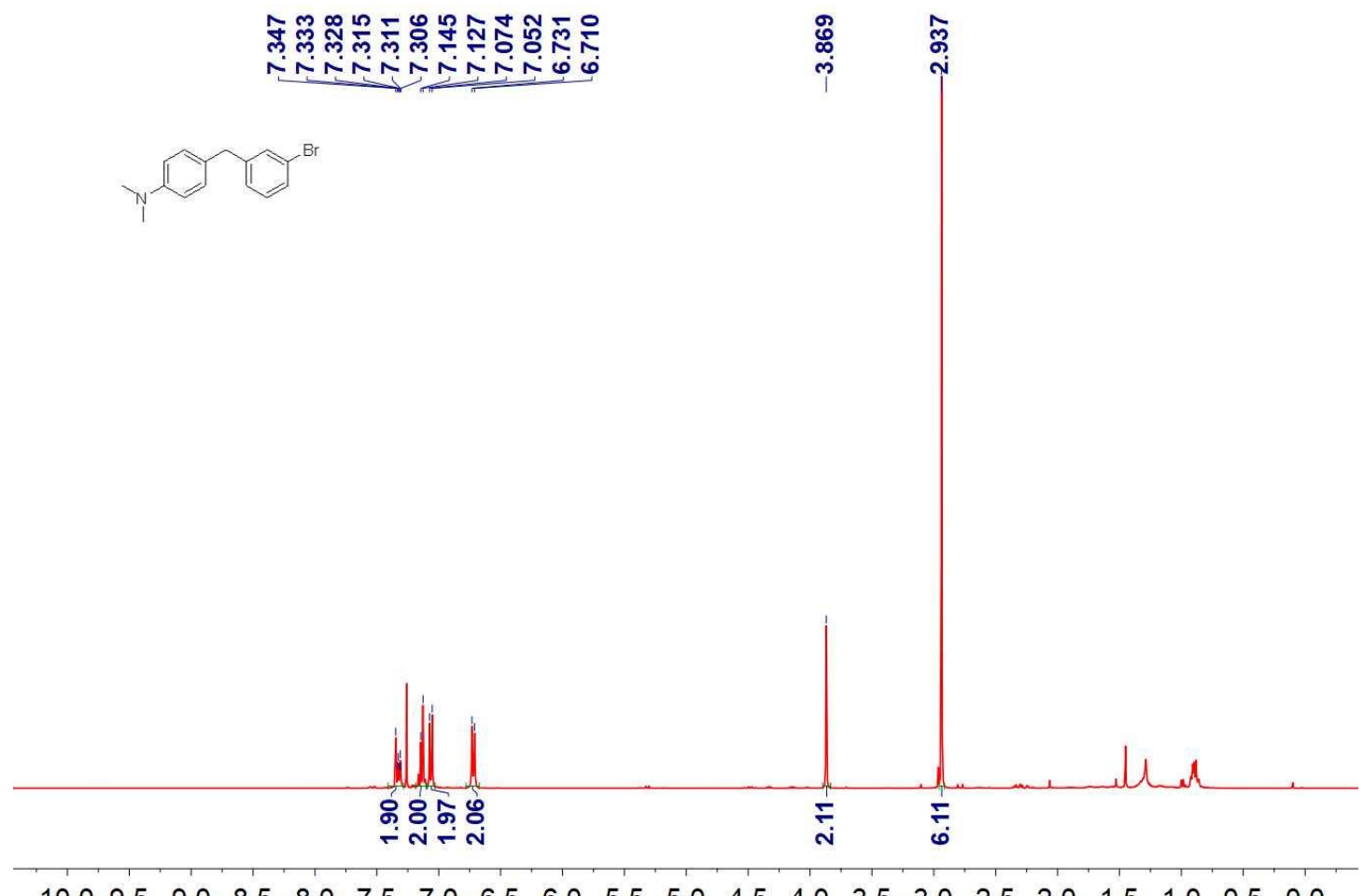

$\begin{array}{lllllllllllllllllllll}10.0 & 9.5 & 9.0 & 8.5 & 8.0 & 7.5 & 7.0 & 6.5 & 6.0 & 5.5 & 5.0 & 4.5 & 4.0 & 3.5 & 3.0 & 2.5 & 2.0 & 1.5 & 1.0 & 0.5 & 0.0\end{array}$

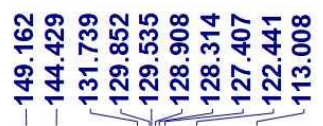

吕店

过

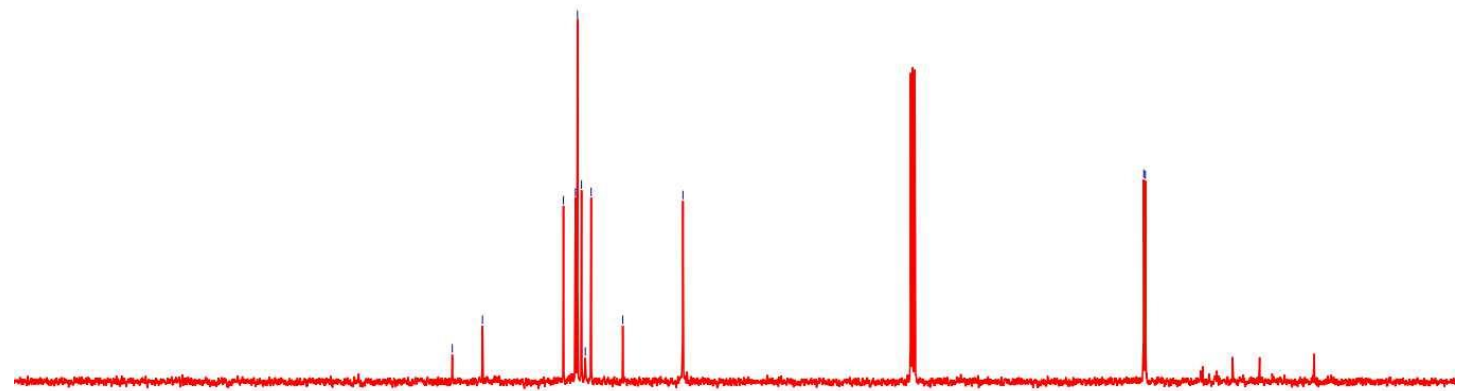

$210200190180170160150140130120110100 \quad 90 \quad 80 \quad 70 \quad 60 \quad 50 \quad 40 \quad 30 \quad 20 \quad 10 \quad 0$ 
4-(2-Bromobenzyl)- $N, N$-dimethylaniline (2z)
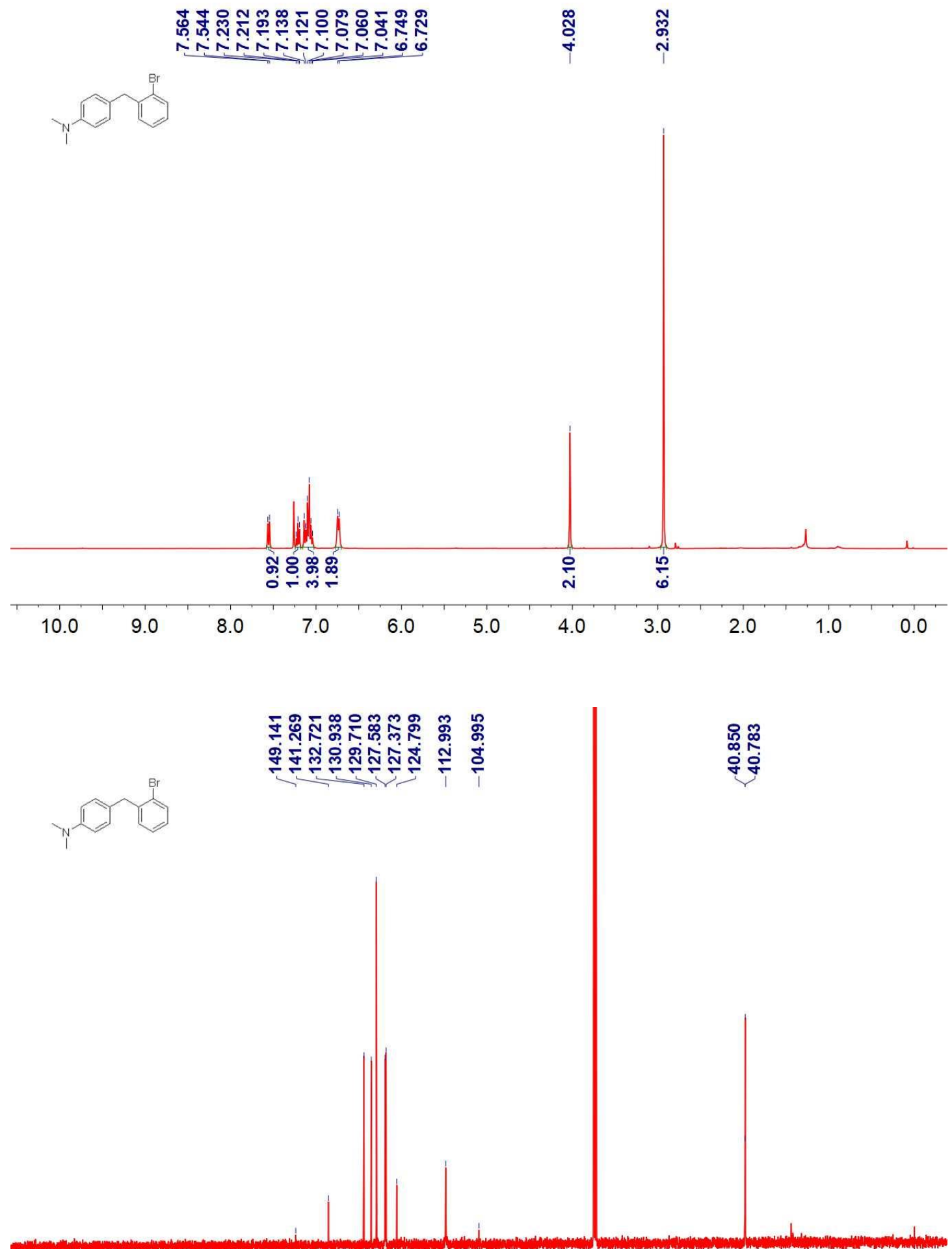

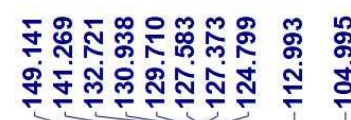

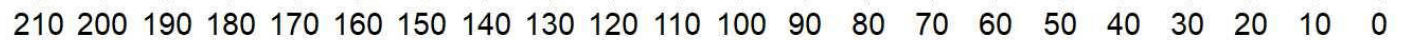


4-(4-lodobenzyl)-N,N-dimethylaniline (2aa)

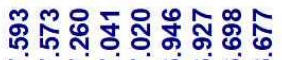

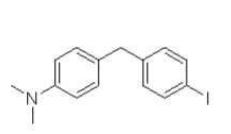

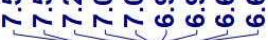

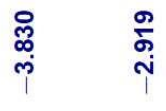
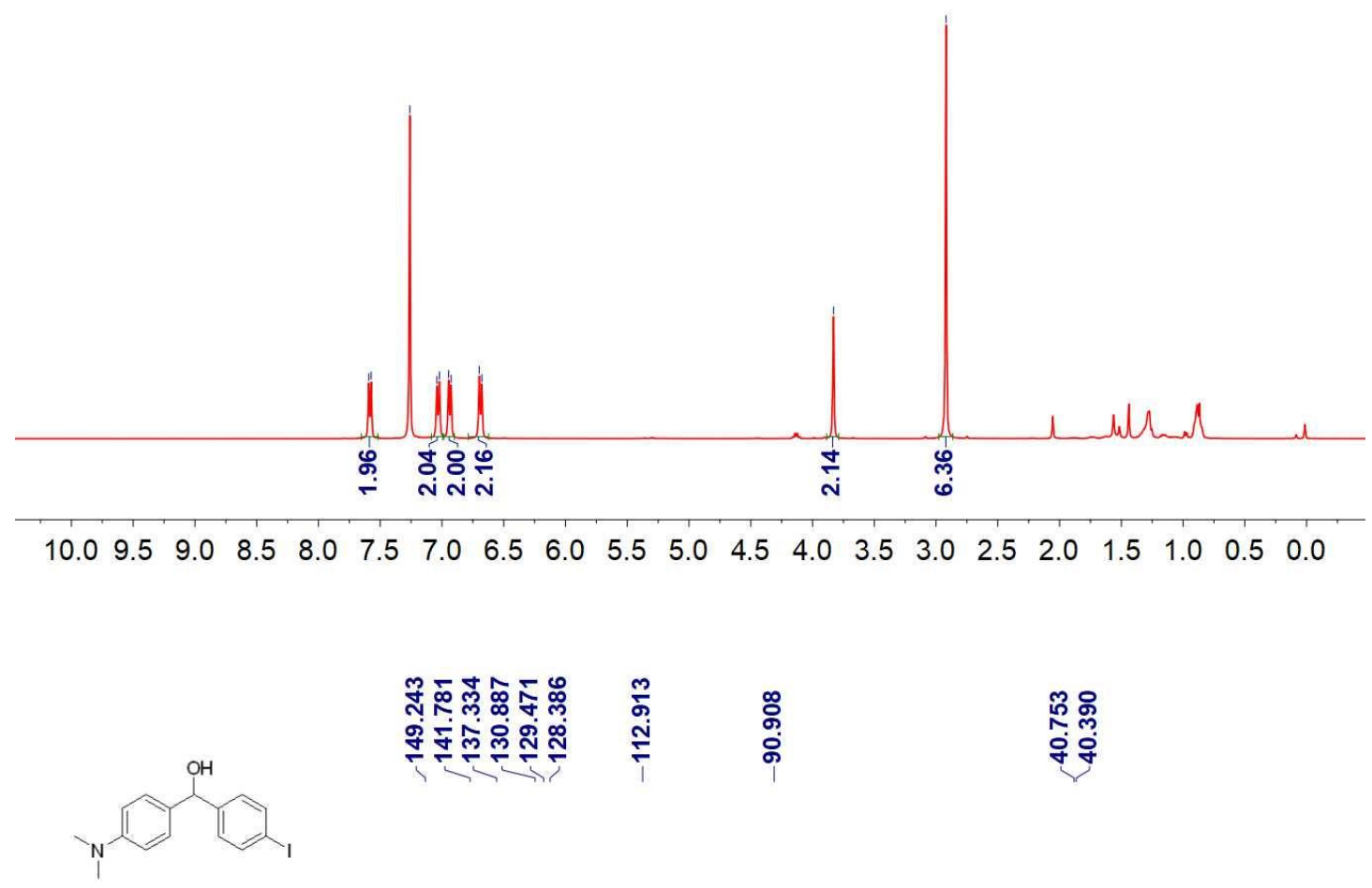

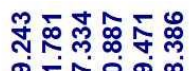

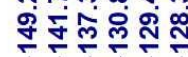

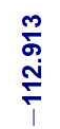

ஃ̊ํ.

ํㅐ용

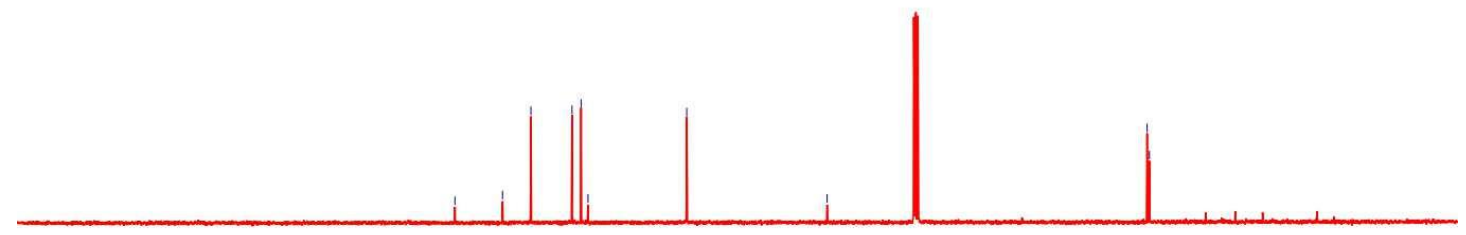

$210200190180170160150140130120110100 \quad 90 \quad 80 \quad 70 \quad 60 \begin{array}{lllllll}40 & 40 & 30 & 20 & 10 & 0\end{array}$ 

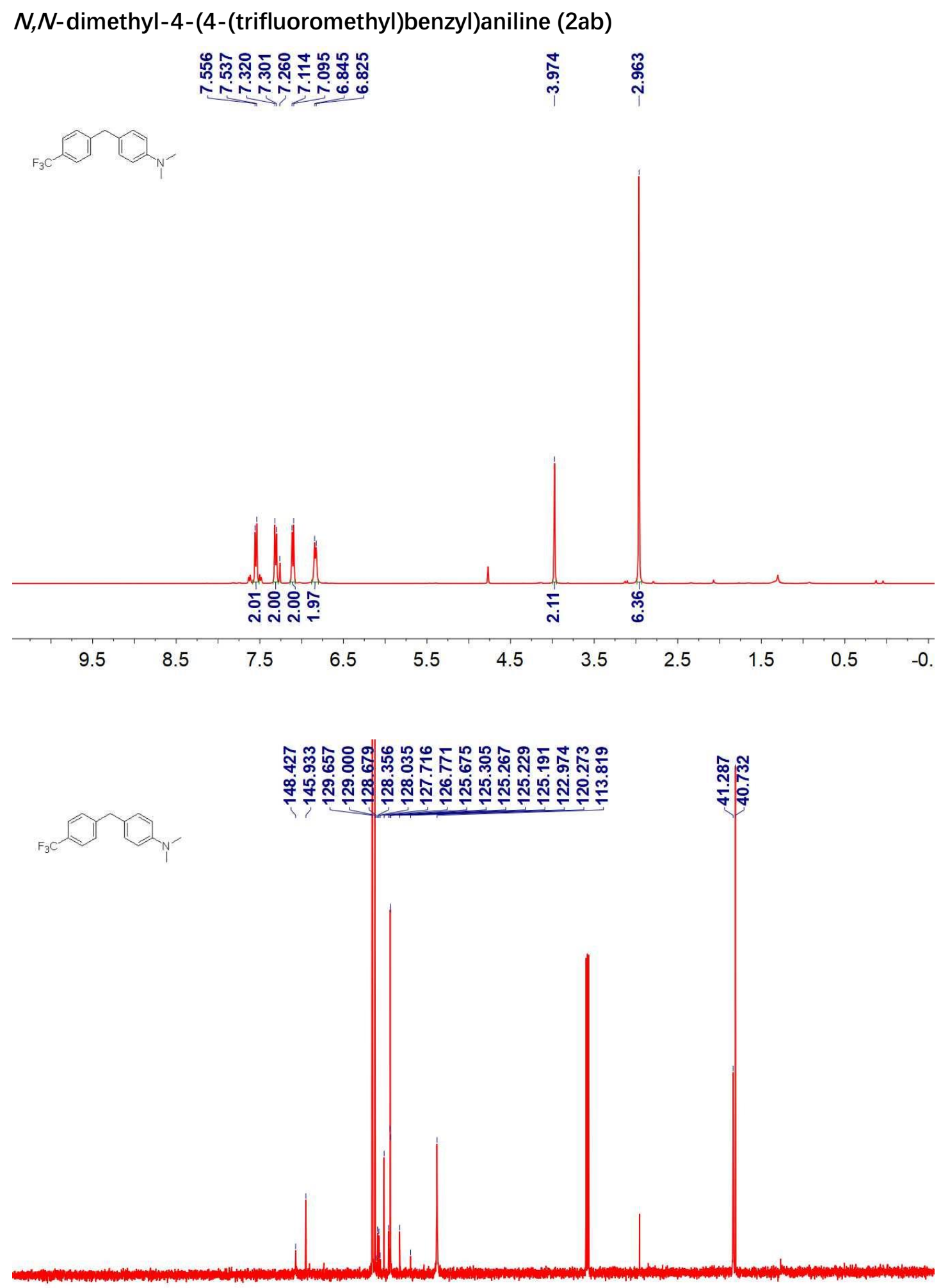

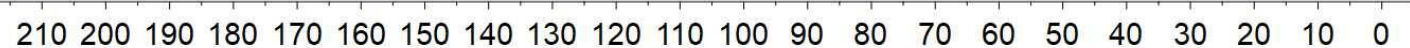


Methyl 4-(4-(dimethylamino)benzyl)benzoate (2ac)
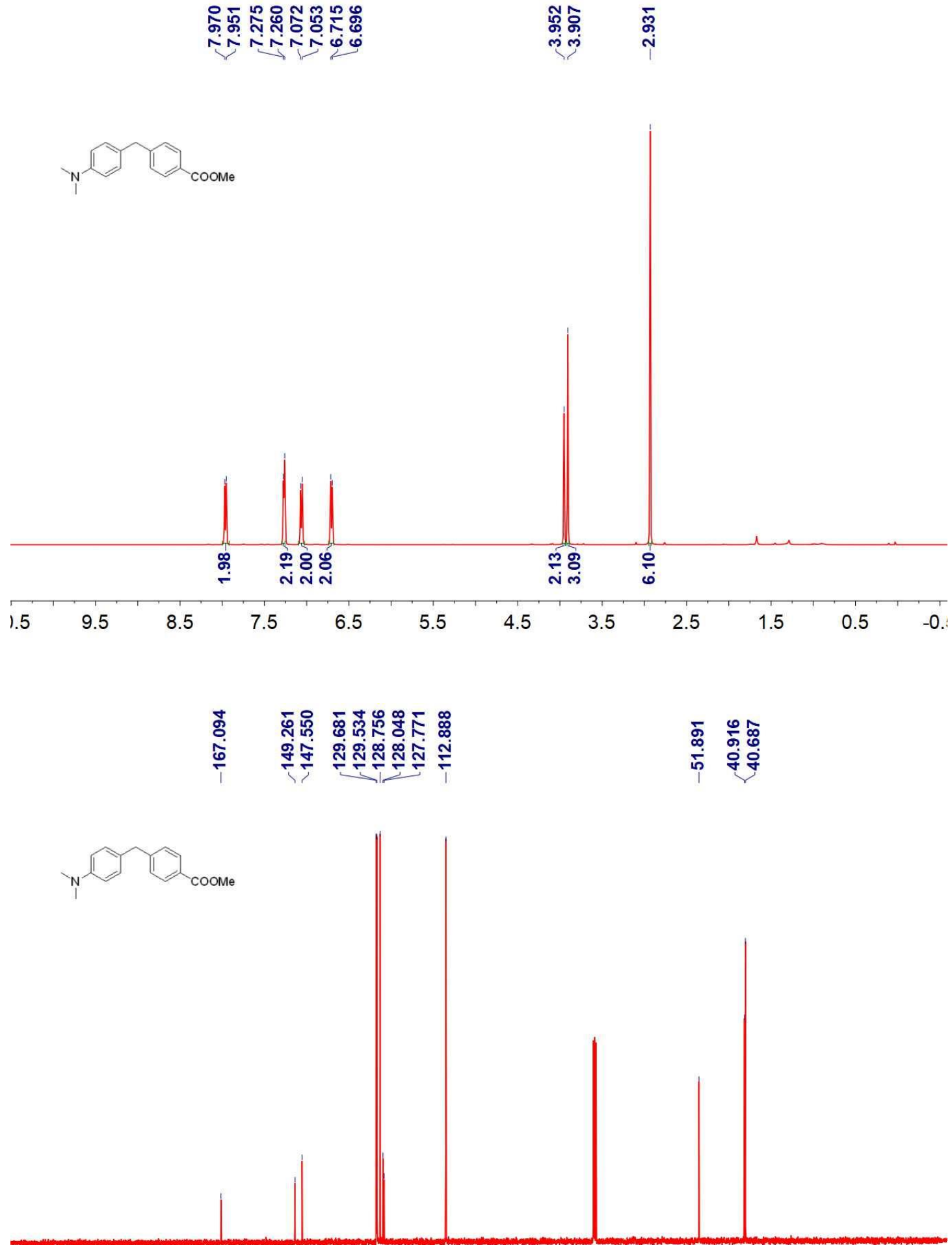

$210200190180170160150140 \quad 130120 \quad 110 \quad 100 \quad 90 \quad 80 \quad 70 \quad 60 \quad 50 \quad 40 \quad 30 \quad 20 \quad 10 \quad 0$ 
4-(4-(Dimethylamino)benzyl)benzonitrile (2ad)

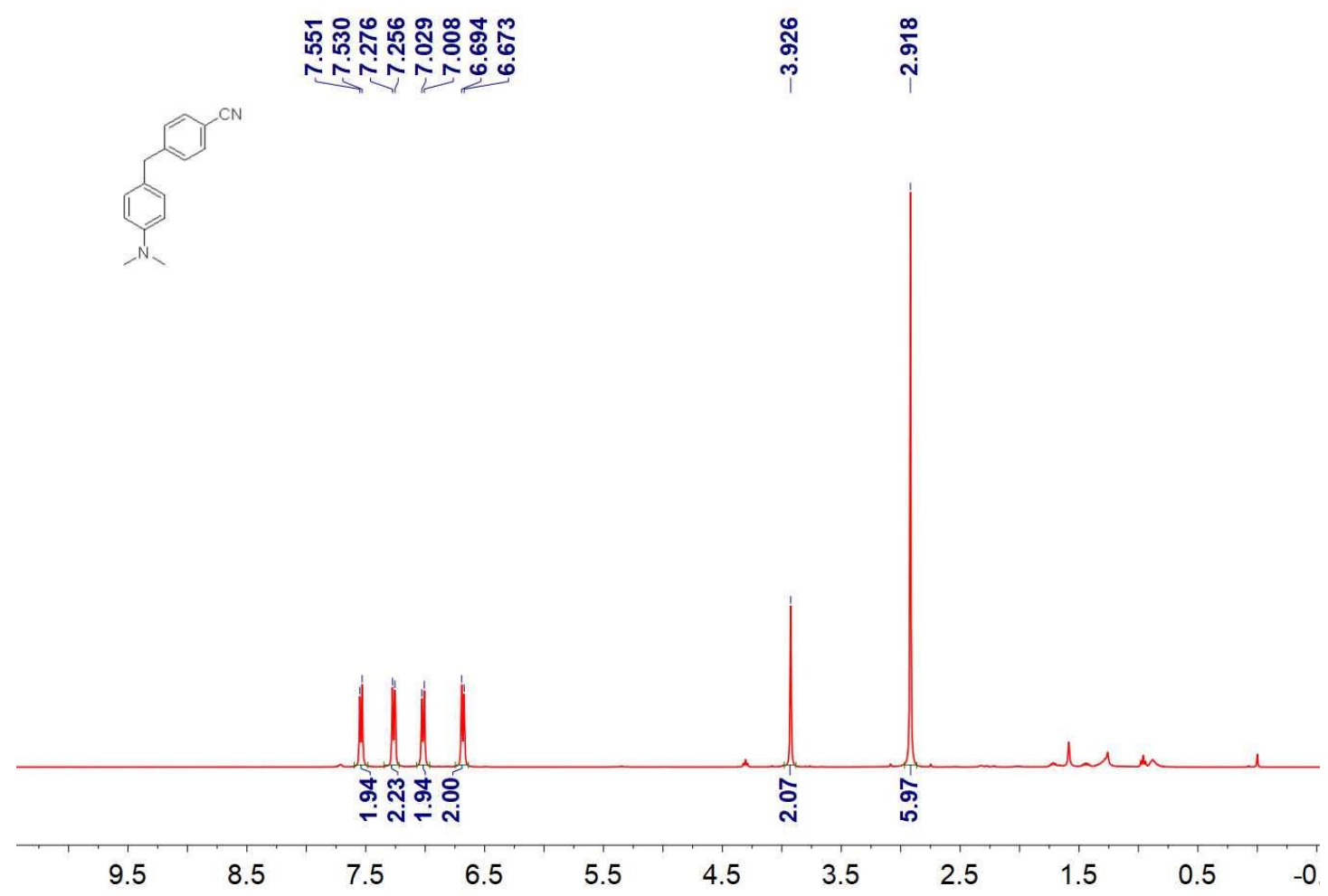

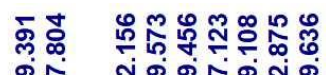

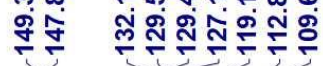

\section{㾸}

did

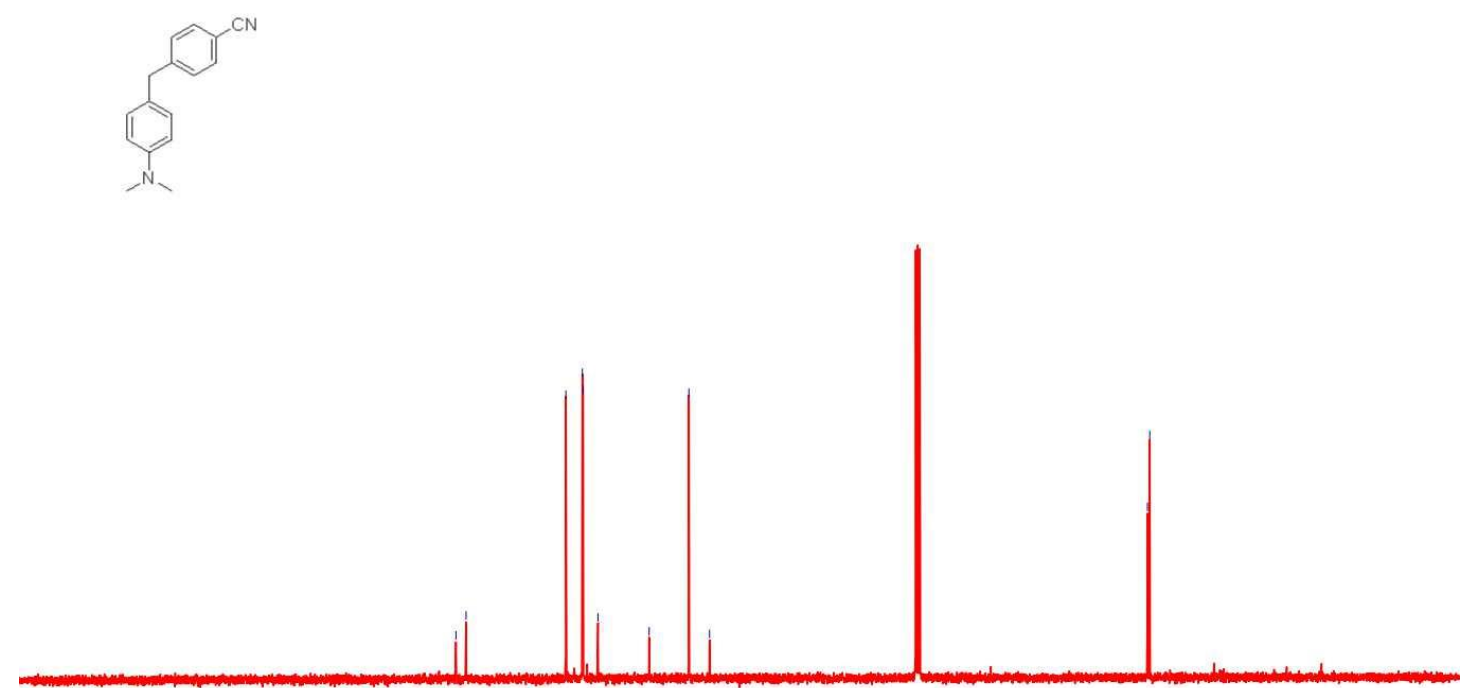

$210200190180170160150140130120110100 \quad 90 \quad 80 \quad 70 \quad 60 \quad 50 \quad 40 \quad 30 \quad 20 \quad 10 \quad 0$ 
N,N-dimethyl-4-(4-nitrobenzyl)aniline (2ae)
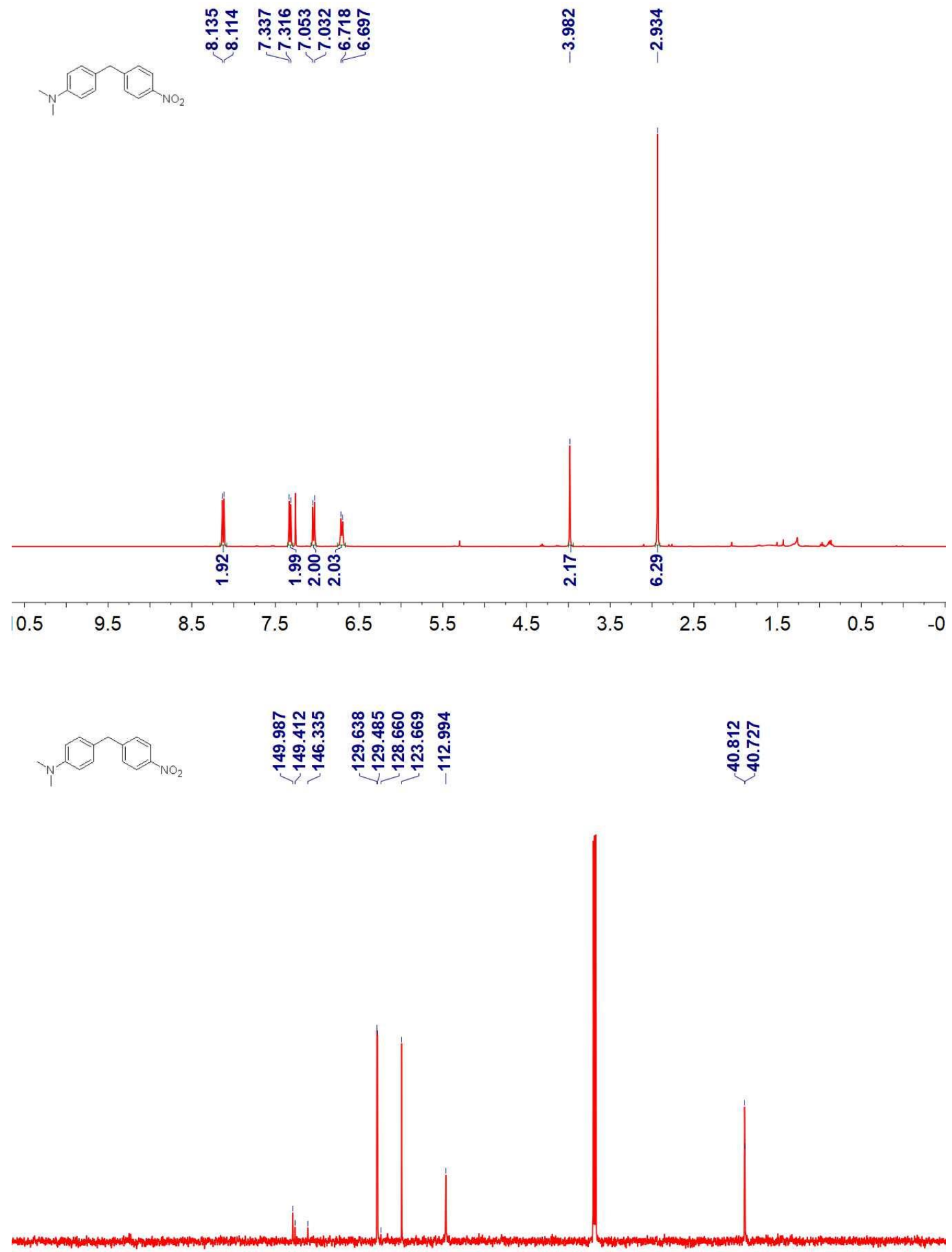

$210200190180170160150140130120110100 \quad 90 \quad 80 \quad 70 \quad 60 \quad 50 \quad 40 \quad 30 \quad 20 \quad 10 \quad 0$ 


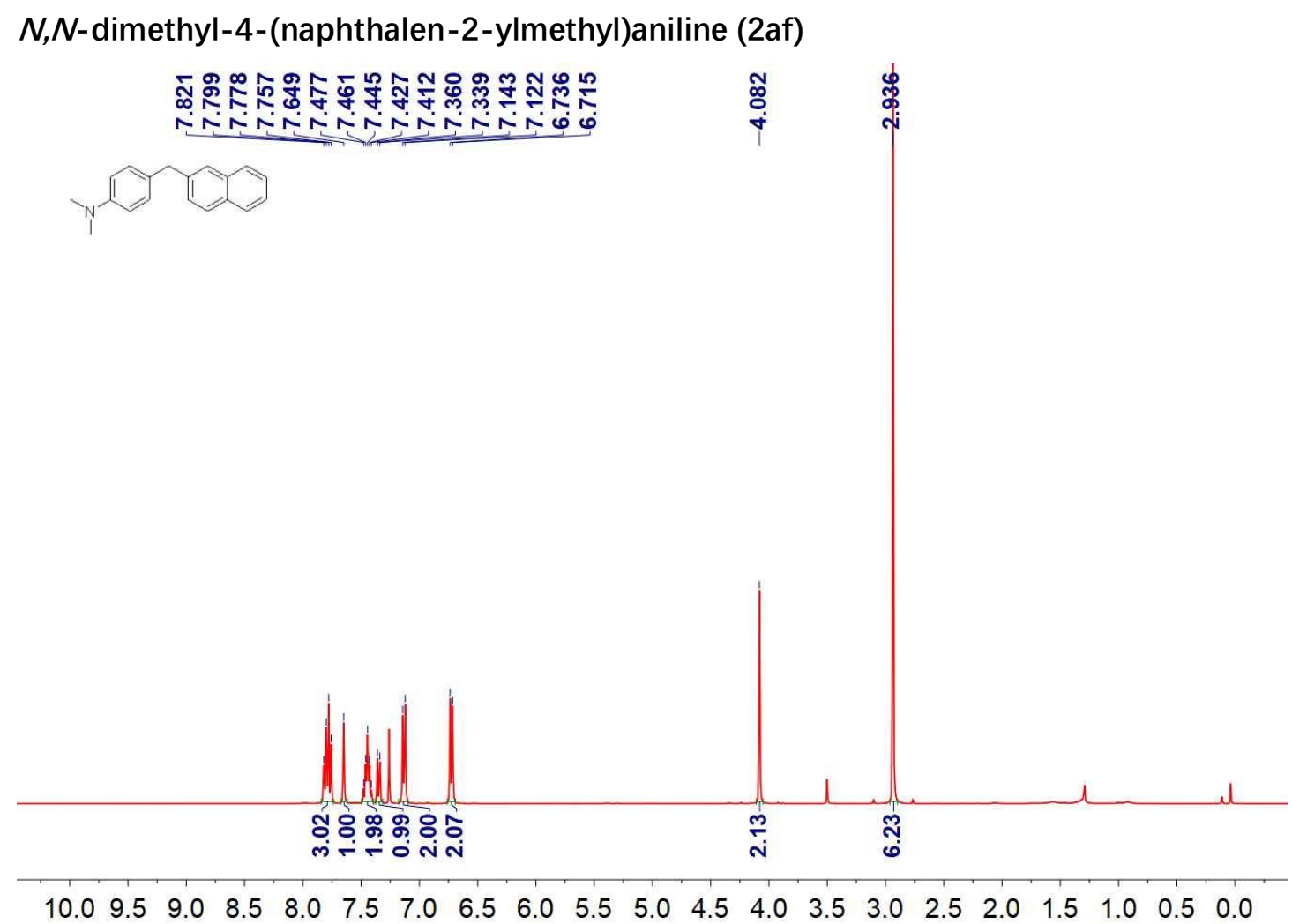

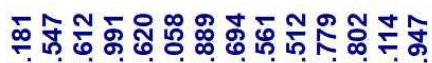

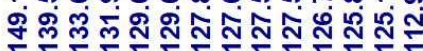

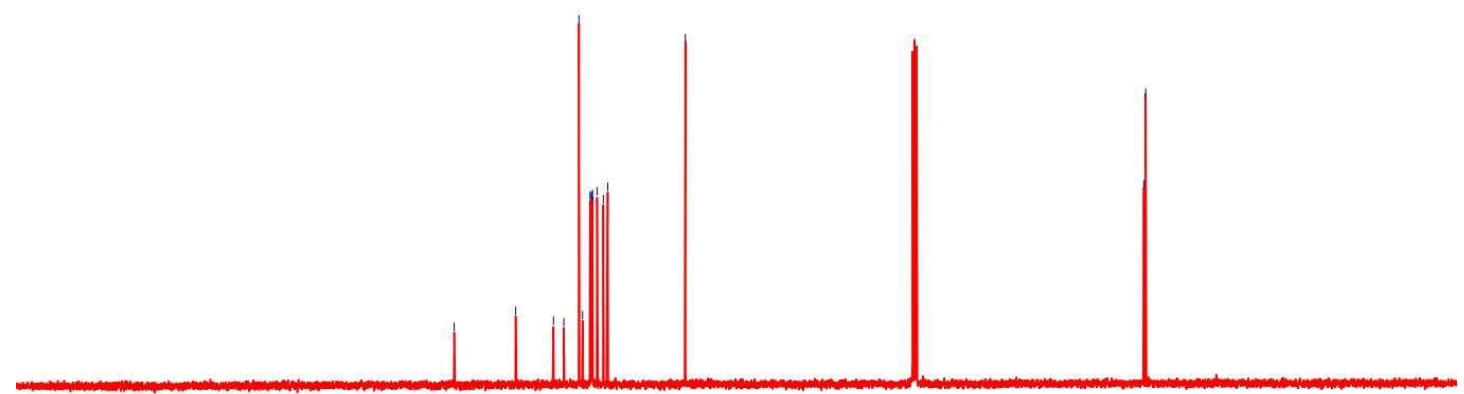

$210200190180170160150140130120110100 \quad 90 \quad 80 \quad 70 \quad 60 \quad 50 \quad 40 \quad 30 \quad 20 \quad 10 \quad 0$ 
4-(Furan-2-ylmethyl)- N,N-dimethylaniline (2ag)

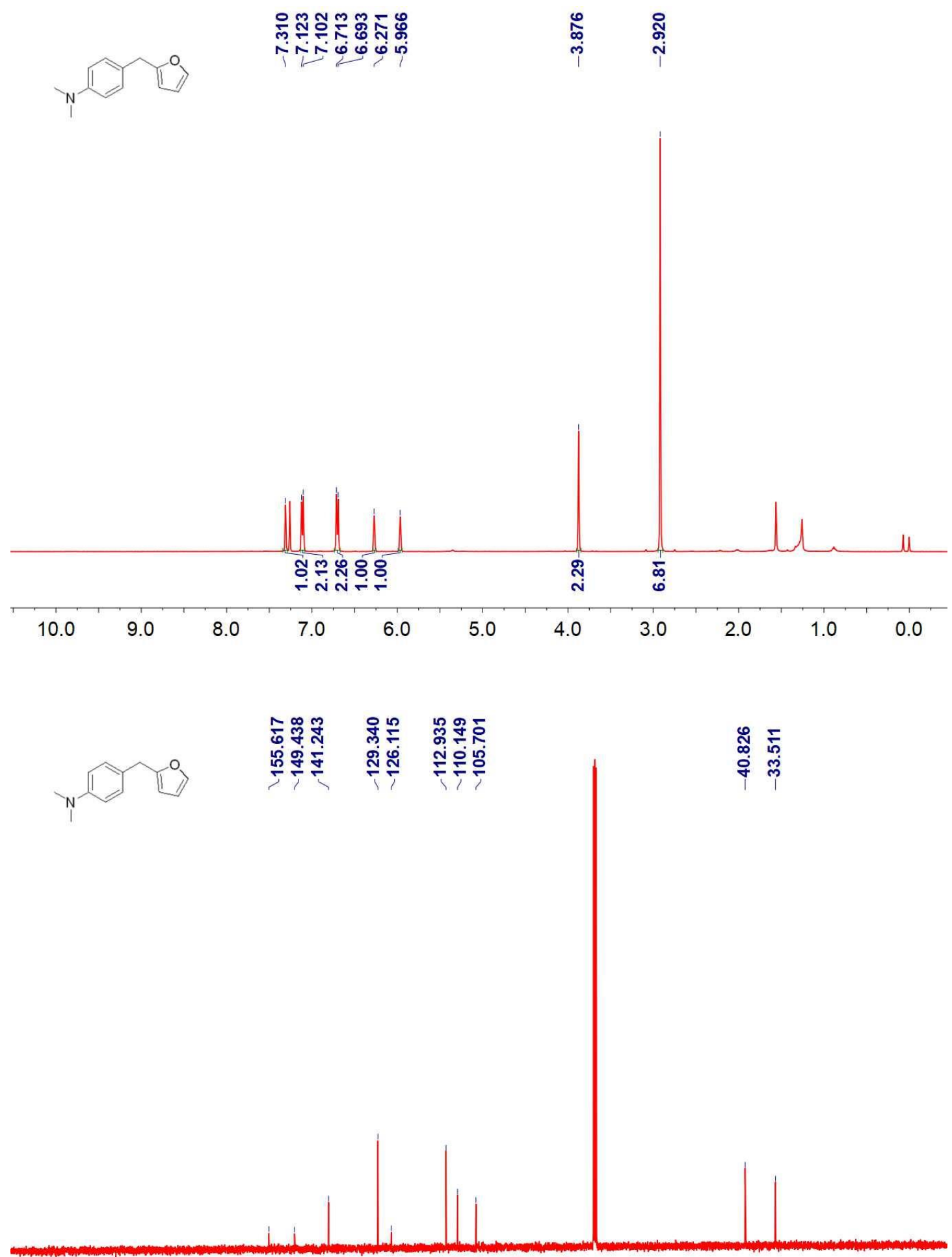

$210200190180170160150140130120110100 \quad 90 \quad 80 \quad 70 \quad 60 \quad 50 \quad 40 \quad 30 \quad 20 \quad 10 \quad 0$ 
N,N-dimethyl-4-(thiophen-2-ylmethyl)aniline (2ah)

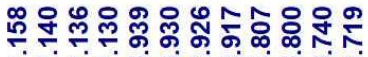

NNN

:

N

i

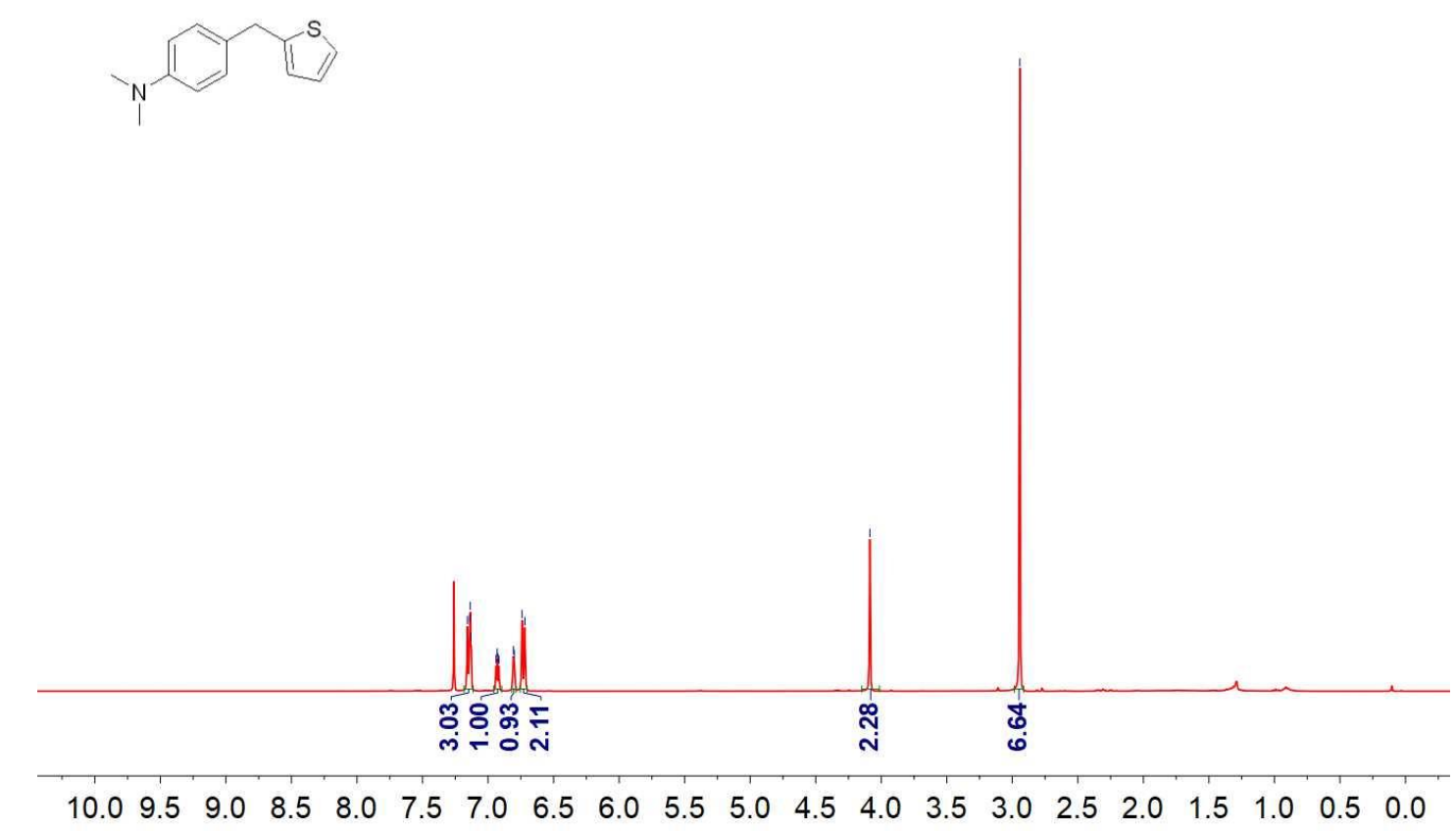

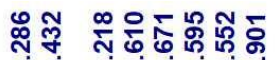

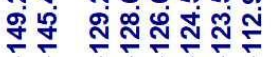

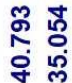

Non

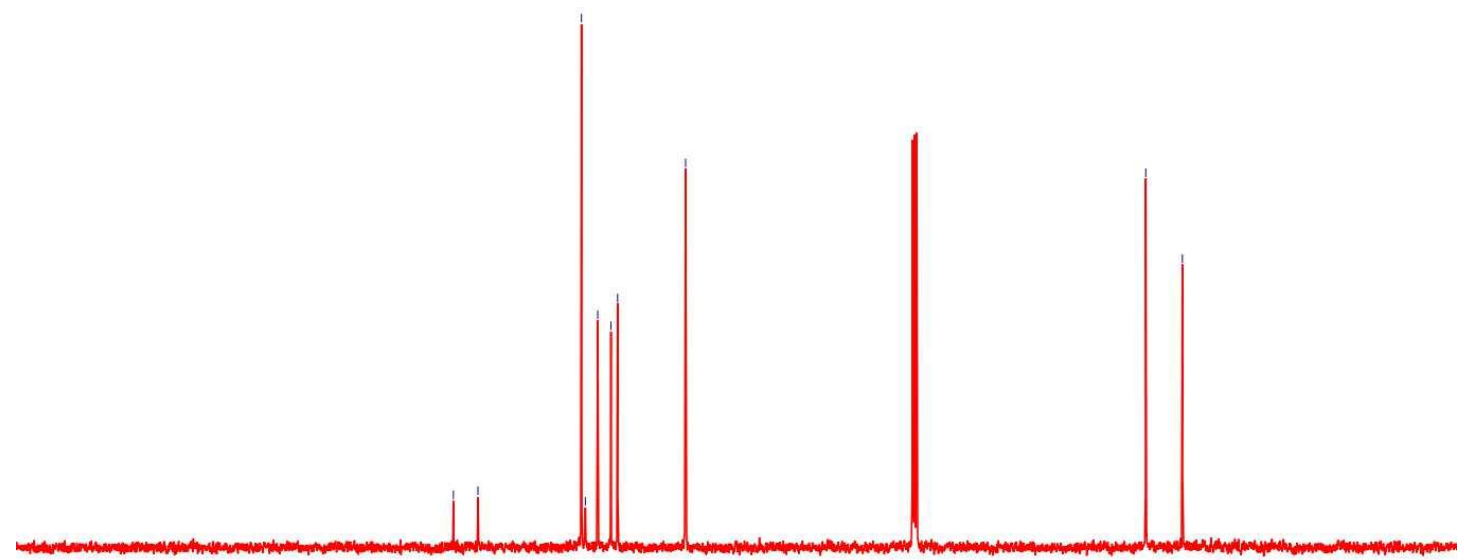

$210200190180170160150140130120110100 \quad 90 \quad 80 \quad 70 \quad 60 \quad 50 \quad 40 \quad 30 \quad 20 \quad 10 \quad 0$ 


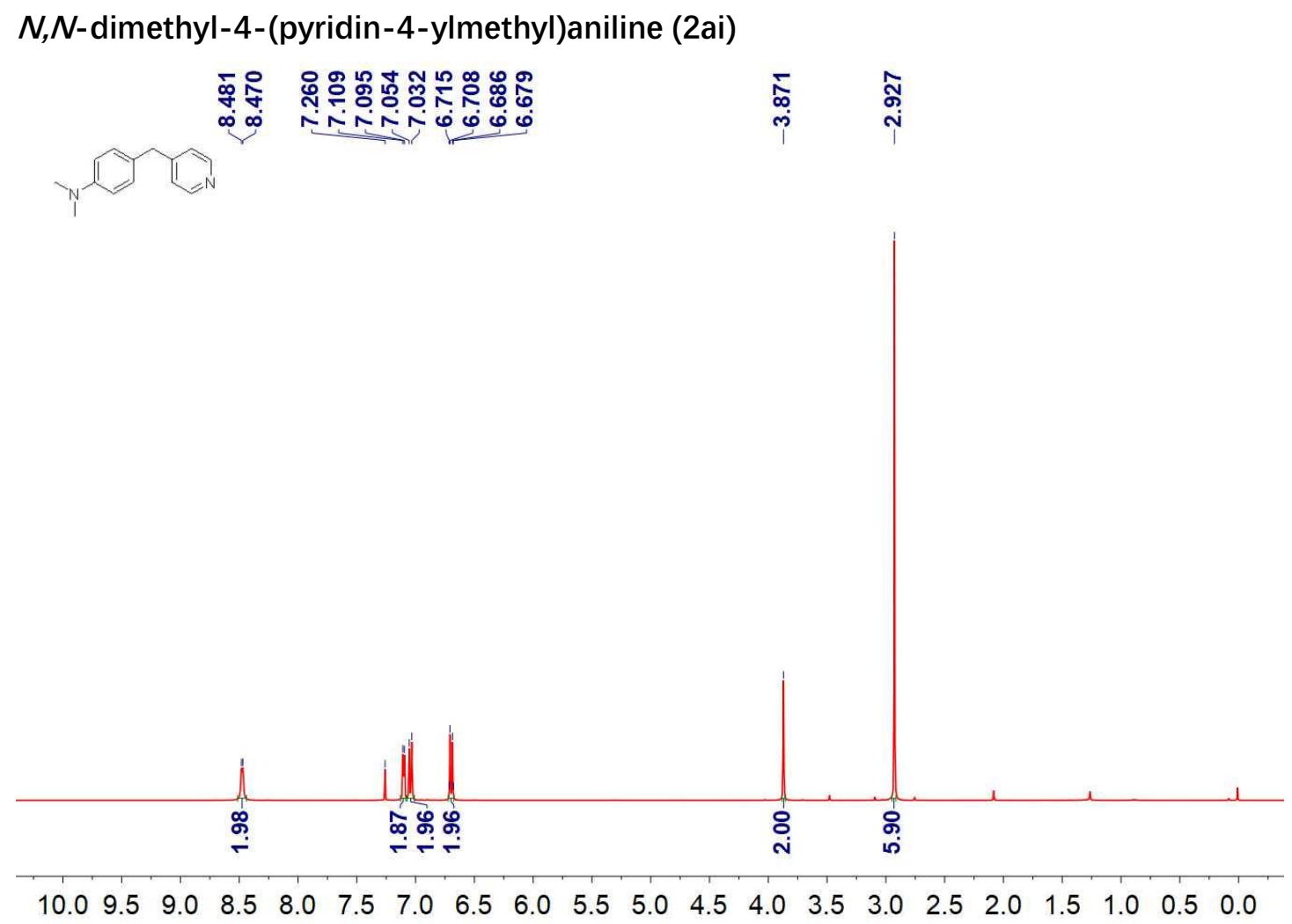

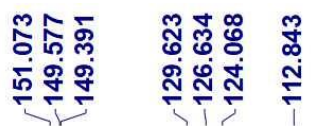

ิิธ

$\prod_{N}^{1}$
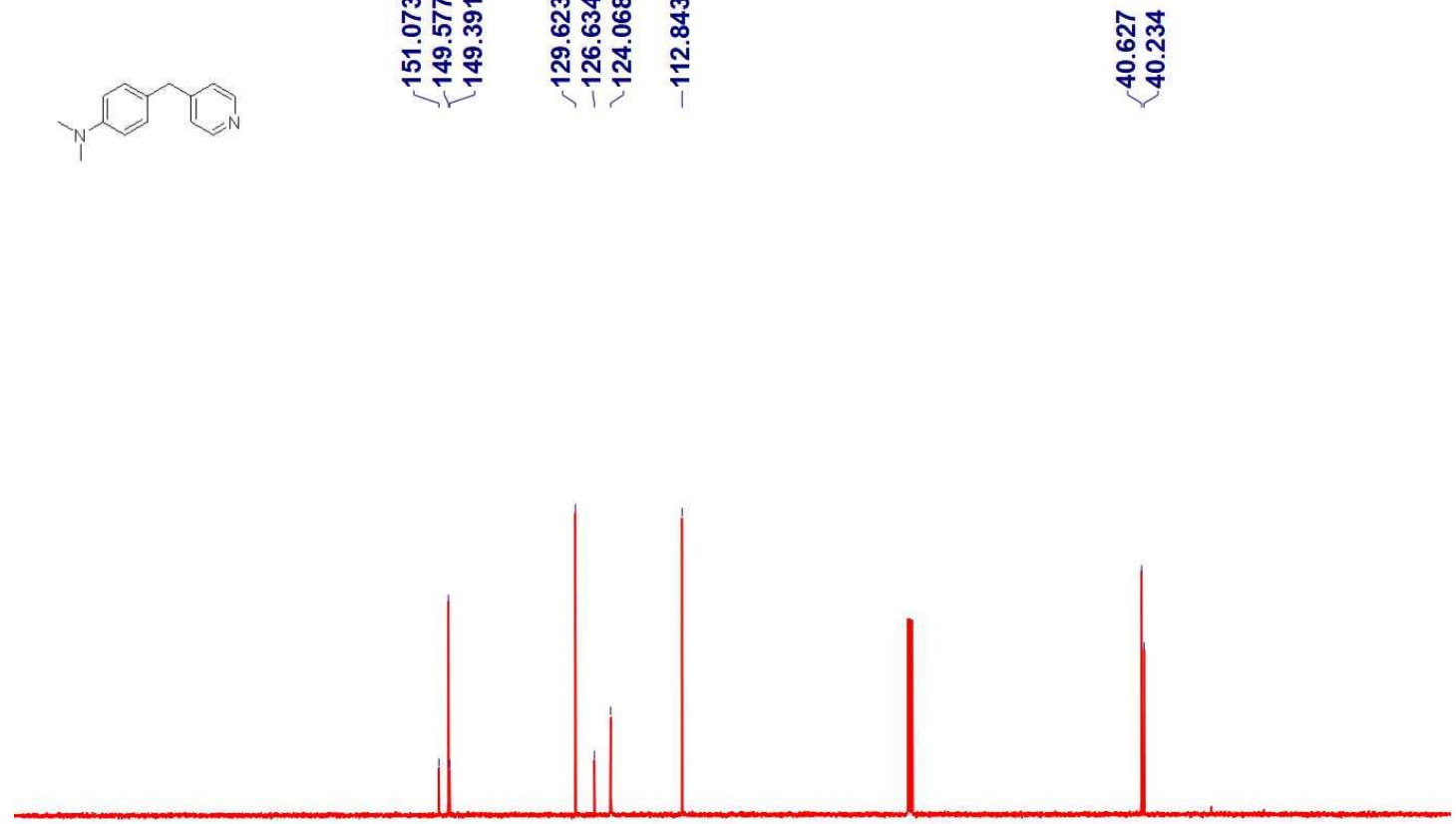

$210200190180170160150140130120110100 \quad 90 \quad 80 \quad 70 \quad 60 \quad 50 \quad 40 \quad 30 \quad 20 \quad 10 \quad 0$ 

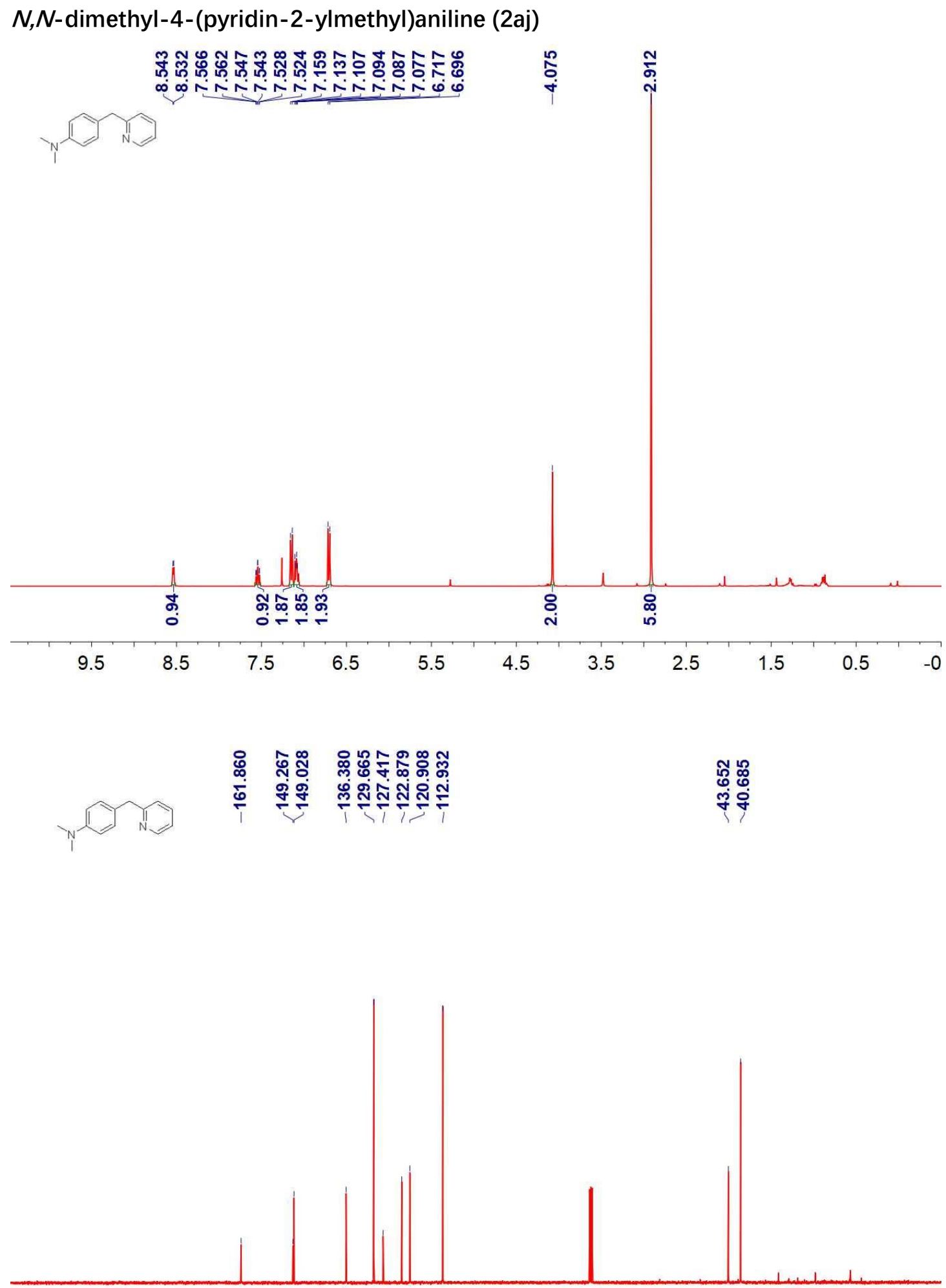

$210200190180170160150140130120110100 \quad 90 \quad 80 \quad 70 \quad 60 \quad 50 \quad 40 \quad 30 \quad 20 \quad 10 \quad 0$ 
$N, N$-dimethyl-4-(4-(5,6,7,8-tetrahydroquinolin-2-yl)benzyl)aniline (2ak)

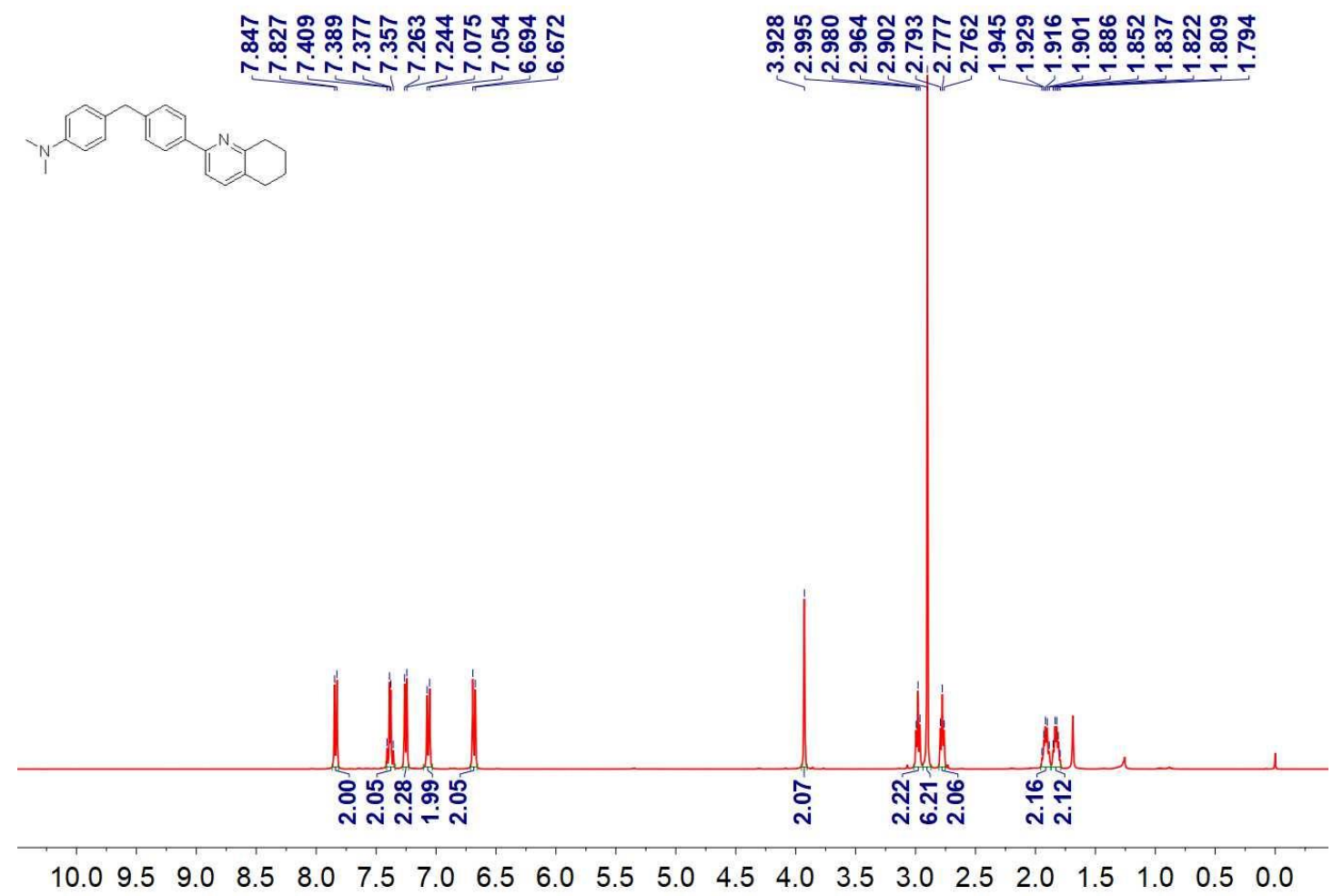

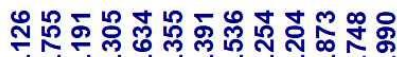

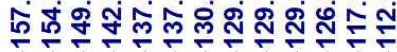

:

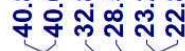

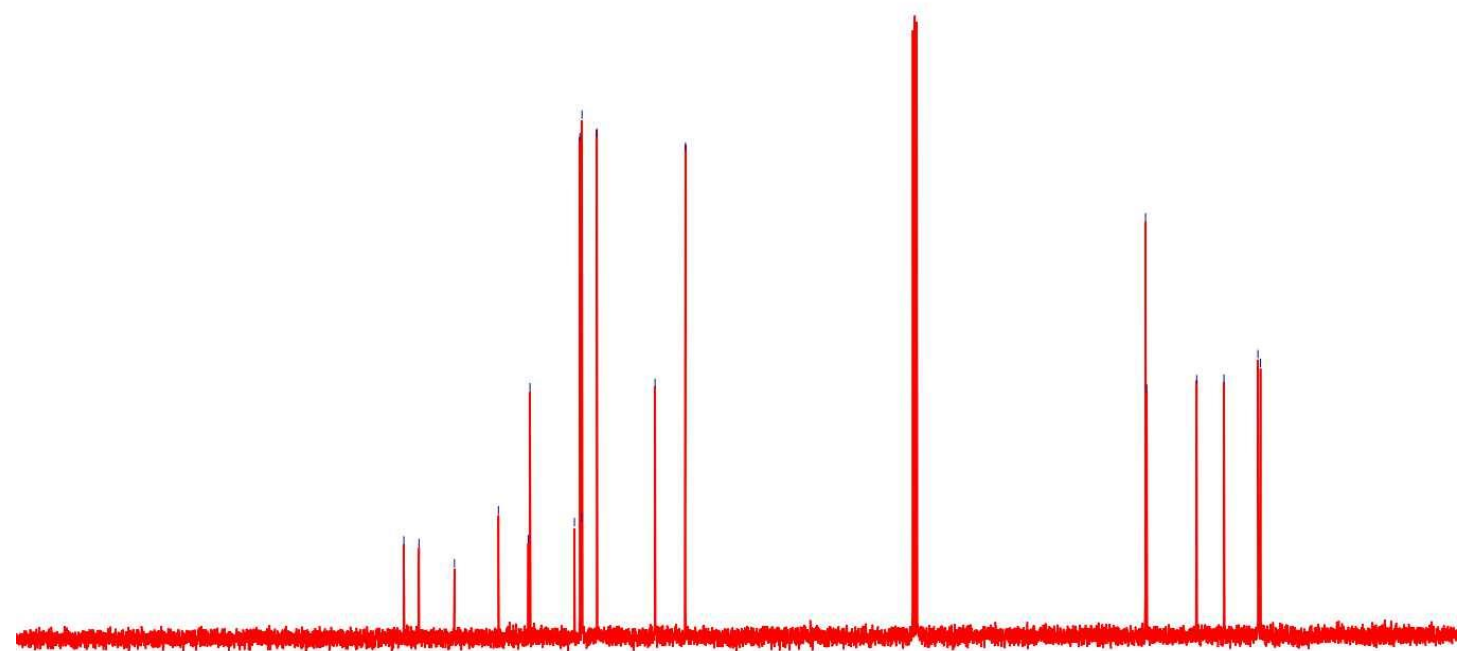

$210200190180170160150140130120110 \quad 100 \quad 90 \quad 80 \quad 70 \quad 60 \quad 50 \quad 40 \quad 30 \quad 20 \quad 10 \quad 0$ 
4-((1H-indol-3-yl)methyl)-N,N-dimethylaniline (2al)

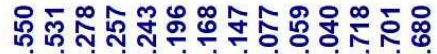

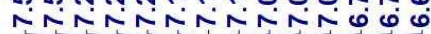

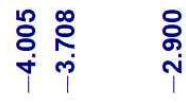

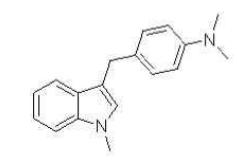

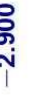
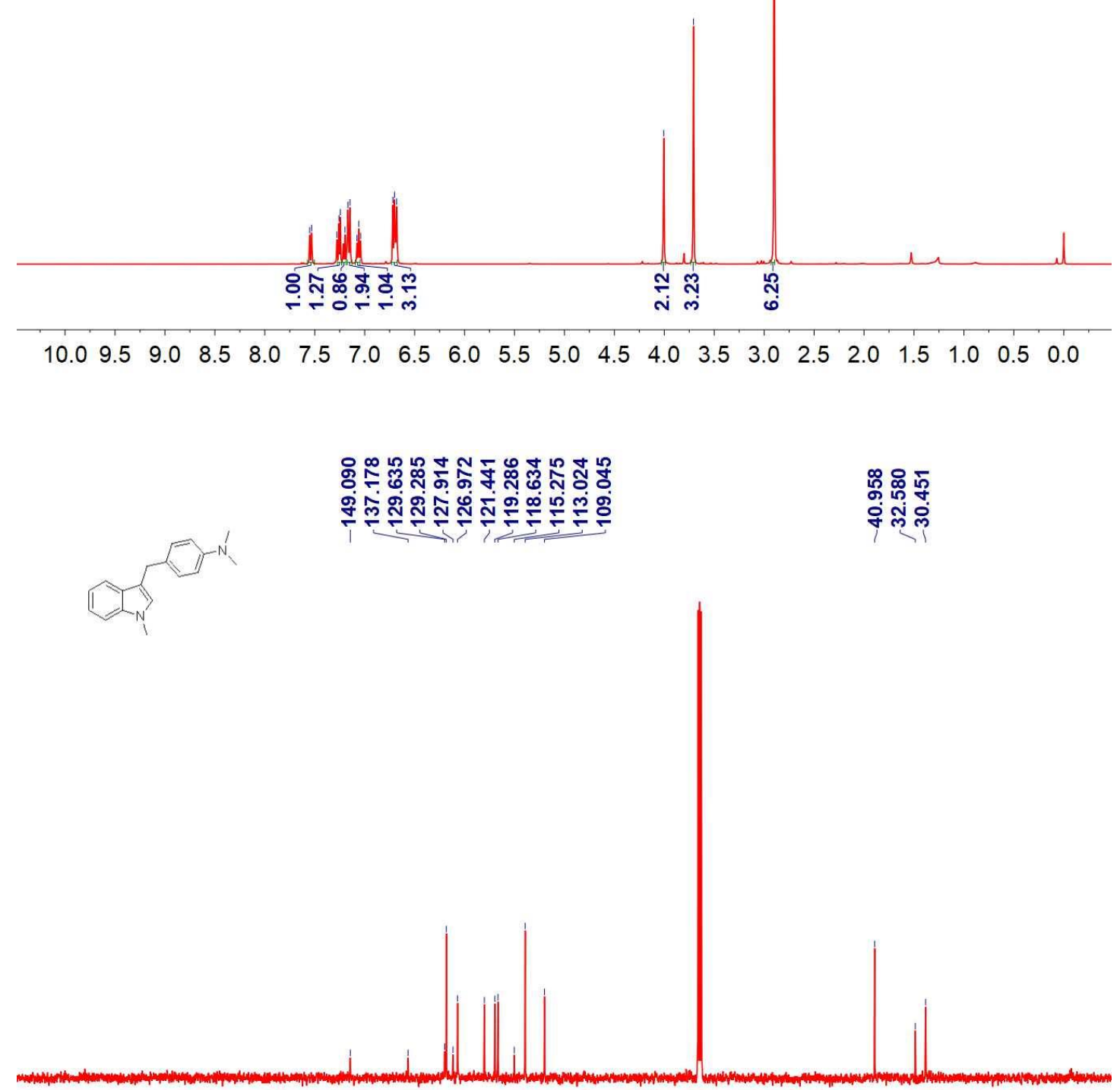

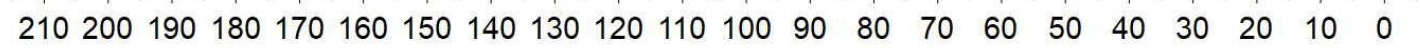


4-((1H-indol-3-yl)methyl)-N,N-dimethylaniline (2am)

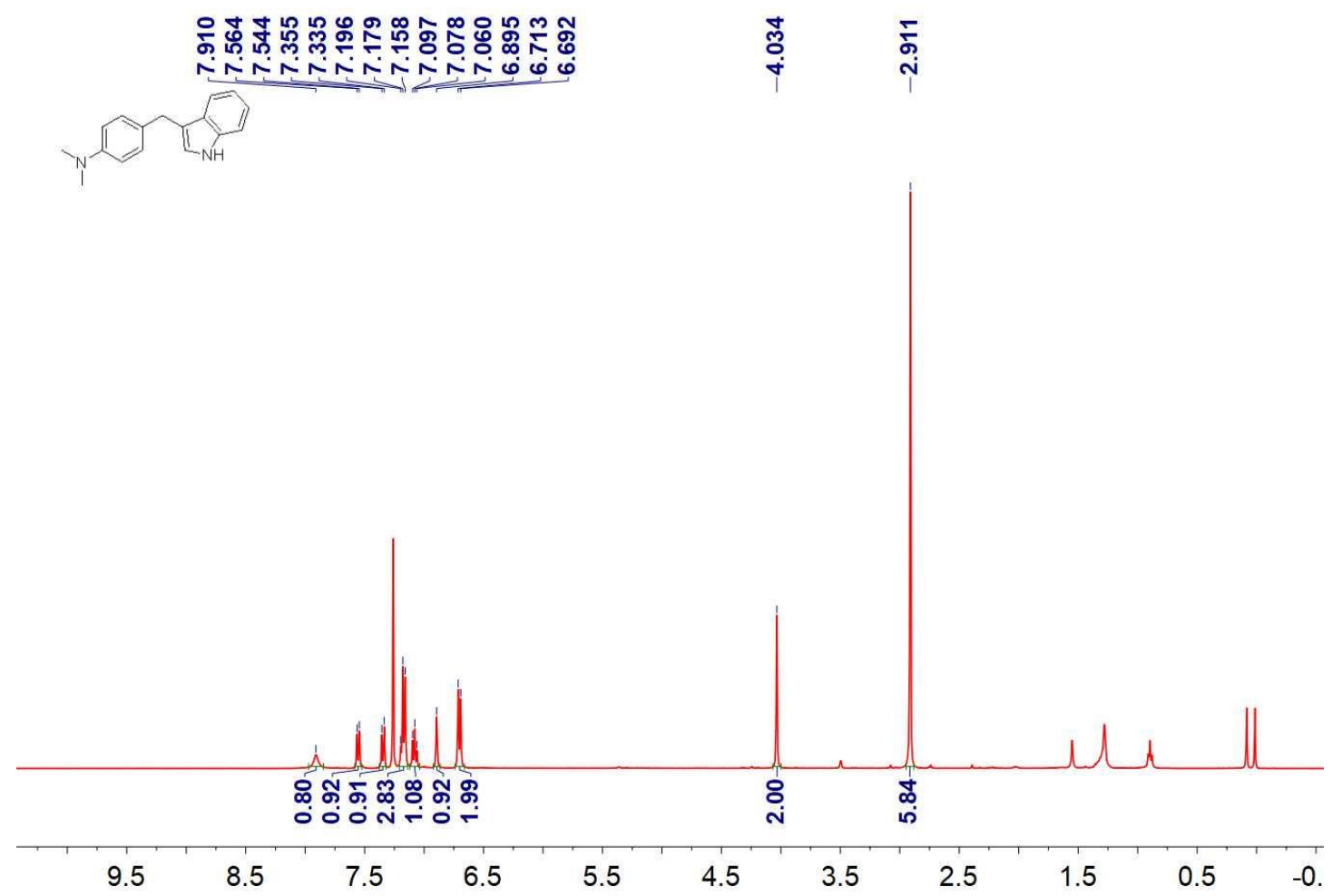

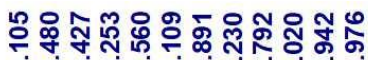

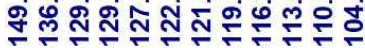

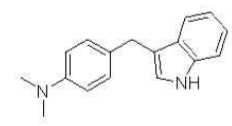

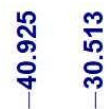

$210200190180170160150140130120110100 \quad 90 \quad 80 \quad 70 \quad 60 \quad 50 \quad 40 \quad 30 \quad 20 \quad 10 \quad 0$ 
4,4' -(1,4-Phenylenebis(methylene))bis( $N, N$-dimethylaniline) (2an)
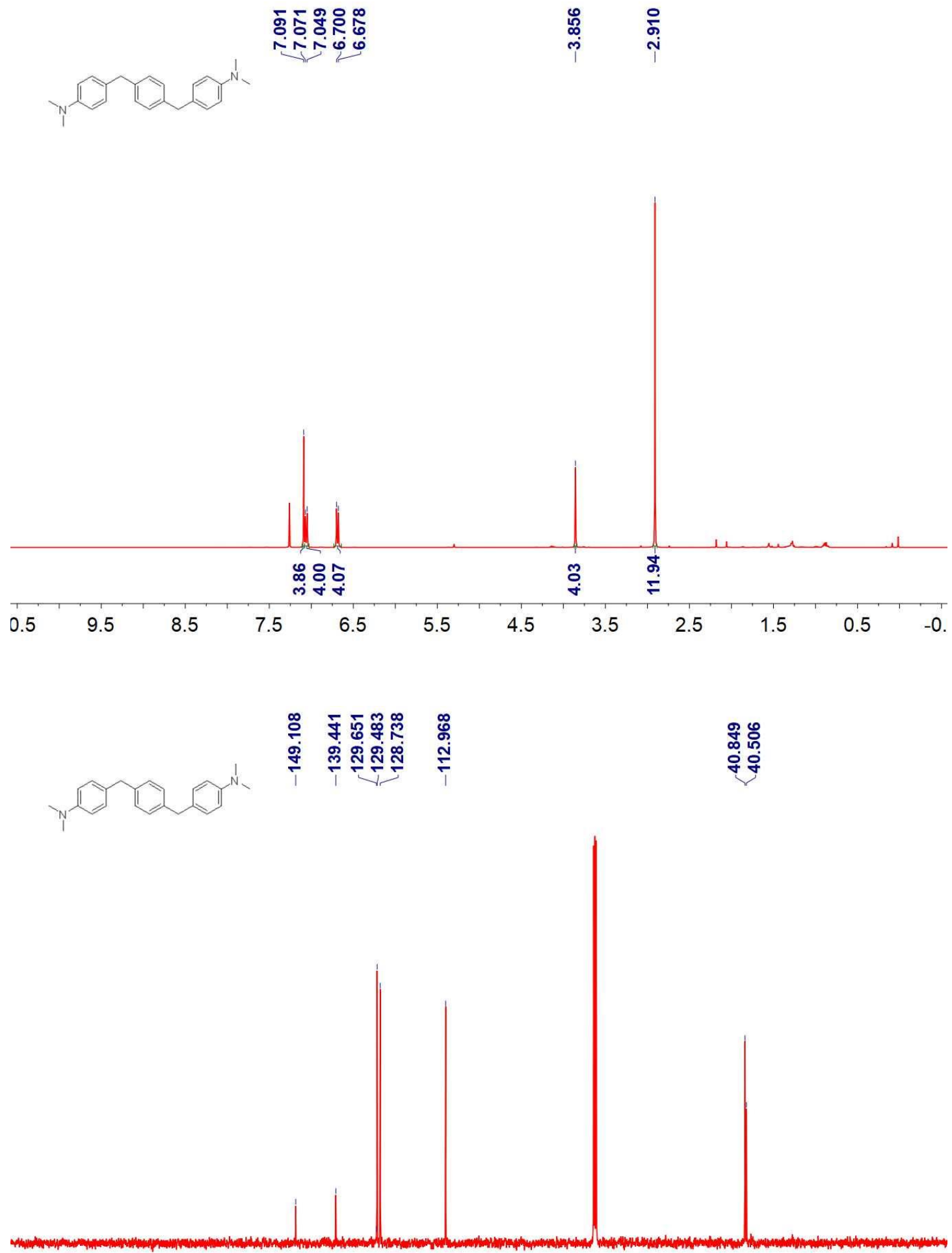

$210200190180170160150140130120110 \quad 100 \quad 90 \quad 80 \quad 70 \quad 60 \quad 50 \quad 40 \quad 30 \quad 20 \quad 10 \quad 0$ 

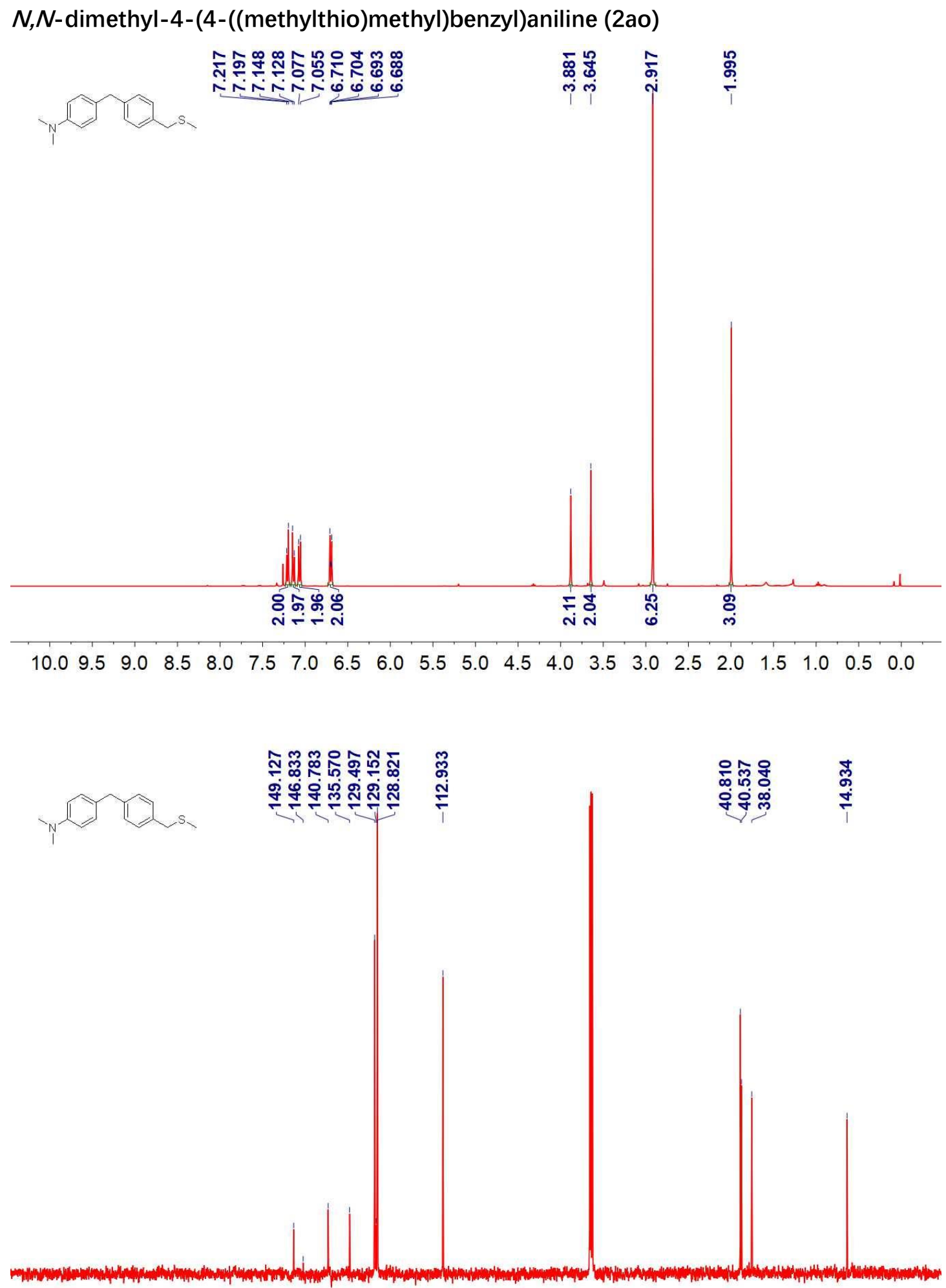

$210200190180170160150140130120110100 \quad 90 \quad 80 \quad 70 \quad 60 \quad 50 \quad 40 \quad 30 \quad 20 \quad 10 \quad 0$ 
4-(4-(methoxymethyl)benzyl)- $N, N$-dimethylaniline (2ap)

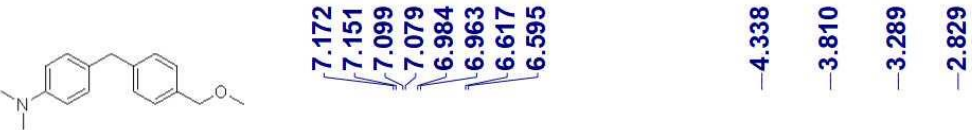

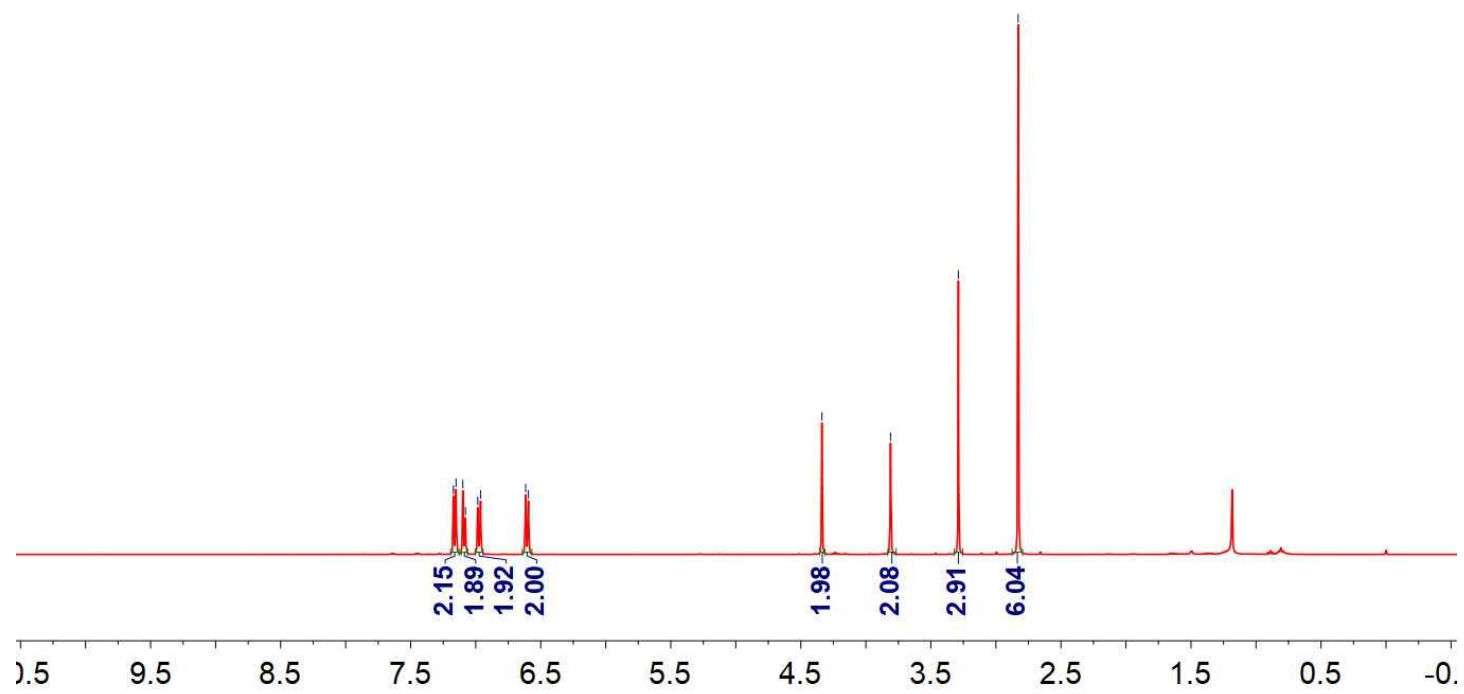

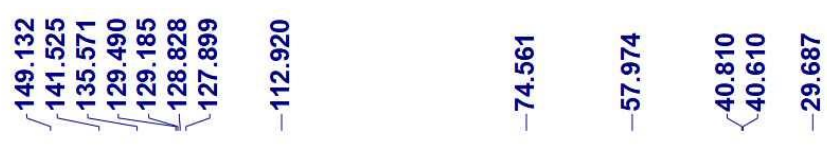

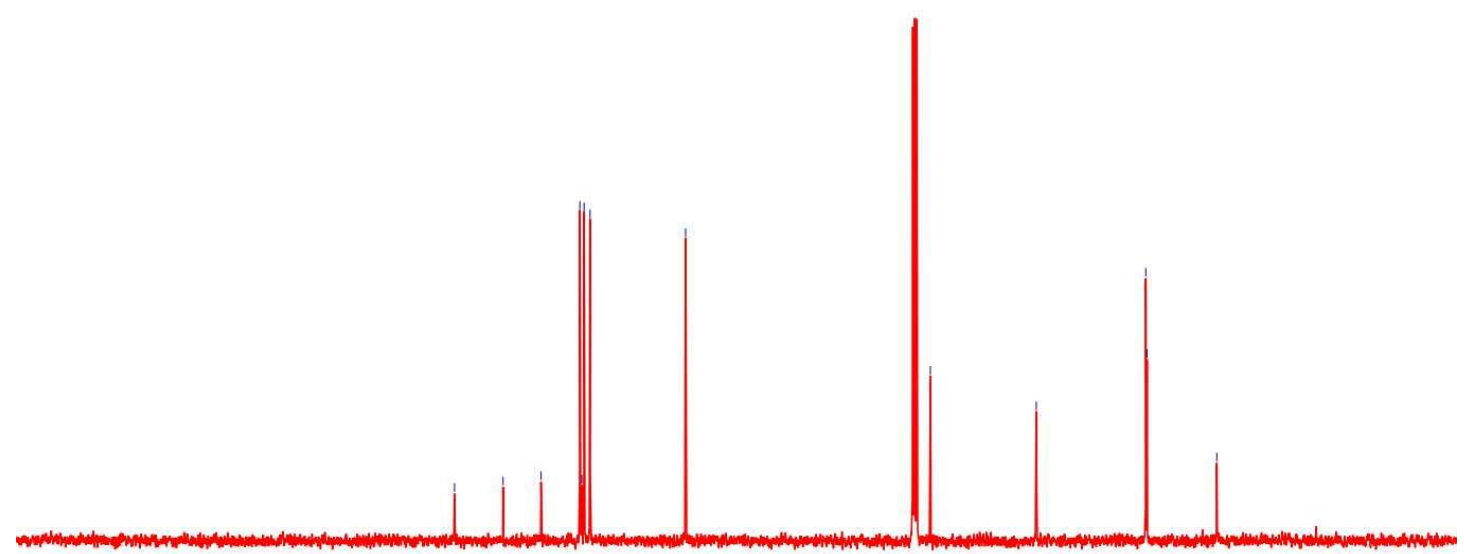

$210200190180170160150140130120110100 \quad 90 \quad 80 \quad 70 \quad 60 \quad 50 \quad 40 \quad 30 \quad 20 \quad 10 \quad 0$ 
4-(4-((dimethylamino)methyl)benzyl)-N,N-dimethylaniline (2aq)

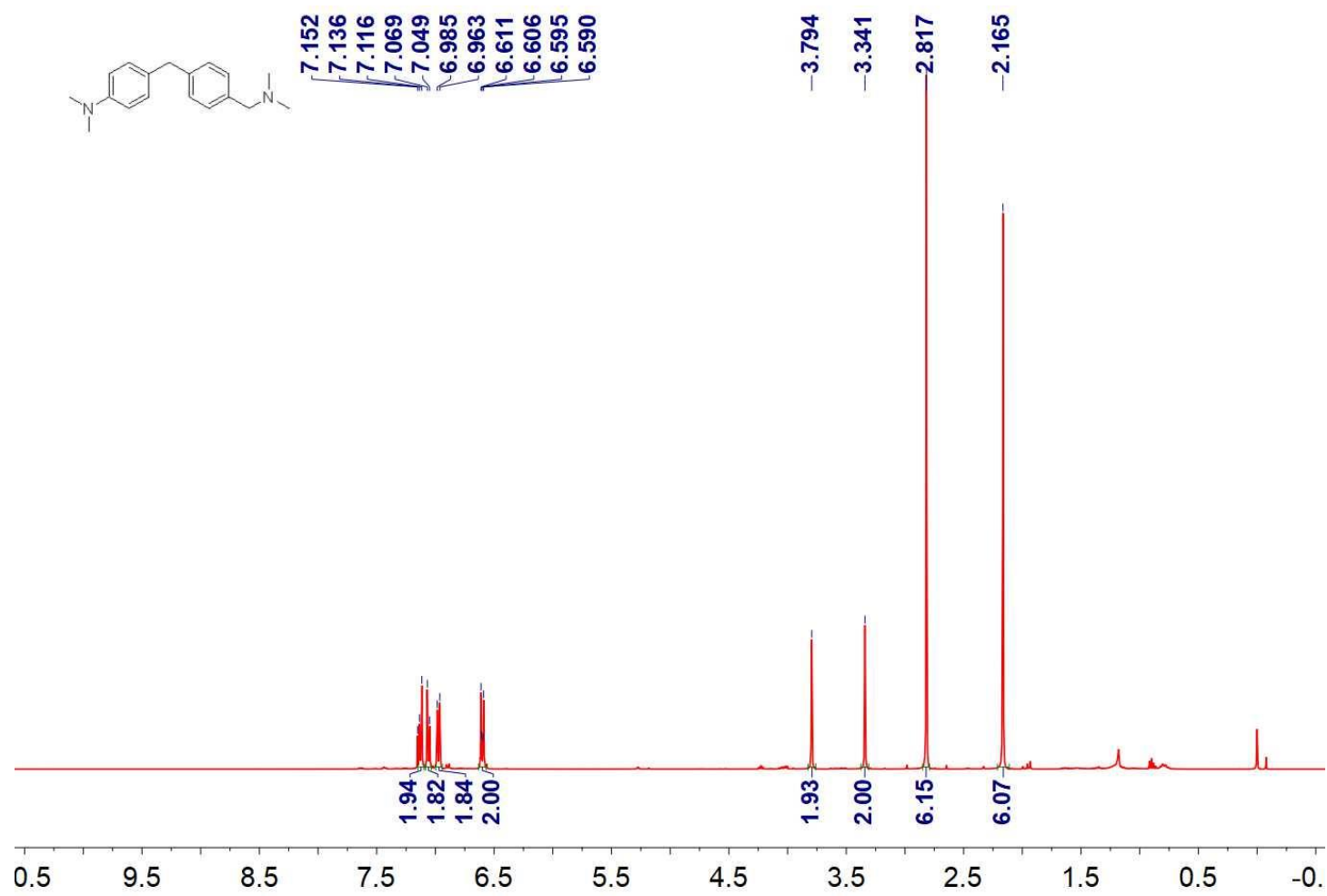

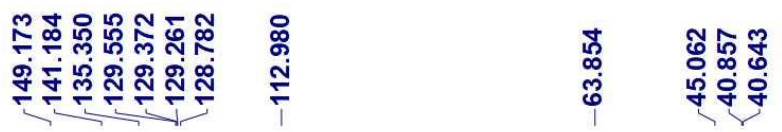

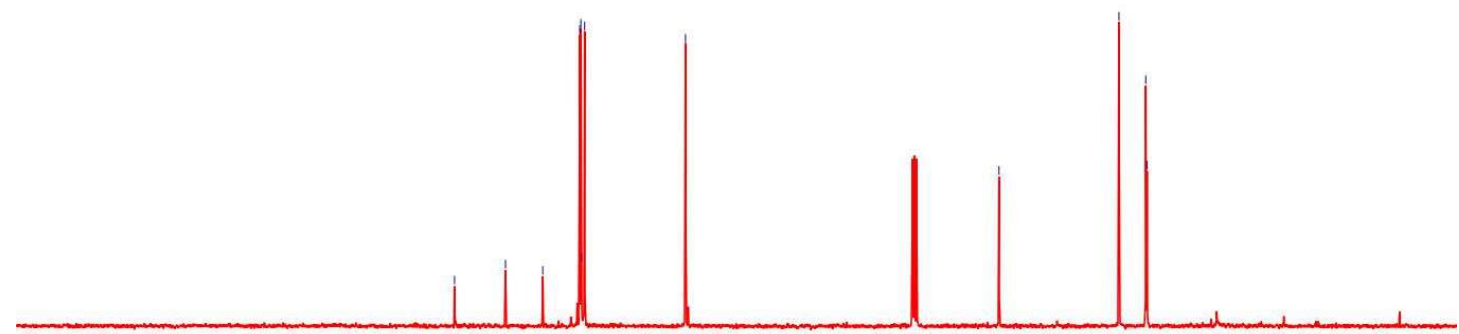

$210200190180170160150140130120110100 \quad 90 \quad 80 \quad 70 \quad 60 \quad 50 \quad 40 \quad 30 \quad 20 \quad 10 \quad 0$ 
N,N-dimethyl-4-(3-phenylprop-2-yn-1-yl)aniline (2ar)

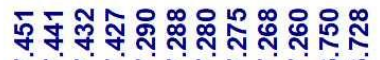

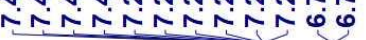
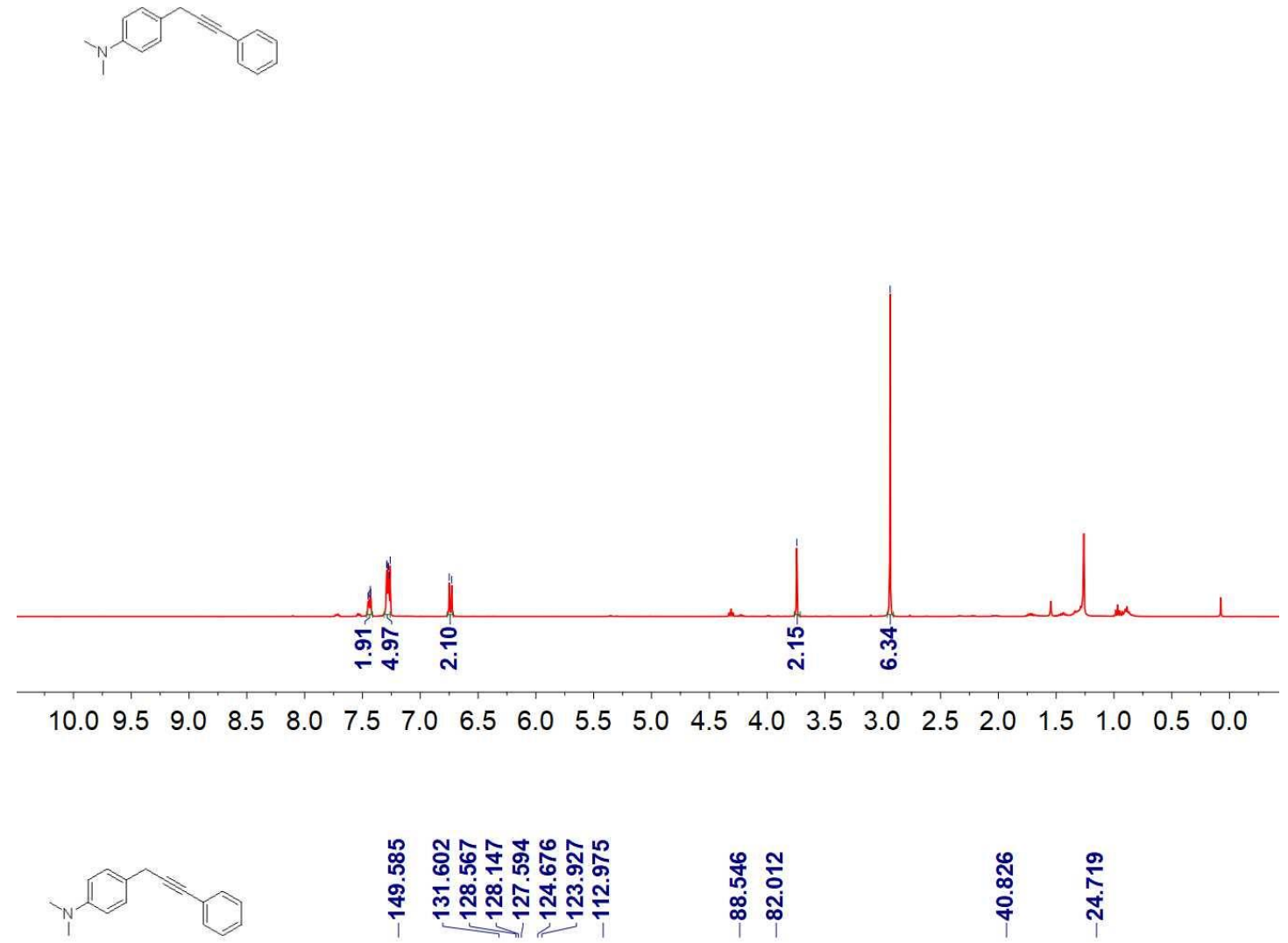

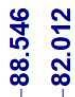

జั.

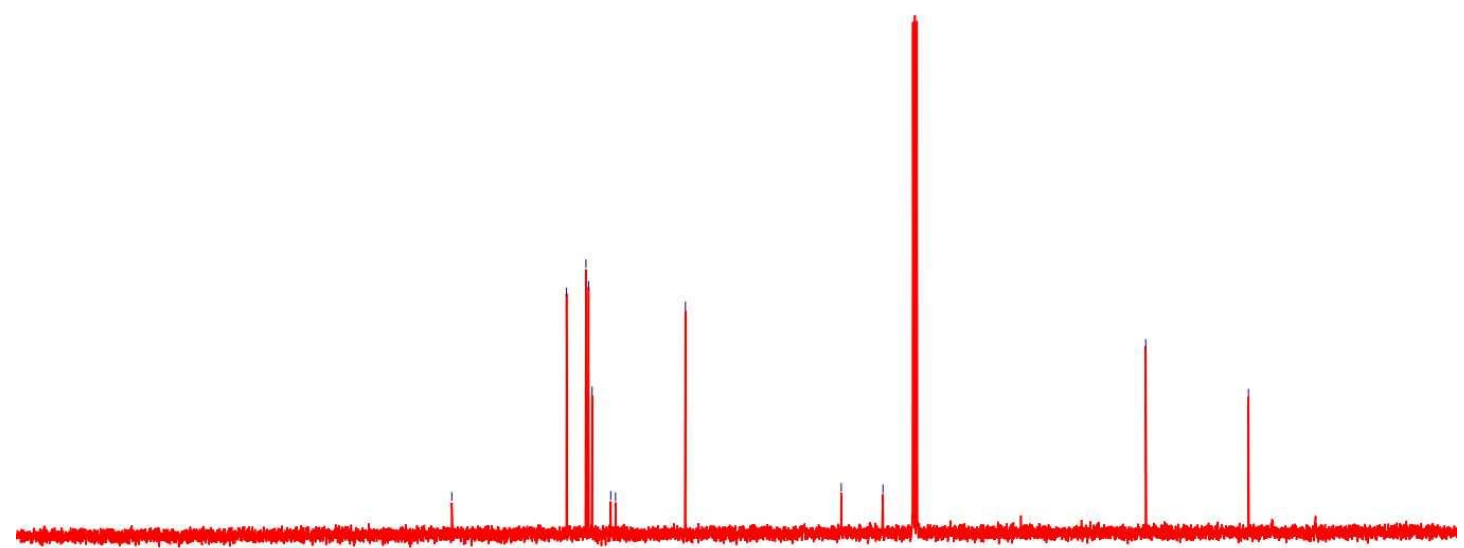

$210200190180170160150140130120110100 \quad 90 \quad 80 \quad 70 \quad 60 \quad 50 \quad 40 \quad 30 \quad 20 \quad 10 \quad 0$ 
Mixture of 4-cinnamyl- $N, N$-dimethylaniline (2as) and (2j) $(80: 20)$

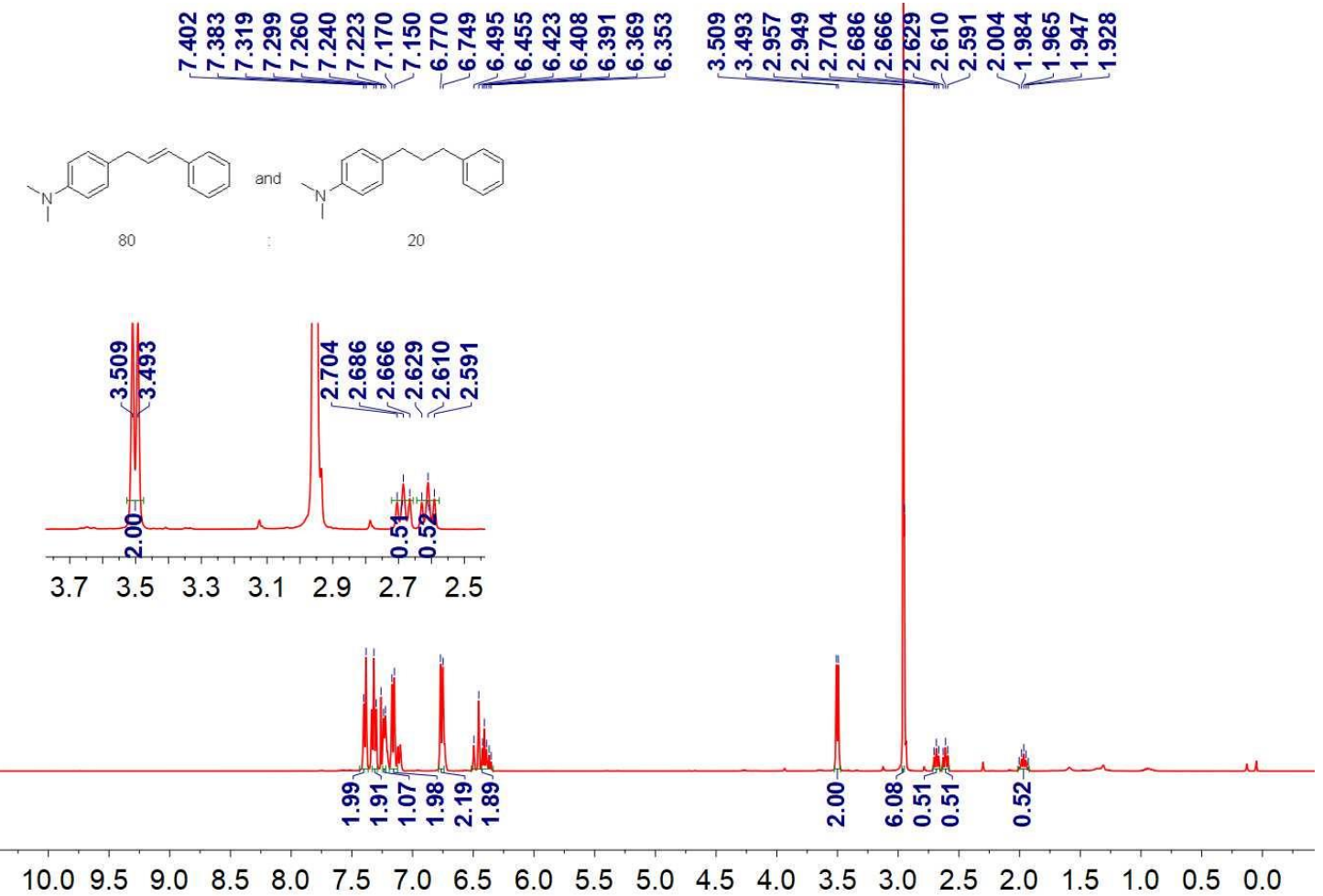

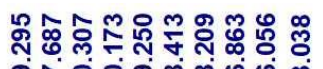

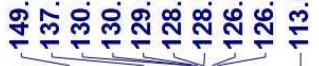

గ్రొ

市œ

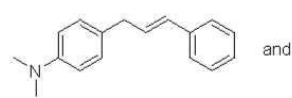

80

20

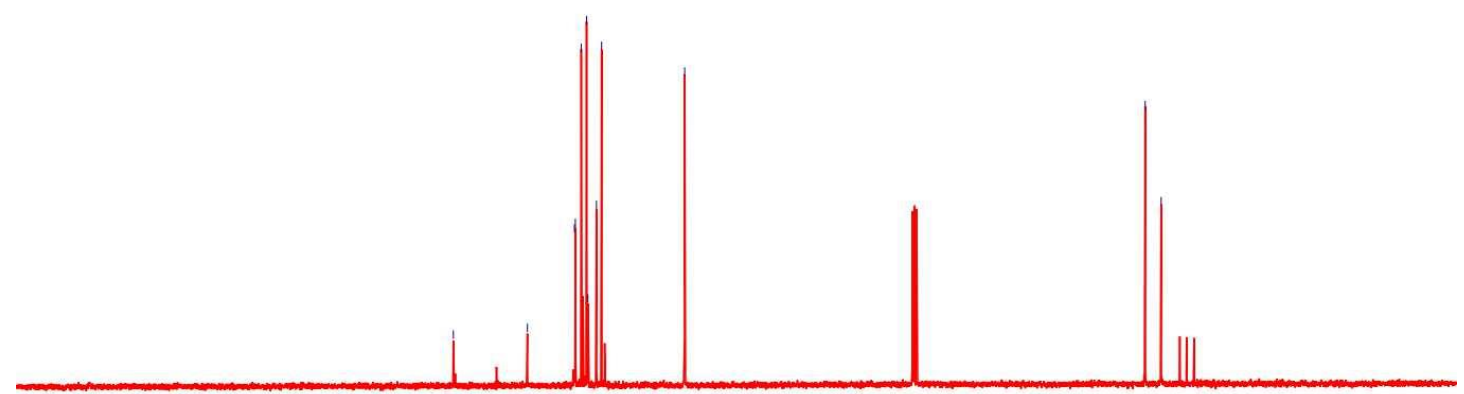

$210200190180170160150140130120110100 \quad 90 \quad 80 \quad 70 \quad 60 \quad 50 \quad 40 \quad 30 \quad 20 \quad 10 \quad 0$ 
4-(4-(Dimethylamino)benzyl)phenol (2at)

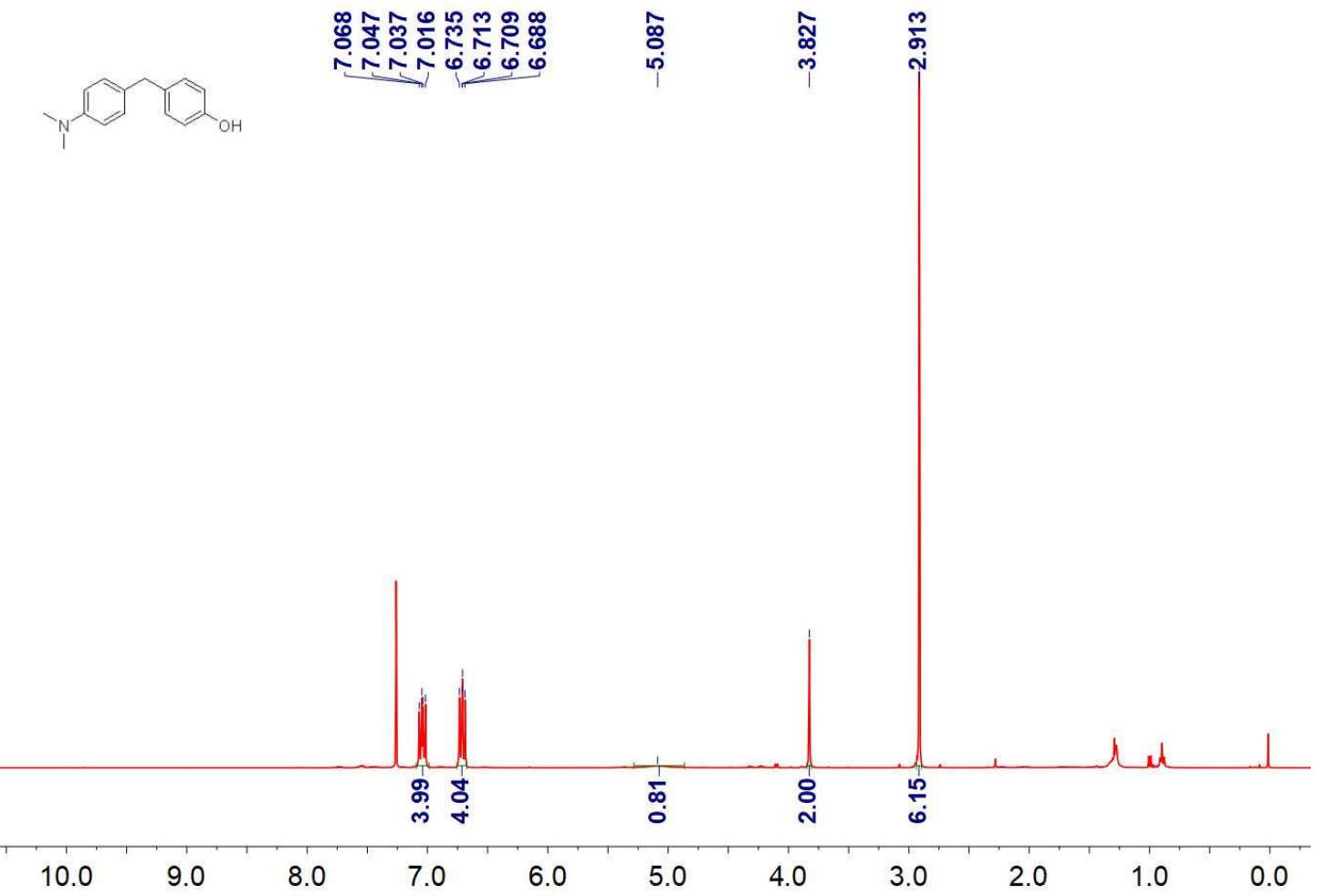

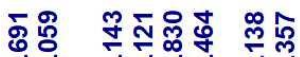

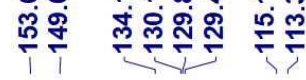

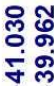
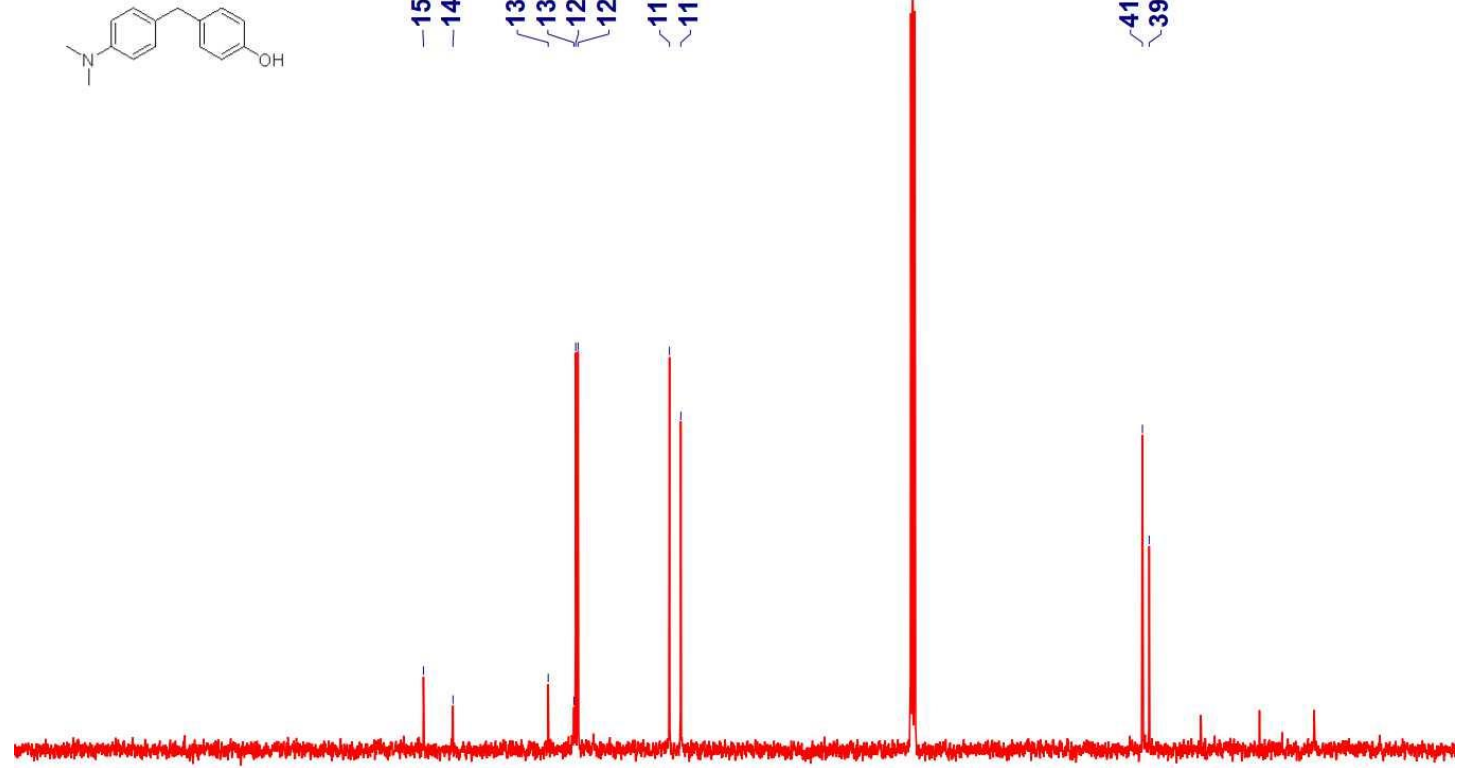

$210200190180170160150140130120110100 \quad 90 \quad 80 \quad 70 \quad 60 \quad 50 \quad 40 \quad 30 \quad 20 \quad 10 \quad 0$ 


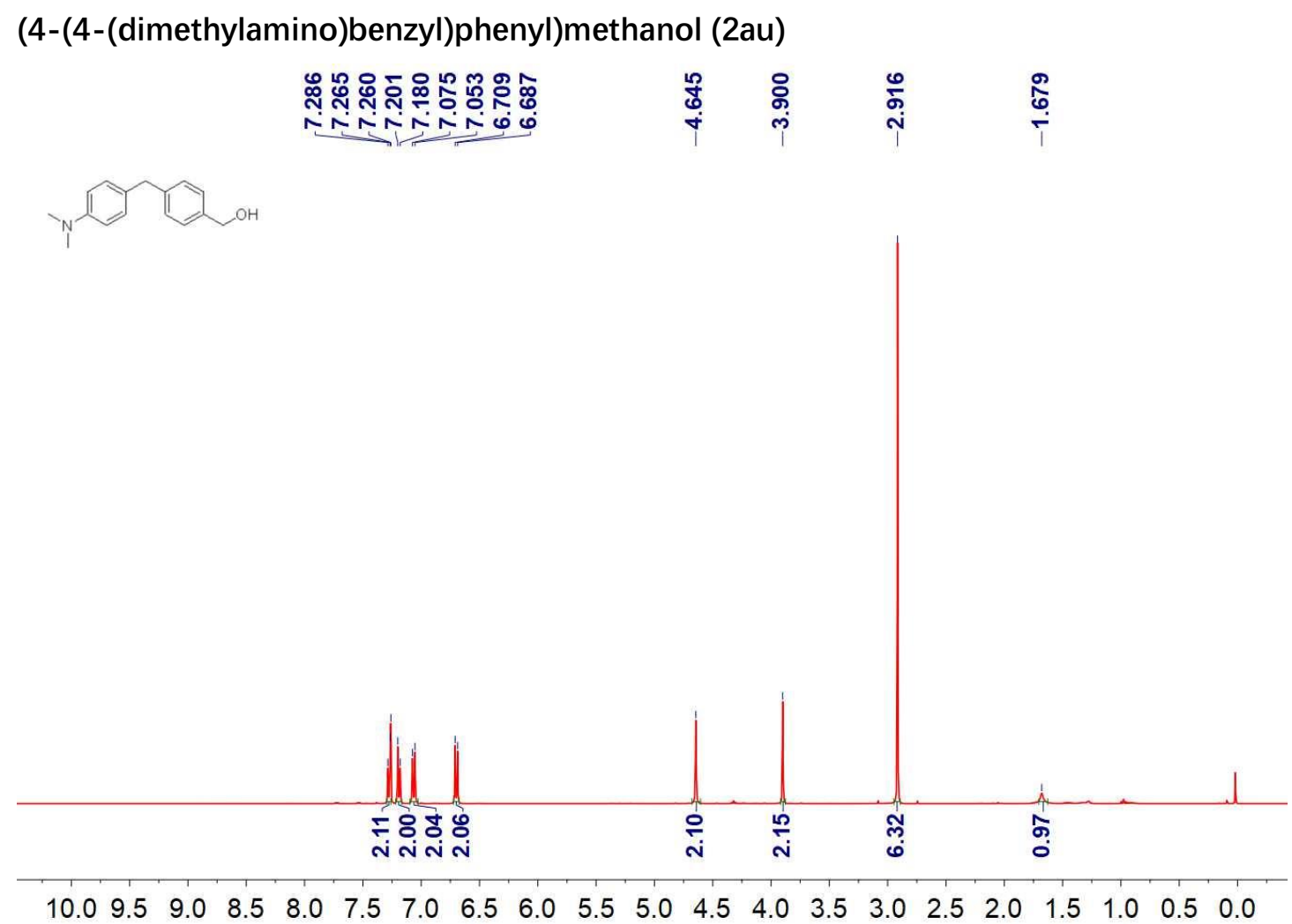

勧

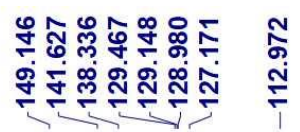

离

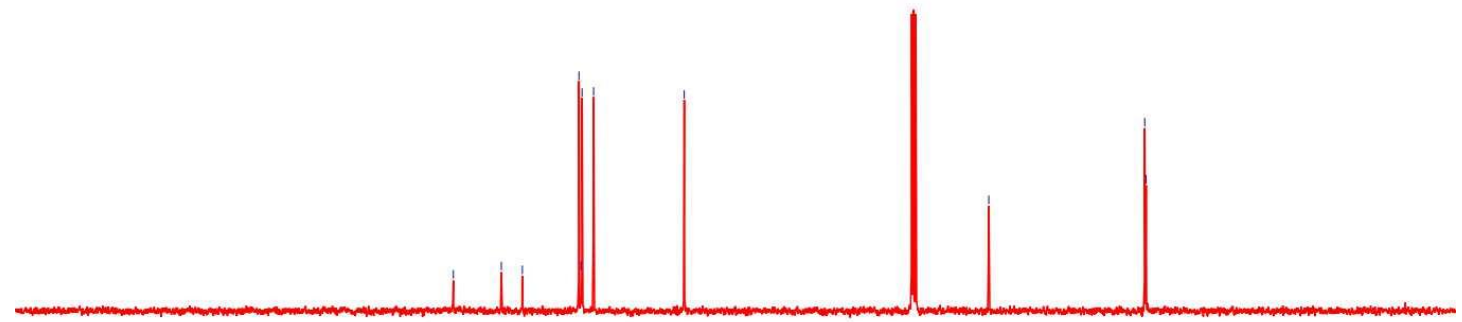

$210200190180170160150140130120110100 \quad 90 \quad 80 \quad 70 \quad 60 \quad 50 \quad 40 \quad 30 \quad 20 \quad 10 \quad 0$ 


\section{4-Isopropyl- $N, N$-dimethylaniline (4a)}
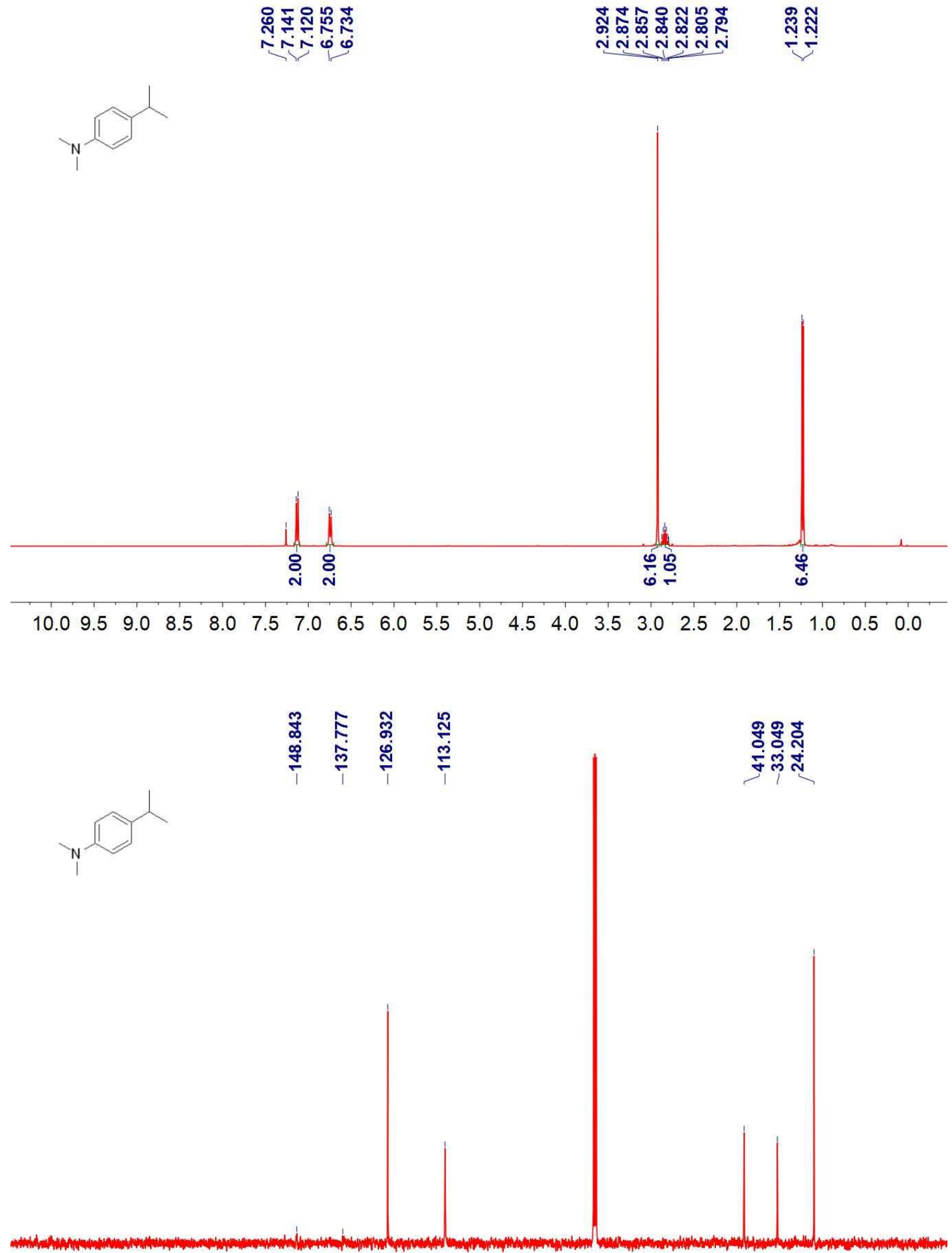

$210200190180170160150140130120110100 \quad 90 \quad 80 \quad 70 \quad 60 \quad 50 \quad 40 \quad 30 \quad 20 \quad 10 \quad 0$ 

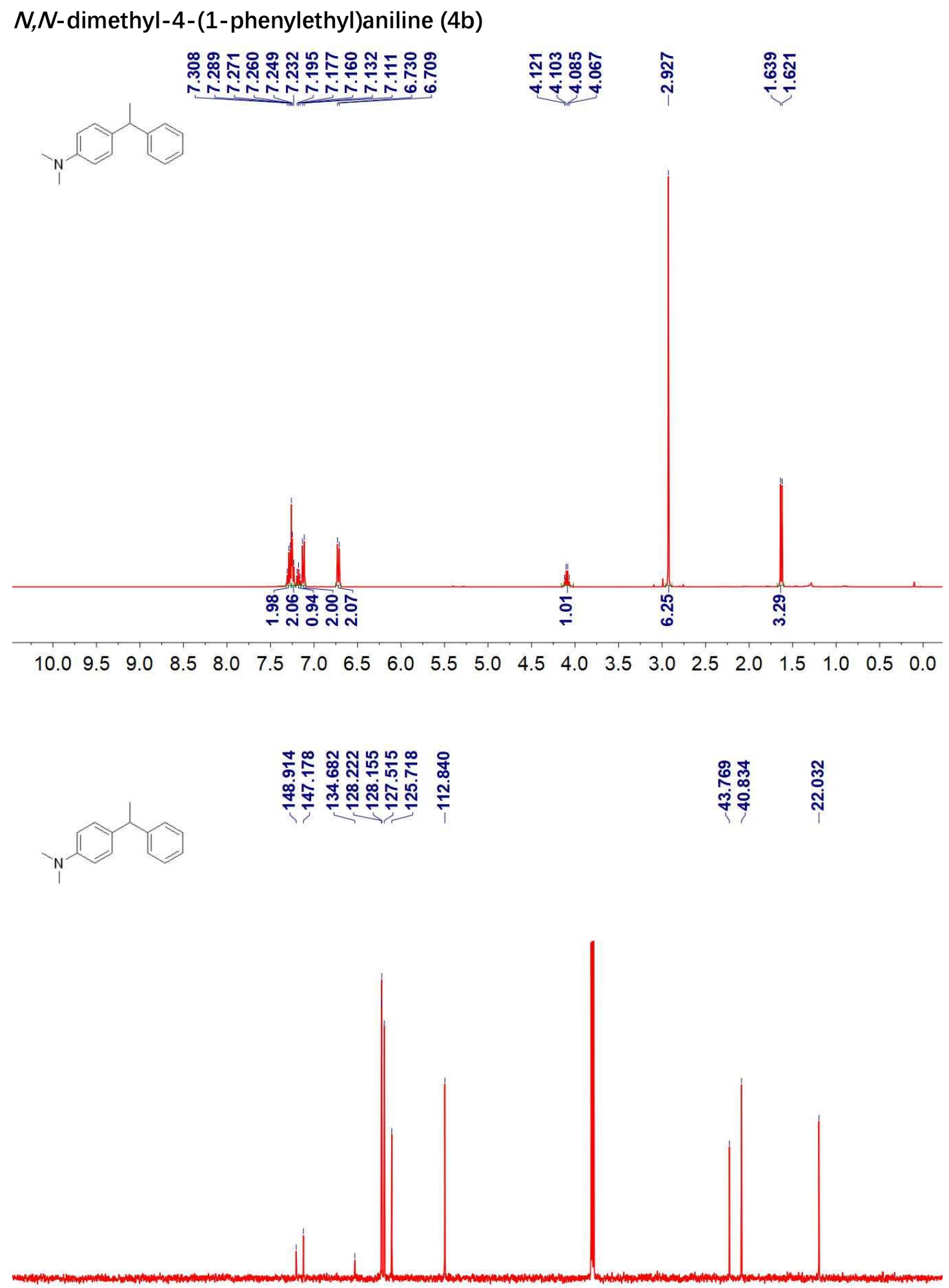

$210200190180170160150140130120110100 \quad 90 \quad 80 \quad 70 \quad 60 \quad 50 \quad 40 \quad 30 \quad 20 \quad 10 \quad 0$ 
4-(1-Cyclopropylethyl)- $N, N$-dimethylaniline (4c)

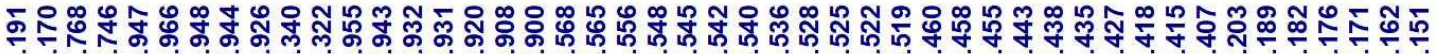
זT

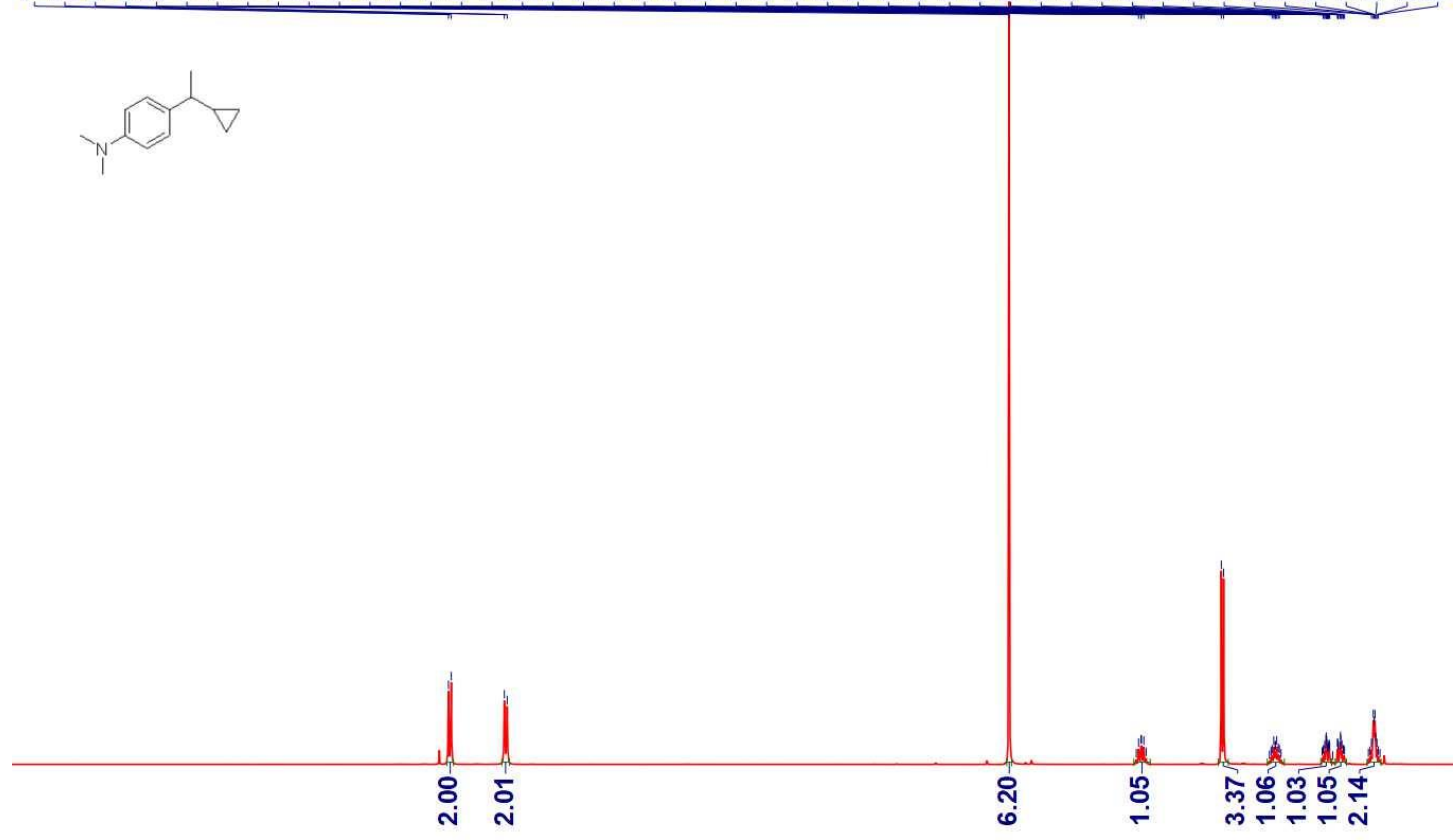

$\begin{array}{lllllllllllllllllllll}10.0 & 9.5 & 9.0 & 8.5 & 8.0 & 7.5 & 7.0 & 6.5 & 6.0 & 5.5 & 5.0 & 4.5 & 4.0 & 3.5 & 3.0 & 2.5 & 2.0 & 1.5 & 1.0 & 0.5 & 0.0\end{array}$

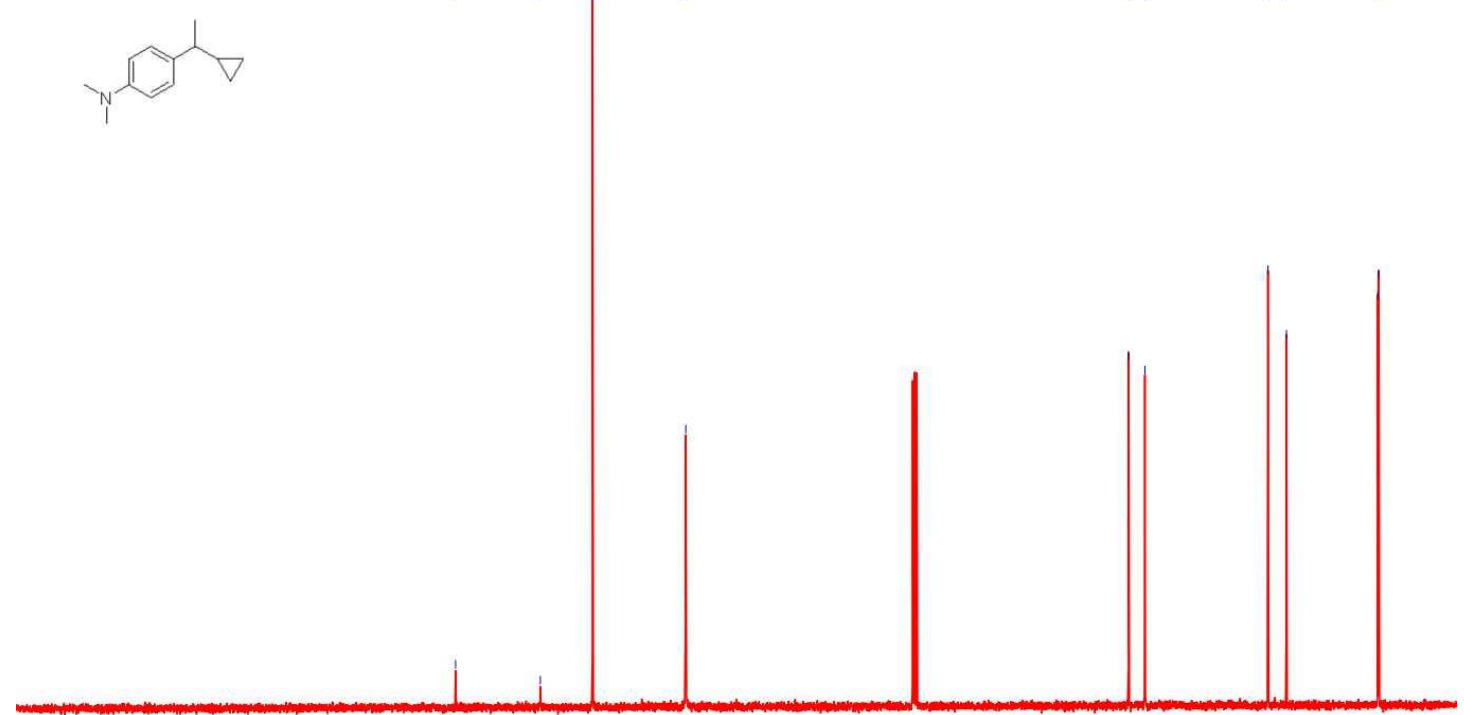

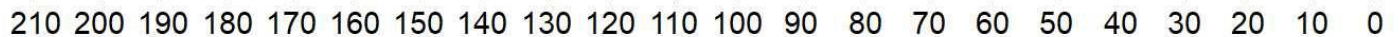


4-(3,3-Dimethylbutan-2-yl)- N,N-dimethylaniline (4d)

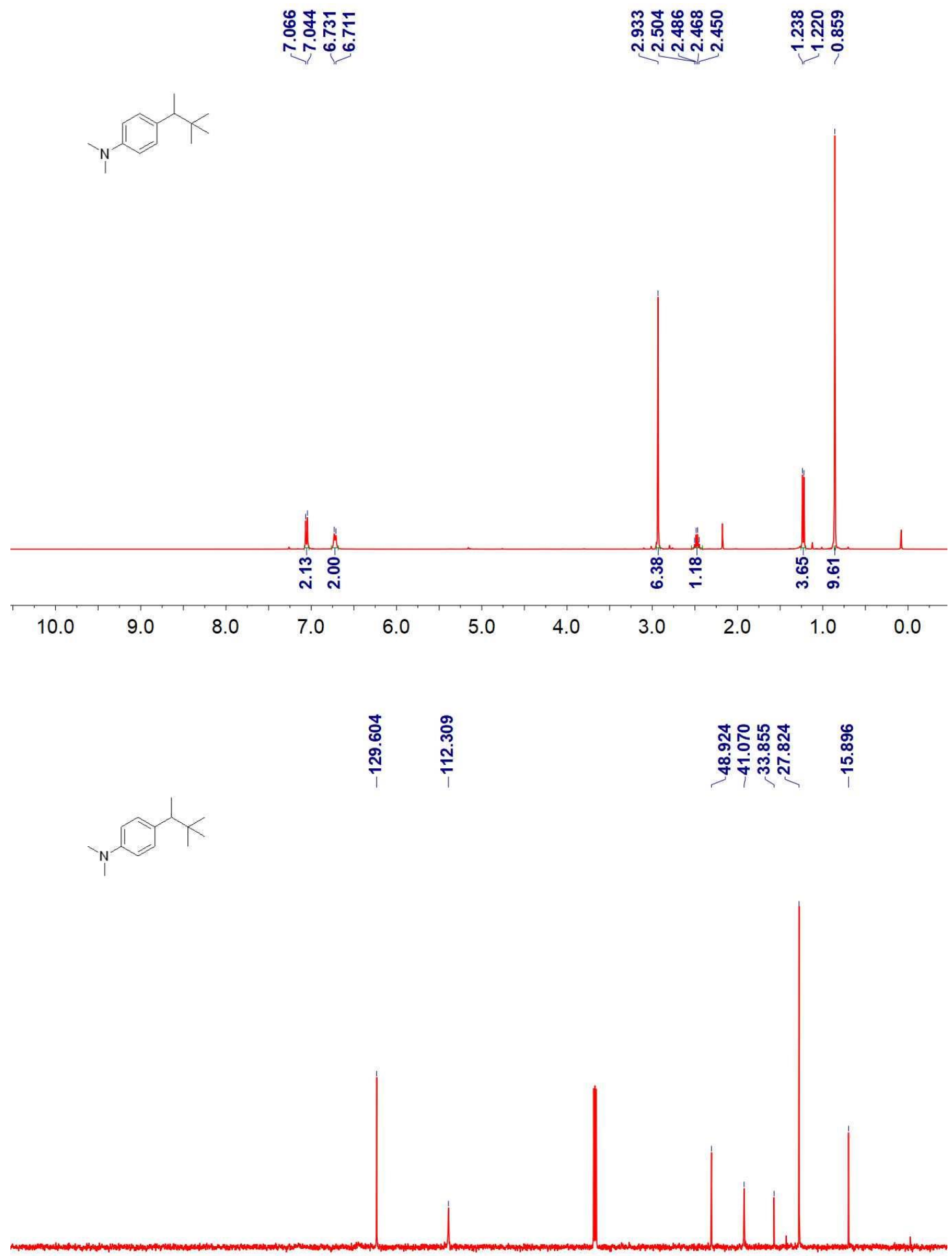

$210200190180170160150140130120110100 \quad 90 \quad 80 \quad 70 \quad 60 \quad 50 \quad 40 \quad 30 \quad 20 \quad 10 \quad 0$ 
4-(2-((1,1,1,3,3,3-Hexafluoropropan-2-yl)oxy)-1-phenylethyl)- $N, N$-dimethylaniline (4e)

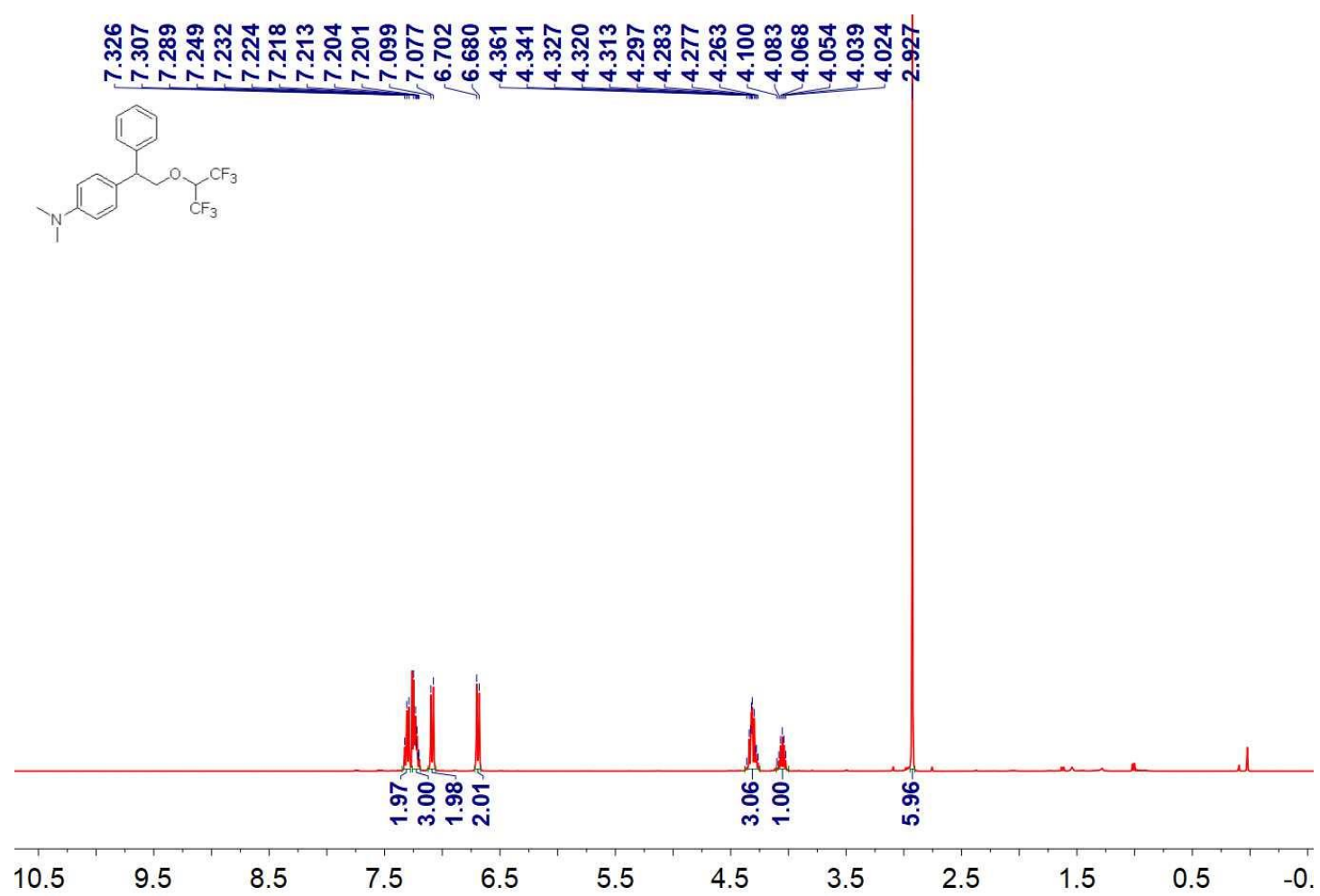

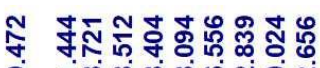

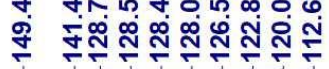

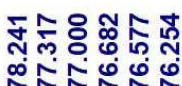

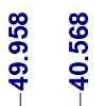

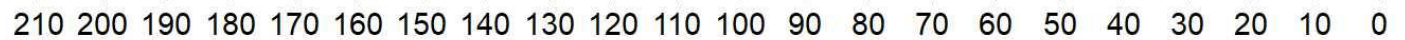


N,N-dimethyl-4-(2-phenoxy-1-phenylethyl)aniline (4f)
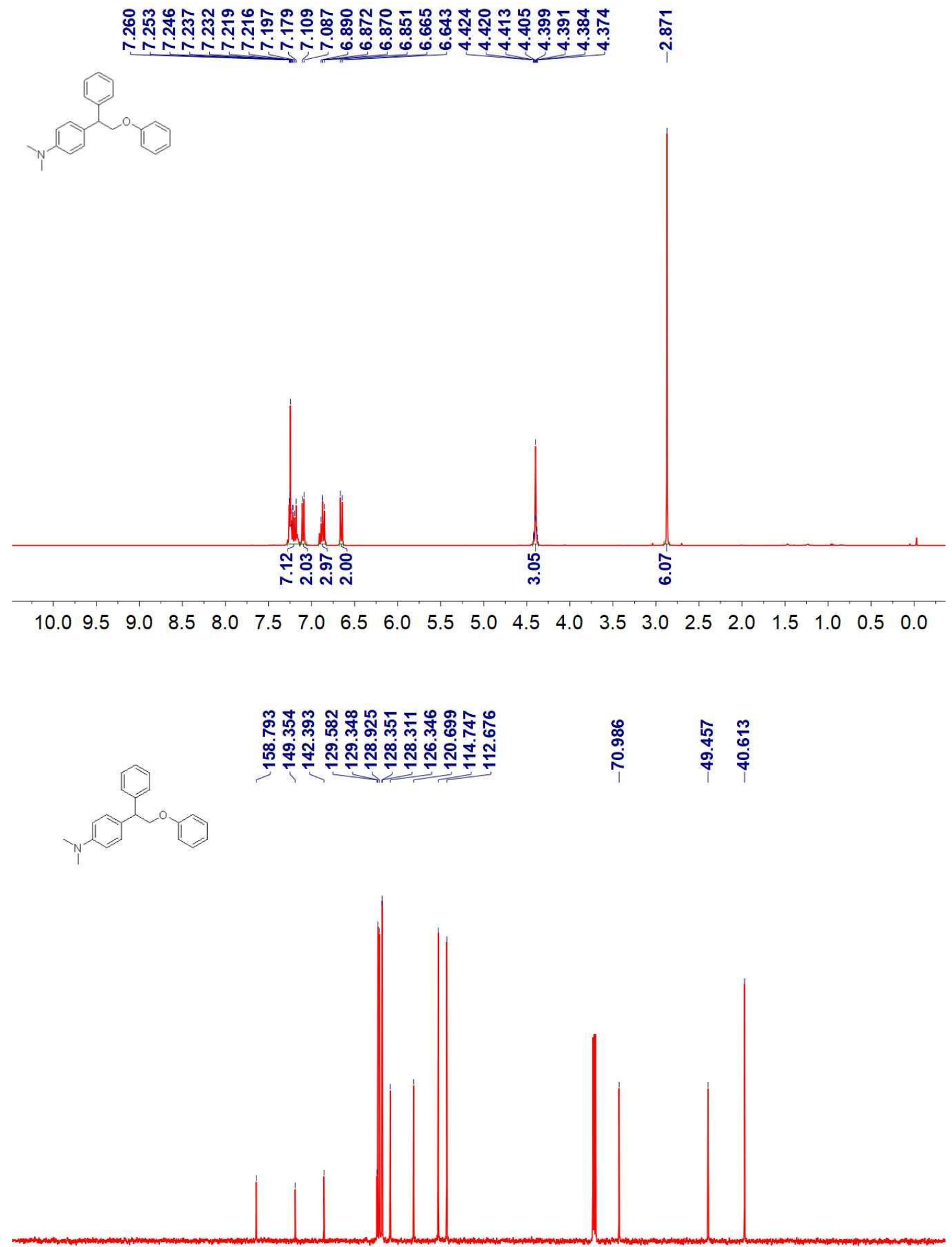

$210200190180170160150140130120110100 \quad 90 \quad 80 \quad 70 \quad 60 \quad 50 \quad 40 \quad 30 \quad 20 \quad 10 \quad 0$ 
4-Cyclobutyl- $N, N$-dimethylaniline (4g)

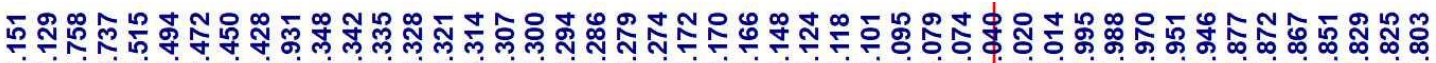
T-
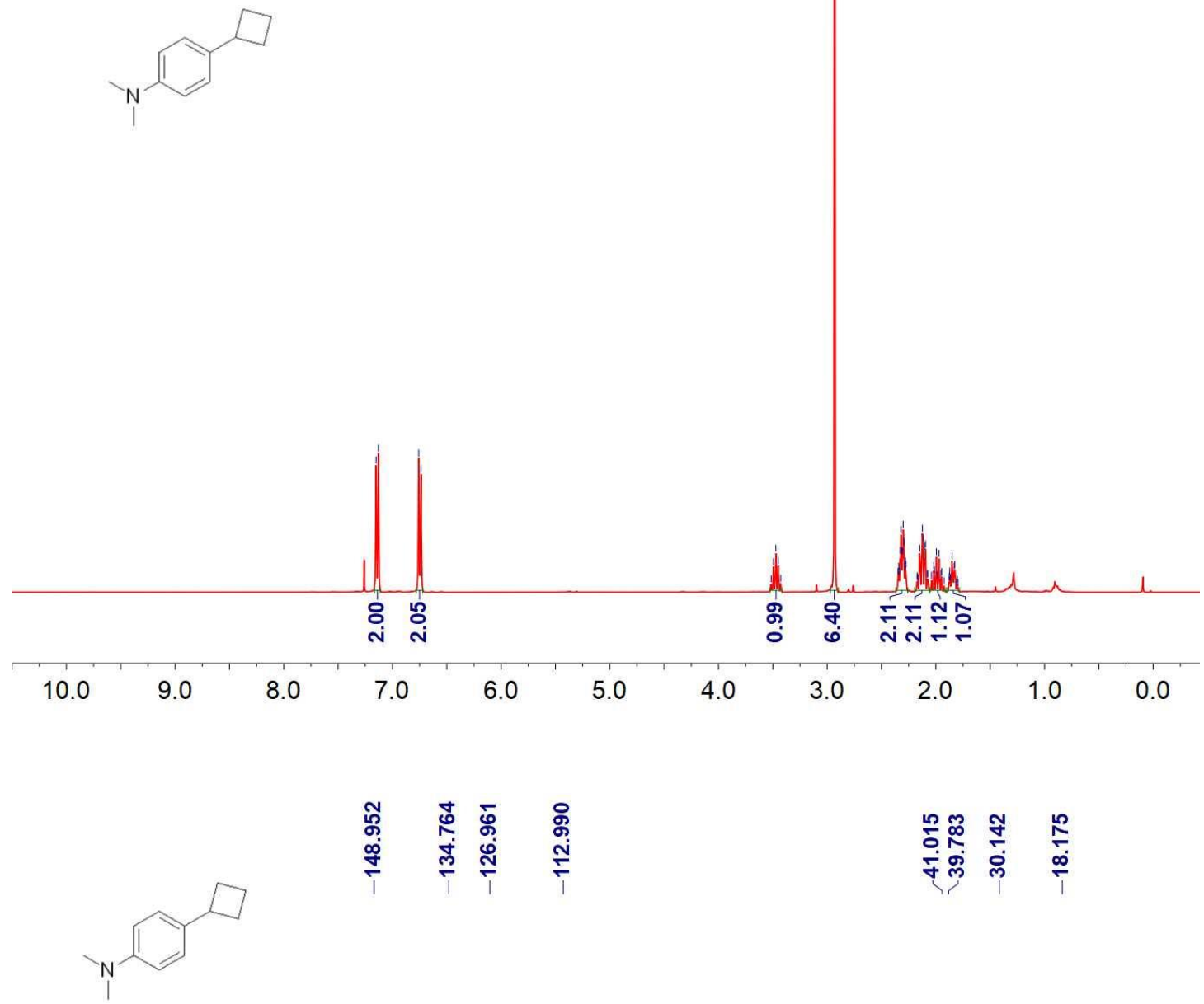

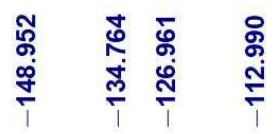

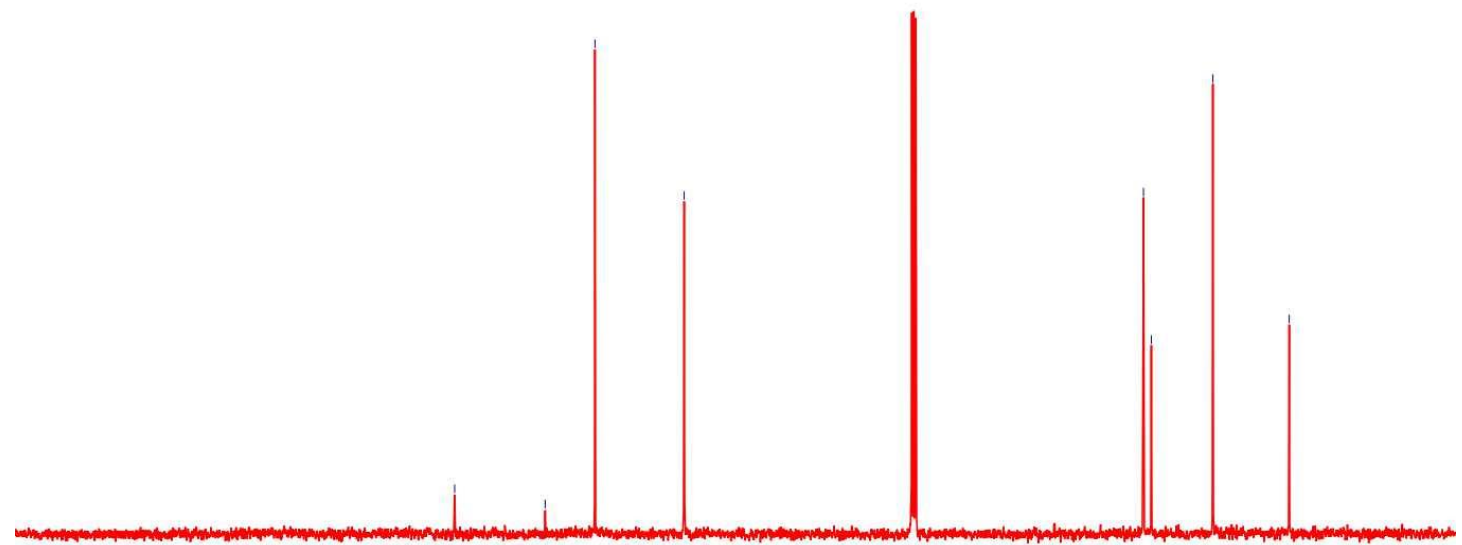

$210200190180170160150140130120110100 \quad 90 \quad 80 \quad 70 \quad 60 \quad 50 \quad 40 \quad 30 \quad 20 \quad 10 \quad 0$ 
4-Cyclopentyl- $N, N$-dimethylaniline (4h)
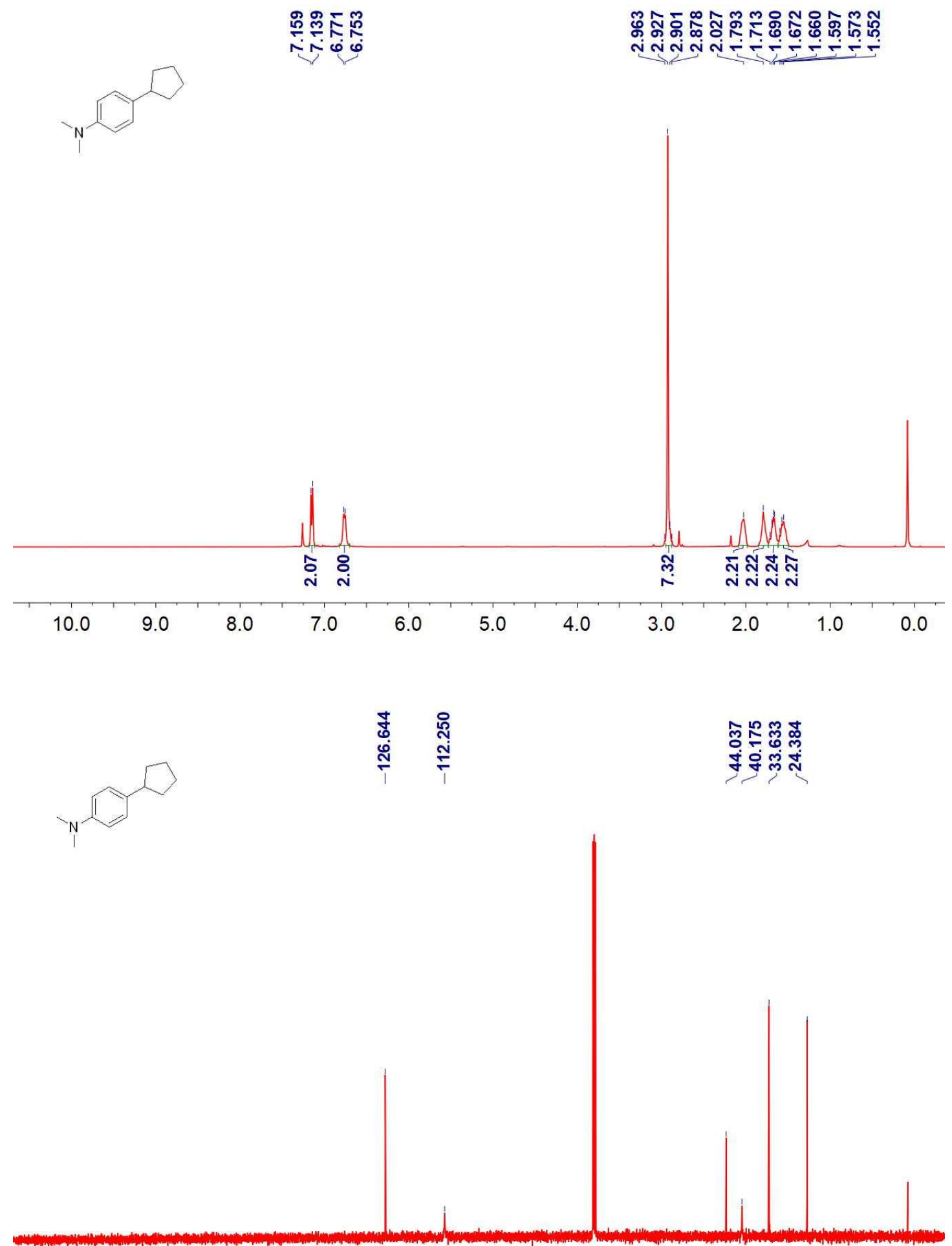

$210200190180170160150140130120110100 \quad 90 \quad 80 \quad 70 \quad 60 \quad 50 \quad 40 \quad 30 \quad 20 \quad 10 \quad 0$ 
4-Cyclohexyl-N,N-dimethylaniline (4i)

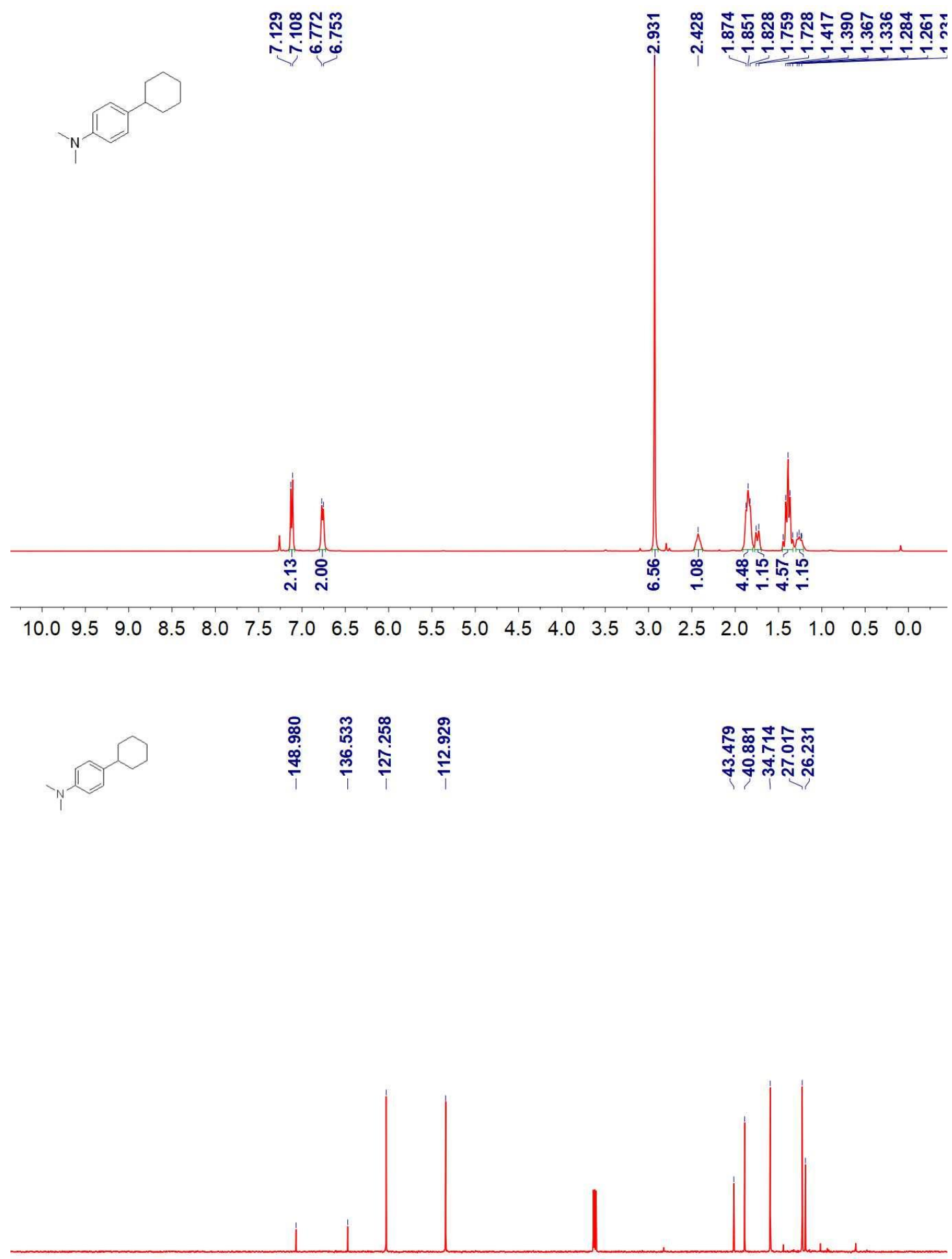

$210200190180170160150140130120110100 \quad 90 \quad 80 \quad 70 \quad 60 \quad 50 \quad 40 \quad 30 \quad 20 \quad 10 \quad 0$ 
4-Cycloheptyl- $N, N$-dimethylaniline (4j)

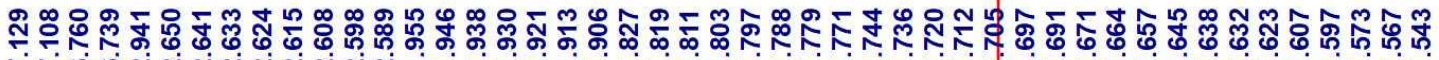

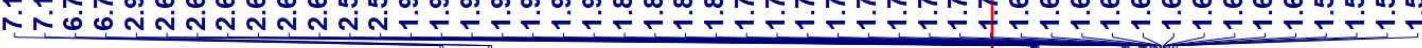
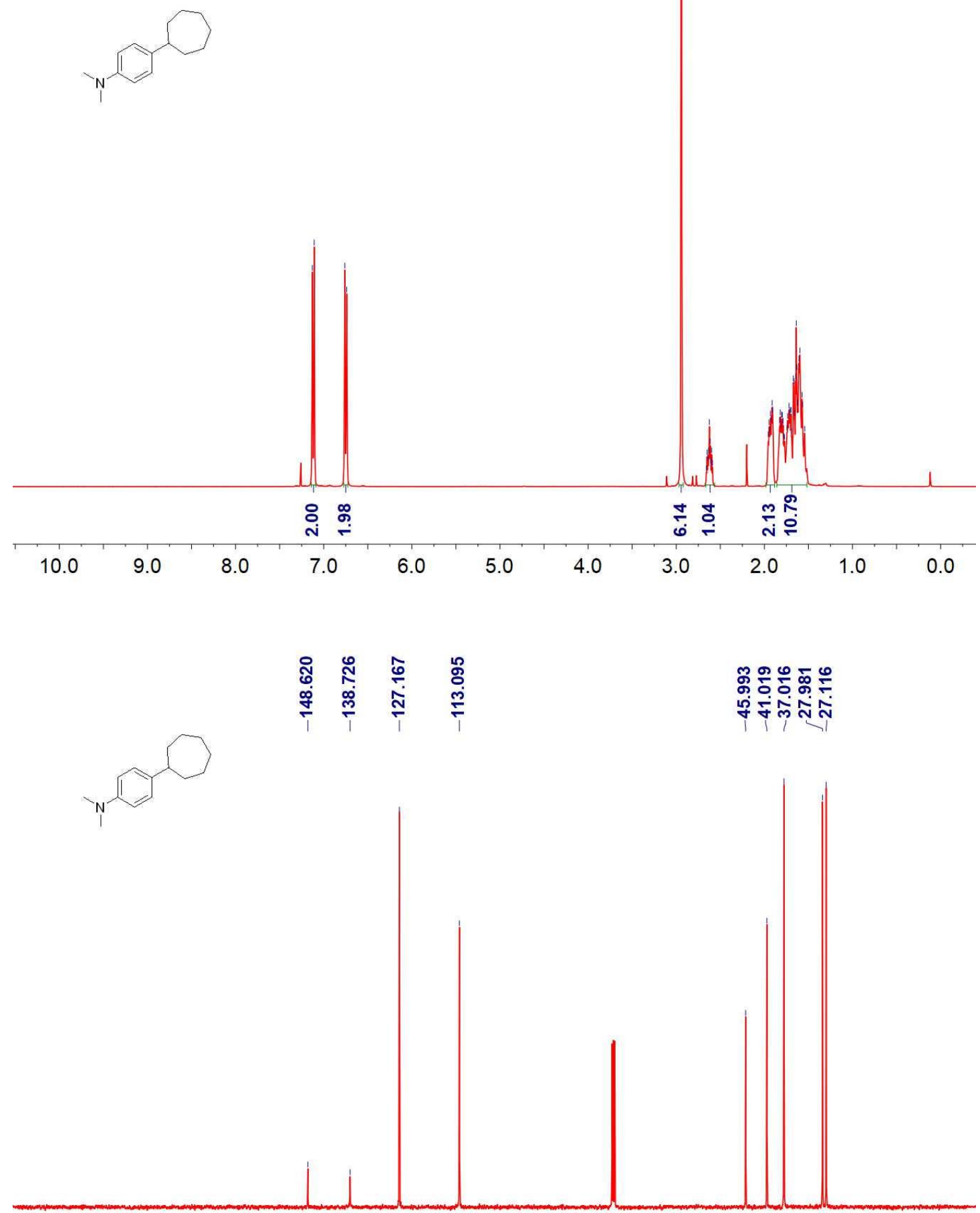

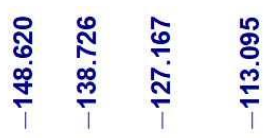

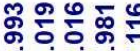

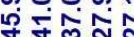

$210200190180170160150140130120110 \quad 100 \quad 90 \quad 80 \quad 70 \quad 60 \quad 50 \quad 40 \quad 30 \quad 20 \quad 10 \quad 0$ 
4-Cyclododecyl-N,N-dimethylaniline (4k)
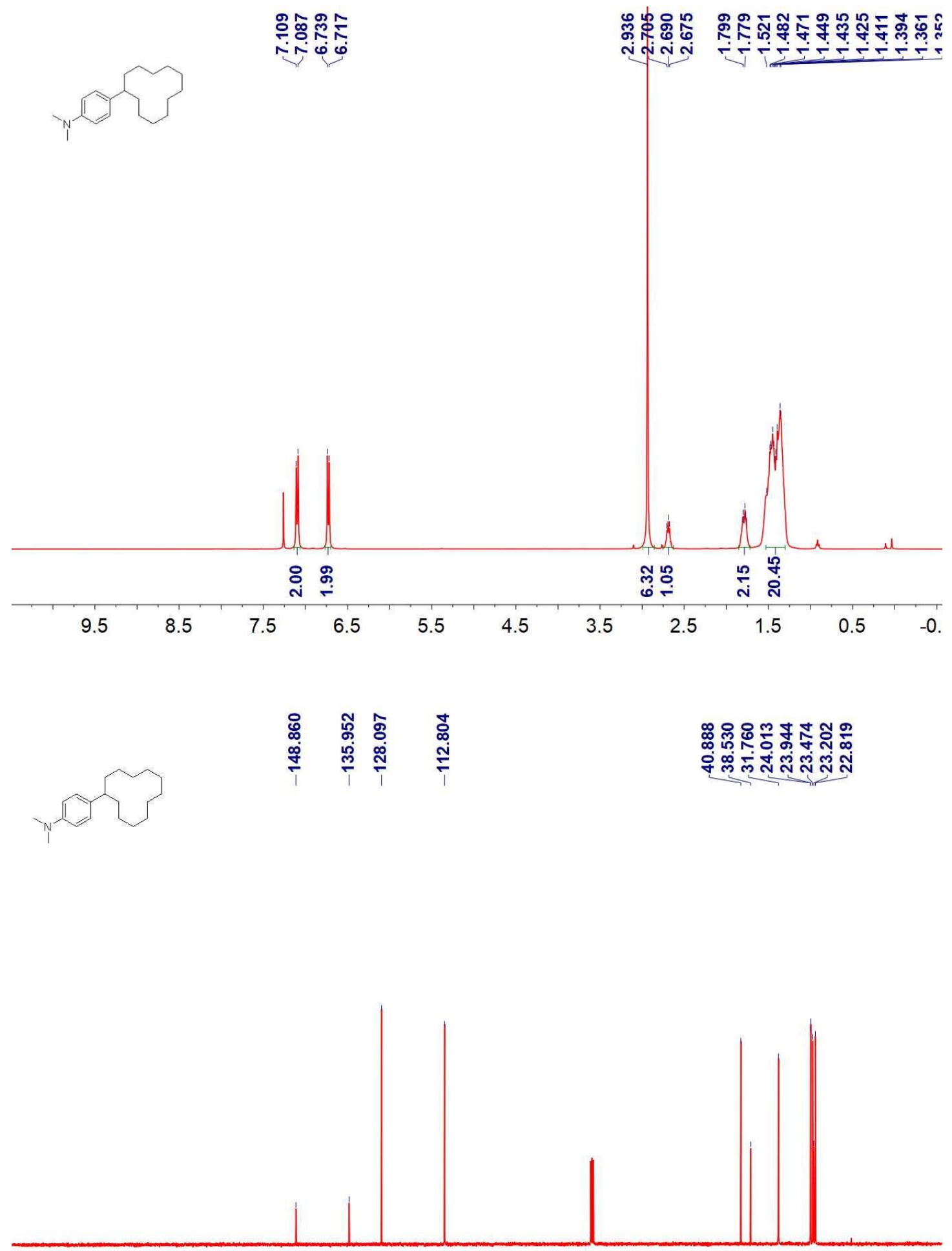

$210200190180170160150140130120110100 \quad 90 \quad 80 \quad 70 \quad 60 \quad 50 \quad 40 \quad 30 \quad 20 \quad 10 \quad 0$ 
$\mathrm{N}, \mathrm{N}$-dimethyl-4-(9H-xanthen-9-yl)aniline (4I)

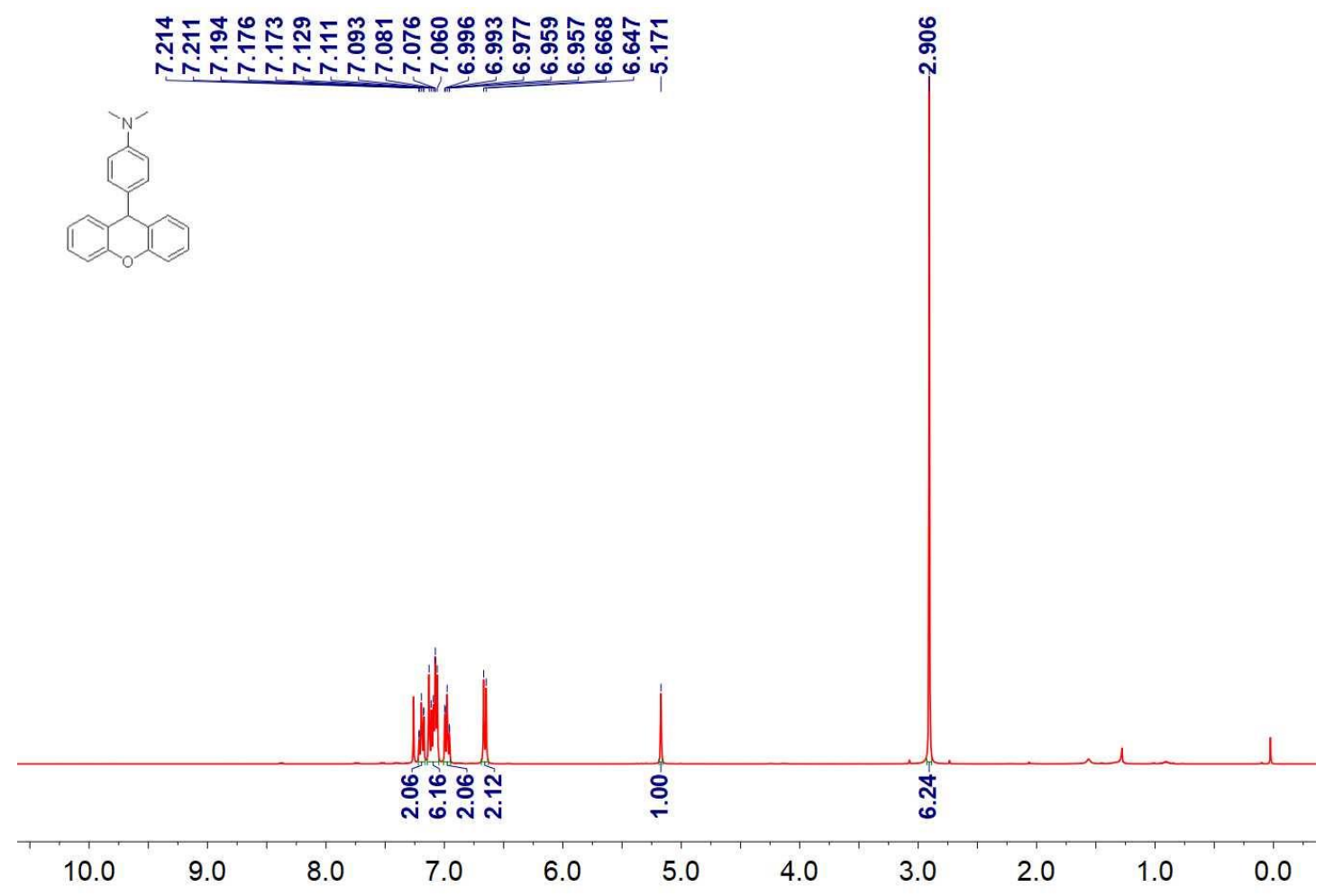

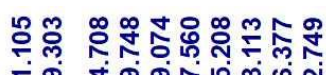

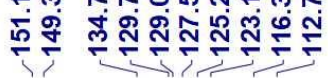

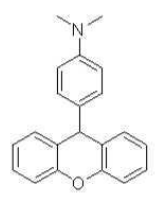

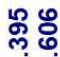

भु่

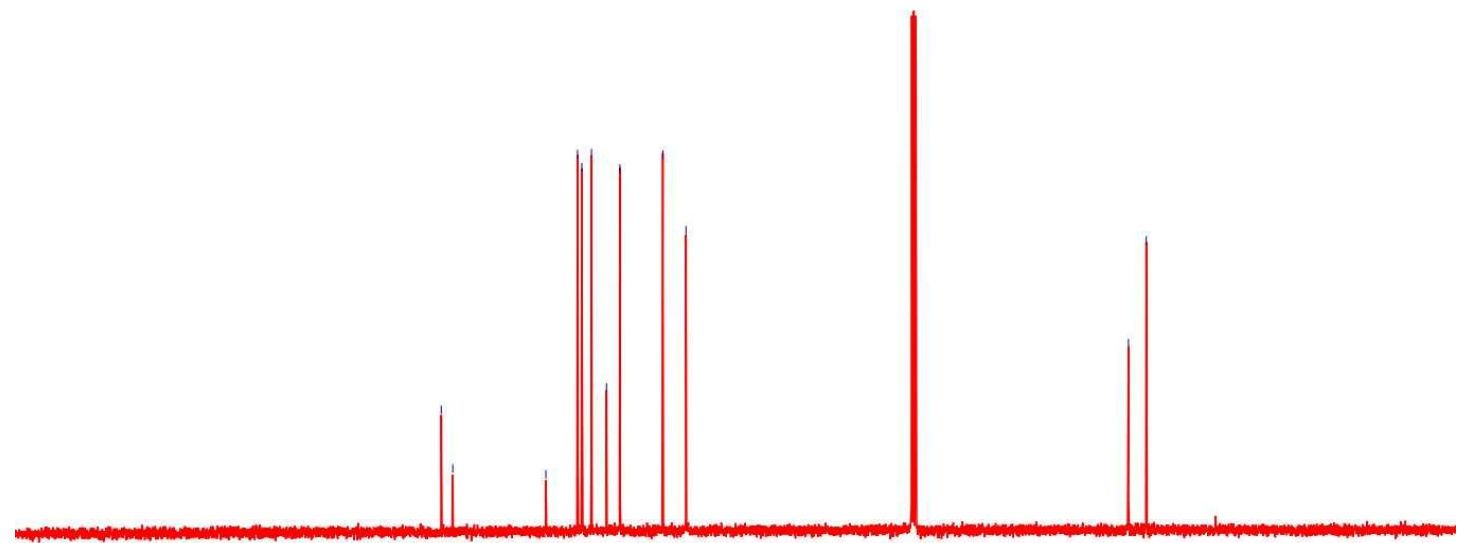

$210200190180170160150140130120110100 \quad 90 \quad 80 \quad 70 \quad 60 \quad 50 \quad 40 \quad 30 \quad 20 \quad 10 \quad 0$ 
$N, N$-dimethyl-4-(1,2,3,4-tetrahydronaphthalen-1-yl)aniline (4m)

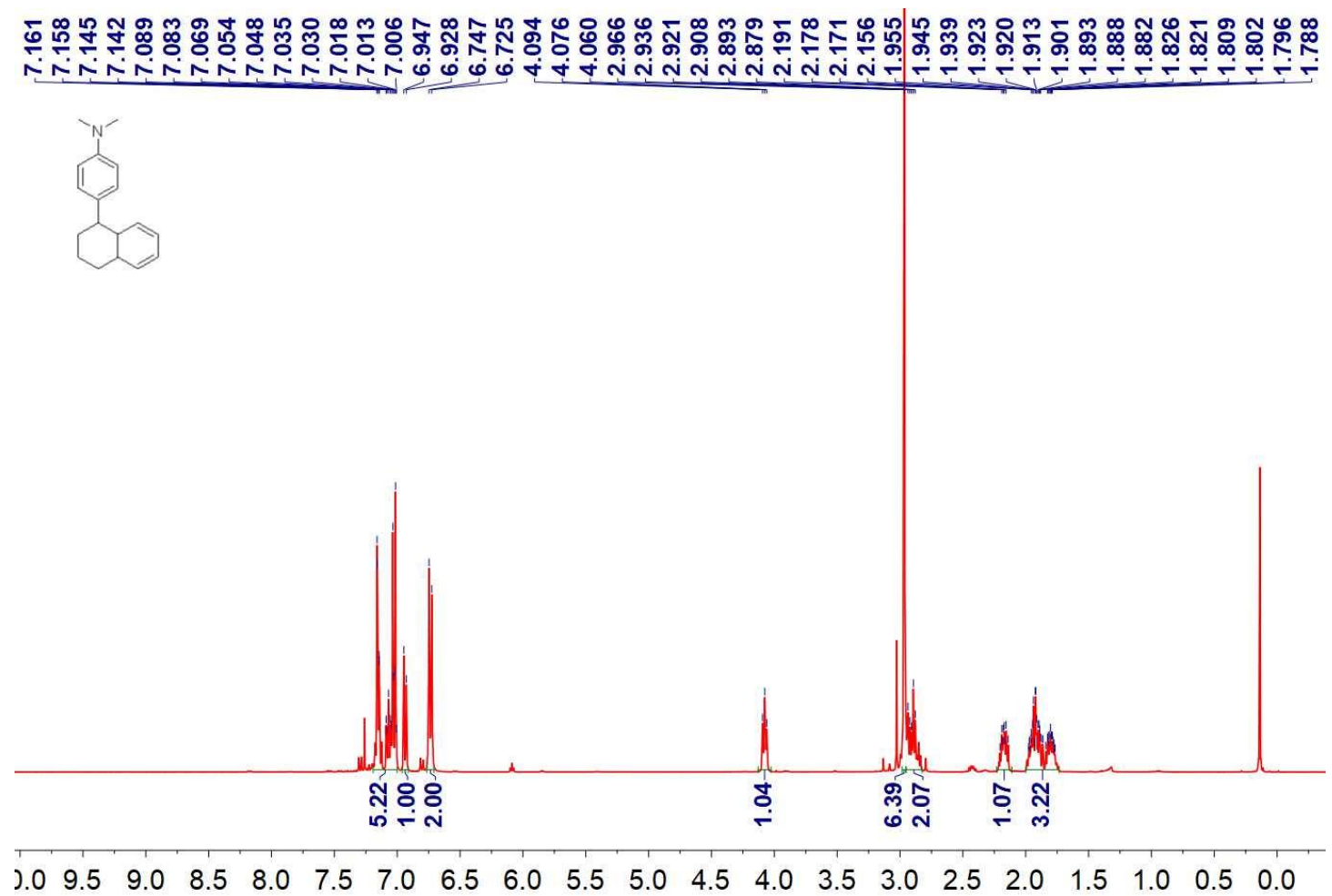

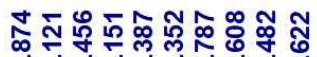

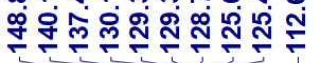

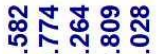

求所
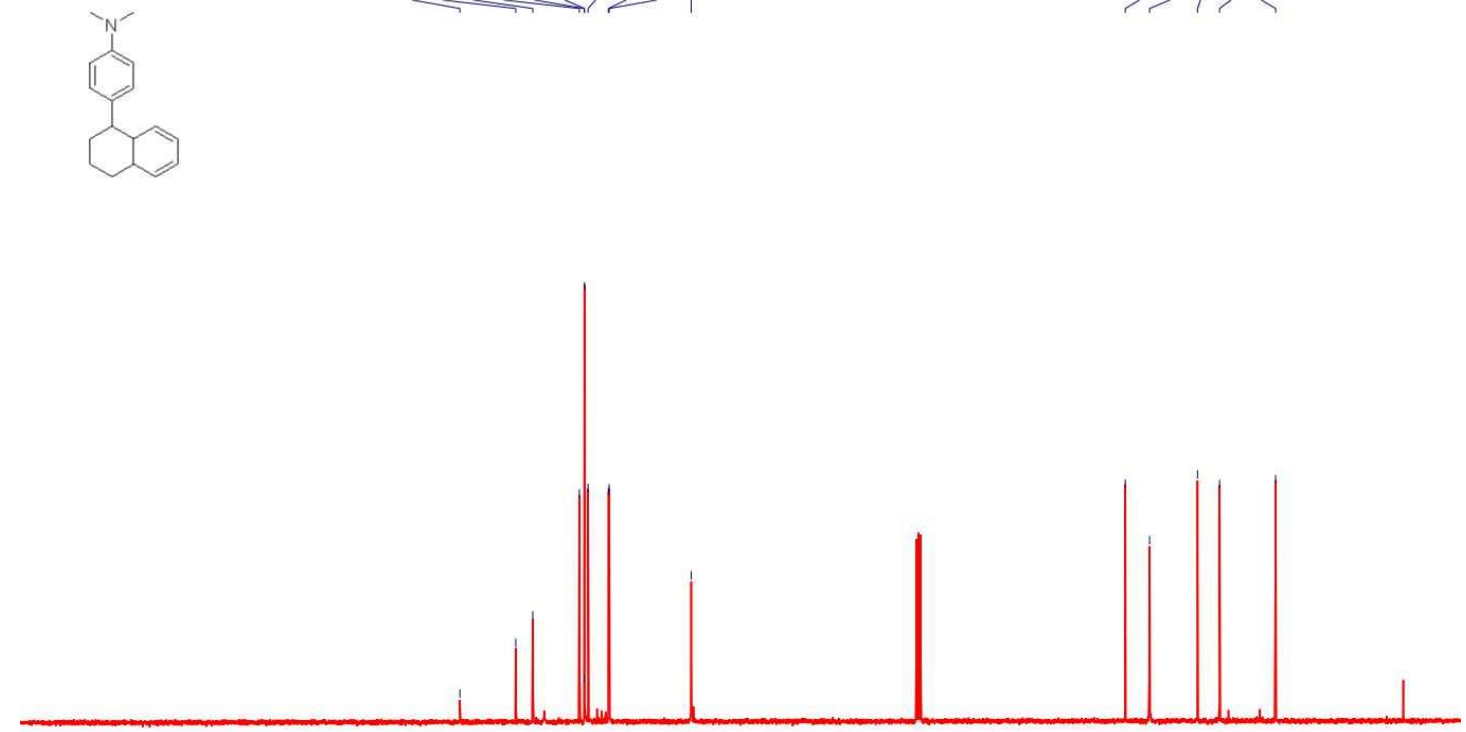

$210200190180170160150140130120110100 \quad 90 \quad 80 \quad 70 \quad 60 \quad 50 \quad 40 \quad 30 \quad 20 \quad 10 \quad 0$ 
4-(Adamantan-2-yl)-N,N-dimethylaniline (4na)

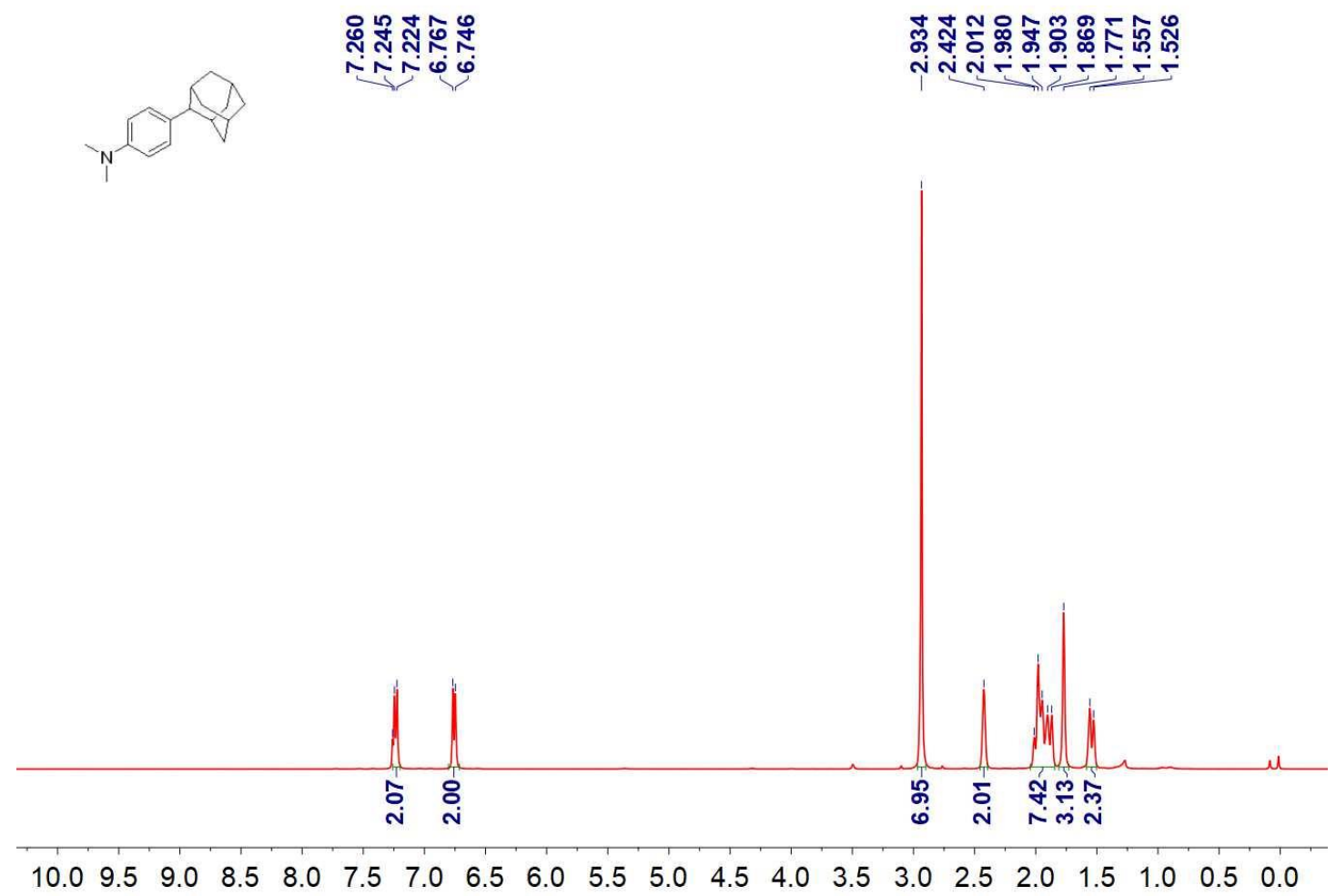

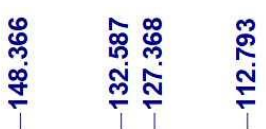

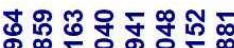

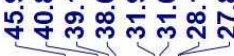

N 10

(1)

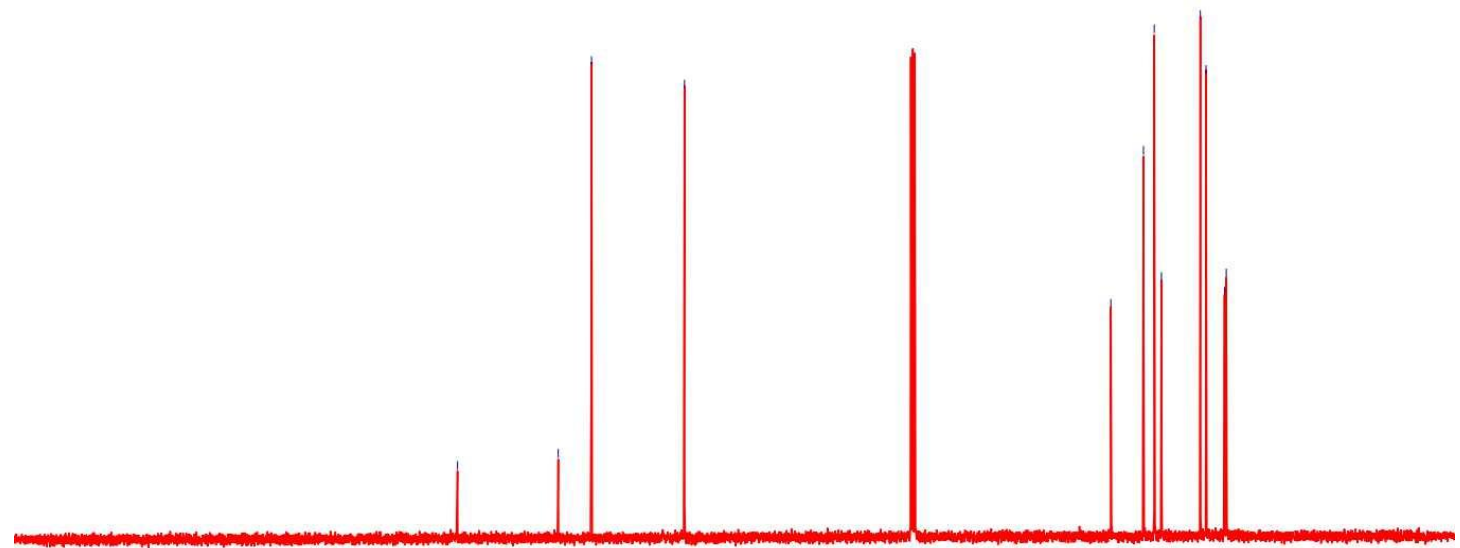

$210200190180170160150140130120110100 \quad 90 \quad 80 \quad 70 \quad 60 \quad 50 \quad 40 \quad 30 \quad 20 \quad 10 \quad 0$ 
1-(4-(Adamantan-2-yl)phenyl)piperidine (4nb)

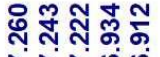

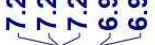

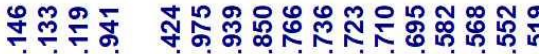

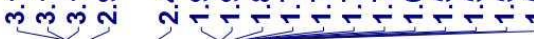

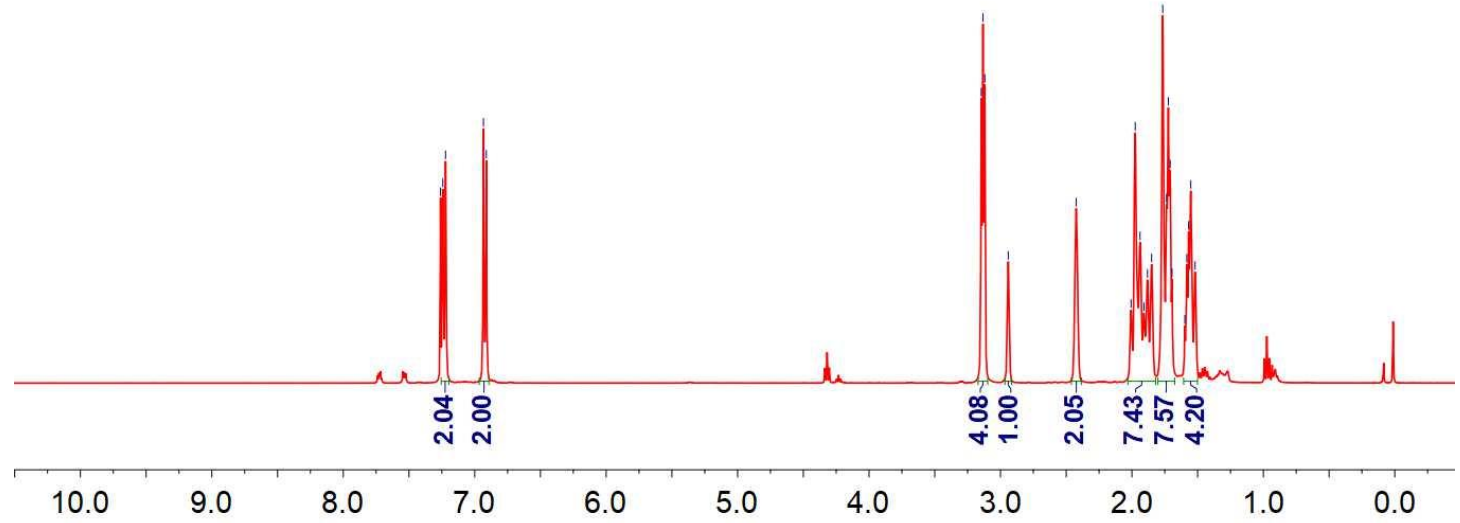

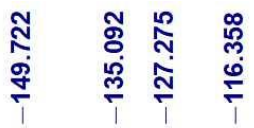

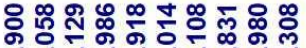

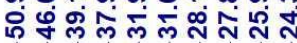

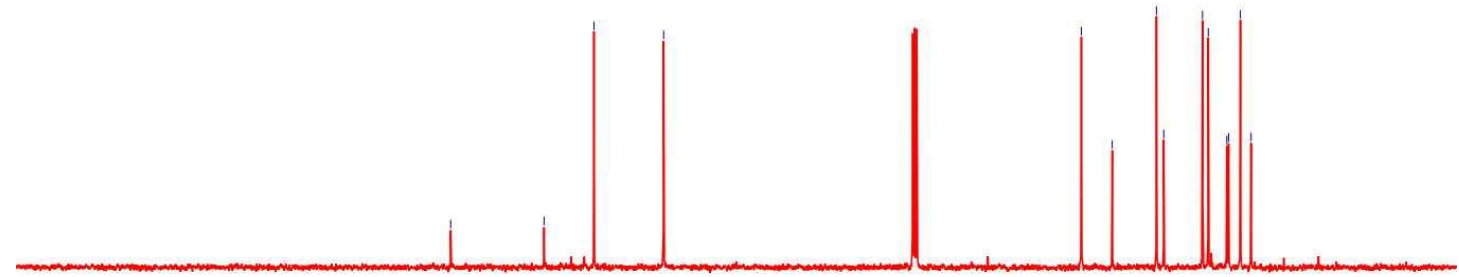

$210200190180170160150140130120110 \quad 100 \quad 90 \quad 80 \quad 70 \quad 60 \quad 50 \quad 40 \quad 30 \quad 20 \quad 10 \quad 0$ 
N,N-dimethyl-4-(1,7,7-trimethylbicyclo[2.2.1]heptan-2-yl)aniline (4o) (mixture of two stereomers, d.r. $=80: 20$ )
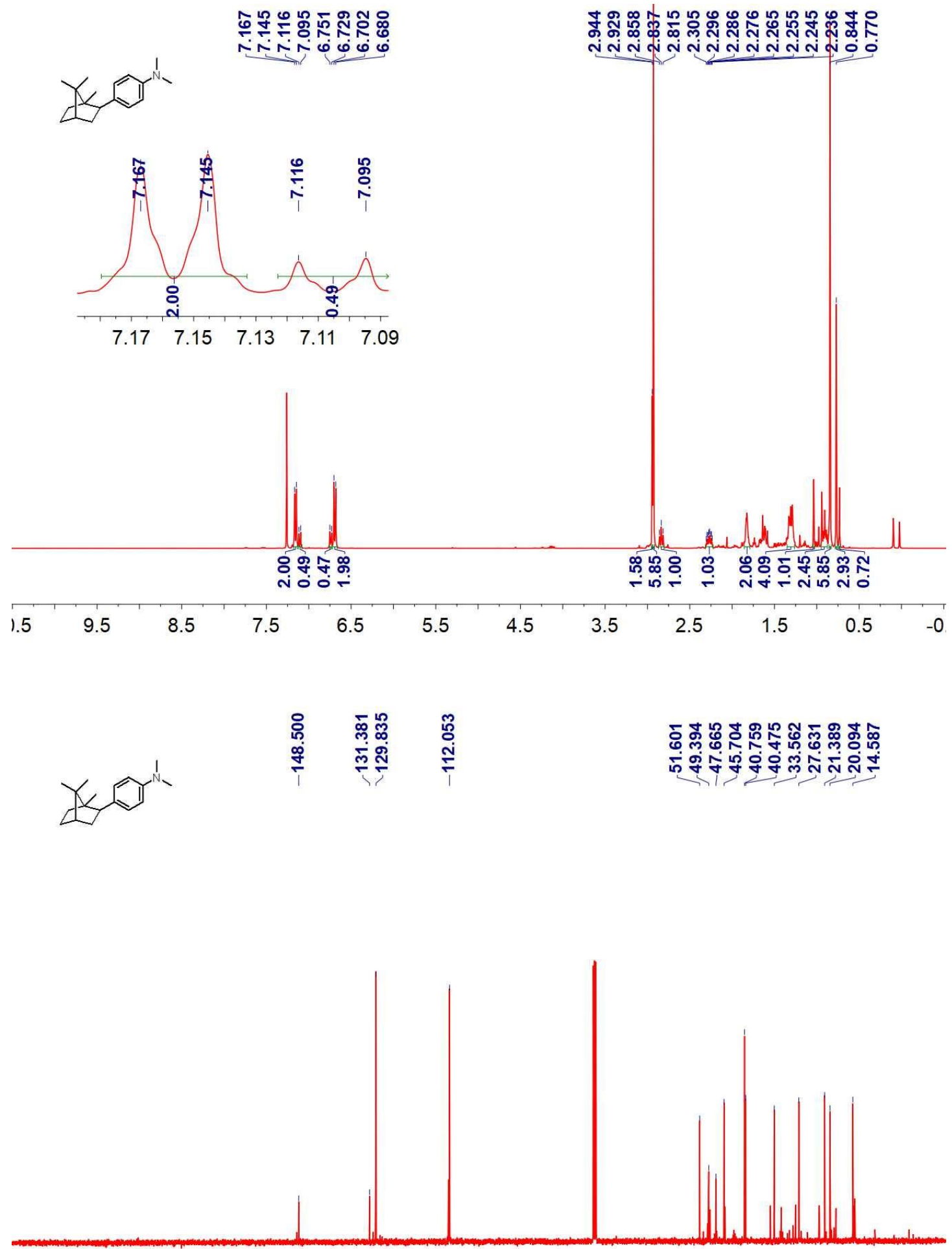

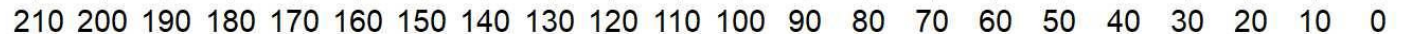


1-(4-((5S)-2-methyl-5-(prop-1-en-2-yl)cyclohexyl)phenyl)piperidine (4p) (mixture of 4 stereomers)

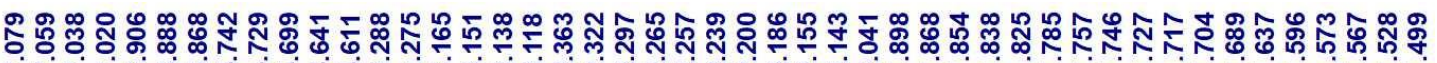

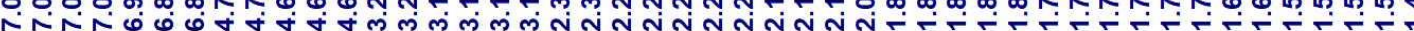

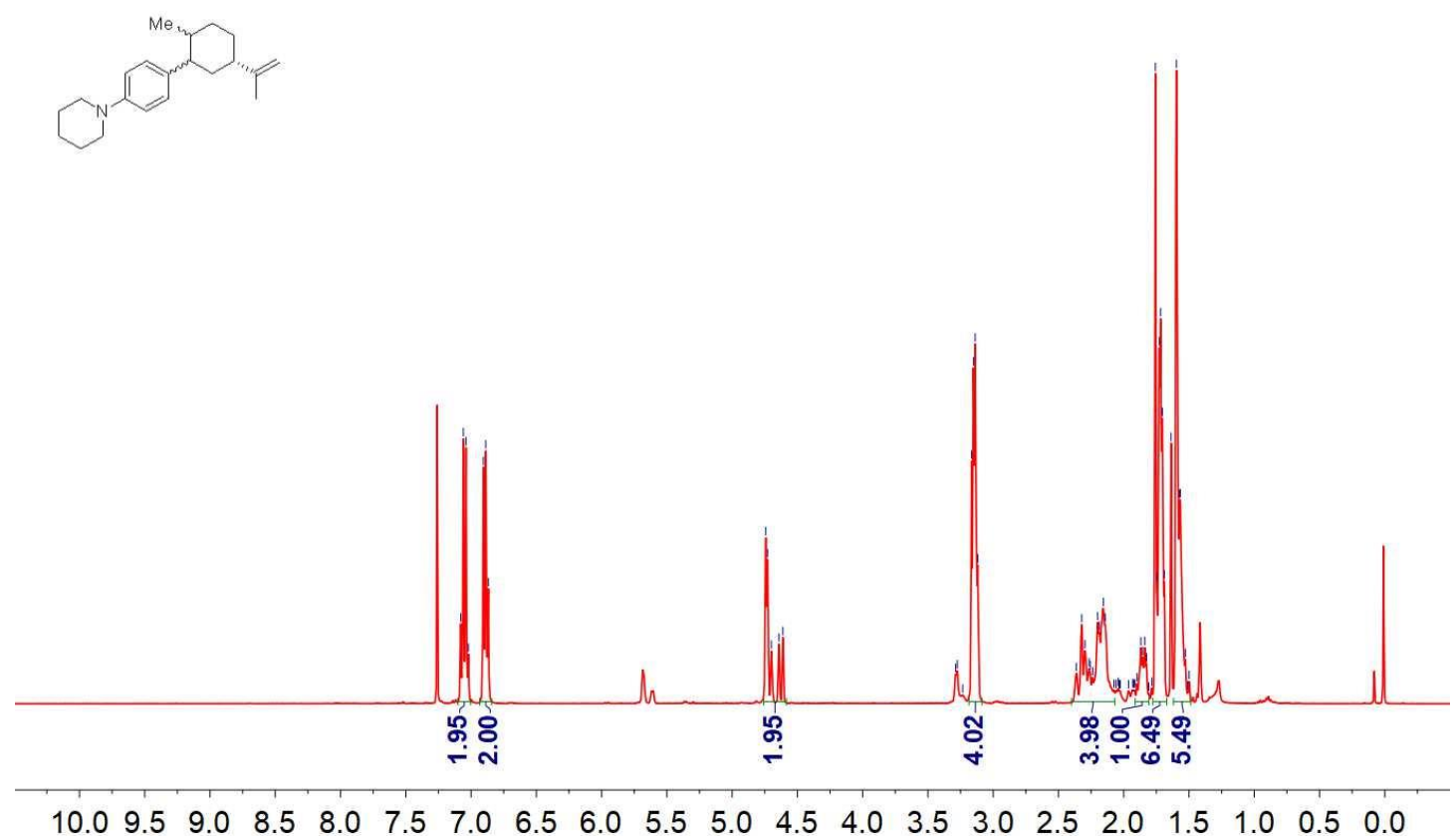


N,N-dimethyl-4-(3-methylcyclopentadecyl)aniline (4q)

(mixture of two stereomers, d.r. $=80: 20$ )

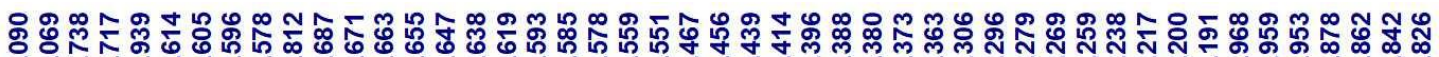
N
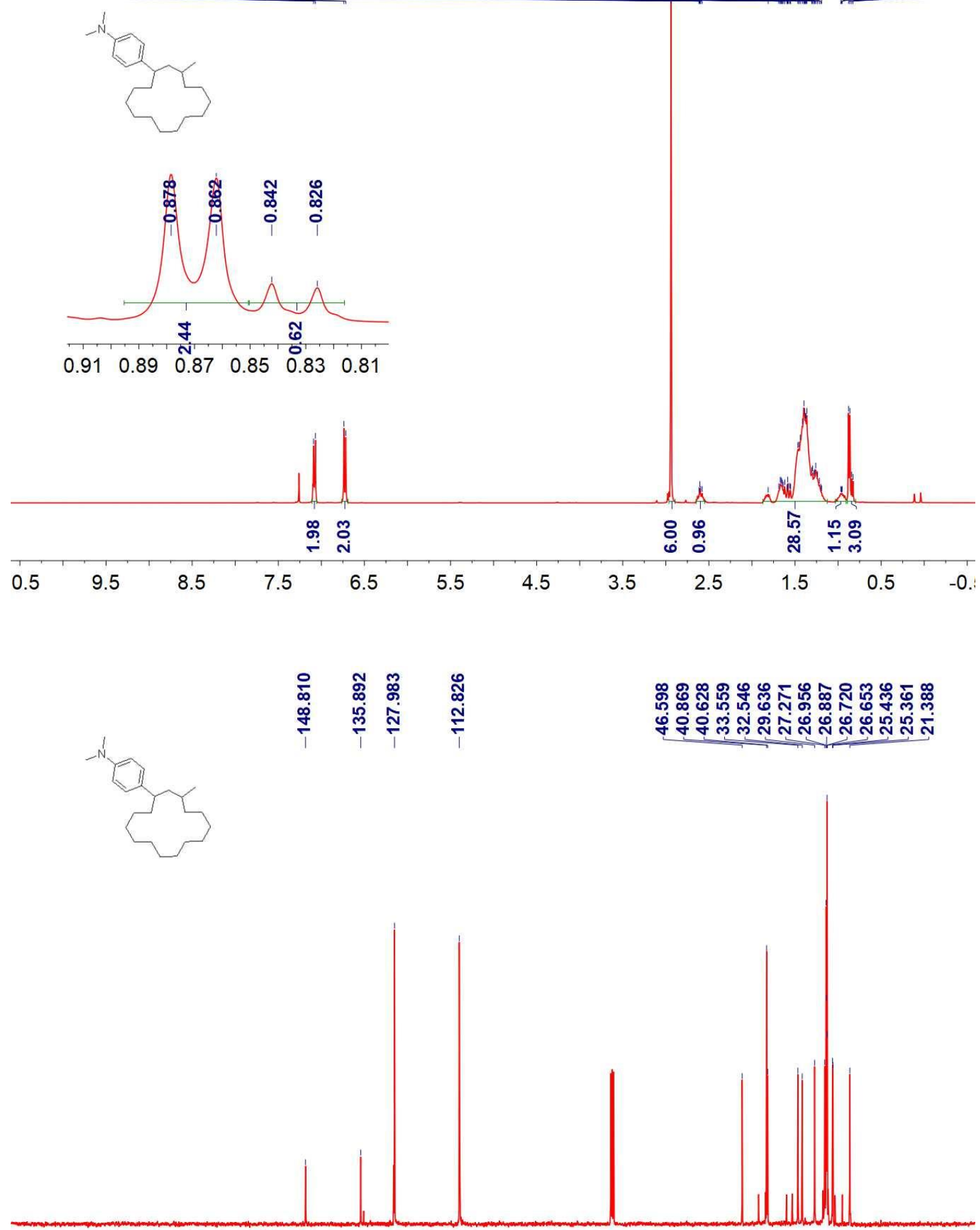

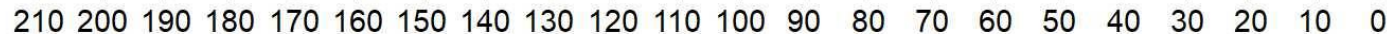


4-(4-(4-Benzhydrylpiperazin-1-yl)-1-(4-fluorophenyl)butyl)- $N, N$-dimethylaniline (4r)

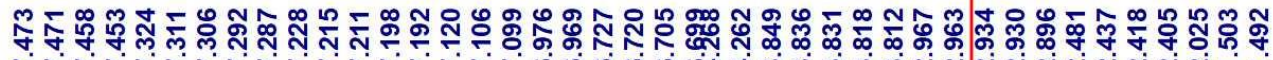

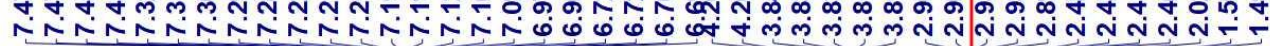

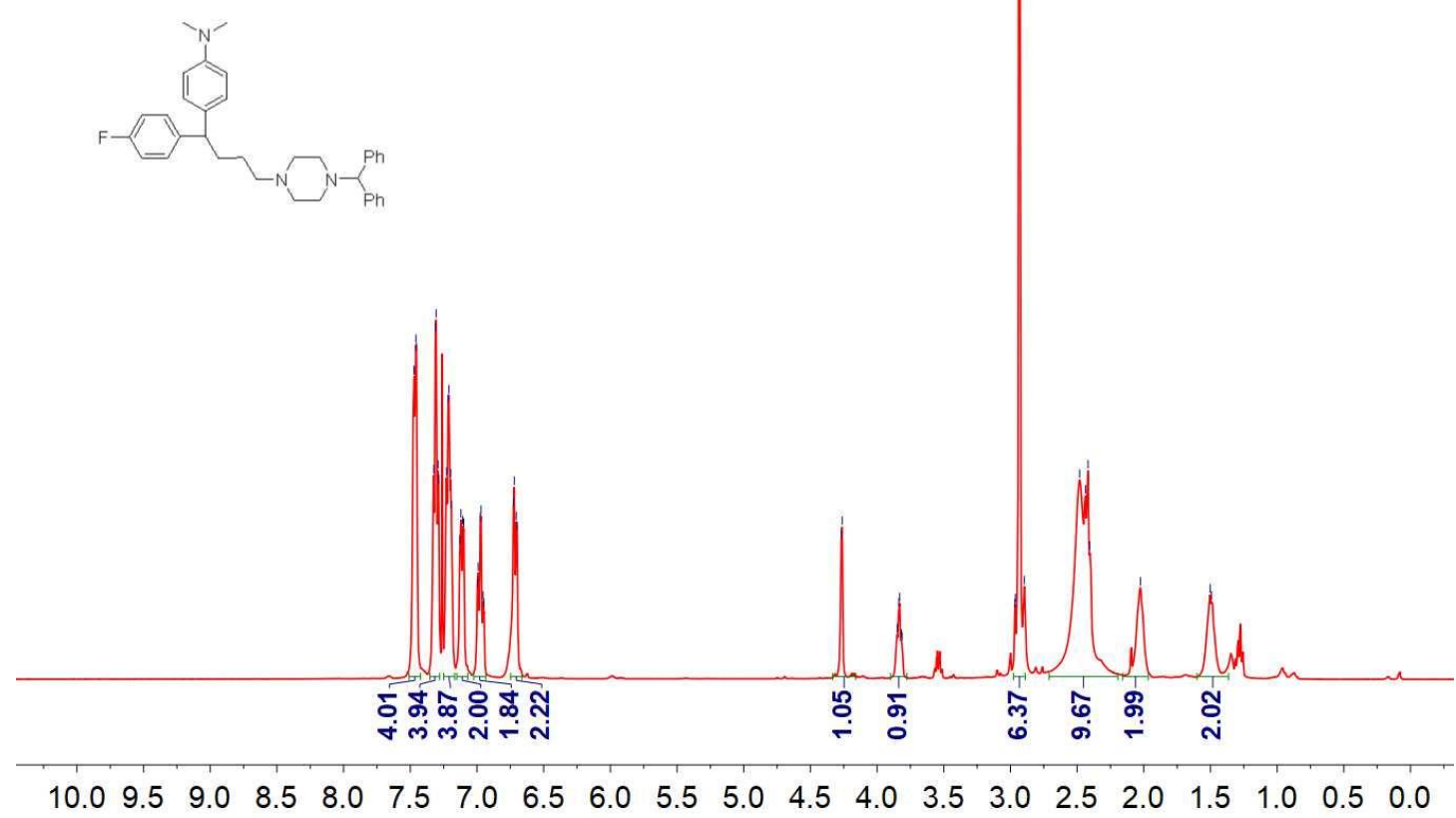

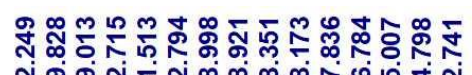

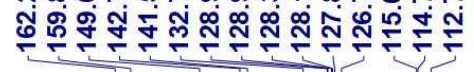
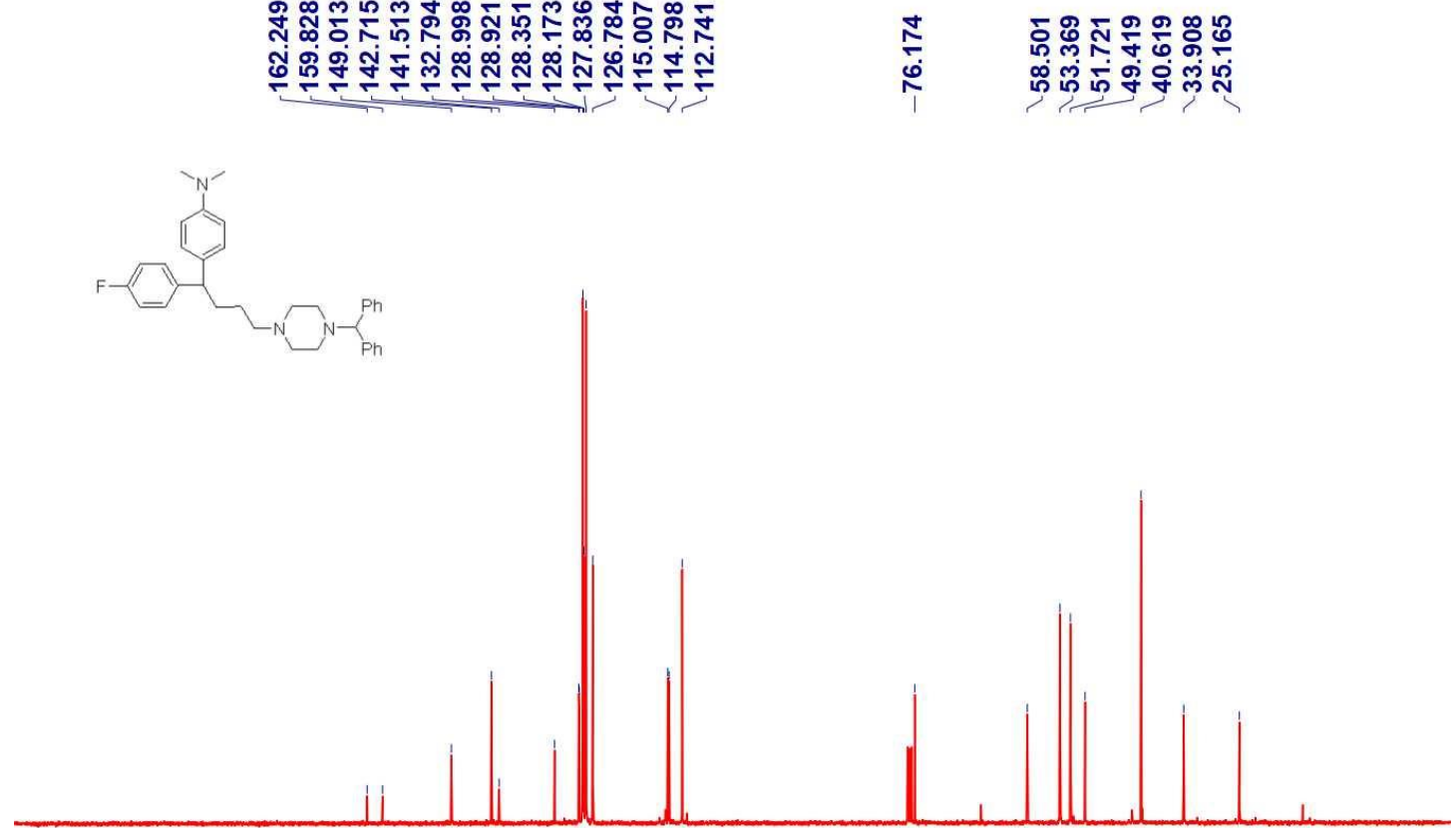

$210200190180170160150140130120110 \quad 100 \quad 90 \quad 80 \quad 70 \quad 60 \quad 50 \quad 40 \quad 30 \quad 20 \quad 10 \quad 0$ 
2-(4-(Dimethylamino)phenyl)-2-phenylethan-1-ol (4s)

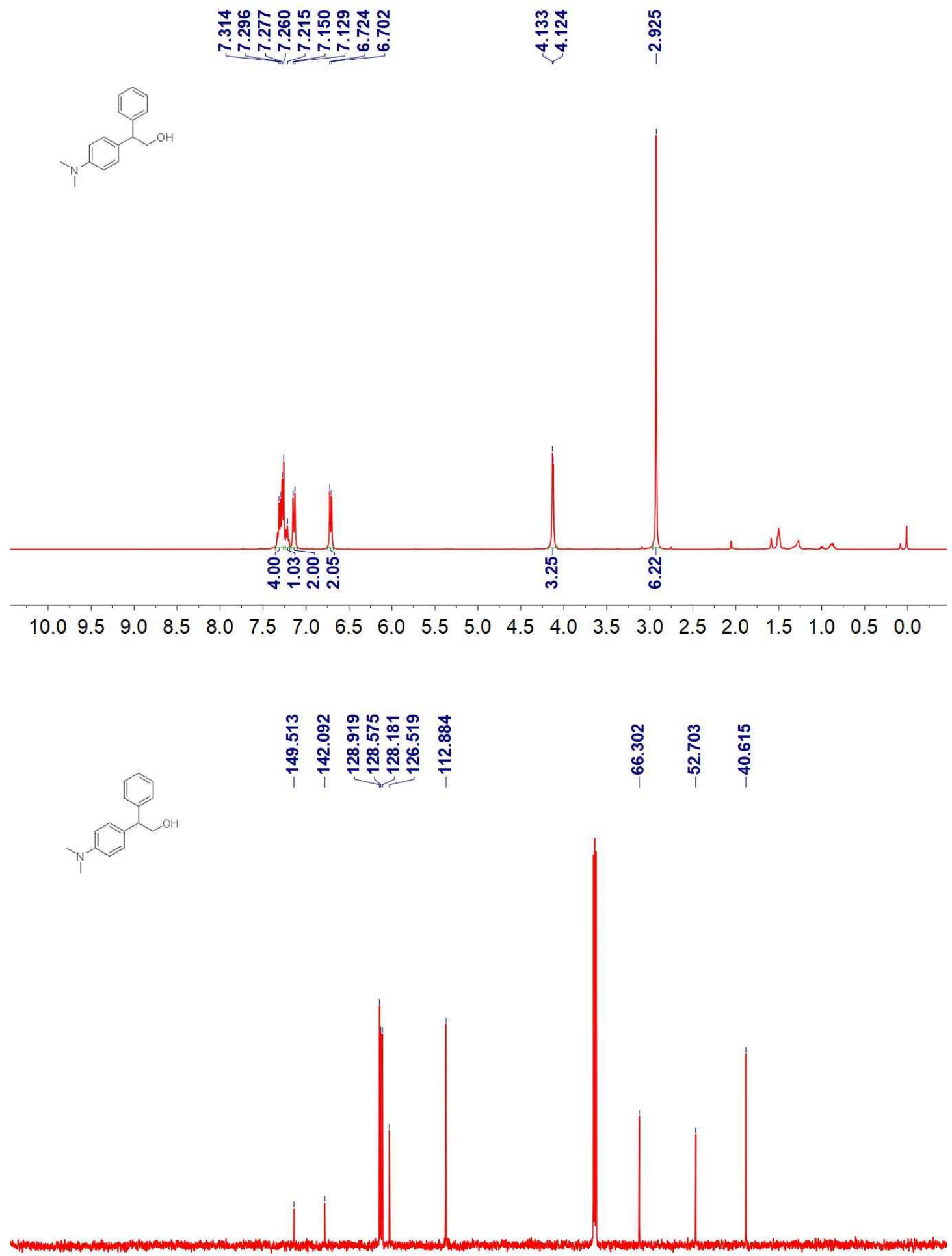

$210200190180170160150140130120110100 \quad 90 \quad 80 \quad 70 \quad 60 \quad 50 \quad 40 \quad 30 \quad 20 \quad 10 \quad 0$ 
Trans-4-(4-(dimethylamino)phenyl)cyclohexan-1-ol (4t)

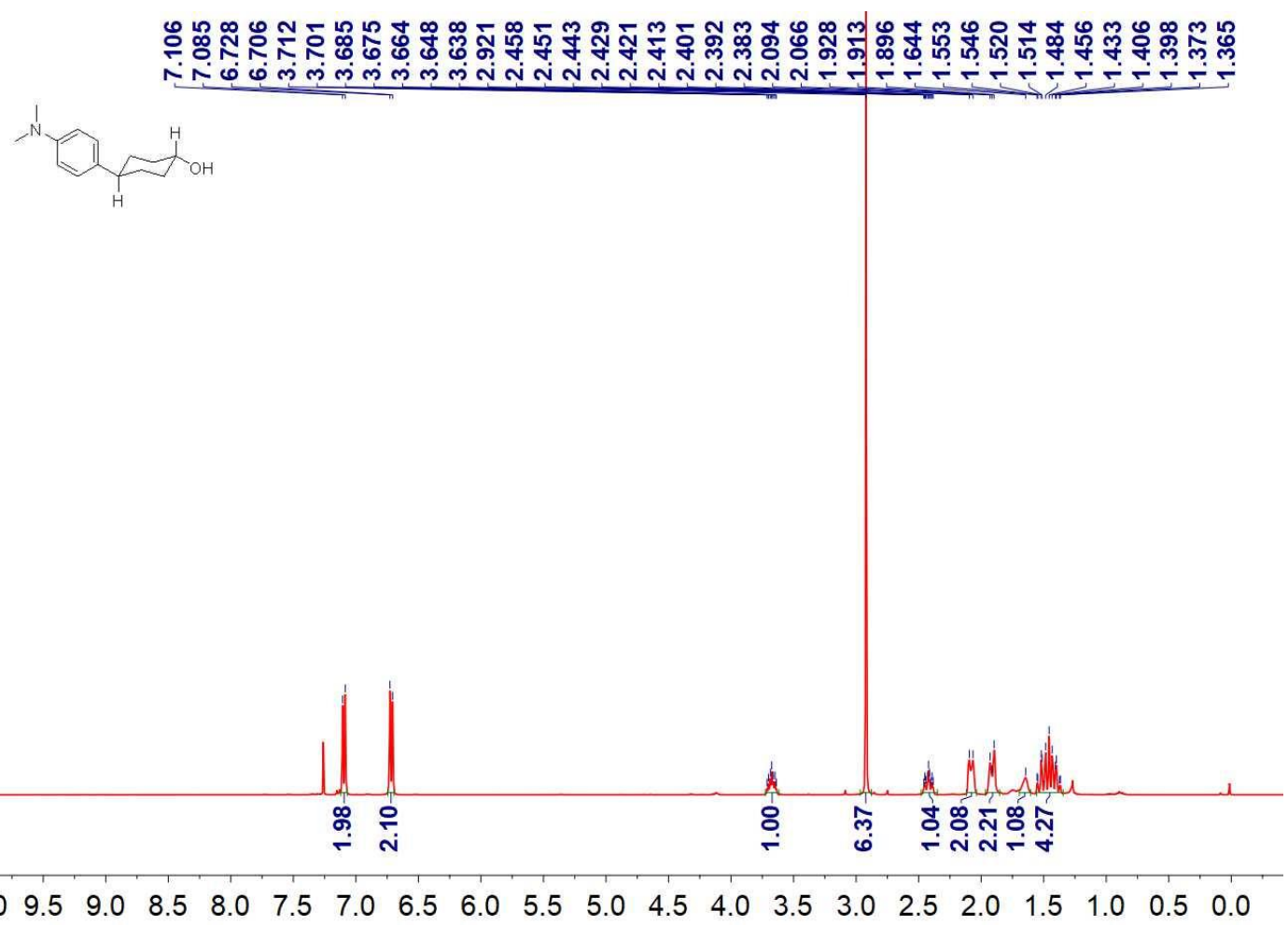

$\begin{array}{lllllllllllllllllllll}10.0 & 9.5 & 9.0 & 8.5 & 8.0 & 7.5 & 7.0 & 6.5 & 6.0 & 5.5 & 5.0 & 4.5 & 4.0 & 3.5 & 3.0 & 2.5 & 2.0 & 1.5 & 1.0 & 0.5 & 0.0\end{array}$
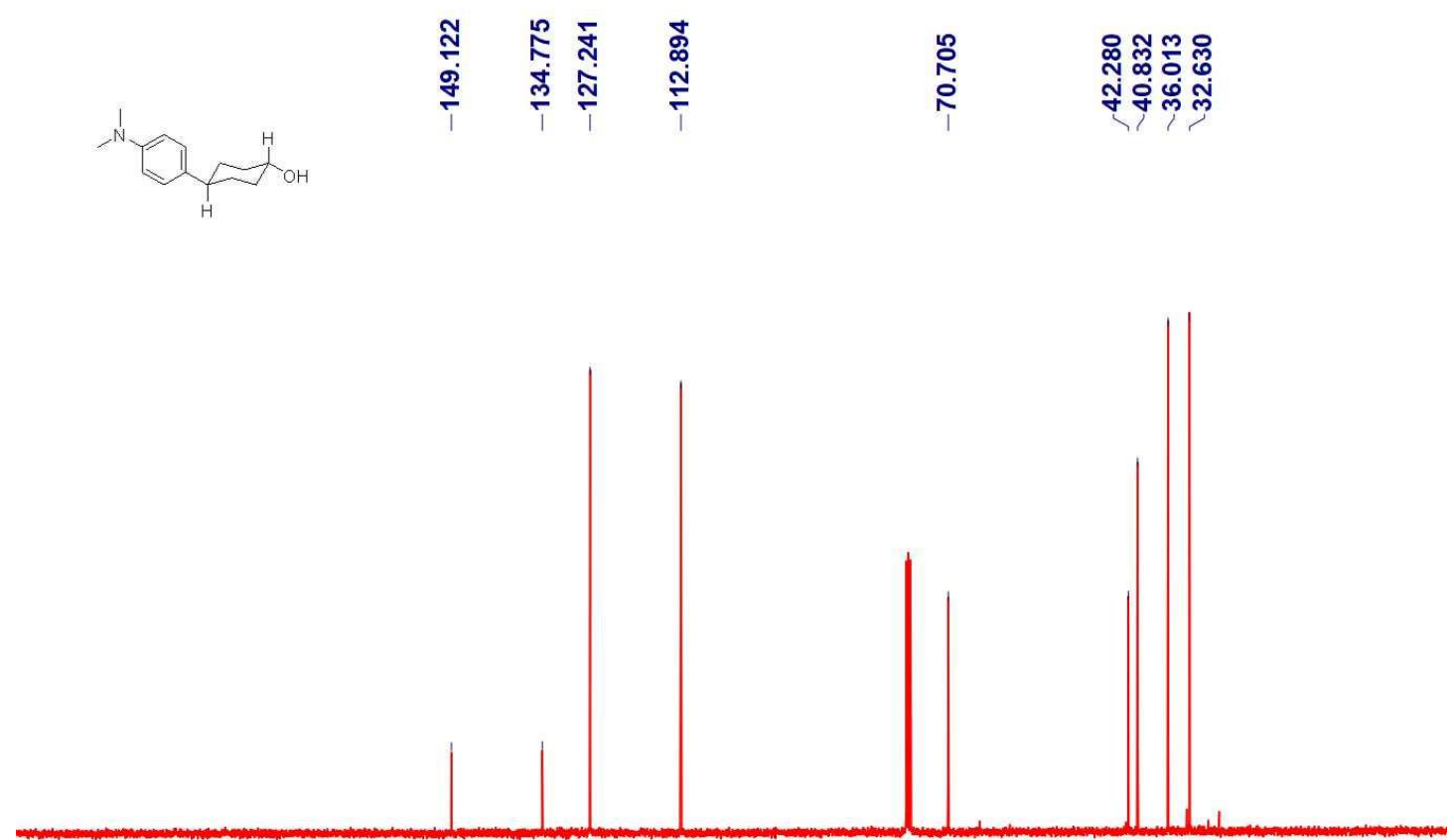

$210200190180170160150140130120110100 \quad 90 \quad 80 \quad 70 \quad 60 \begin{array}{llllllll}40 & 30 & 20 & 10 & 0\end{array}$ 
Cis-4-(4-(dimethylamino)phenyl)cyclohexan-1-ol (4t')

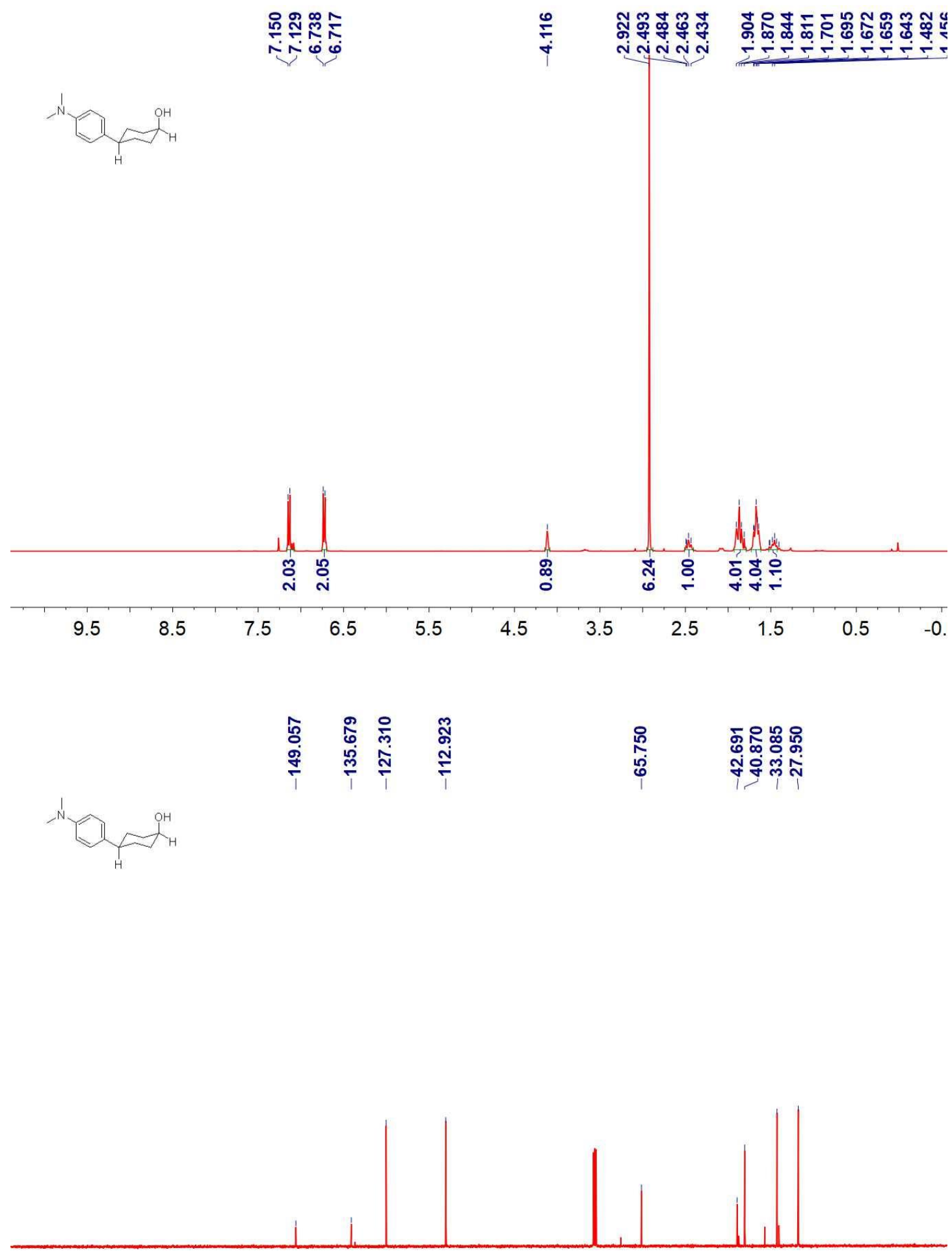

$210200190180170160150140130120110100 \quad 90 \quad 80 \quad 70 \quad 60 \quad 50 \quad 40 \quad 30 \quad 20 \quad 10 \quad 0$ 
3-(4-(Dimethylamino)phenyl)butan-2-one (4u)
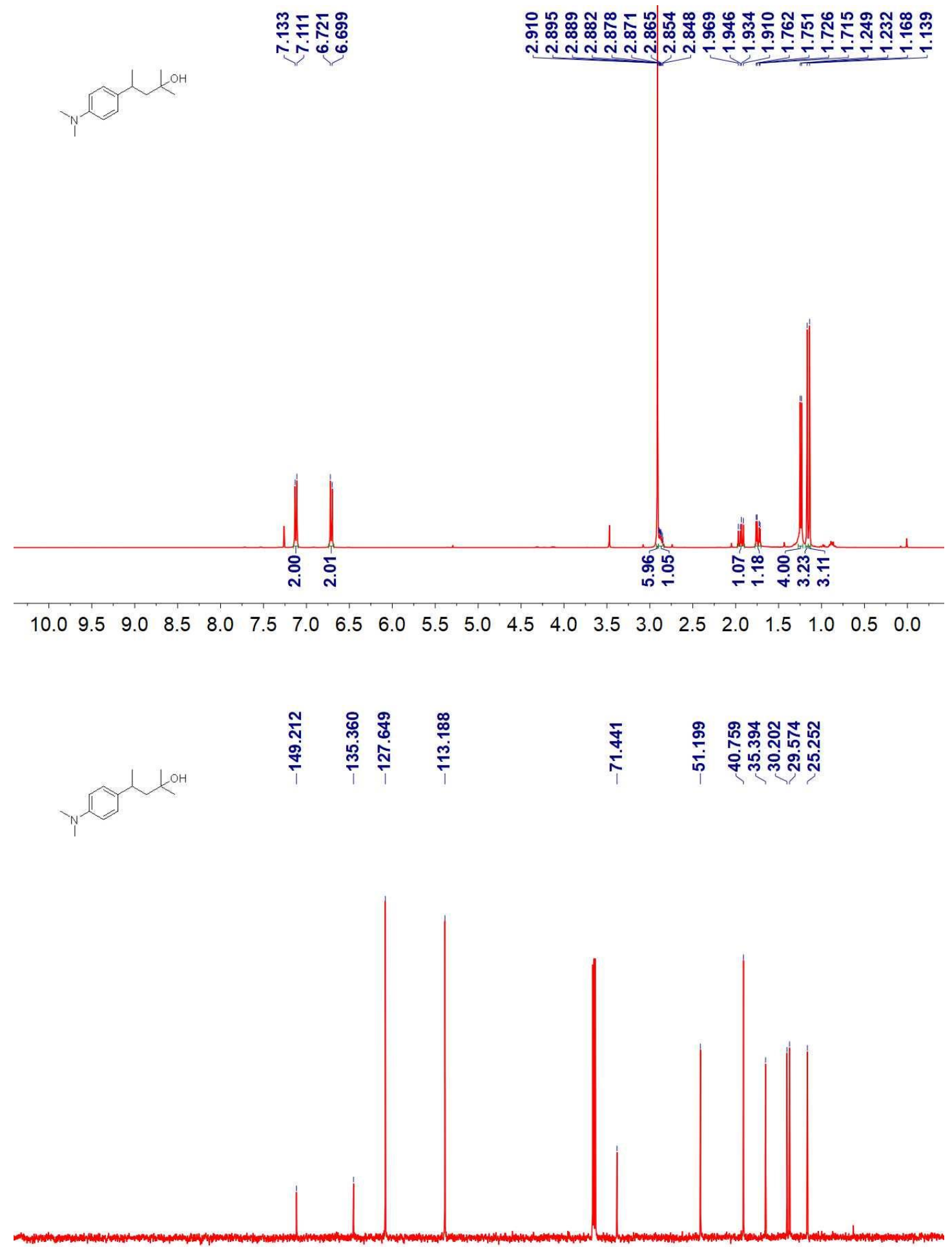

$210200190180170160150140130120110100 \quad 90 \quad 80 \quad 70 \quad 60 \quad 50 \quad 40 \quad 30 \quad 20 \quad 10 \quad 0$ 
3-(4-(Dimethylamino)phenyl)butan-2-one (4v)

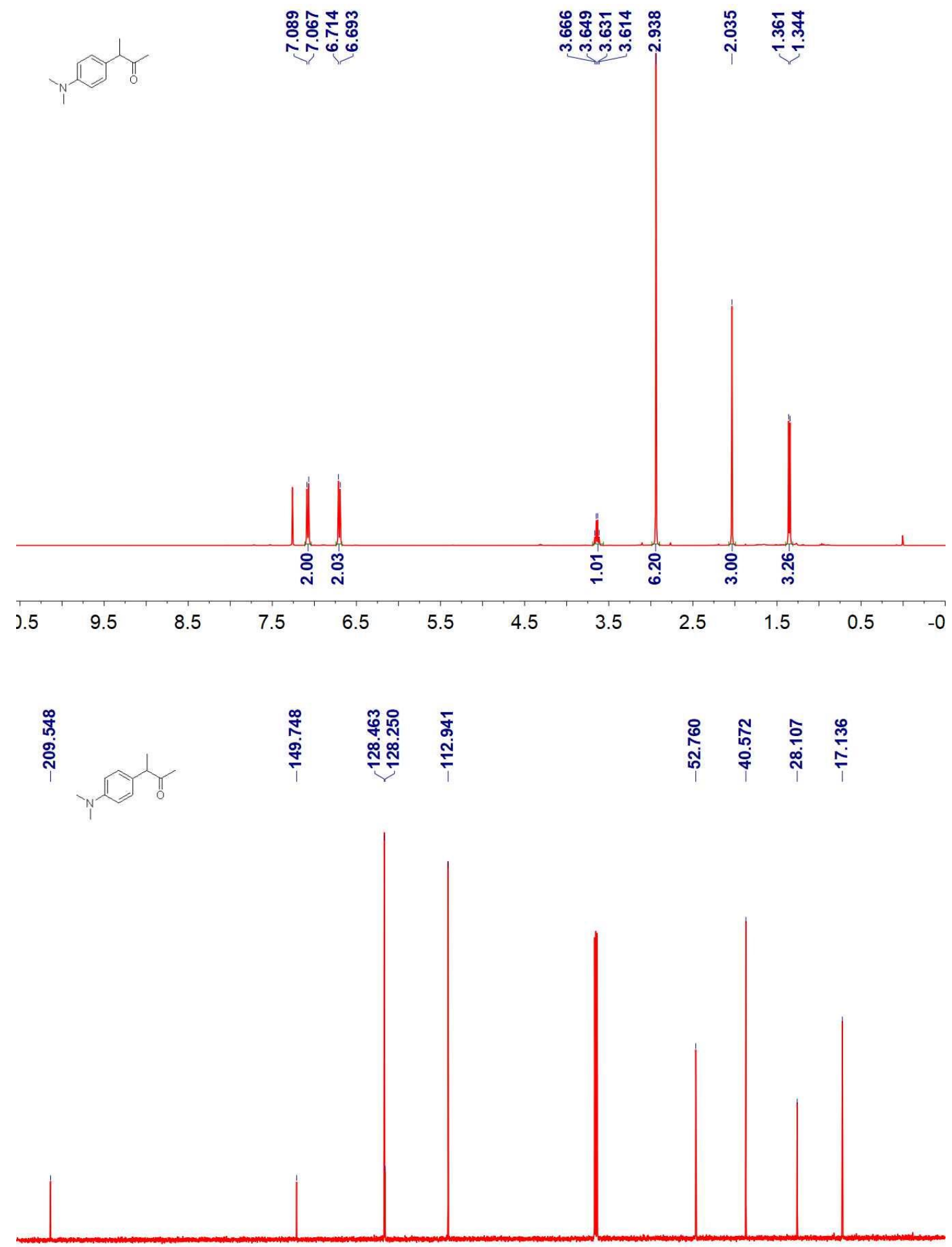

$210200190180170160150140130120110100 \quad 90 \quad 80 \quad 70 \quad 60 \quad 50 \quad 40 \quad 30 \quad 20 \quad 10 \quad 0$ 
(3S,8S,9S,10R,13S,14S,17R)-10,13-dimethyl-17-((S)-1-(4-(piperidin-1-yl)phenyl)ethyl) $-2,3,4,7,8,9,10,11,12,13,14,15,16,17$-tetradecahydro- $1 H$-cyclopenta[a]phenanthren-3ol (8a)

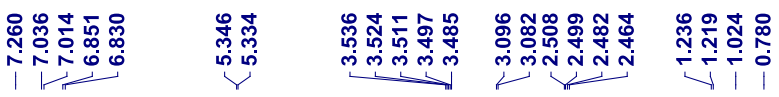
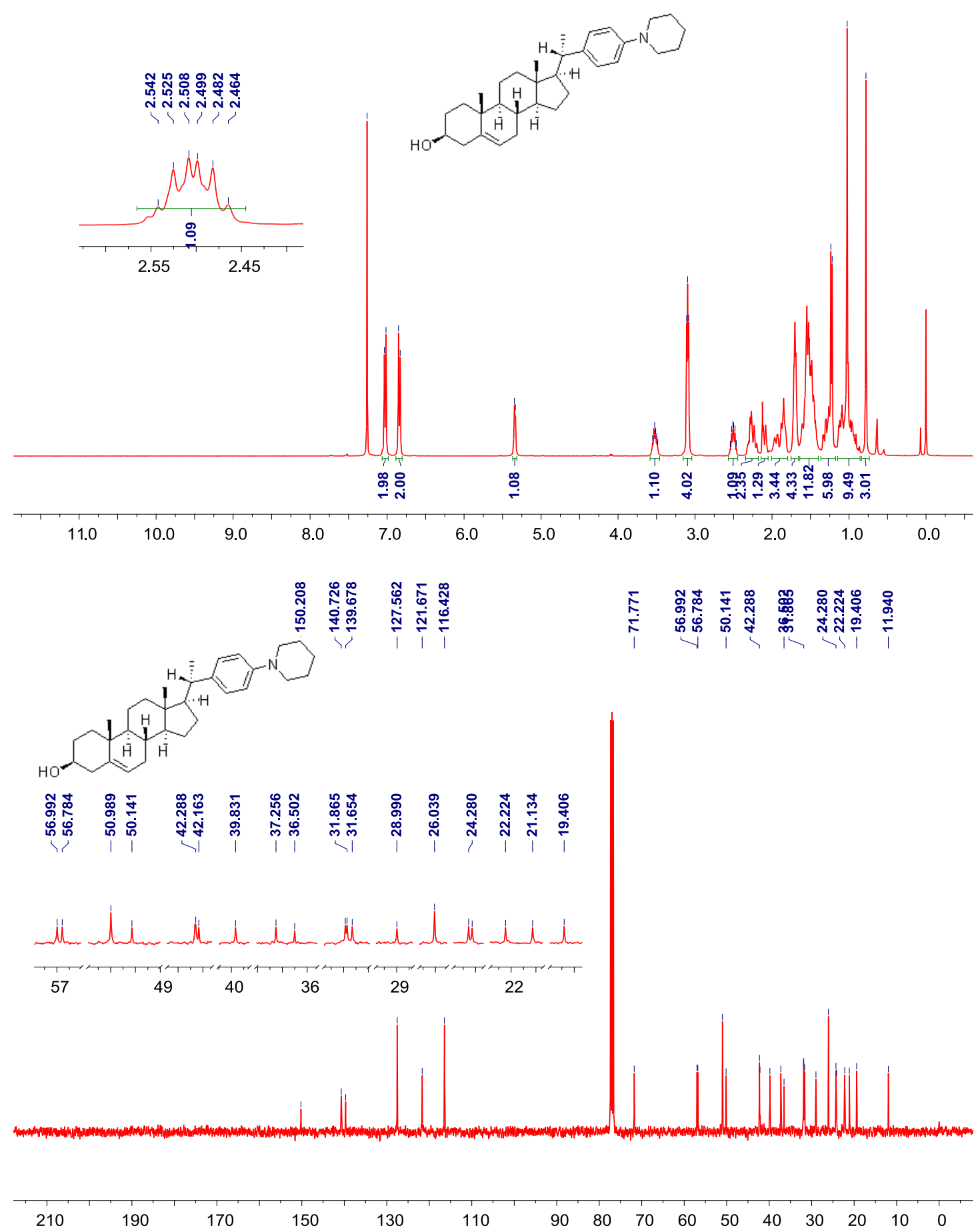


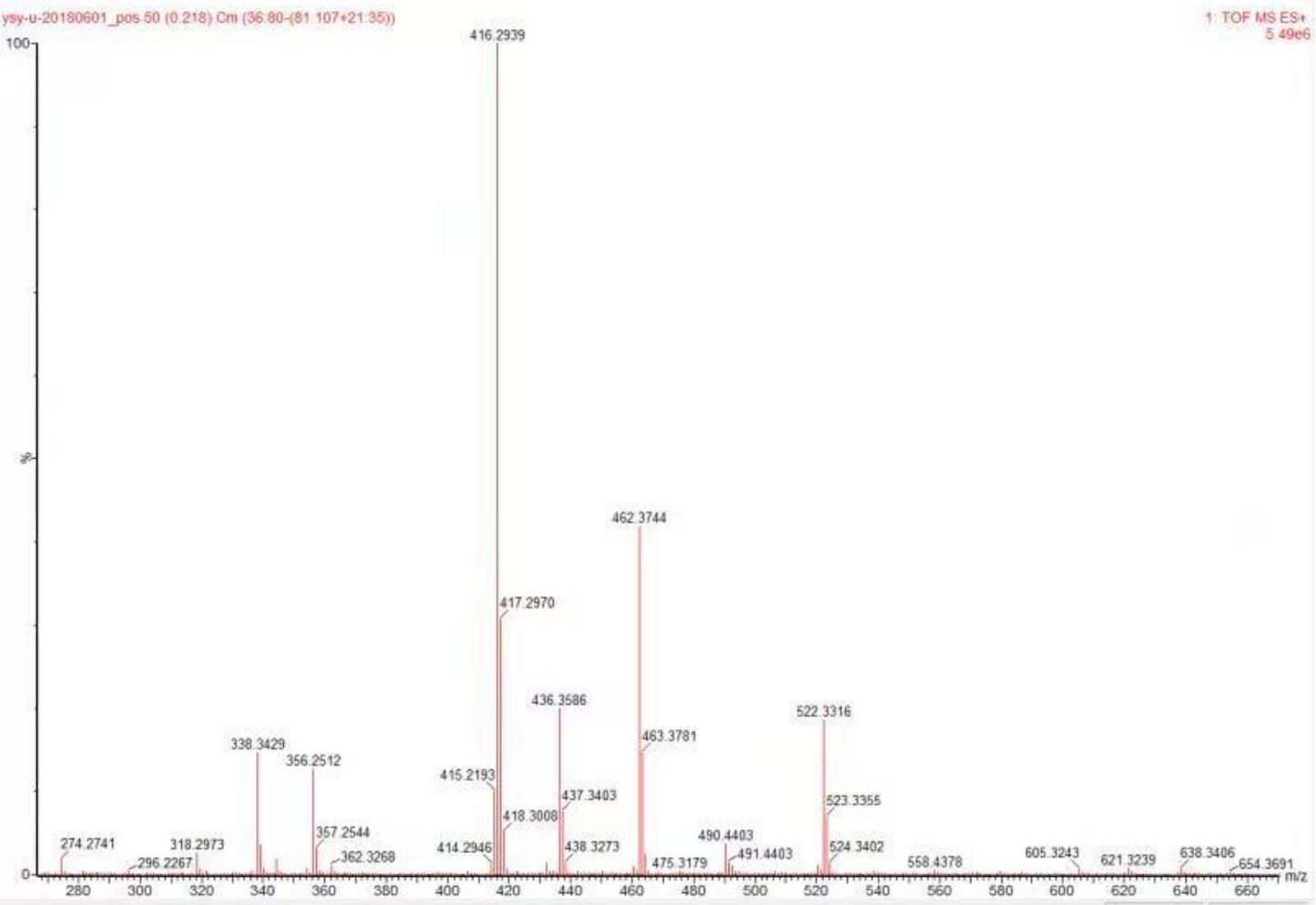


(8S,9S,13S,14S,17S)-13-Methyl-17-(4-(piperidin-1-yl)phenyl)-7,8,9,11,12,13,14,15,16, 17-decahydro-6H-cyclopenta[a]phenanthren-3-ol (8b)

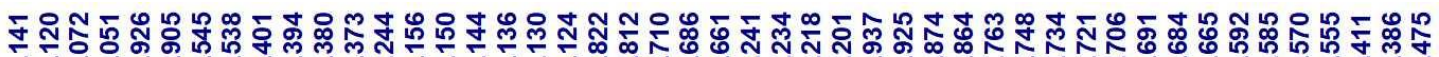

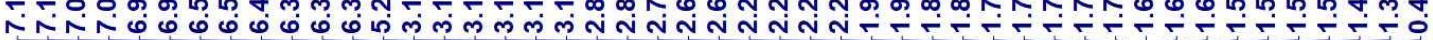

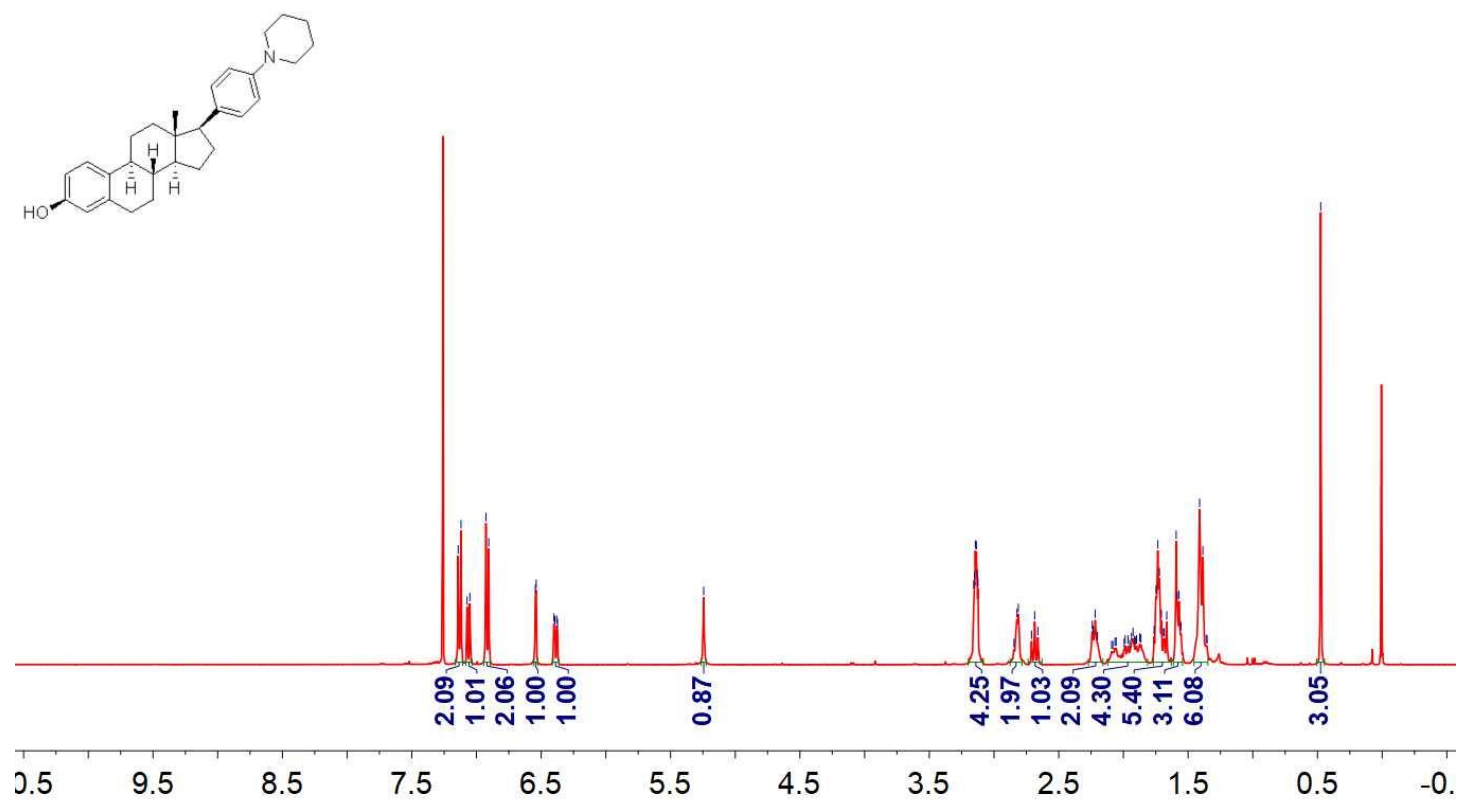

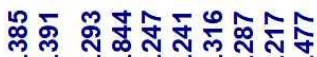

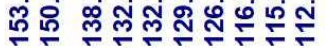

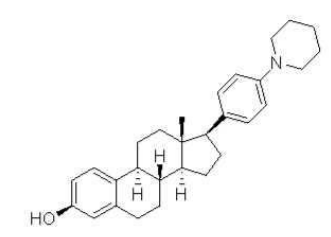

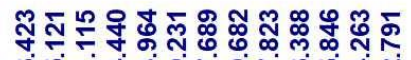

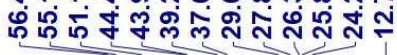

$210200190180170160150140130120110 \quad 100 \quad 90 \quad 80 \quad 70 \quad 60 \quad 50 \quad 40 \quad 30 \quad 20 \quad 10 \quad 0$ 
(3S,8R,9S,10S,13S,14S,17S)-10,13-Dimethyl-17-(4-(piperidin-1-yl)phenyl)hexadecahy dro-1H-cyclopenta[a]phenanthren-3-ol (8c)
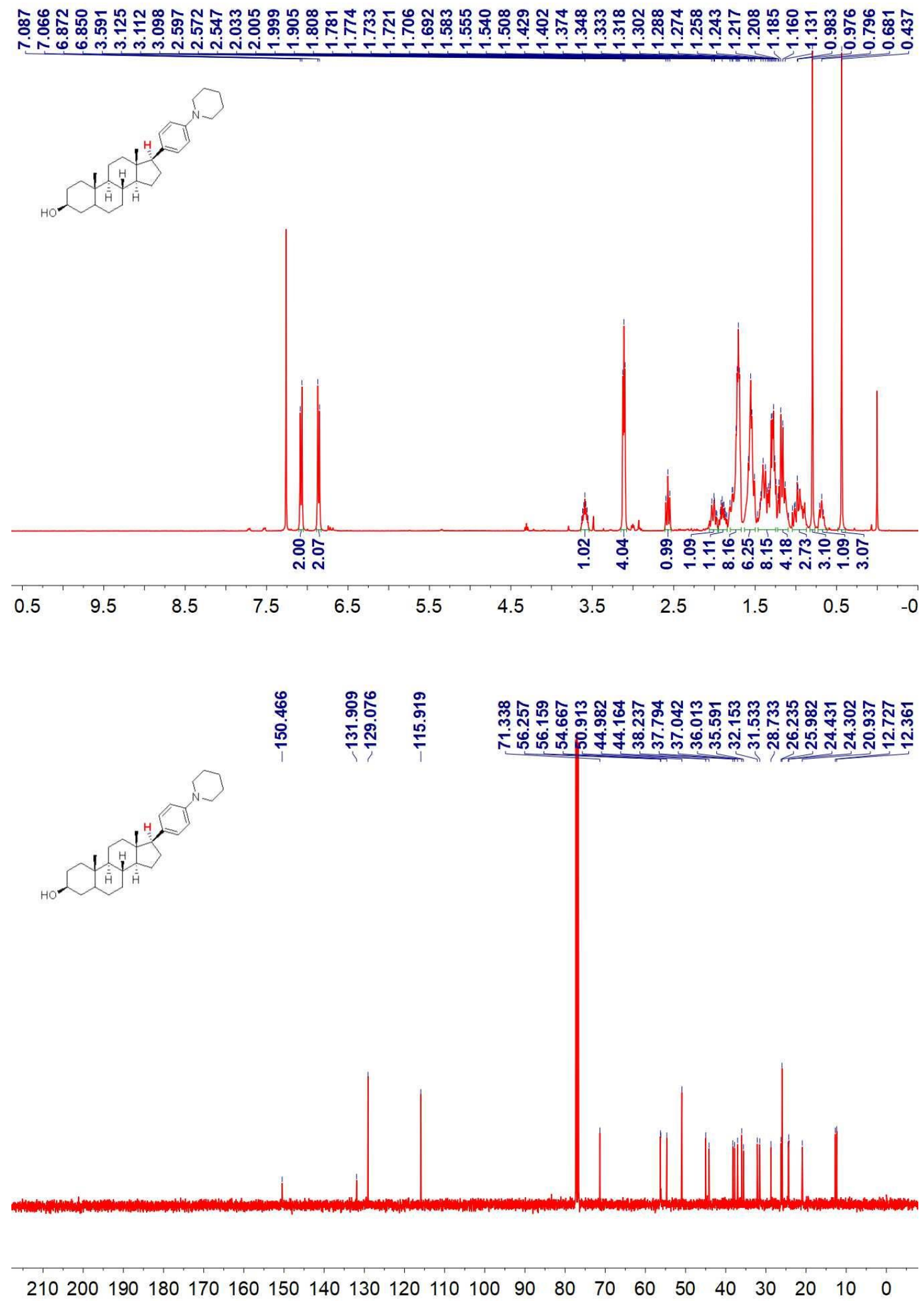
(8S,9S,10R,13R,14S,17R)-3-(4-(dimethylamino)phenyl)-10,13-dimethyl-17-((R)-6-met hylheptan-2-yl)-2,3,4,7,8,9,10,11,12,13,14,15,16,17-tetradecahydro-1H-cyclopenta[a]p henanthren-3-ol (10)

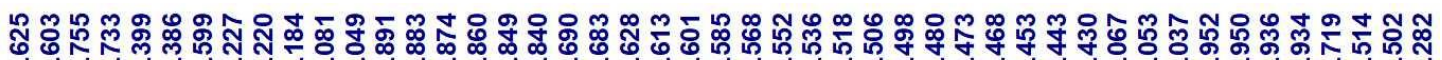
숩
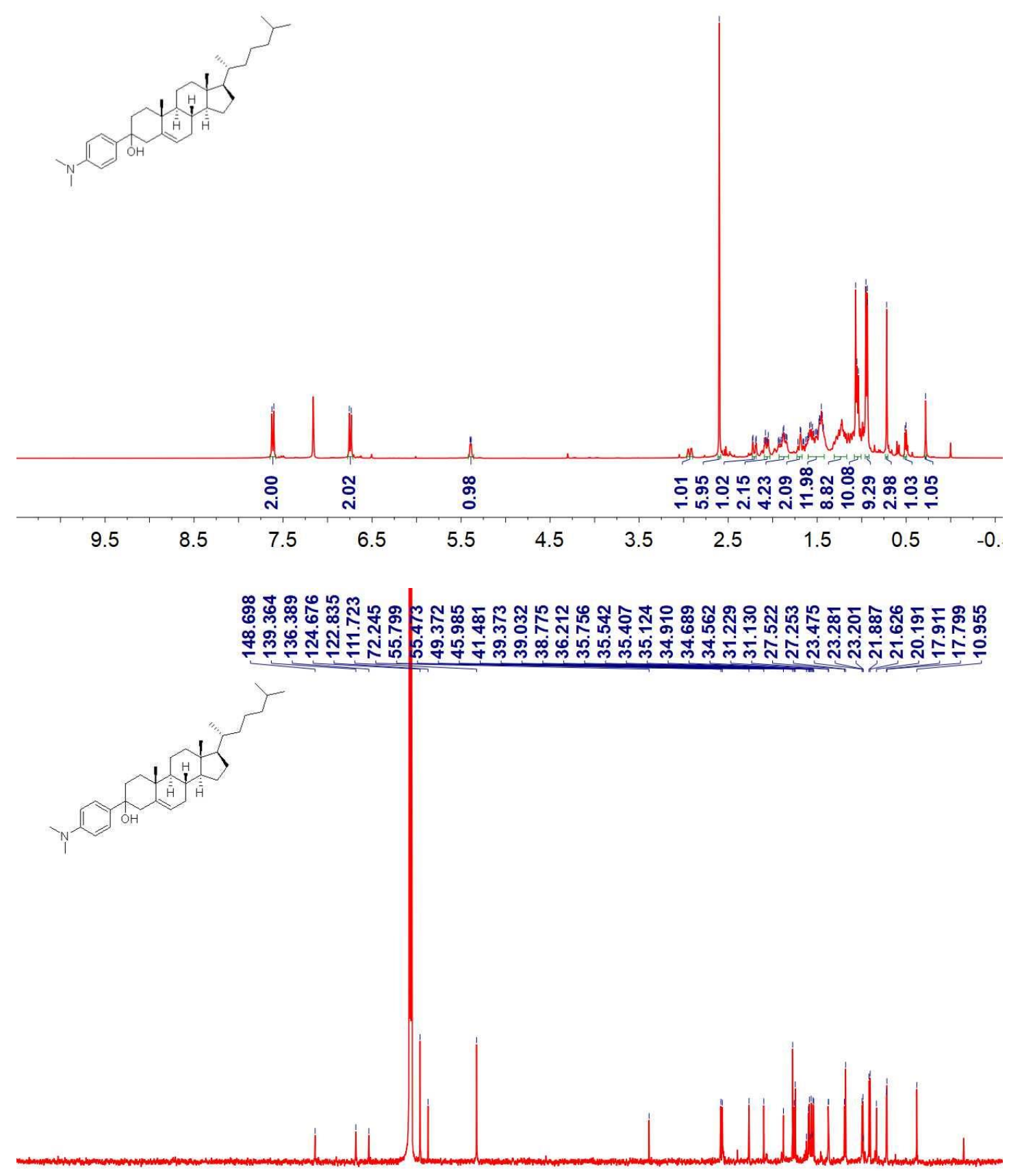

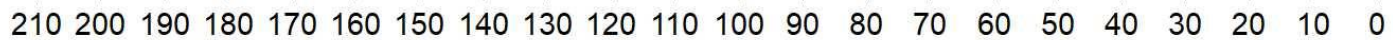


4-((8S,9S,10R,13R,14S,17R)-10,13-Dimethyl-17-((R)-6-methylheptan-2-yl)-2,3,4,7,8,9, $10,11,12,13,14,15,16,17$-tetradecahydro-1 $H$-cyclopenta[a]phenanthren-3-yl)- $N, N$-dim ethylaniline (11)

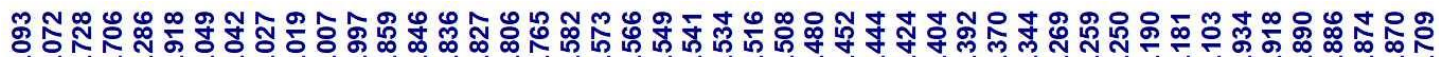
ヘ

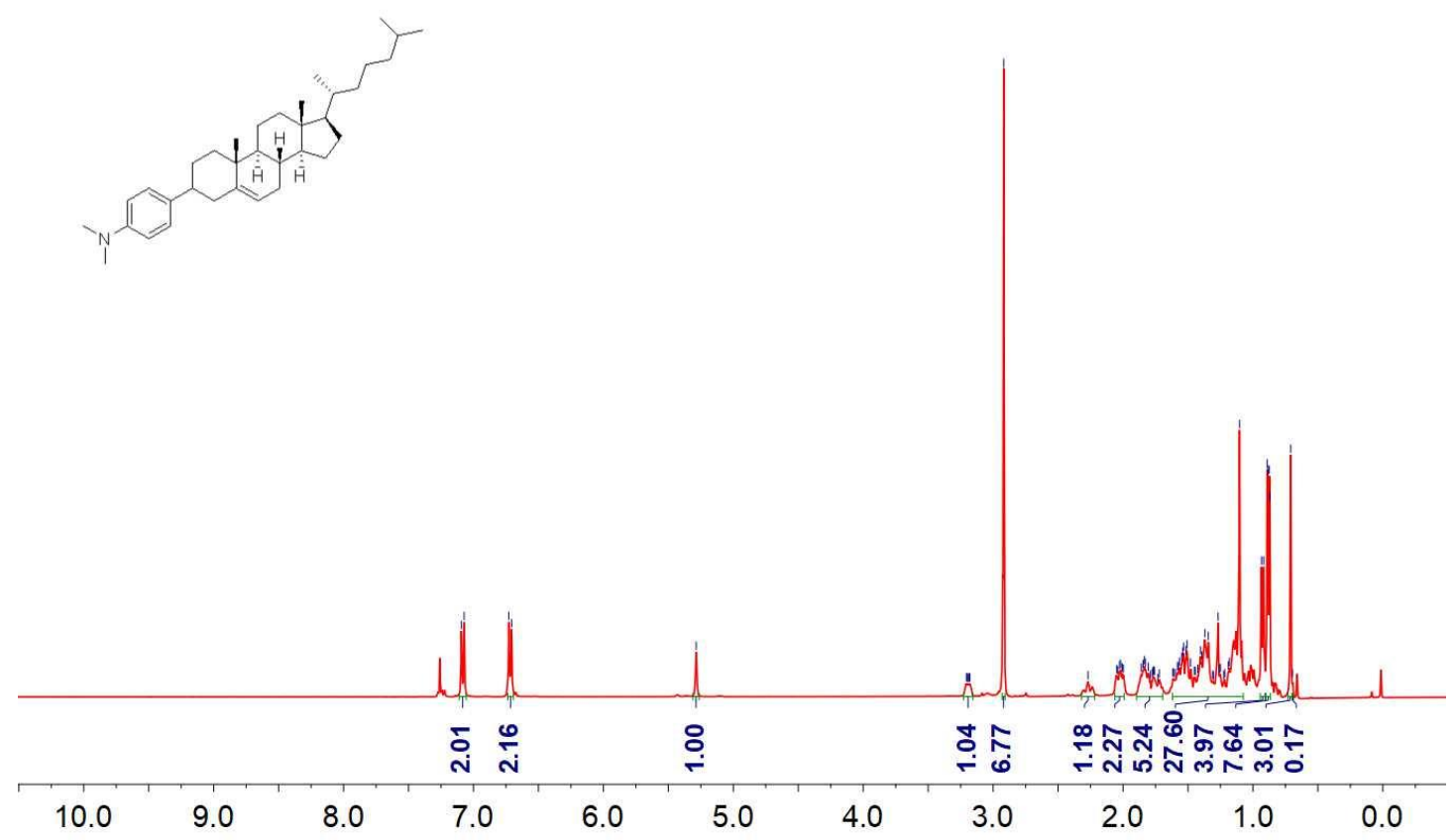

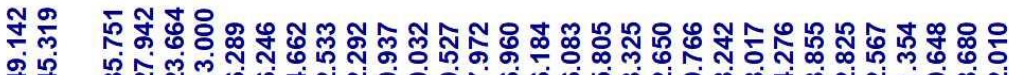

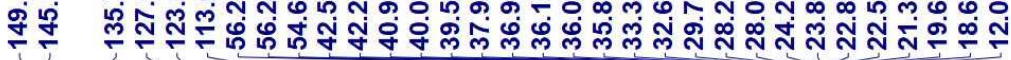

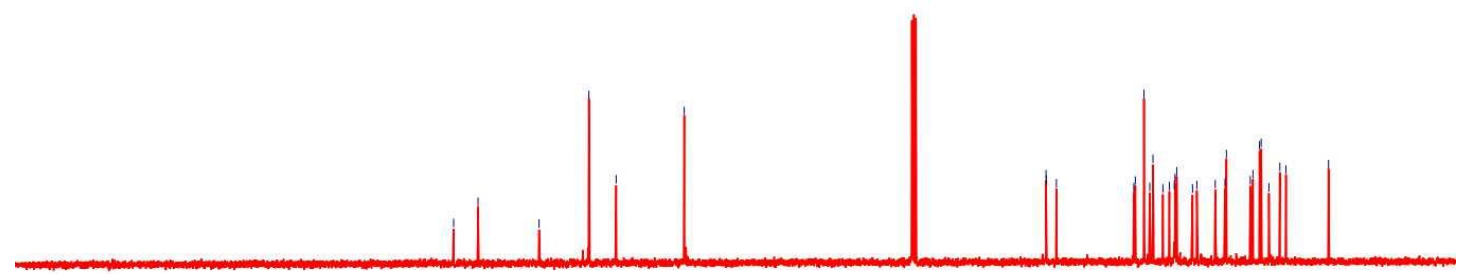

$210200190180170160150140130120110 \quad 100 \quad 90 \quad 80 \quad 70 \quad 60 \quad 50 \quad 40 \quad 30 \quad 20 \quad 10 \quad 0$ 
9-([1,1'-Biphenyl]-4-yl)-9H-xanthene (14)

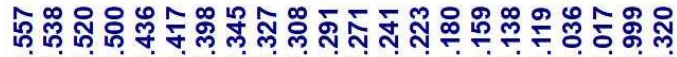

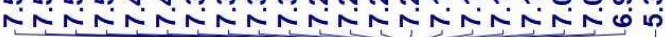
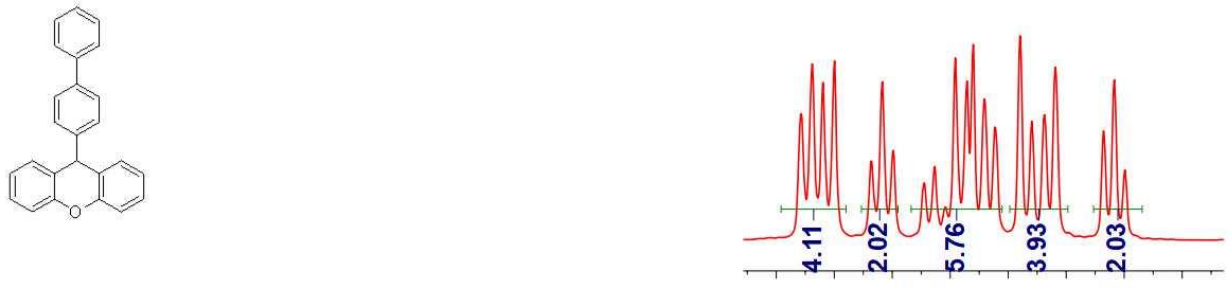

$\begin{array}{lllllll}7.6 & 7.5 & 7.4 & 7.3 & 7.2 & 7.17 .0 & 6.9\end{array}$

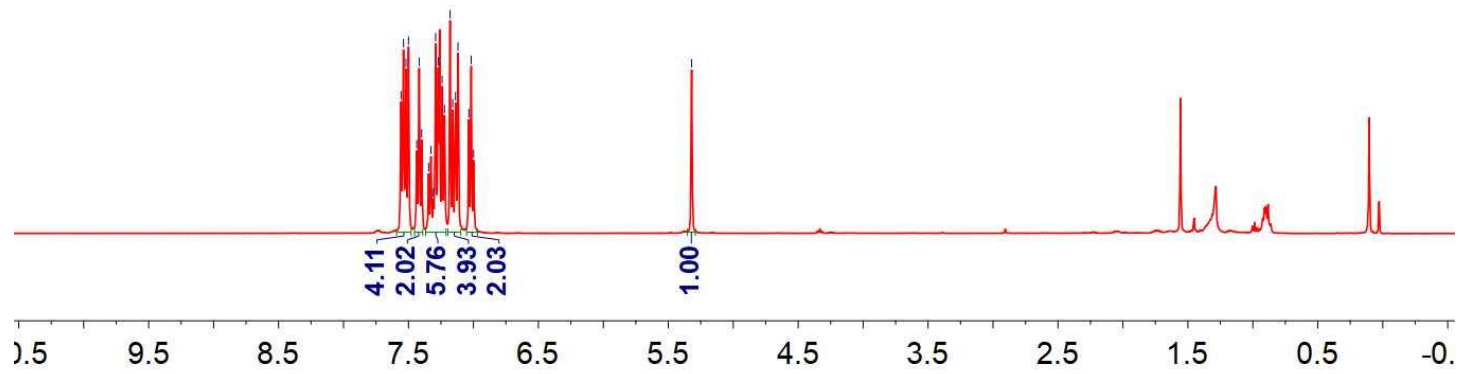

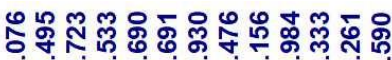

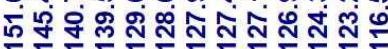

ষั

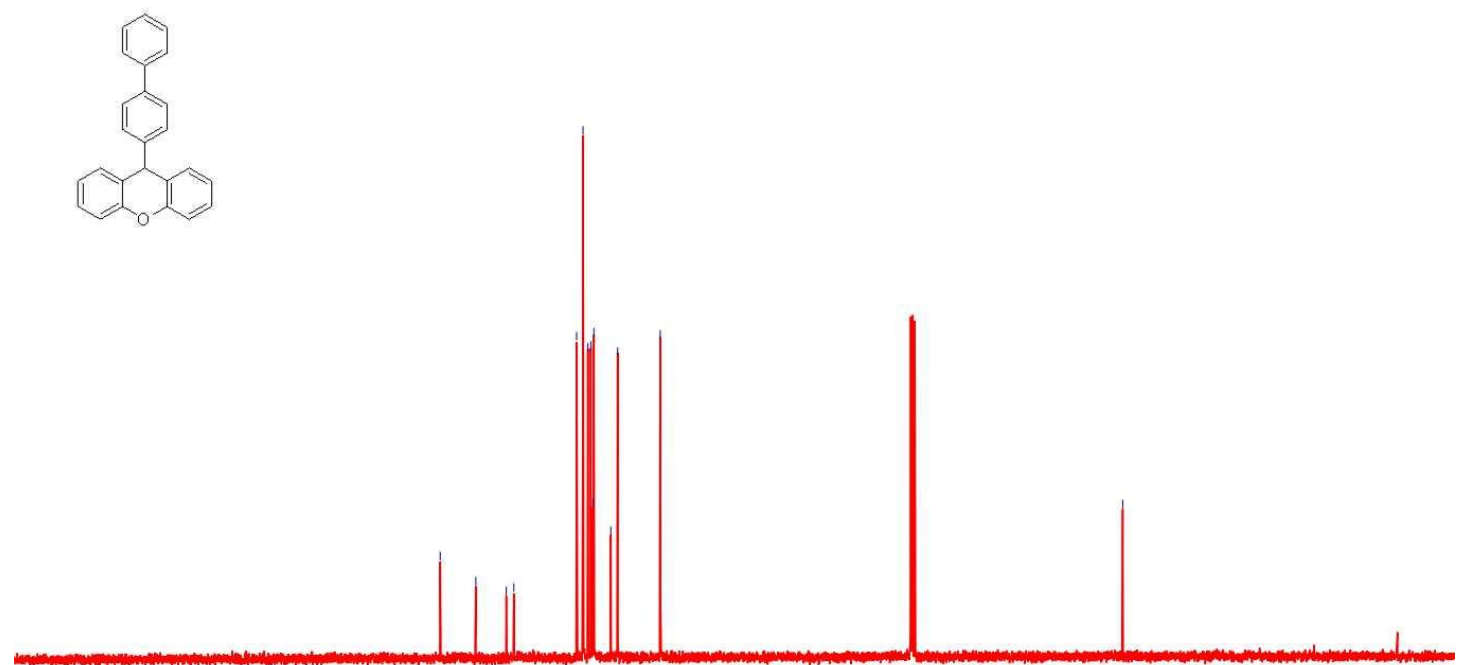

$210200190180170160150140130120110100 \quad 90 \quad 80 \quad 70 \quad 60 \quad 50 \quad 40 \quad 30 \quad 20 \quad 10 \quad 0$ 


\section{9-(4-(Dibenzo[b,d]furan-4-yl)phenyl)-9H-xanthene (16)}

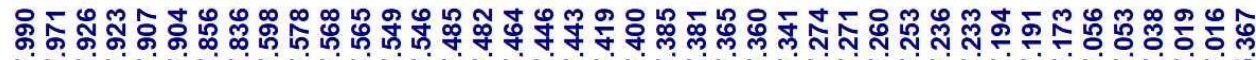

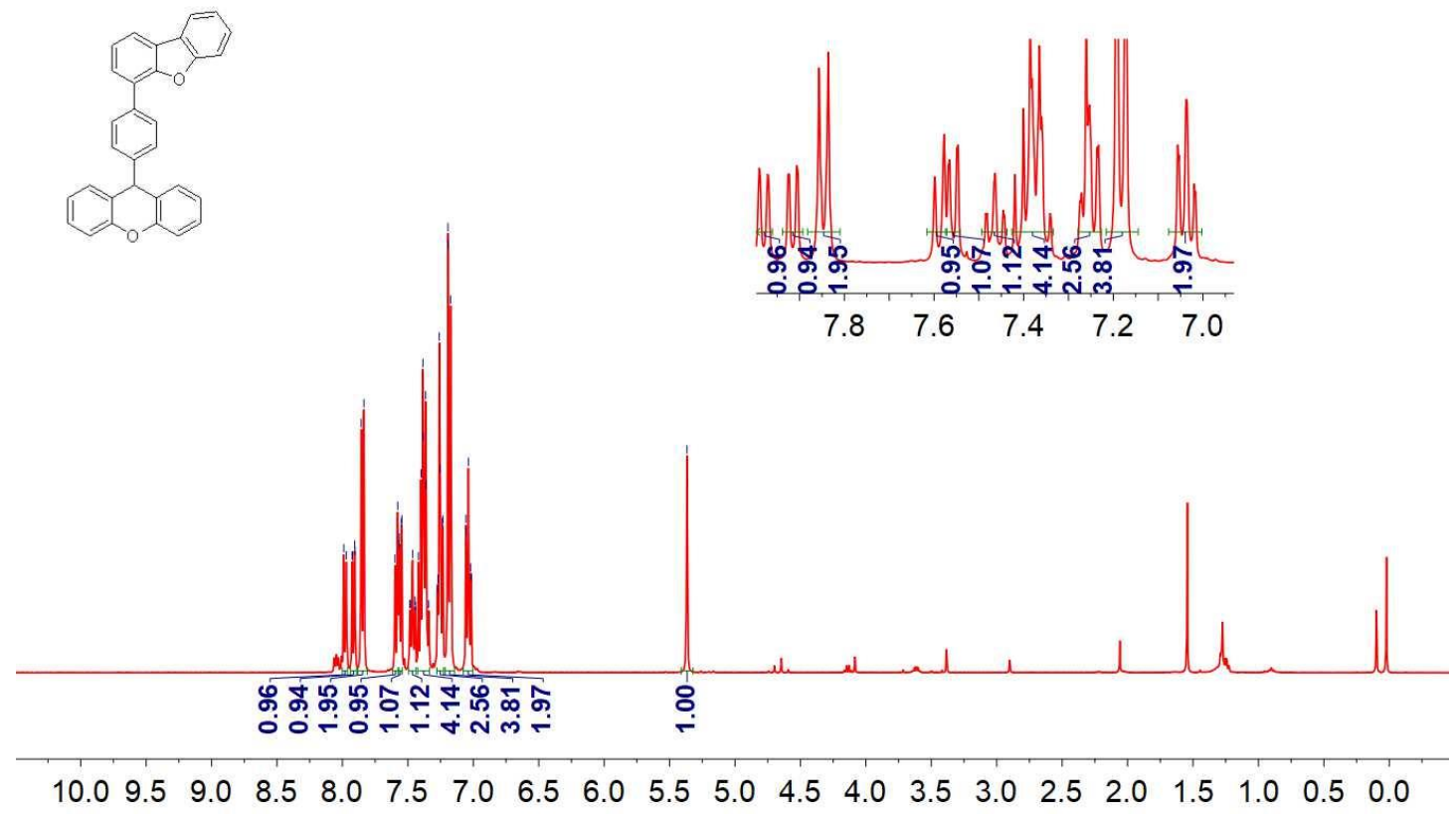

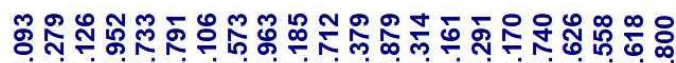

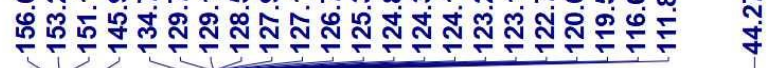

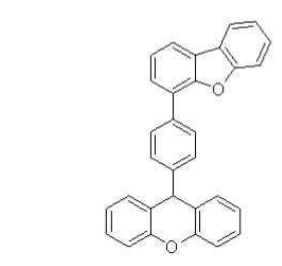

ำ

$210200190180170160150140130120110100 \quad 90 \quad 80 \quad 70 \quad 60 \quad 50 \quad 40 \quad 30 \quad 20 \quad 10 \quad 0$ 
N,N-dimethyl-2',3',4',5' -tetrahydro-[1,1'-biphenyl]-4-amine (5)

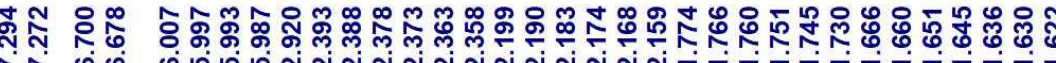

Nั
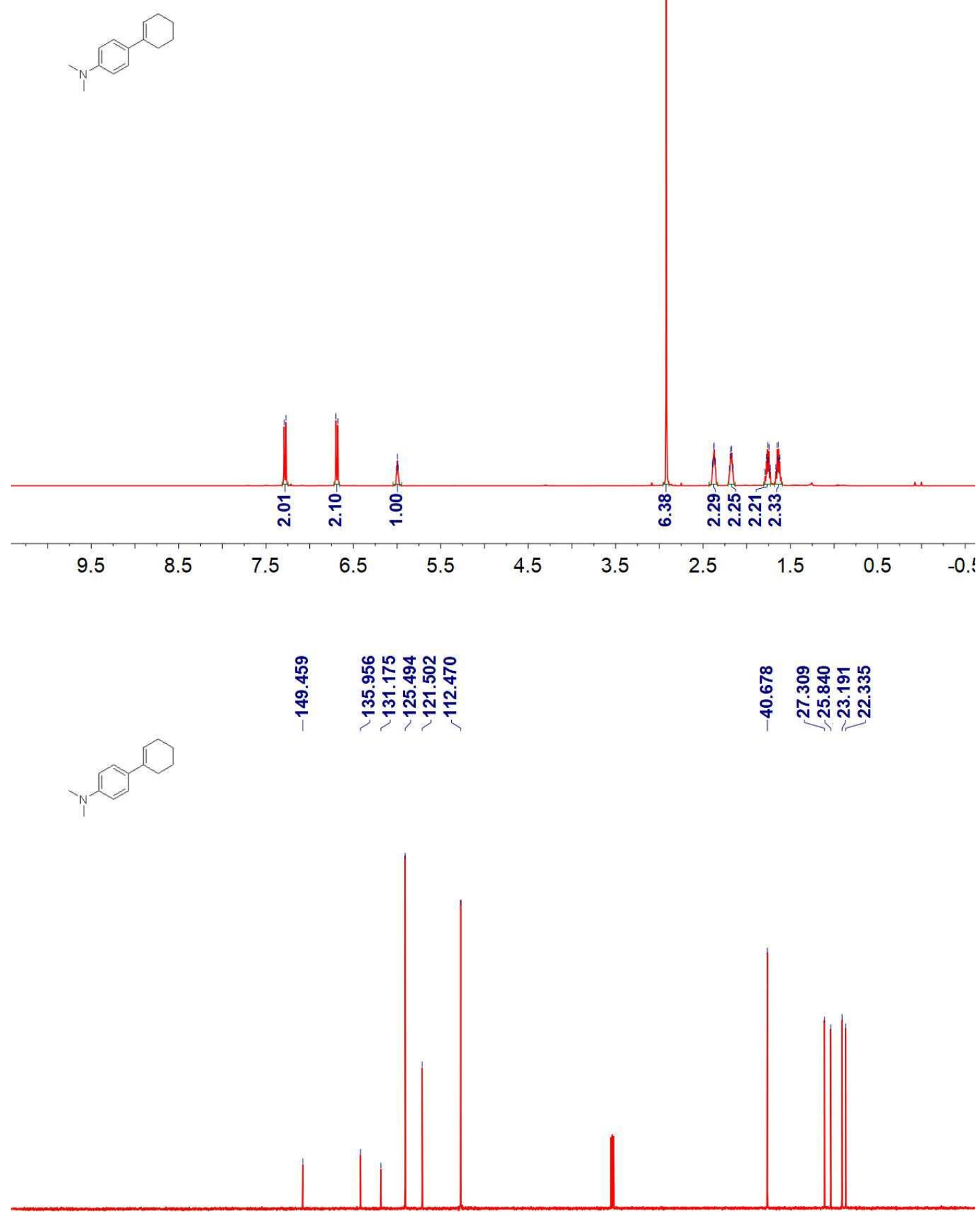

$210200190180170160150140130120110100 \quad 90 \quad 80 \quad 70 \quad 60 \quad 50 \quad 40 \quad 30 \quad 20 \quad 10 \quad 0$ 
4-(((4-Bromophenyl)thio)(phenyl)methyl)-N,N-dimethylaniline (6)
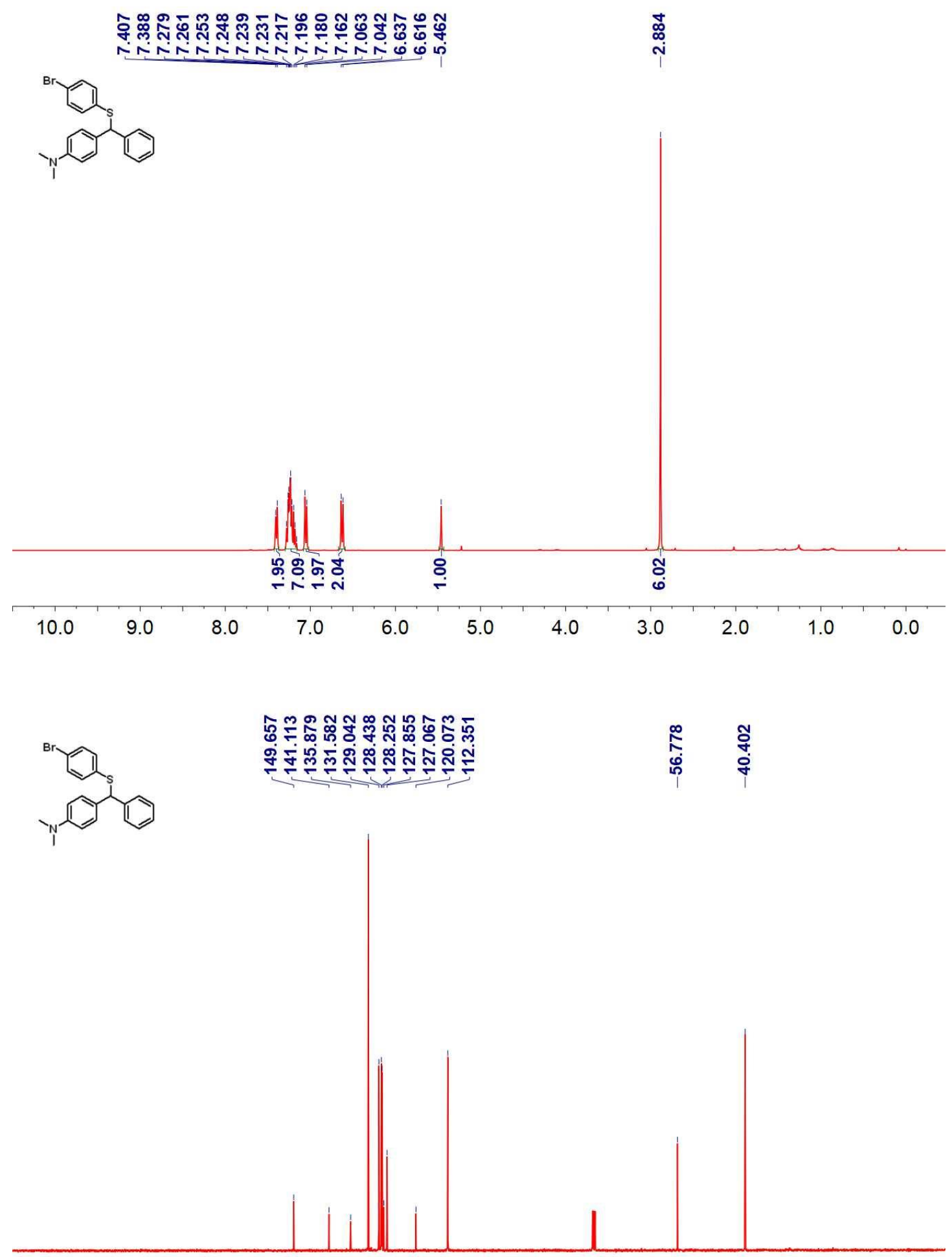

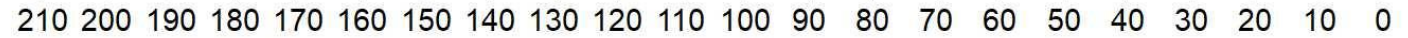




\section{NOE spectra}

\section{Derivative of pregnenolone (7a)}

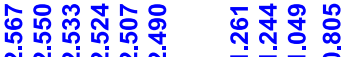

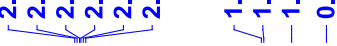

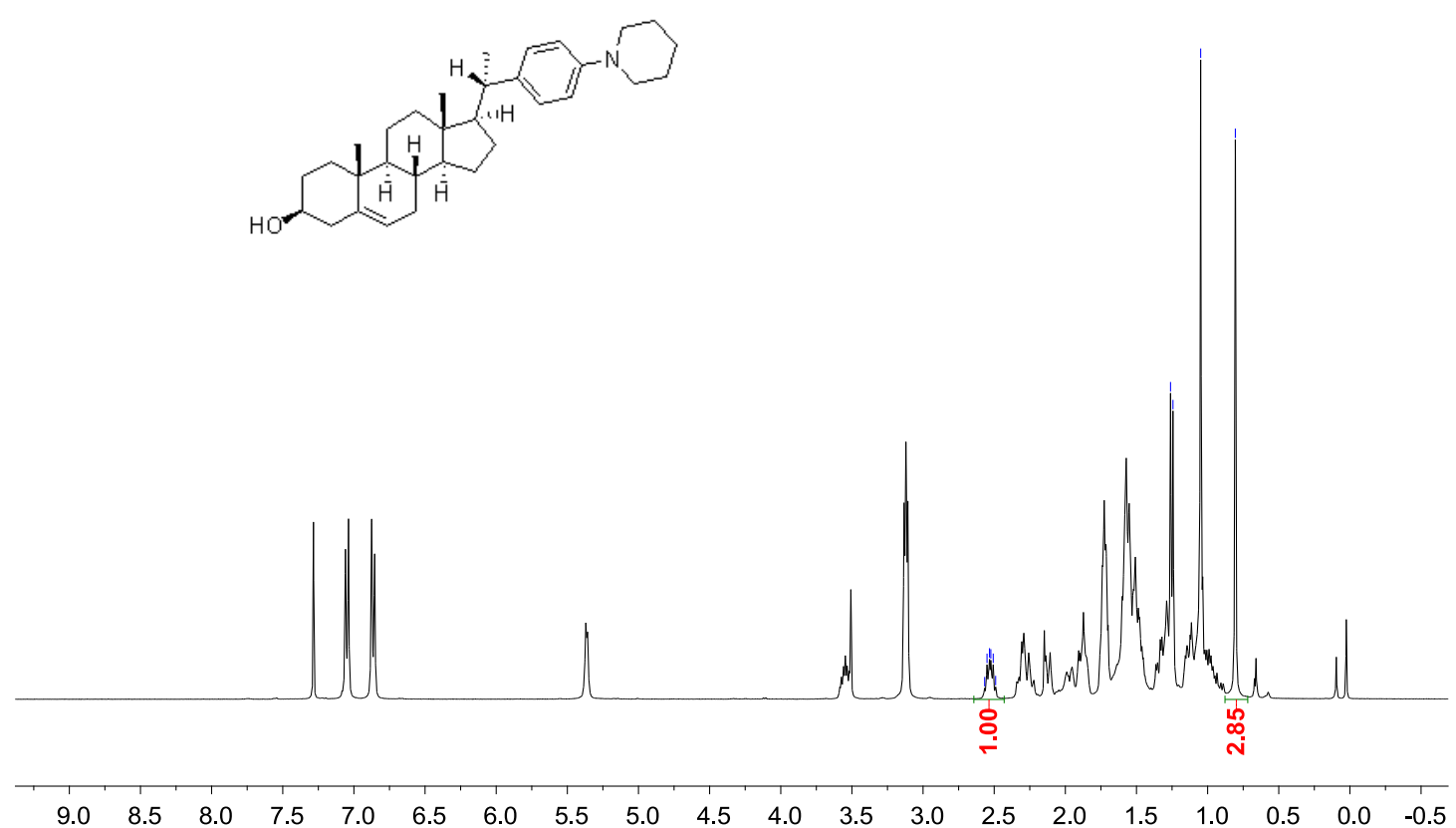

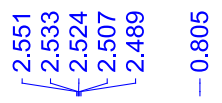

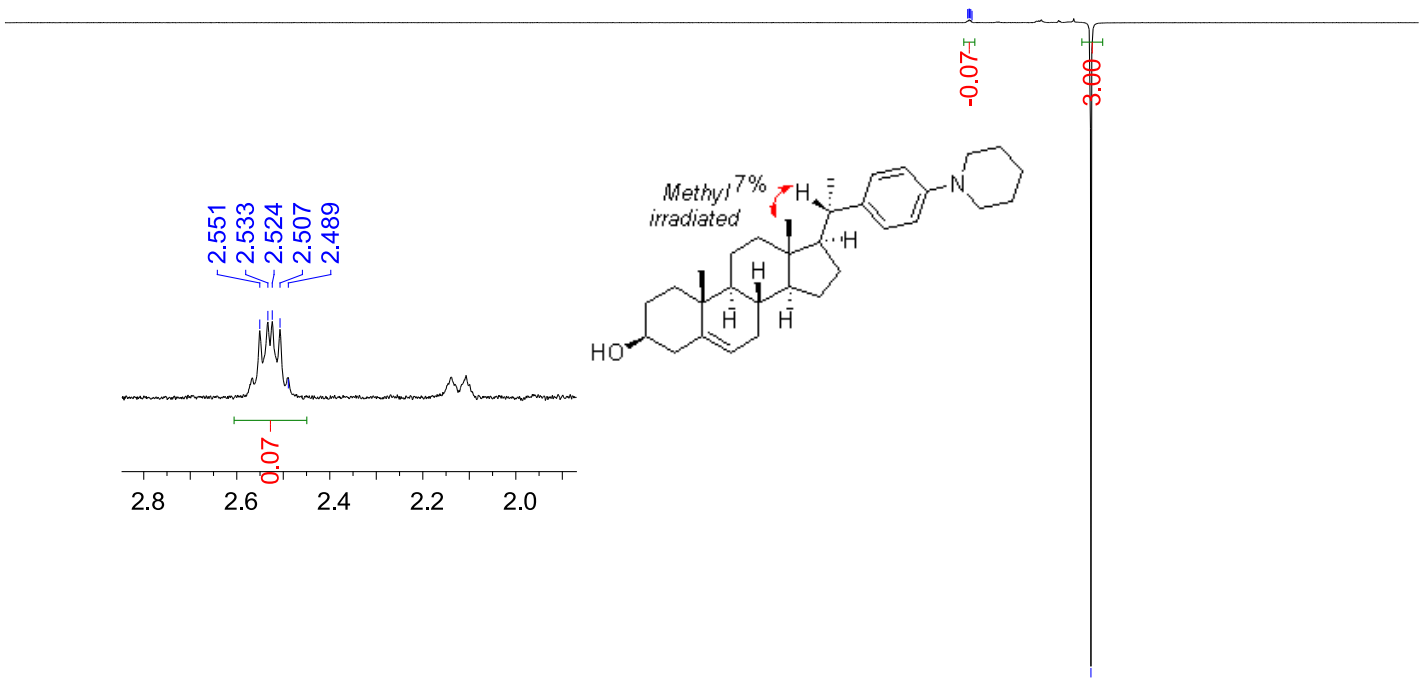

$16 \quad 15$ 


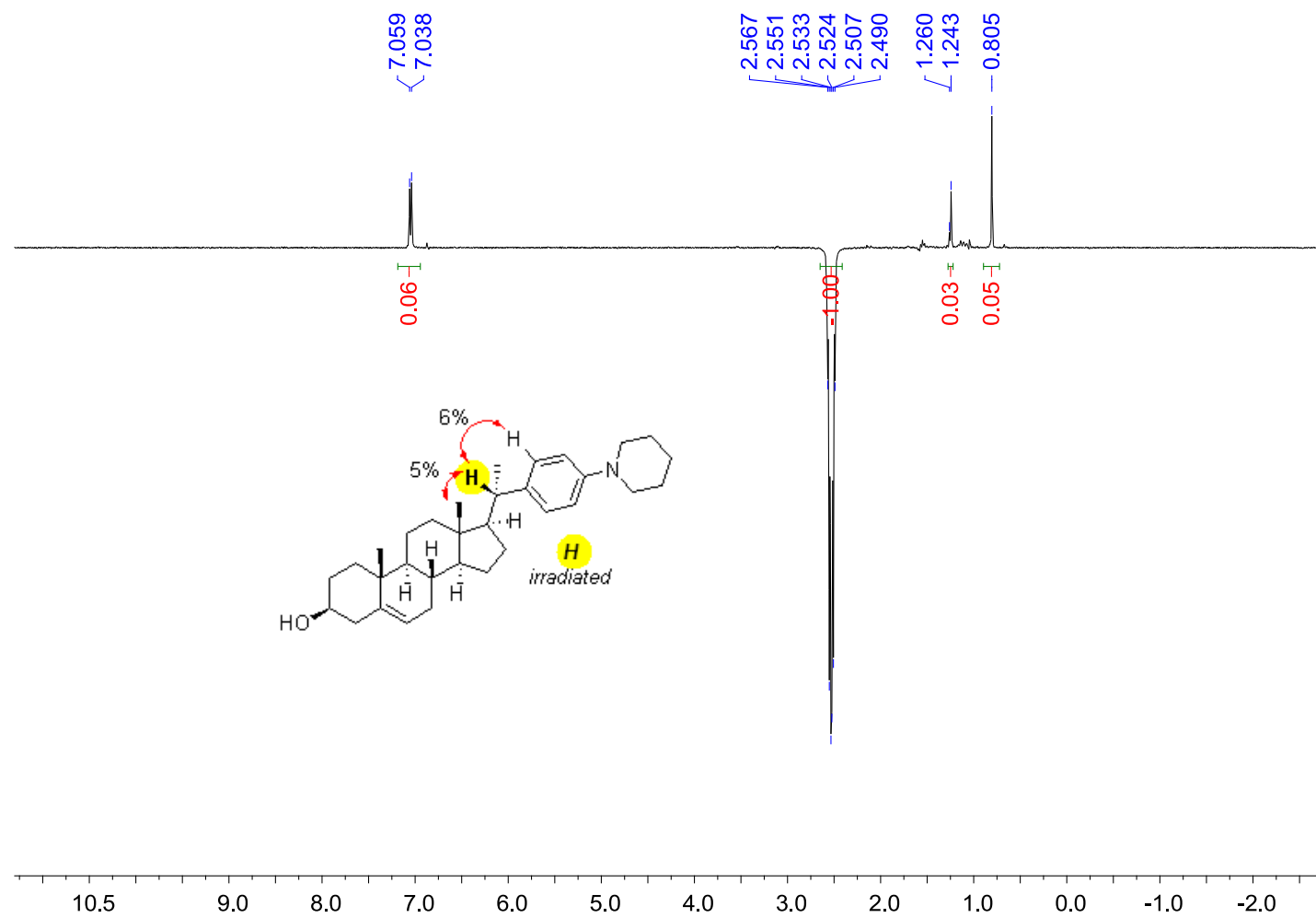




\section{Derivative of estrone (7b)}
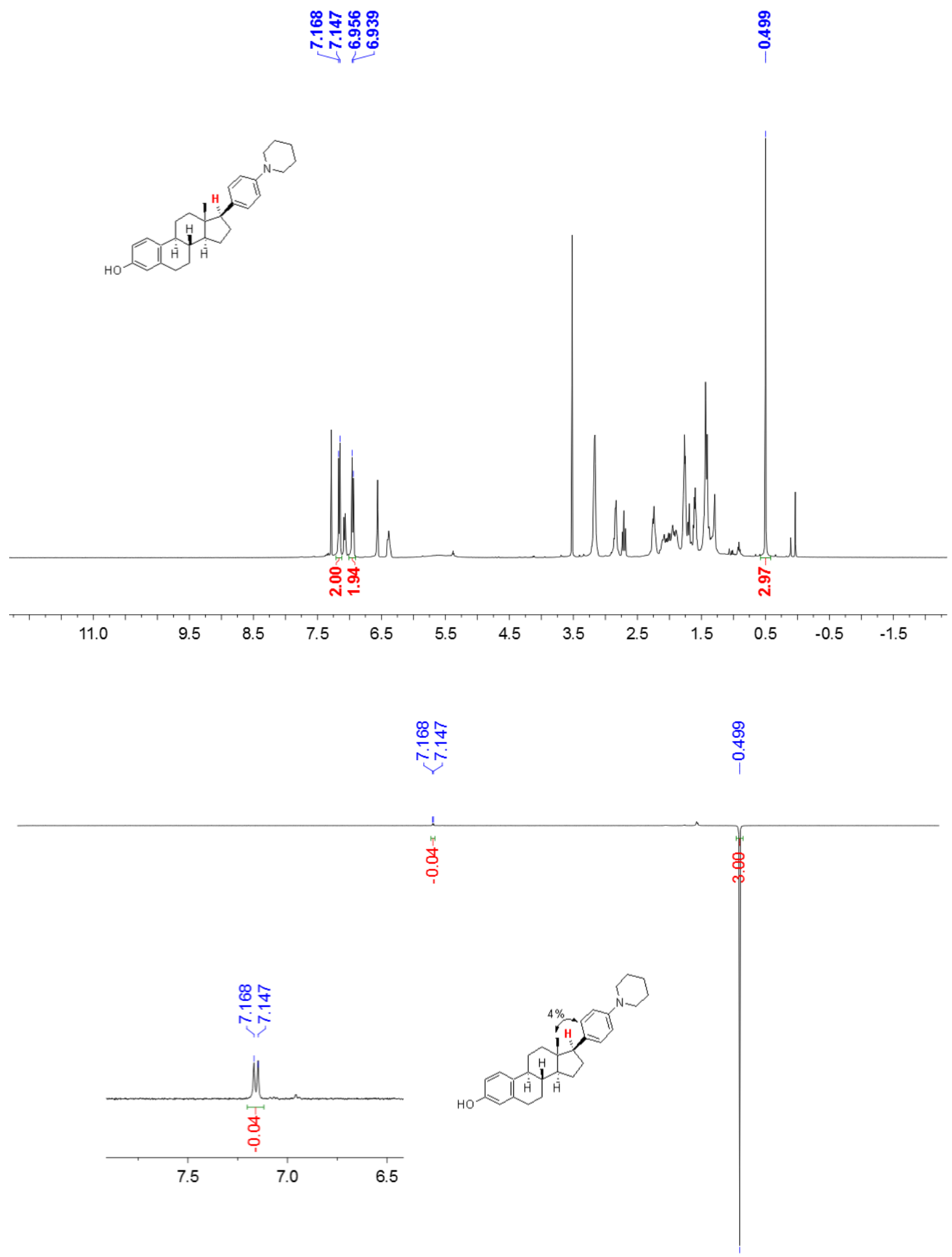

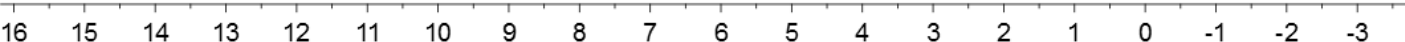




\section{Derivative of epiandrosterone (7c)}

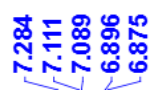

ळᄒ̣
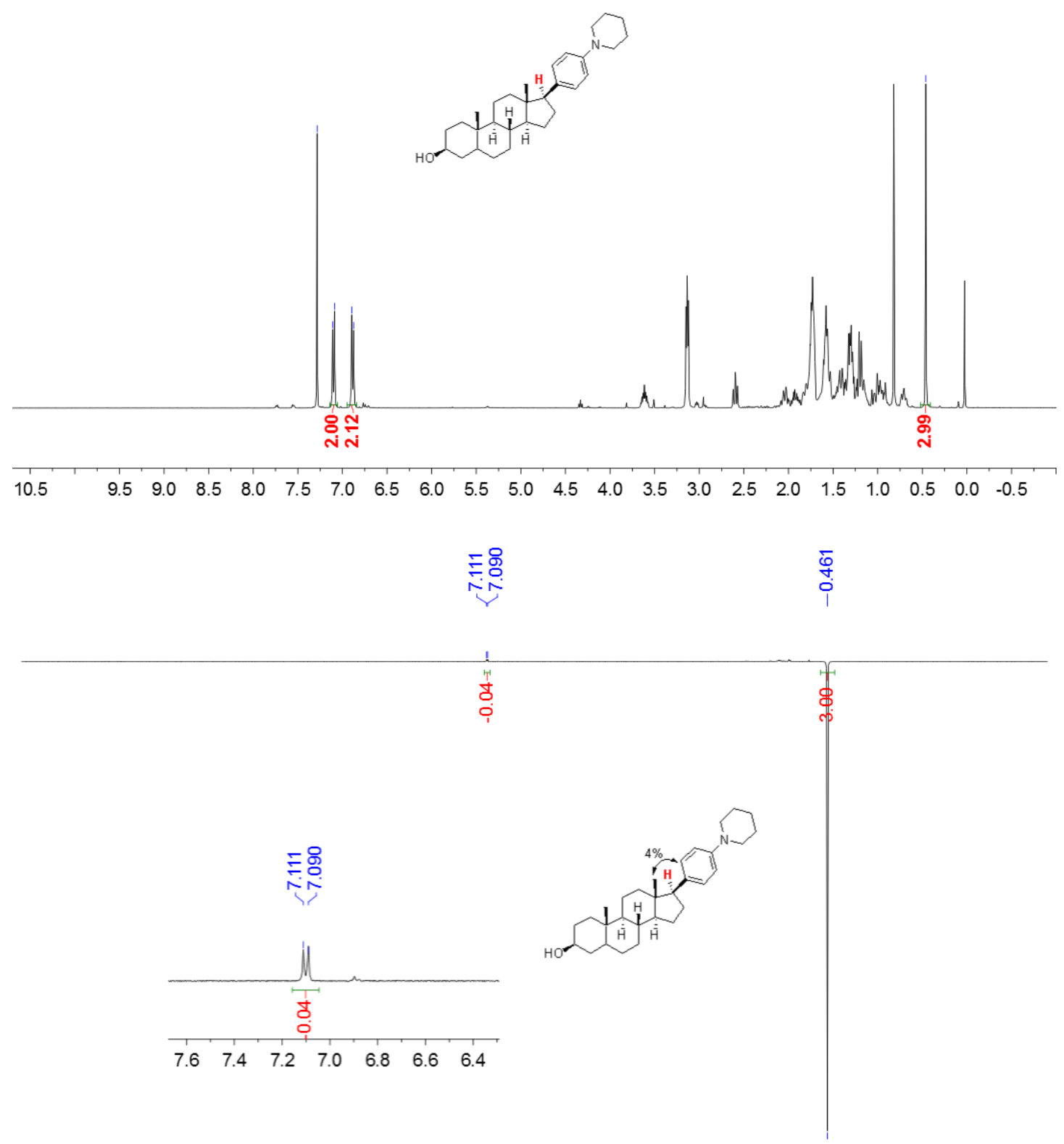

$\begin{array}{lllllllllll}16 & 15 & 14 & 13 & 12 & 11 & 10 & 9 & 8 & 7\end{array}$ 
13. ${ }^{1} \mathrm{H}$ NMR of the crude reaction mixtures in optimization of the reaction conditions, $\mathrm{pH}-\mathrm{yield}$ correlation, isotope tracing, and KIE studies

For LC-1, yield 31\%
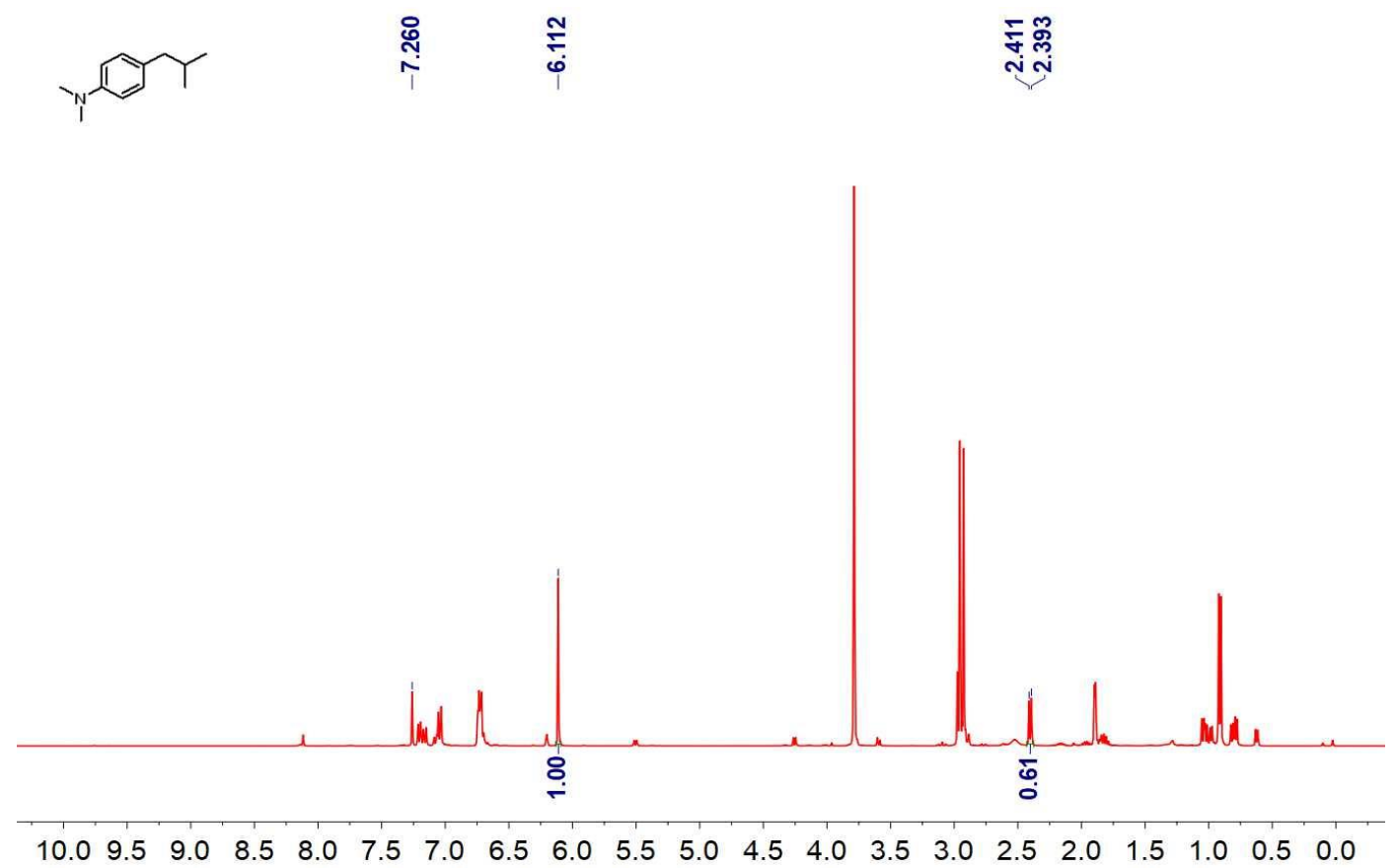

For LC-2, yield 50\%
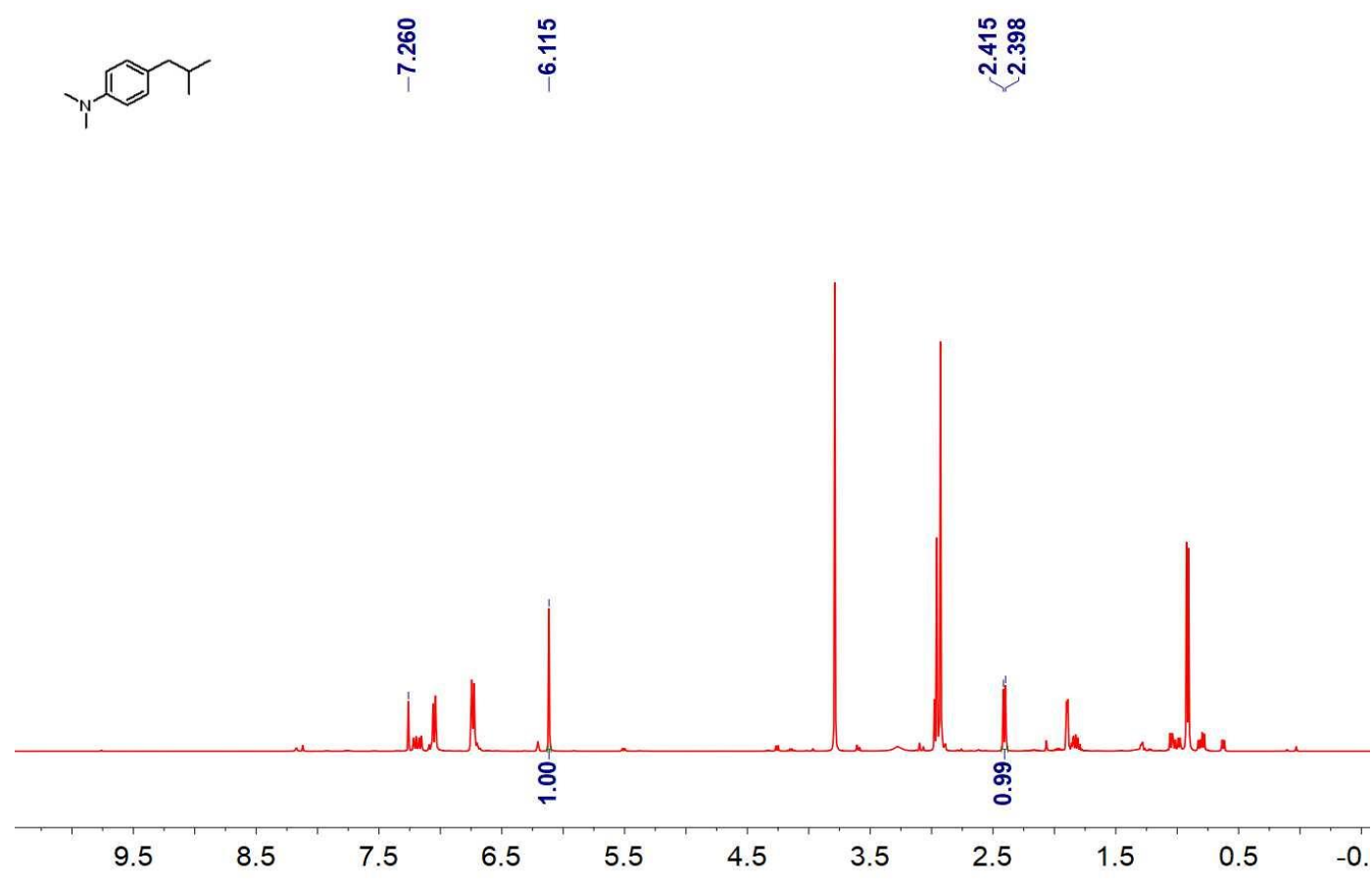


\section{For LC-3, yield 41\%}<smiles>CC(C)Cc1ccc(N(C)C)cc1</smiles>

กิ

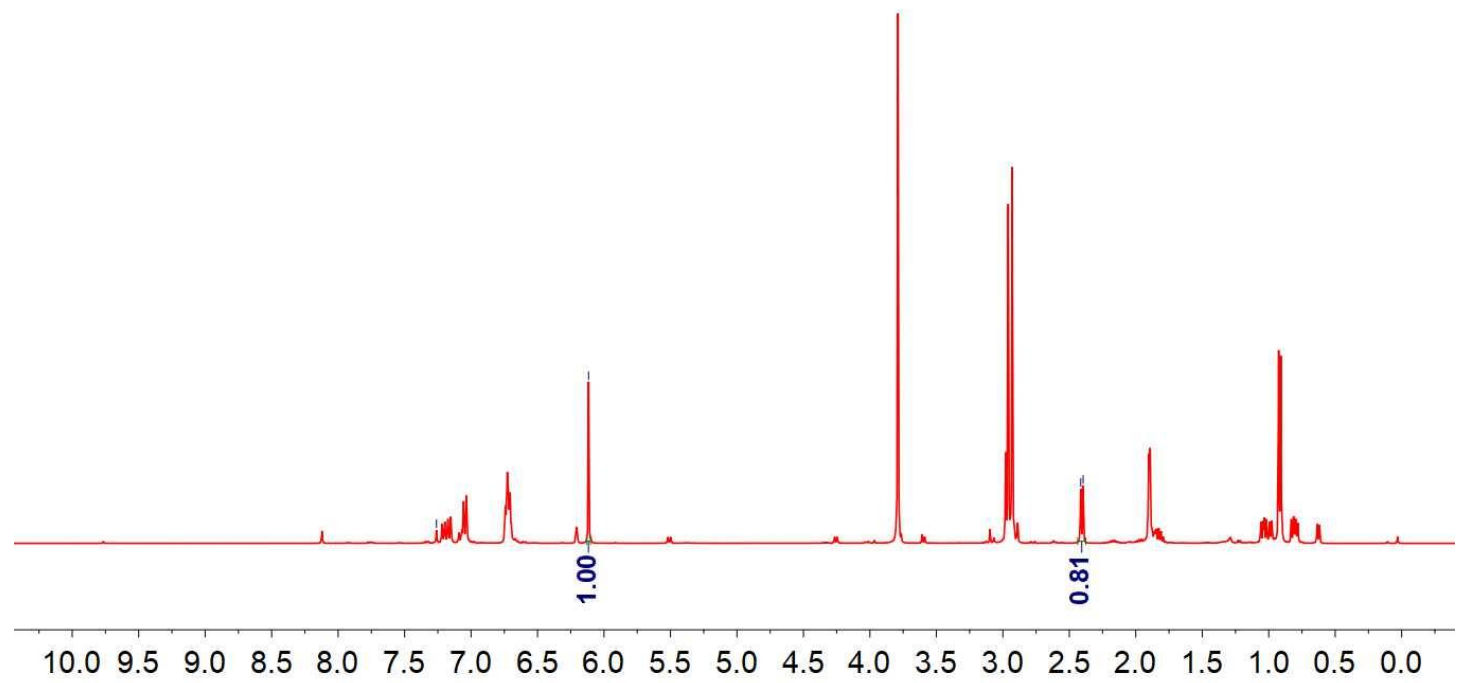


For yield 92\%, $\mathrm{pH}=1.56$

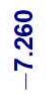

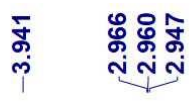

贷

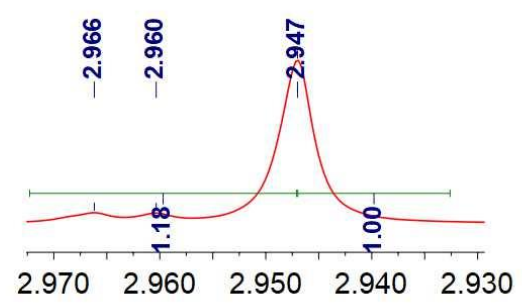

\section{$\stackrel{0}{=}$}

$\begin{array}{llllllllllllllllllllll}10.0 & 9.5 & 9.0 & 8.5 & 8.0 & 7.5 & 7.0 & 6.5 & 6.0 & 5.5 & 5.0 & 4.5 & 4.0 & 3.5 & 3.0 & 2.5 & 2.0 & 1.5 & 1.0 & 0.5 & 0.0\end{array}$

For yield $70 \%, \mathrm{pH}=3.03$

$\underset{\substack{\text { N } \\ \text { † }}}{ }$
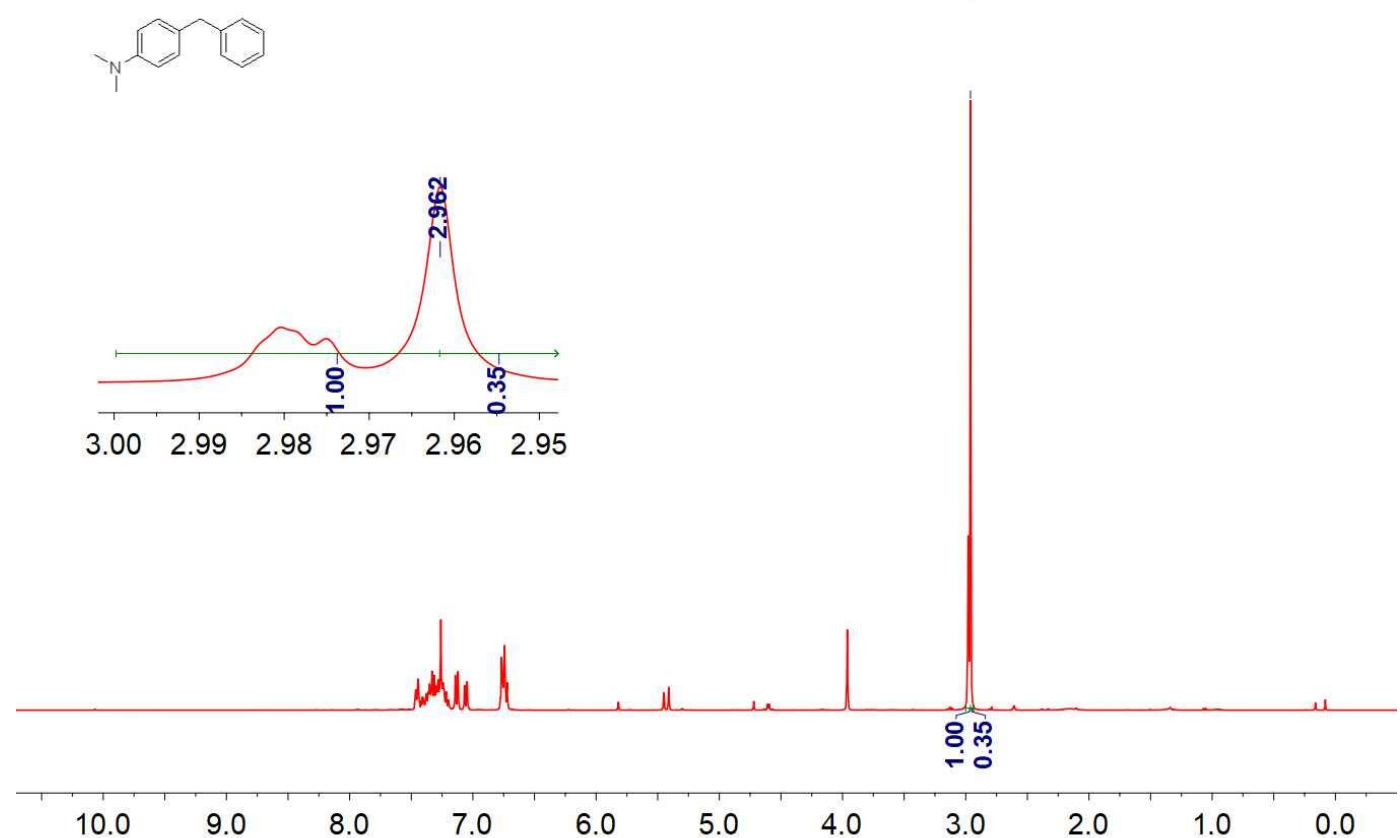
For yield $68 \%, \mathrm{pH}=3.52$

ஸ্ষু
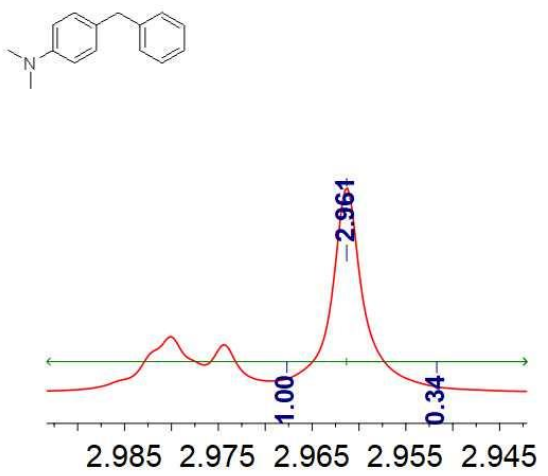

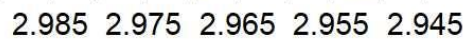

Wha

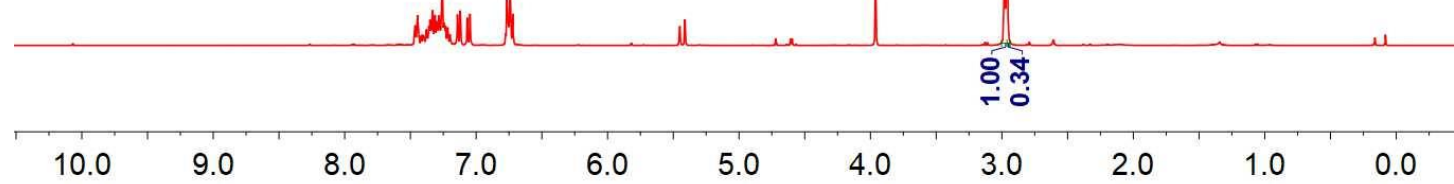

For yield 50\%, $\mathrm{pH}=3.95$

กำ
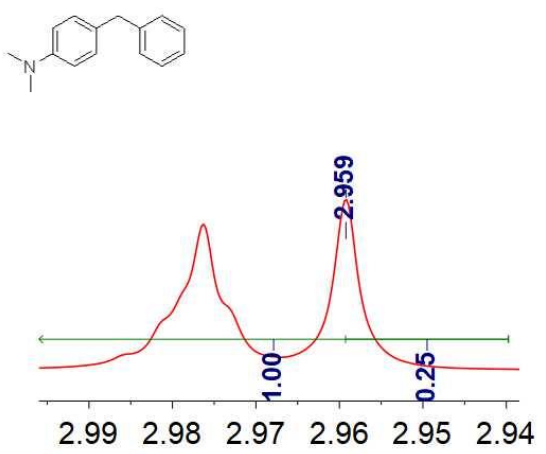

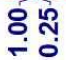

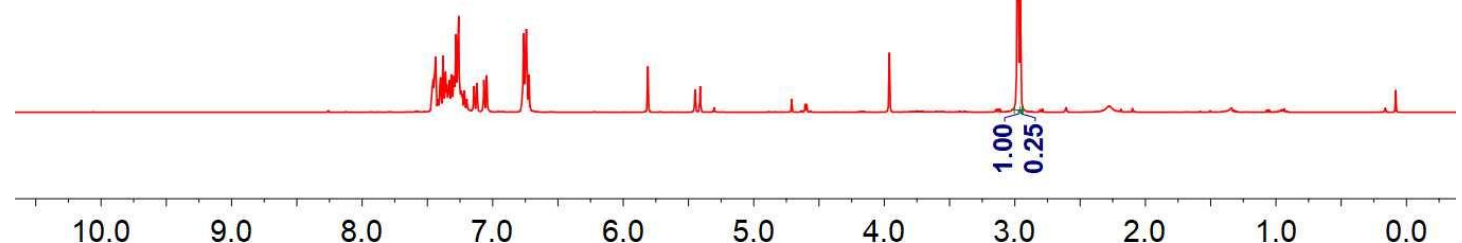


For yield 32\%, $\mathrm{pH}=4.44$

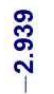
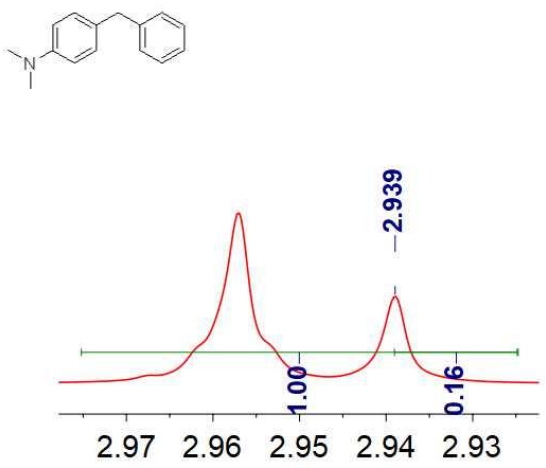

웅

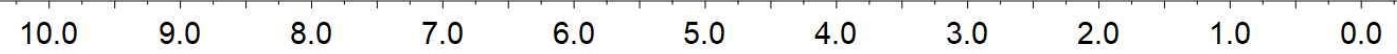

For yield 1\%, pH = 7.15

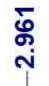
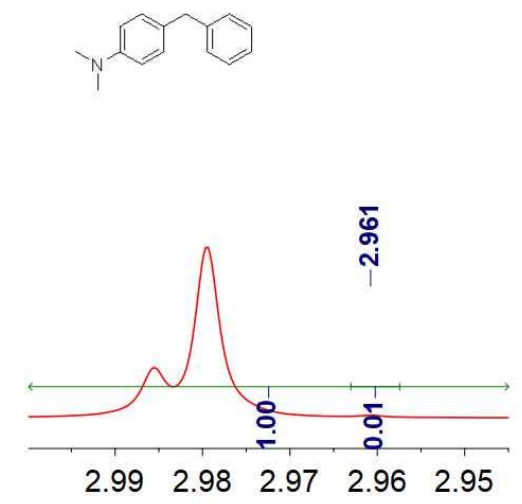

șo

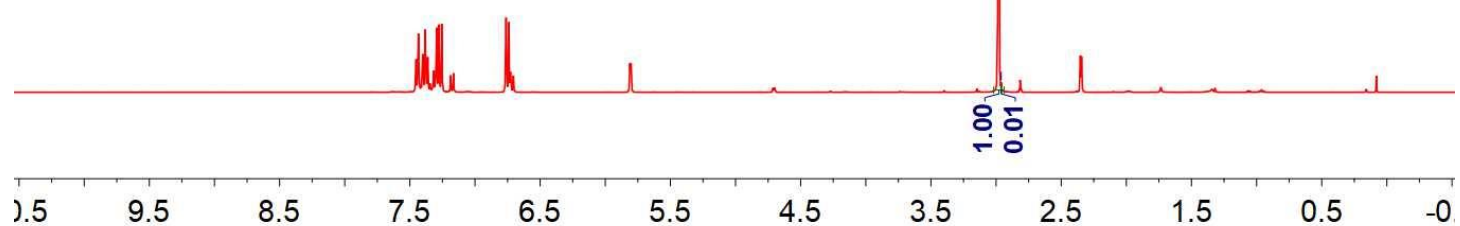


4-((4-lodophenyl)methyl-d)-N,N-dimethylaniline (d-2aa)

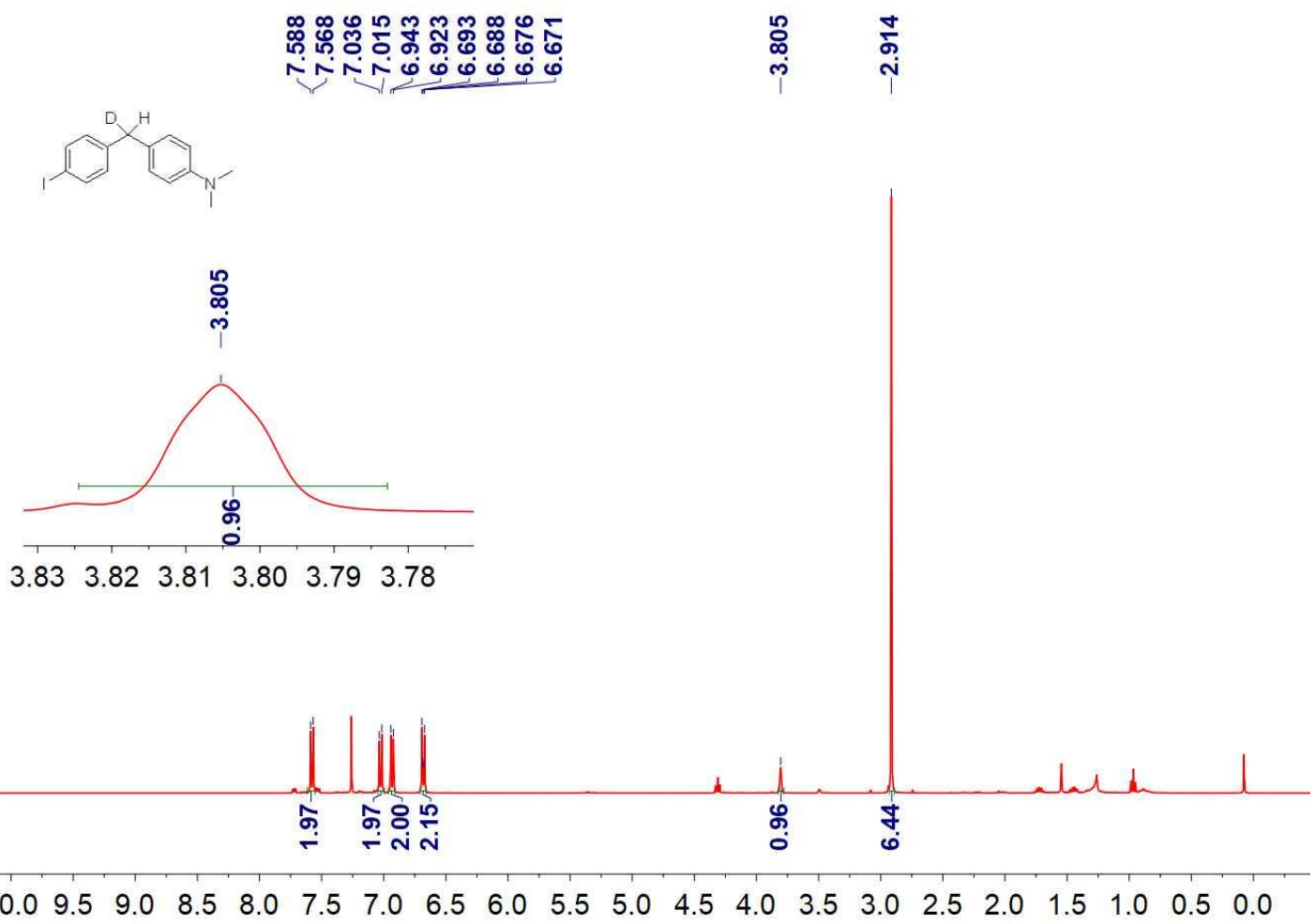

Mixture of (2aa) and (d-2aa): H/D = 85:15

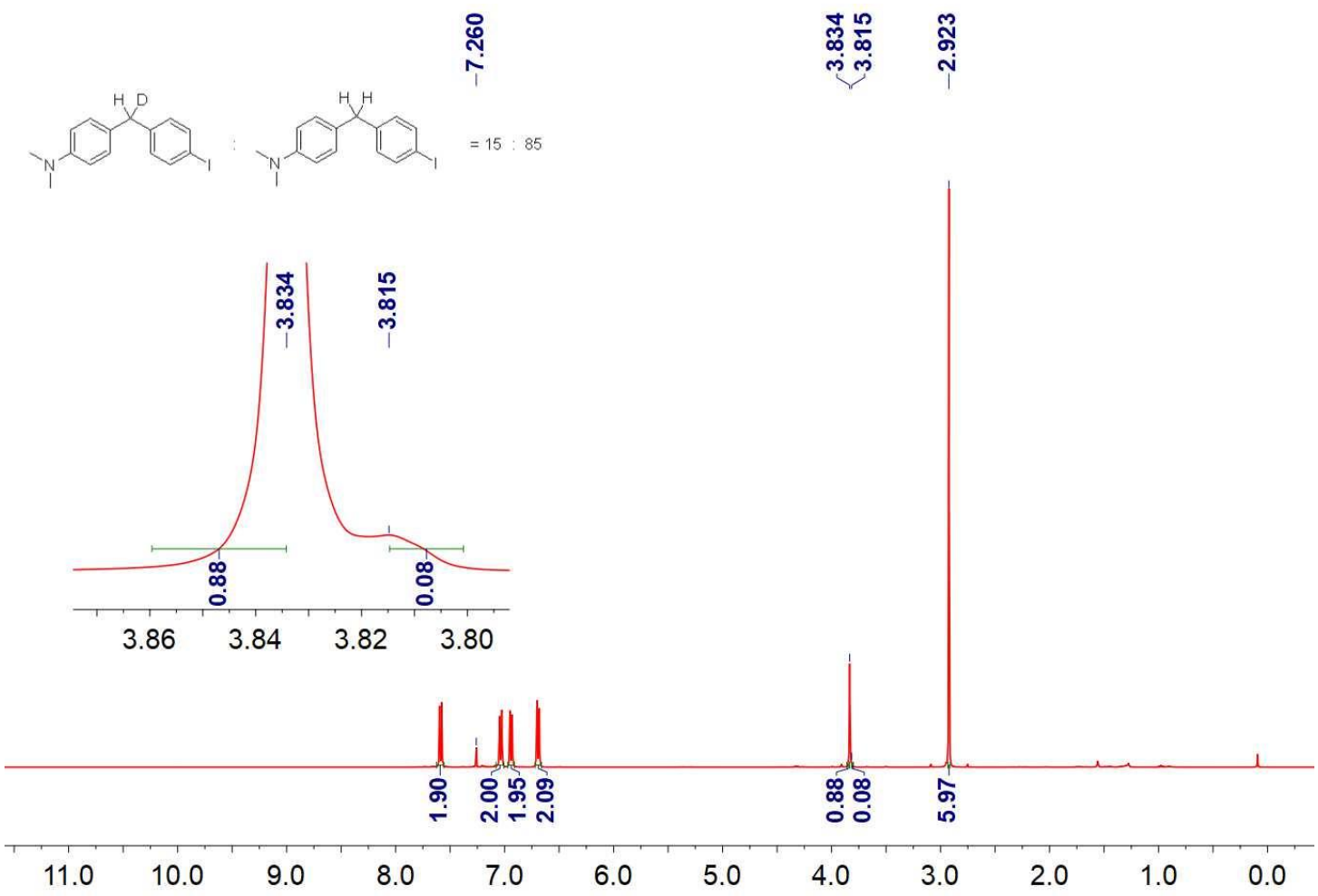


For mixture of (2aa) and (d-2aa) $=8$ to 92

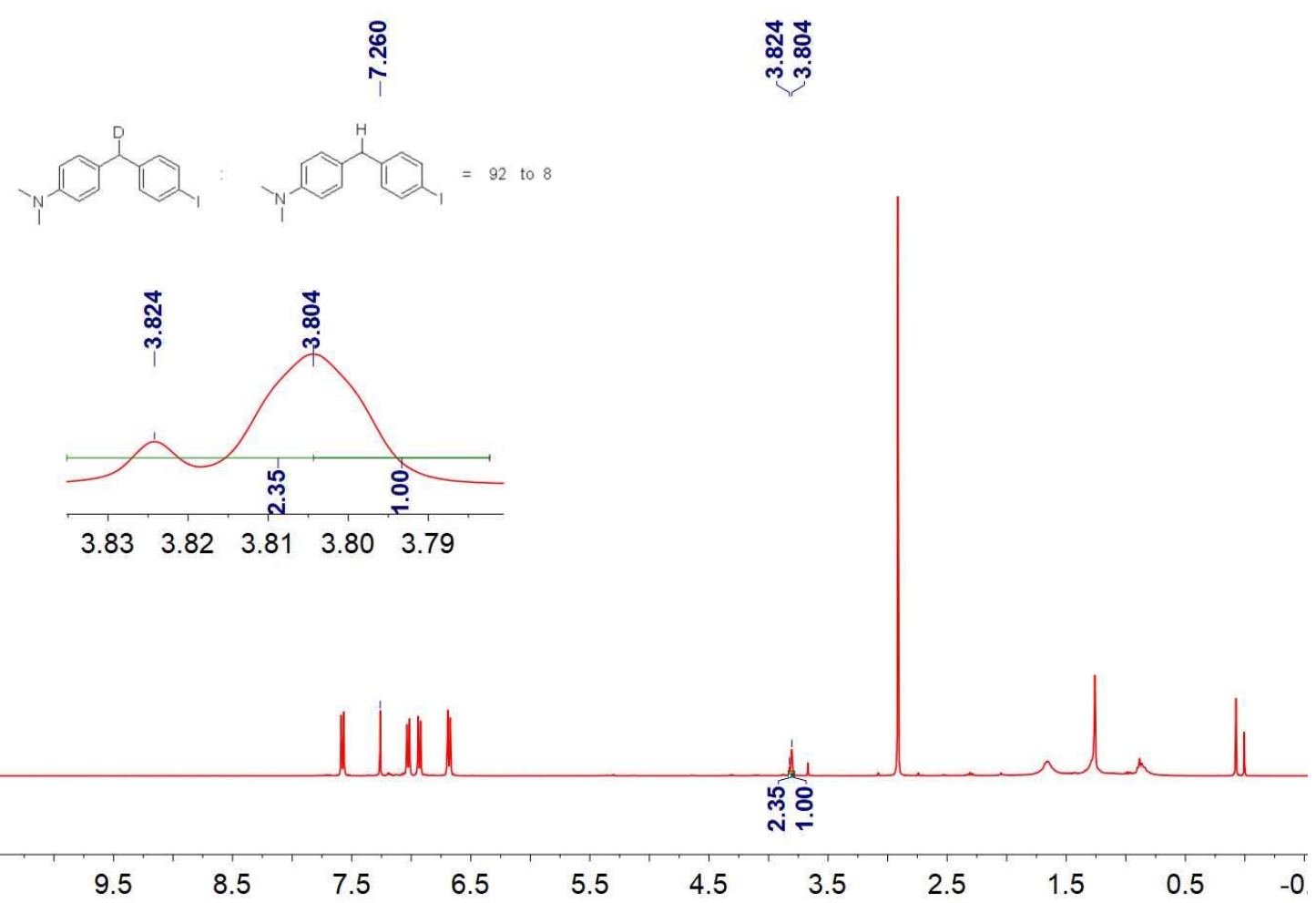

For yield $28 \%, \mathrm{k}_{\mathrm{H}} / \mathrm{k}_{\mathrm{D}}=2.63$

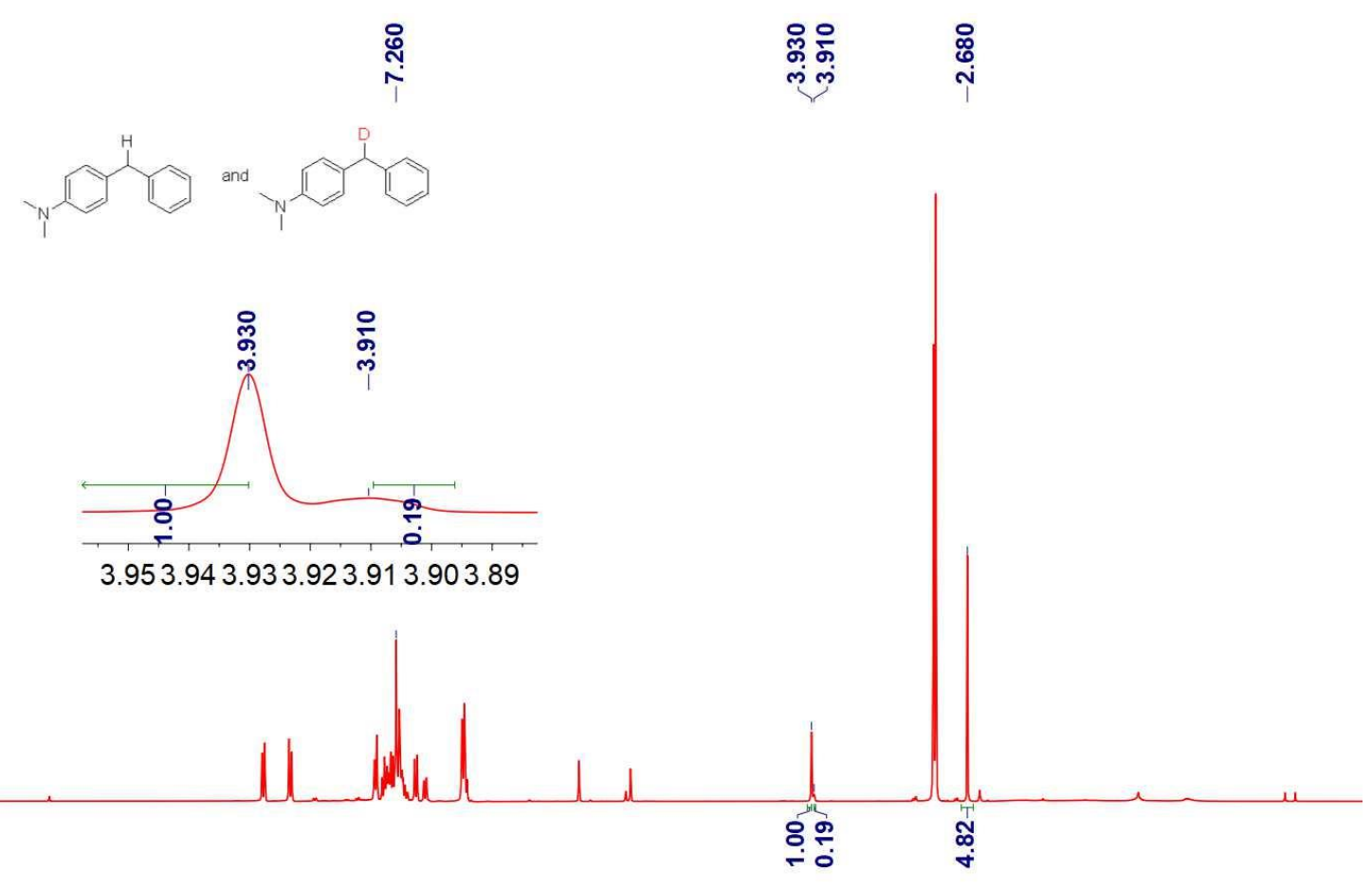

$\begin{array}{llllllllllllllllllllll}10.0 & 9.5 & 9.0 & 8.5 & 8.0 & 7.5 & 7.0 & 6.5 & 6.0 & 5.5 & 5.0 & 4.5 & 4.0 & 3.5 & 3.0 & 2.5 & 2.0 & 1.5 & 1.0 & 0.5 & 0.0\end{array}$ 
For yield $19 \%, \mathrm{k}_{H} / \mathrm{k}_{\mathrm{D}}=1.25$

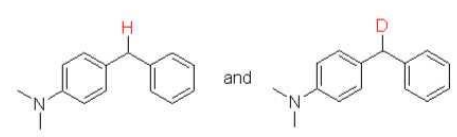

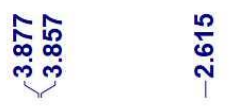

¿̊̀

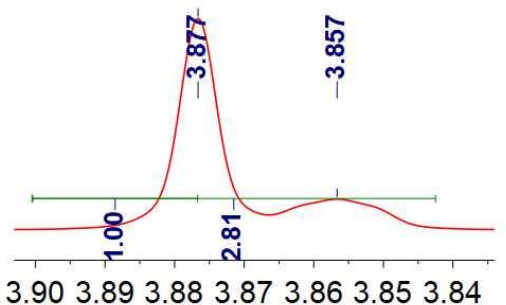

3.903 .893 .883 .873 .863 .853 .84

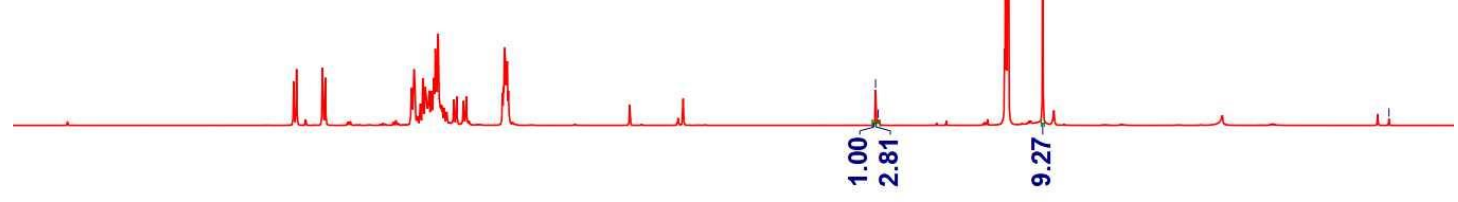

$\begin{array}{llllllllllllllllllllll}10.0 & 9.5 & 9.0 & 8.5 & 8.0 & 7.5 & 7.0 & 6.5 & 6.0 & 5.5 & 5.0 & 4.5 & 4.0 & 3.5 & 3.0 & 2.5 & 2.0 & 1.5 & 1.0 & 0.5 & 0.0\end{array}$ 


\section{References}

1. (a) Liu, J.-t.; Yang, S.; Tang, W.; Yang, Z.; Xu, J. Iridium-Catalyzed Efficient Reduction of Ketones in Water with Formic Acid as a Hydride Donor at Low Catalyst Loading. Green Chem. 2018, 20, 2118-2124. (b) Yang, Z.; Zhu, Z.; Luo, R.; Quu, X.; Liu, J.-t.; Yang, J.-K.; Tang, W. Iridium-Catalyzed Highly Efficient Chemoselective Reduction of Aldehydes in Water Using Formic Acid as the Hydrogen Source. Green Chem. 2017, 19, 3296-3301.

2. (a) Wang, Z.; Lu, S.-M.; Li, J.; Wang, J.; Li, C. Unprecedentedly High Formic Acid Dehydrogenation Activity on an Iridium Complex with an $N, N^{\prime}$-Diimine Ligand in Water. Chem. Eur. J. 2015, 21, 12592-12595. (b) Lu, S.; Wang, Z.; Wang, J.; Li, J.; Li, C., Hydrogen Generation from Formic Acid Decomposition on a Highly Efficient Iridium Catalyst Bearing a Diaminoglyoxime Ligand. Green Chem. 2018, 20, 1835-1840.

3. Singh, C., Kavala, V., Samal, A. and Patel, B. Aqueous-Mediated N-Alkylation of Amines. Eur. J. Org. Chem. 2007, 1369-1377.

4. Antilla, J. C.; Buchwald, S. L. Copper-Catalyzed Coupling of Arylboronic Acids and Amines. Org. Lett. 2001, 3, 2077.

5. Domingo, V.; Prieto, C.; Castillo, A.; Silva, L.; Quilez del Moral, J. F.; Barrero, A. F. lodine-Promoted Metal-Free Aromatization: Synthesis of Biaryls, Oligo p-Phenylenes and A-Ring Modified Steroids. Adv. Synth. Catal. 2015, 357, 3359-3364.

6. Qin, C.; Davies, H. M. L. Role of Sterically Demanding Chiral Dirhodium Catalysts in Site-Selective C-H Functionalization of Activated Primary C-H Bonds. J. Am. Chem. Soc. 2014, 136, 9792-9796.

7. Reeves, J. T.; Fandrick, D. R.; Tan, Z.; Song, J. J.; Lee, H.; Yee, N. K.; Senanayake, C. H. Room Temperature Palladium-Catalyzed Cross Coupling of Aryltrimethylammonium Triflates with Aryl Grignard Reagents. Org. Lett., 2010, 12, 4388-4391.

8. Blakey, S. B.; MacMillan, D. W. C. The First Suzuki Cross-Couplings of Aryltrimethylammonium Salts. J. Am. Chem. Soc. 2003, 125, 6046-6047.

9. Curtin, D. Y. Anomalous Oxidations of 1-(p-Methoxyphenyl)-1-Phenylethylene with Performic and Perbenzoic acid. J. Am. Chem. Soc. 1954, 76, 5777-5779.

10. Singh, A. Intramolecular Nucleophilic Participation. Effect of Certain Ortho Substituents on Solvolysis Rates of Benzyl and Benzhydryl Bromides. J. Am. Chem. Soc. 1962, 84, 1179-1185.

11. Sachs, F.; Weigert, W. p-Dimethylaminobenzaldehyde. VII. Ber. dtsch. chem. Ges. 1908, 40, 4361-4367.

12. Stiles, M.; Sisti, A. J. A Convenient Method for the Preparation of Aldehydes. J. Org. Chem. 1960, 25, 1691-1693.

13. Puckowski, R. T.; Ross, W. A. Preparation and Reactions of Some Optically Active Substituted Benzhydrols. J. Chem. Soc. 1959, 3555-3565. 
14. Schmidlin, J.; von Escher, R. Hexahydrotriphenyl Carbinol. Ber. dtsch. chem. Ges. 1908, 41, 447-450.

15. Anthony, S.; Julia, B.; Murray, F. Convenient General Method for Preparation of Aldehydes. II. J. Org. Chem. 1962, 27, 279-281.

16. Rybakova, M. N. Reaction of N-Dimethylaminodiarylcarbinolates with Esters. Uch. Zap. Permsk. Gos. Univ. 1961, 19, 71-74.

17. Mihailescu M. A.; St. Caragea, P. The Grignard (Organomagnesium) Compounds of Certain Polyhalogenated Derivatives of Benzene and Their Condensation Products with Aromatic Aldehydes and Ketones. Bulletin Section Scien. Academ. Roum. 1929, 12, 7-18.

18. Siegfried H.; Hans S.; Hans, S. Color and Constitution. I. Dyes with Crossed Absorption Systems. Liebigs Ann. 1954, 587, 132-145.

19. Lin M. H., Silver J. E., Le Noble W. J. Search for Nucleophilicity Effect on the Face Selectivity of Addition to a Aterically Unbiased Ketone. J. Org. Chem. 1988, 53, 5155-5158.

20. Harold K. o-p-Isomerism in the Preparation of Diaminodiphenylmethane. J. Chem. Soc. Trans. 1920, 117, 988-992.

21. Katritzky A. R.; Lan, X.; Lam, J. N. Benzotriazole as a Synthetic Auxiliary: Advantageous Syntheses of Substituted Diarylmethanes and Heterocyclic Analogs. J. Org. Chem. 1991, 56, 4397-403.

22. Smith L. H., Welch K. N. The Chemistry of the Knoevenagel and Similar Reactions. J. Chem. Soc. 1934, 1136-1140.

23. Sami B. A.; Nadia F. A.; Sadek E. A. Xanthenyl and Thioxanthenyl derivatives. Reactions of Xanthenyl and Thioxanthenyl Halides with Substituted Ethylenes. Bull. Chem. Soc. Jpn. 1975, 48, 2200-2202. 